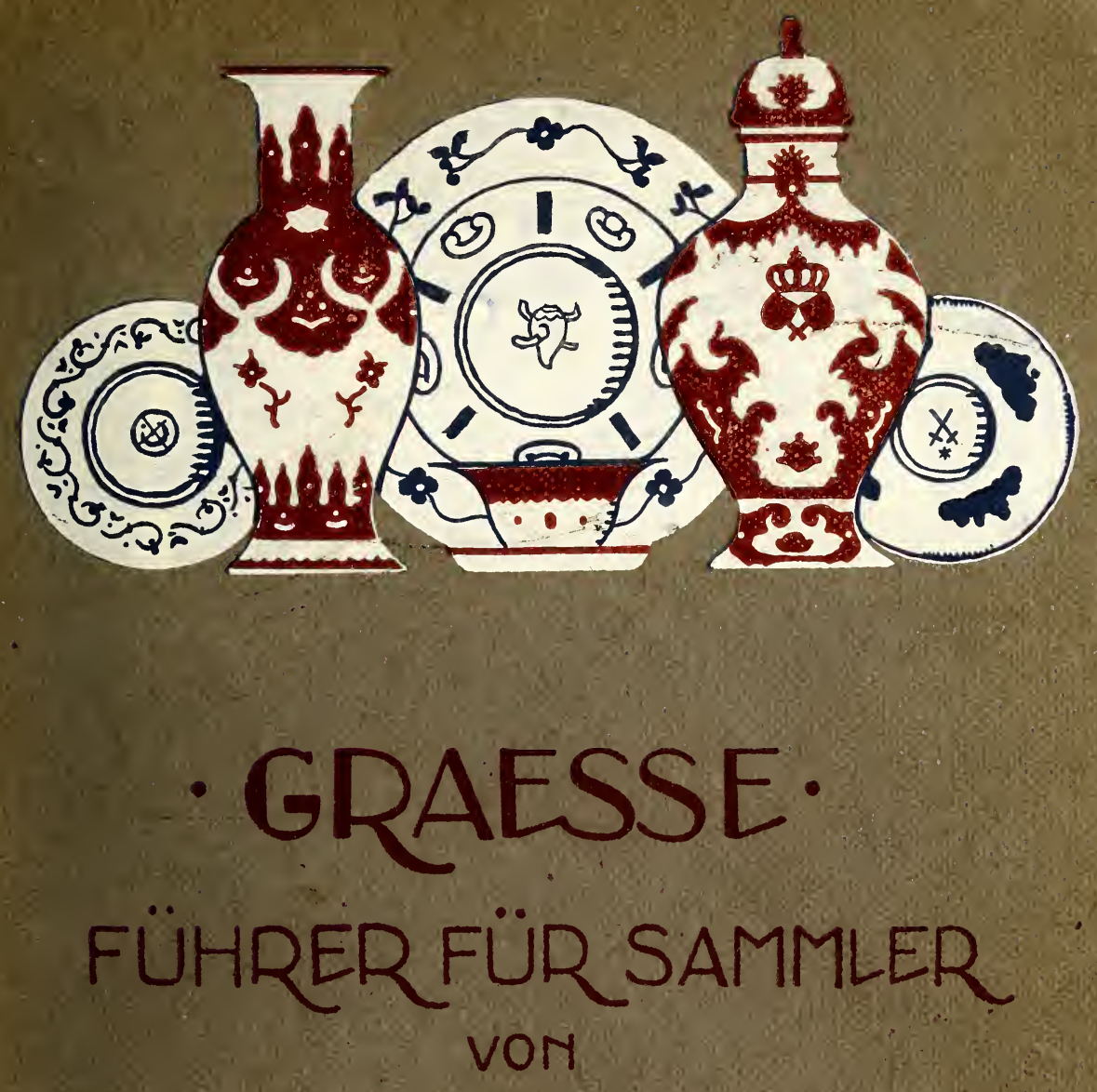

PORZELLAM, FAYEMCE ETC.

$$
\begin{gathered}
\text { I5.AUFLAGE } \\
\text { BEARBEITET VON } \\
\text { E. ZIMTERMAMI }
\end{gathered}
$$$$
\text { BERLIT } 1919
$$ 


\section{SMITHSONIAN singrinting INSTITUTION LIBRARIES}

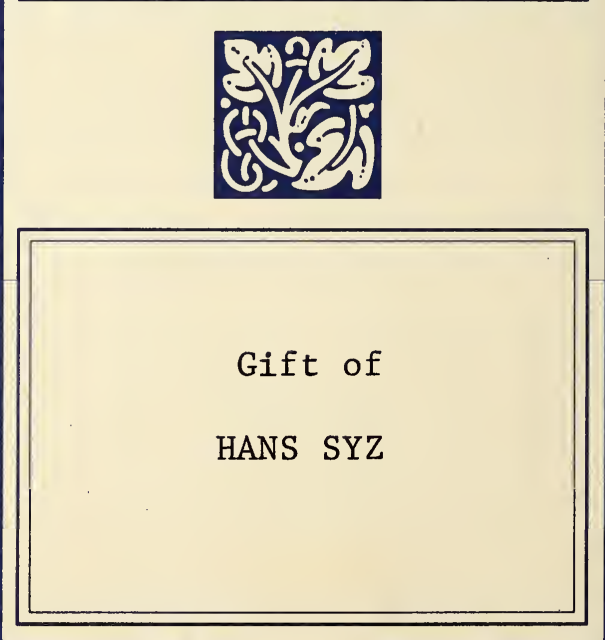

HANS SYZ 


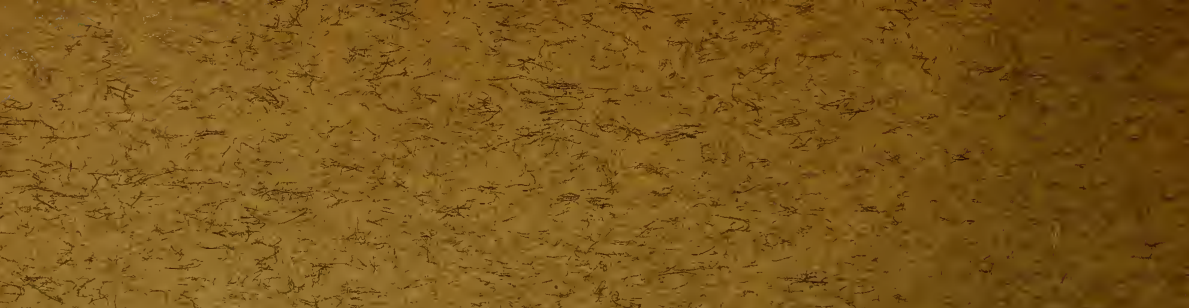
ats

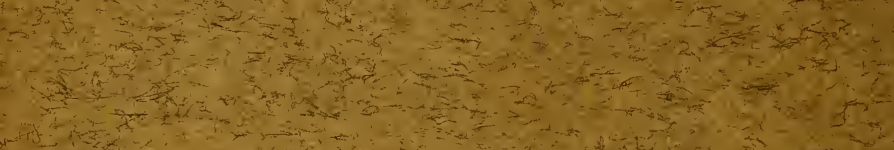
20.

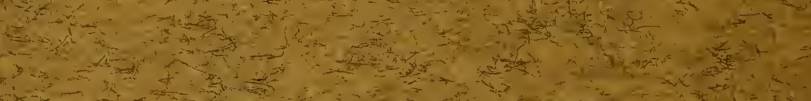

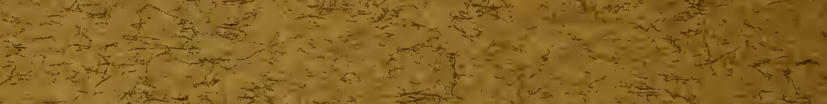
सह

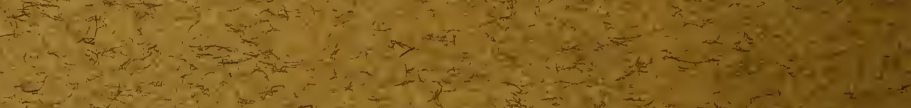

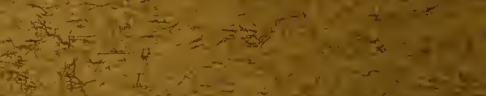

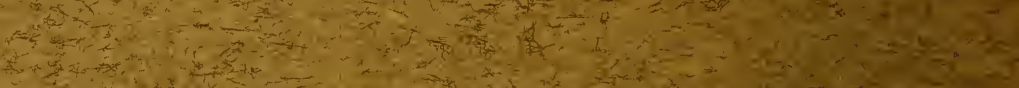

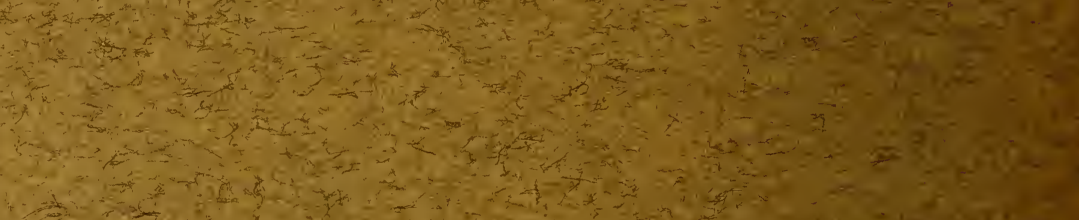
(2) a a

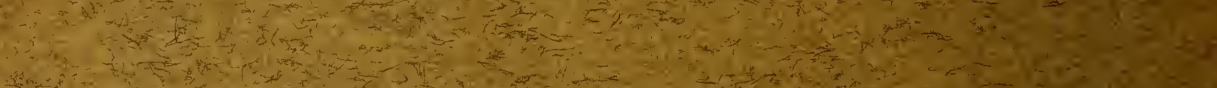

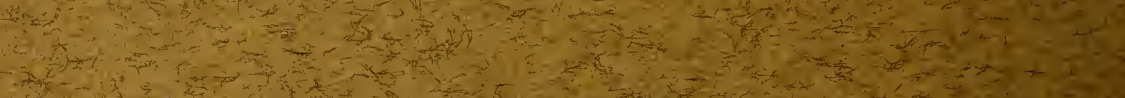
(1)

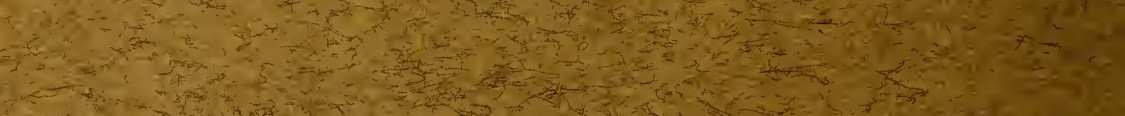

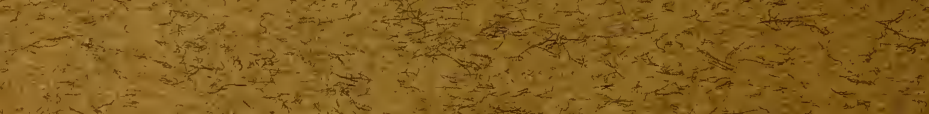
and

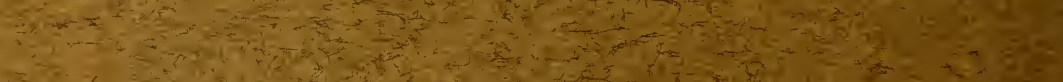

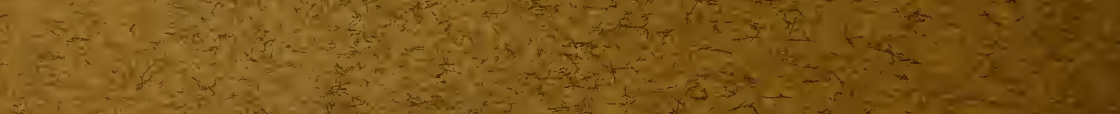

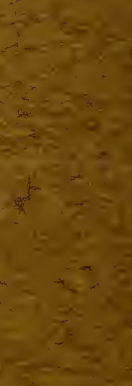


Führer für Sammler von

Porzellan und Fayence,

Steinzeug, Steingut usw. 
Verlagsbuchhandlung Richard Carl Schmidt \& Co. Tel. Amt Lützow, 5147 Berlin W 62 Tel. Amt Lützow, 5147

\section{BIBLIOTHEK}

für

\section{Kunst- und Antiquitättensammmler}

Band 1. Bernhart, M. Medaillen und Plaketten. M. 6.-

Band 2. Kuemmel, O. Kunstgewerbe in Japan. M. 6.-

Band 3. Schnorr v. Carolsfeld, L. Porzellan. 2. Aufl. M. 8.-

Band 4. Haenel, E. Alte Waffen. M. 6.-

Band 5. Schmidt, Robert. Möbel. 4. Aufl. M.9.Band 6. Schuette, M. Alte Spitzen. M. 8.-

Band 7. Bassermann-Jordan, Ernst. Uhren. M. 6.-

Band 8. Ruth-Sommer, $\boldsymbol{H}$. Alte Musikinstrumente. M. 6.-

Band 9. Donath, A. Psychologie des Kunstsammelns. 2. Aufl. M. 6.-

Band 10. Schulze, $P$. Alte Stoffe. M. 6.-

Band11. v. Berchem, E. Siegel. M. 8.-

Band 12. Schottmüller, $F$. Bronzestatuetten und Geräte. M. 8.-

Band 13. Martin, W. Alt-Holländische Bilder. M. 10.-

Band 14. Schottenloher, K. Das alte Buch. M.12.-Weitere Bande sind in Vorbereitung. 


\section{Führer für Sammler}

\section{von \\ Porzellan und Fayence, Steinzeug, Steingut usw.}

Vollständiges Verzeichnis der auf älterem Porzellan, Fayence, Steingut usw. befindlichen Marken

von

Dr. J. G. Th. Graesse .und E. Jaennicke

Vollständig umgearbeitet, vermehrt und mit wissenschaftlichen Belegen, Erläuterungen und Registern ausgestattet von

\section{Professor Dr. E. Zimmermann}

Direktor der Porzellansammlung in Dresden

Fünfzehnte Auflage

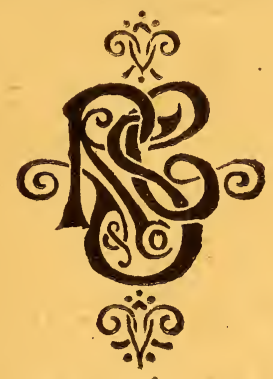

BERLIN W 62

Richard Carl Schmidt \& Co.

1919 


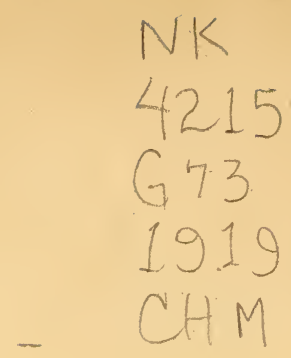

Alle Rechte, auch das der Übersetzung, vorbehalten Nachdruck der Tafeln wird gerichtlich veriolgt 


\section{Vorwort zur XIII. Auflage.}

Vorliegende neue Auflage des Graesseschen Markenbuches erscheint fast in ganz neuer Gestalt. Als ich vor einiger Zeit von der Verlagsbuchhandlung gebeten wurde, die neue Auflage dieses. Werkes zu besorgen, war mir sofort klar, daß diesem, obwohl es ganz unbestreitbar immer das am meisten benutzte keramische Markenbuch der Welt gewesen ist, dennoch zwei Dínge bisher eigentlich immer gefehlt hatten: Klarheit und Übersichtlichkeit der An. ordnung, sowie wissenschaftliche Grundlage. Was die fehlende Klarheit anbetrifft, so wird sie jeder nur zu sehr bemerkt haben, der die letzten Auflagen dieses Werkes in Benutzung gezogen hat. Durch das beständige Einfiigen neuer Marken in die schon von früher her feststehenden und schwer umzuändernden Markengruppierungen war schließlich das Bild jeder Seite so verwirrt und unübersichtlich geworden, $\mathrm{da} B$ vielfach ein nicht ungewöhnliches $\mathrm{Ma} B$ von Geduld erforderlich war, wollte man aus diesem Wirrwarr eine bestimmte Marke herausfinden. Diese. Mühe wurde bisher auch durch kein Register erleichtert. In wissenschaftlicher Beziehung aber war das Werk insofern völlig unzureichend, als bei so gut wie keiner der in demselben wiedergegebenen Marken ihr Ursprung, ihre Quelle genannt, mithin auch nirgends die. Möglichkeit einer Nachprüfung gegeben war. So mußte, wer dieses Buch benutzen wollte, dem Verfasser unbedingten Glauben schenken.

Diesen beiden Úbelständen abzuhelfen, schien mir daher bei Durchführung vorliegender Arbeit meine erste Pflicht zu sein, dann aber weiter das ganze Werk noch einmal gründlich durchzukontrollieren, um möglichst alle im Laufe der Zeit entstandenen Fehler auszumerzen, schließlich aber vor allem auch die jüngsten Resultate der keramischen Forschung, die ja in der letzten Zeit auf gewissen Gebieten besonders lebhaft gewesen ist, aufzunehmen.

Wie weit dies alles gelungen ist, möge die vorliegende Auflage selber zeigen. Doch muß hier gleich bemerkt werden, daß die Kürze der Zeit, die zur Bearbeitung derselben zur Verï̈gung stand, noch nicht gestattete, alle $\mathrm{Ab}$ teilungen des Werkes schon in völlig gleicher Weise durchzuarbeiten. Es mußte daher hier je nach ihrer Wichtigkeit für den Benutzer ein Unterschied gemacht werden. So wurde einerseits der Ursprung aller Marken festgestellt, soweit dies heute noch möglich war, mit alleiniger Ausnahme der japanischen, deren Zahl bekanntlich Legion ist, weshalb von diesen doch nur einige wenige Beispiele gegeben werden konnten. Wer hier wirklich ernste Studien machen will, der muß das treffliche Werk von Morse, Catalogue of Japanese Pottery (Museum of fine Arts, Boston I897) zur Hand nehmen, woselbst er diese Marken finden kann. Ưberhaupt muß hier gleich betont werden, daß wirkliche Vollständigkeit hinsichtlich der bisher festgestellten keramischen Marken in einem Werke; das als Handbuch einen gewissen Umfang nicht uiberschreiten

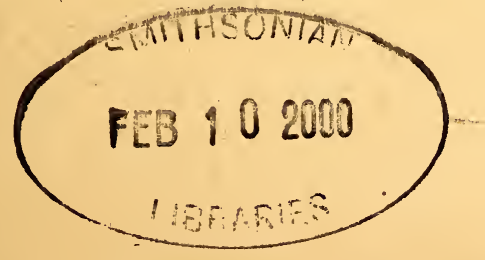


darf, nicht erwartet werden kann. Dies gilt ganz besonders von den Marken unserer eigenen Zeit, die allein ein so starkes Werk bilden würden, wie das hier vorliegende, von denen daher hier nur mehr oder weniger zufällige Proben gegeben werden konnten. Ein wirklich vollstäıdiges keramisches Markenbuch gibt es ja überhaupt noch nicht und wird es wohl auch sobald noch nicht geben. Auf der anderen Seite aber wurden dann alle Marken der neu bearbeiteten Teile in möglichst klaren Systemen angeordnet mit Ausnahme eines Teiles der Majoliken, welcher Mangel jedoch wohl in Anbetracht der geringen Anzahl vou. Majolikensammlern kaum sehr empfunden werden wird. Giänzlich neu bearbeitet wurde die Porzellanabteilung und die Abteilung der deutschen Fayencen, Steinzeuge usw. Dann aber ward auch das bisher immer so sehr vermị Bte alphabetische Verzeichnis der Buchstabenmarken hinzugefügt, ohne welehes ein derartiges Wrrk eigentlich ziemlich nutzlos ist. Schließlich wurde hinsichtlich der Angaben des Ursprungs der einzelnen Marken, die im Anhange wiedergegeben sind, das Prinzip durchge ührt, daß bei allen Stïcken, die in öffentlichen Museen od. dgl. sich befinden, nur diese angegeben wurden, bei allen übrigen dagegen die Werke, die die Titel der Marken zuerst in die Öffentlichkeit gebracht haben. Nur bei den immner wiederkehrenden Marken, den eigentlichen Fabrikmarken, wurde kein besonderer Beleg gegeben, dafür aber uhr ständiges Vorkommen erwälııt.' Wo kein Beleg zu einer Marke sich befindet, konnte demnach bisher ihr Ursprung nicht aufgefunden werden. Der Verfasser ist aber jedem Benutzer dieses Werkes zu großem Dank verpflichtet, der ihm für solche Marken den Ursprung so nachzuweisen in der Lage ist, $\mathrm{da} ß$ er für wissenschaftlich gesichert gelten kann, wie gleichfalls jedem, der ihn auf Fehler und Irrtümer aufmerksam macht, die bei der Kürze der Zeit, die zur Bearbeitung dieser Auflagc zur Vẹrfügung stand; nicht ganz werden ḑusgeblieben sein. Schon jetzt aber möchte er seinen besten Dank allen denen sagen, die ihm bereits bei dieser Auflage geholfen haben, ohne deren Hilfe sie nicht so rasch hätte vollendet werden können, ganz besonders aber den Herren Dir. von Falke, Berlin, Dir. Brinckmann, Hamburg, Dir. Graul und Kurzwelly, Leipzig, Dir. Lenz, Zürich, Dir. Pollazek, Straßburg, Staatsanwalt Riesebieter, Odenburg, Prof. Scherer, Braunschweig und Architêkt Stöhr, Würzburg.

Zum ersten Male wird mit dieser Auflage auch der Versuch gemacht, das bisher immer nur in französischer Sprache herausgegebene Werk auch in deutscher erscheinen zu lassen. Die Schwierigkeit, die mit einer solchen Um. arbeitung verbunden, möge entșchuldigen, wenn hier sich noch vielfach Ungleichheiten und Inkonsequenzen bemerkbar machen werden, die bei einer späteren Auflage verschwinden werden.

Allen Anfängern keramischen Sammelns aber sei zum Schluß gesagt, daß das Auffinden von Marken in diesem Buche noch keine.Gewähr gibt für die Echtheit der Stücke; die solche tragen, da, wenn man ganze Stücke, so auch Marken auf denselben fälschen kann.

Dresden, Januar Igro,

Ernst Zimmermann. 
 \\ Vorwort zur XIV. Auflage.}

Für die vorliegende XIV. Auflage wurden die Abteilungen der deutschen Fayence, des deutschen Porzellans und der chinesischen Keramik, zu denen in den letzten Jahren eine reiche Fülle neuer Marken durch emsige Forschung hinzugekommen war, völlig umgearbeitet und ergänzt, sowie die bisher in keiner Weise ausreichenden Abteilungen der englischen und japanischen Keramik völlig neu angelegt. Daneben fanden auf Grund der Forschungen dèr letzten Jahre mannigfache Ergänzungen aller übrigen Abteilungen statt. Auch wurde den bisherigen Registern ein solches mit den in den Marken sich vorfindenden bildiichen Darstellungen hinzugefügt, so daß sich nun wohl mit Hilfe der Register so ziemlich jede Marke auf dell Tafeln mit leichter Miihe wird auffinden lassen.

Auch bei Abfassung dieser Auflage ward mir wiederum die bereitwilligste Unterstützung vieler zuteil, denen ich hier wieder meinen aufrichtigen Dank aussprechen möchte, ganz besonders aber dem leider inzwischen verstorbenen Dir. Brinckmann in Hamburg, Dir. Pollazek in Straßburg, Dir. Pazaurek in Stutgart, Kustos Stelljes in Eisenach, Dir. Ballanti in Faenza, Dir. Stöhr in Würzburg, Staatsanwalt Riesebieter in Oldenburg und Rentner Lockner in Würzburg.

März 1915, Porzellansammlung

Ernst Zimmermann.

\section{Vorwort zur XV. Auflage.}

Die vorliegende, nunmehr I5. A uflage dieses Markenbuches, stellt nur einen unveränderten Wiederabdruck der I4. Ausgabe vor. Bearbeiter und Verlag atten nicht damit gerechnet, daß noch vor der endgültigen Beendigung des großen Krieges sich eine Neuauflage nötig machen würde. Um ein vollständiges Fehlen dieses sehr begehrten Nachschlagebuches $z u$ vermeiden und da es unter den jetzigen Verhältnissen außerordentlich schwer, ja fașt un= möglich war, das für eine Neubearbeitung nötige Material zu erlangen, hielten es daher Bearbeiter und Verlag im Interesse der Sammler und sonstiger Käufer des Buches am zweckmäßigsten, für diesmal nur einen Wiederabdruck der vorhergehenden Auflage zu bringen

Berlin W 62, Februar rgrg

Richard Carl Schmidt \& Co. 


\section{Inhaltsverzeichnis.}

\section{Europäische Keramik.}

r. Marken auf Fayence, Steingut, Steinzeug usw.

$$
\begin{array}{r}
\text { Seite } \\
1-192 \\
1-66 \\
07, \quad 68 \\
69 \\
70-102 \\
103-143 \\
144, \quad 145 \\
146-164 \\
165, \quad 166 \\
167-169 \\
170-185 \\
186, \quad 187 \\
188-190 \\
r 190 \\
191, \quad 192 \\
193-262 \\
193-215 \\
216-219 \\
220 \\
220, \quad 221 \\
221, \quad 222 \\
222-224 \\
224 \\
225-241 \\
242-252 \\
253-257 \\
257-259 \\
259 \\
260-262
\end{array}
$$$$
\text { Italien. }
$$$$
\text { Spanien }
$$$$
\text { Portugal . }
$$$$
\text { Frankreich . }
$$$$
\text { Deutschland }
$$$$
\text { Österreich-Ungam }
$$$$
\text { Holland }
$$$$
\text { Belgien }
$$$$
\text { Schweiz. }
$$$$
\text { England . }
$$

Dänemark

Schwedeu

Norwegen

Rußland und Polen.

Marken auf Porzellan

Deutschland

Holland

Relgien

Dänemar

Schweden

Frankreich

England .

Italien.

Spanien

Portugal .

Rußland und Polen.

II. Ostasiatische Keramik.

China $263-277$ Japan .

Belege und Erläuterungen

$299-350$

Verzeichnis der auf Marken vorkommenden einzelnen Buchstabeı,

Monogramme usw. ............. . 35ז-359

Verzeichnis der Fabrikationsortc . . . . . . . . . . . . . . . 360-366

Verzeichnis der Künstler, Maler, Dekorateure usw. . . . . . . . 367-382

Verzeichnis der auf Marken vorkommenden bildlichen Darstellıngen $\quad 383, \quad 384$ 


\title{
EUROPÄISCHE KERAMIK
}

\author{
I. \\ MAJOLIKA, FAYENCE, \\ STEINGUT usw.
}

(MAJOLIQUE, FAIENCE, GRĖ, POTERIE etc.) 

Italienische Majolika

Majolique thatiense

Florenz (Florence)

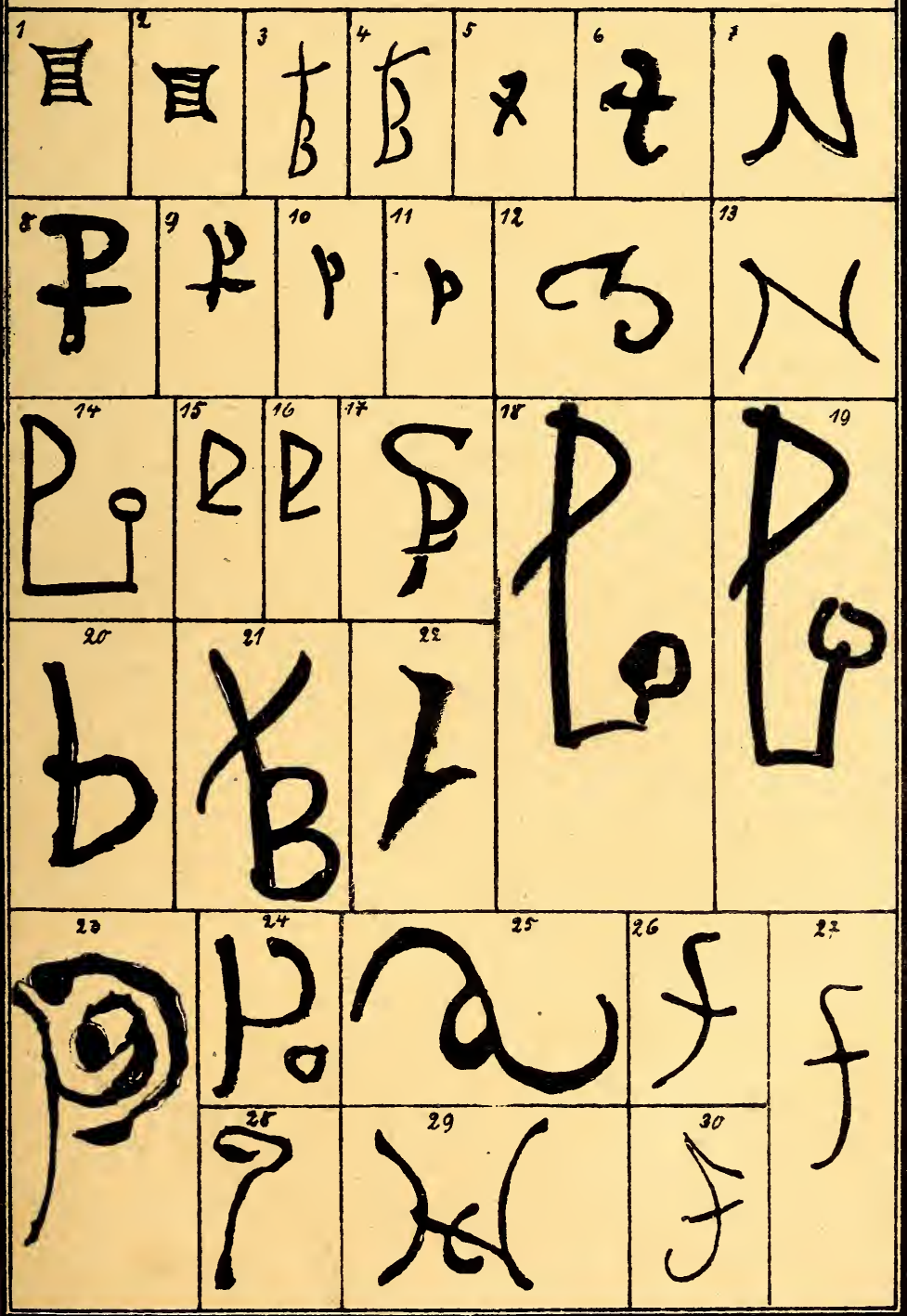




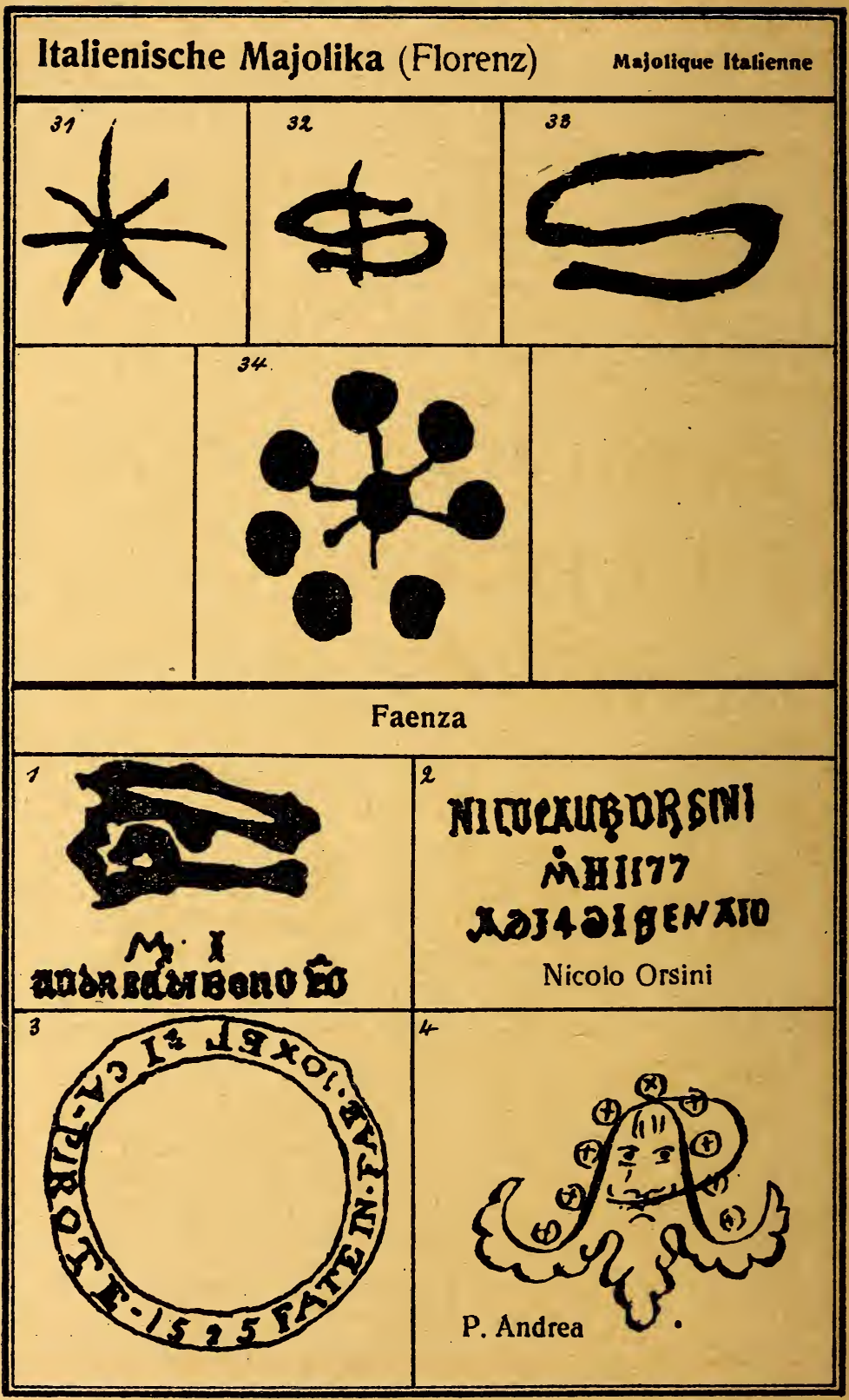


Italienische Majolika

Majollque Itallenne

(Faenza)
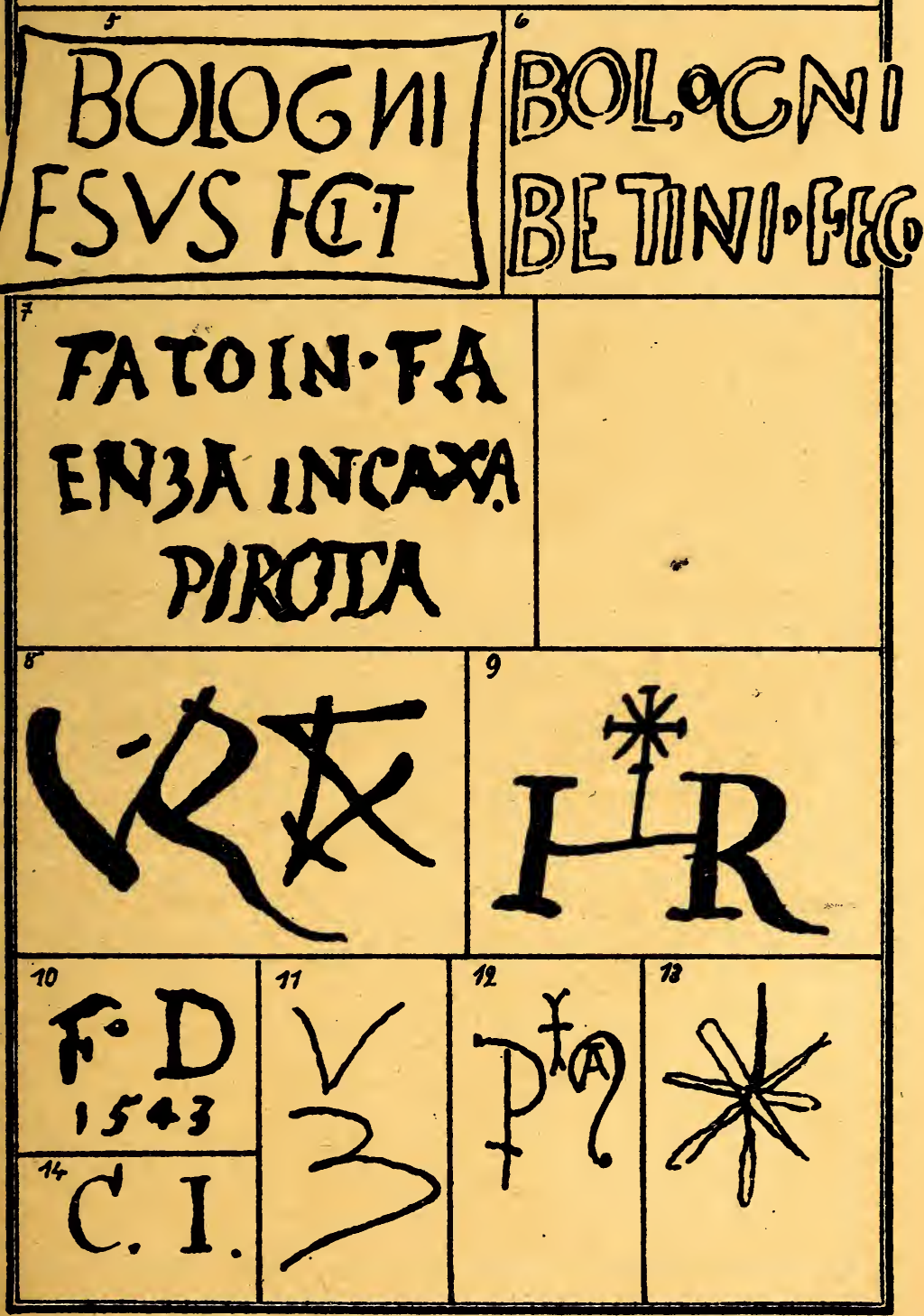


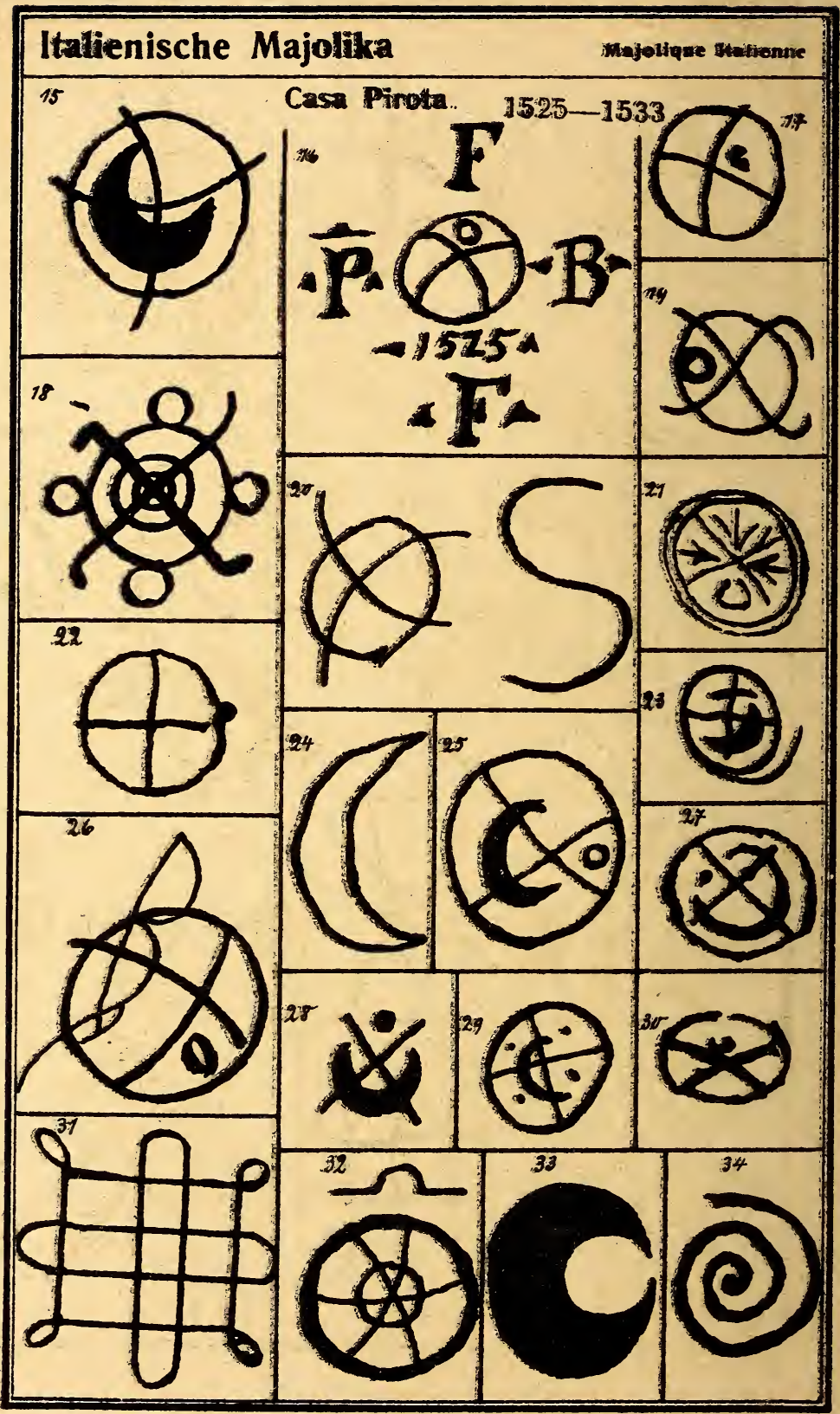




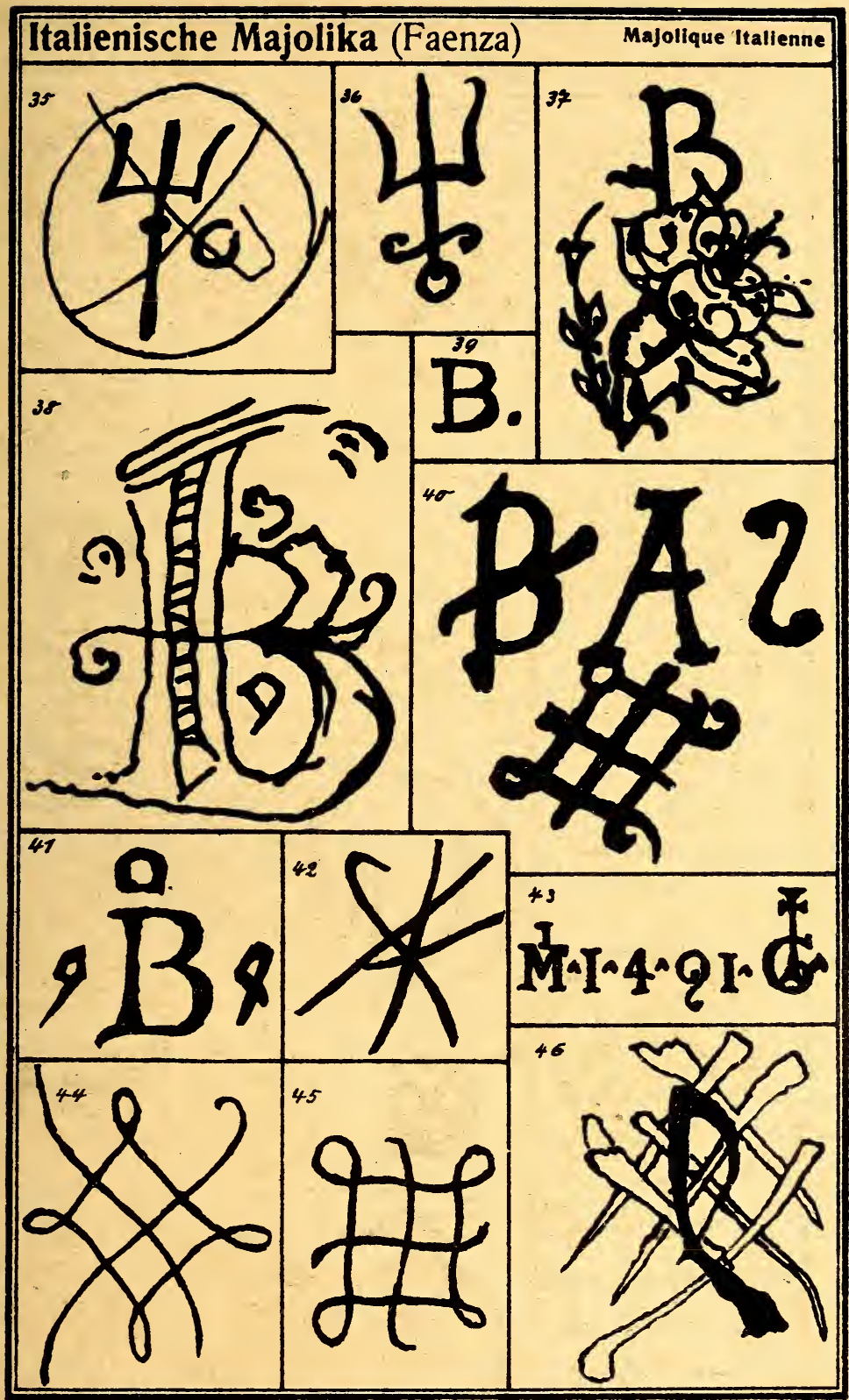




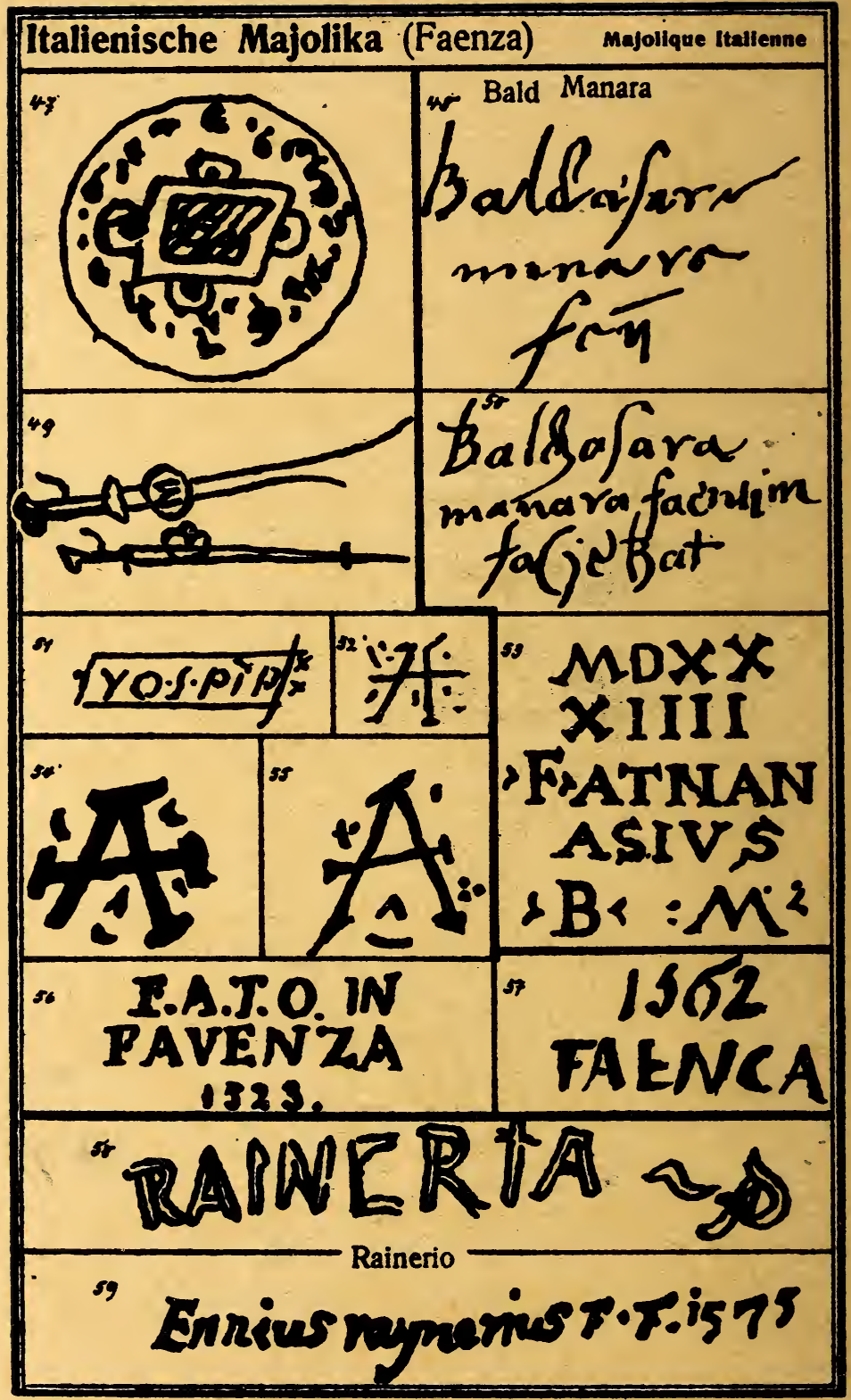




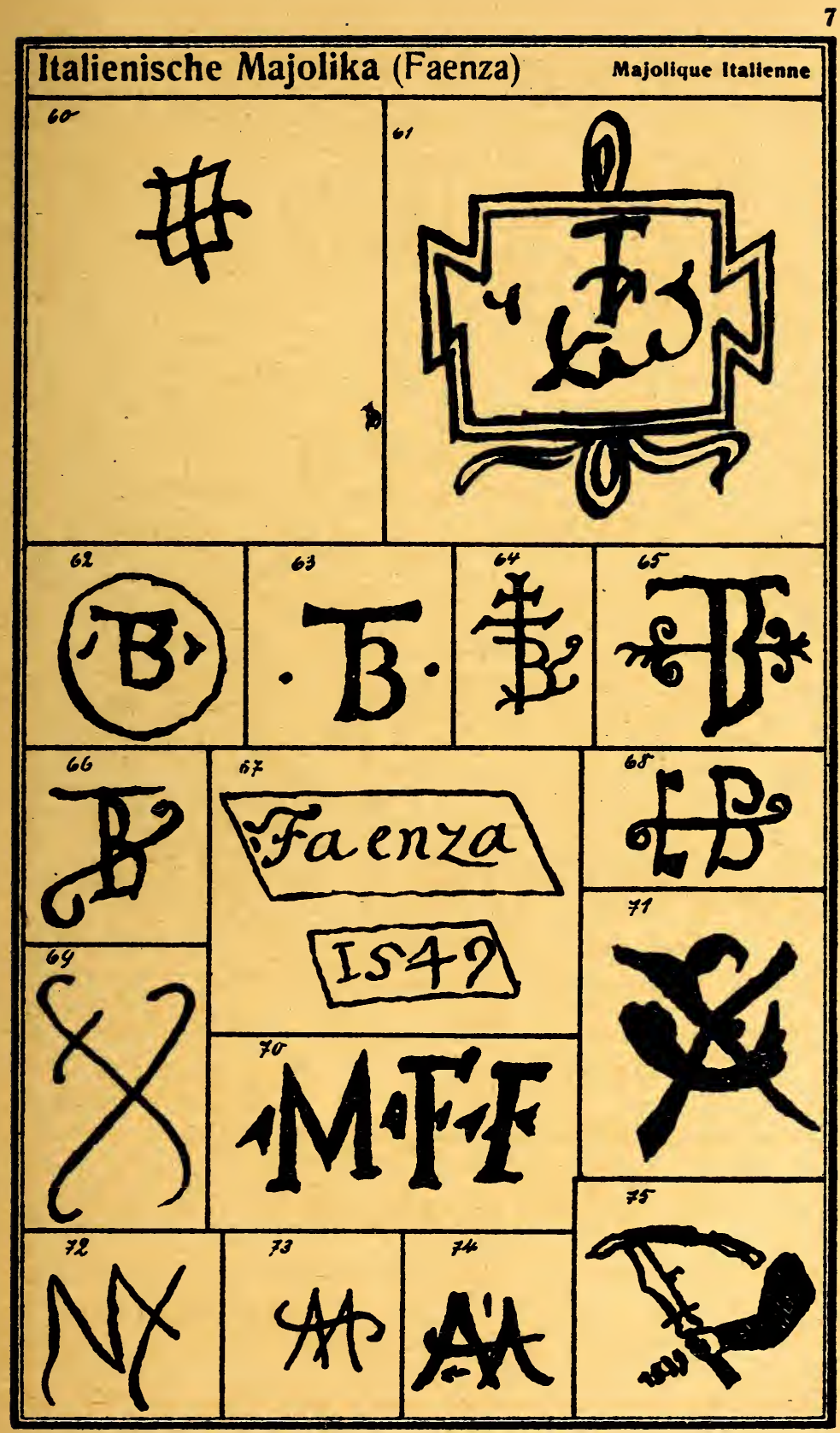




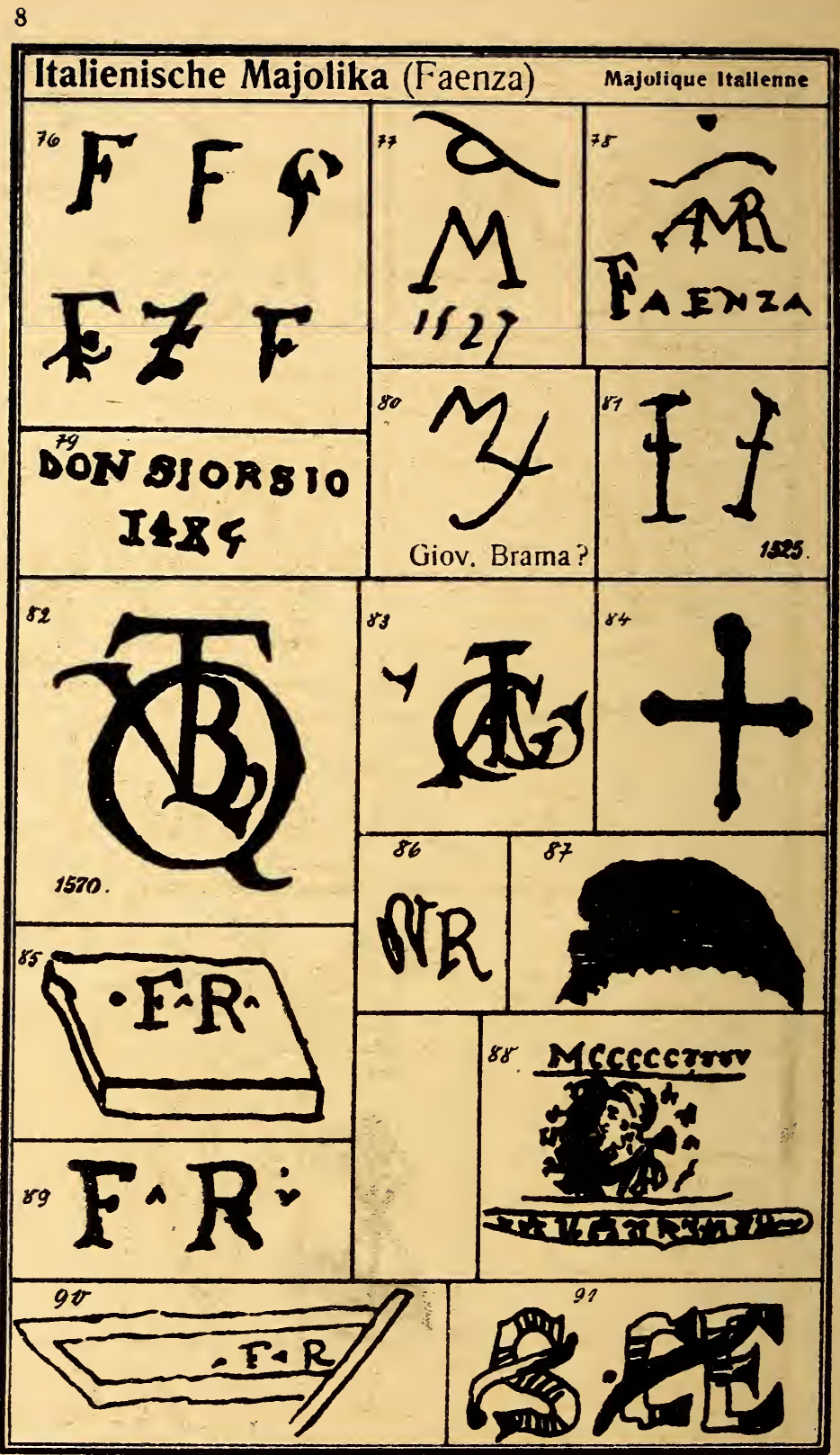




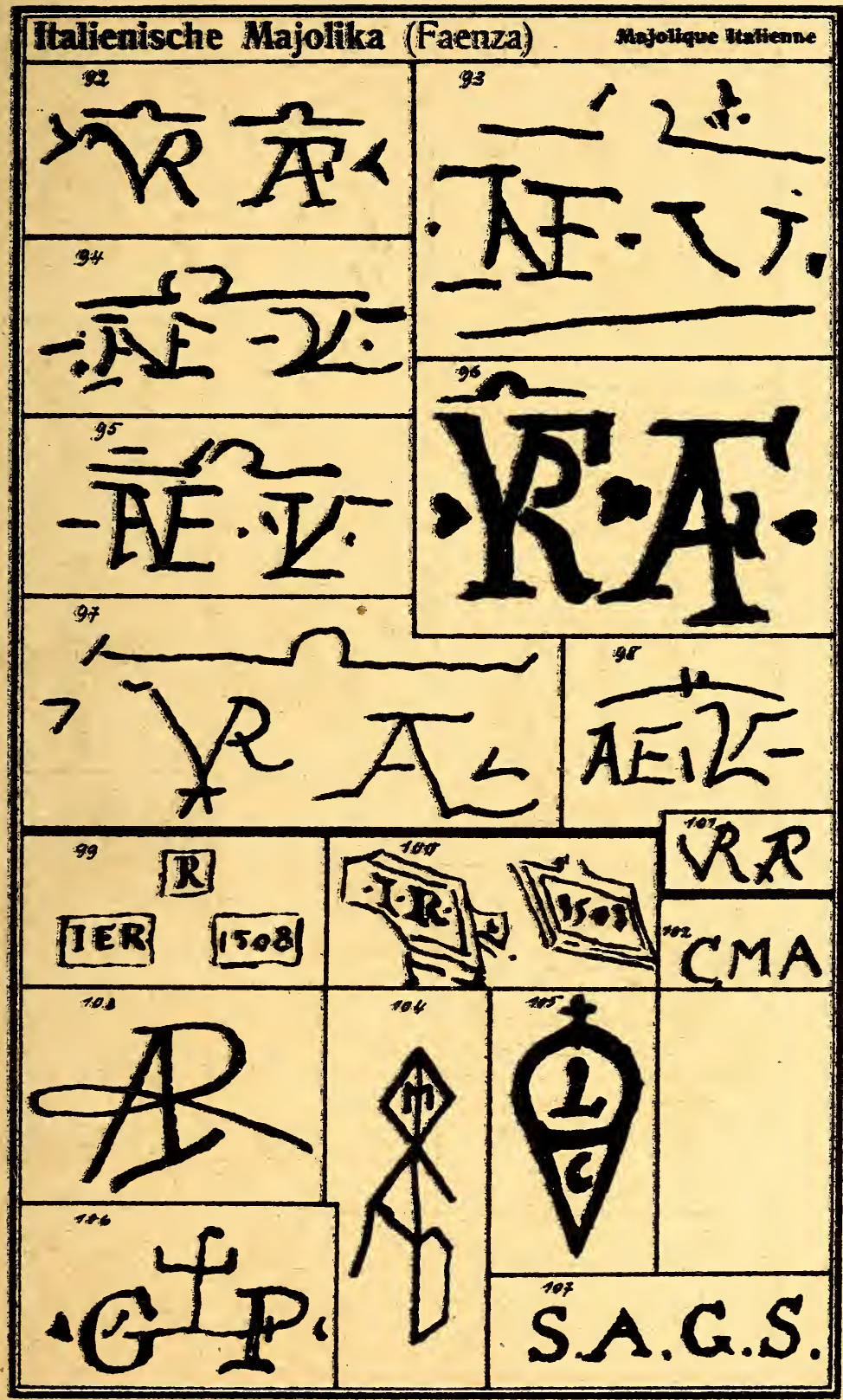




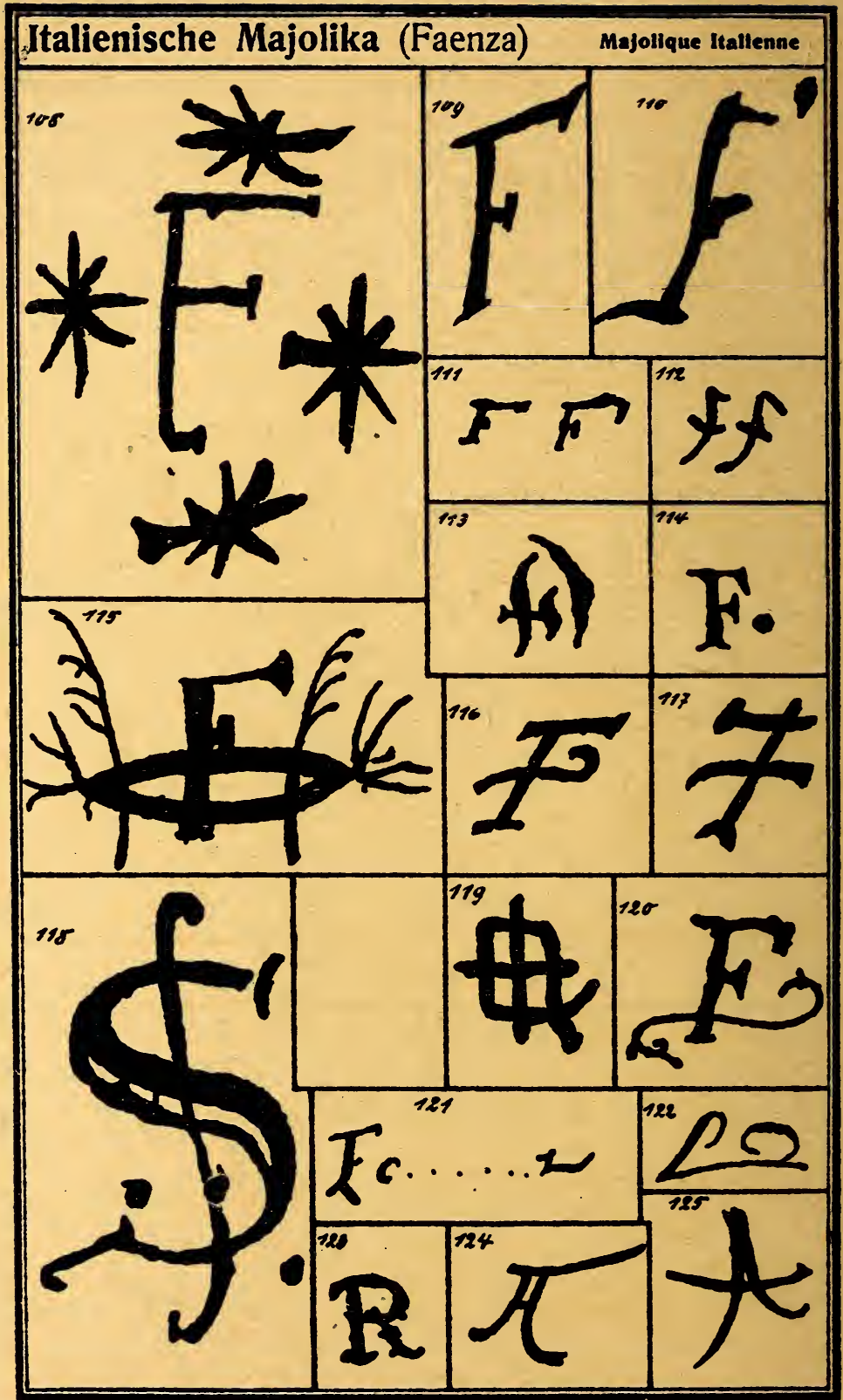




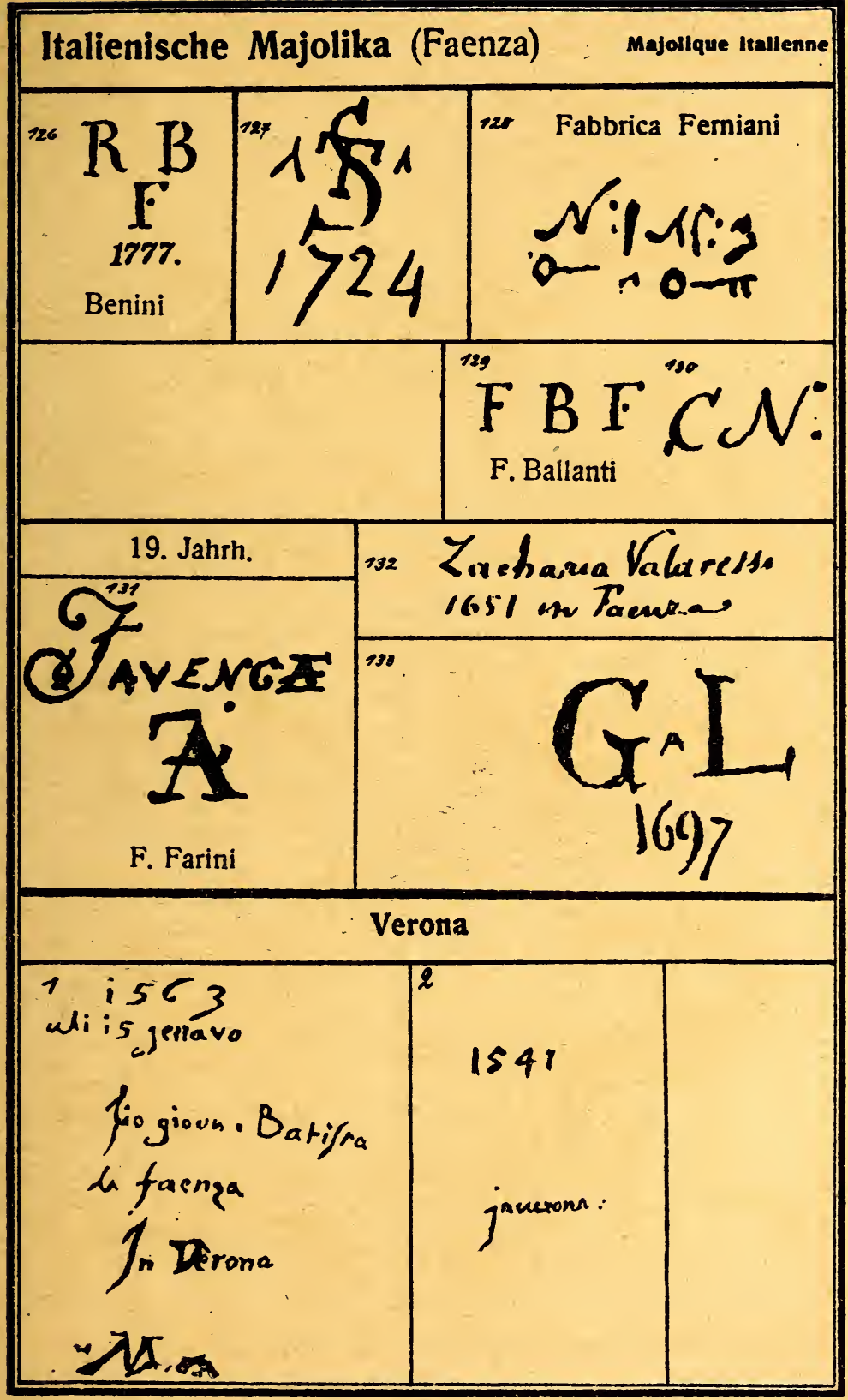




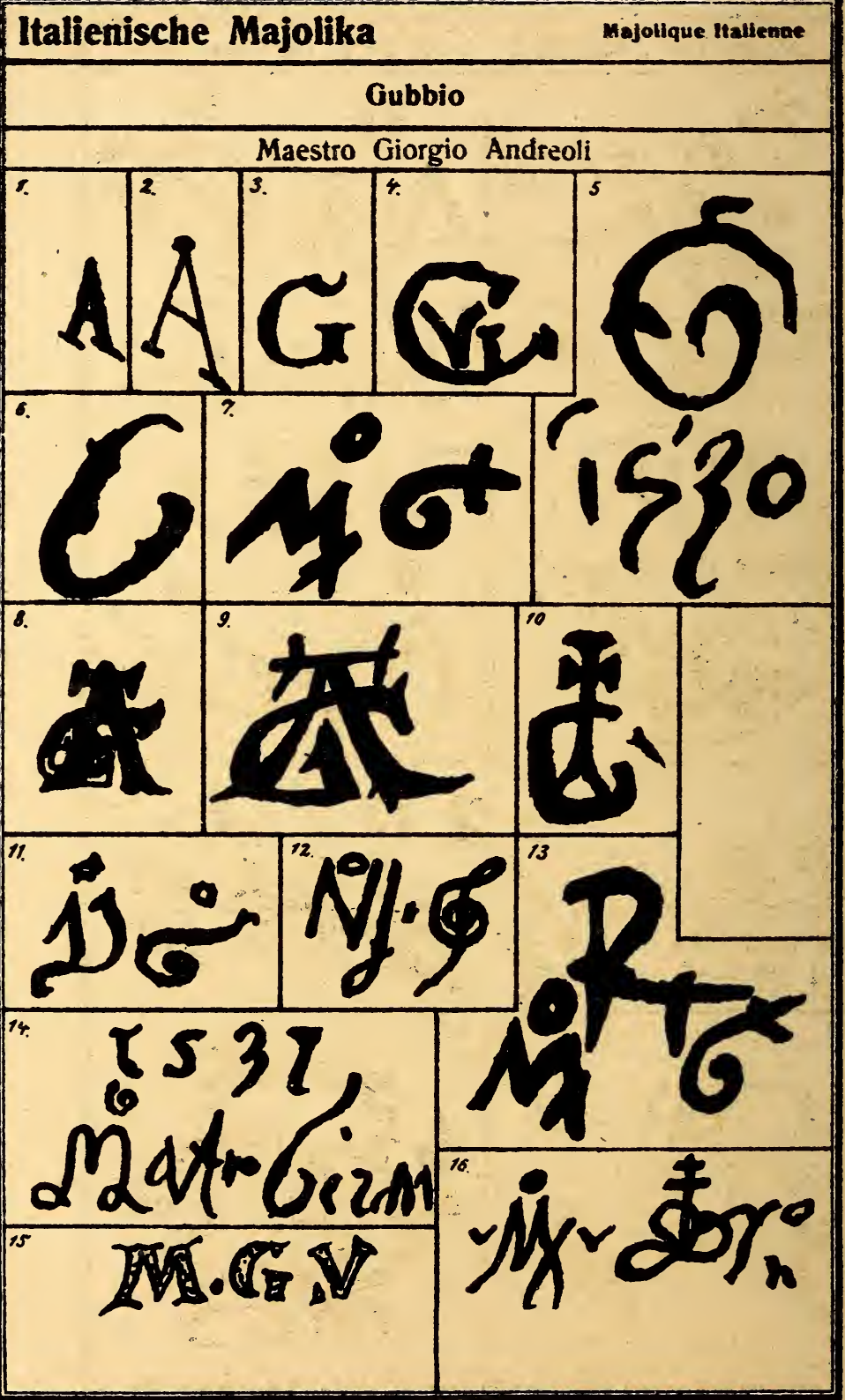




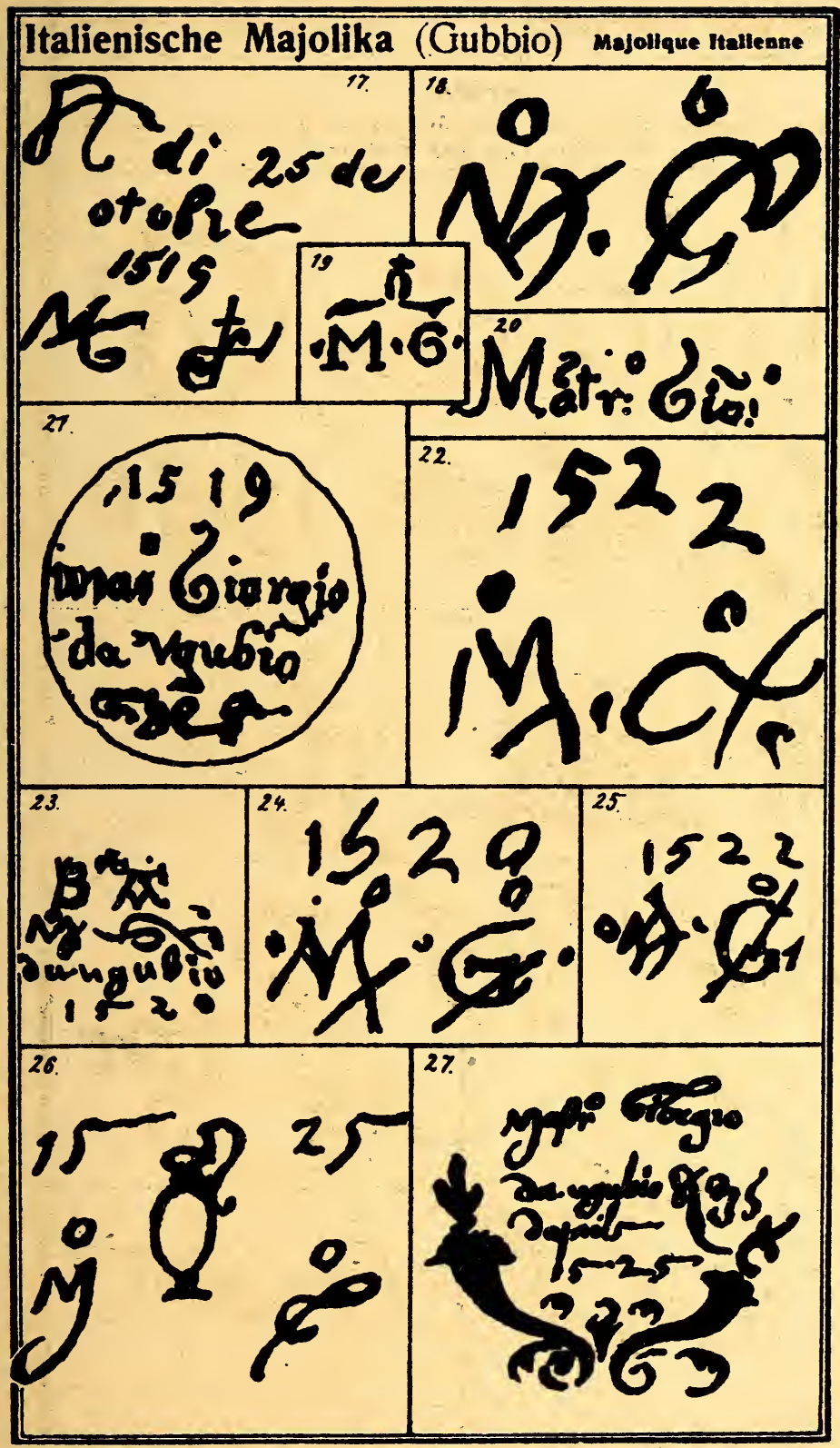




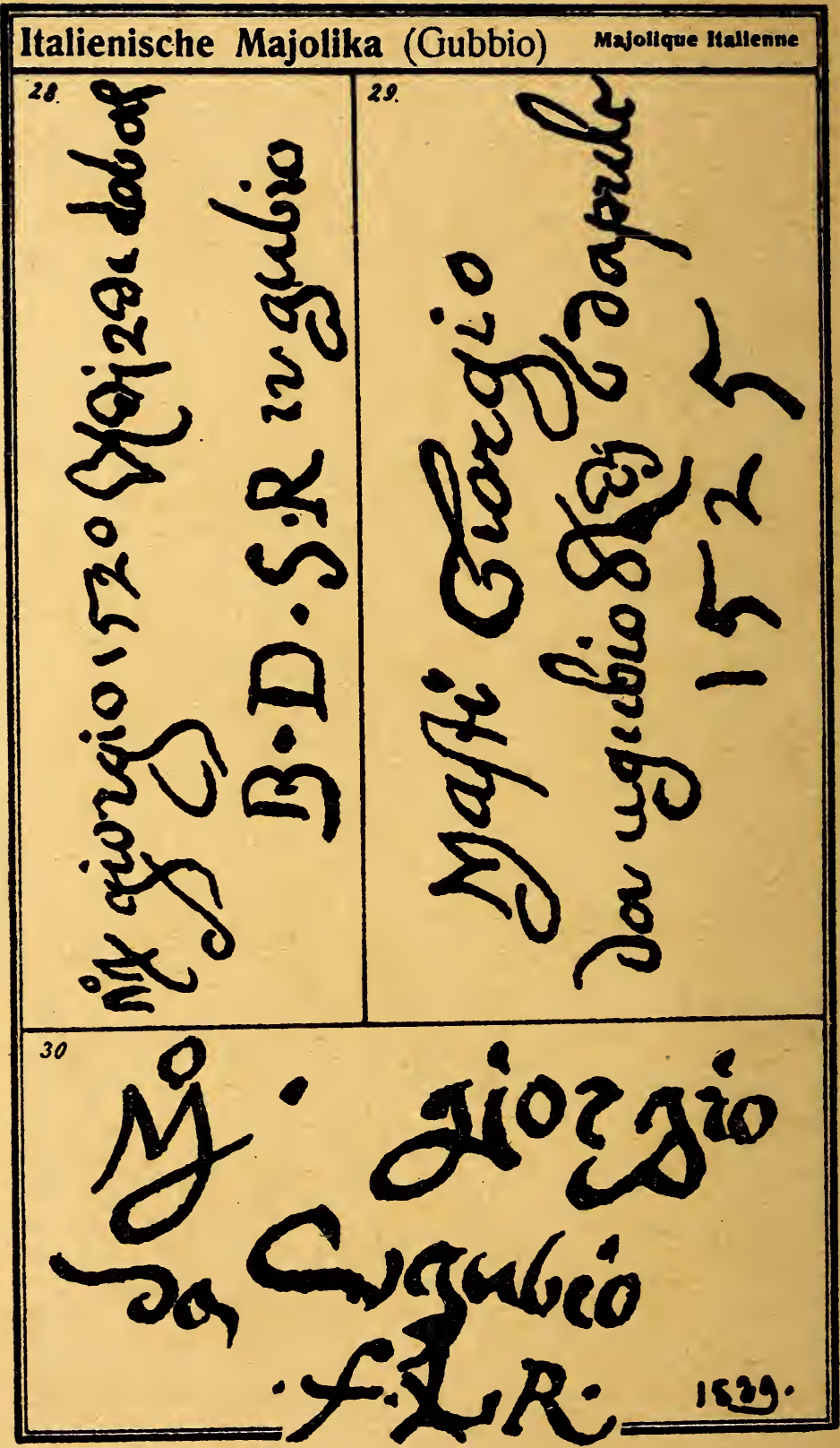




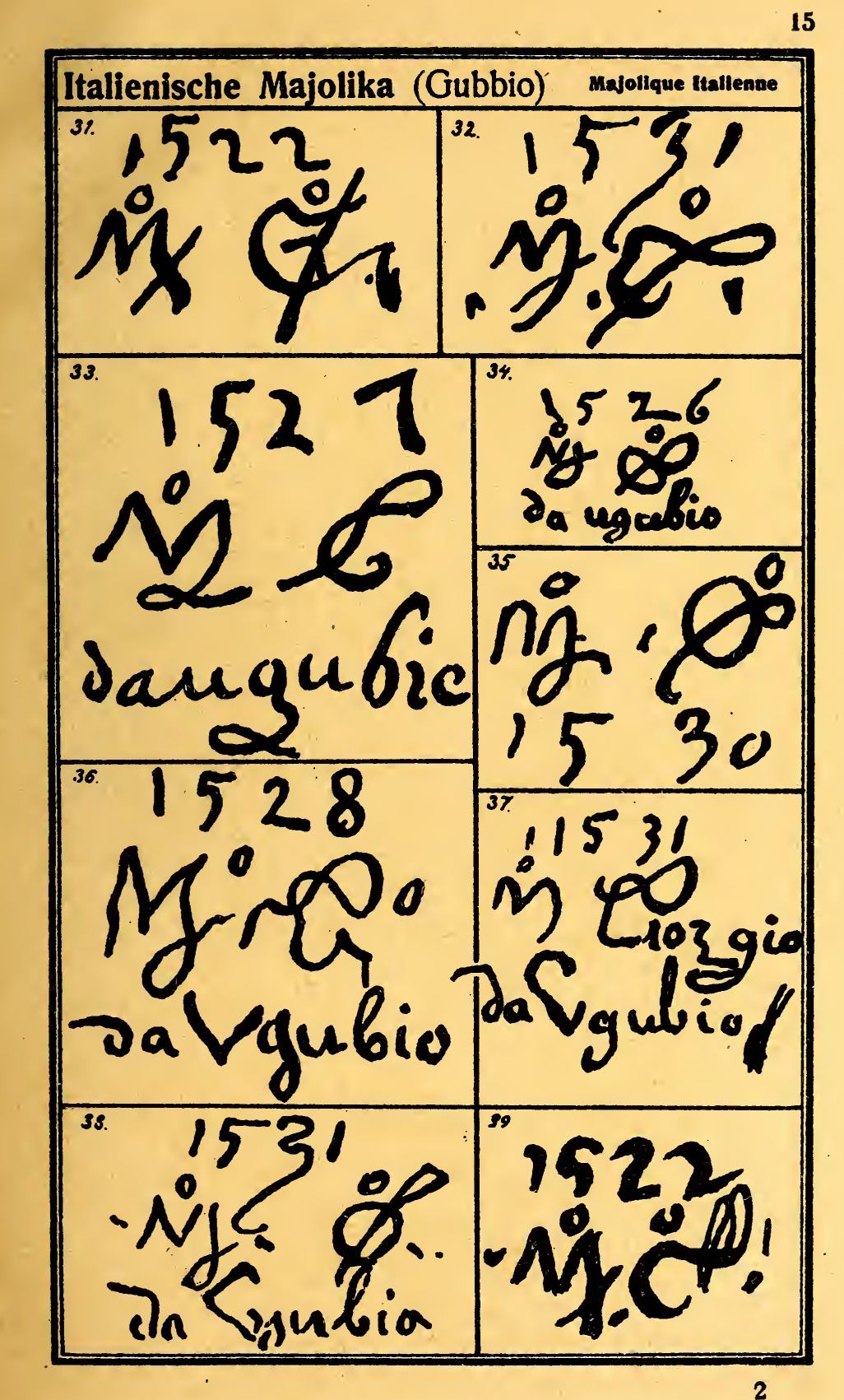




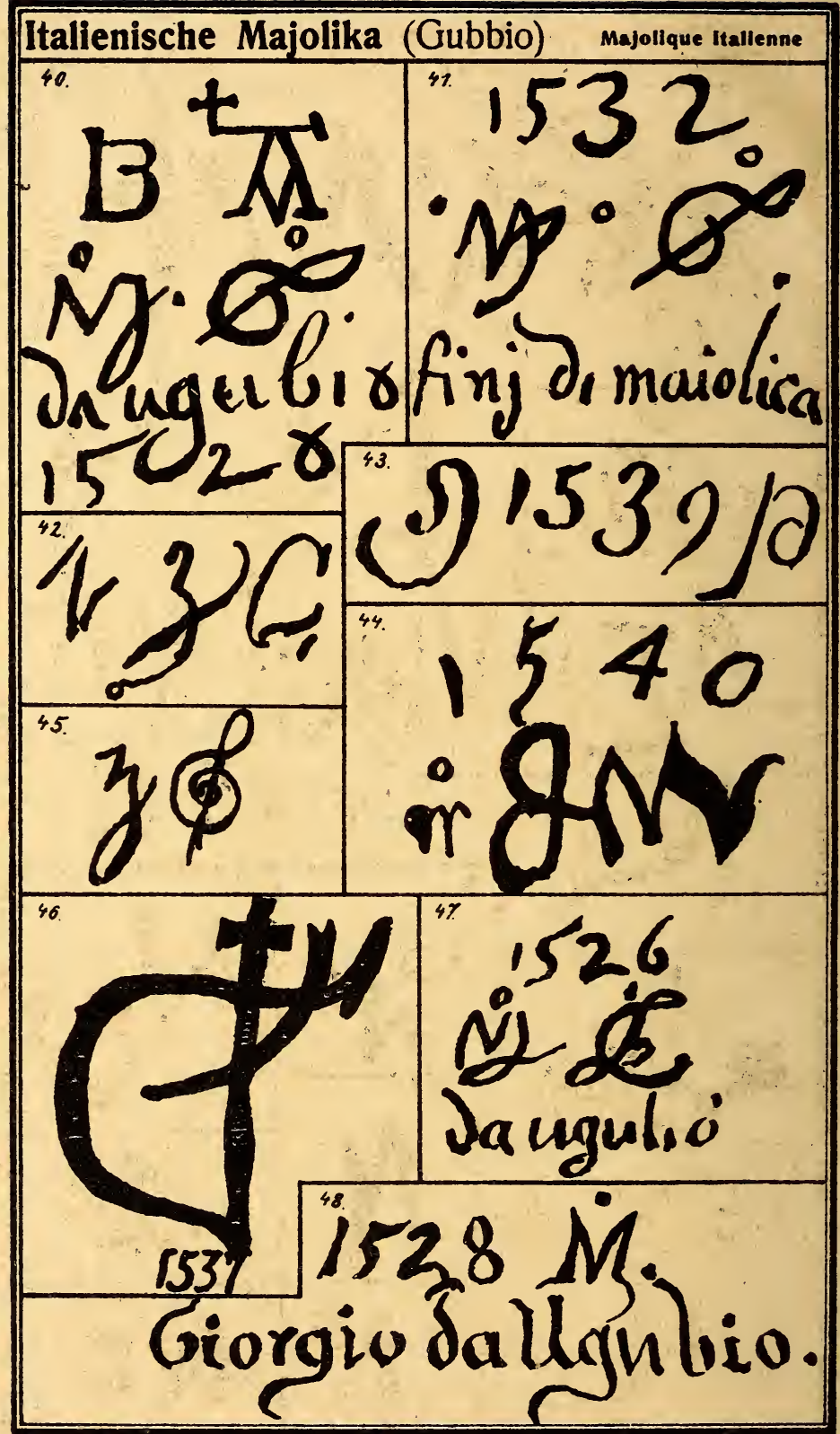




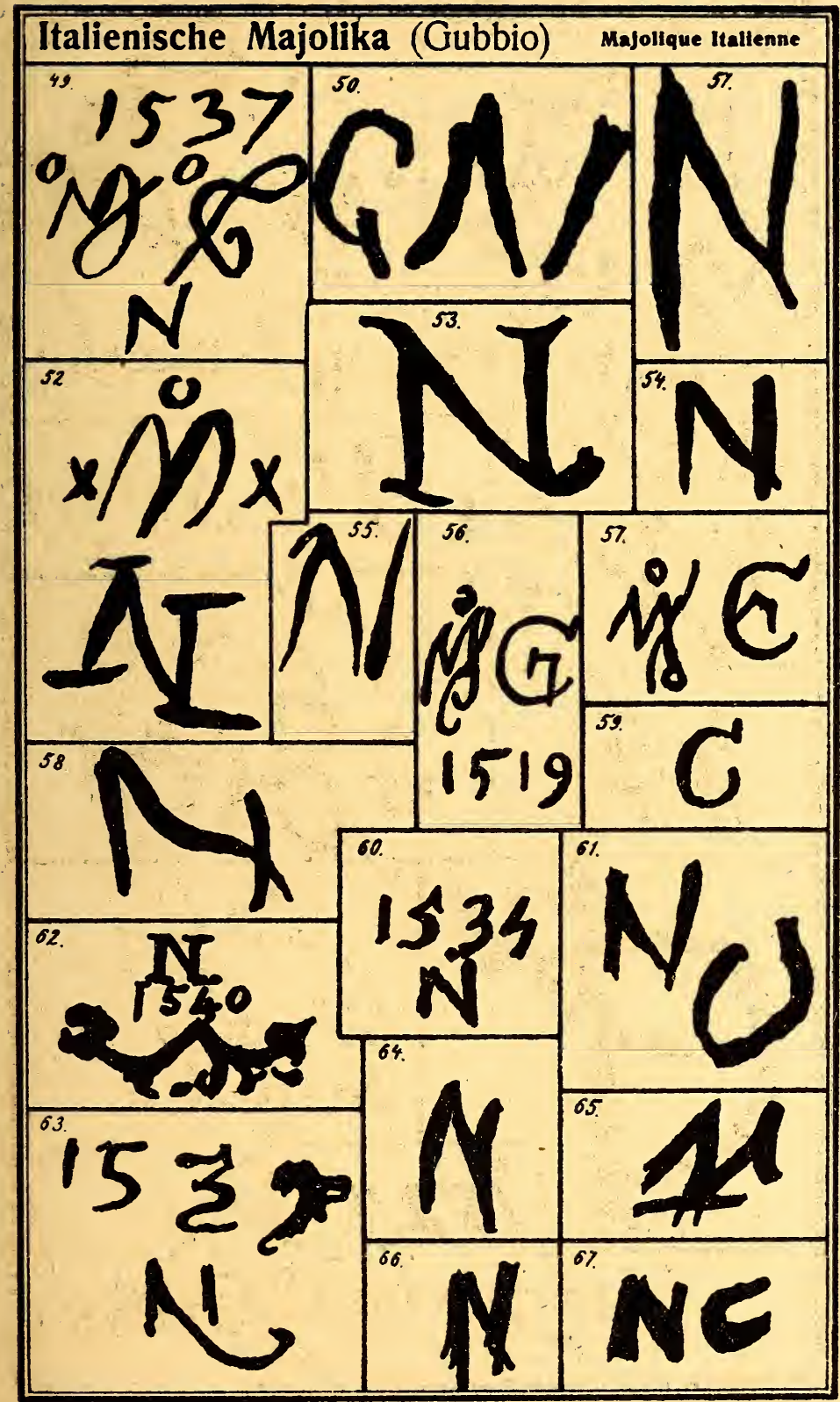




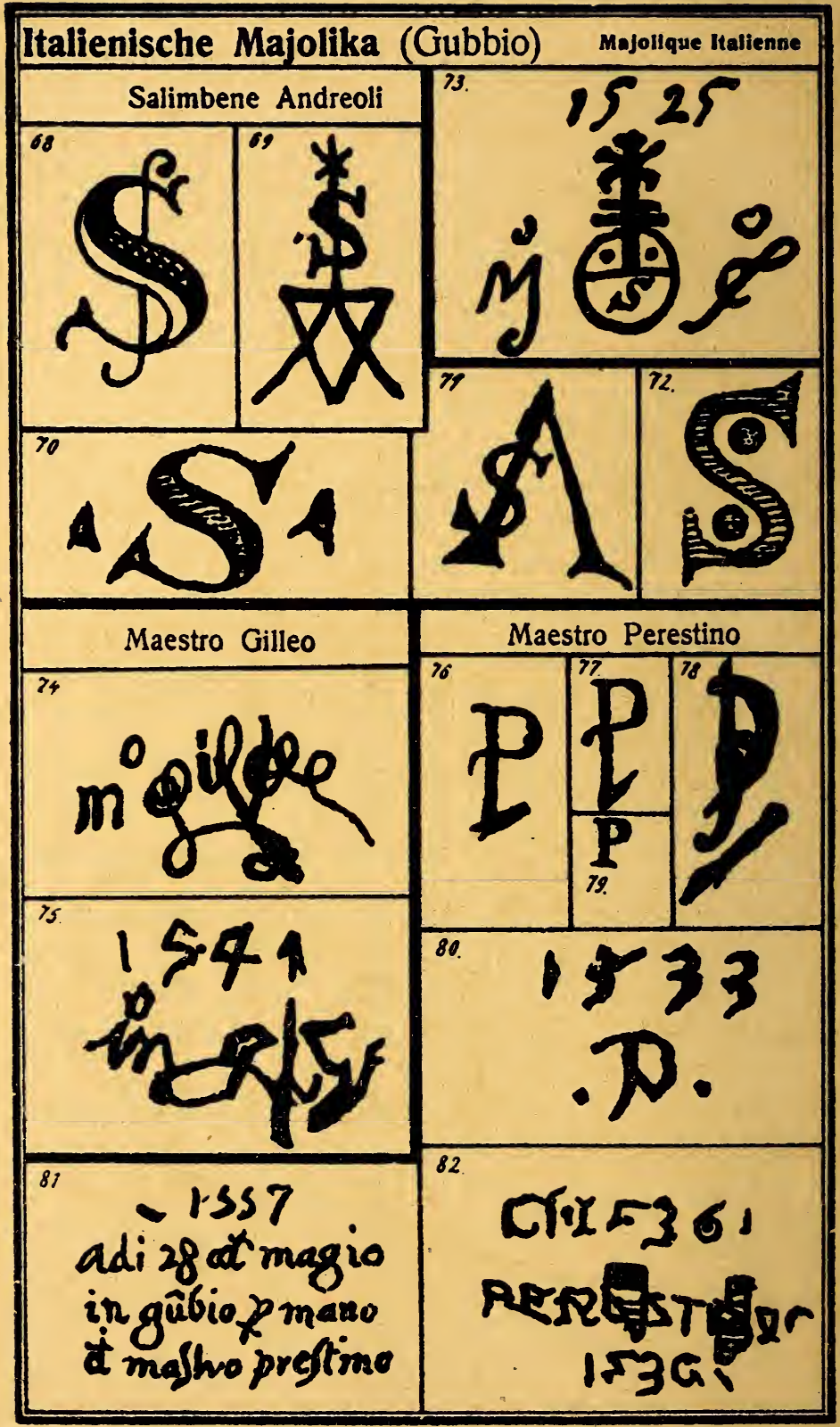




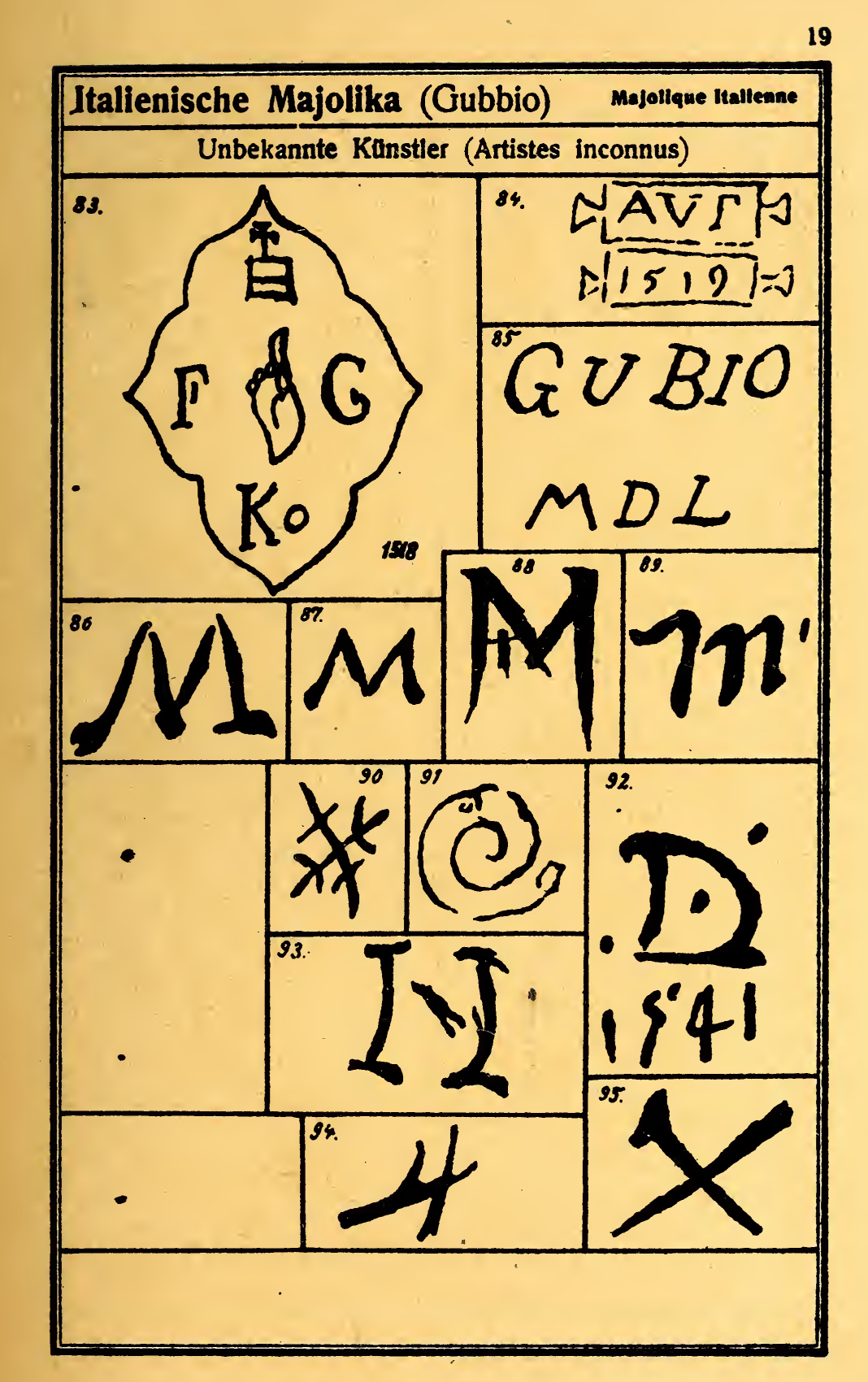




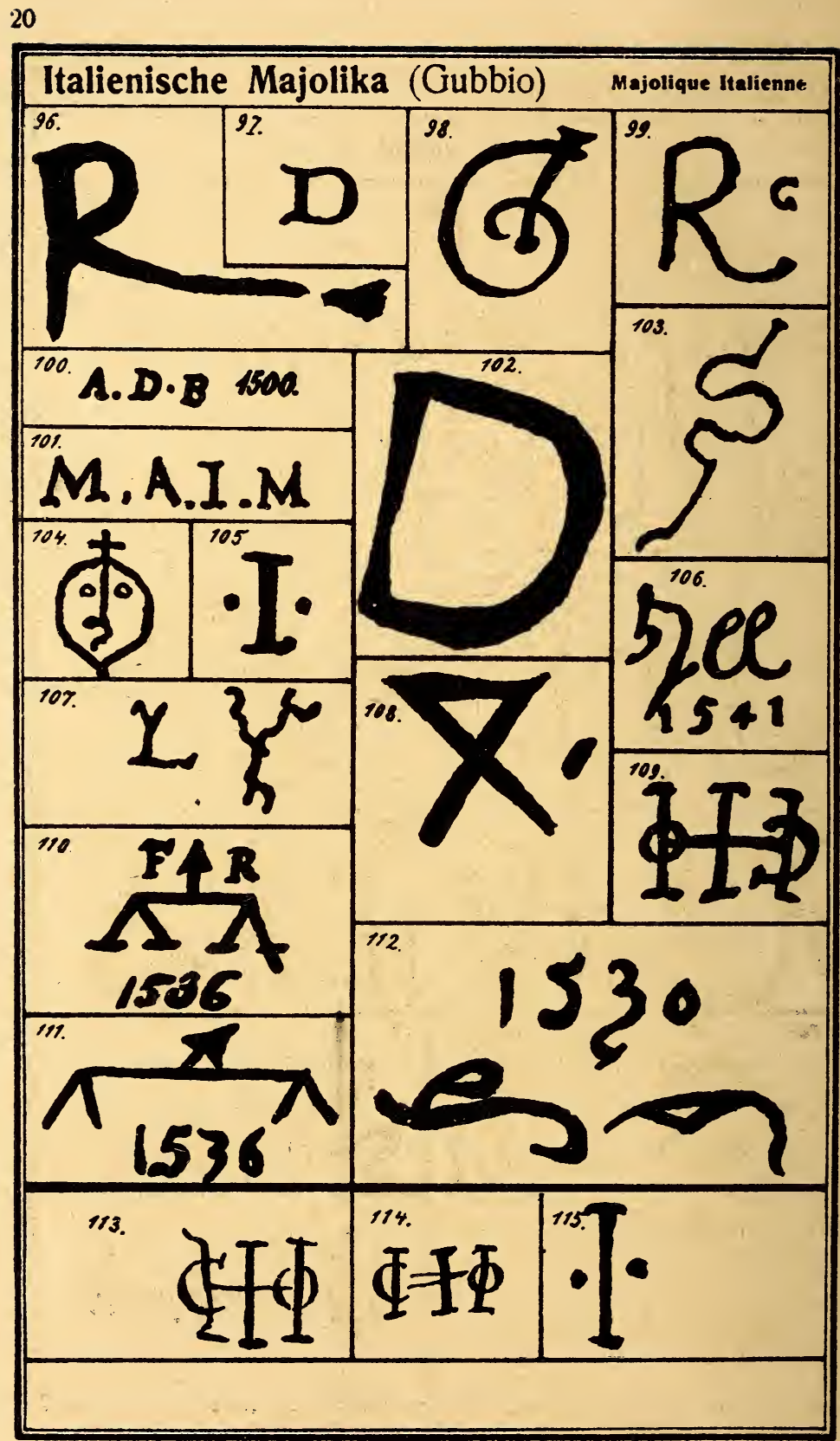




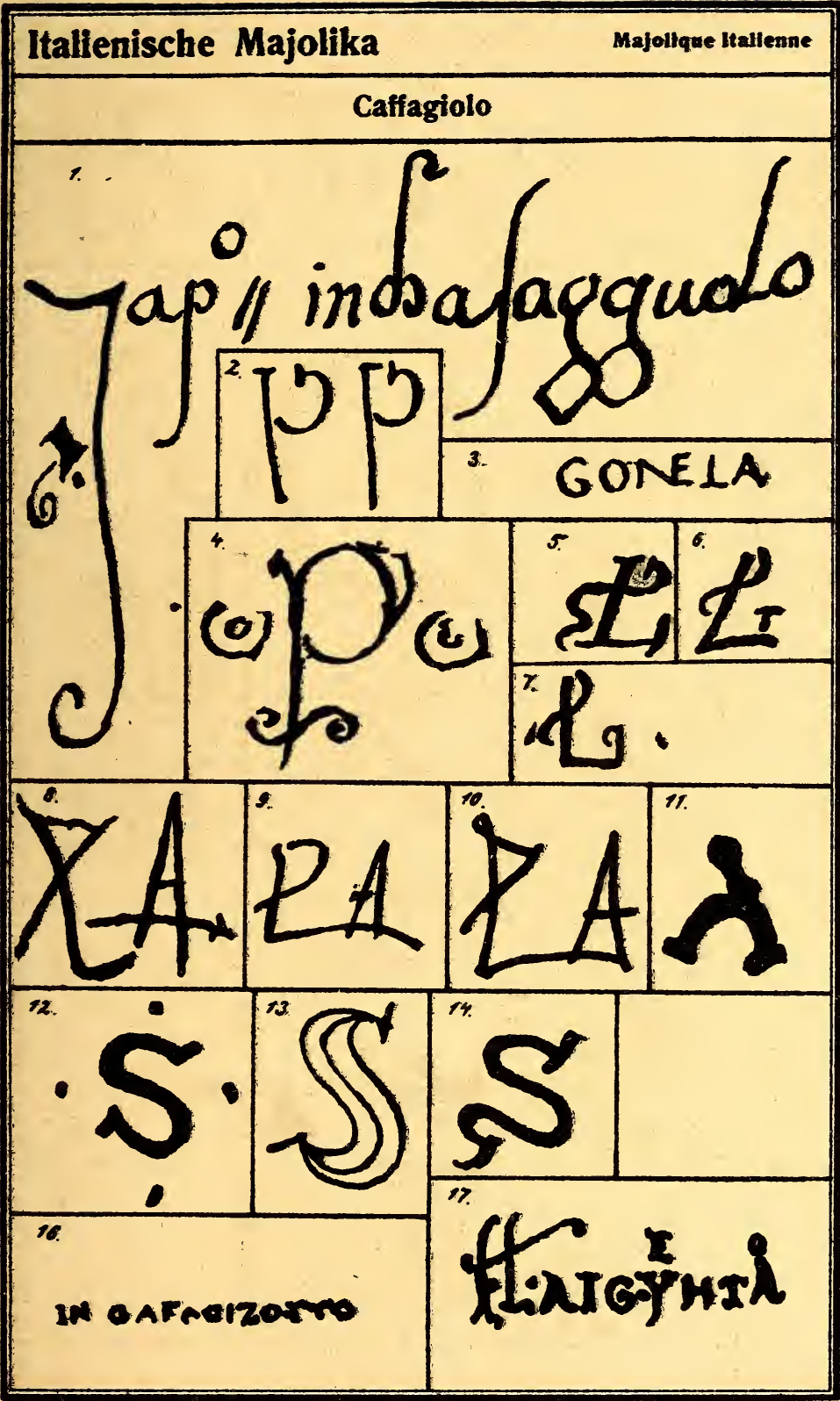




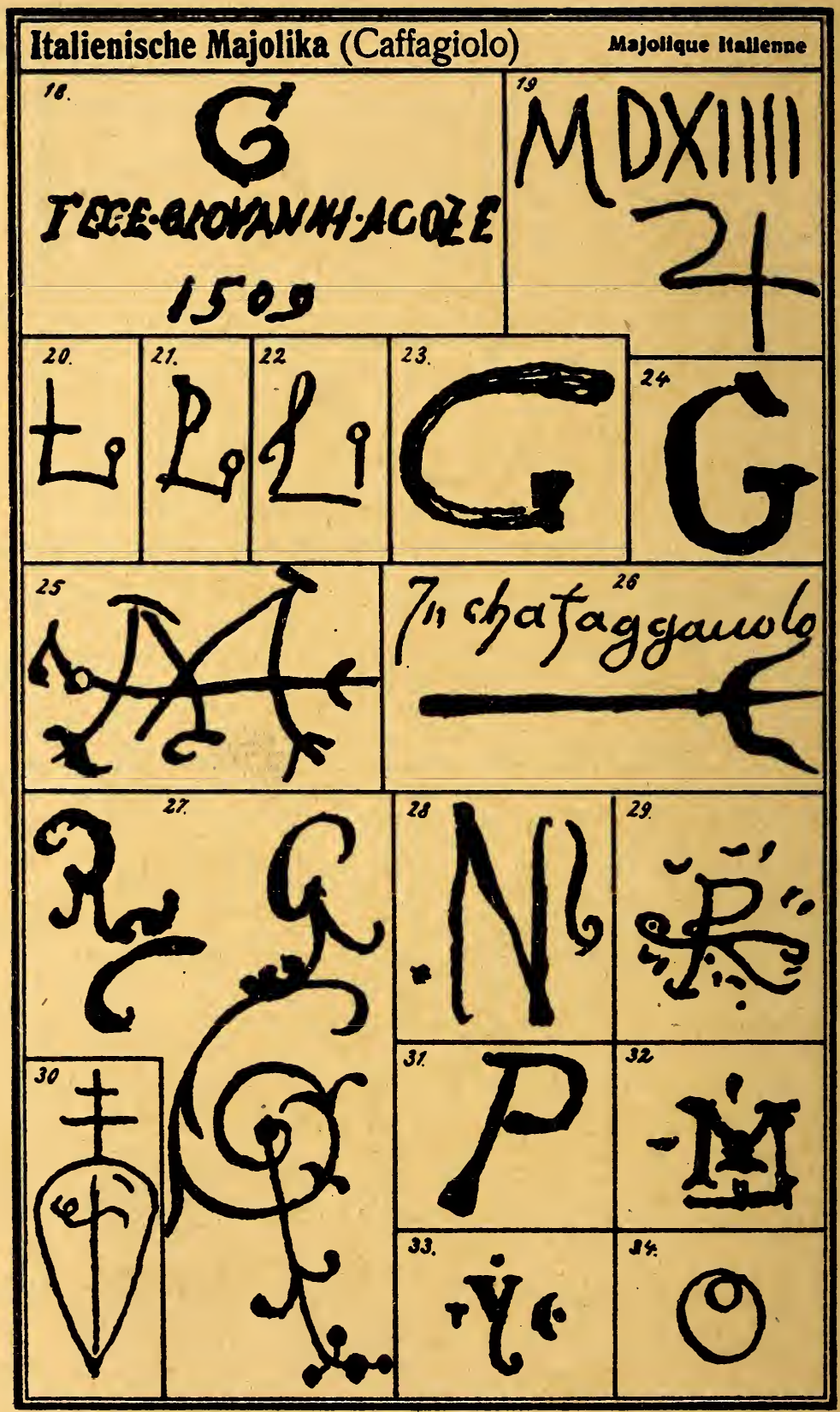




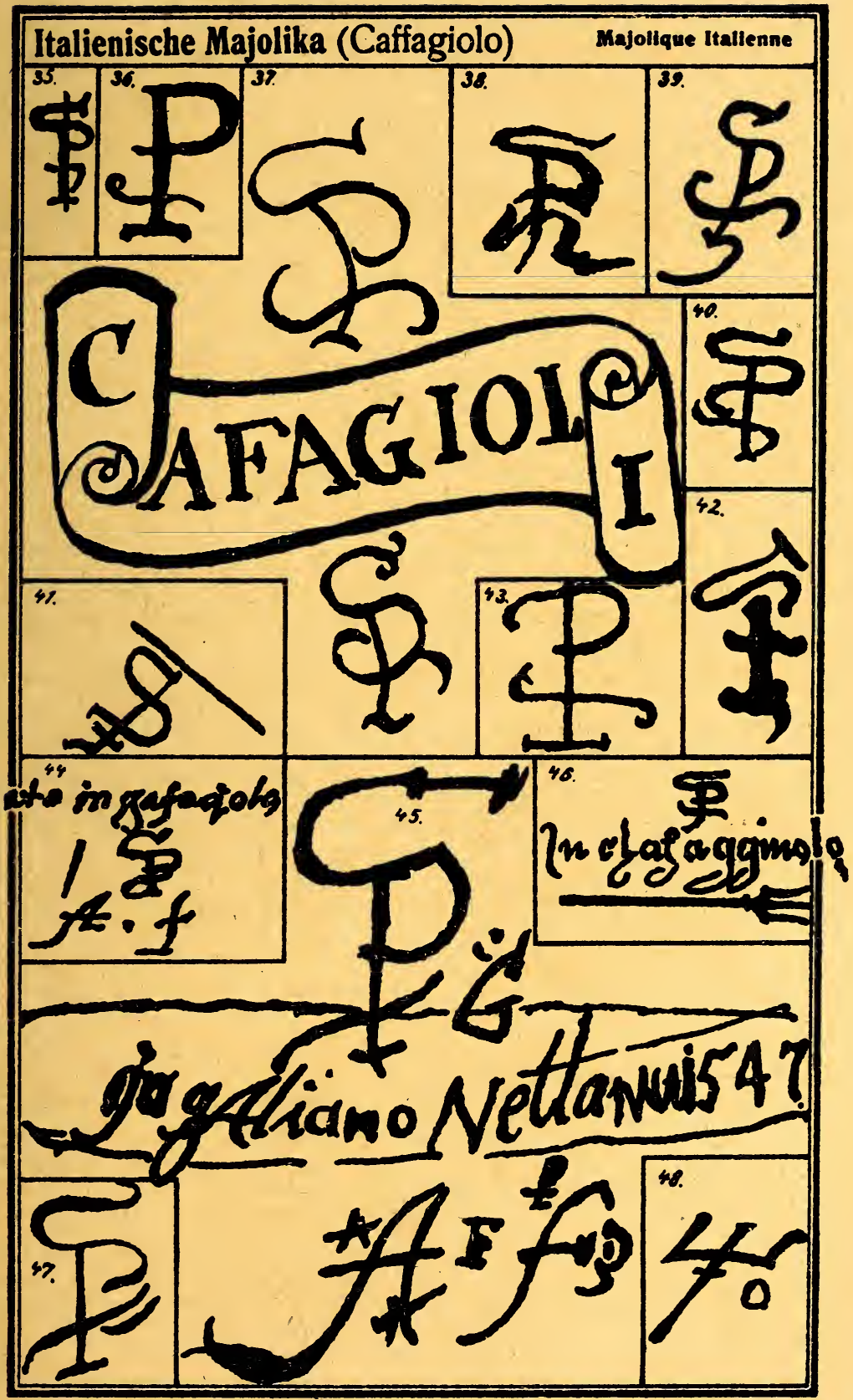




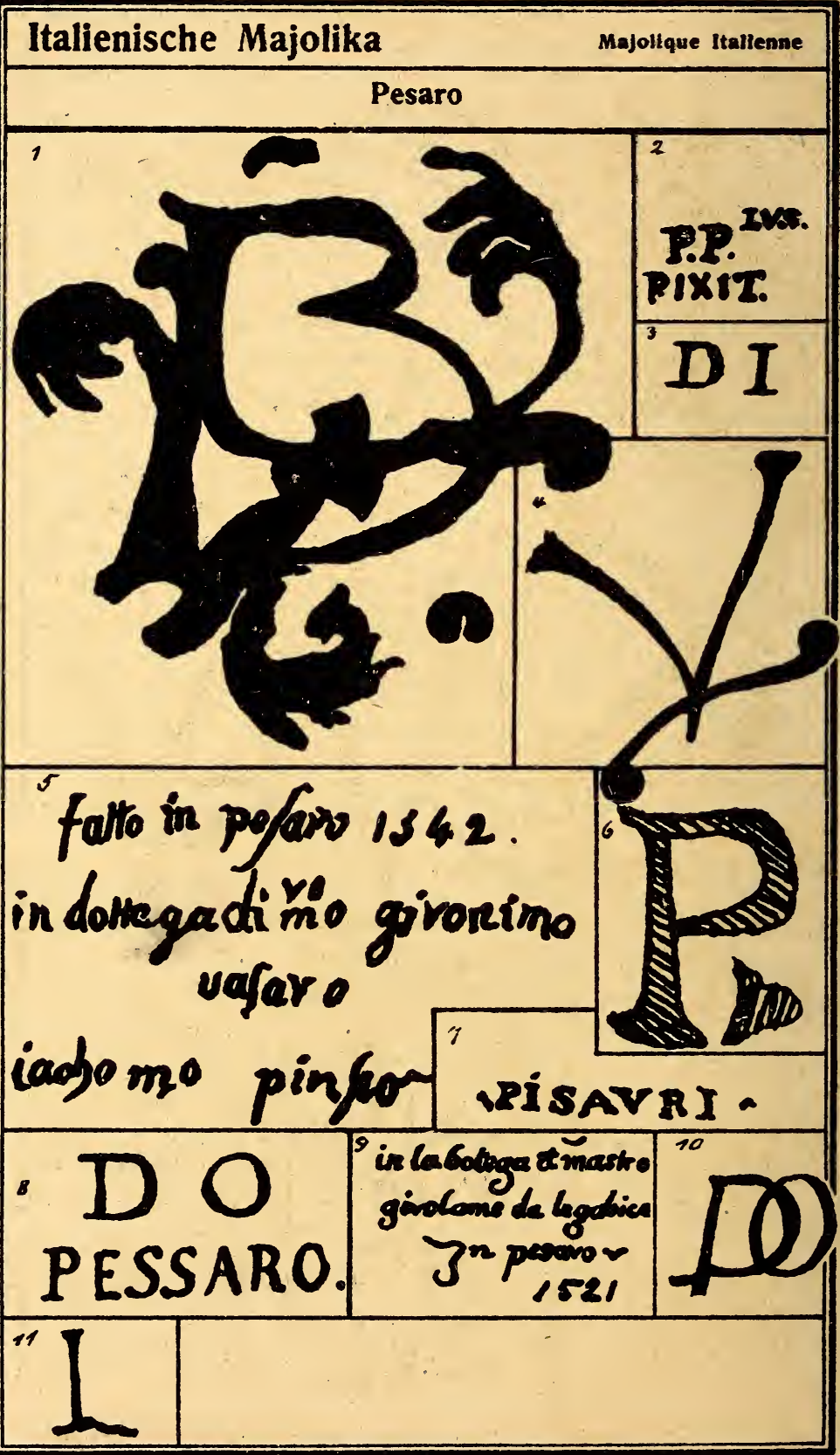




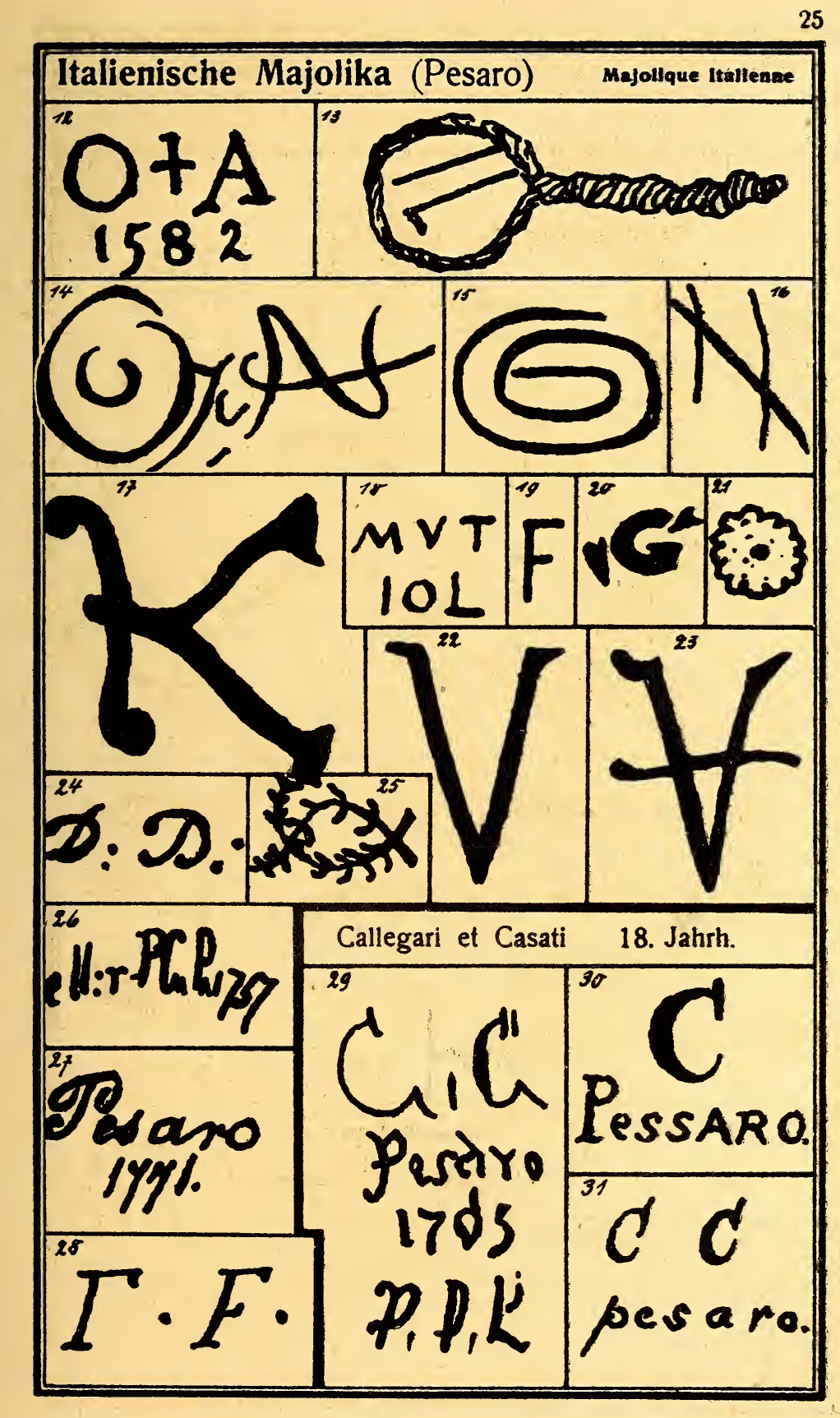




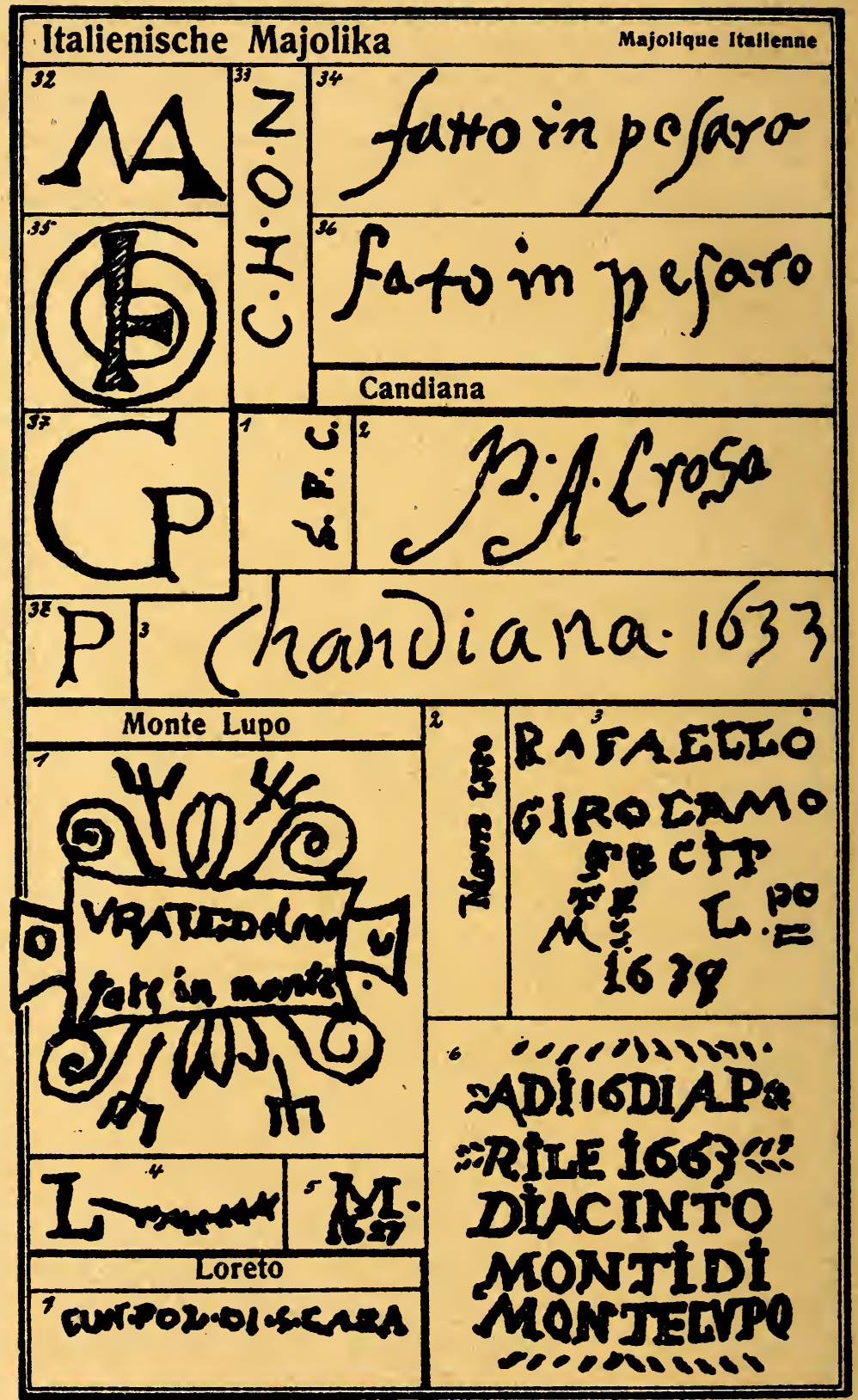


27.

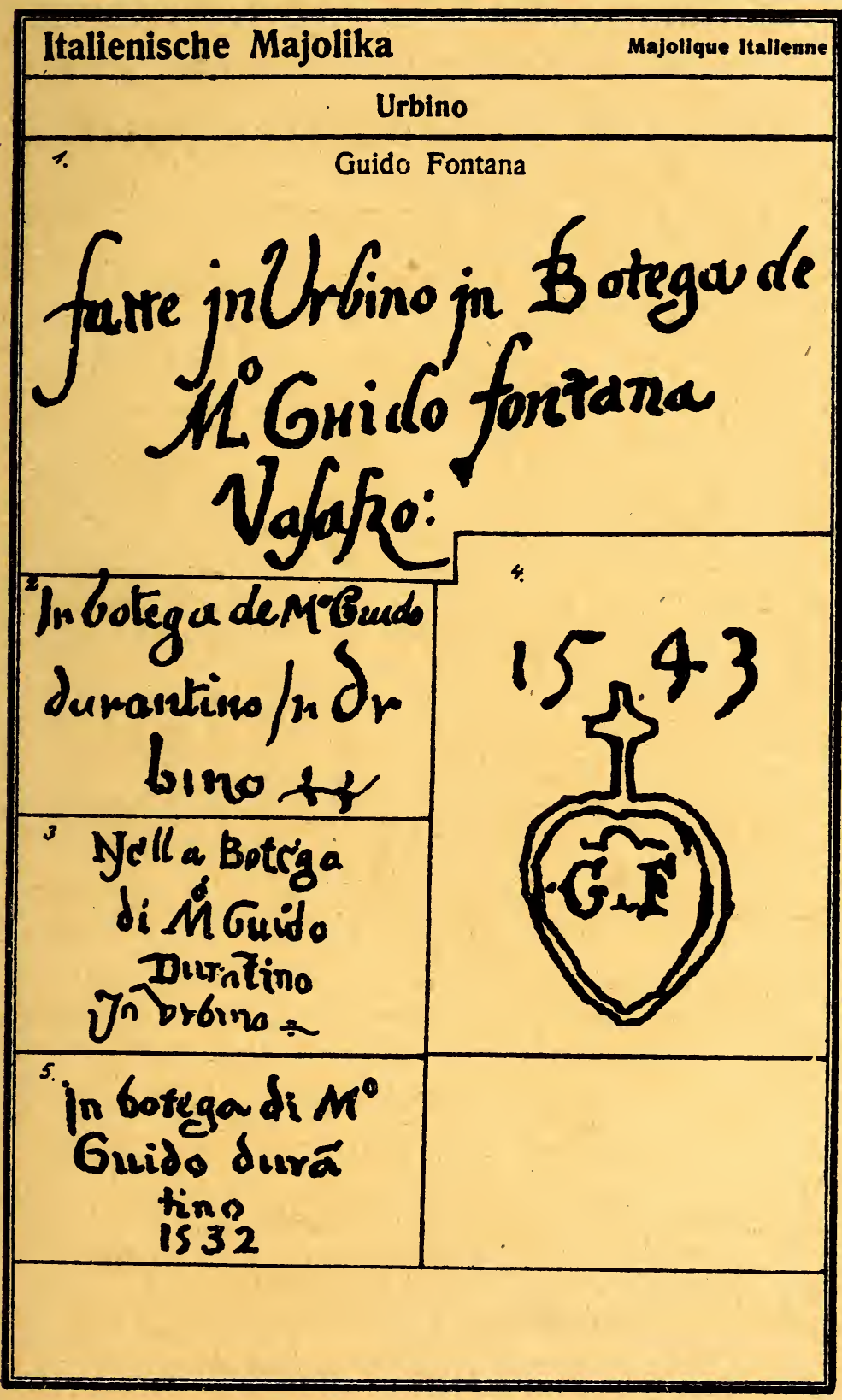




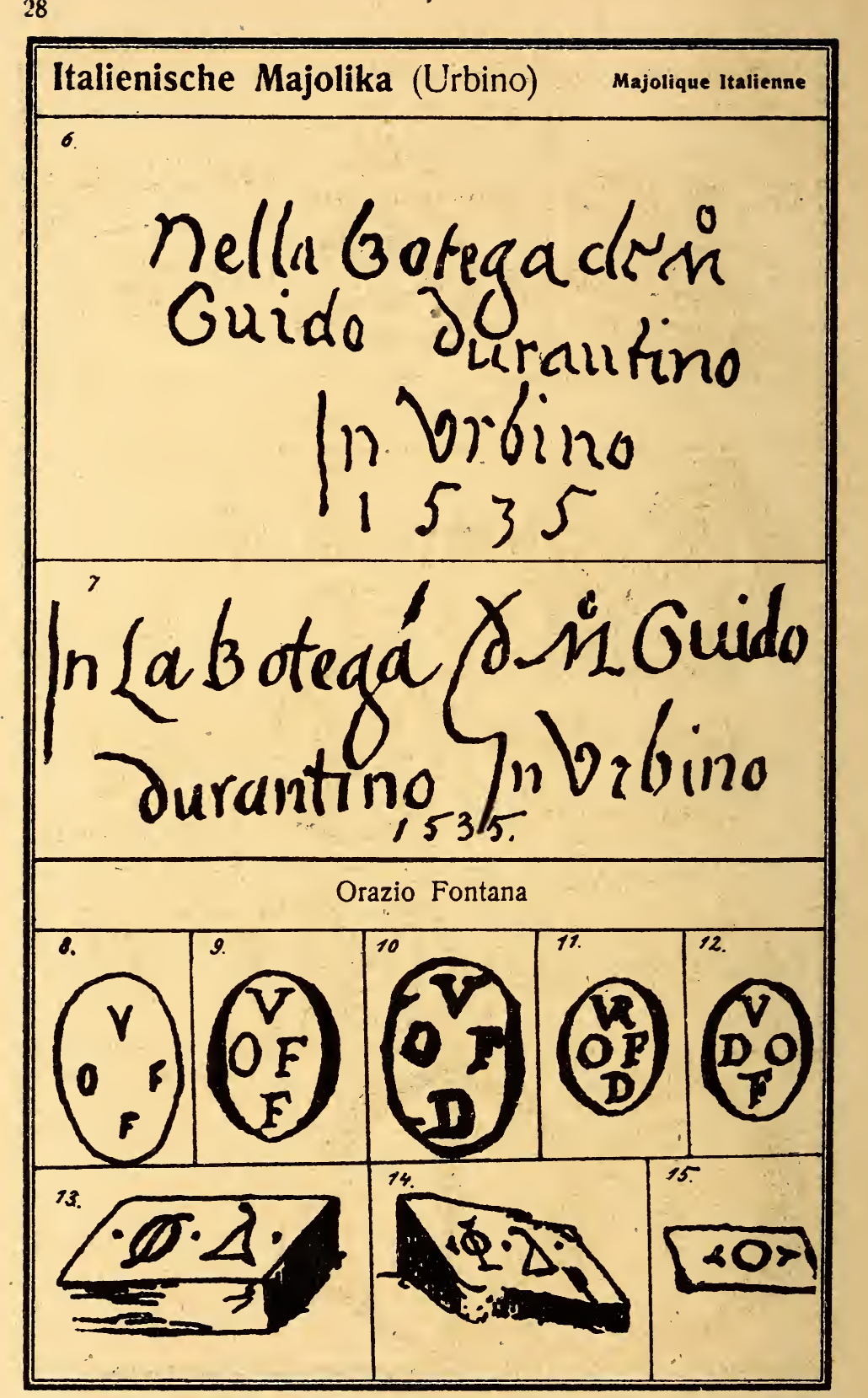




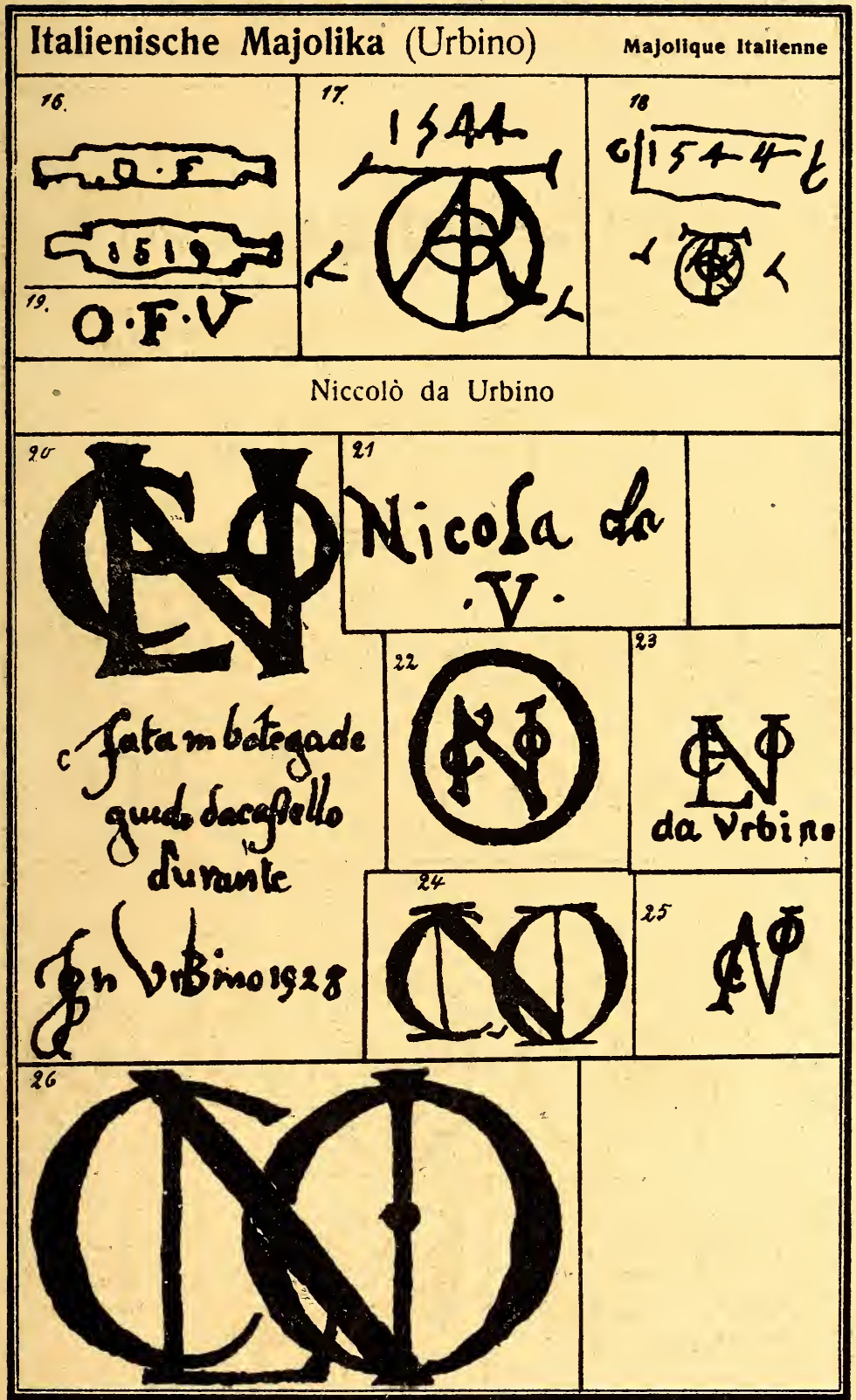




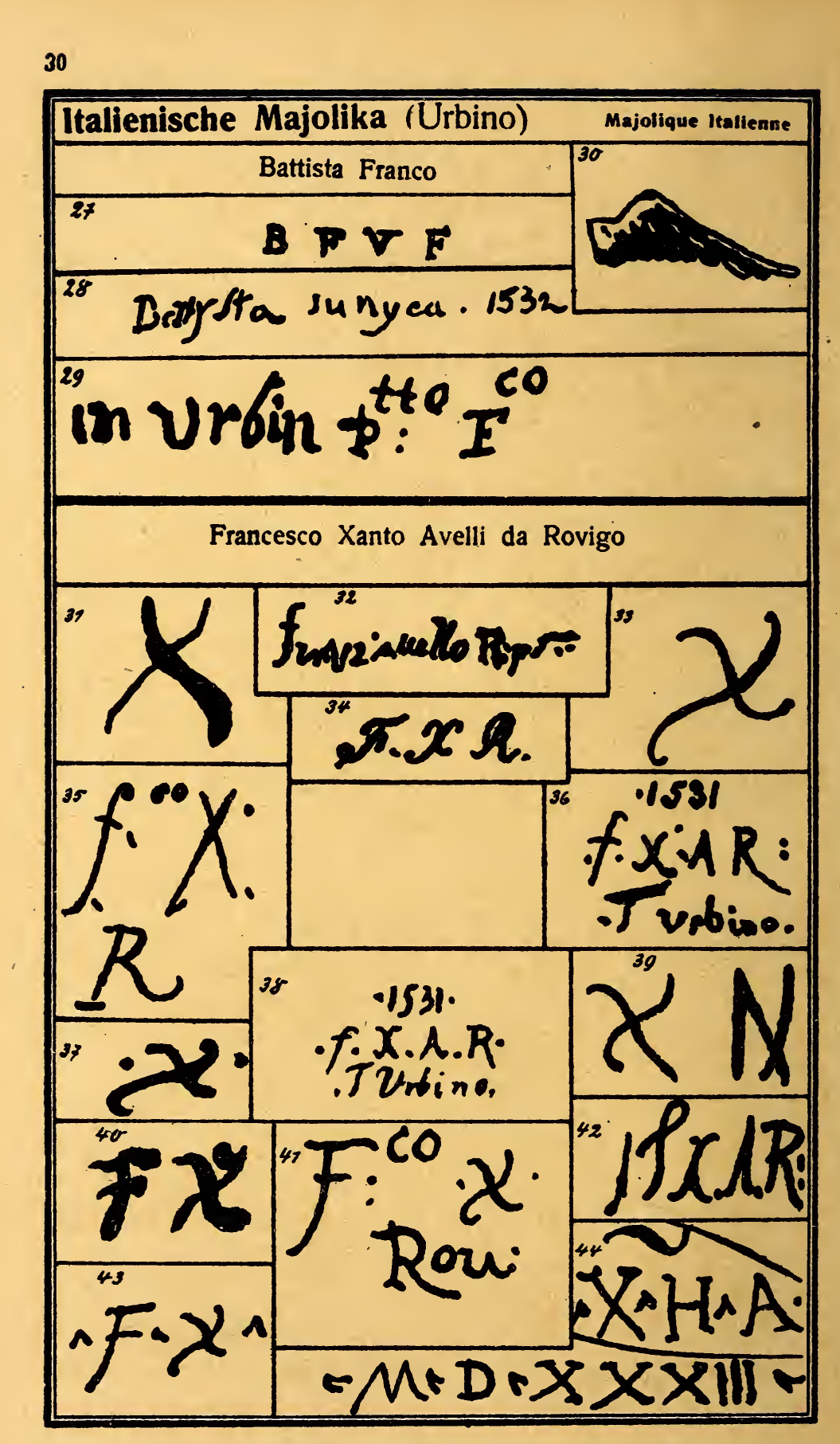




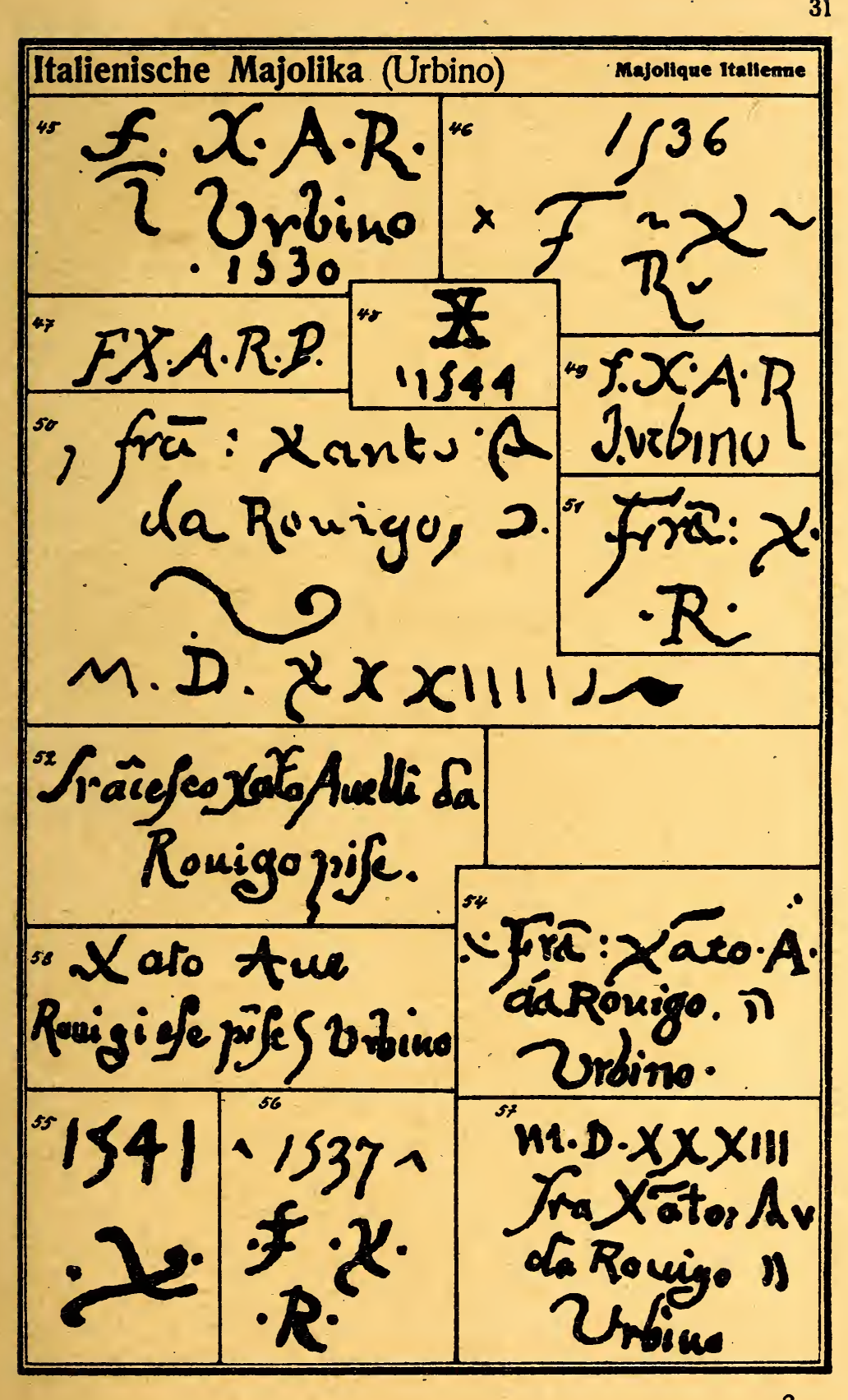




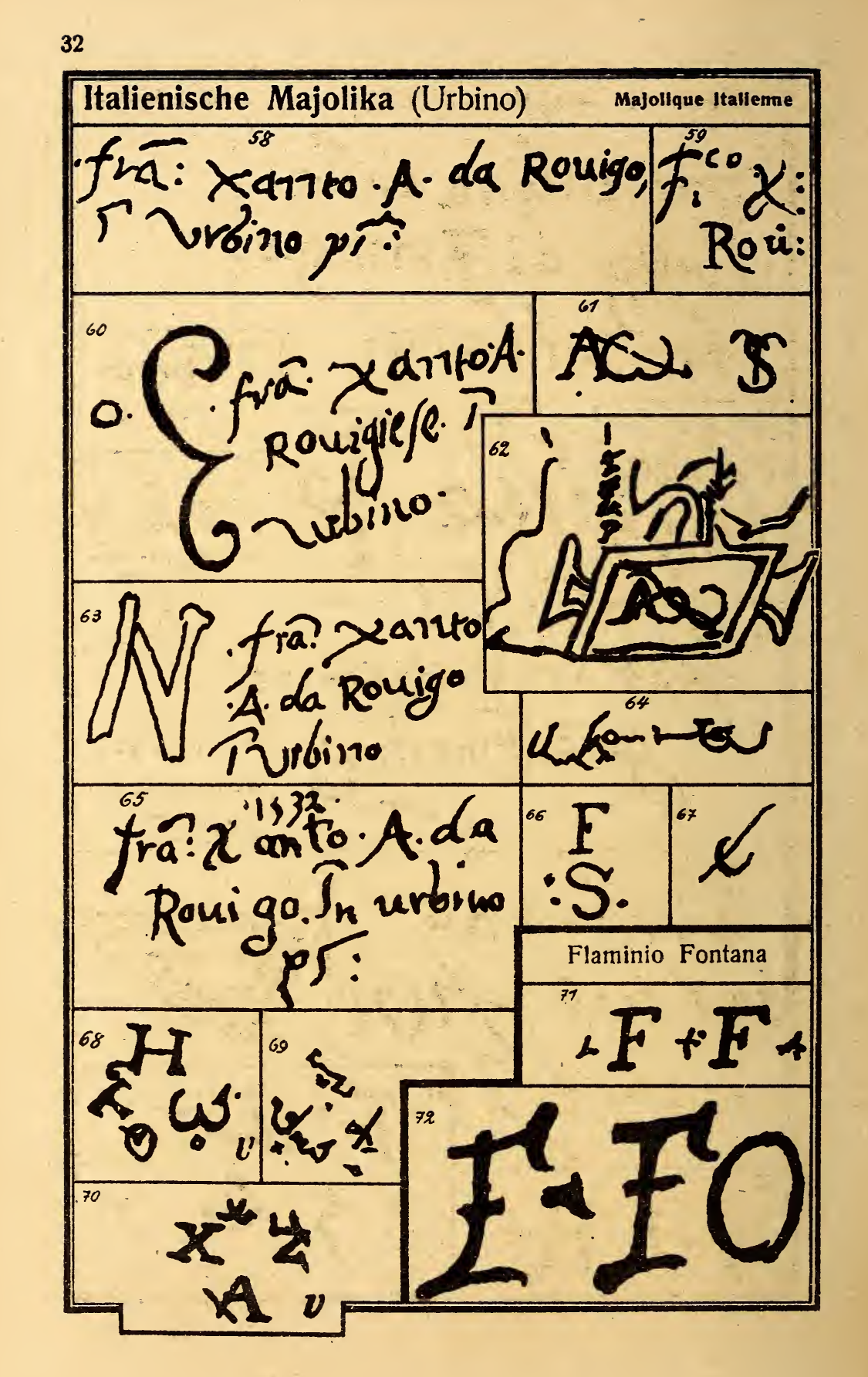




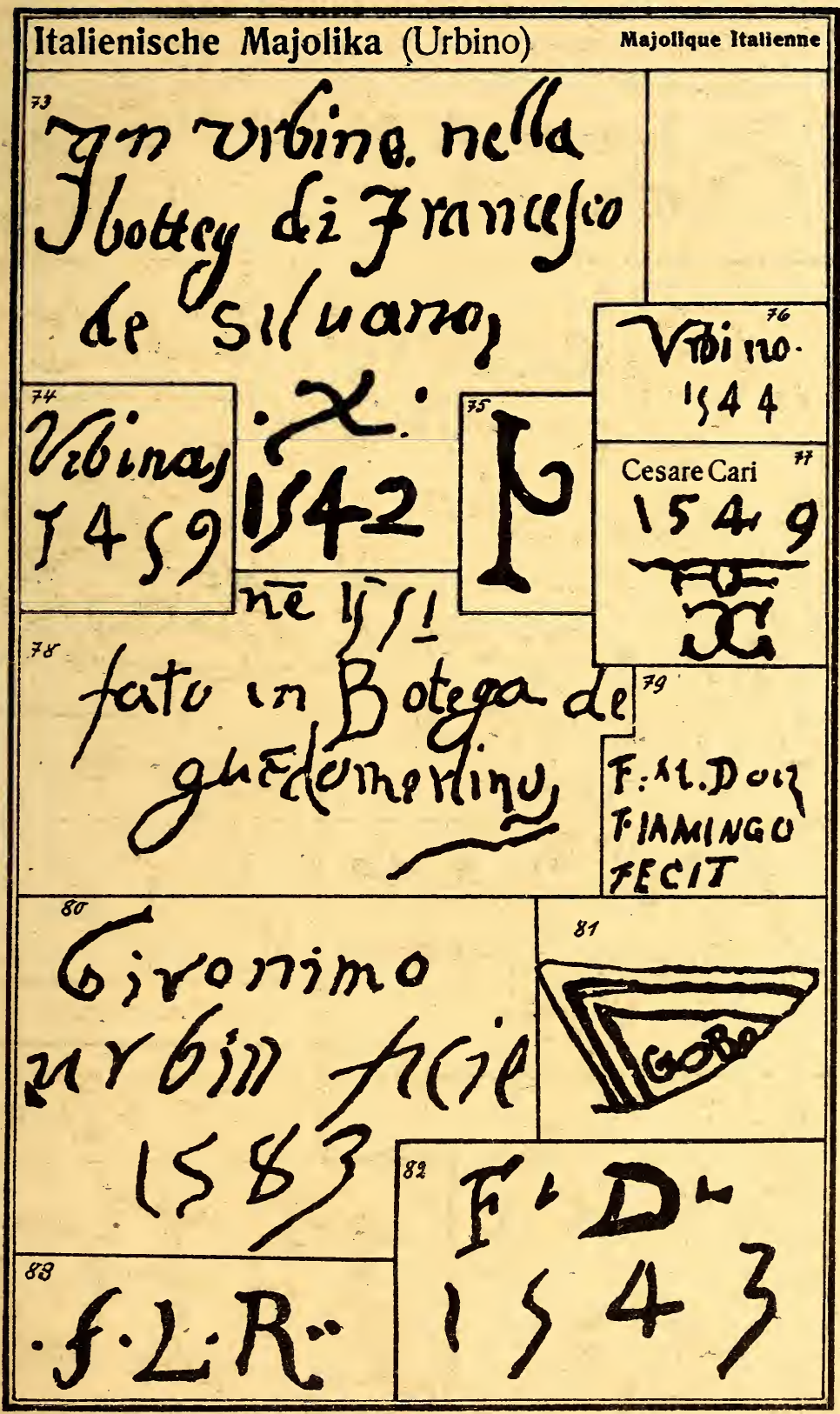




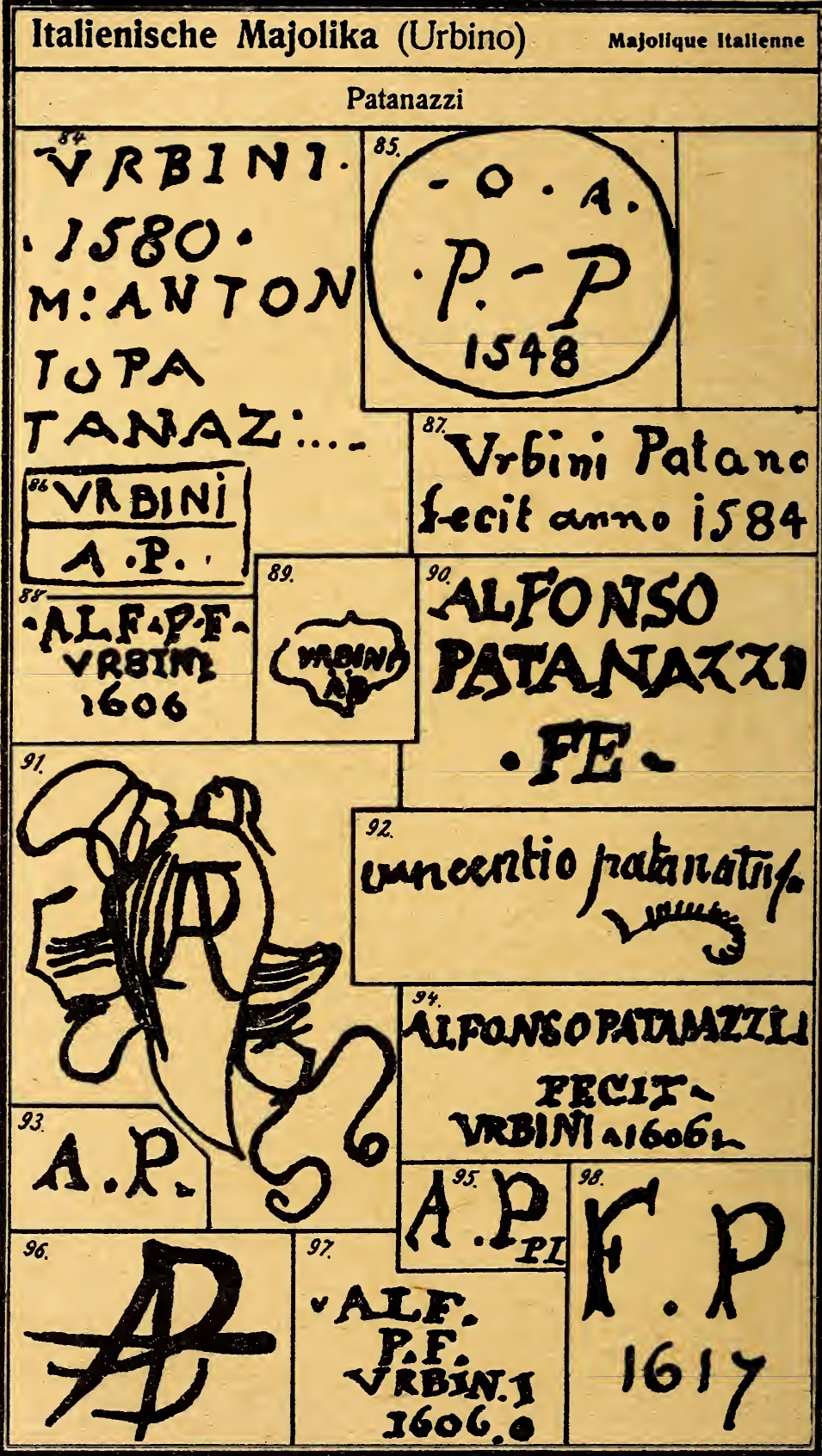




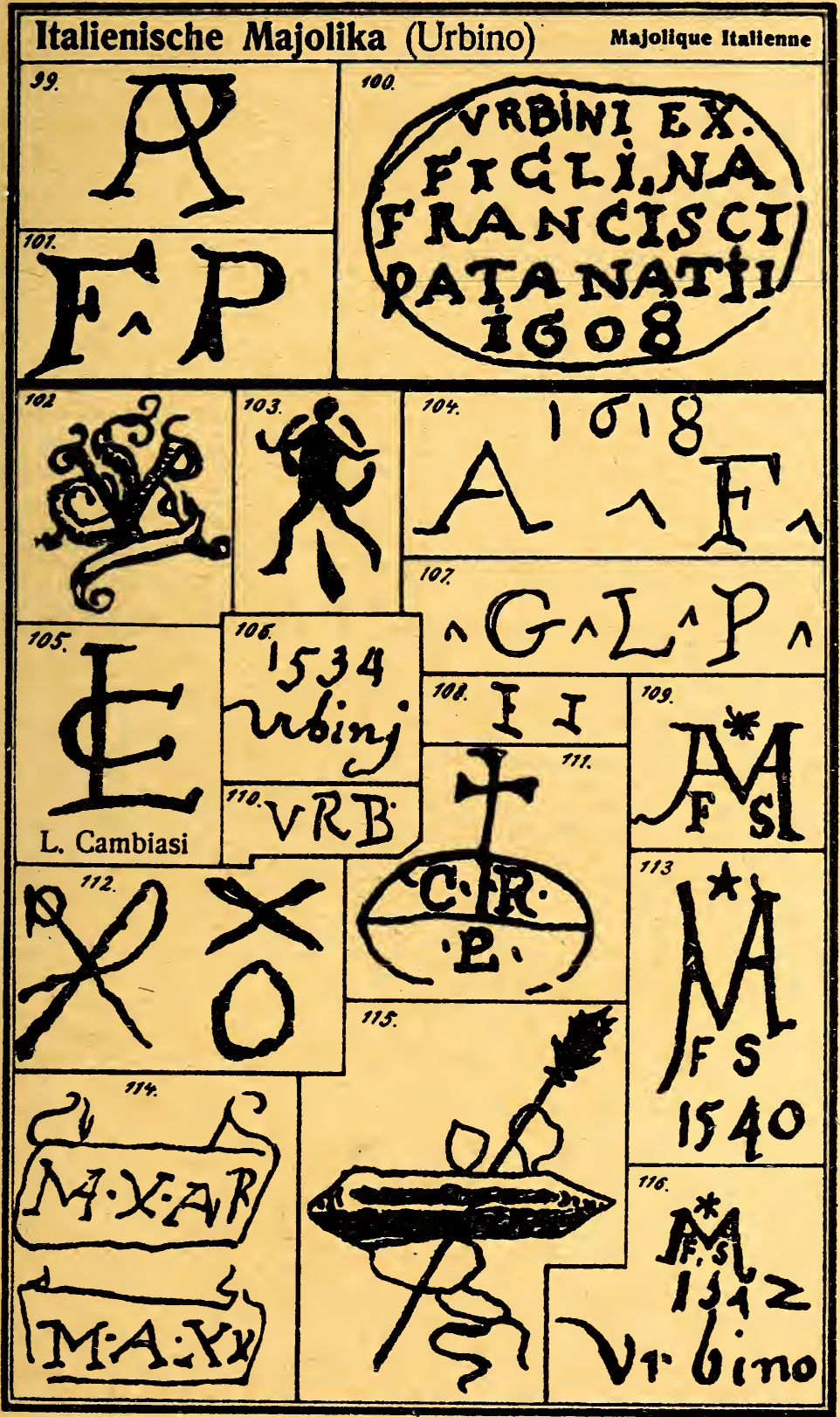




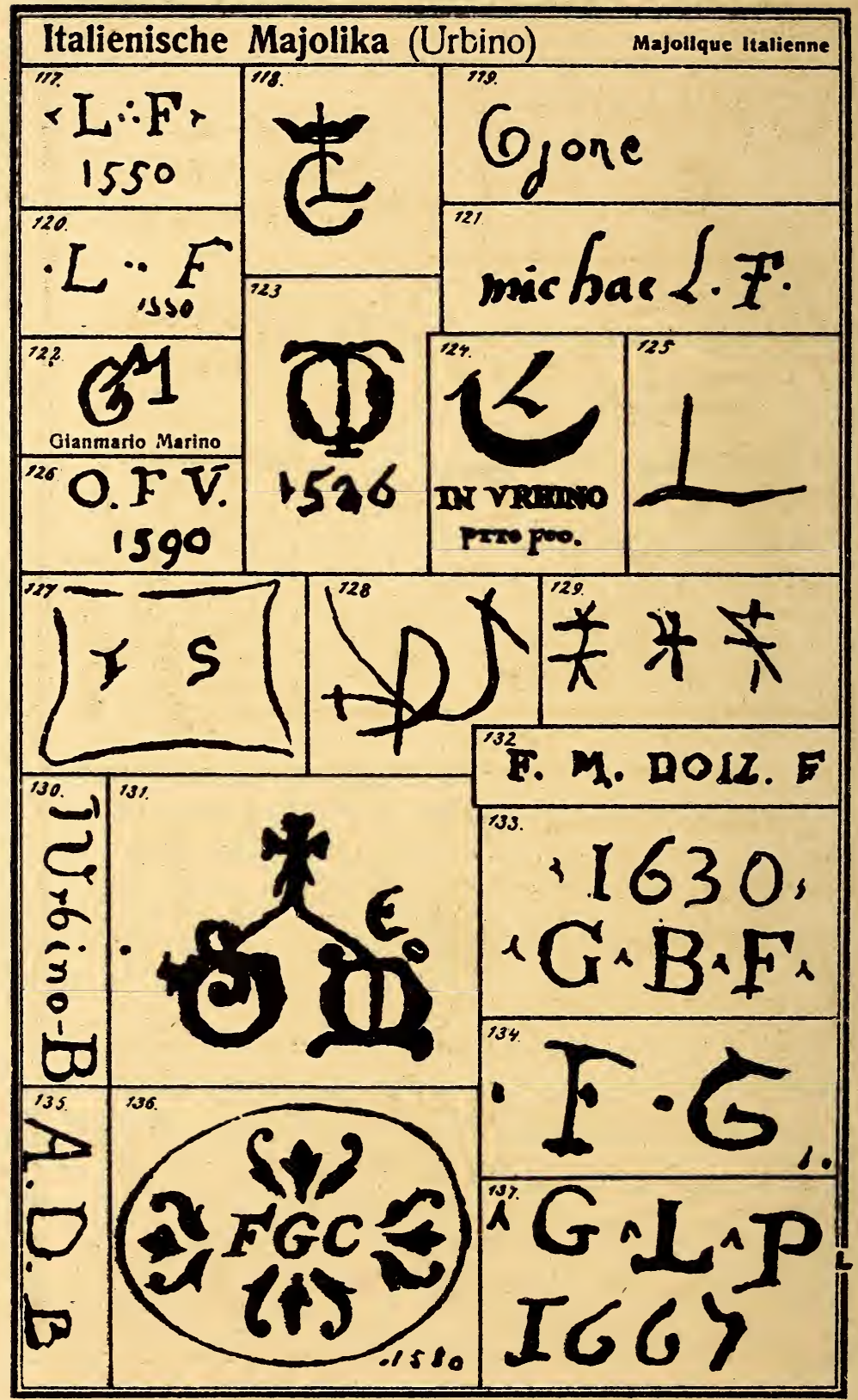




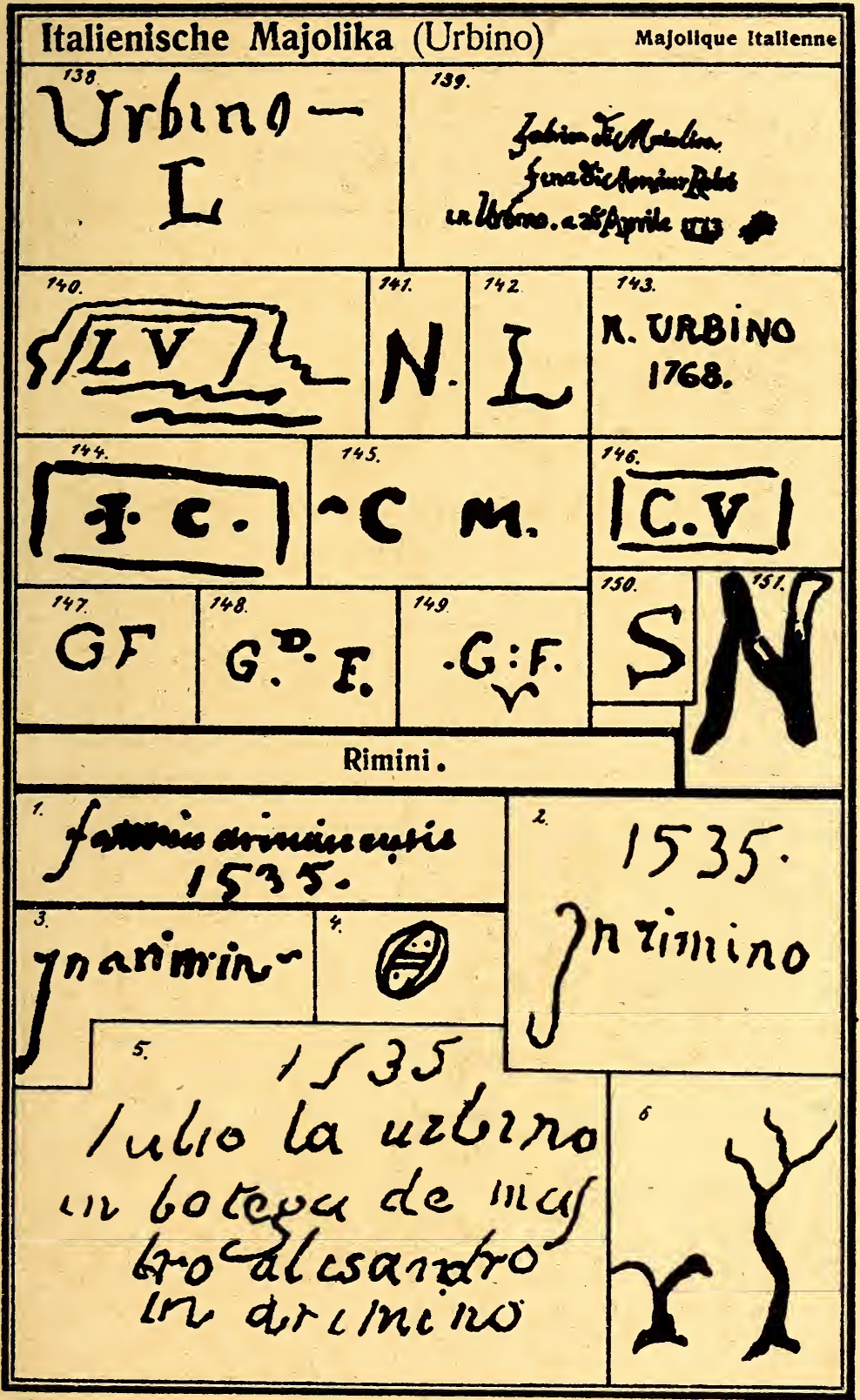




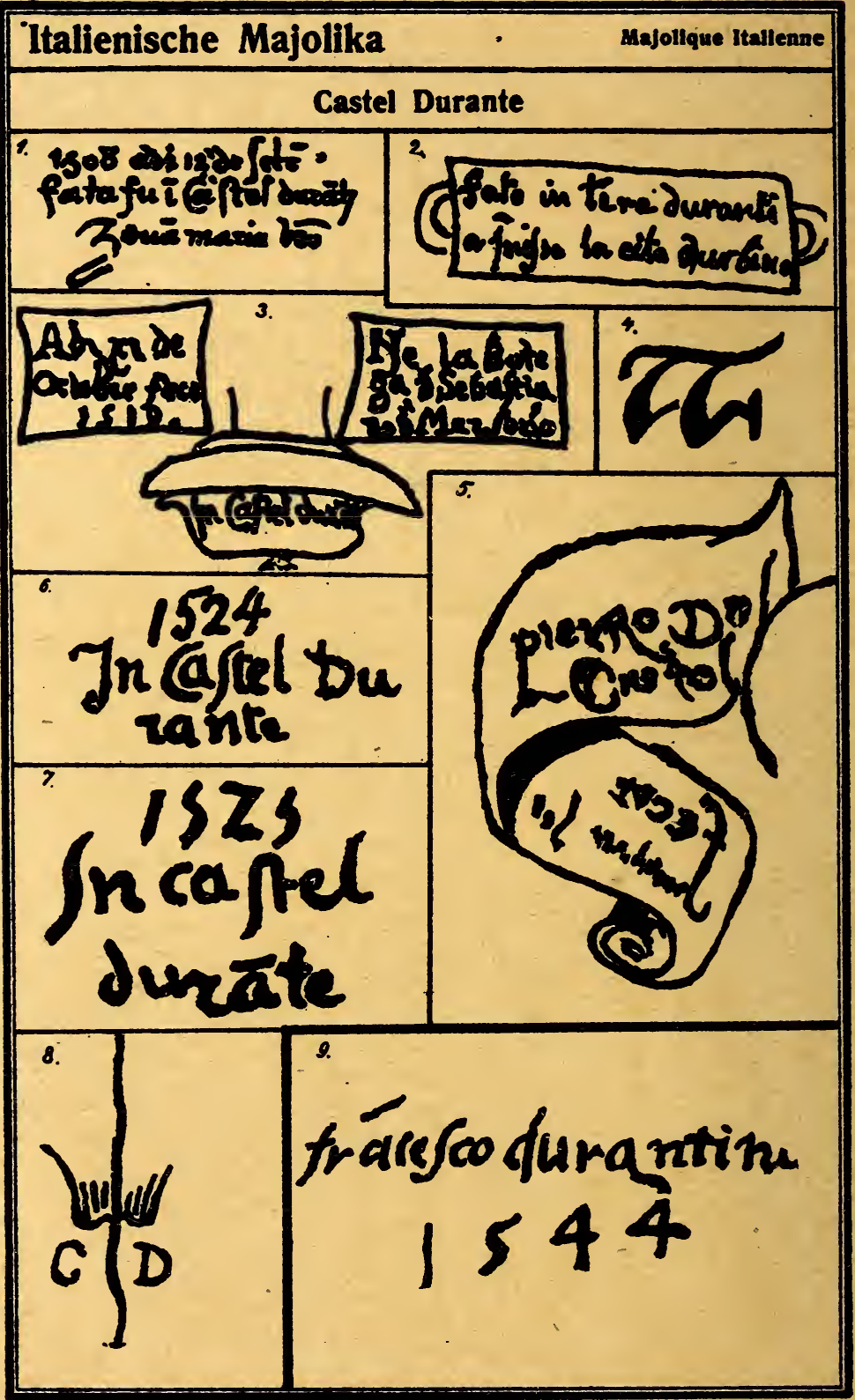




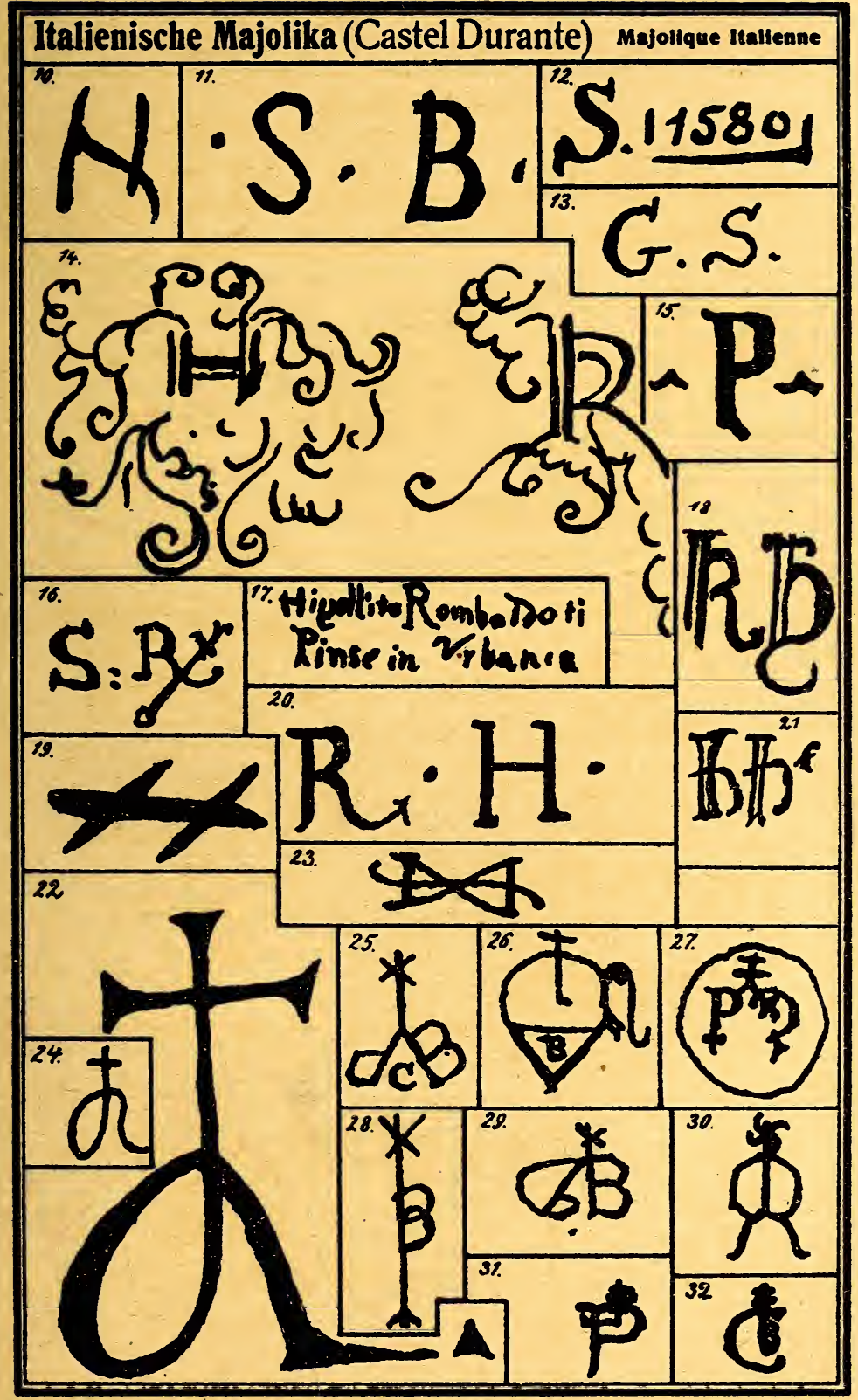




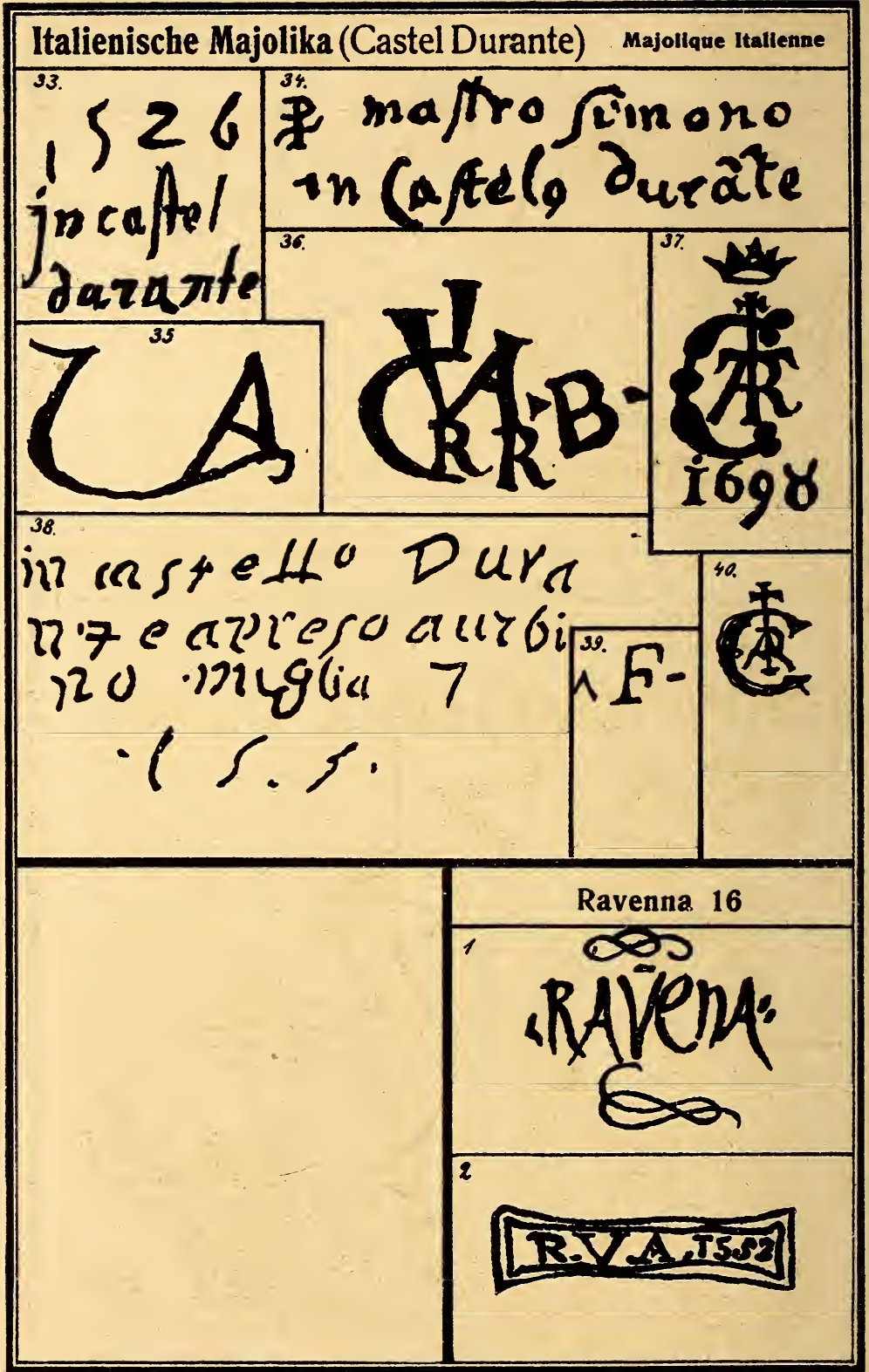




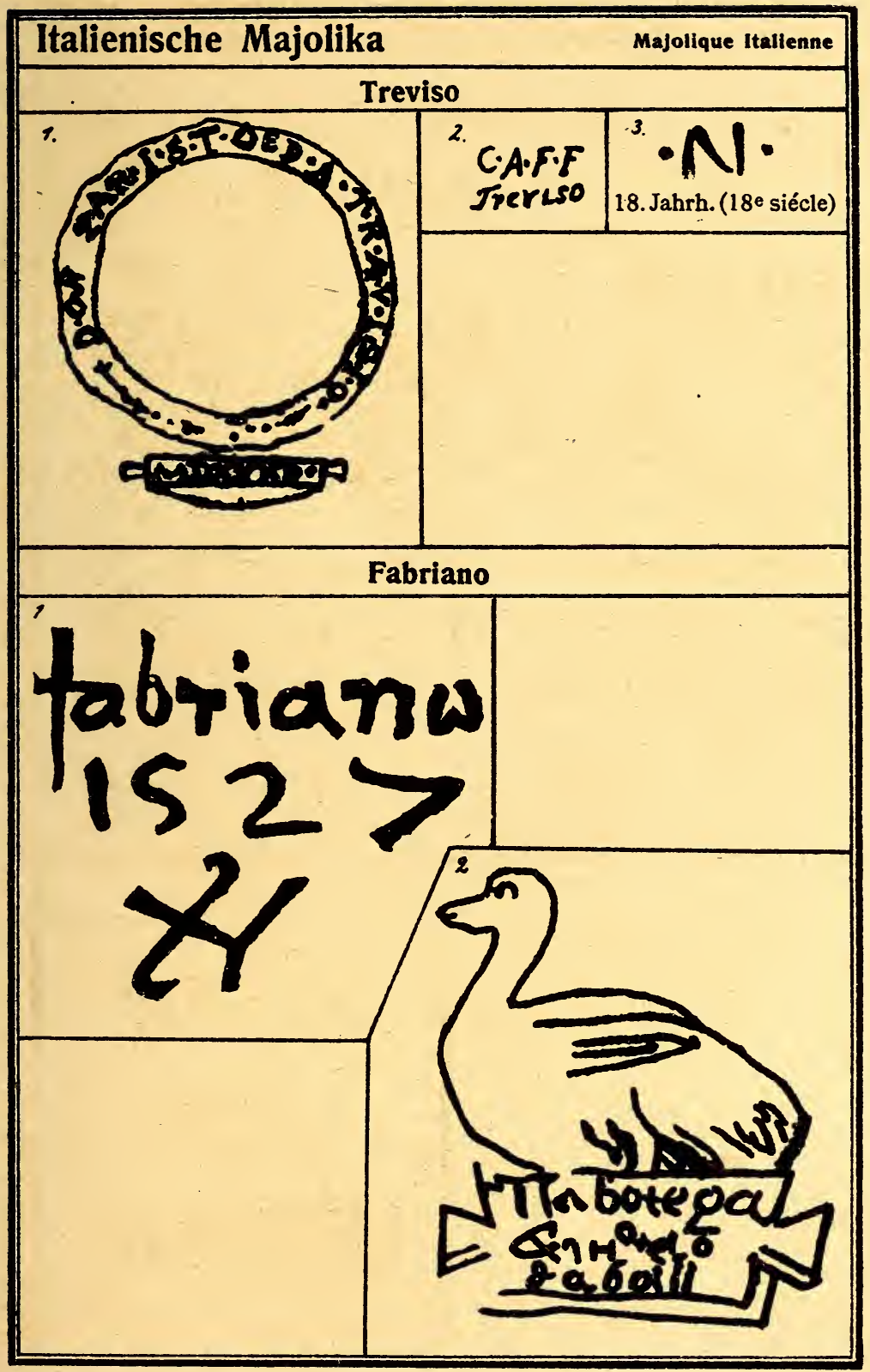




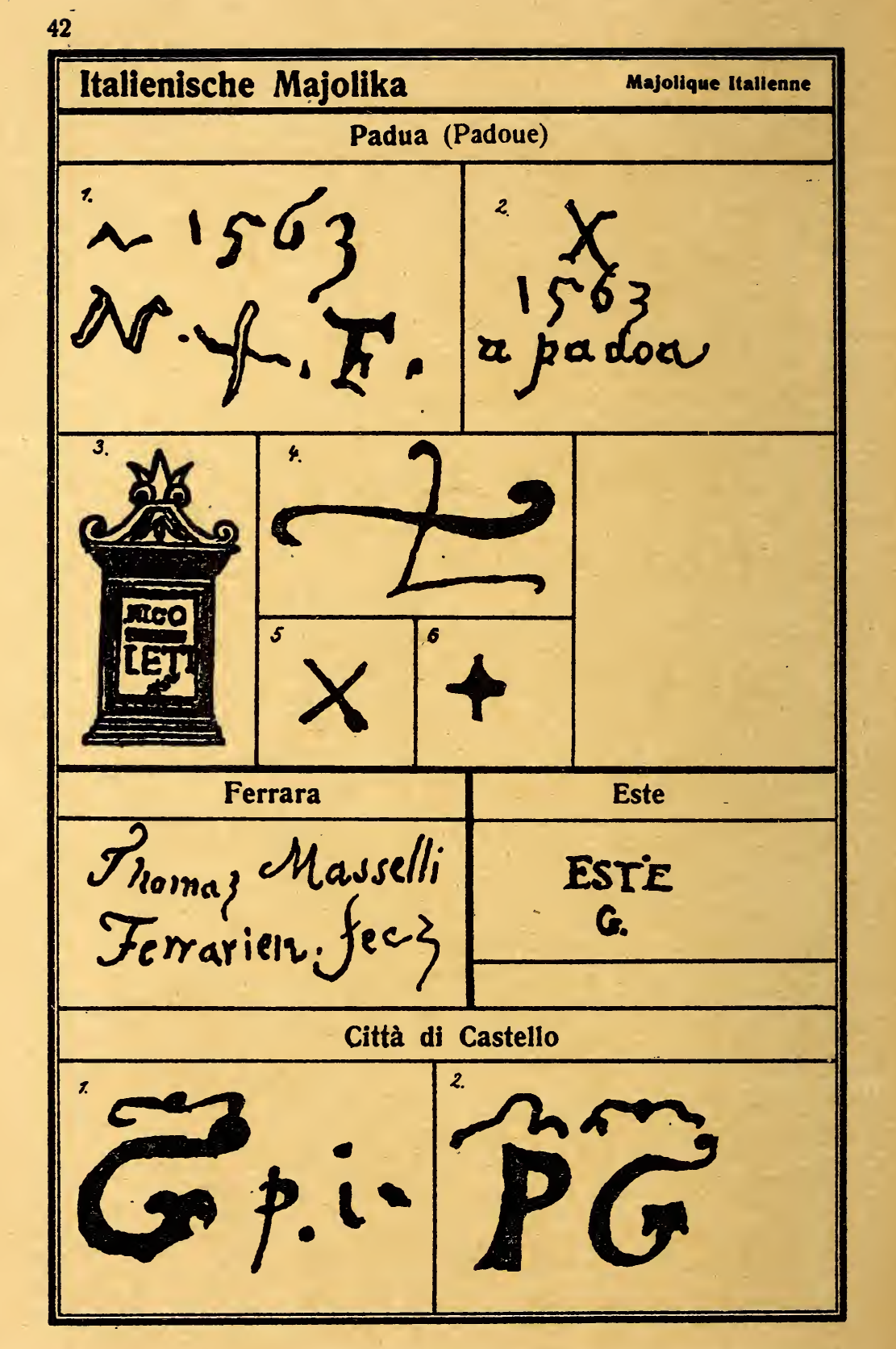




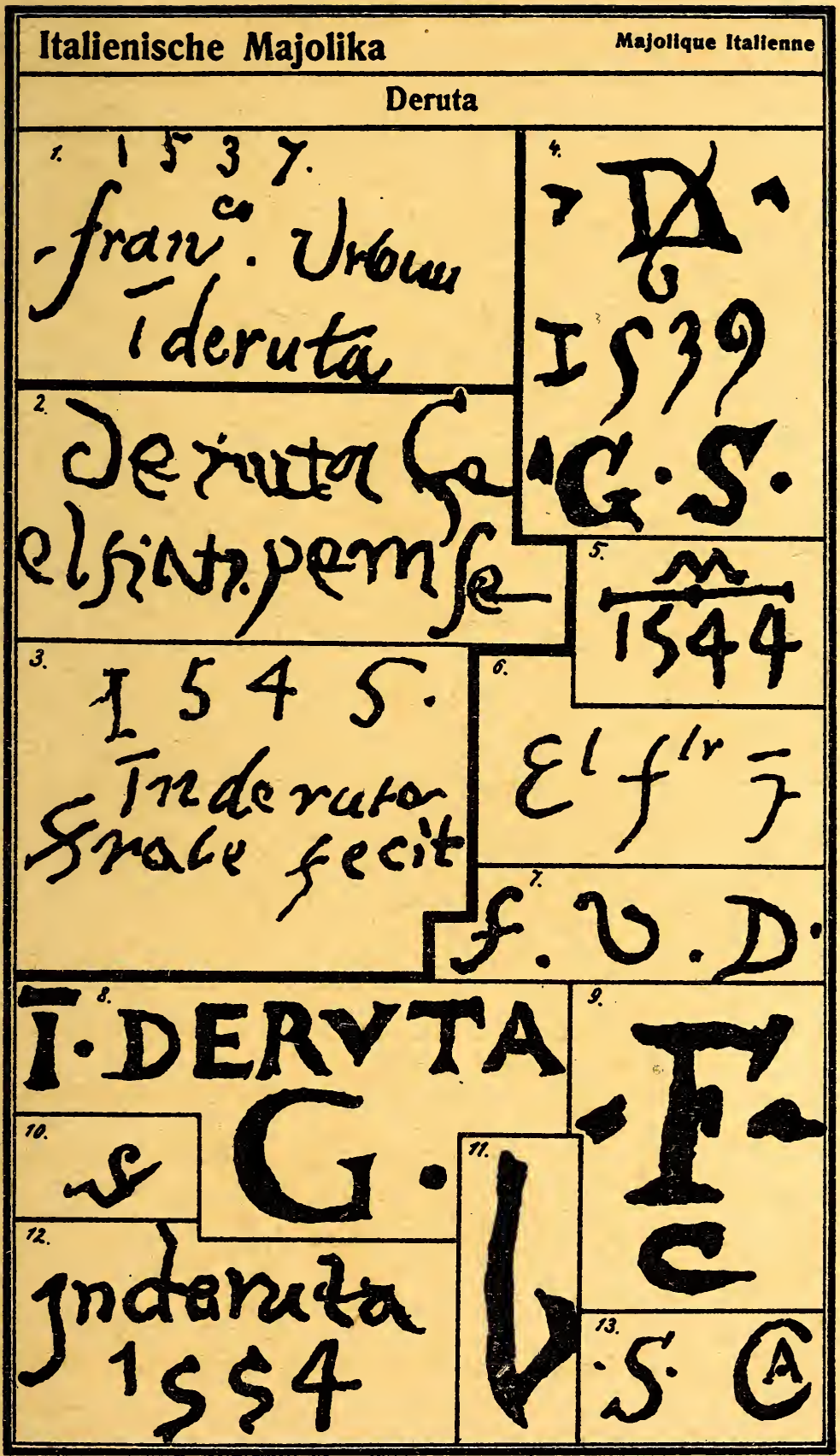




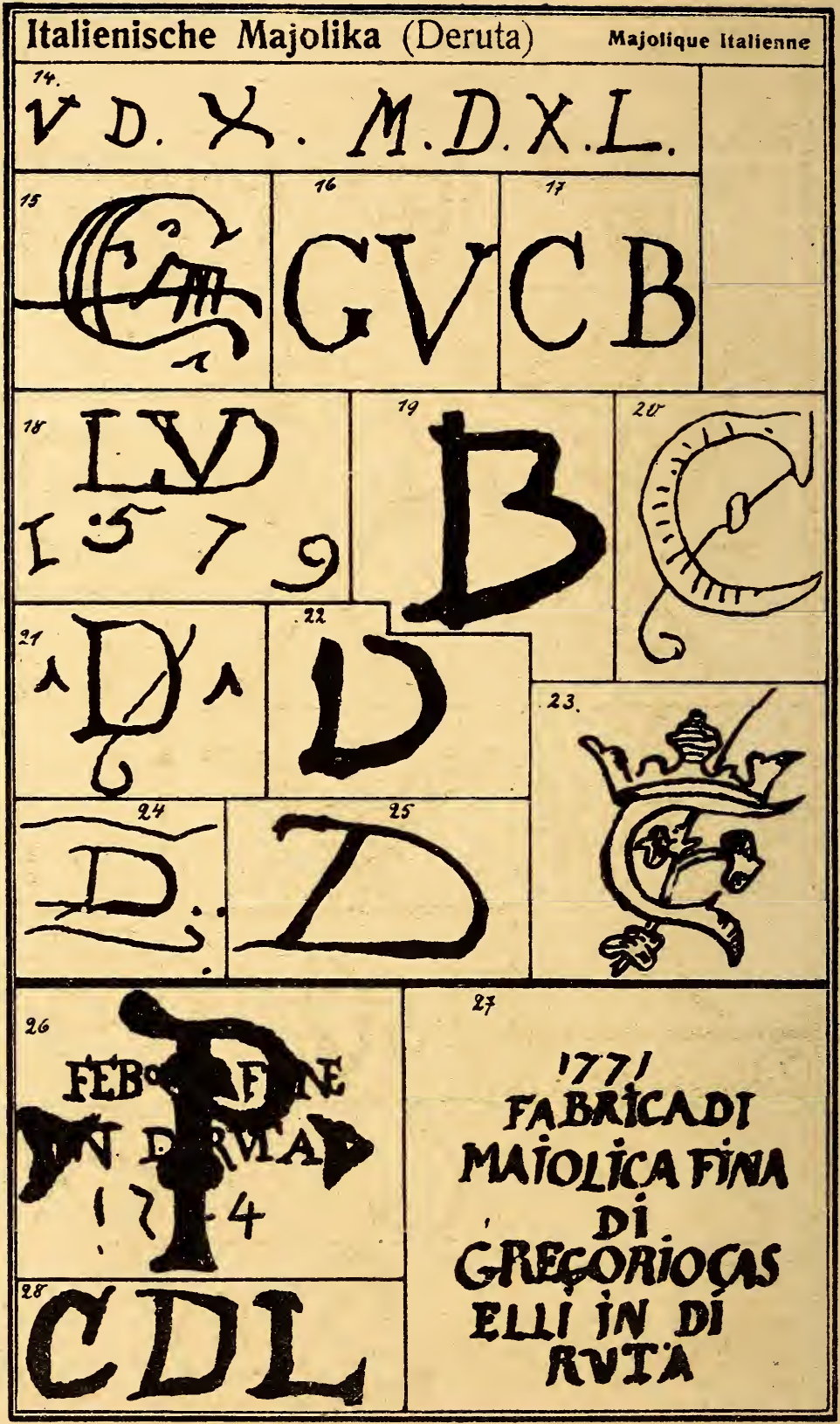


Italienische Majolika

Majolique Itallenne

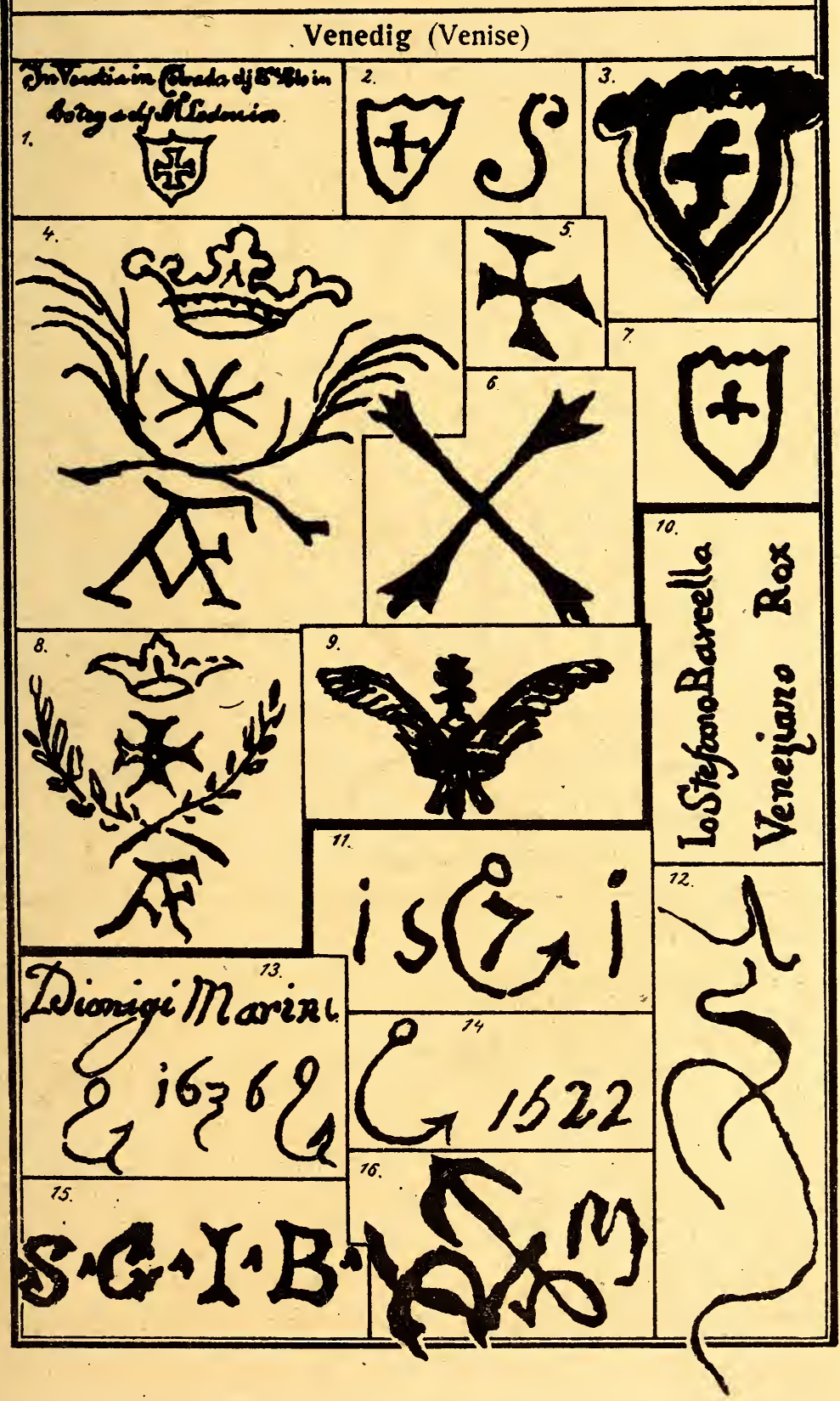




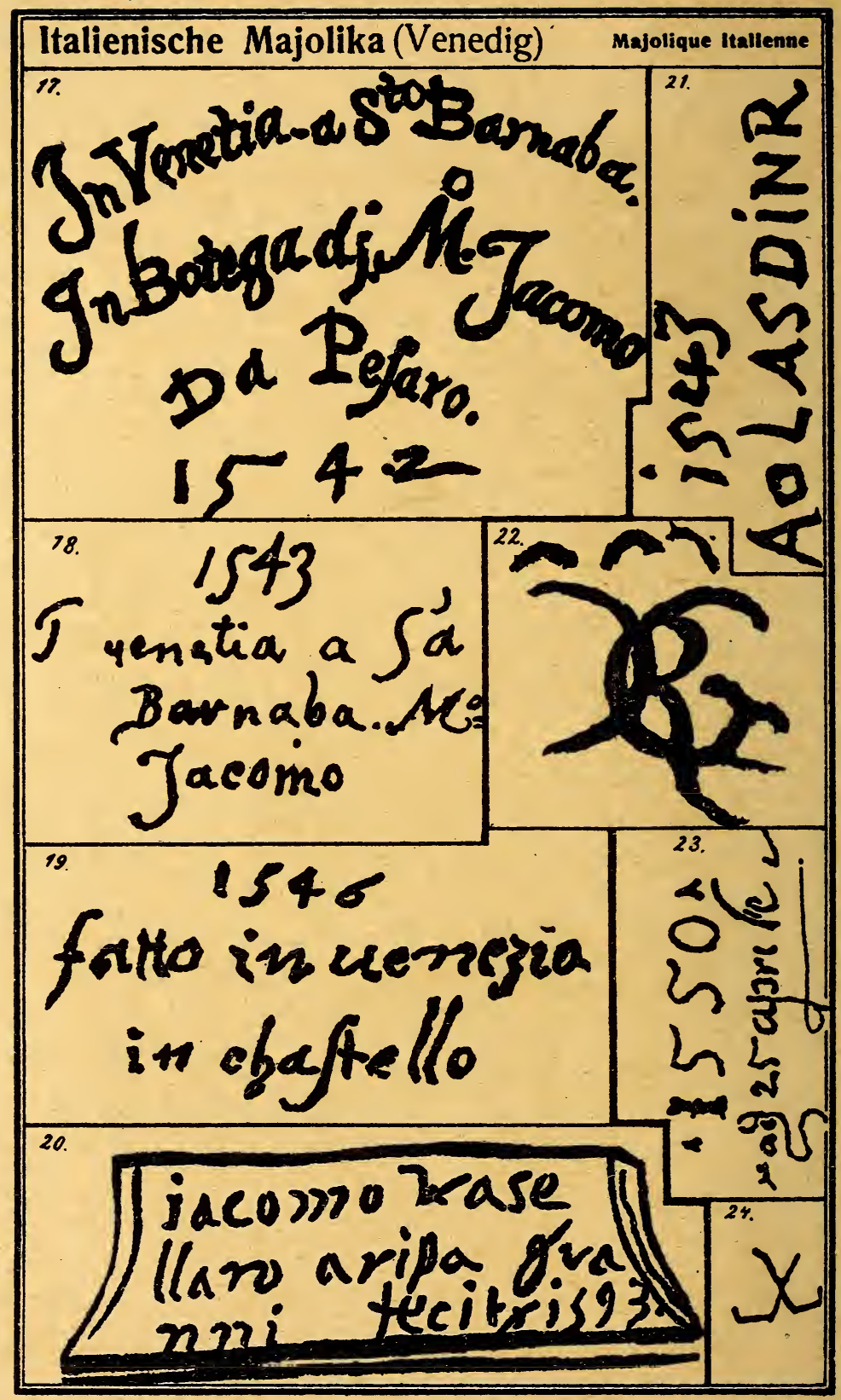




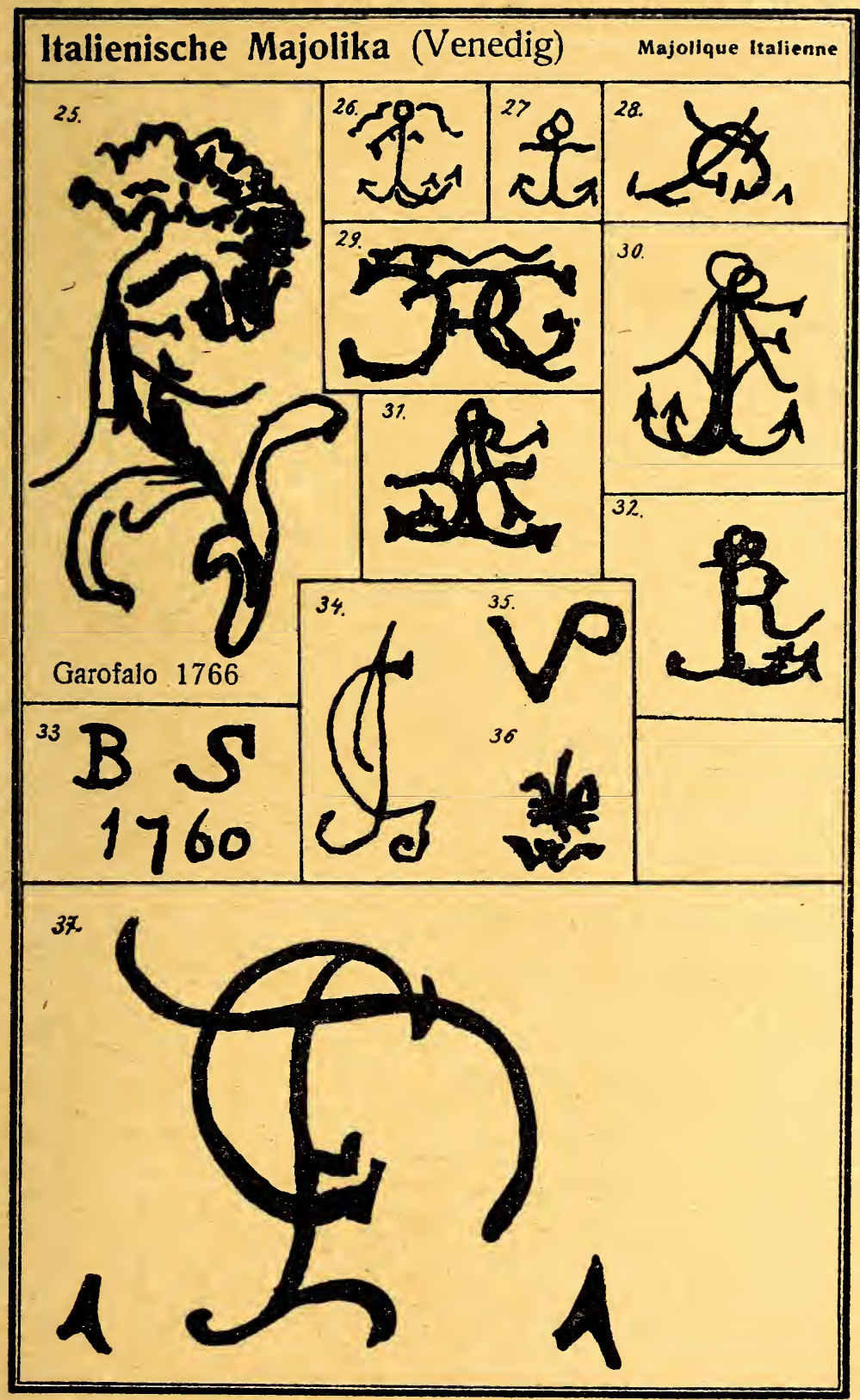




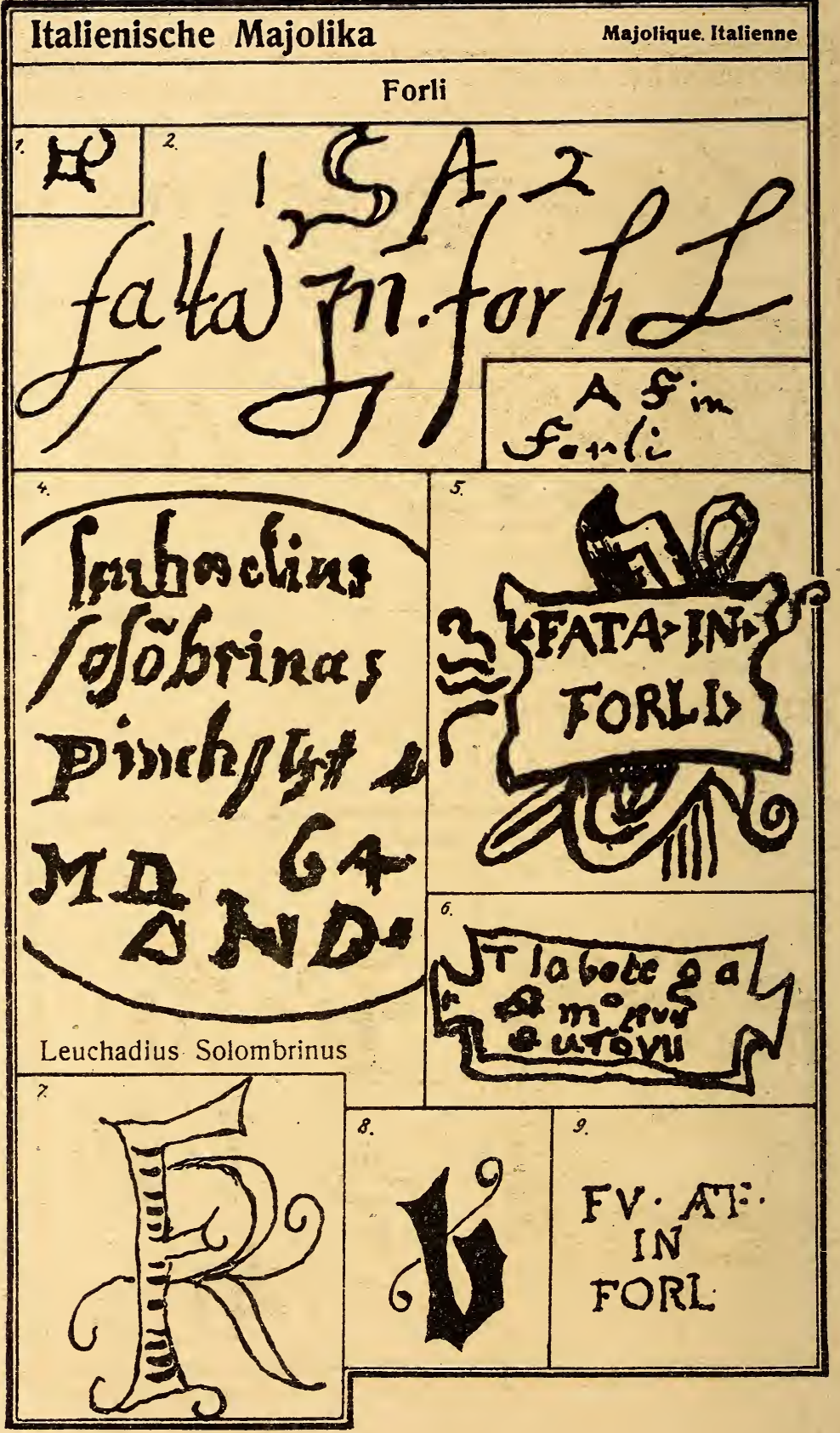




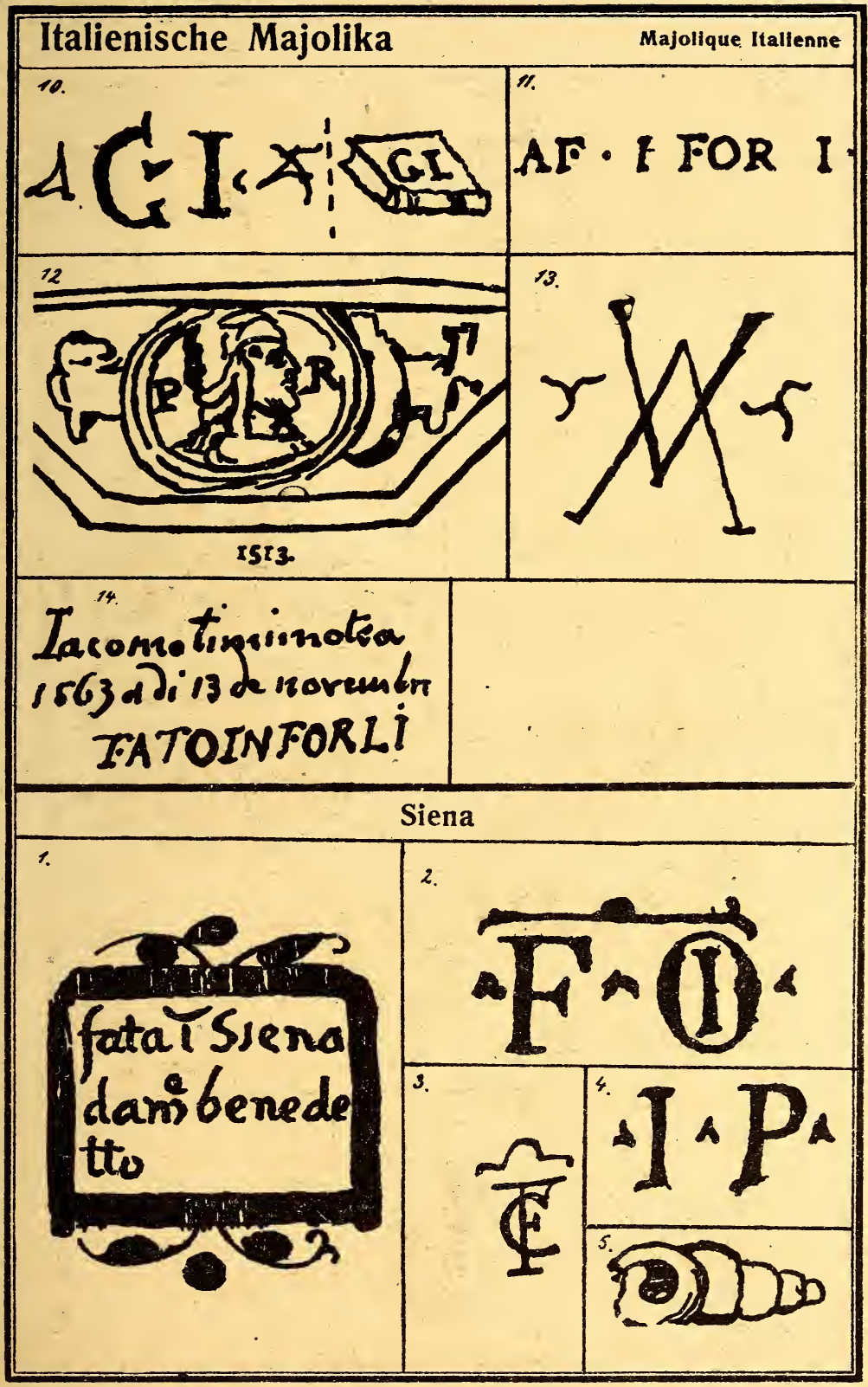


Italienische Majolika

$\sigma$.

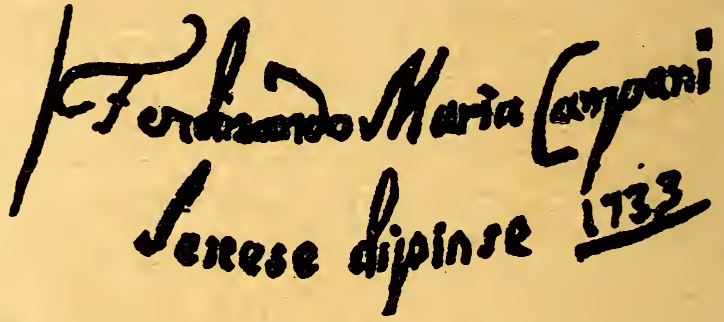

San Quirico (Siena)

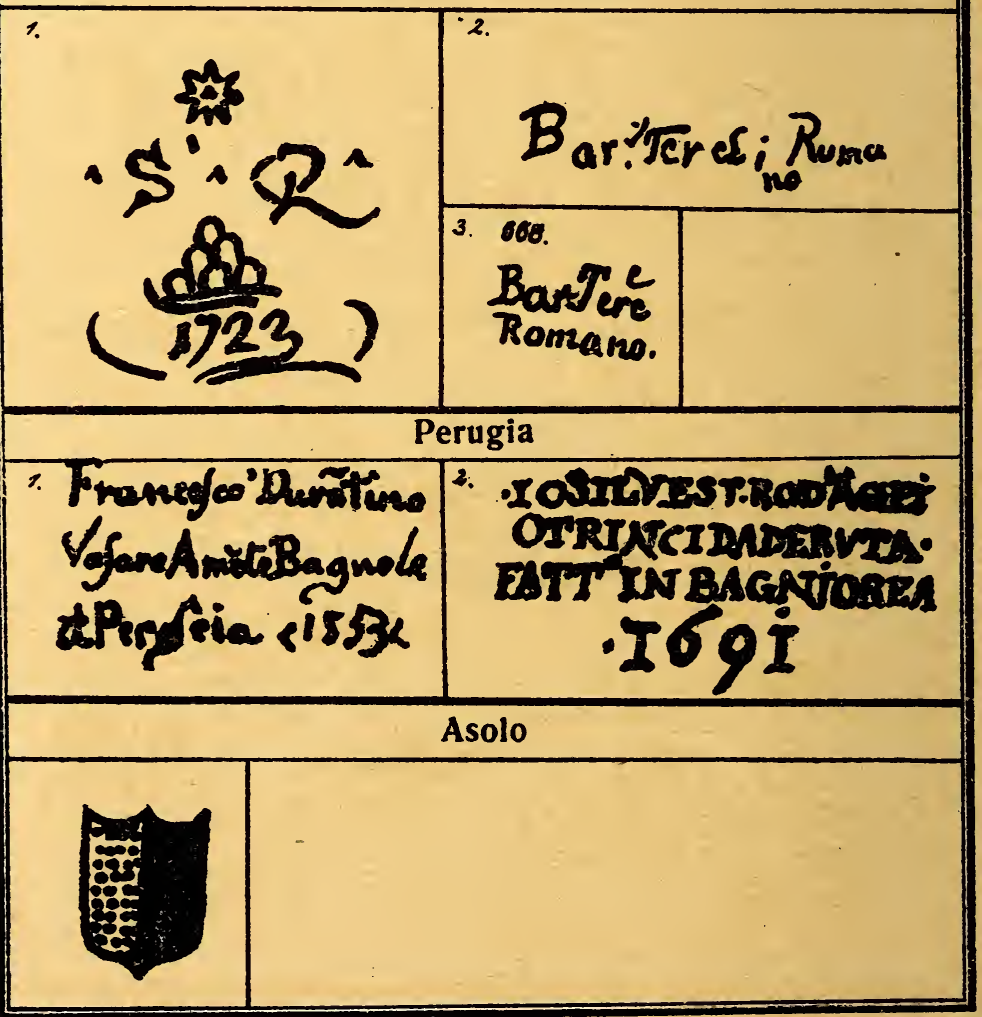


51

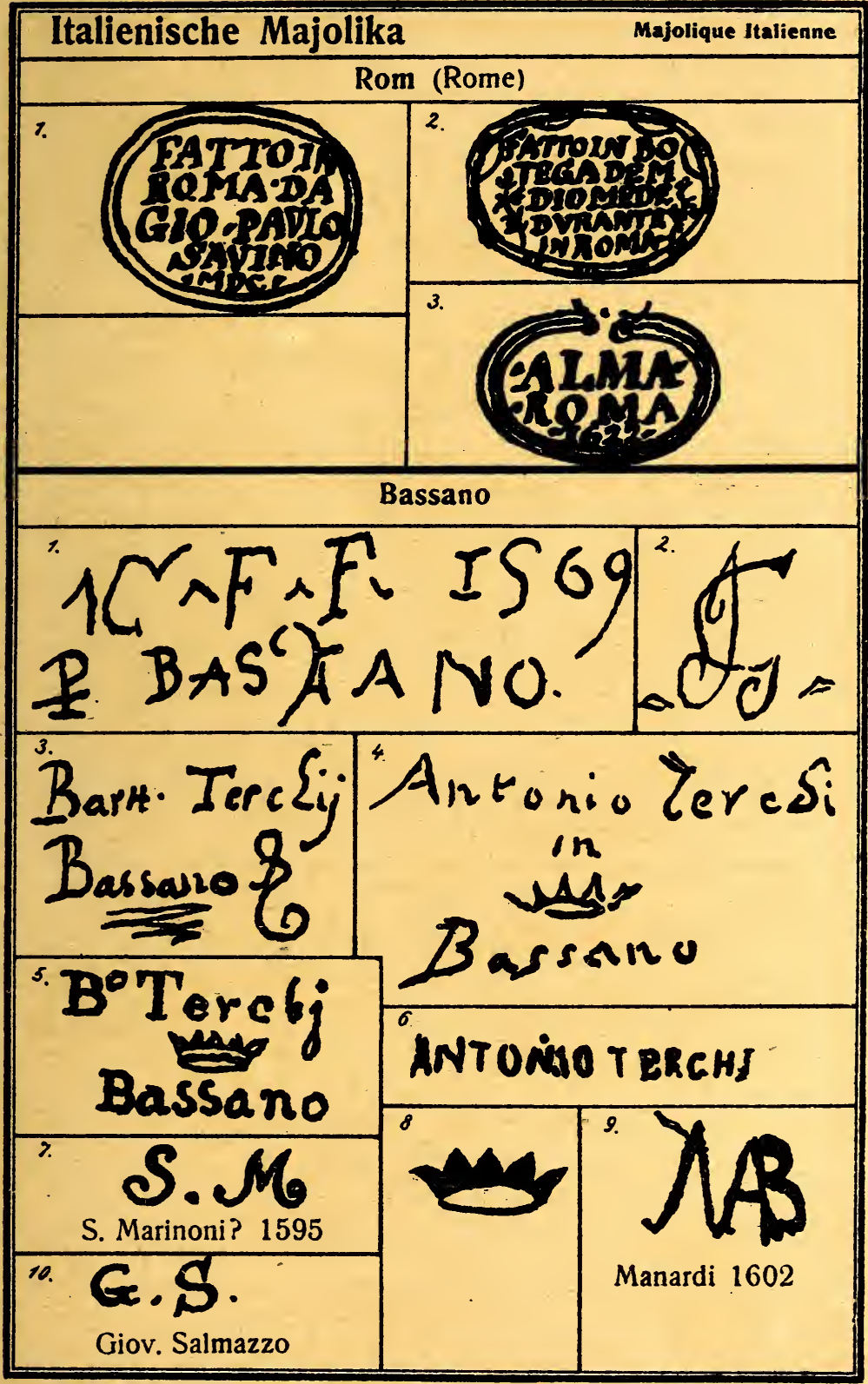


53

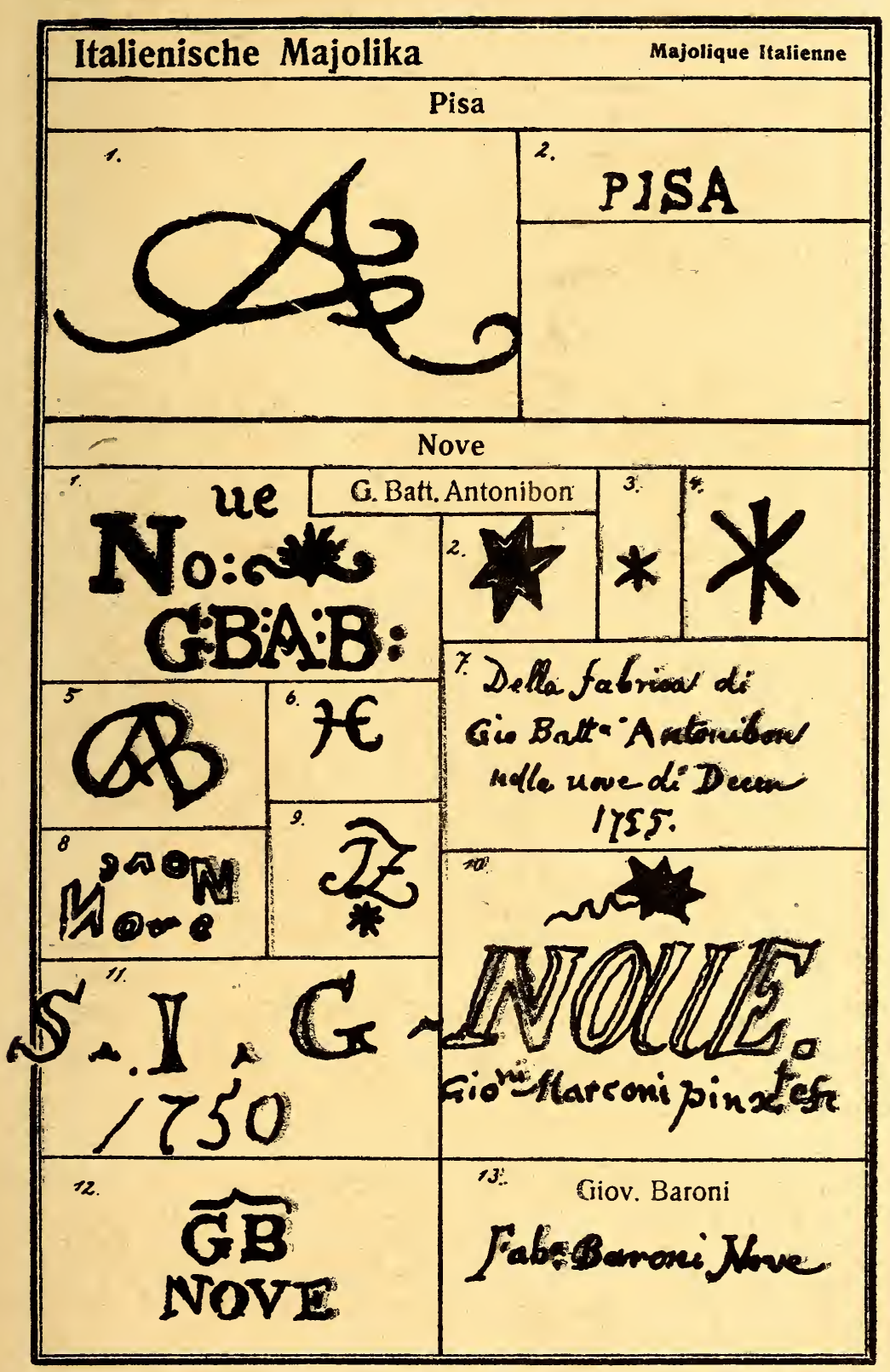


54

Italienische Majolika Majolique Italienne

\section{Borgo S. Sepolcro}

CEOBNTAOMER CAM

$$
1649
$$
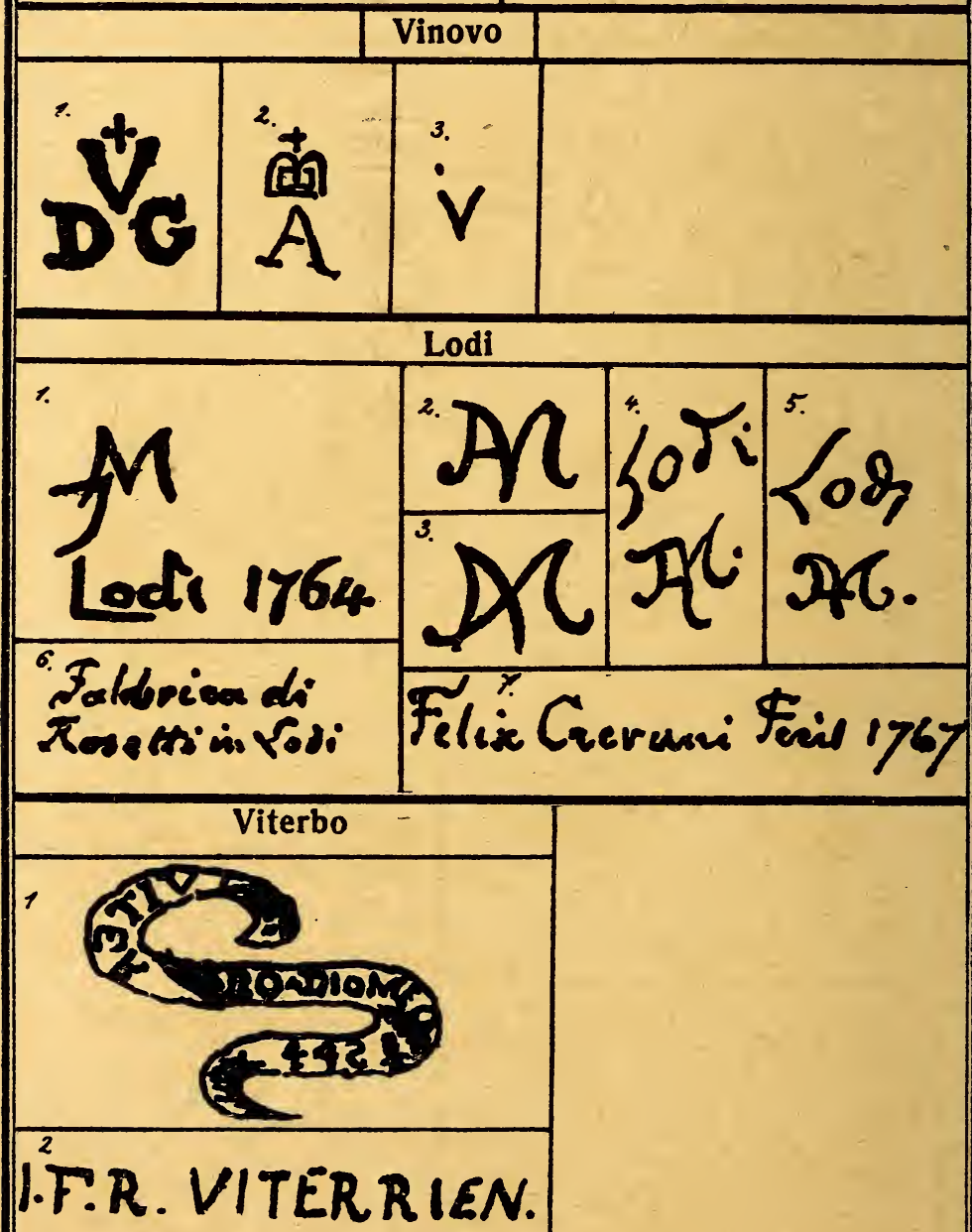


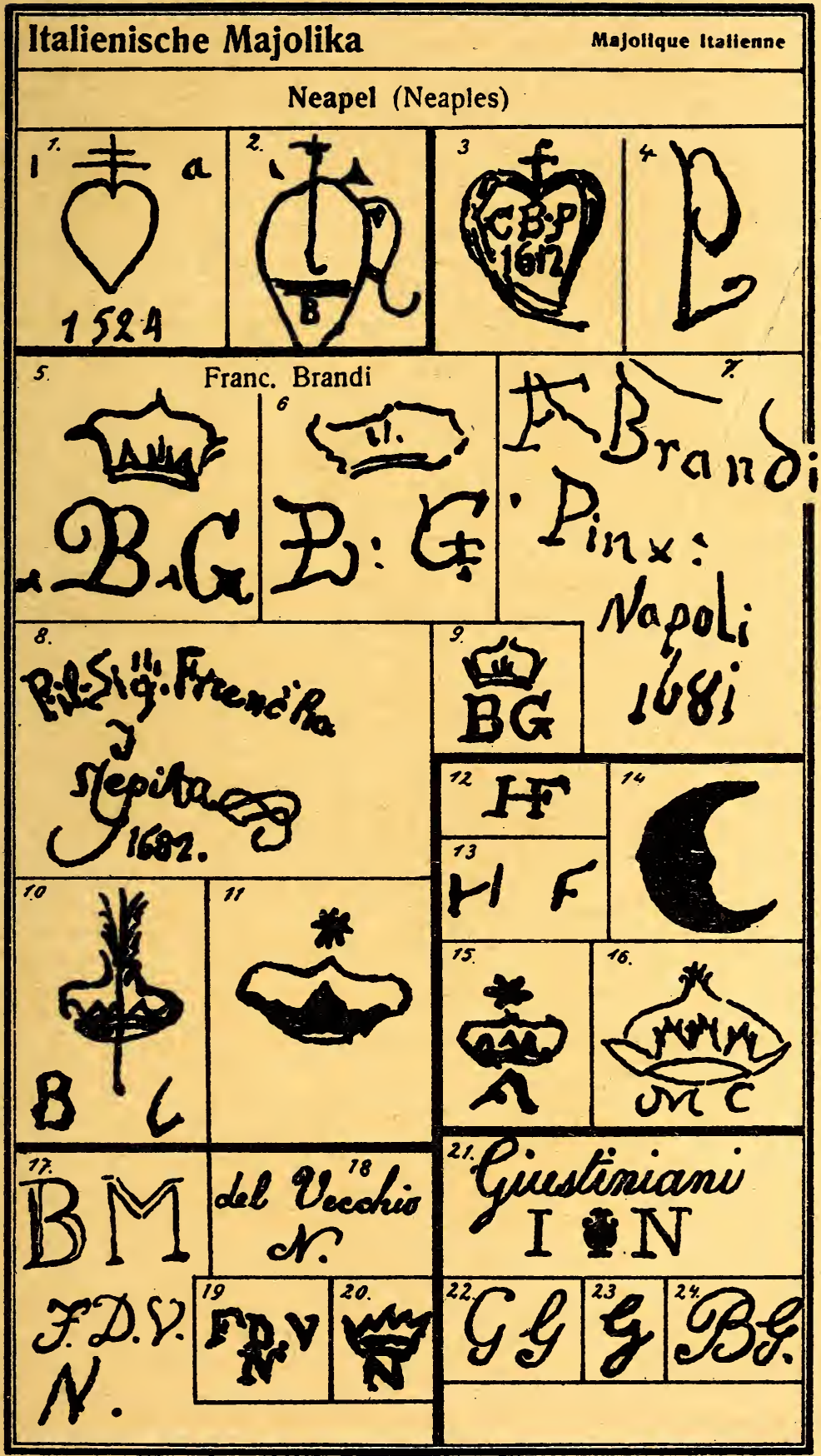




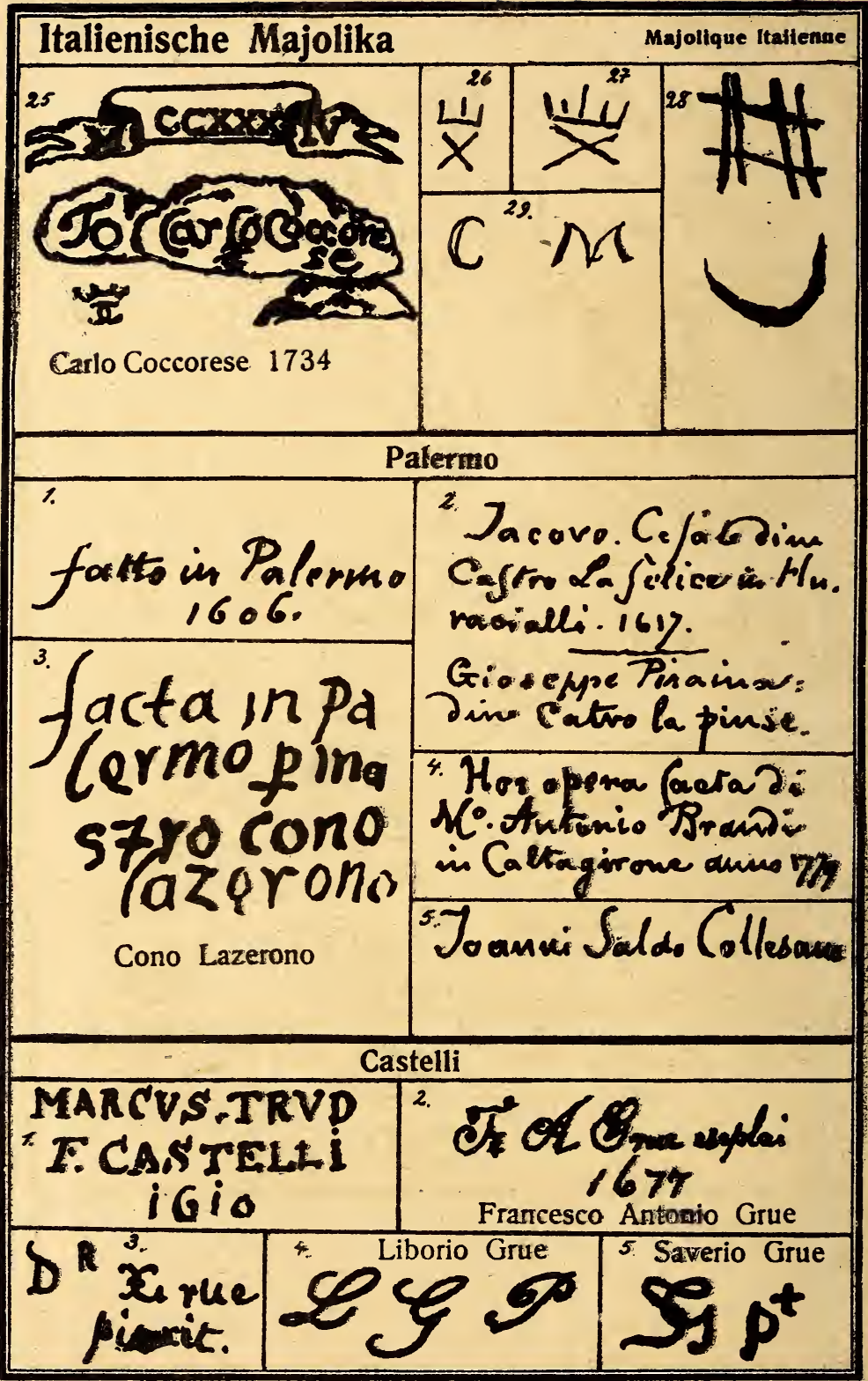




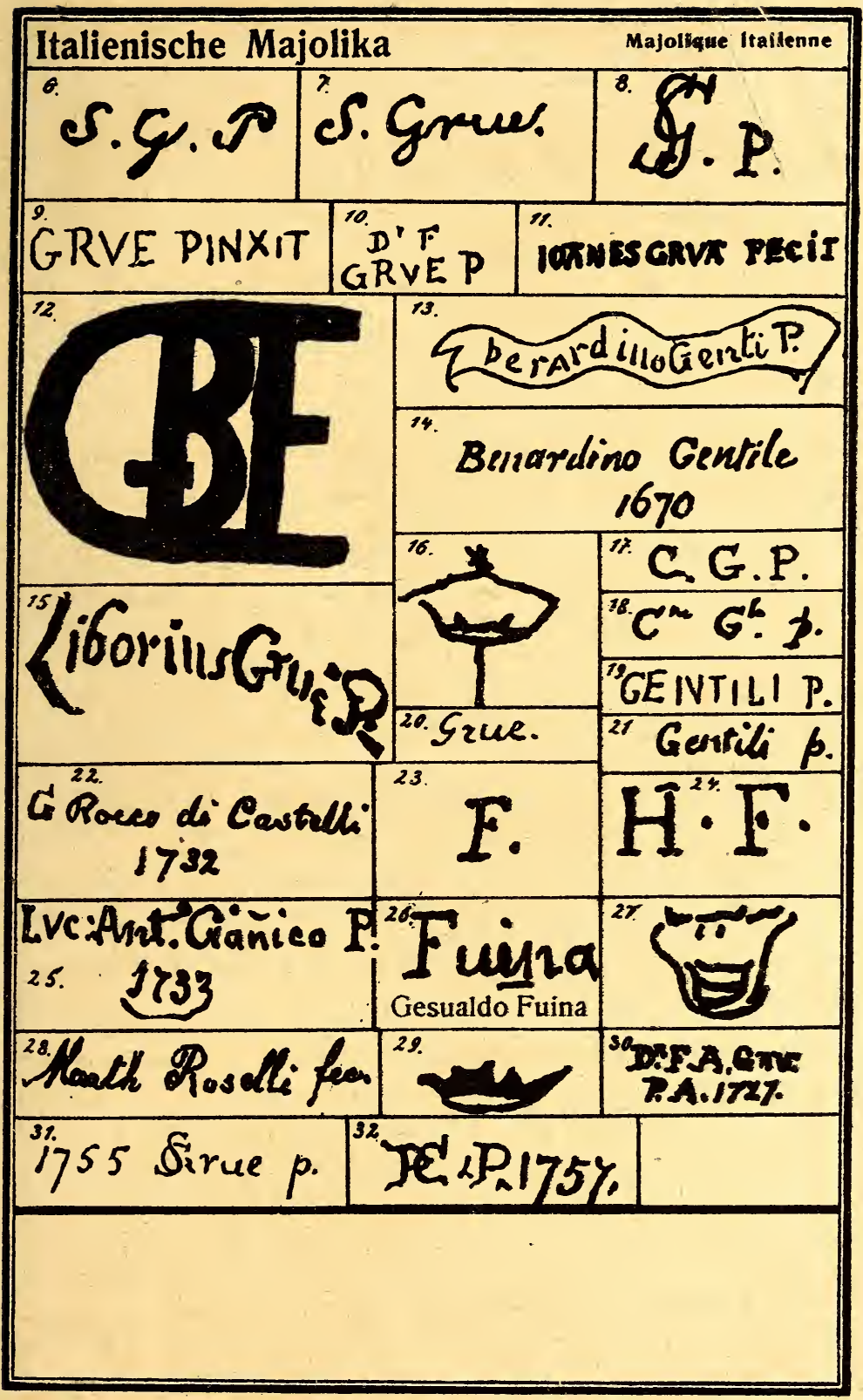



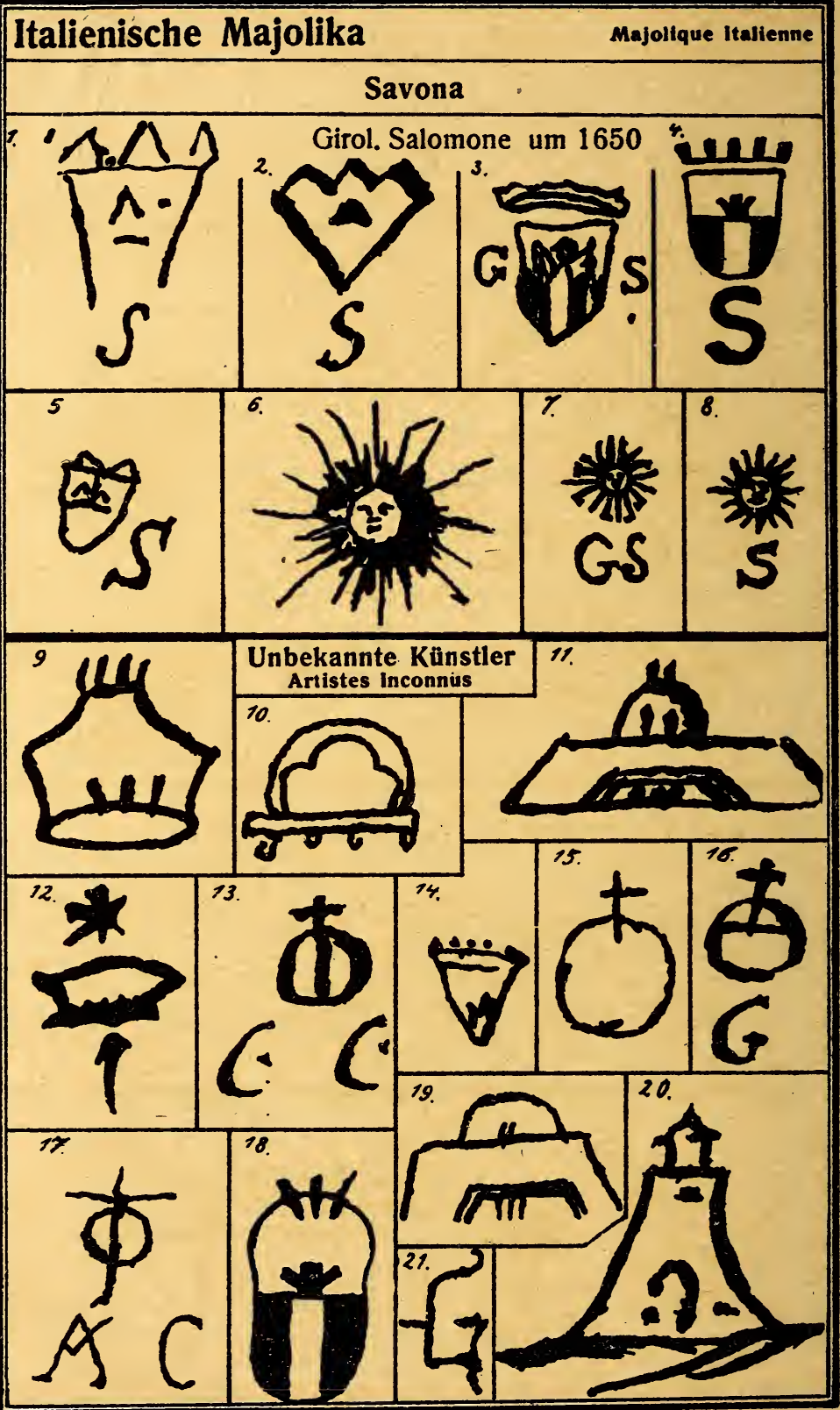


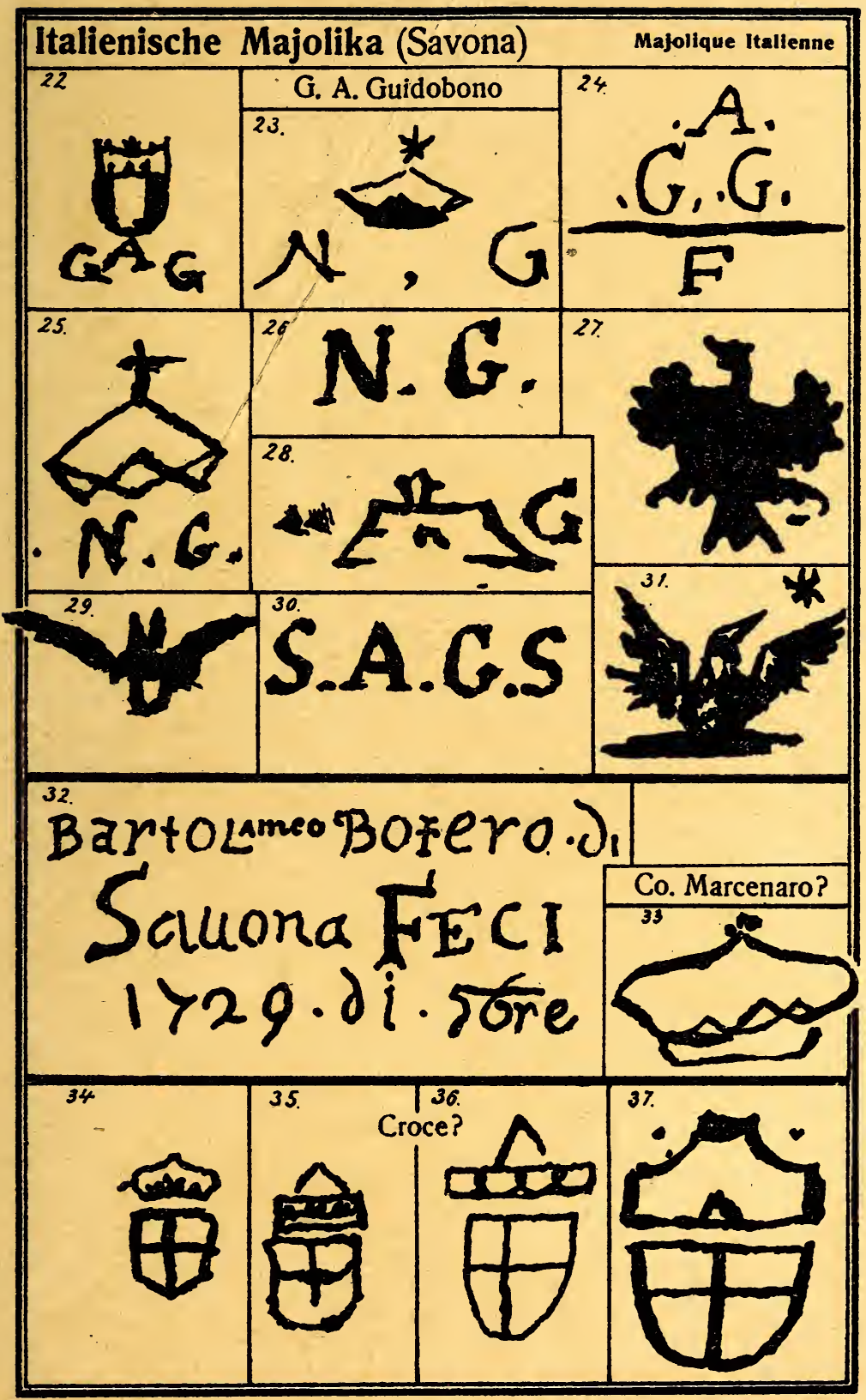




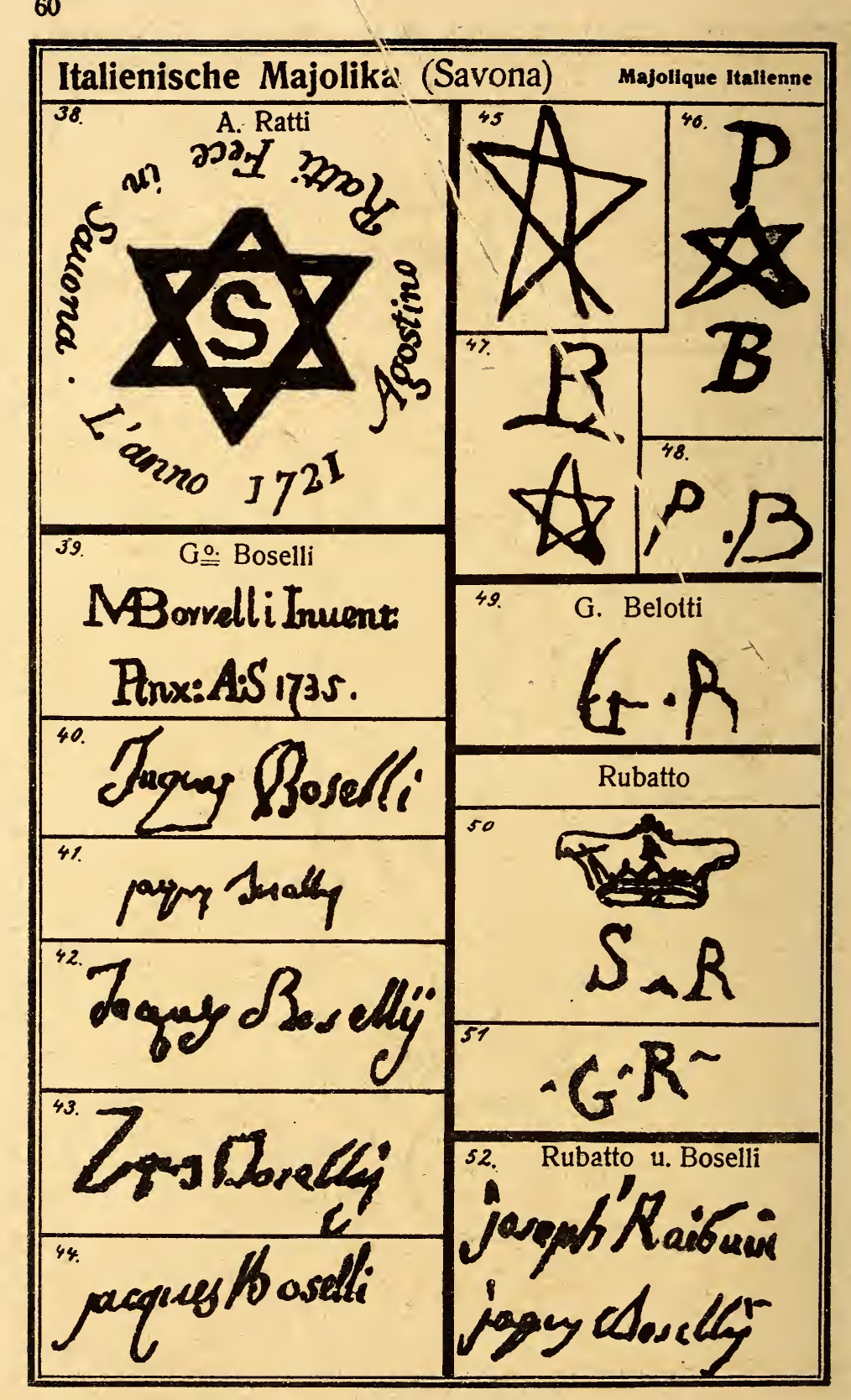




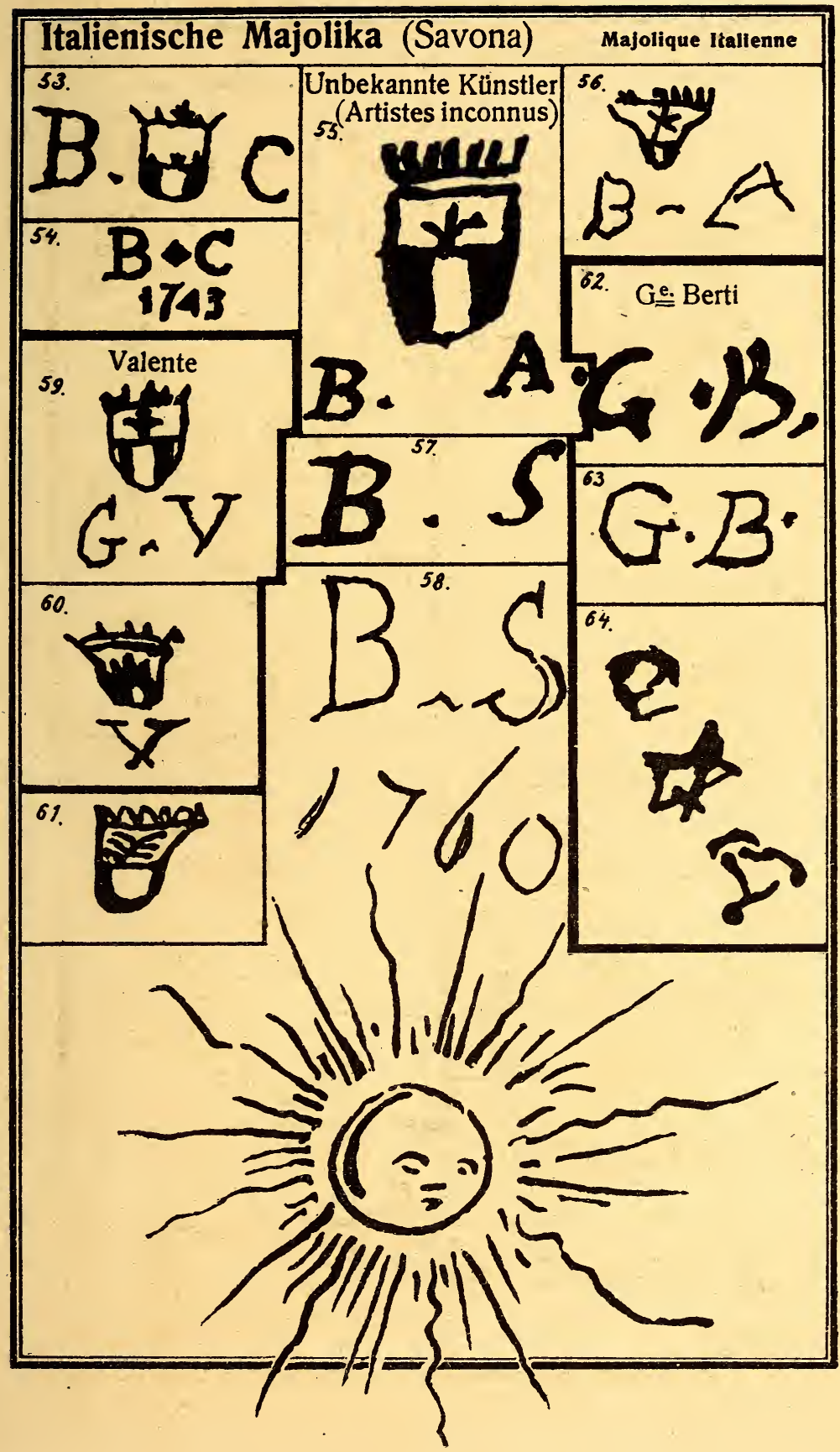




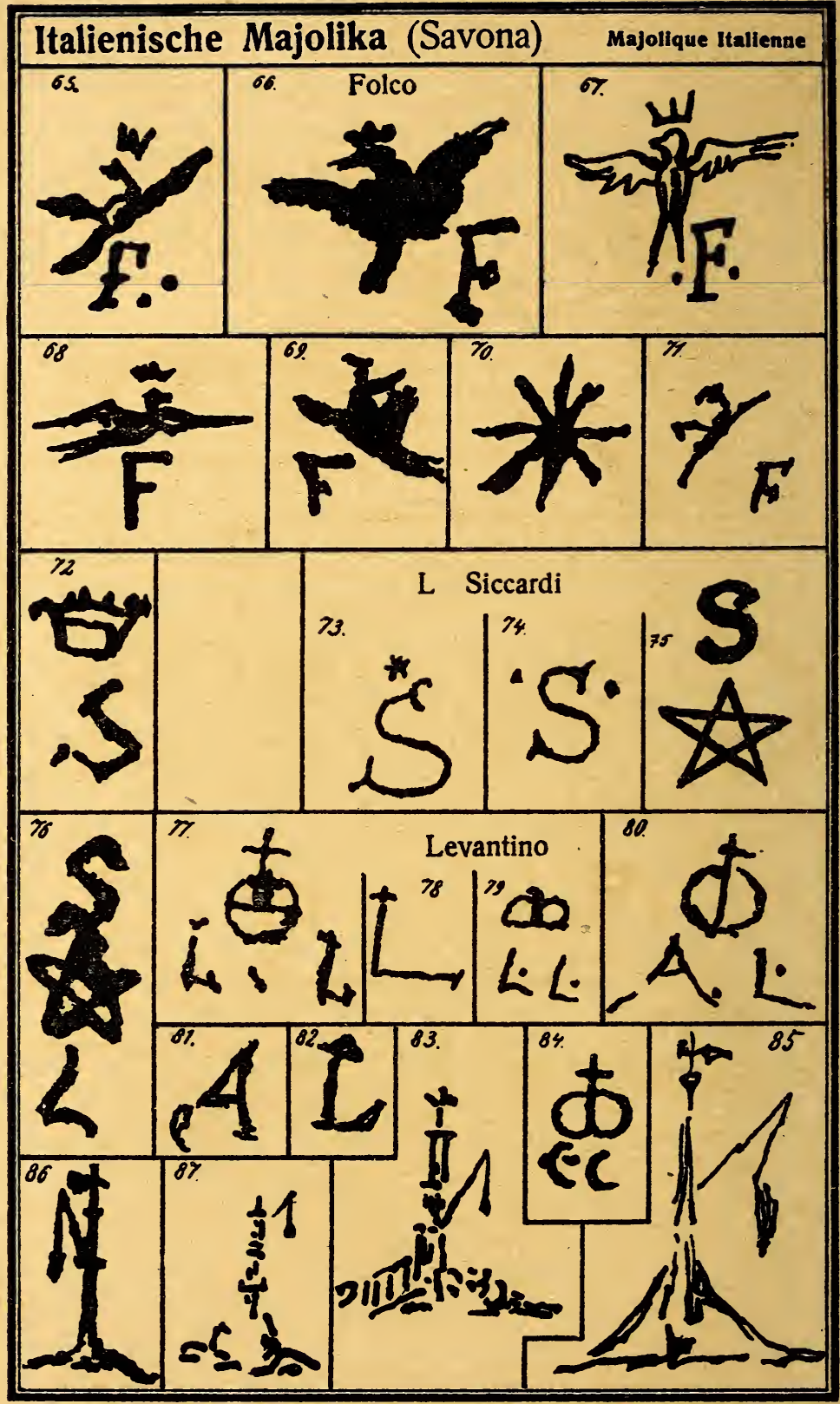




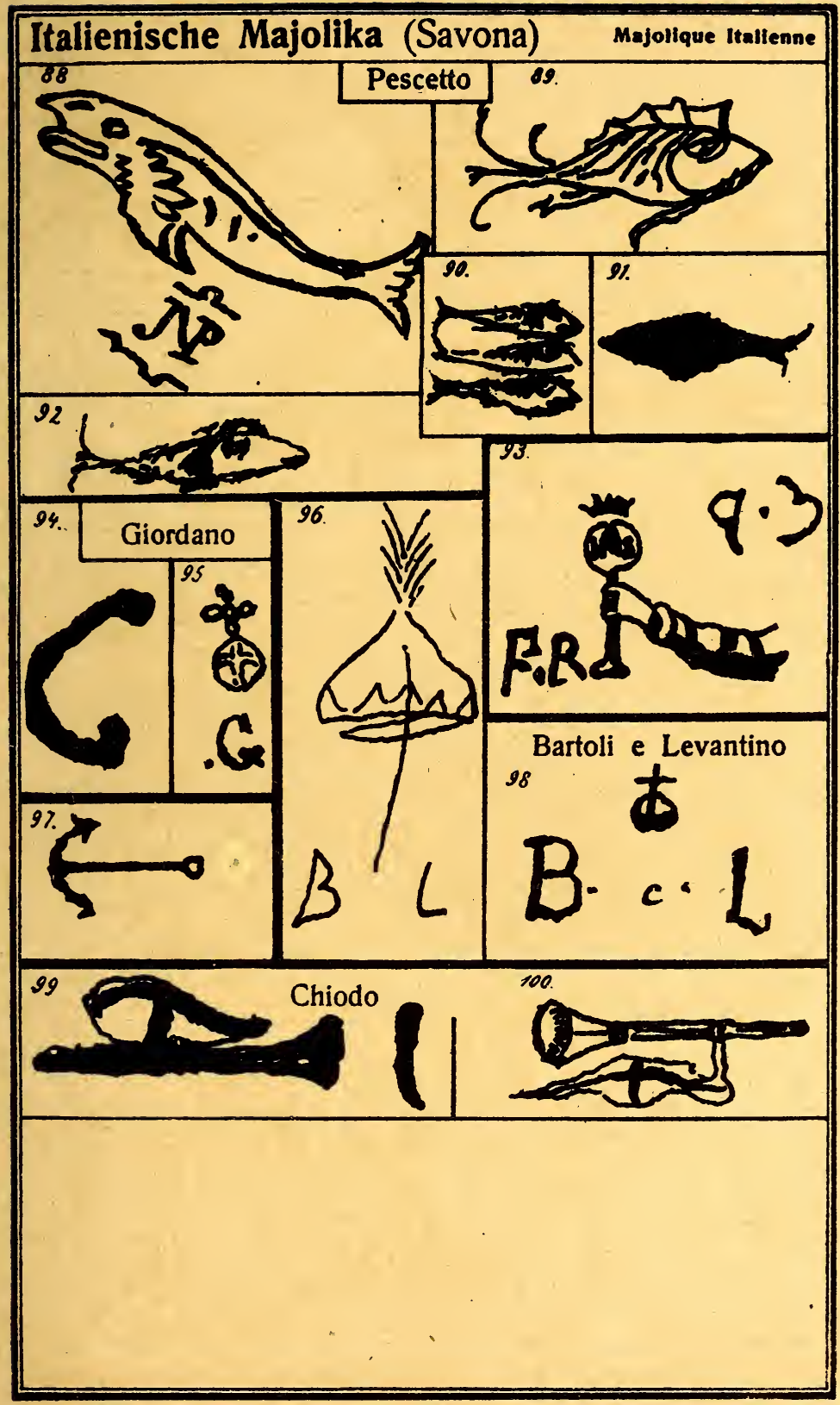




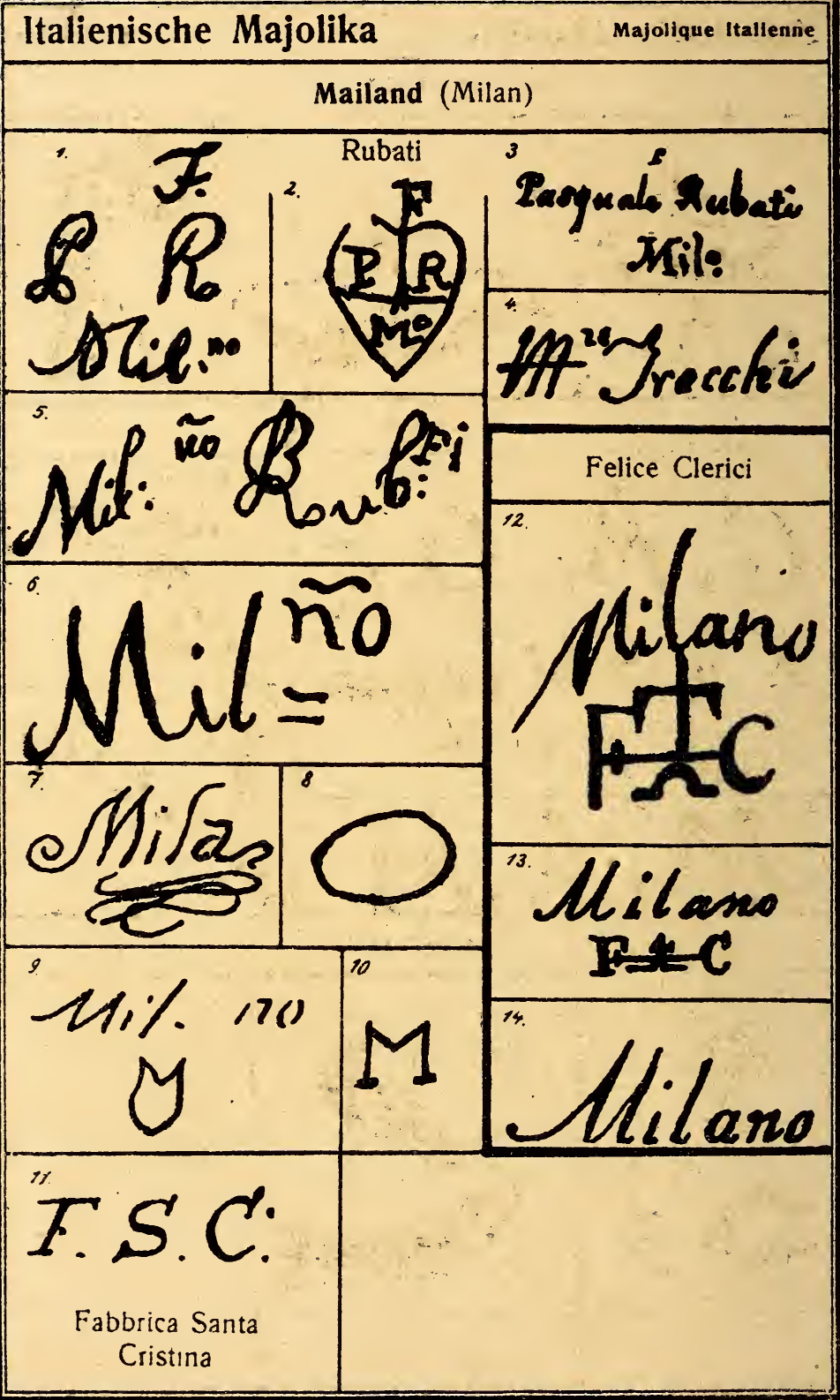




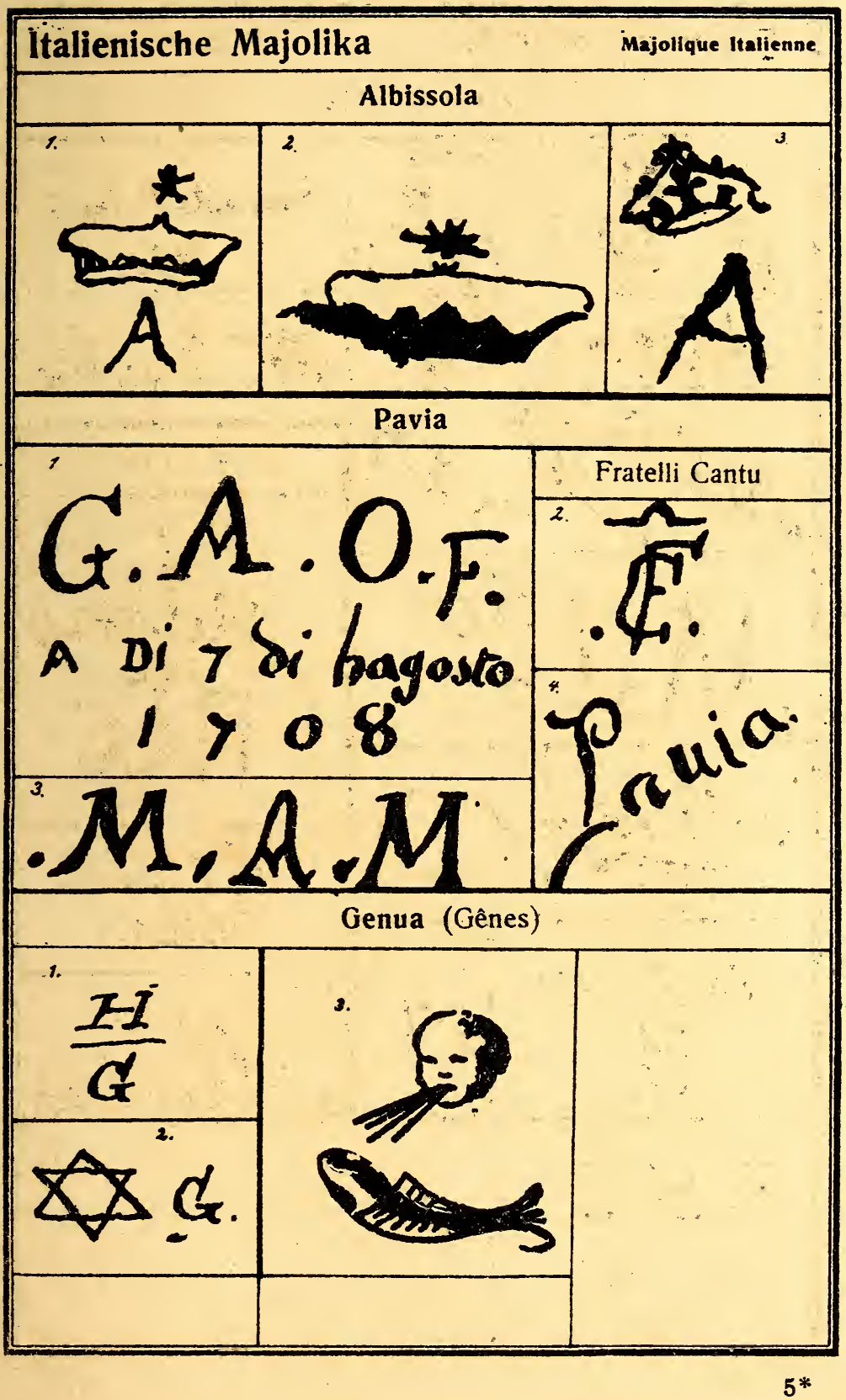


66

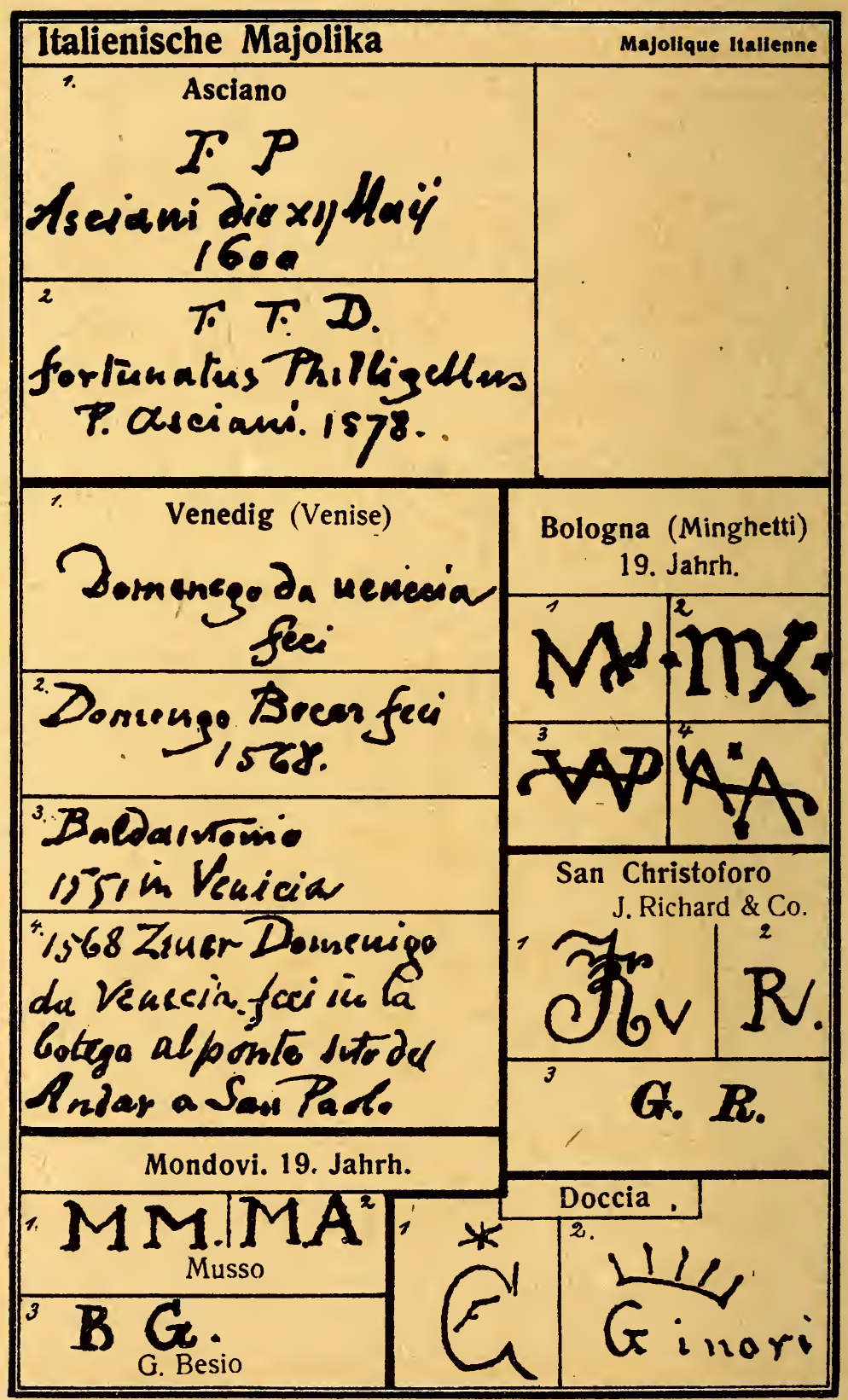




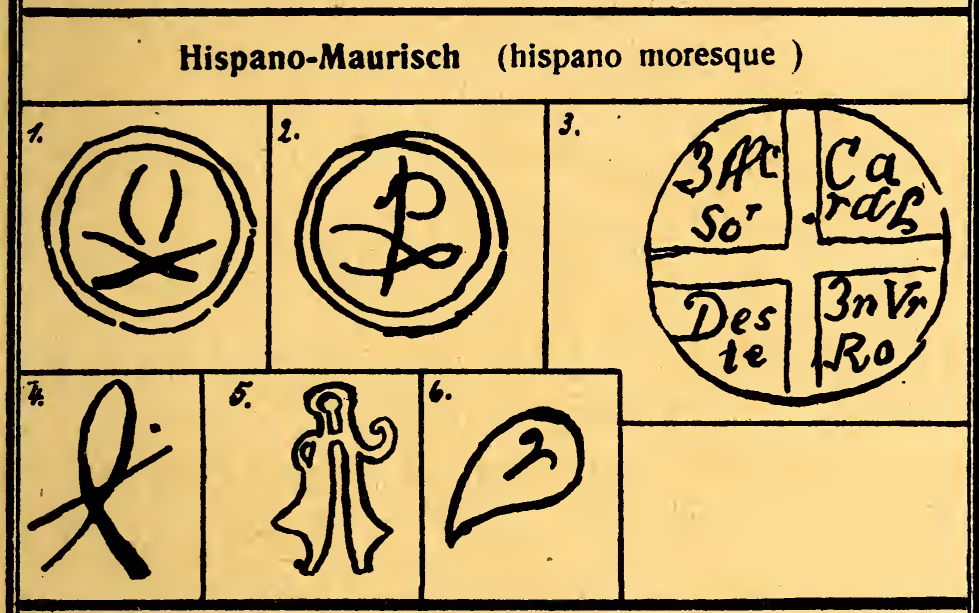

Puente di Arzobispo

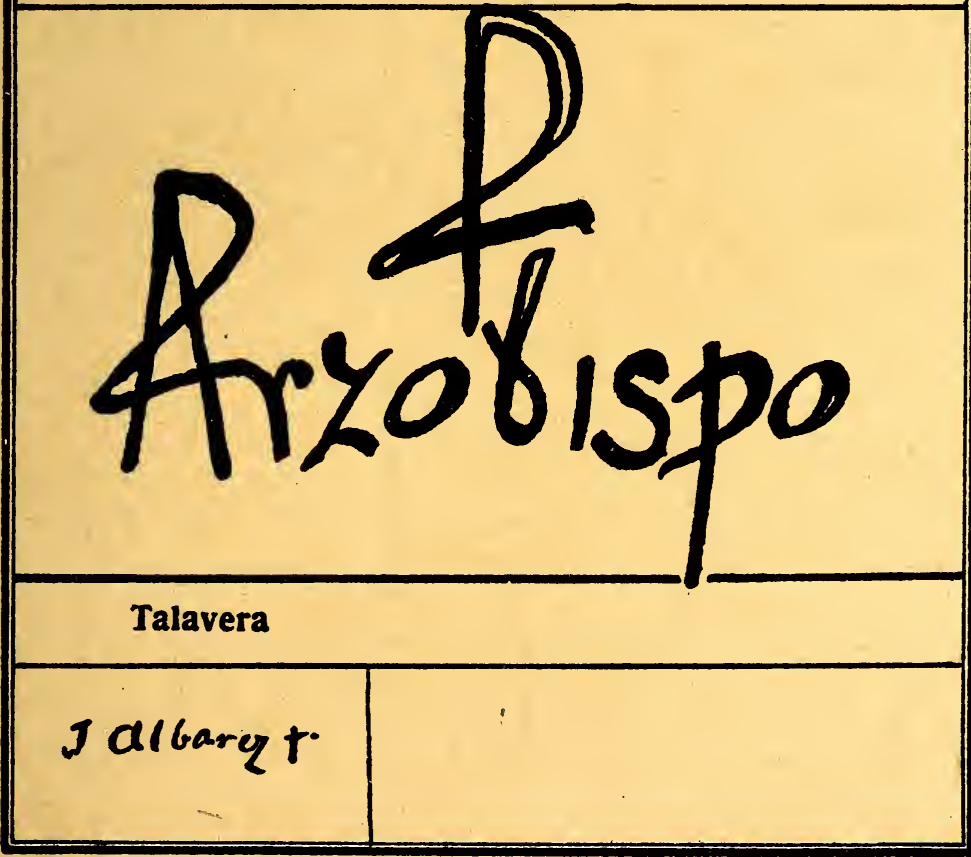




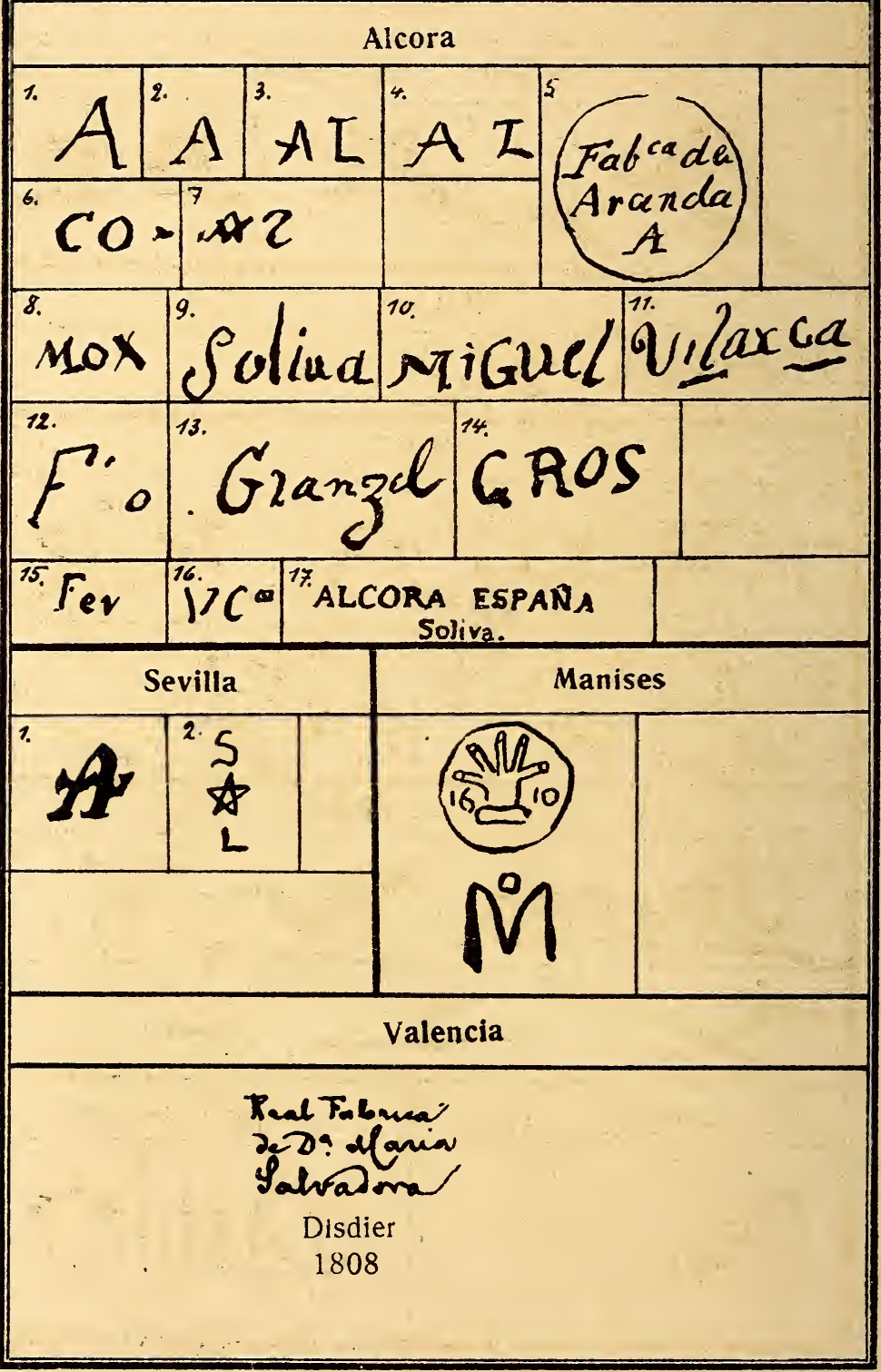




\section{Portugiesische Fayencen}

Falence Portugalse

\section{Porto}

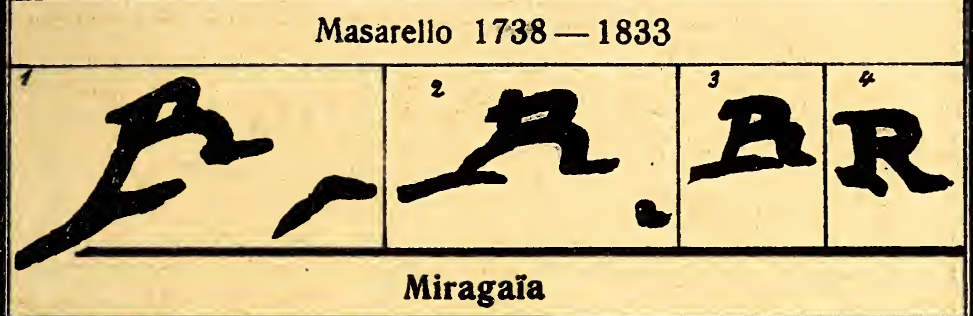

'Miragatia. 'M.P.

\section{Rato}
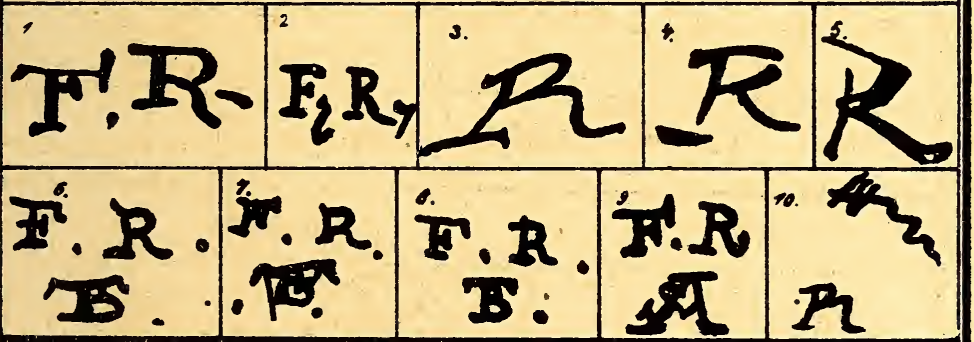

Viana de Castello

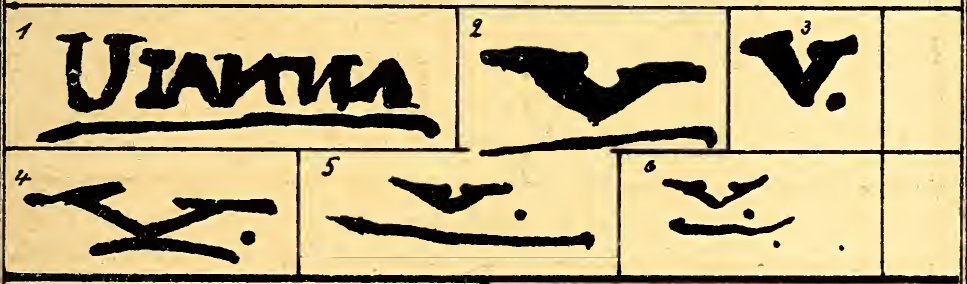

\begin{tabular}{|c|l|}
\hline Coimbra & \multicolumn{2}{|c|}{ Lissabon (Lisbonne) } \\
\hline $\begin{array}{l}\text { Rossi } \\
\text { 1yS5 }\end{array}$ & GAG \\
\hline
\end{tabular}




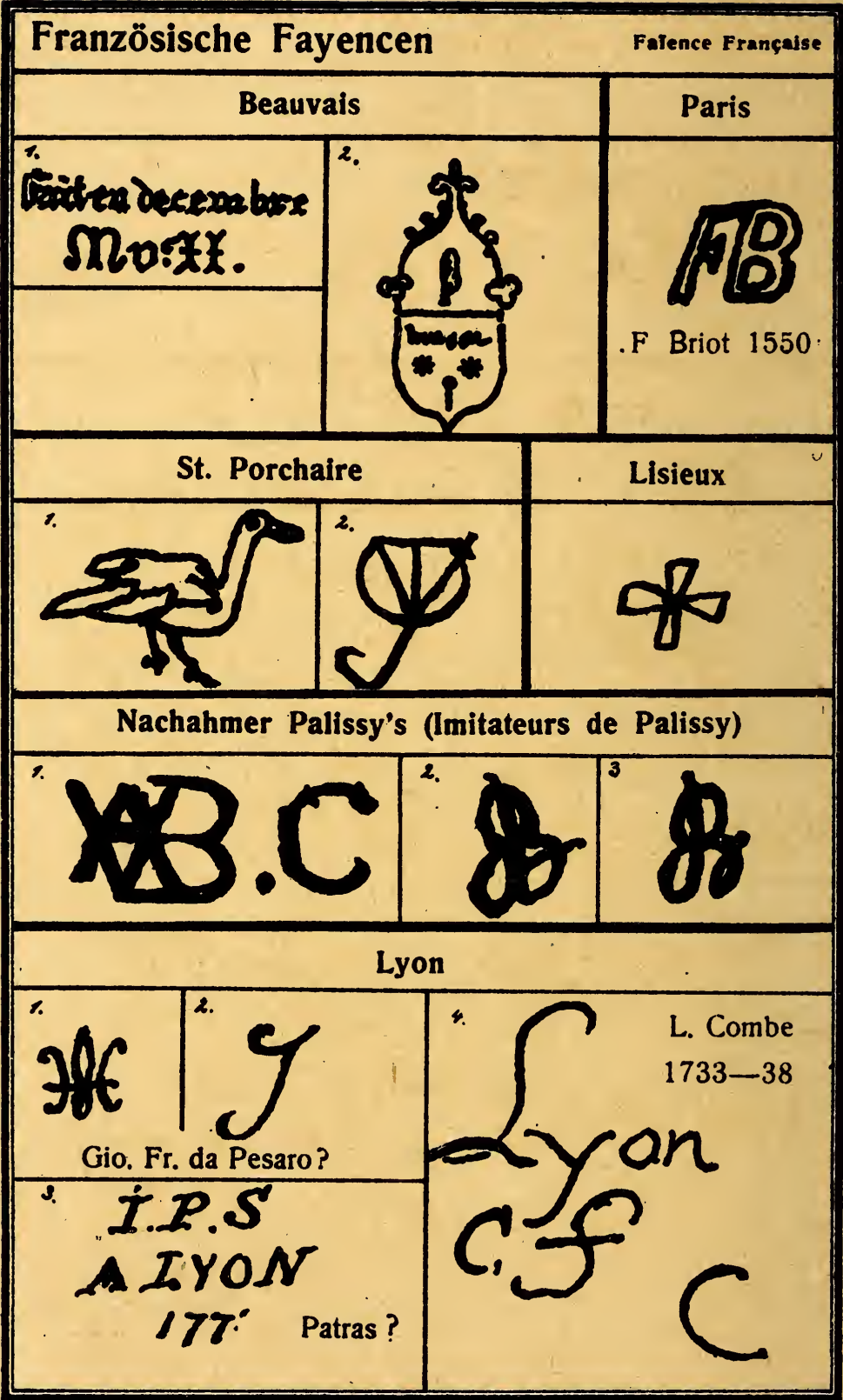




\section{Französische Fayencen \\ FR-ElTAdedV: \\ I6 $\$ 3$}

Fatence Française

Nantes

Nevers

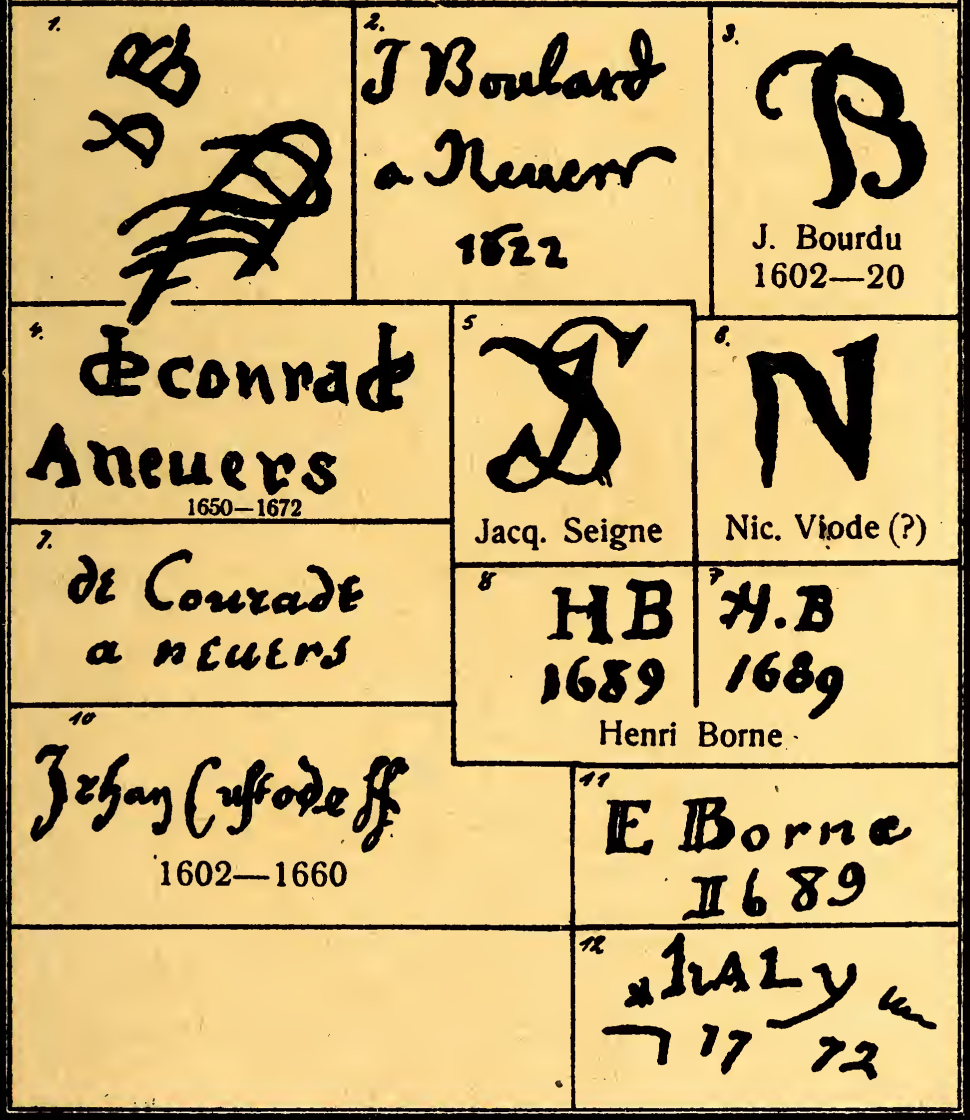




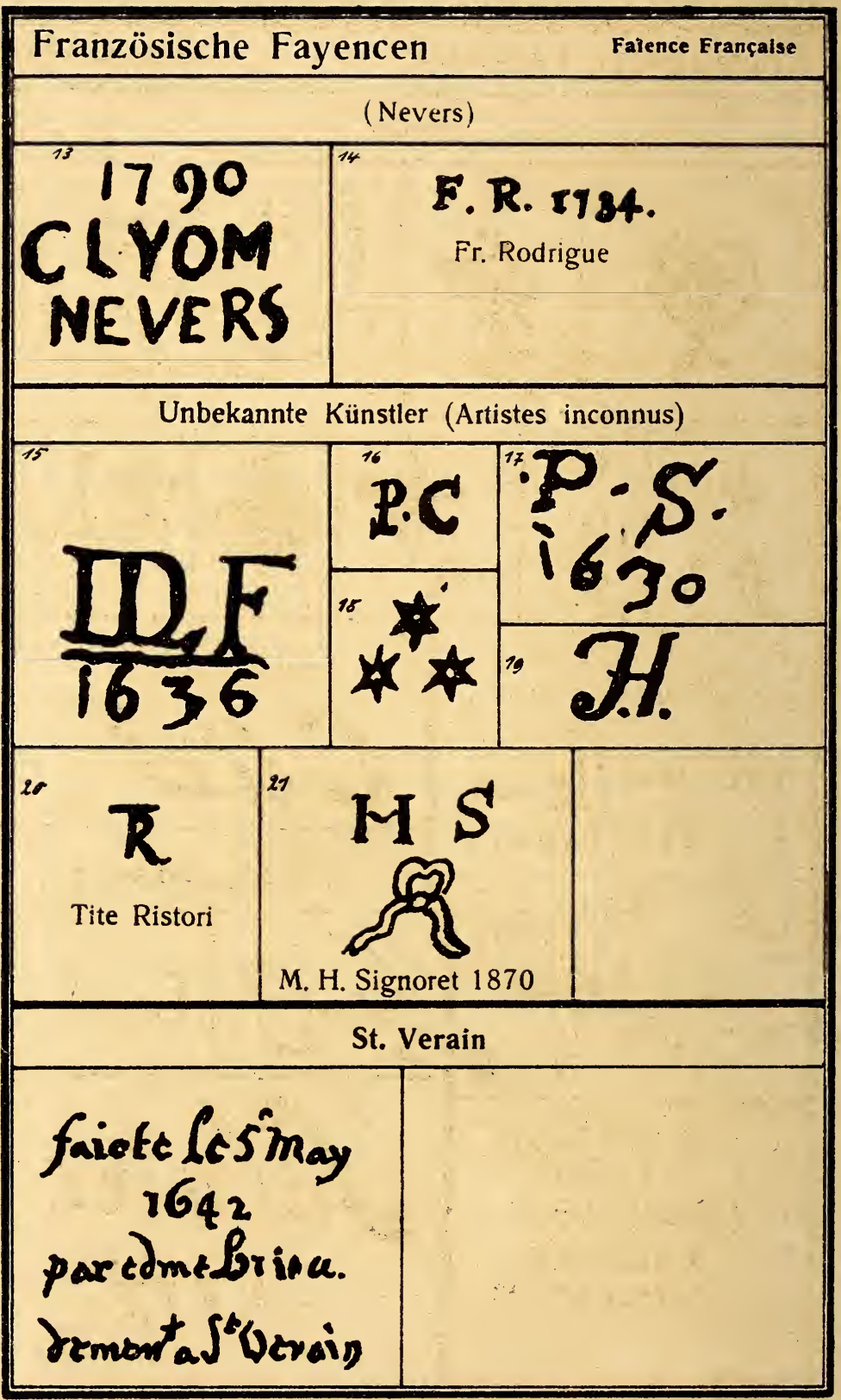




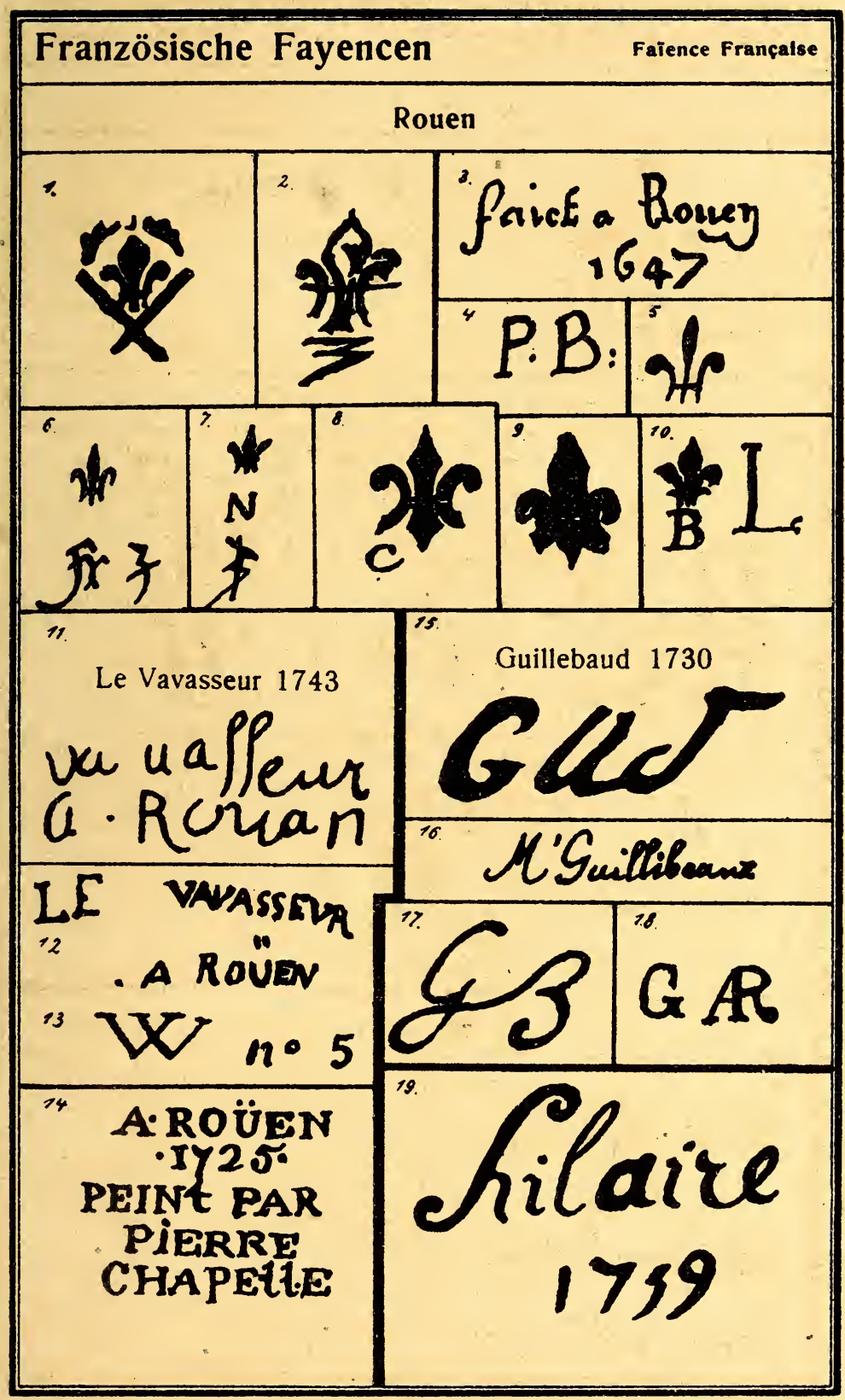




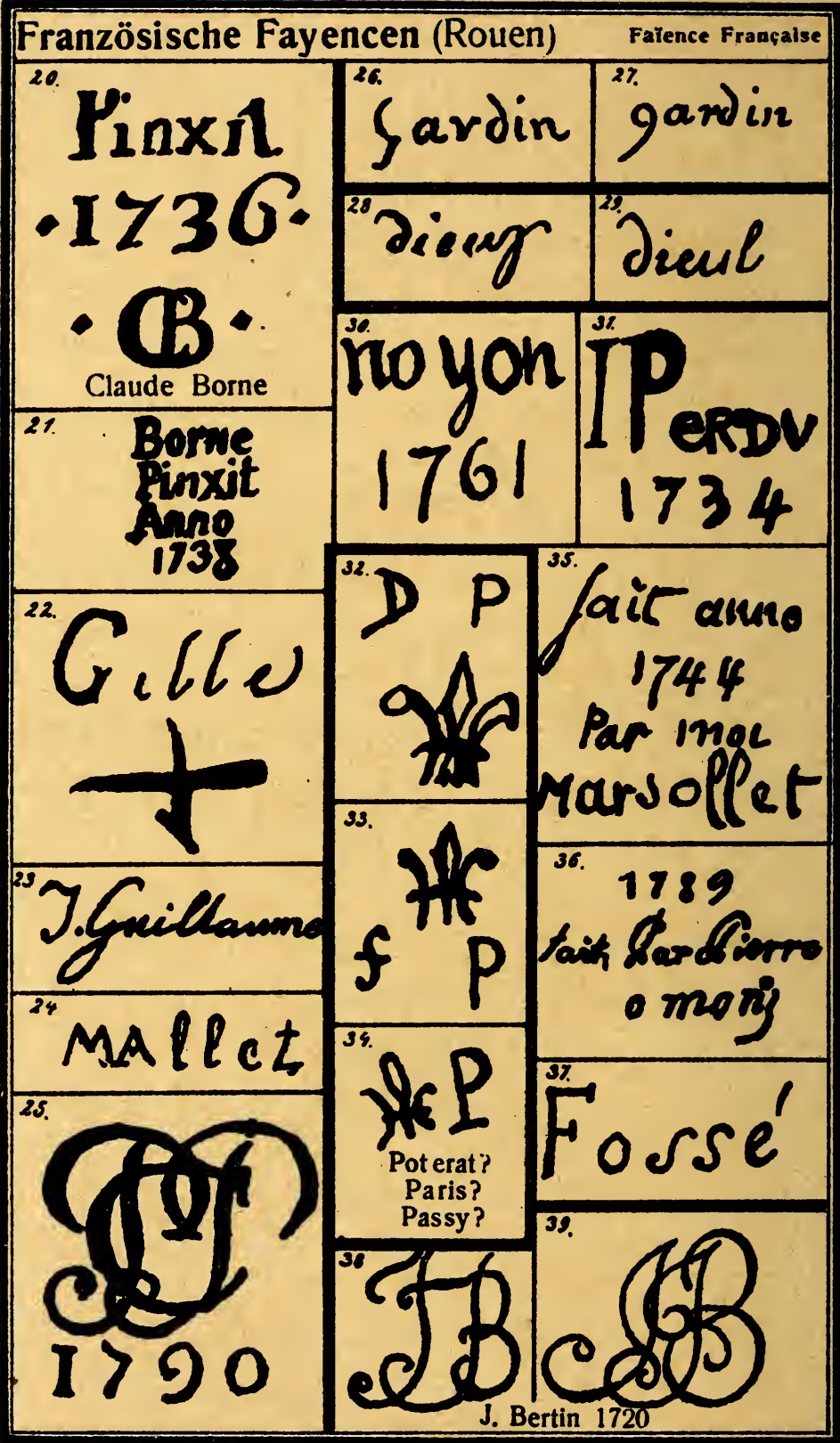




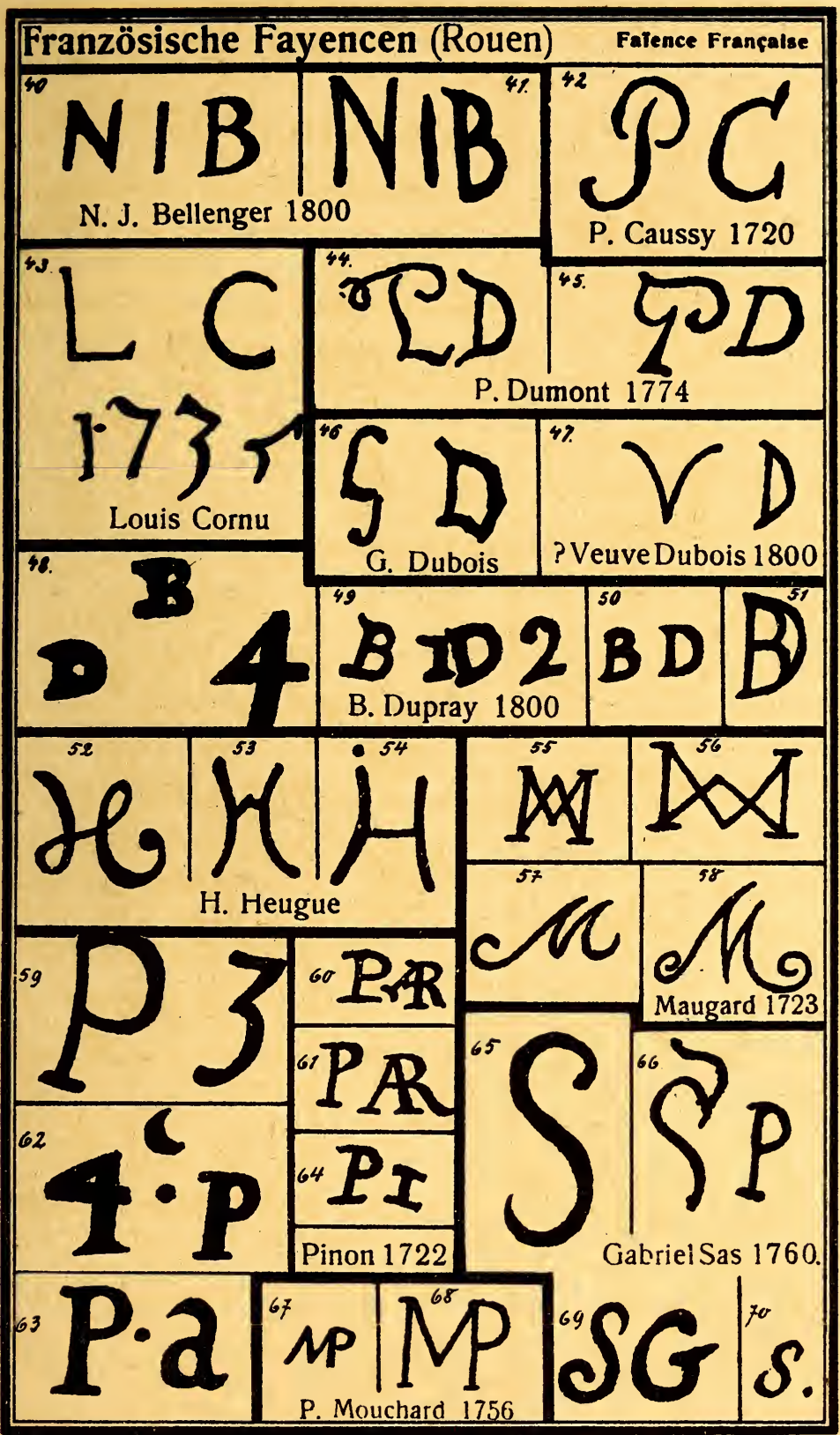




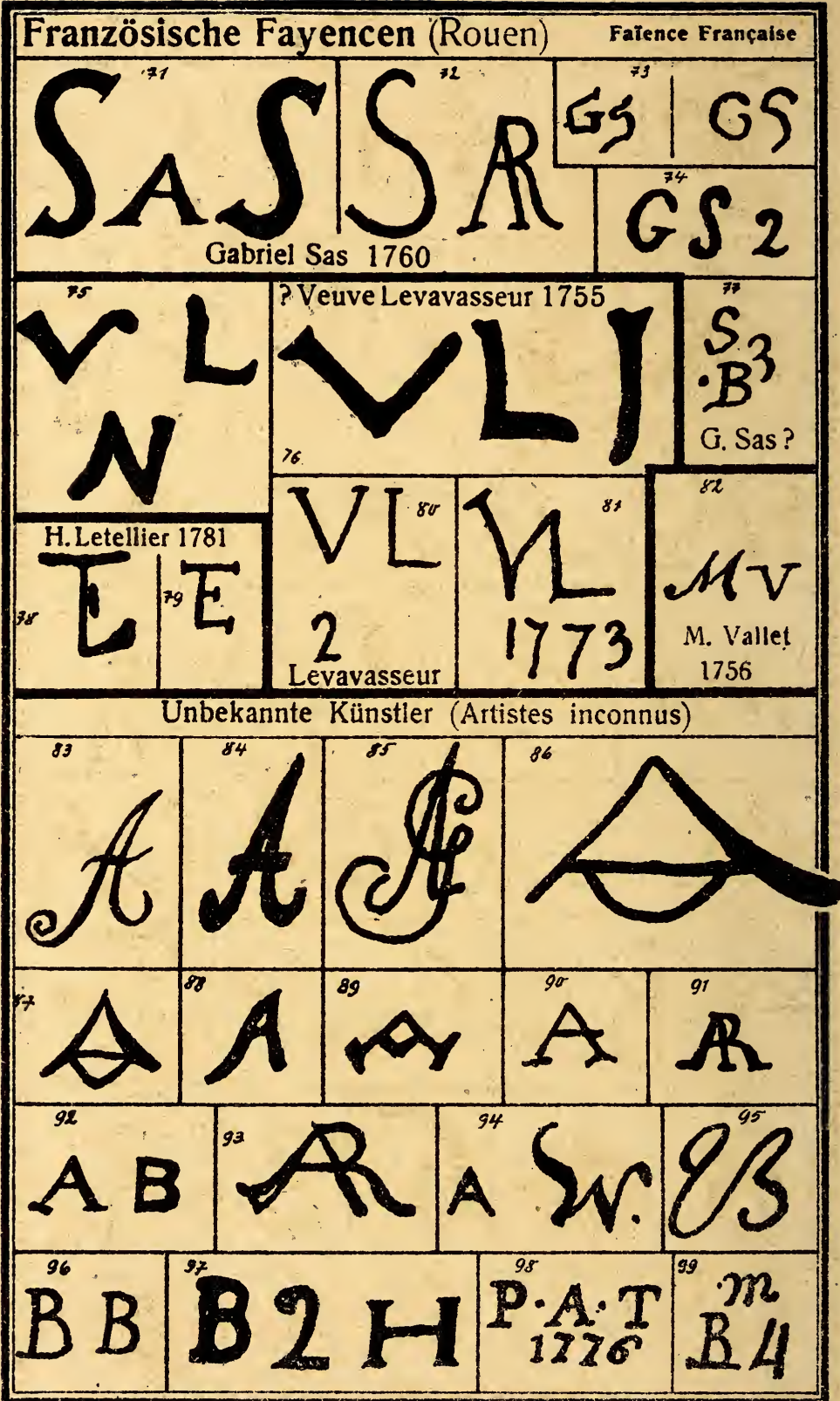




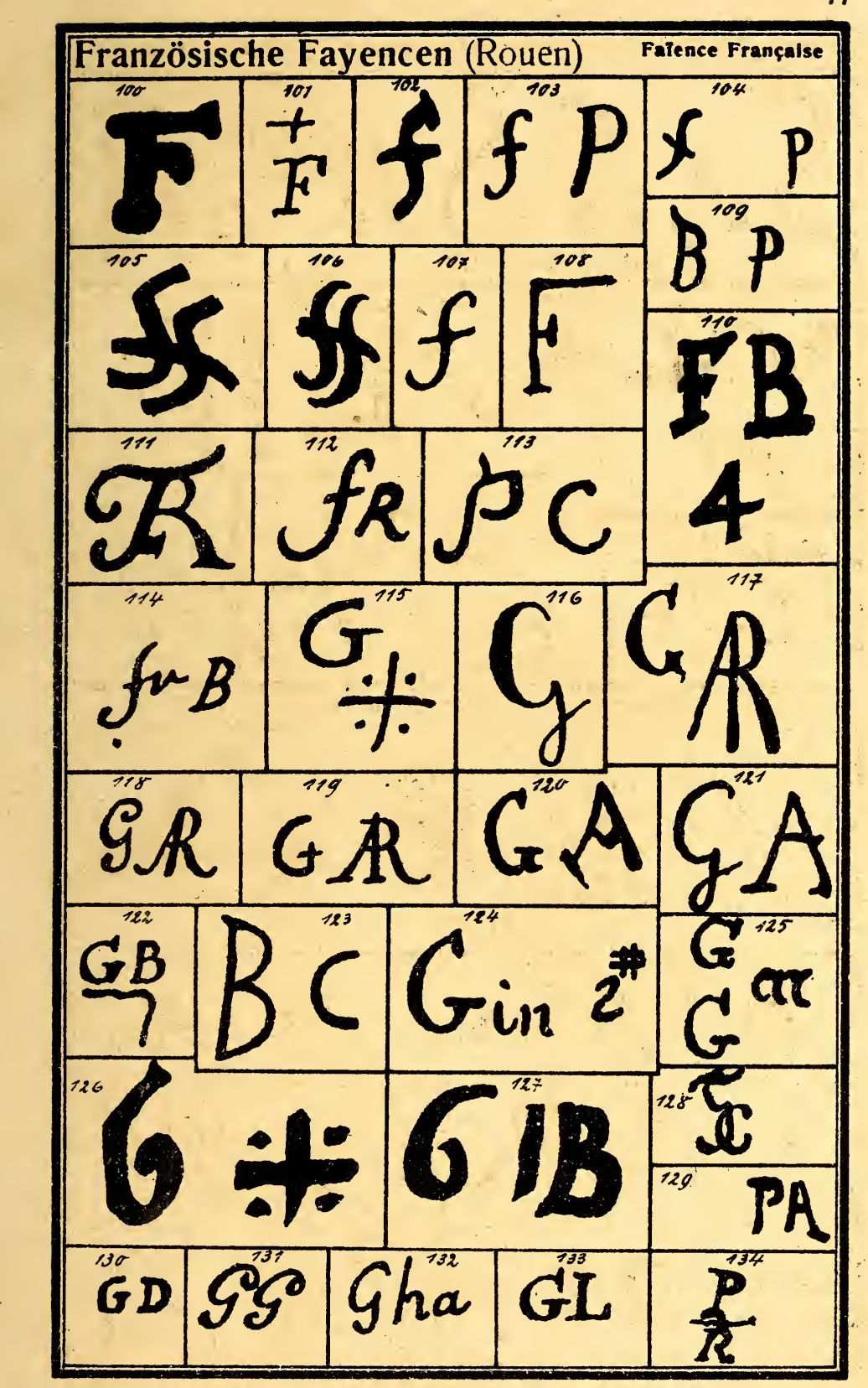




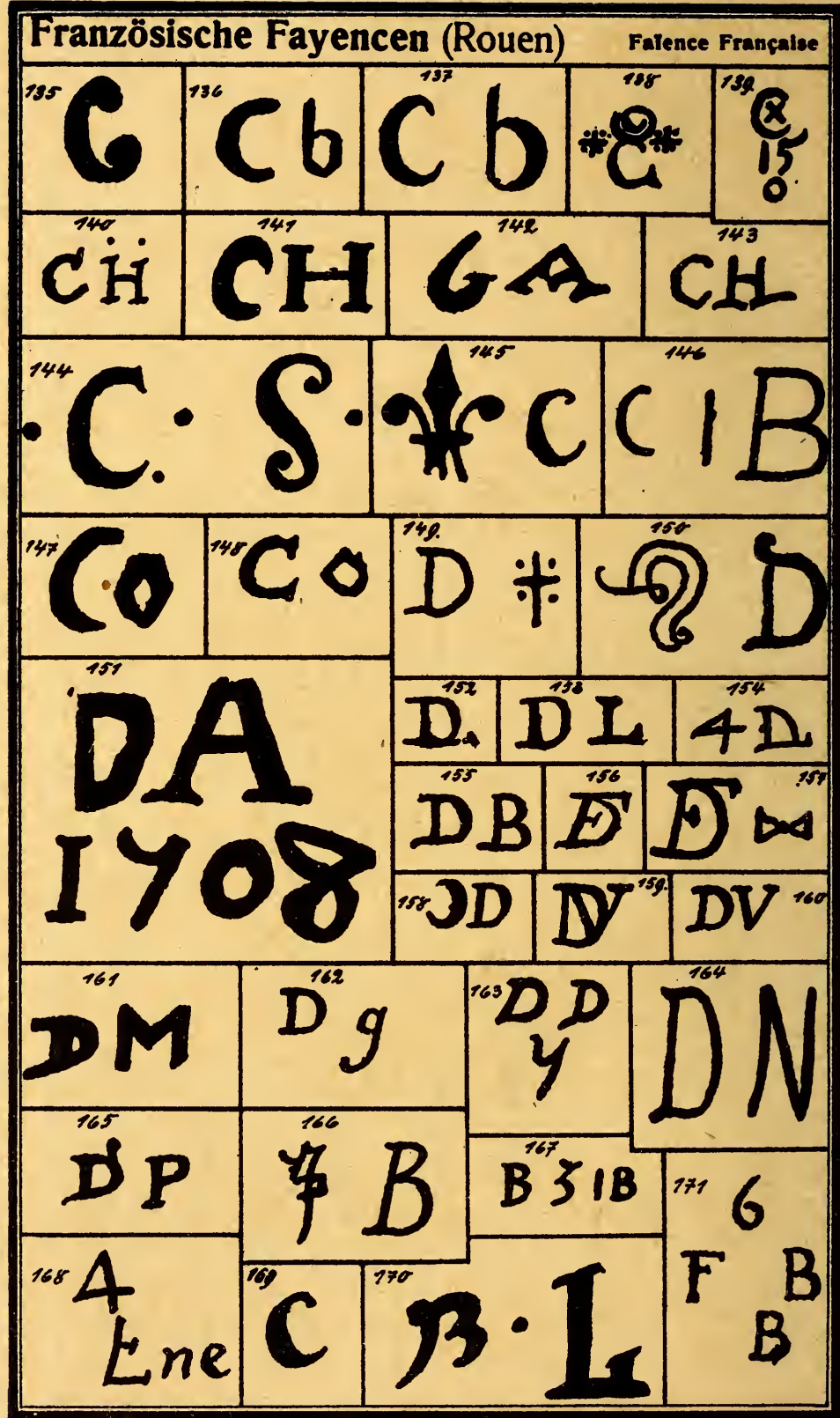




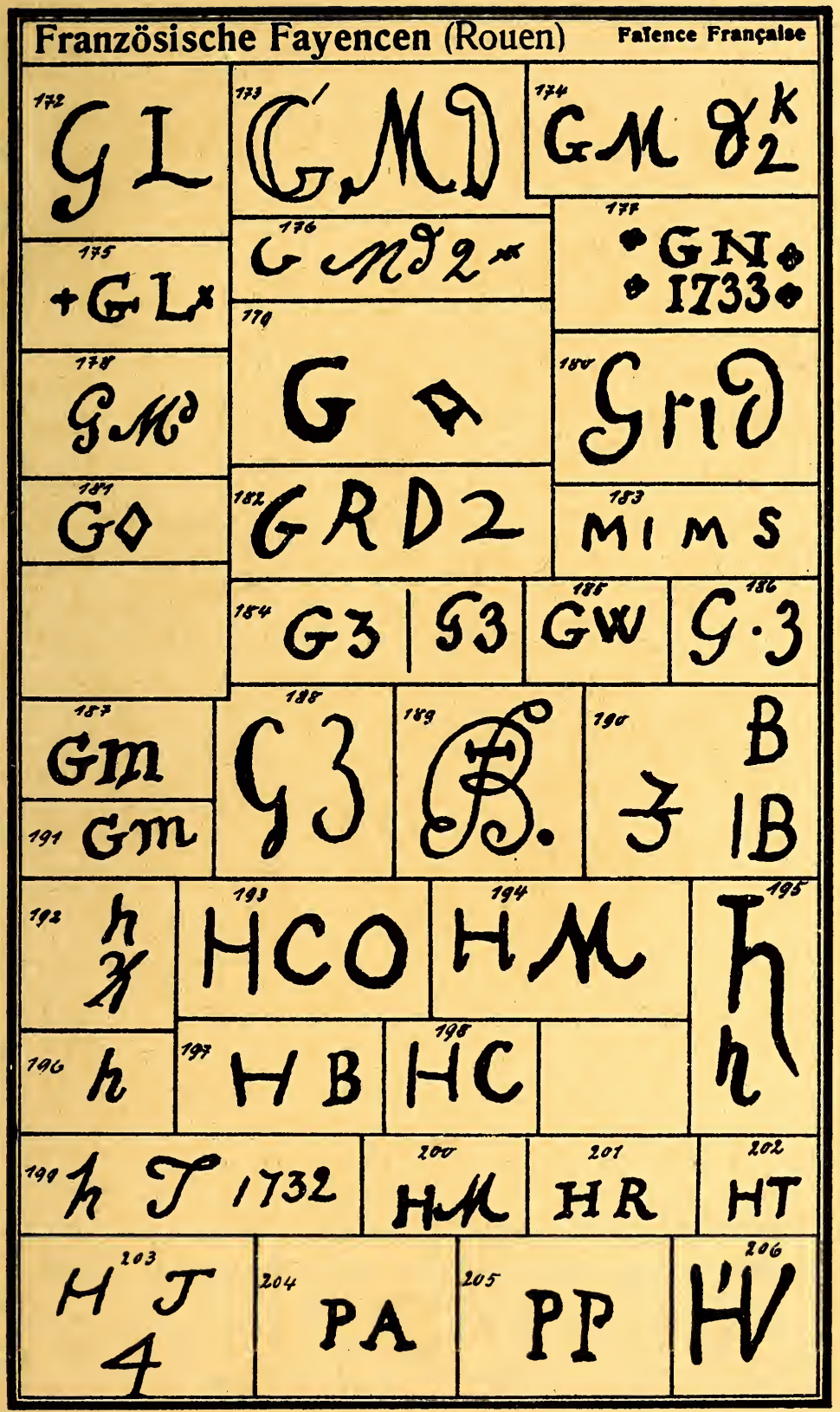




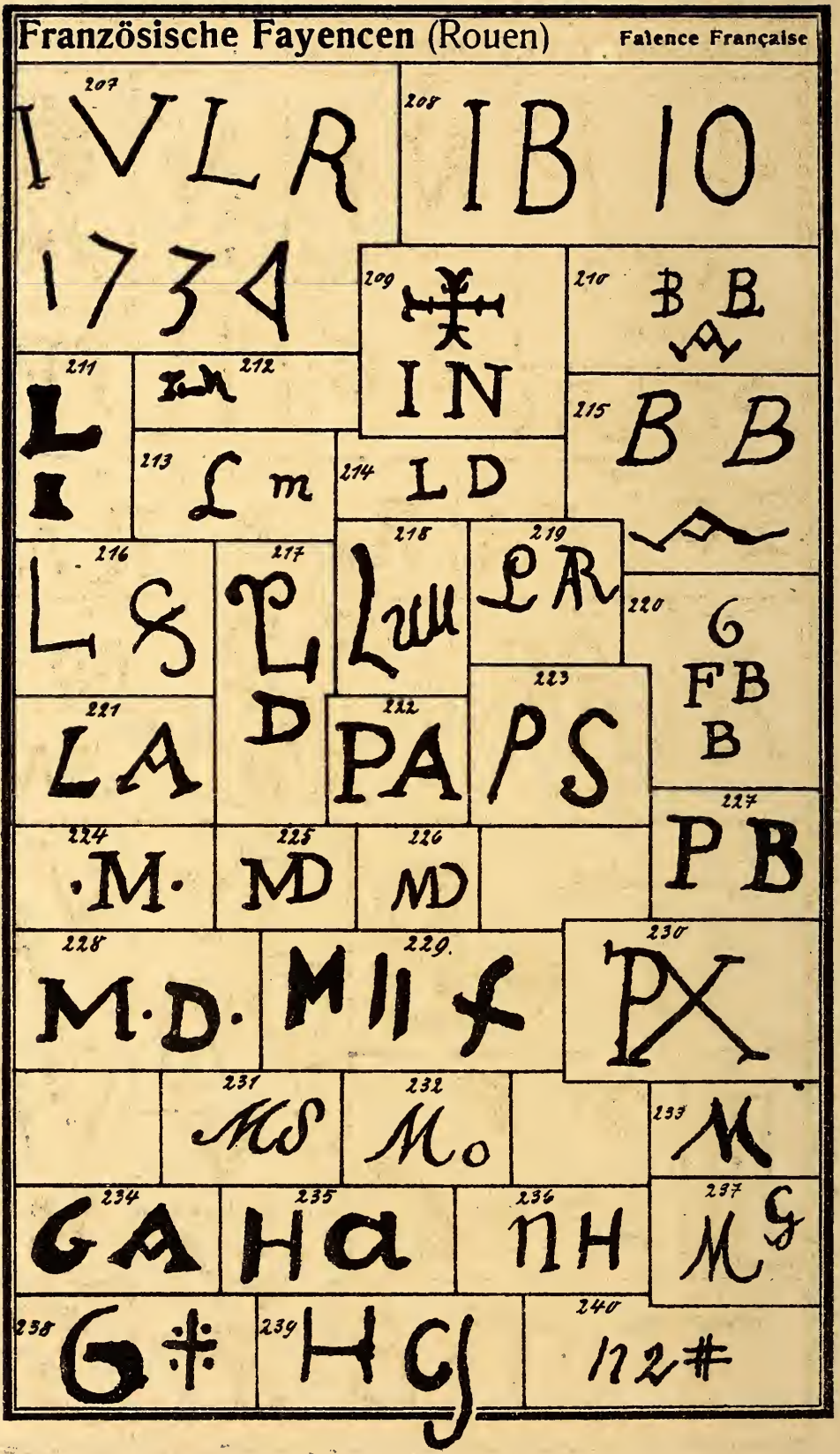




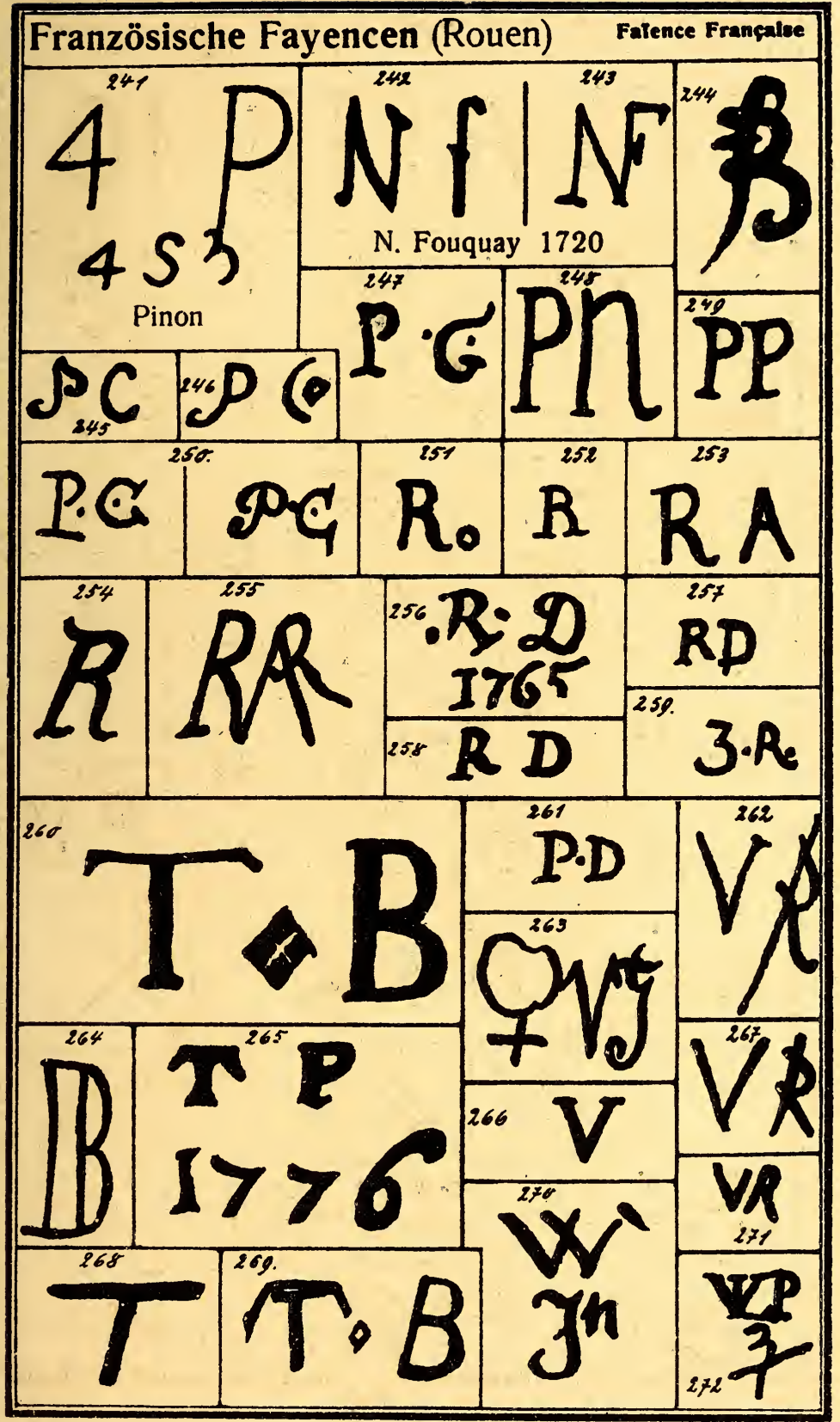




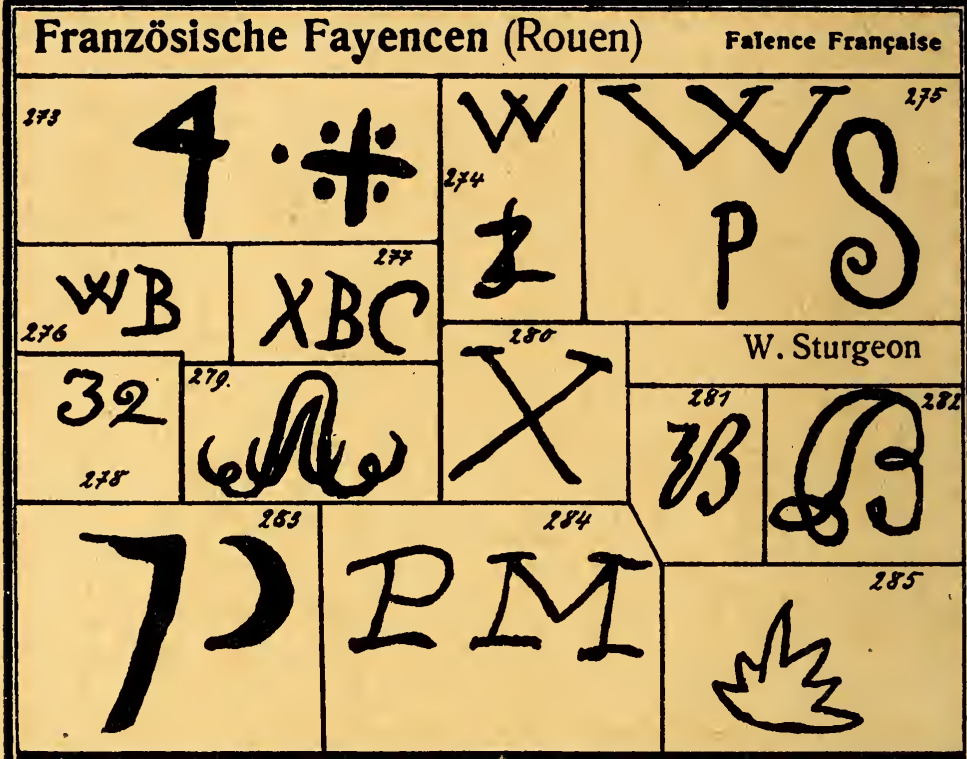

Moustiers

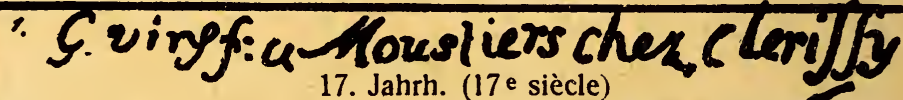
2

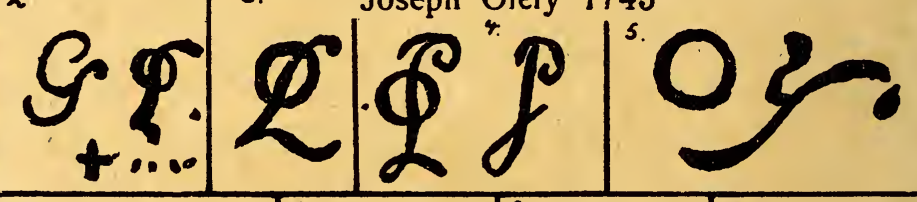

6 .

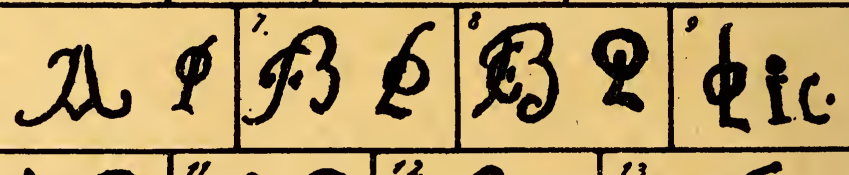

"

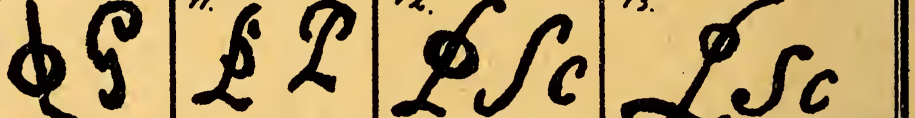

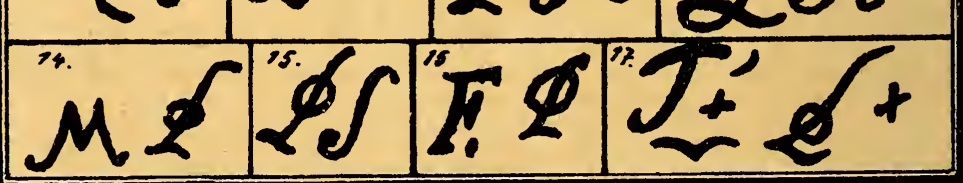




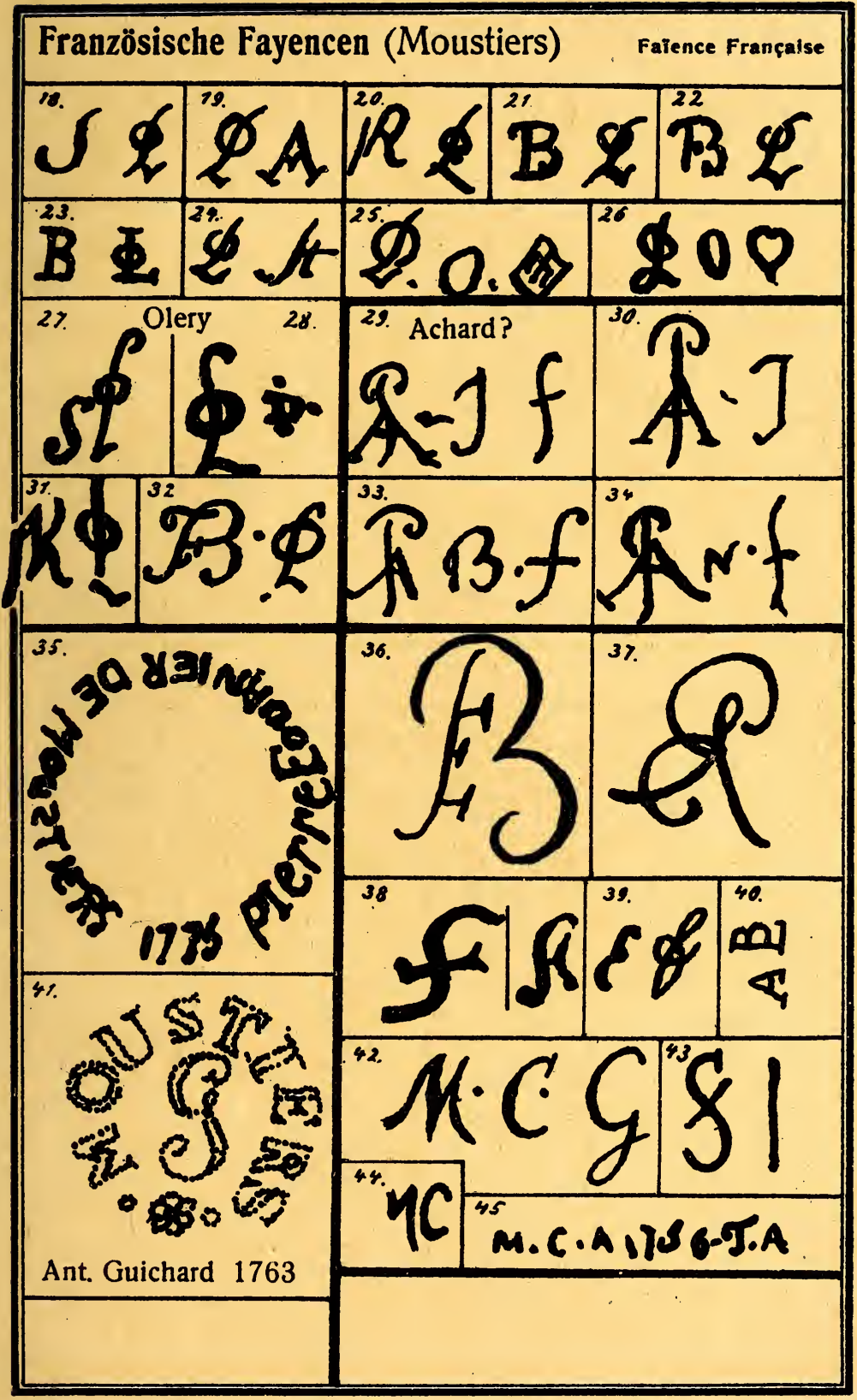


Französische Fayencen (Moustiers) Faience Française

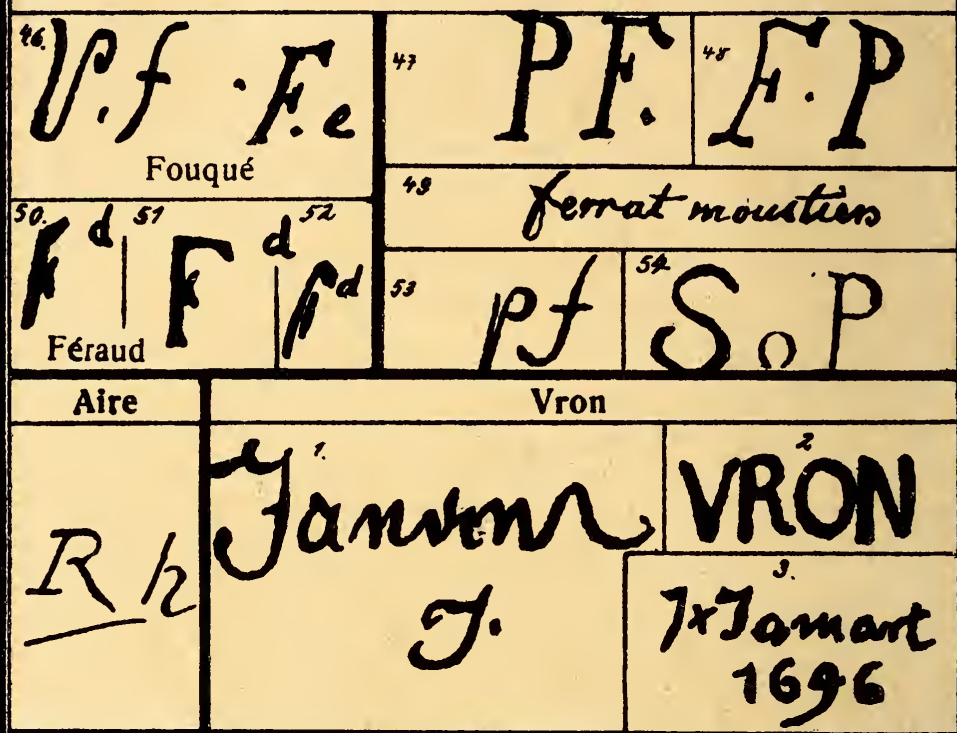

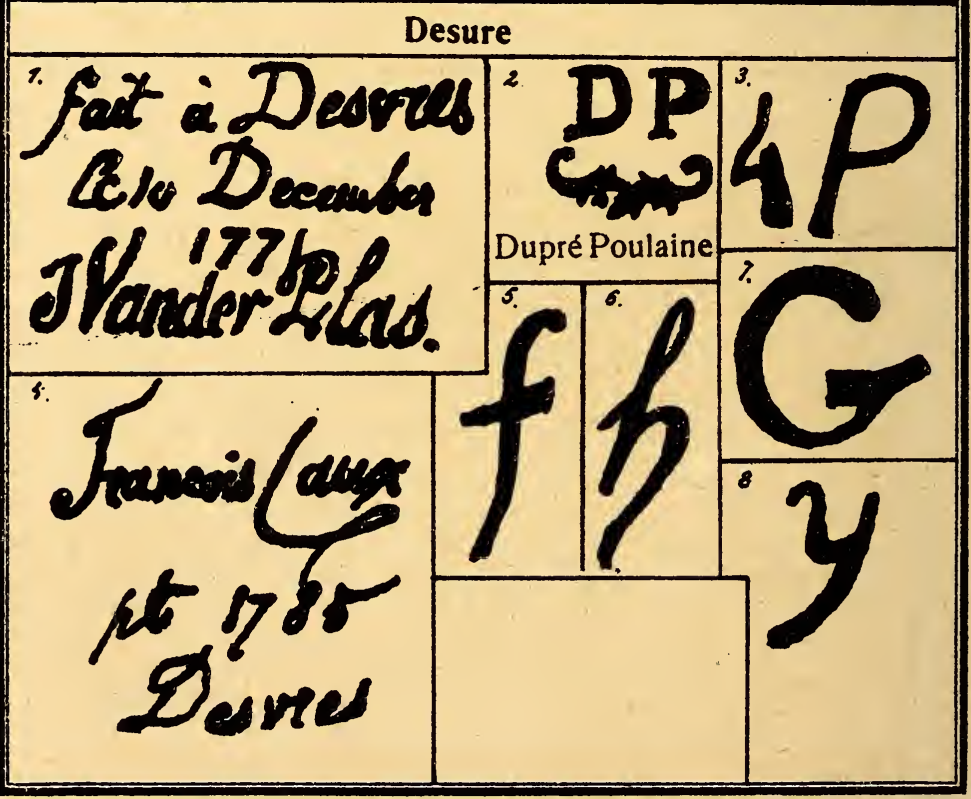




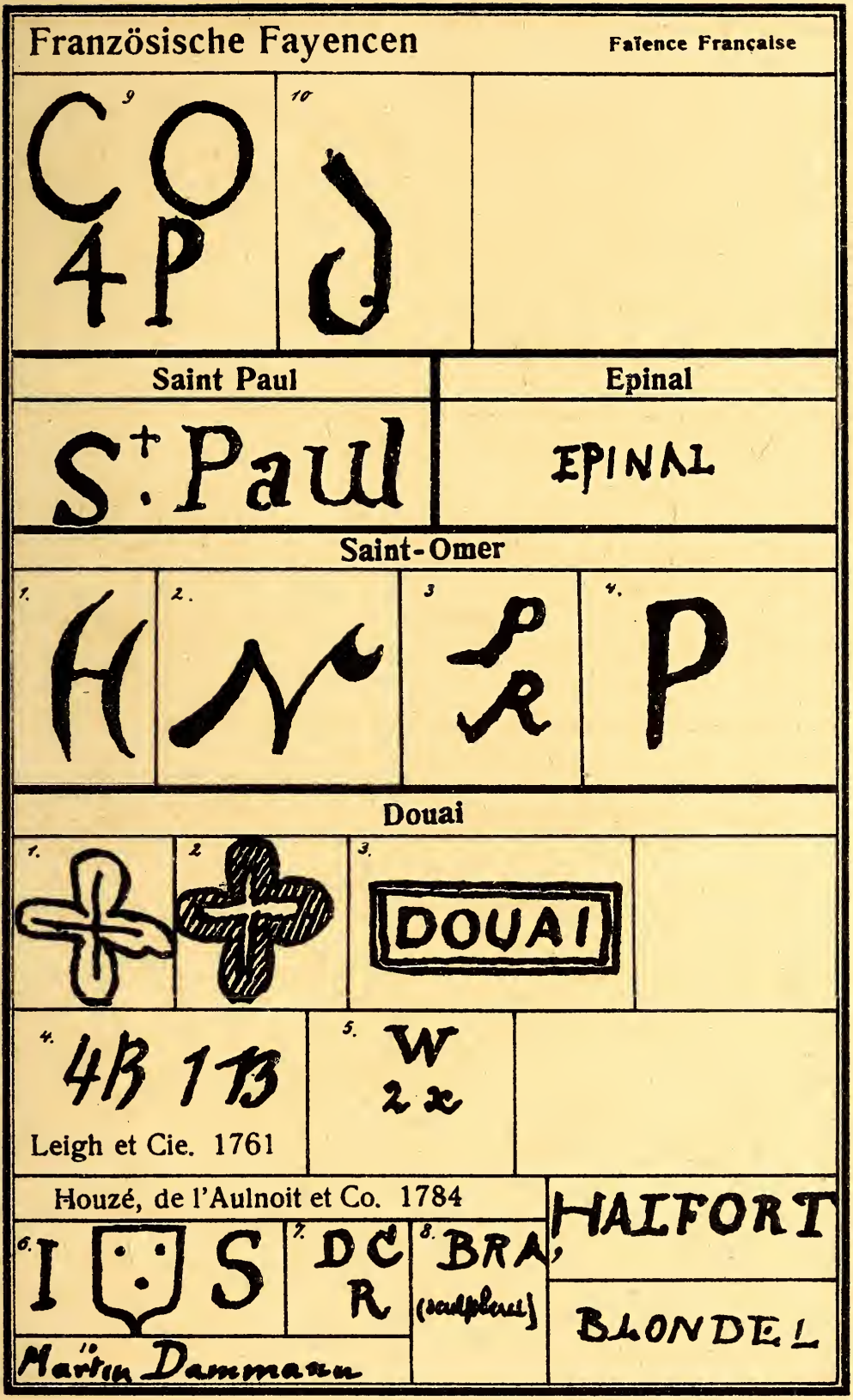




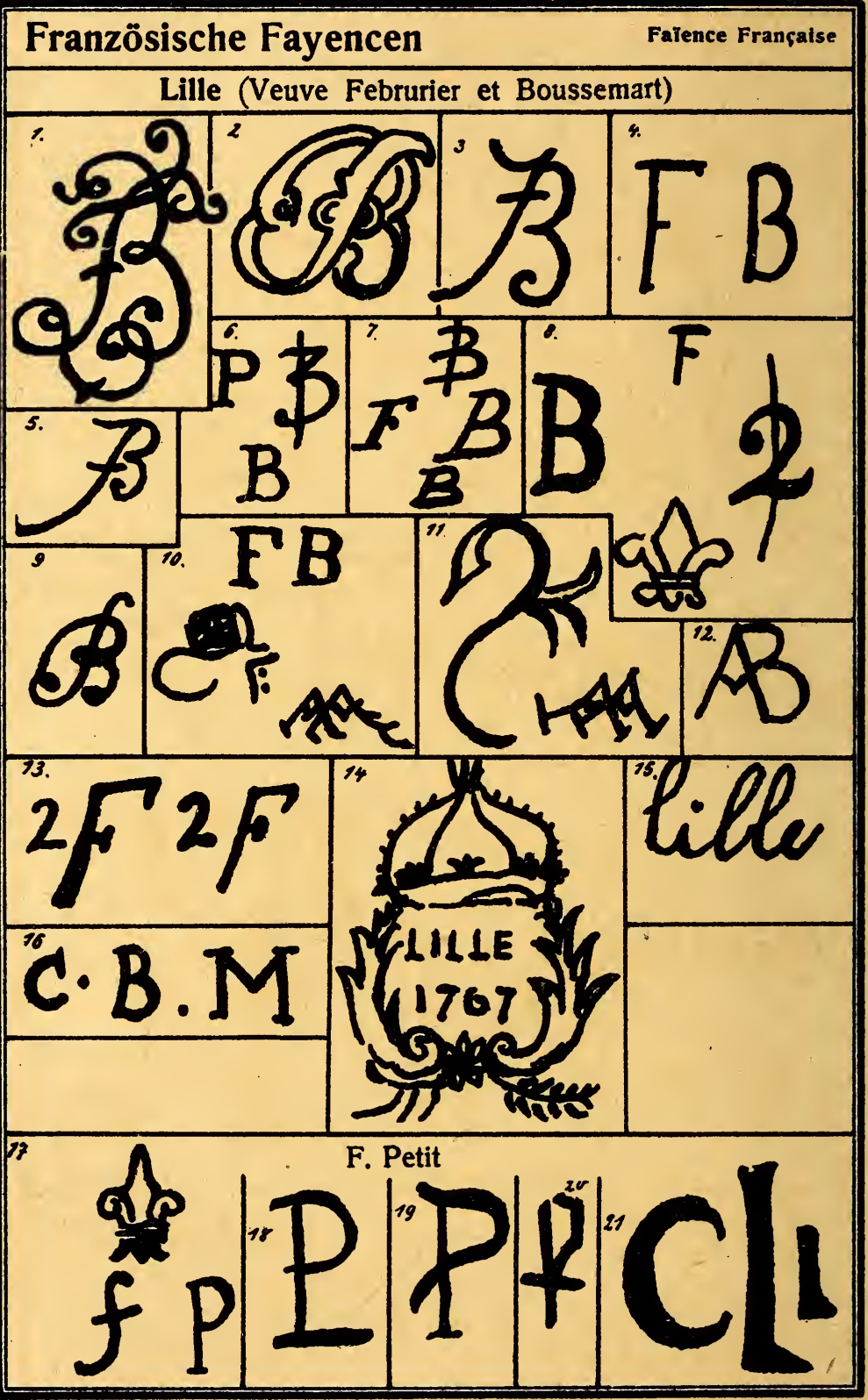




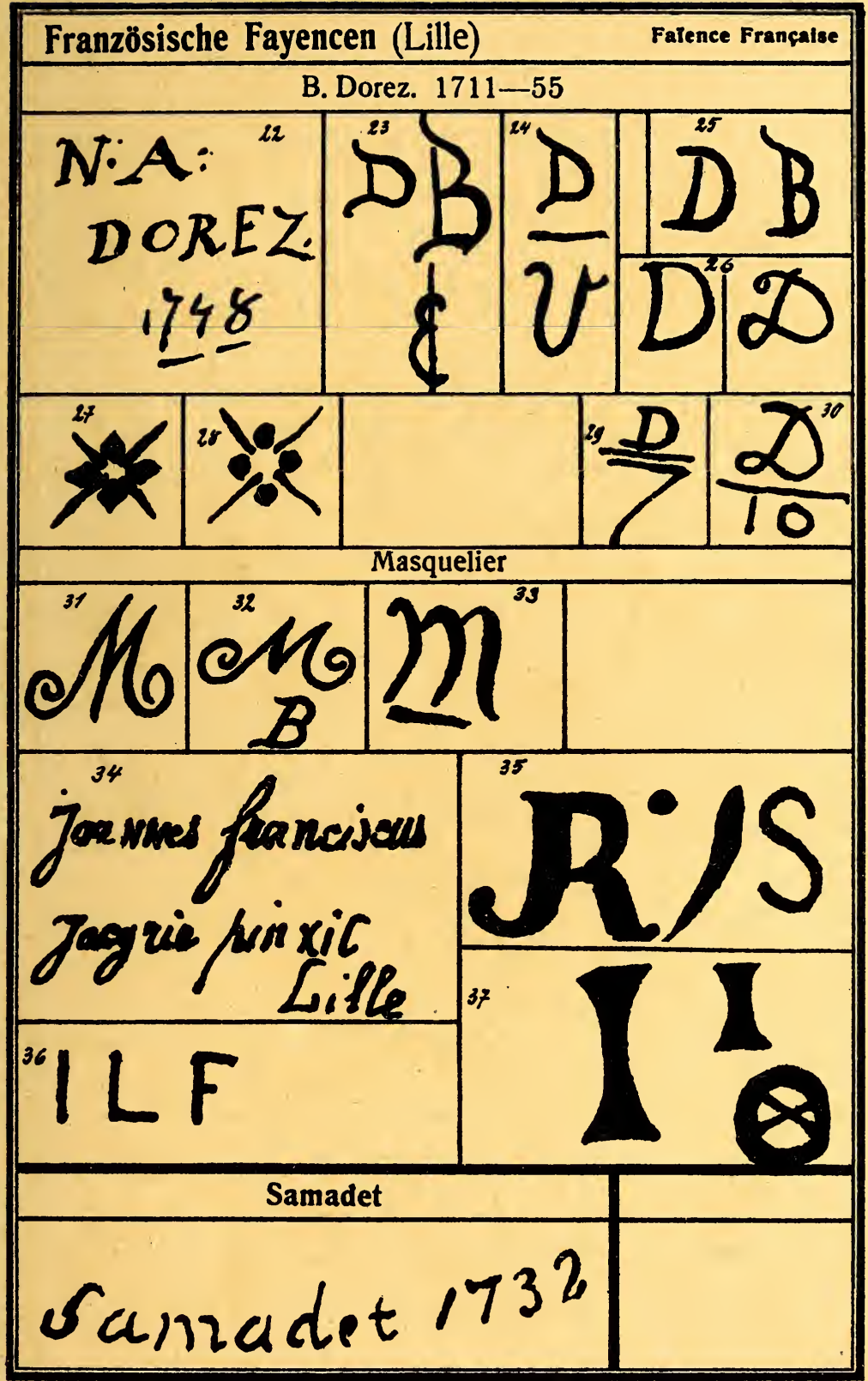


Französische Fayencen

Faience Française

St. Amand

7. P. Fauquez $1718-73$

achenugaw rig 2 jullicte 1885 2.

$C^{2}$ ansars Bepartevere 3. $s_{4} \operatorname{lin}_{11}$ s. a te. dumoz s. asec:a sxe 5.254

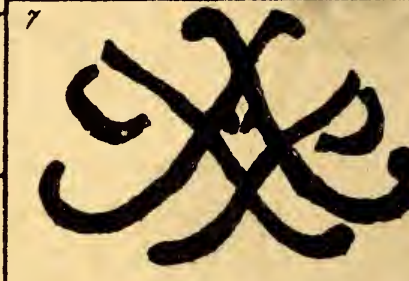

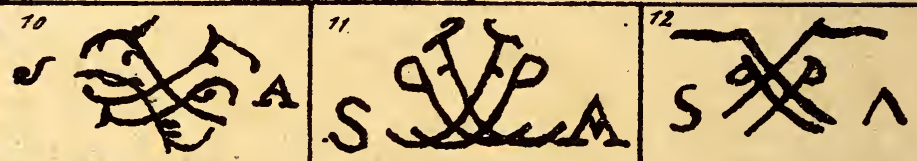
\% $S \cup \cdot A$ 77

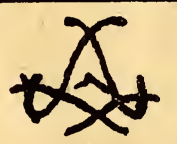

20 Grasea

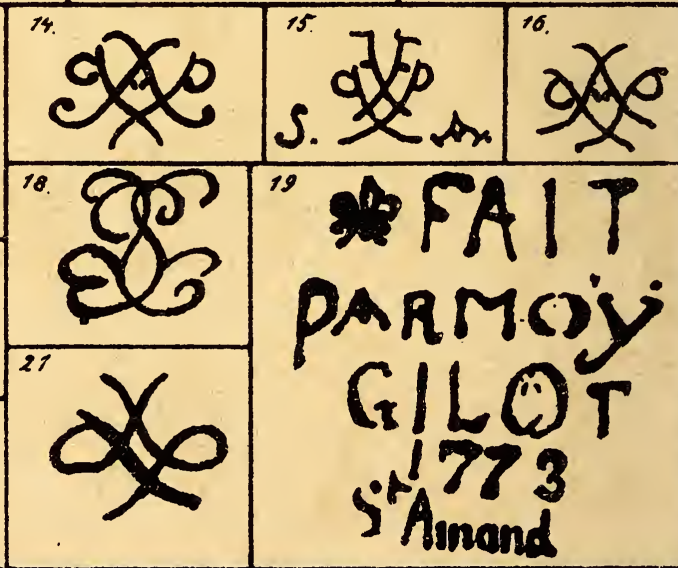




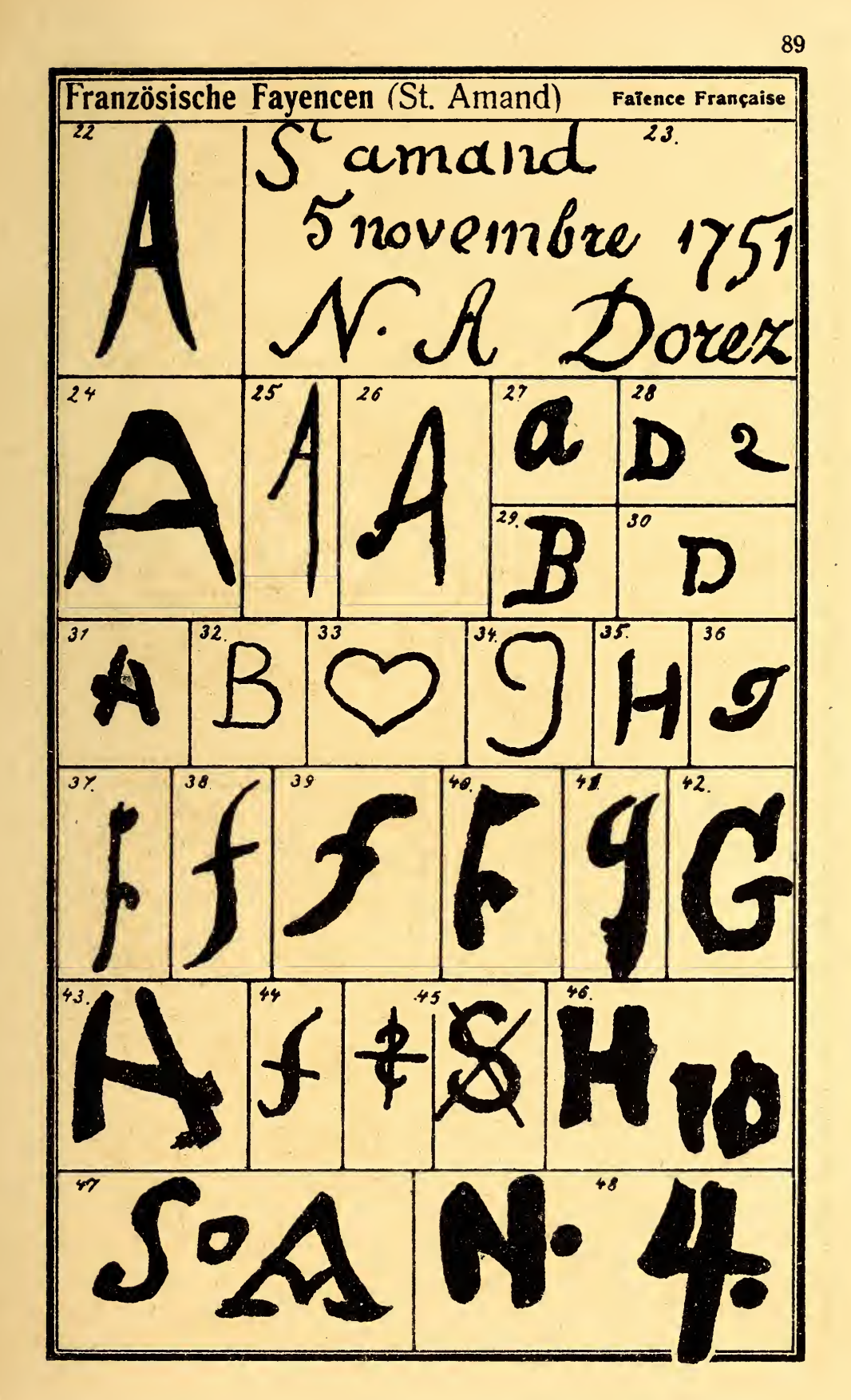




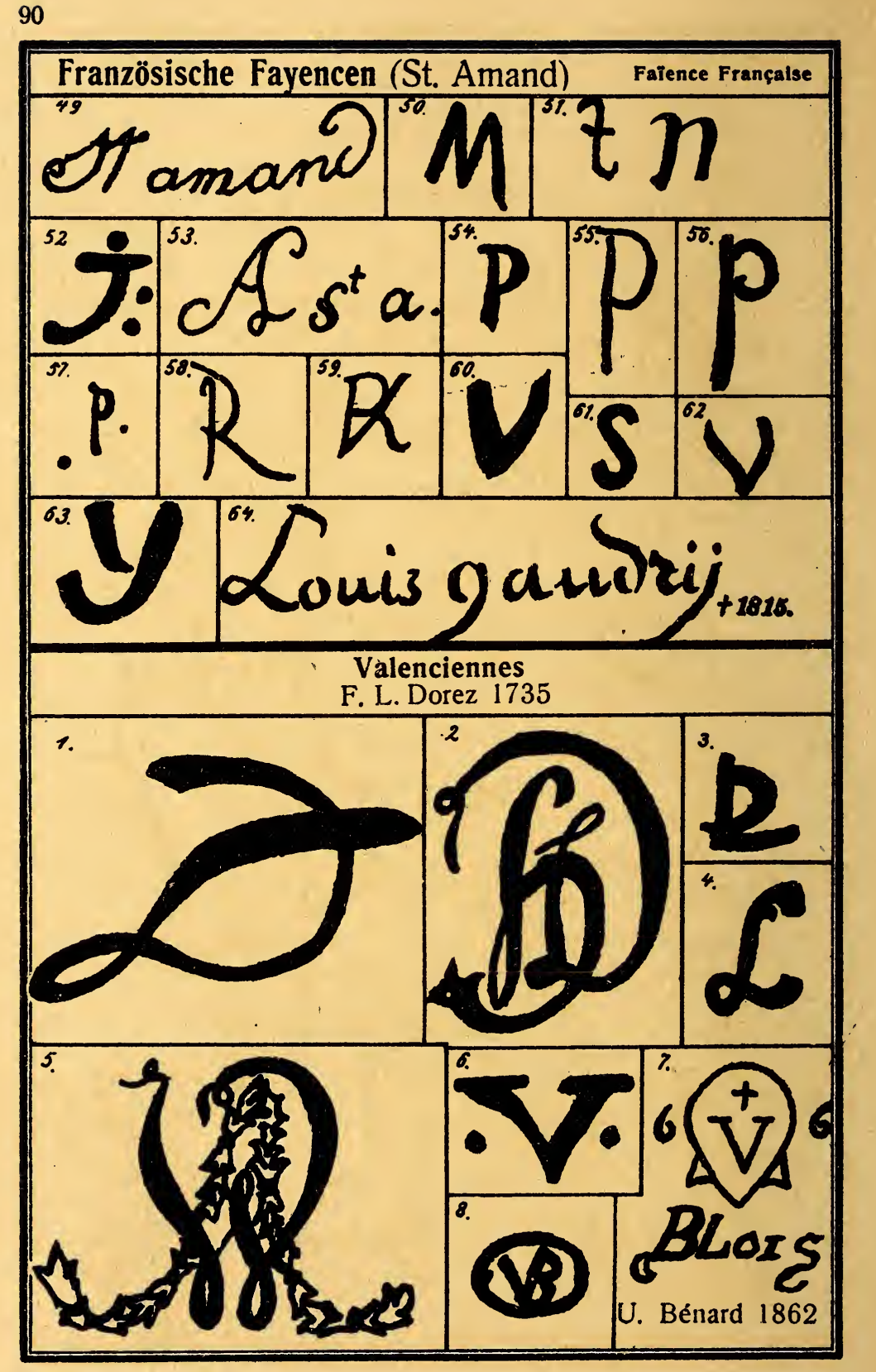




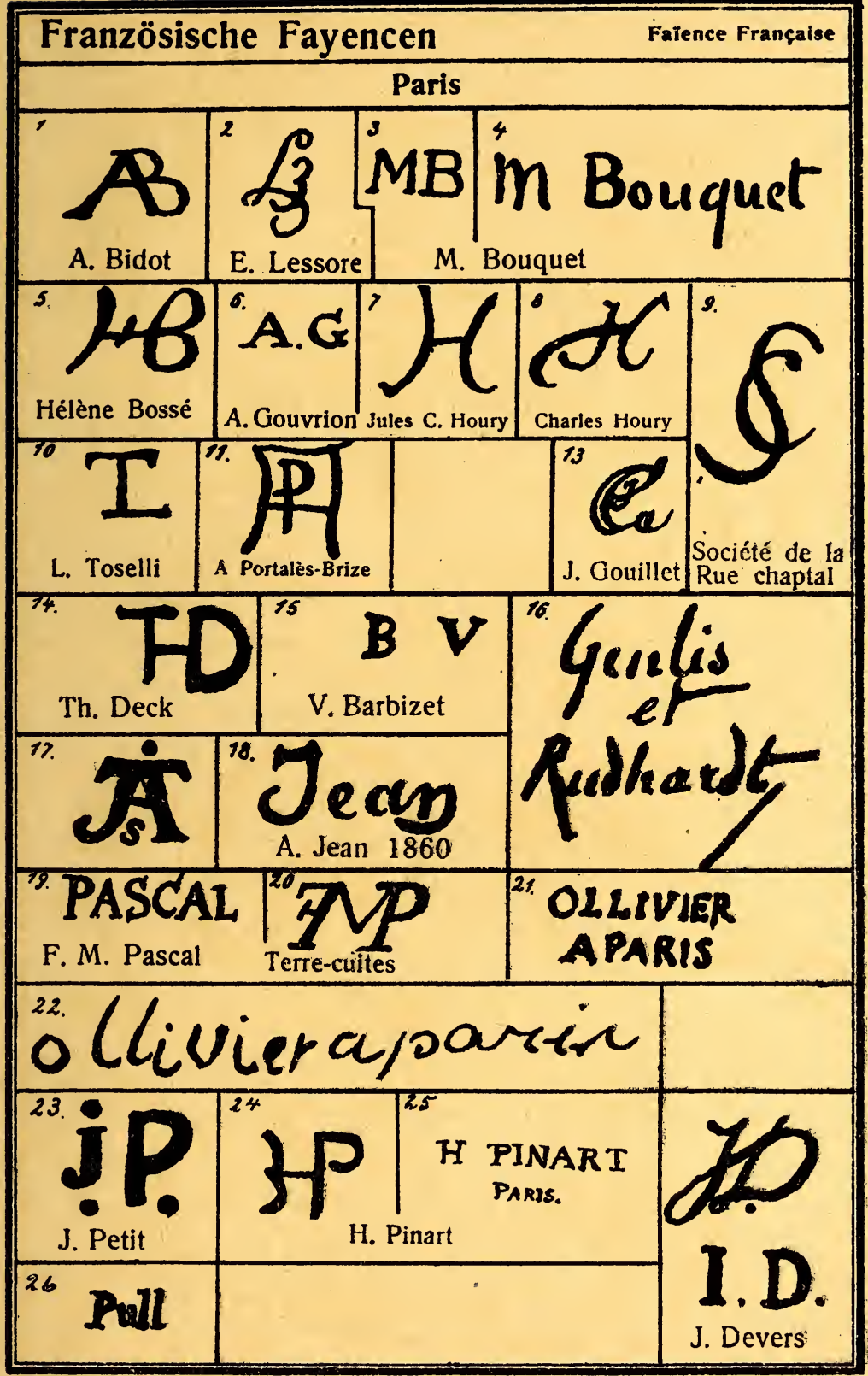




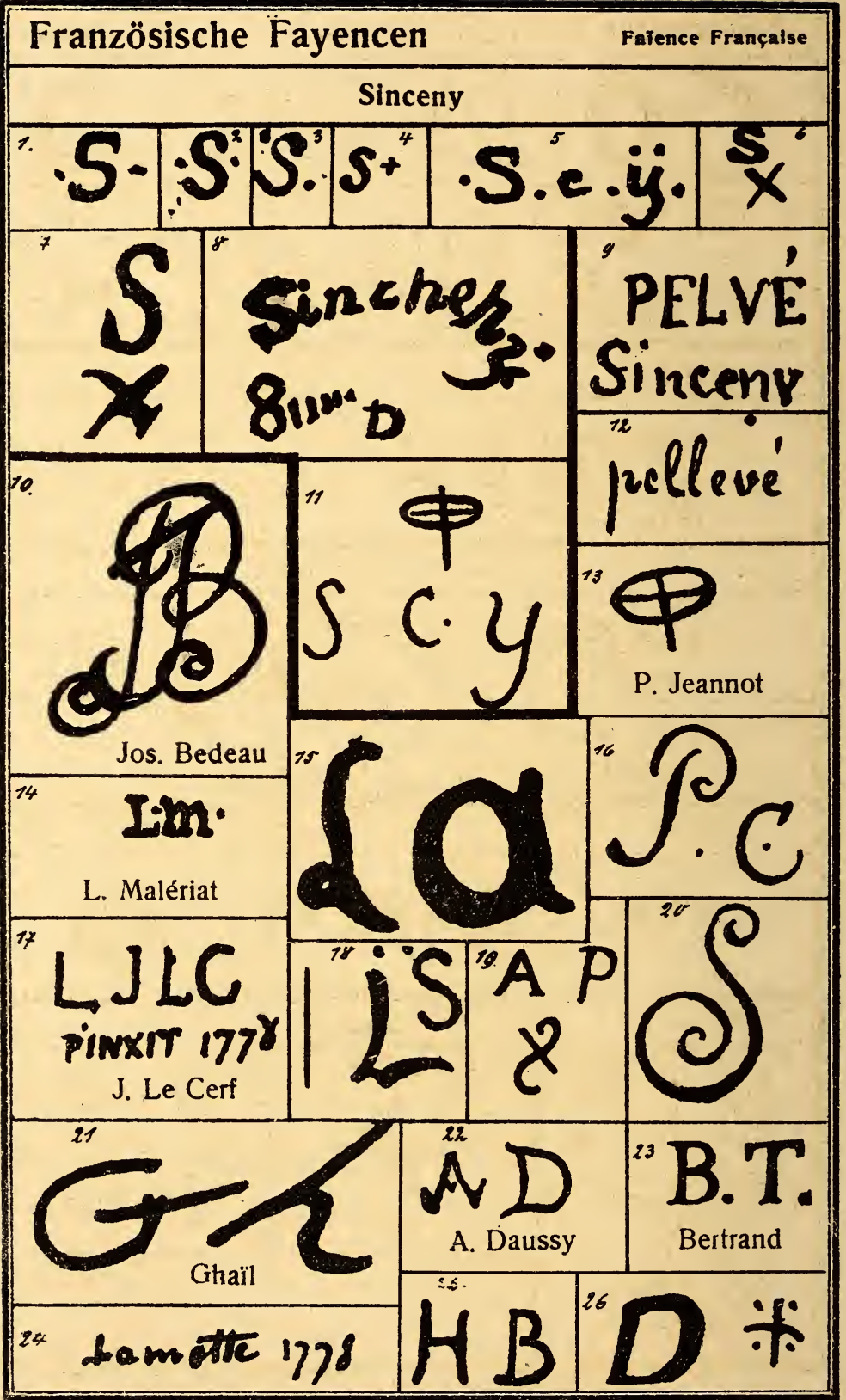




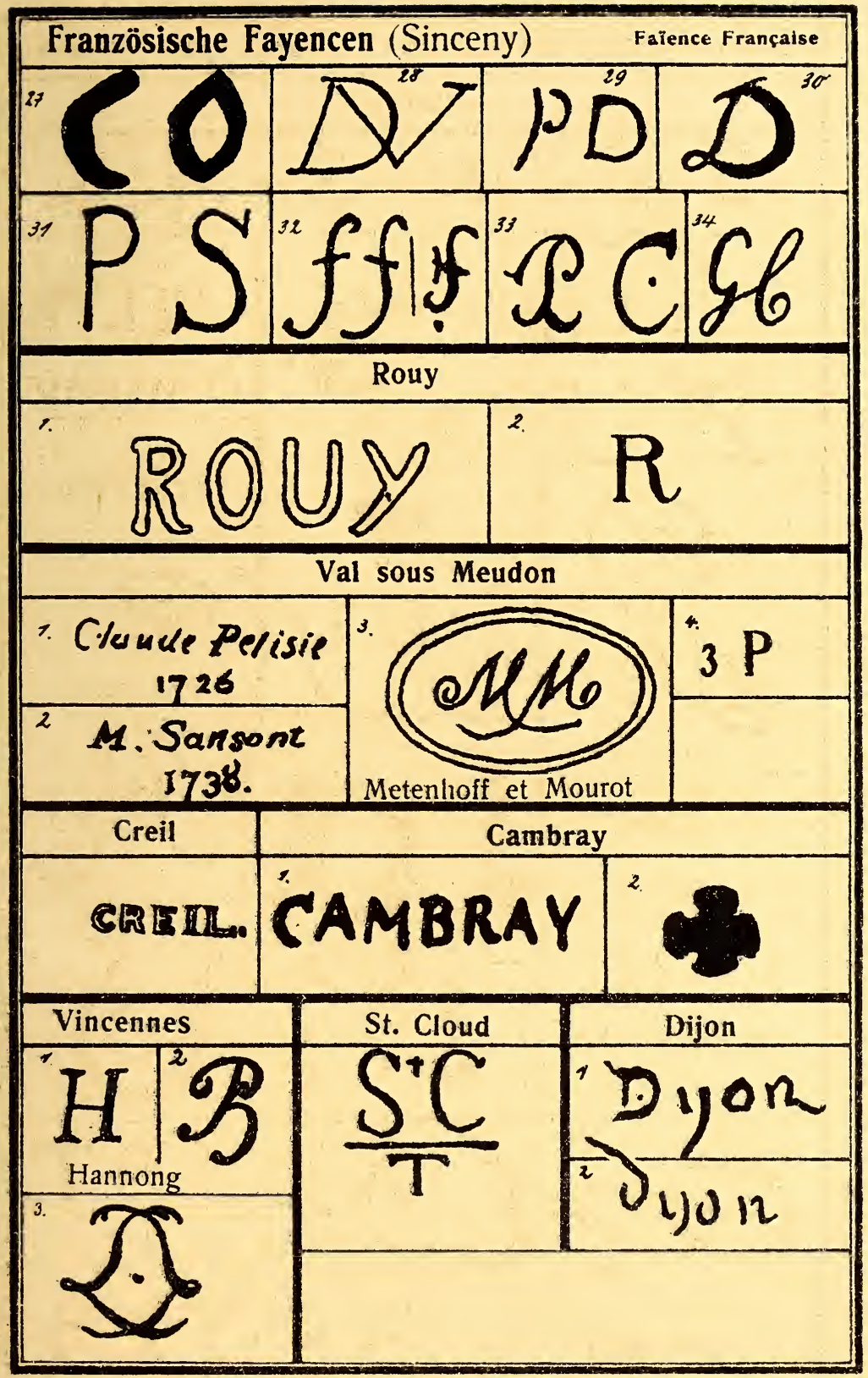




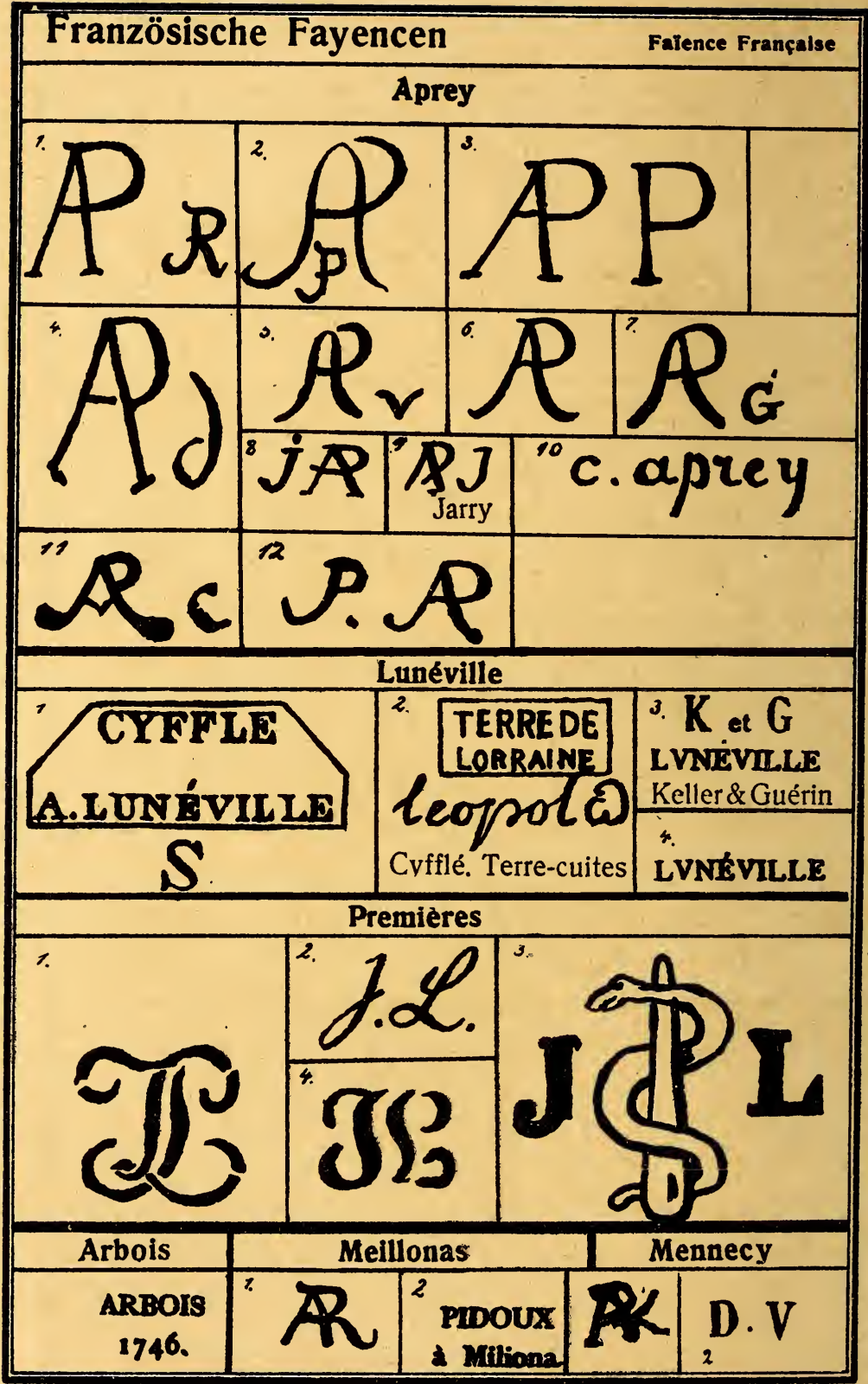




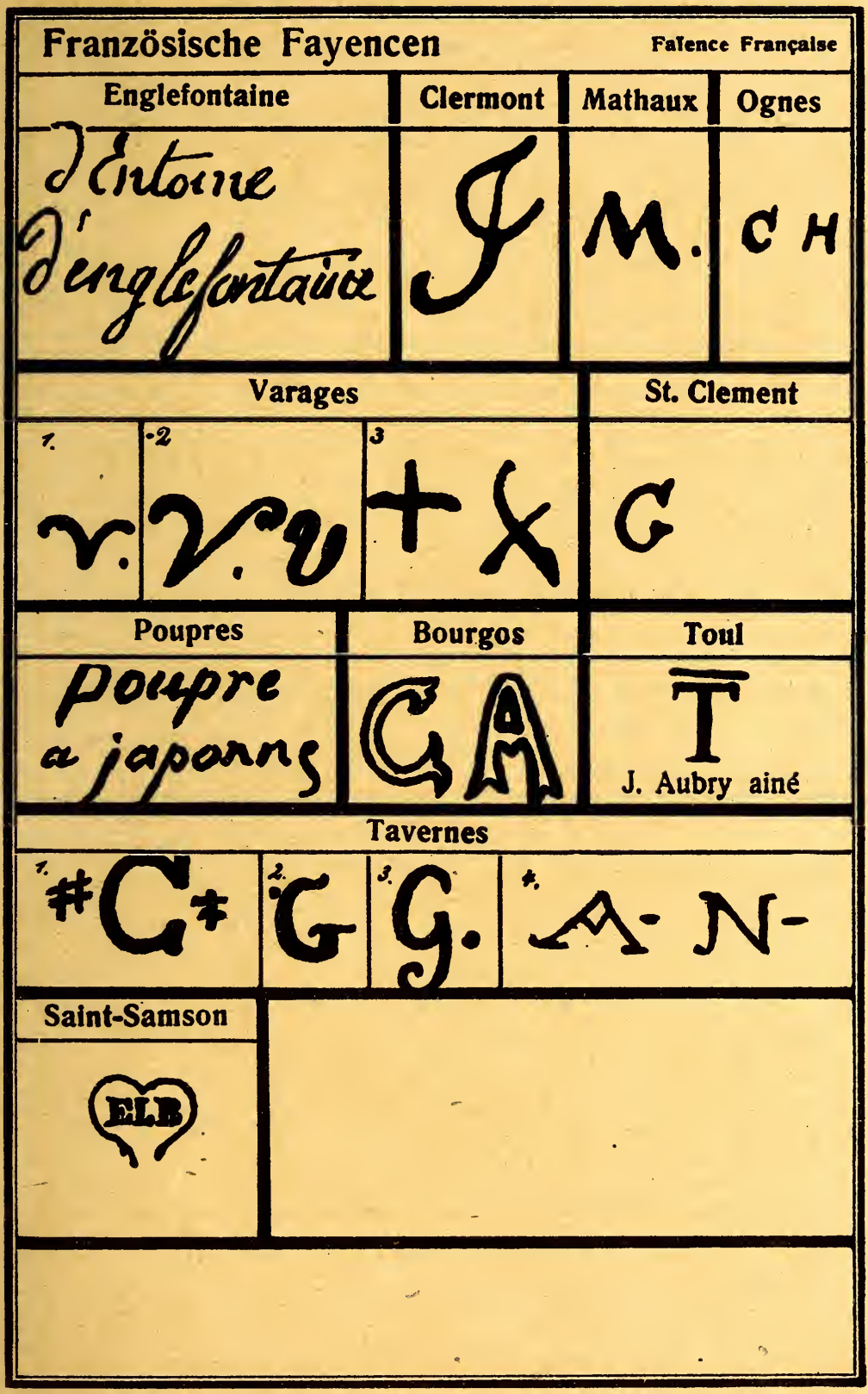




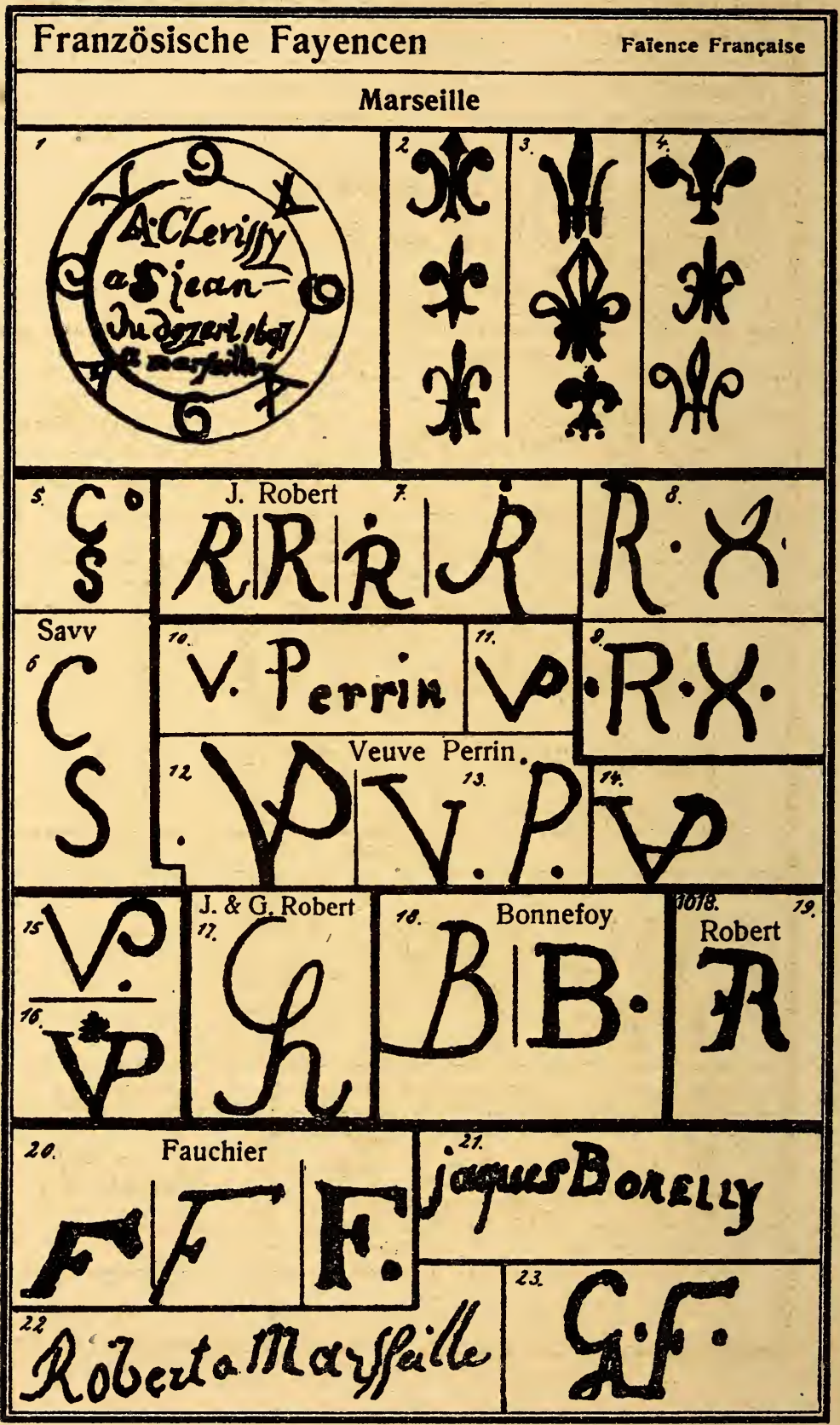




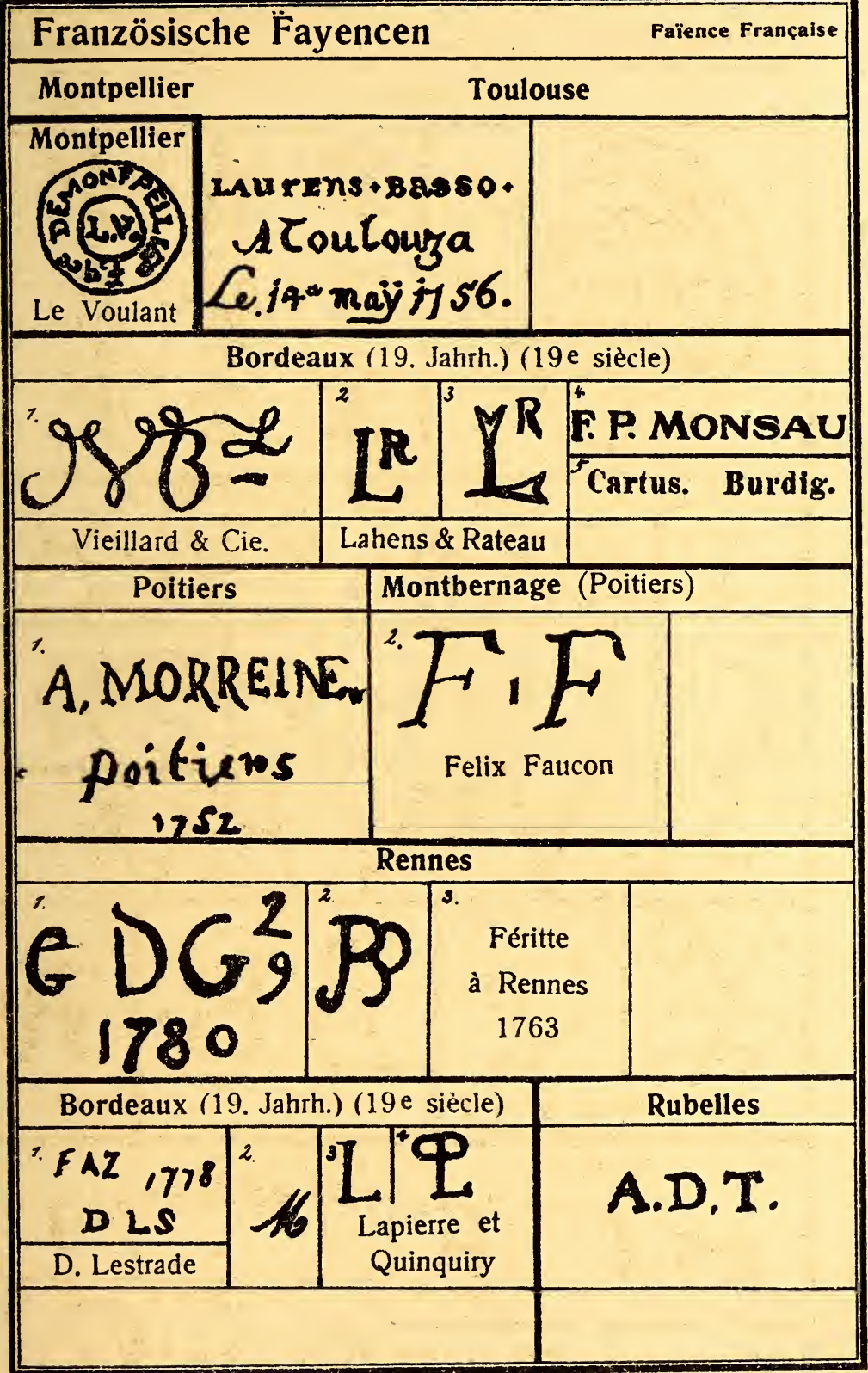




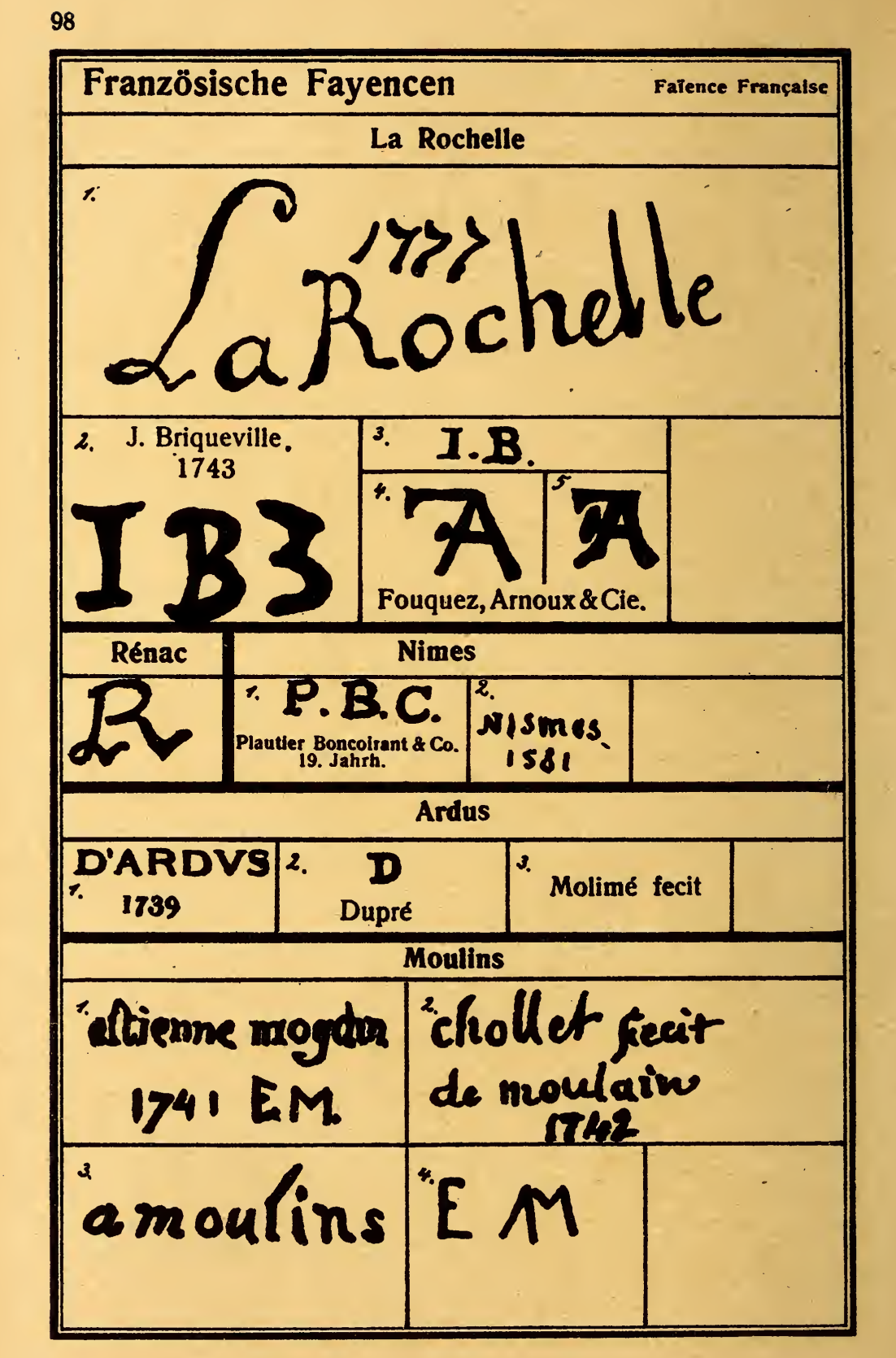




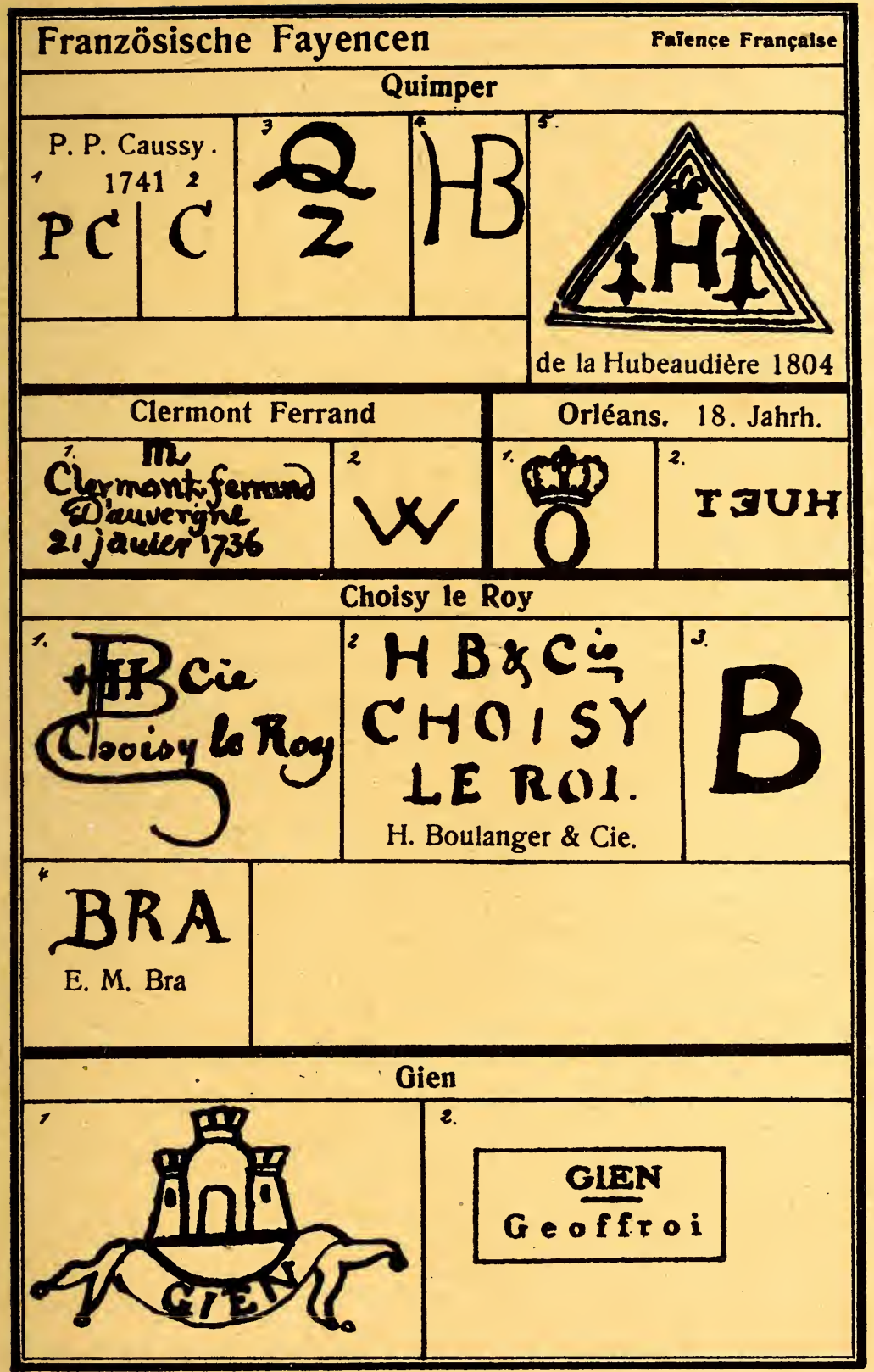




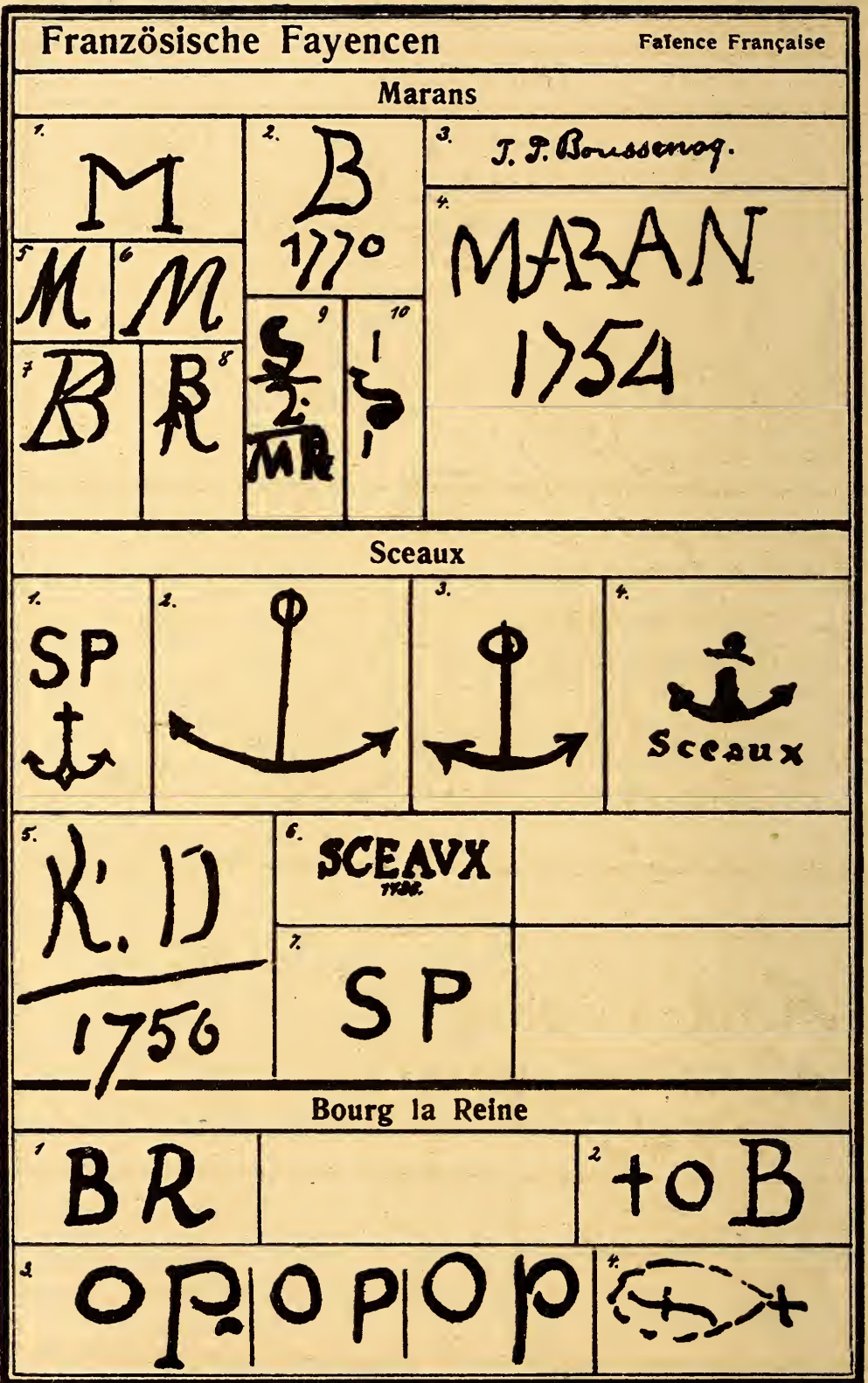




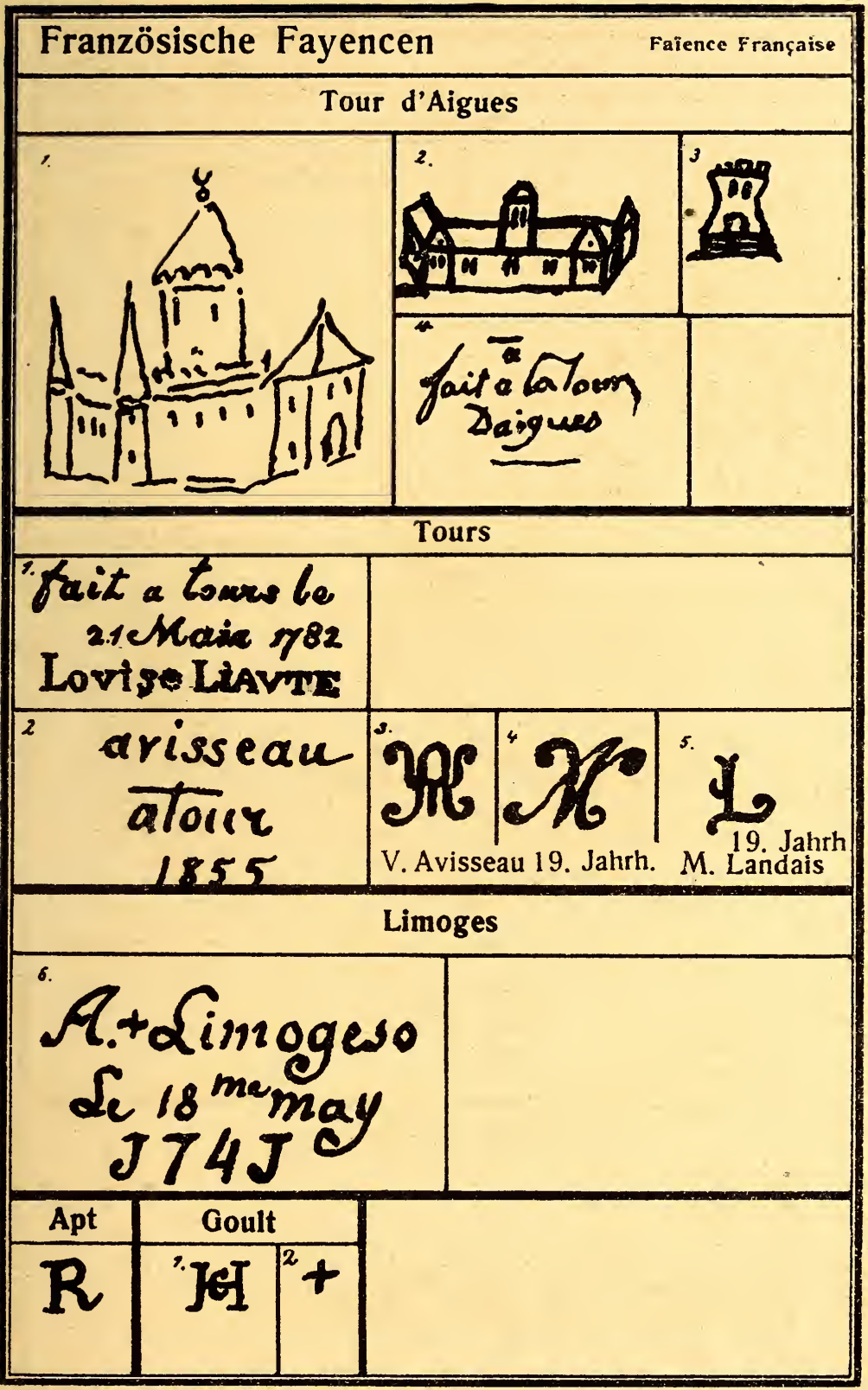


Französische Fayencen

Lamarque $[$ XIX. Jahrh.

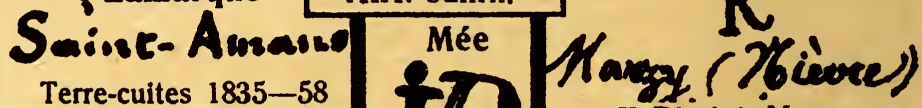

A. MAJORELLE

a INANCY.

$186 \%$

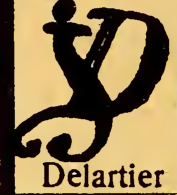

T. Ristòri, Marzy

Montereau

L. L. et $T$.

ONNAING (Nord YAUZAS.

Voisinlieu (Grès)
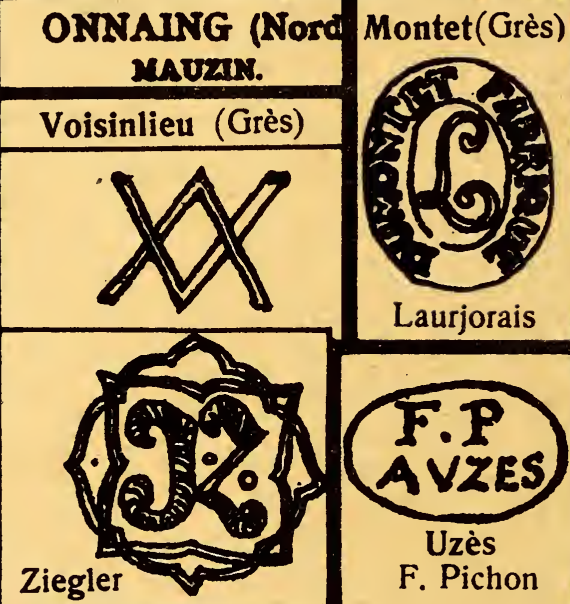

Leboeuf et Thibaut

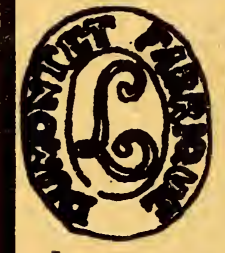

Laurjorais

Rubelles

$S$ et $M$

8. Clement.

Manufiecture

do Saint CKement.

GALTS

NA MCI.

Uzès

F. Pichon

Saint Clément

(Savoyen)

St. Jean de Kaurienne. Cean. 9 oxy

La Forèst.

Bouchard.

la forid

s sarsy

1745 


\section{Deutsche Fayencen}

Farence Allemande

\section{StraBburg (Strasbourg)}

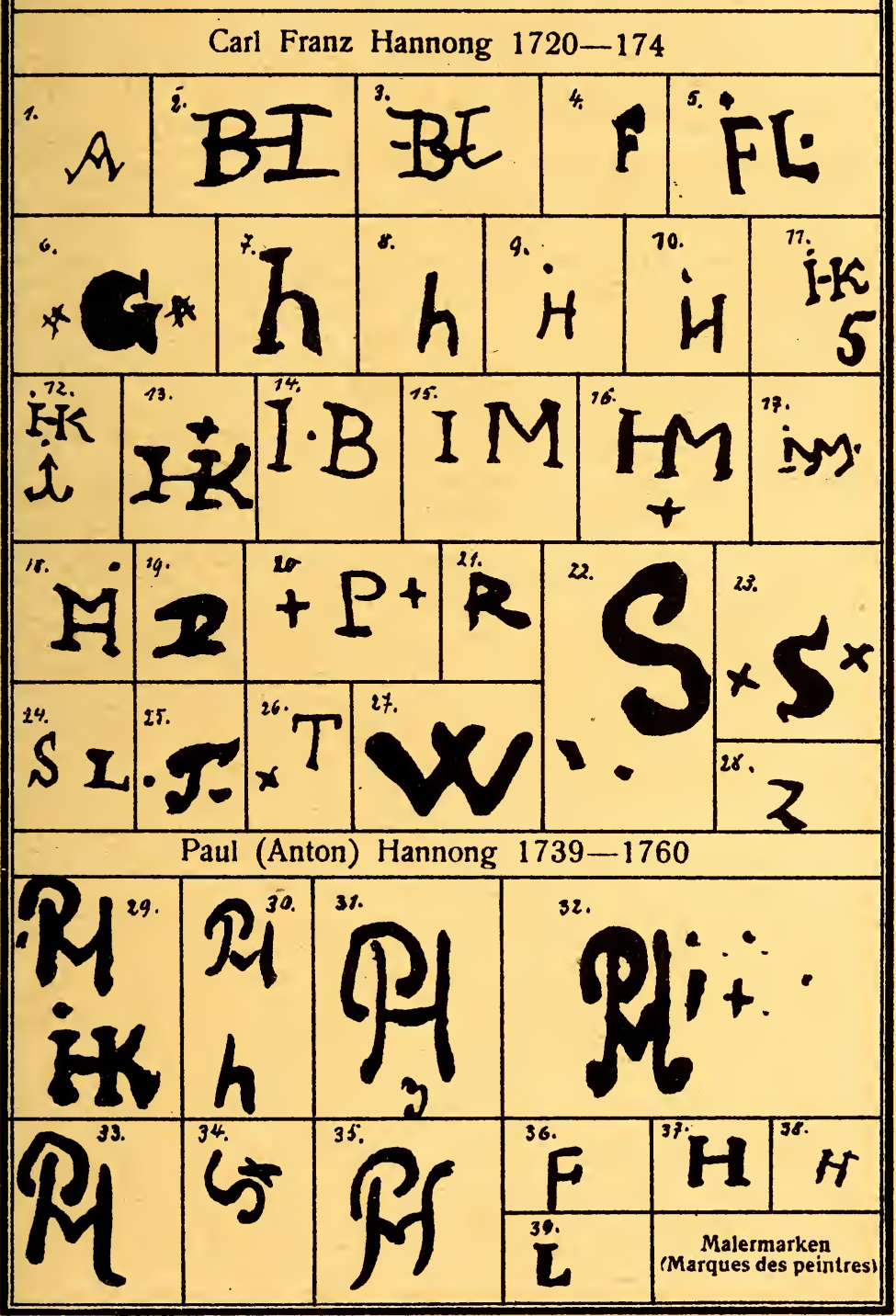




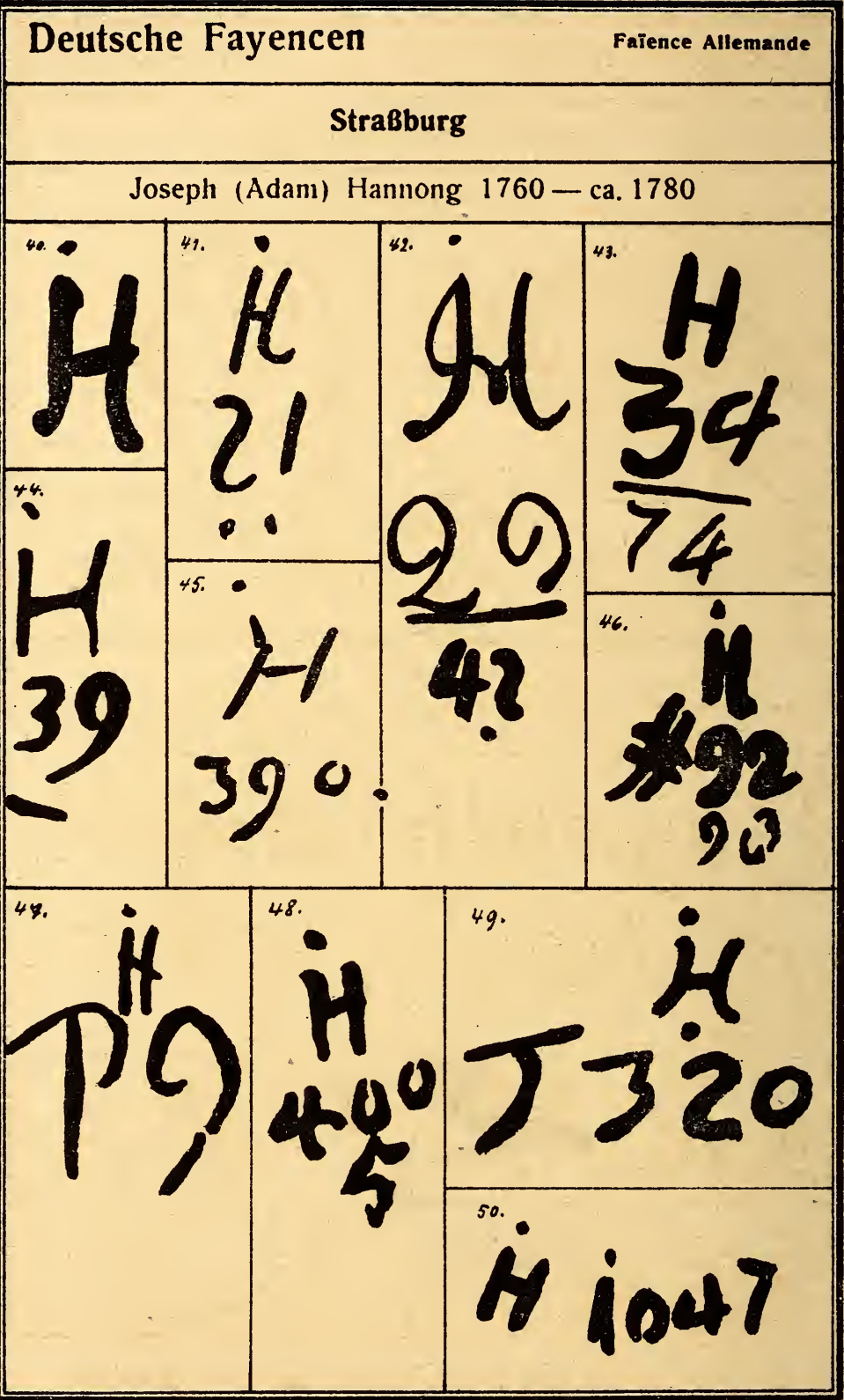




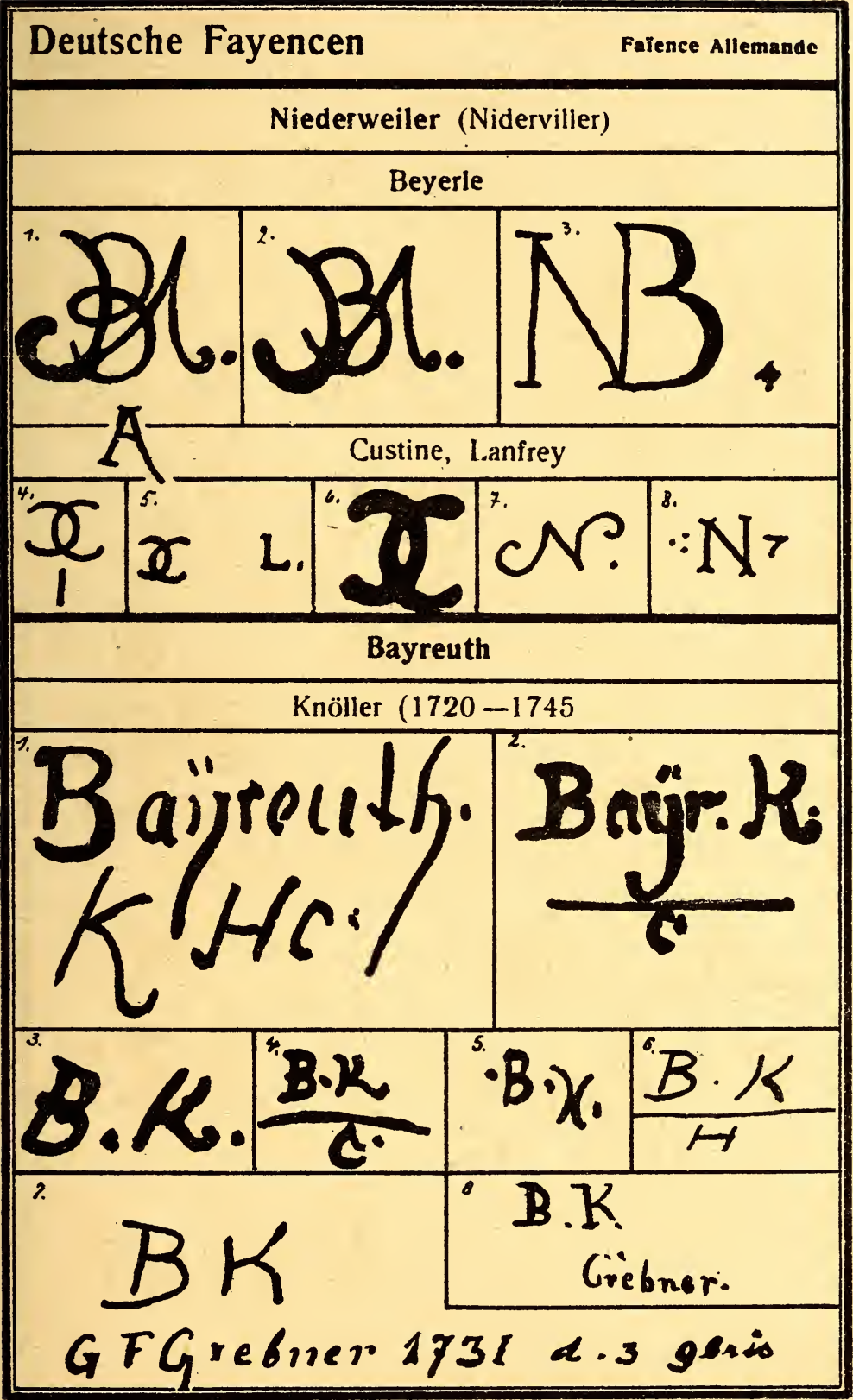




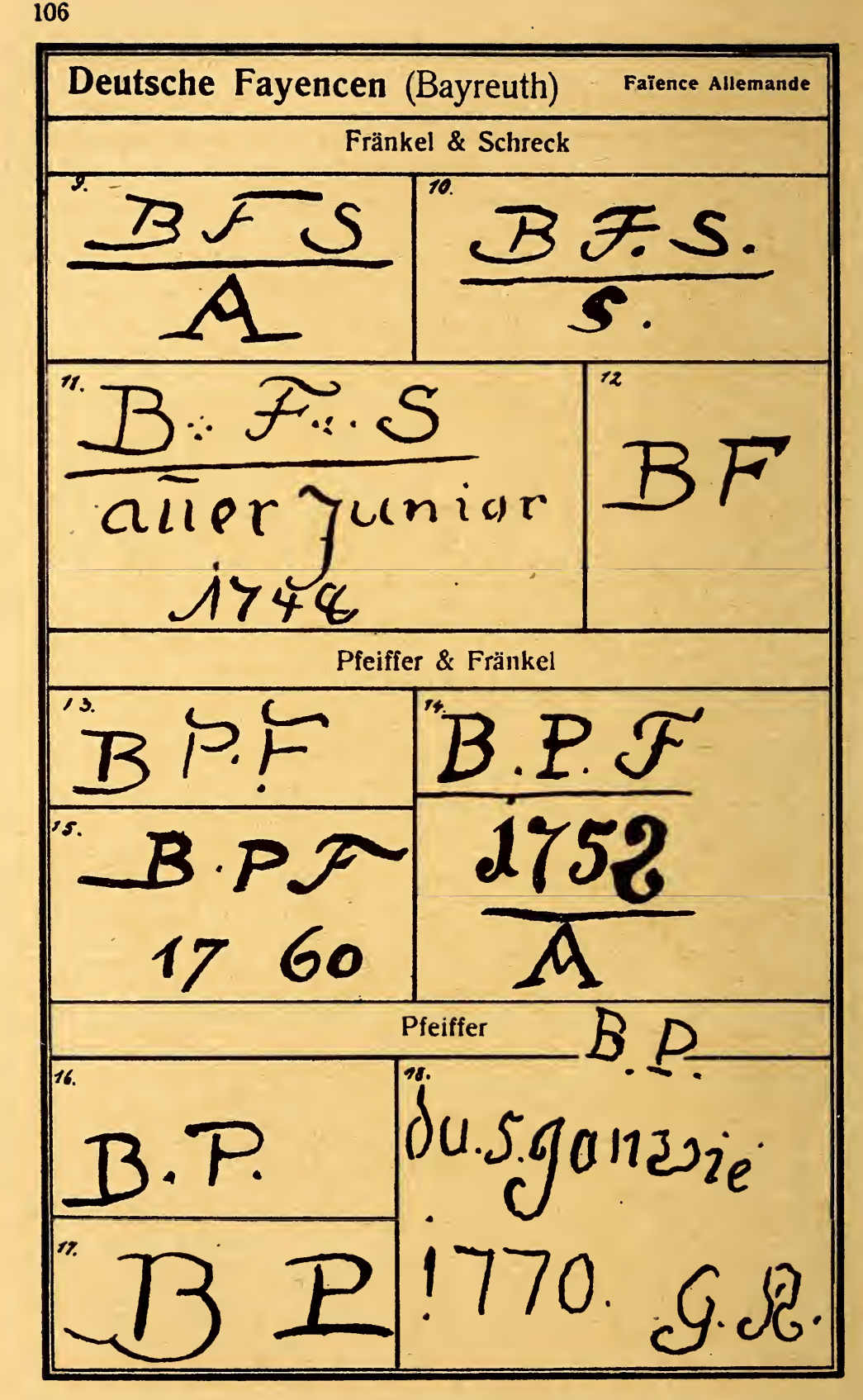




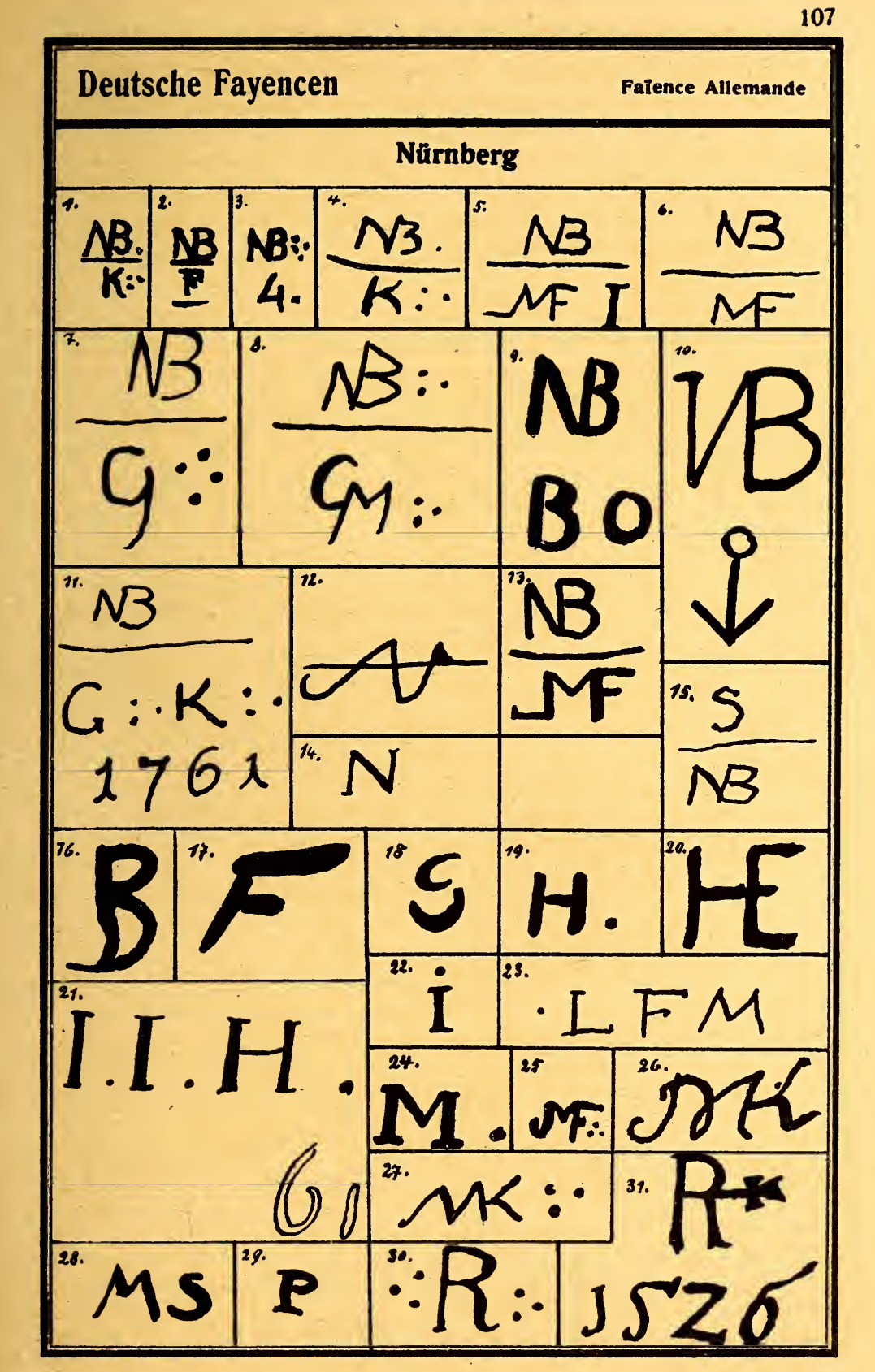




\section{Deutsche Fayencen}

Fafence Allemande

(Nürnberg)

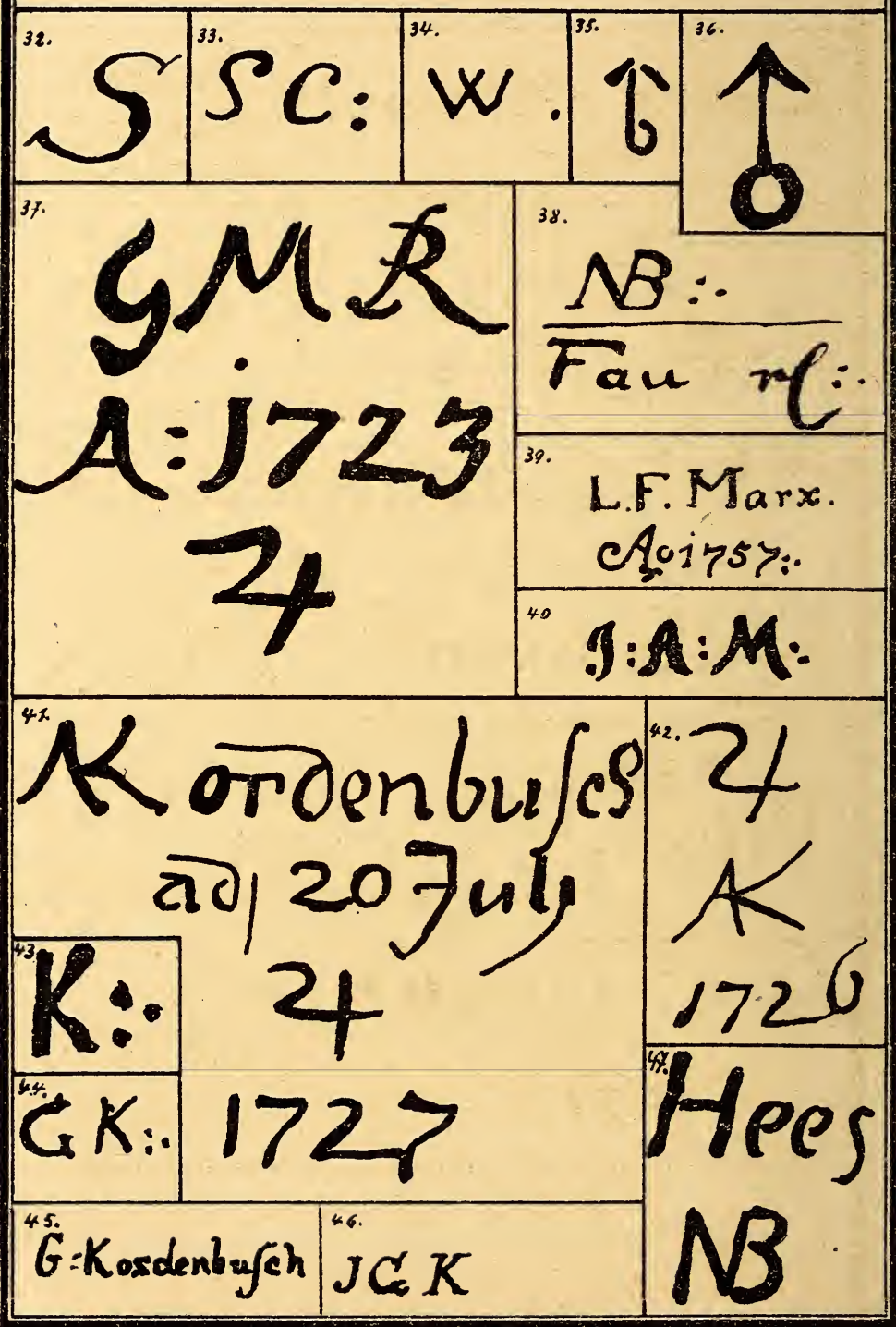


(Nünberg)

$4 \%$

S'eorg Micha el "Btrobbel: Taliber restreviovis Stebizer $177 i$

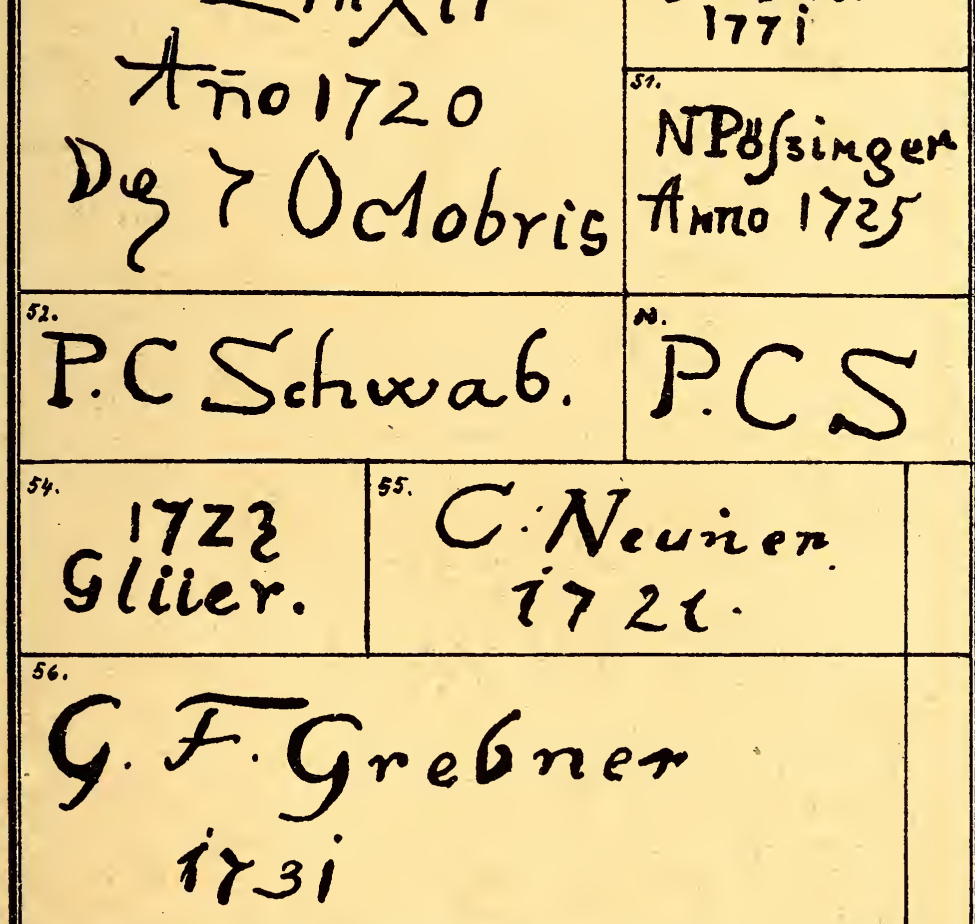




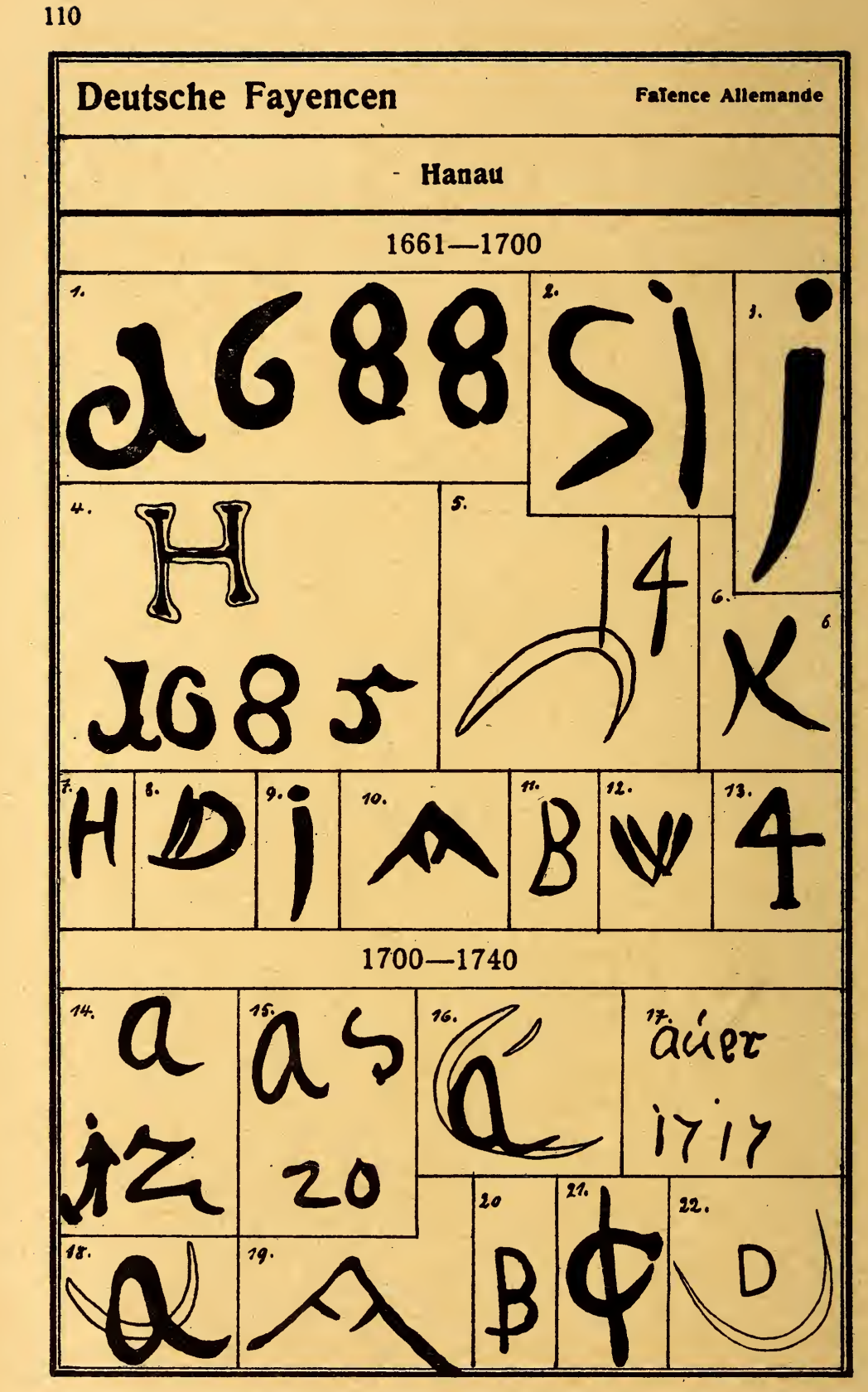




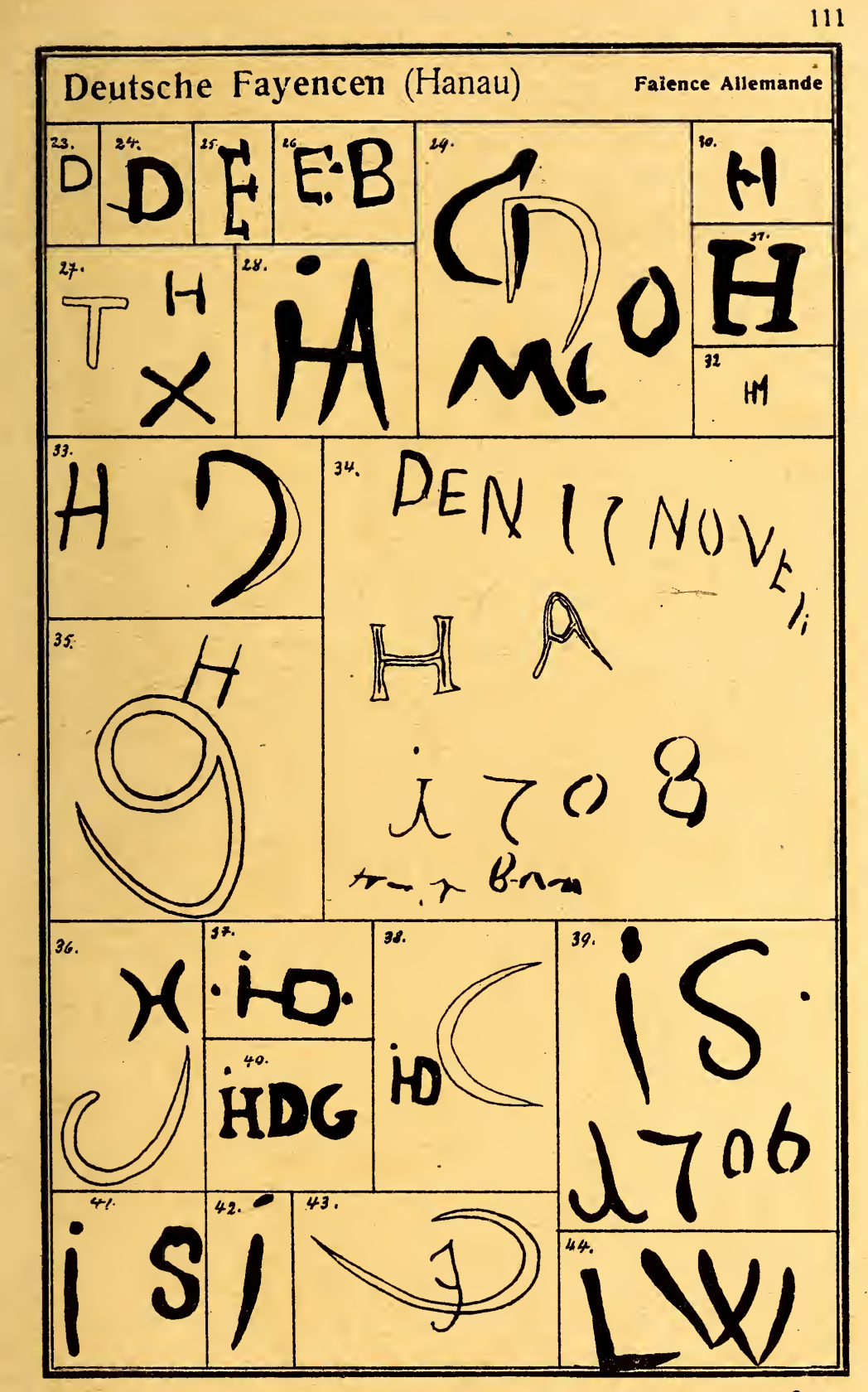




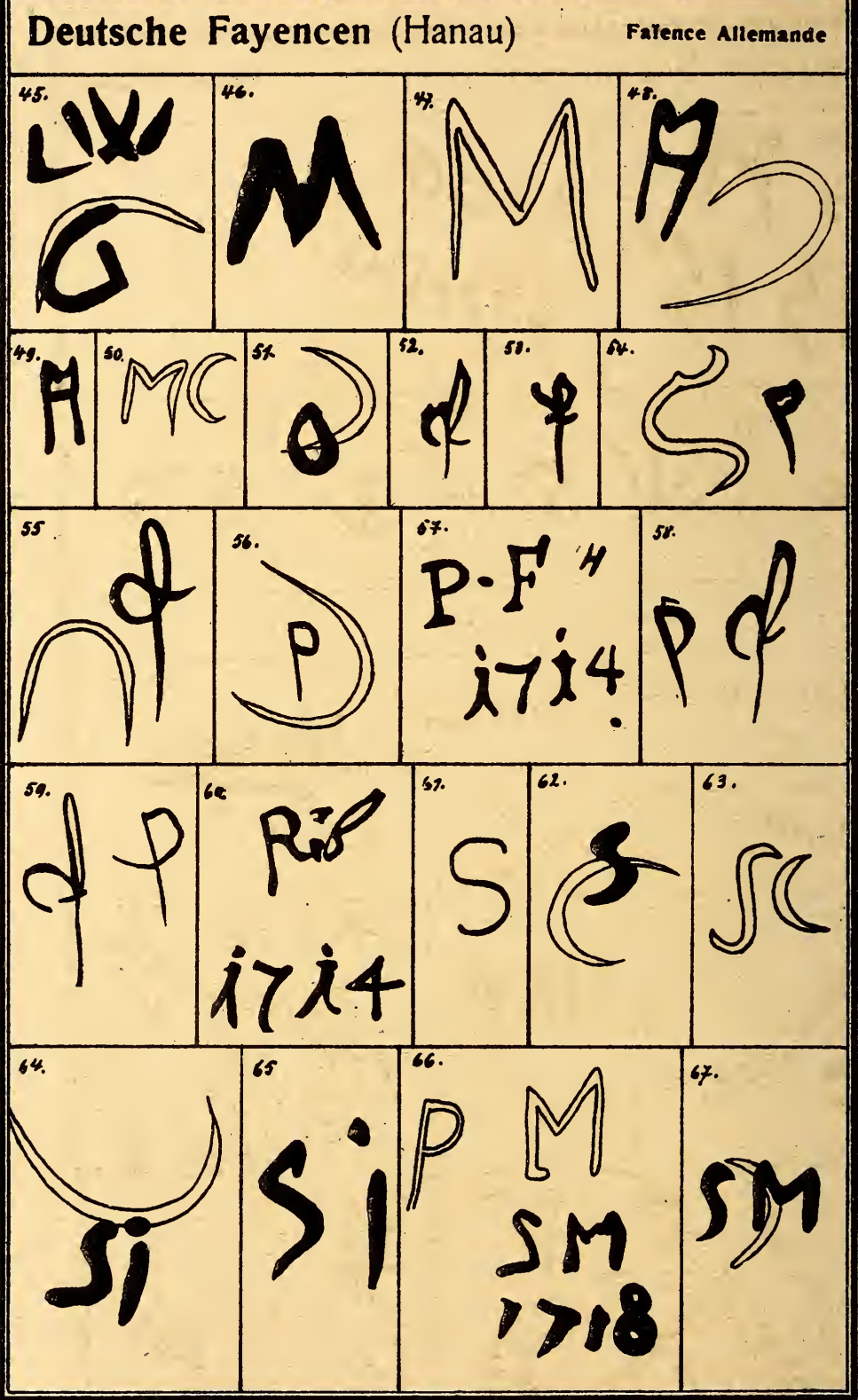




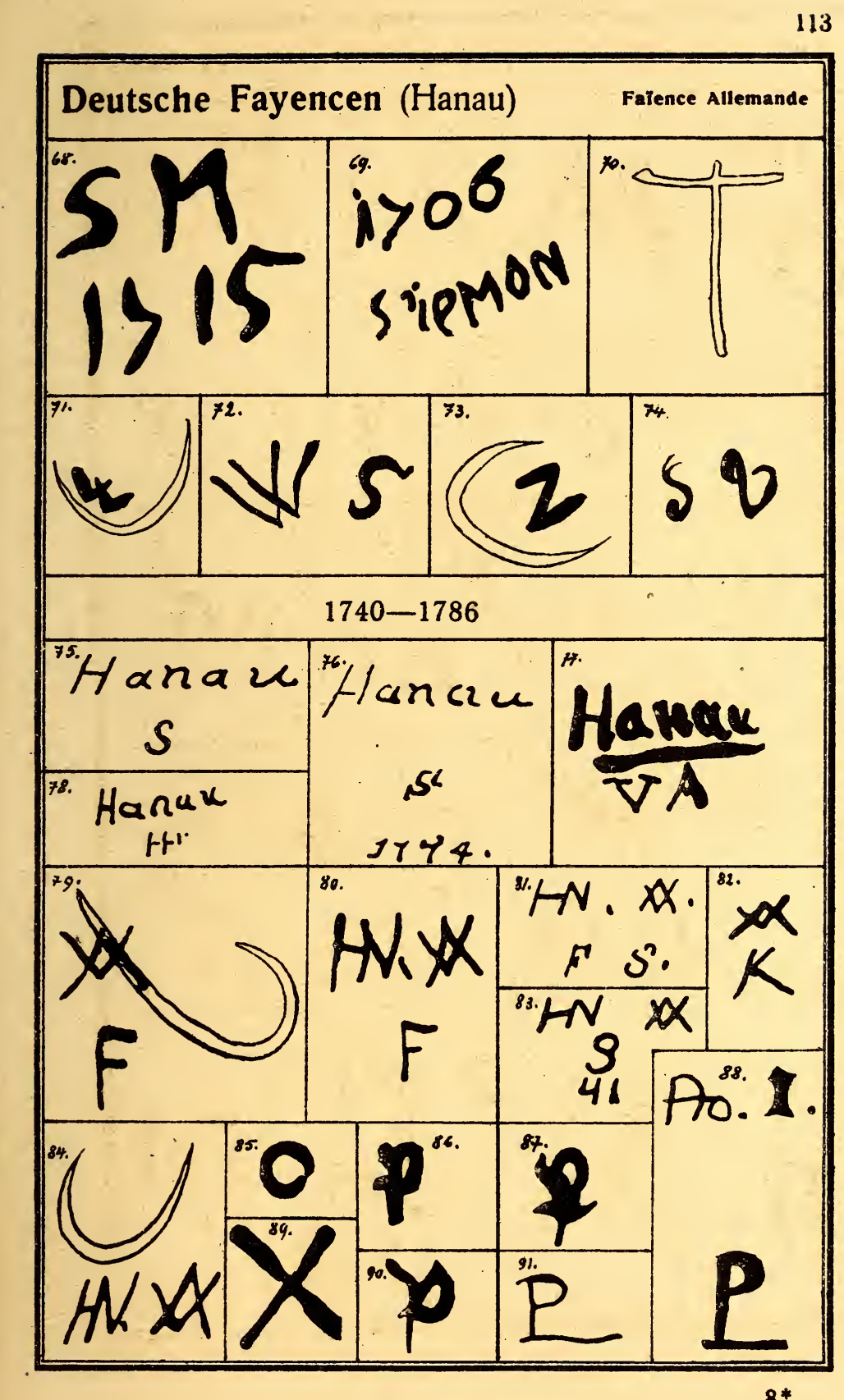




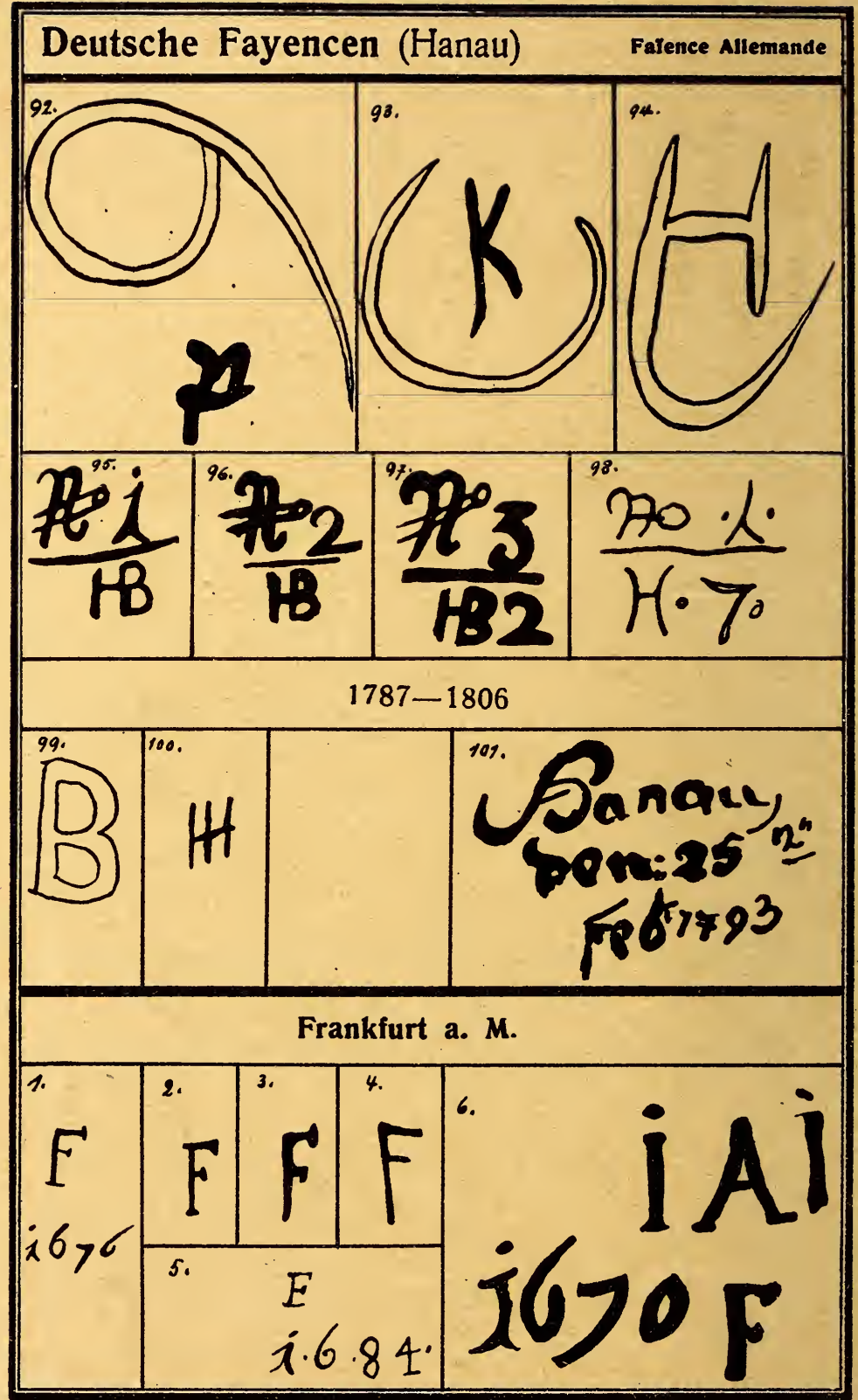




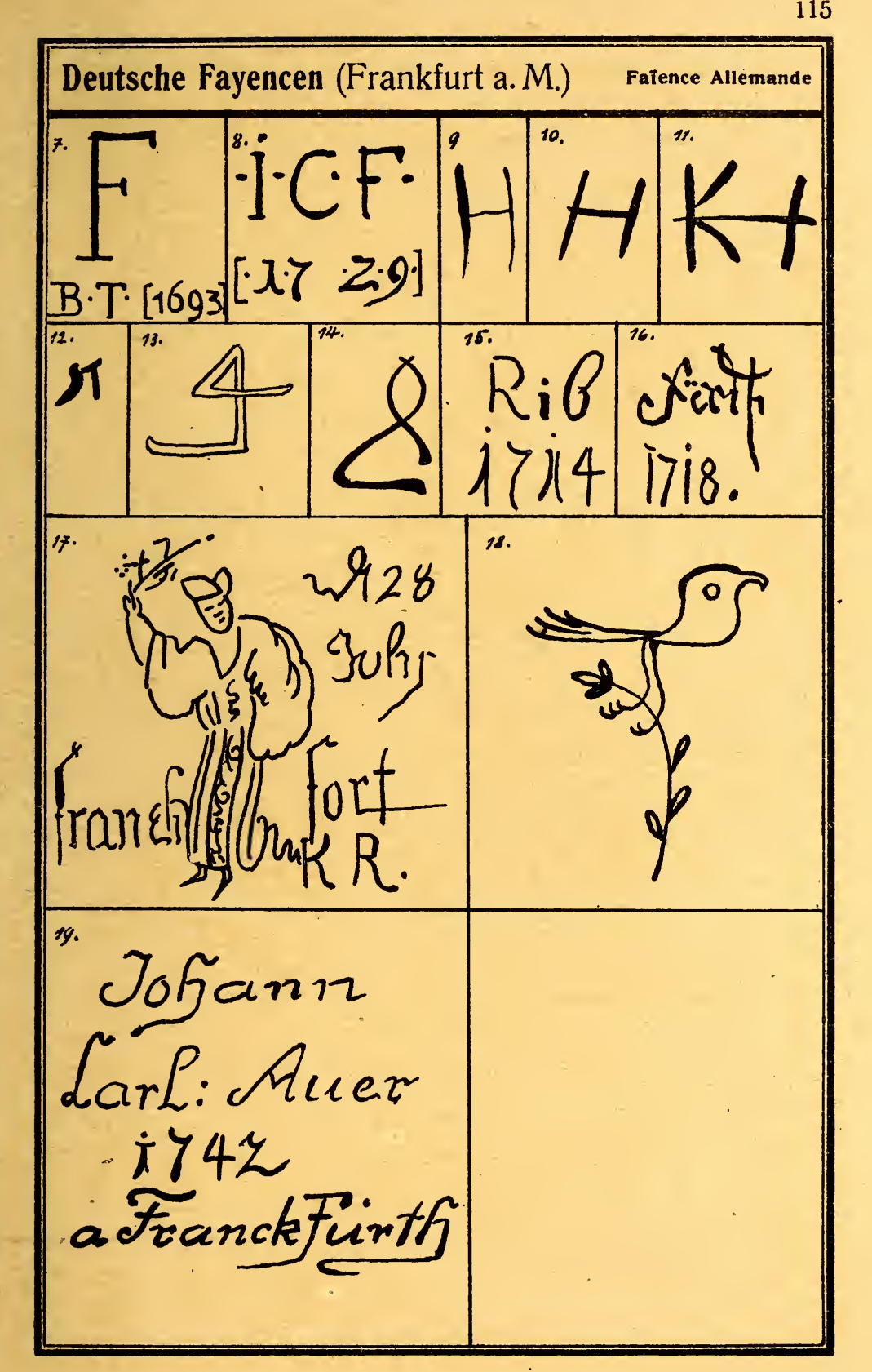




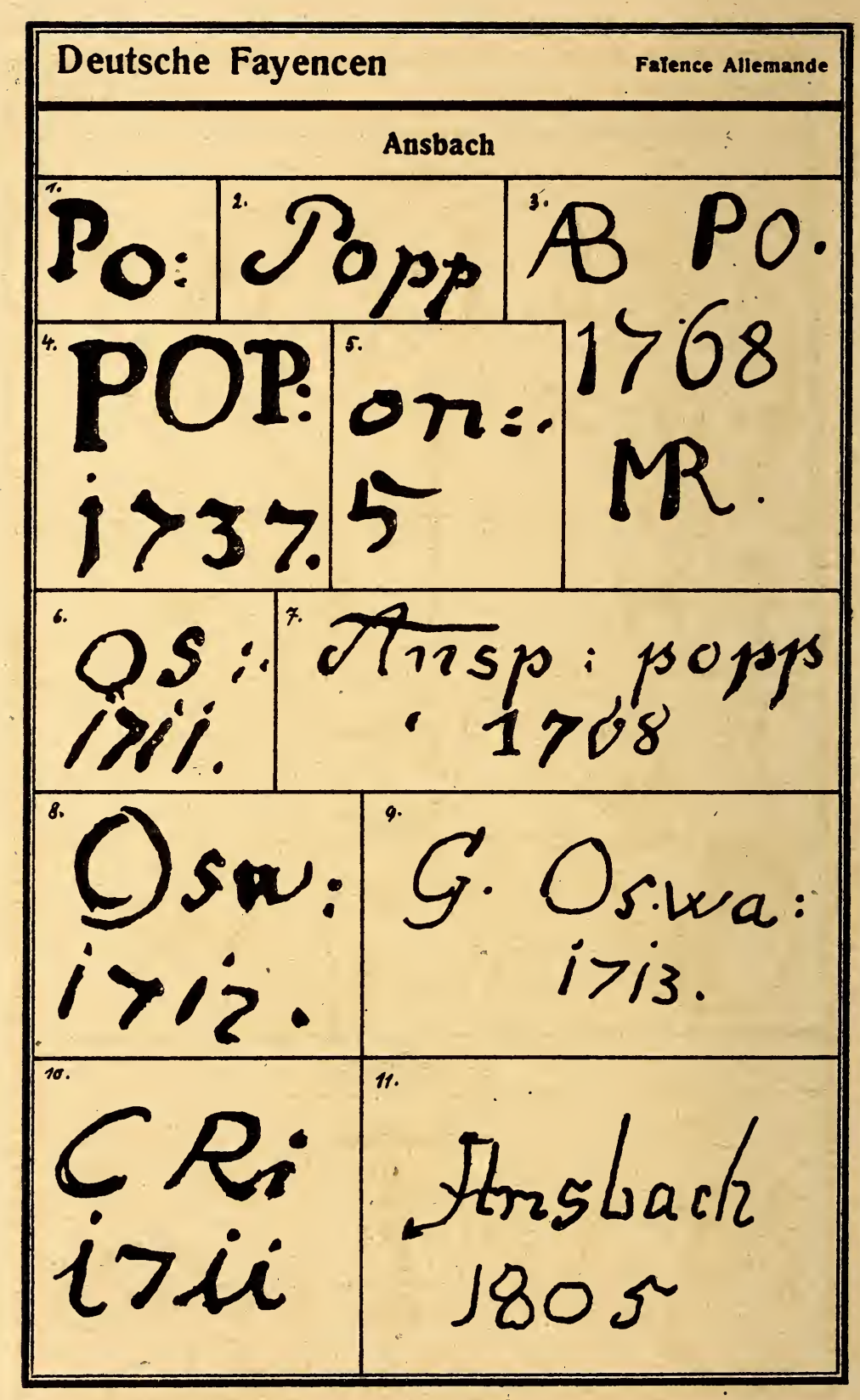




\section{Deutsche Fayencen Falence Allemande}

\section{(Ansbach)}
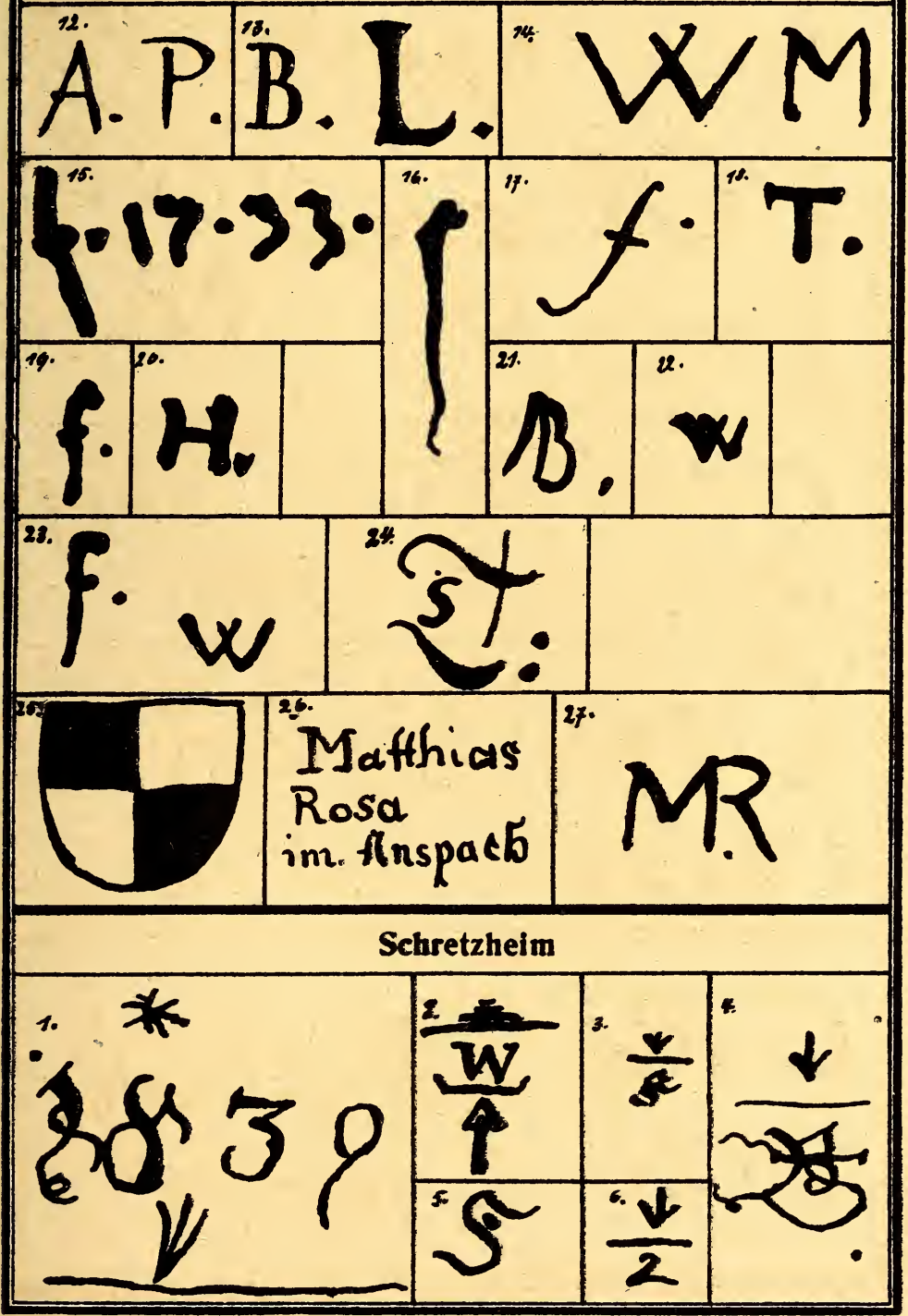


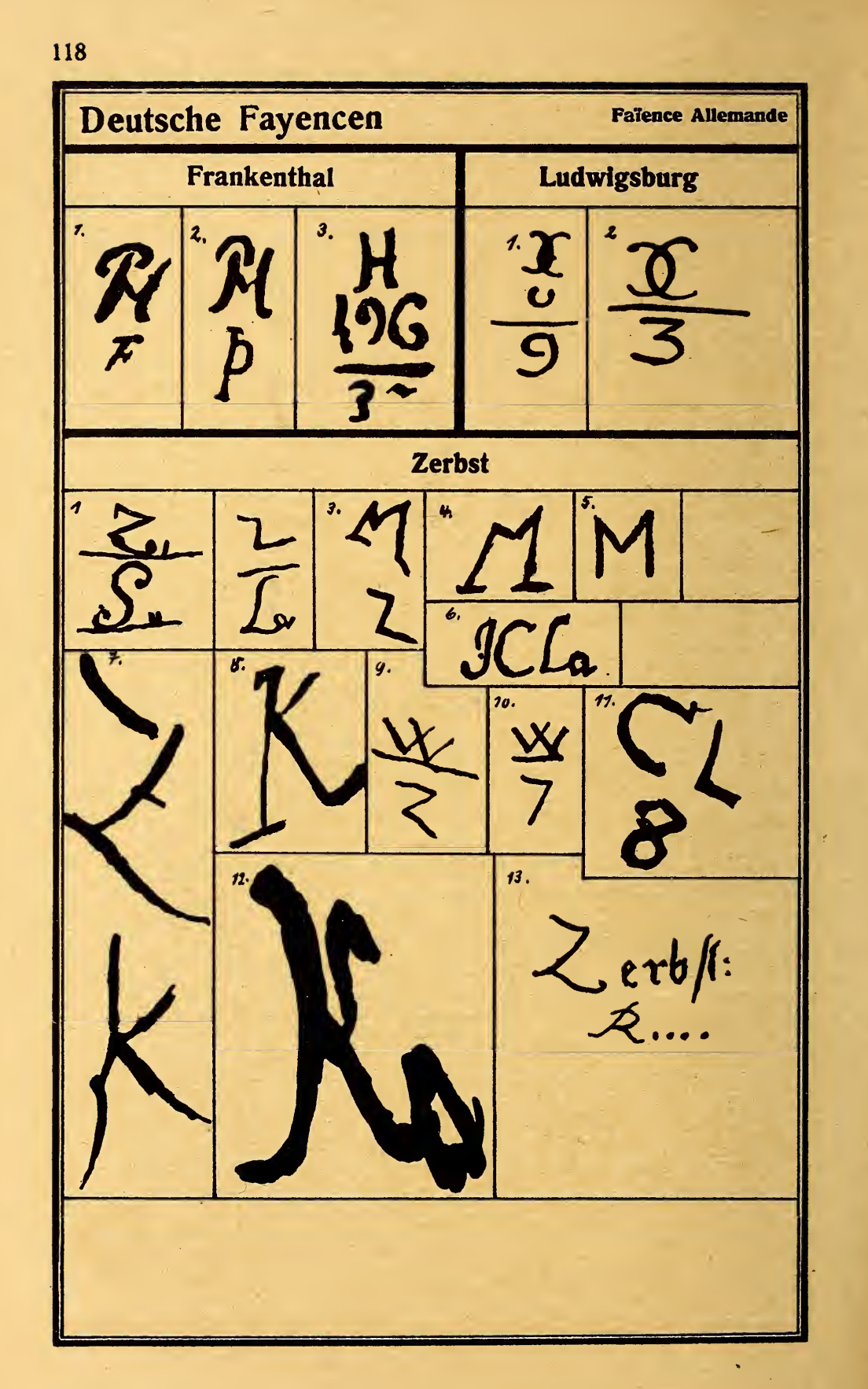




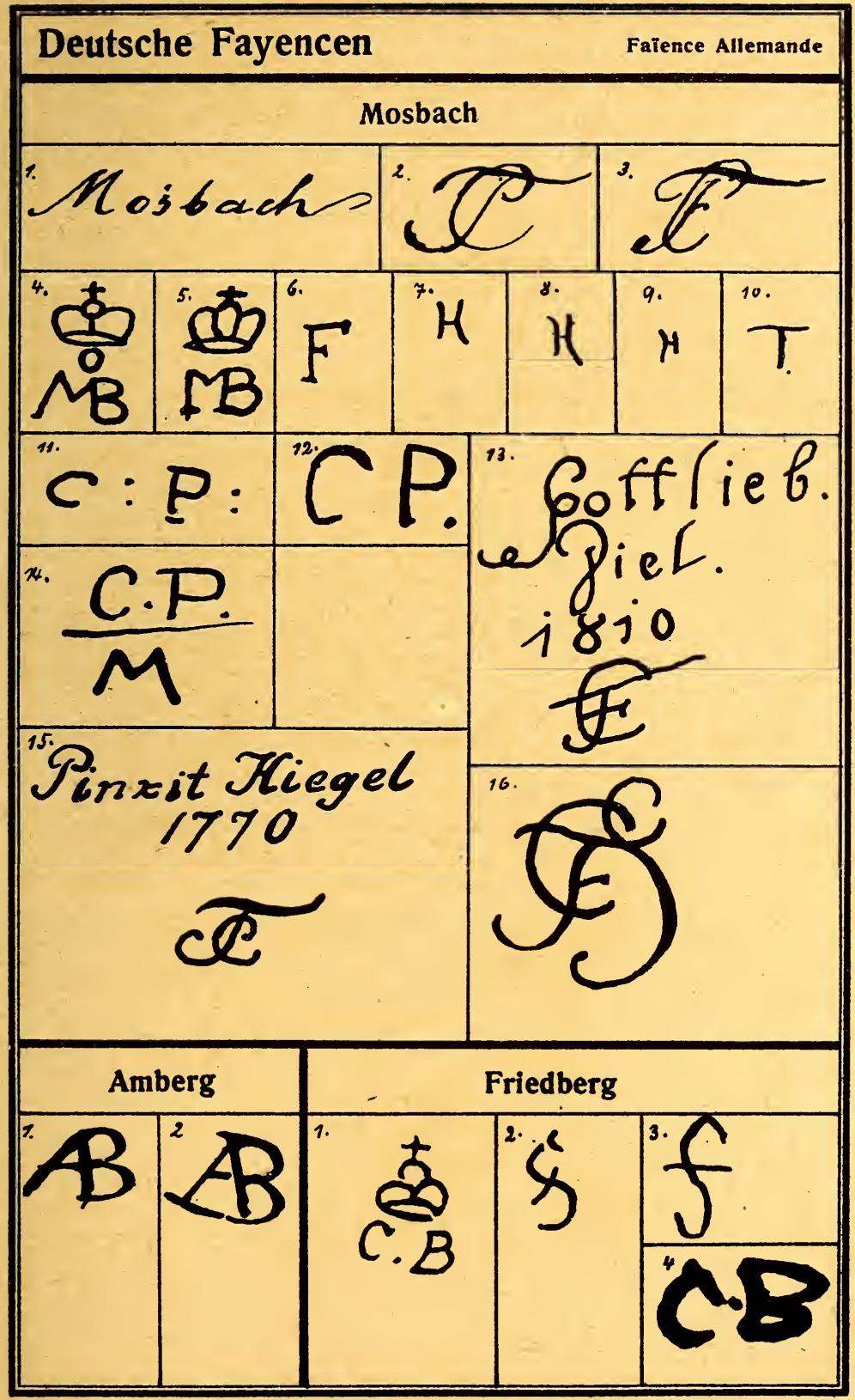




\section{Deutsche Fayencen}

Falence Allemande

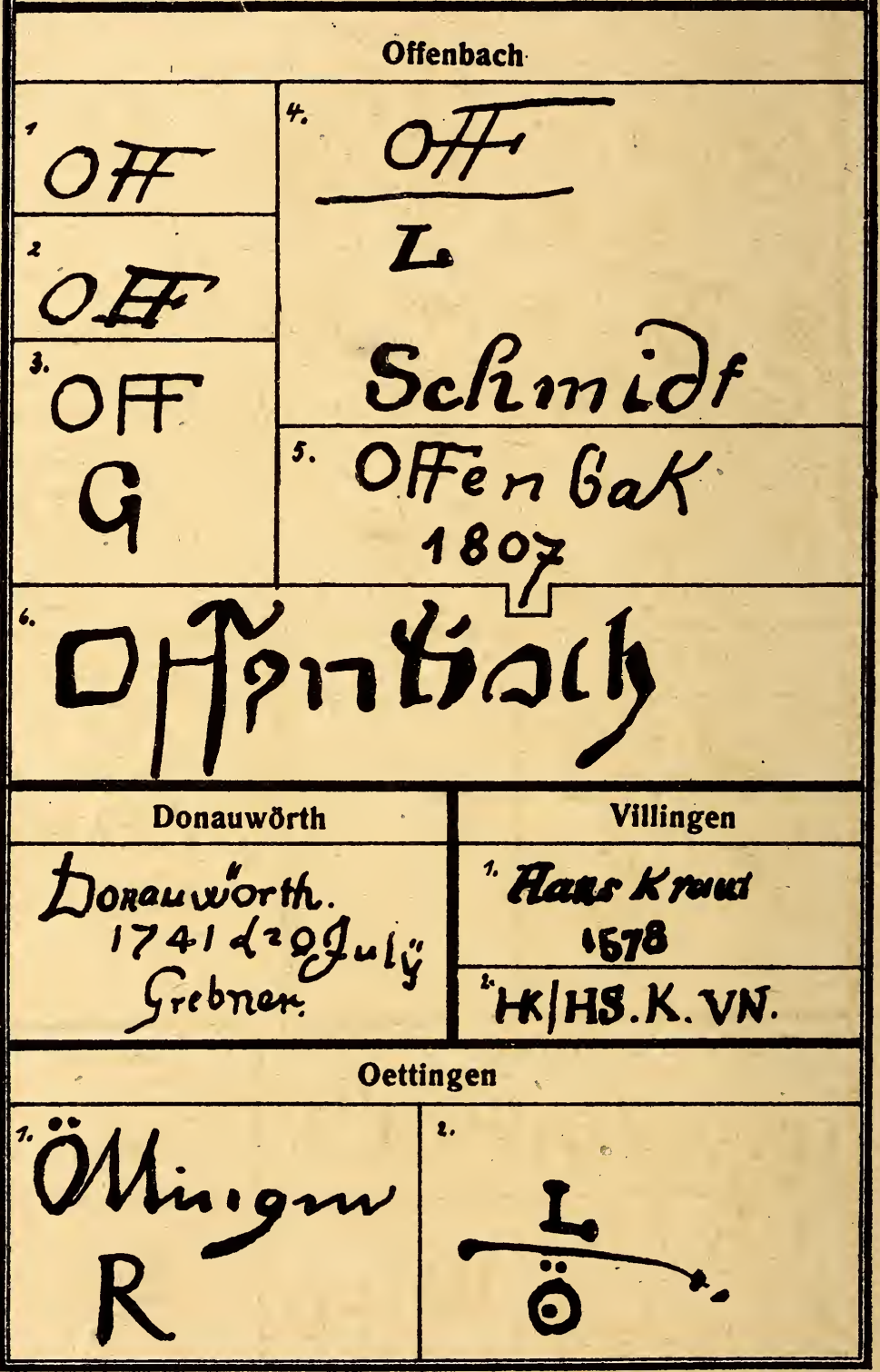




\section{Deutsche Fayencen}

Farence Allemande

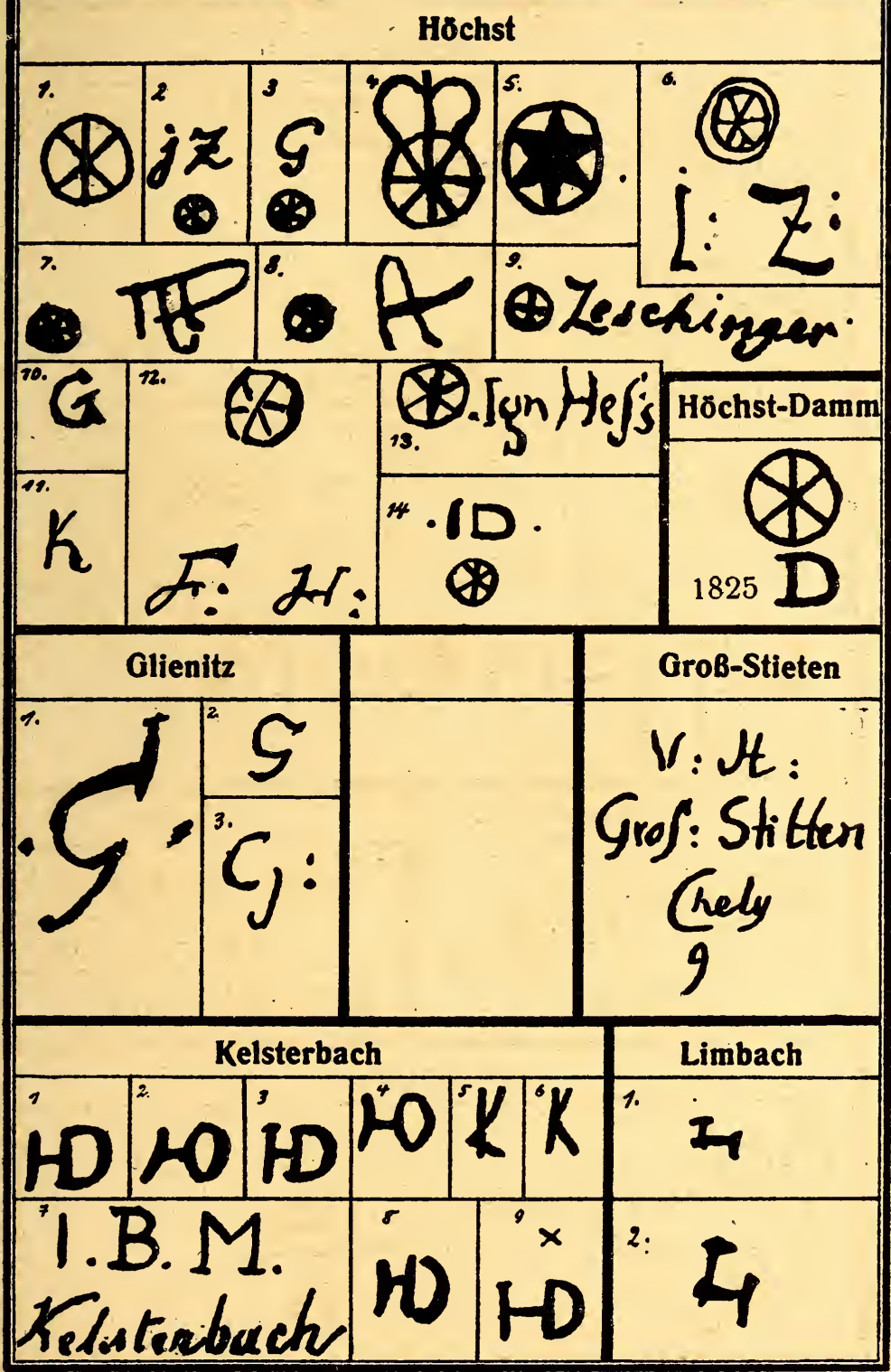




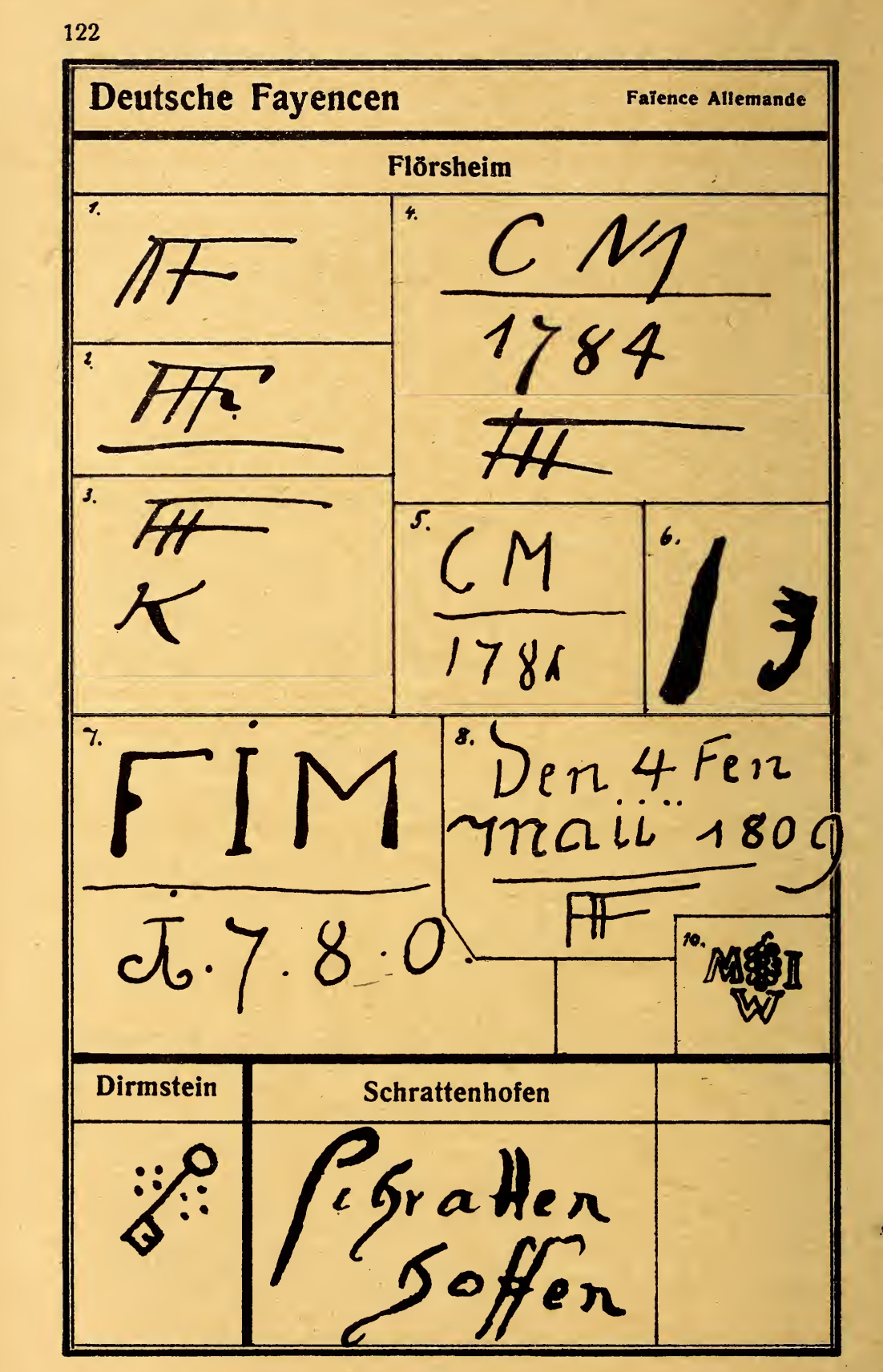




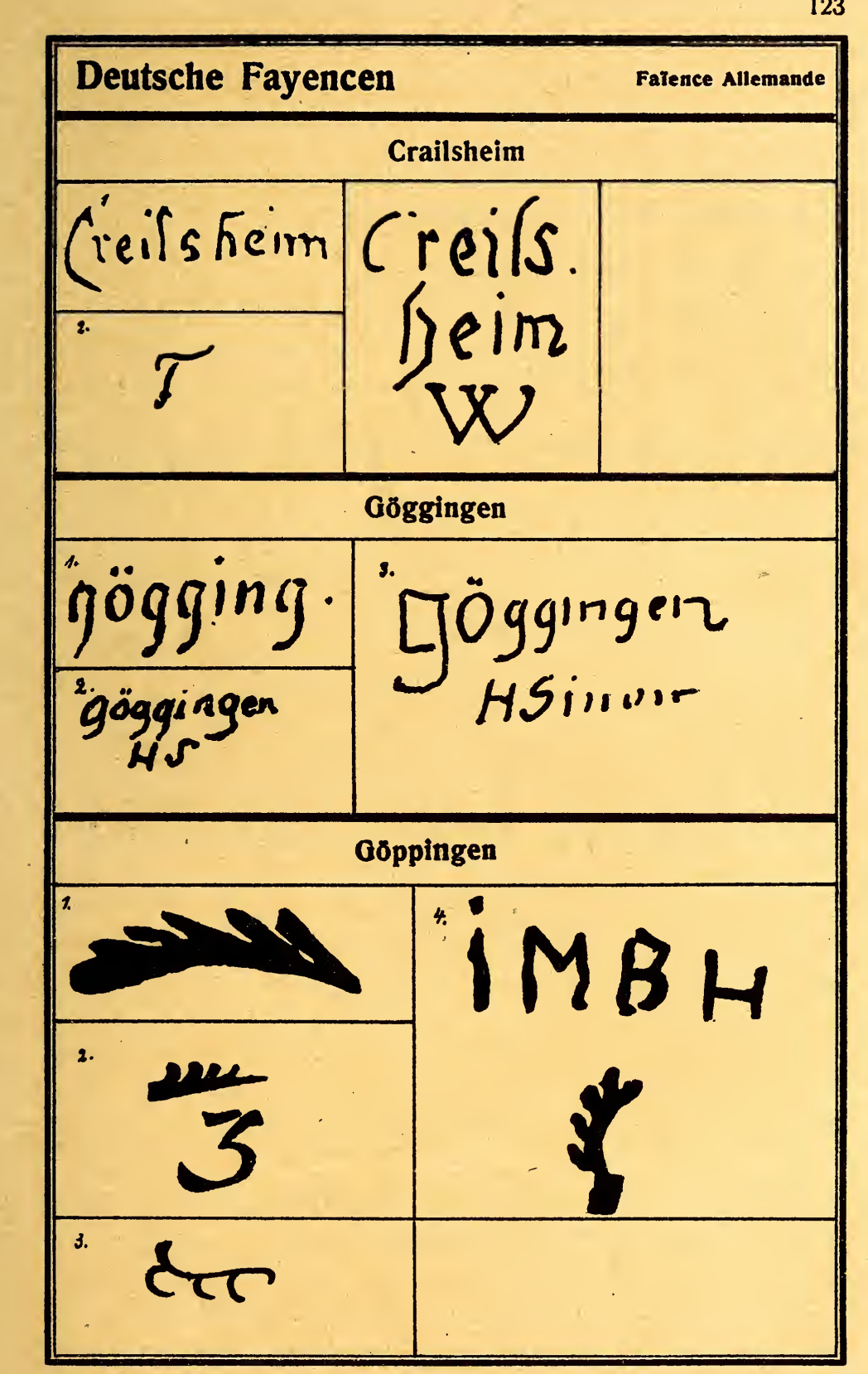




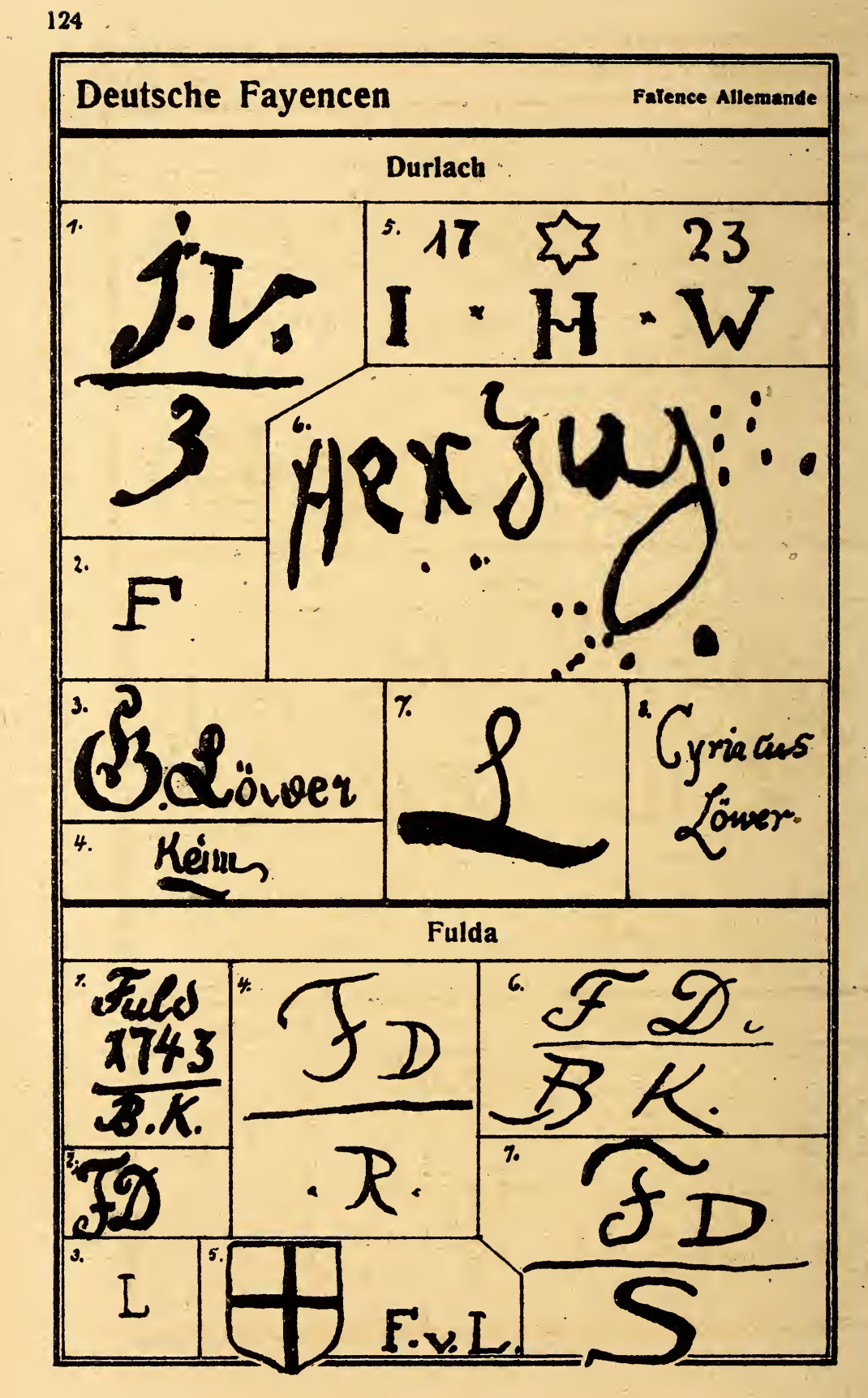




\section{Deutsche Fayencen}

Fafence Allemande

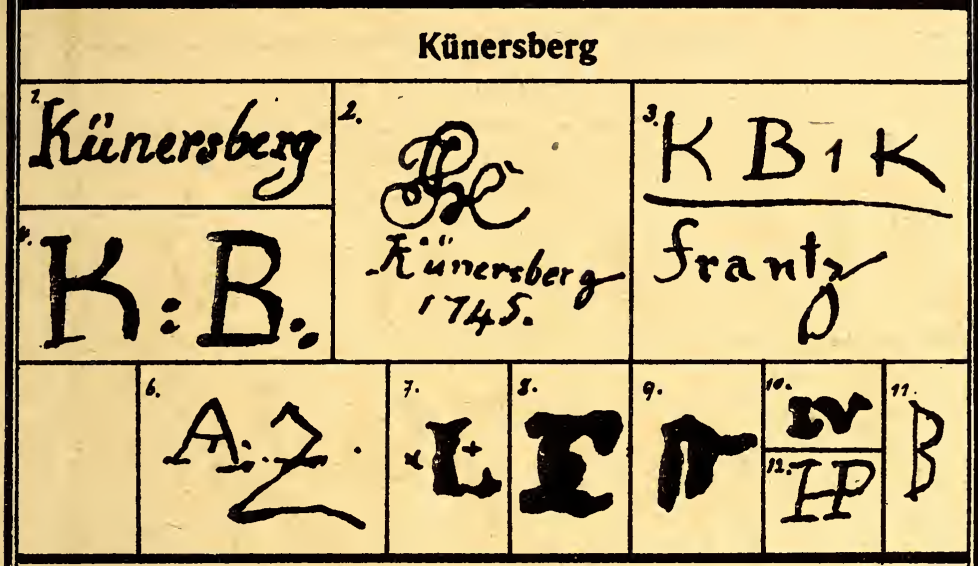

\section{Cassel}

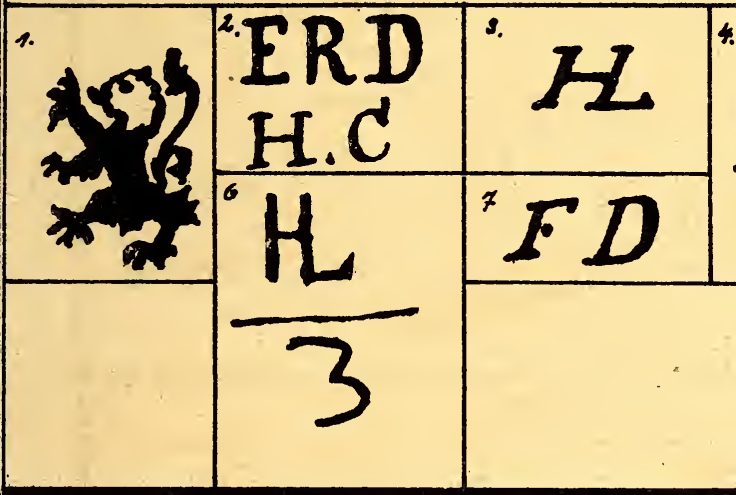

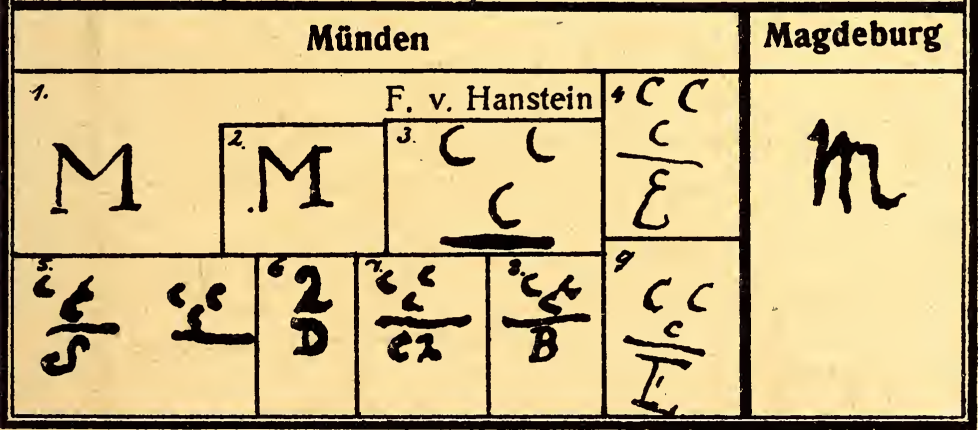




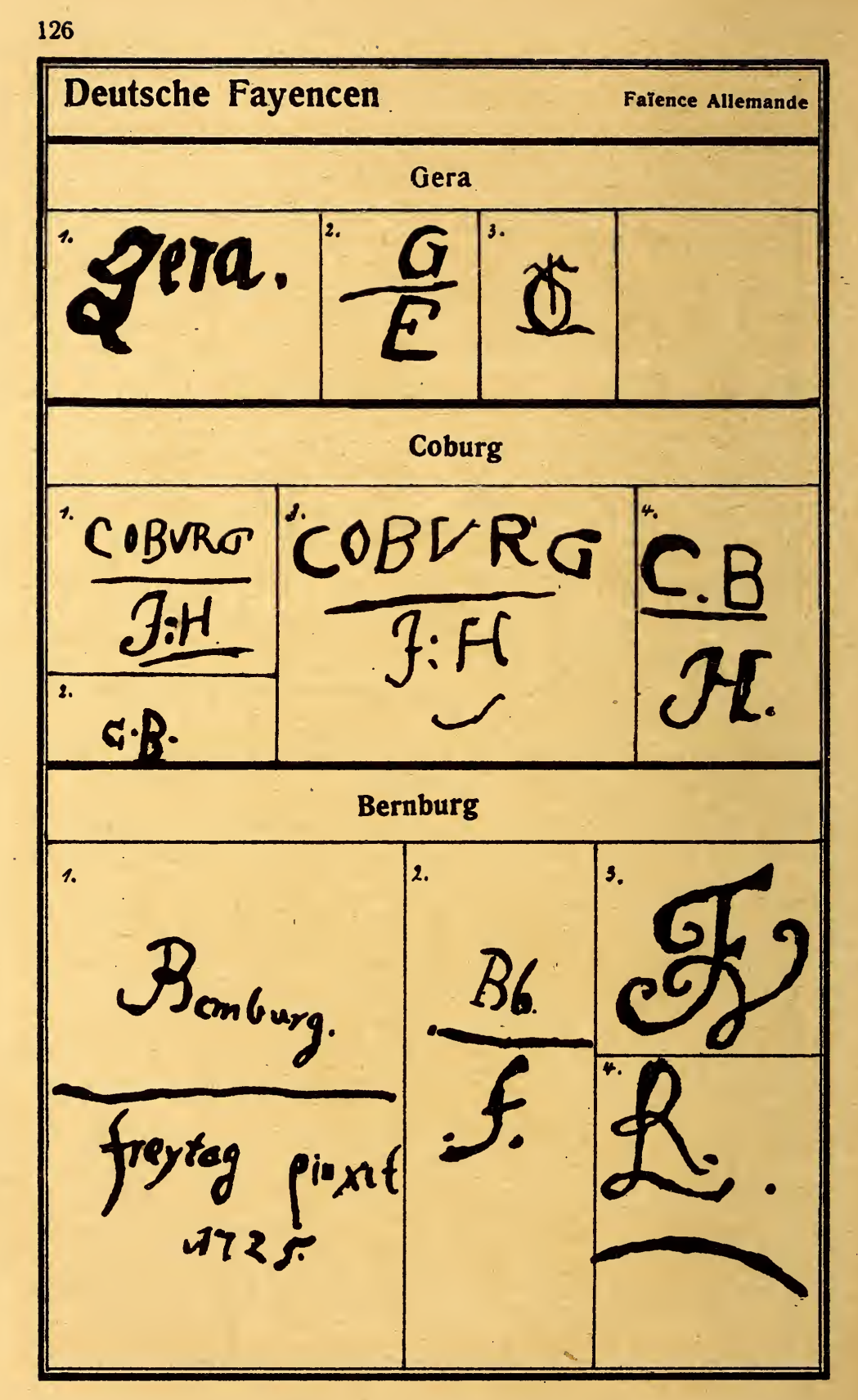




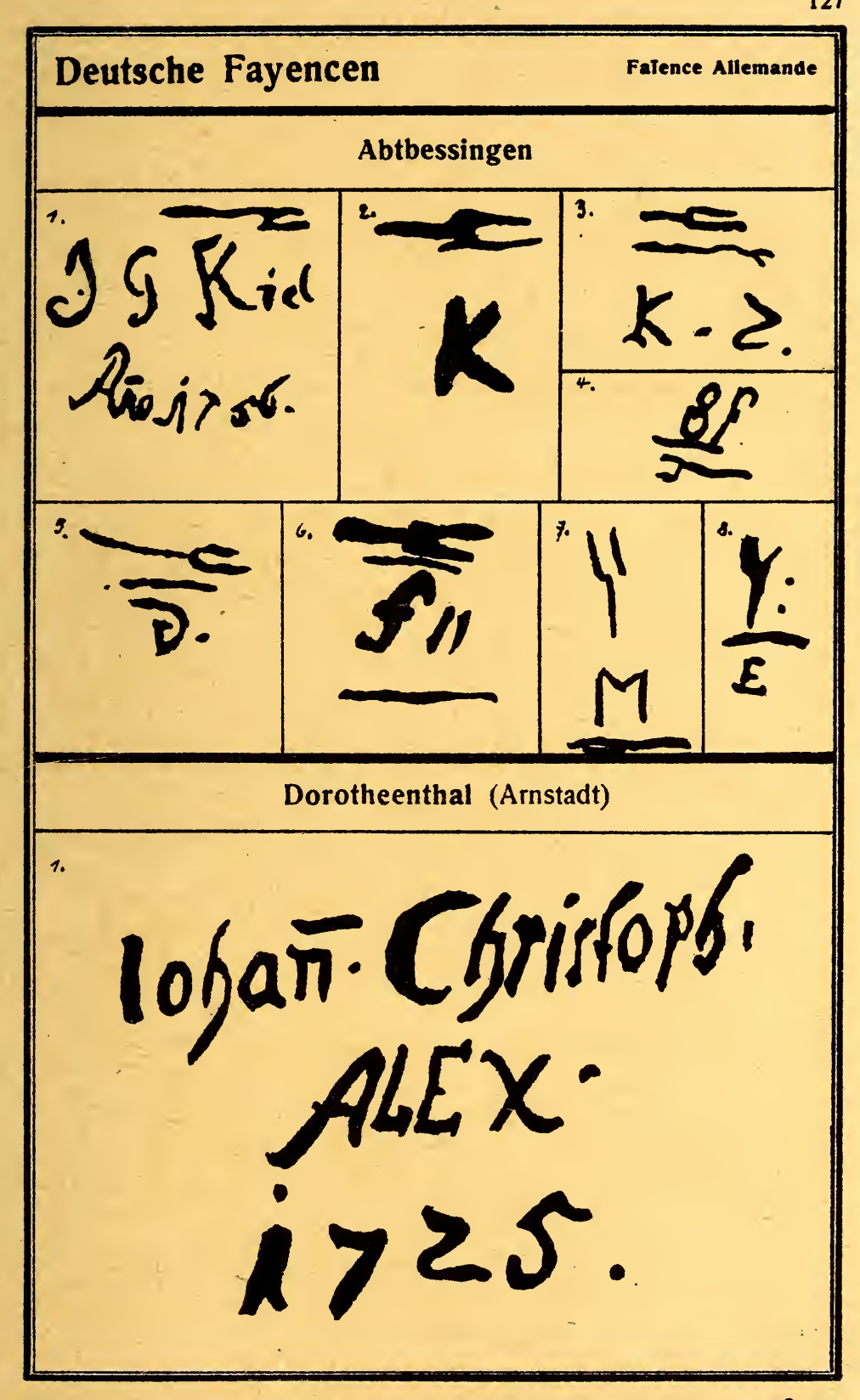


Deutsche Fayencen (Dorotheental-Arnstadt) Faience Allemande

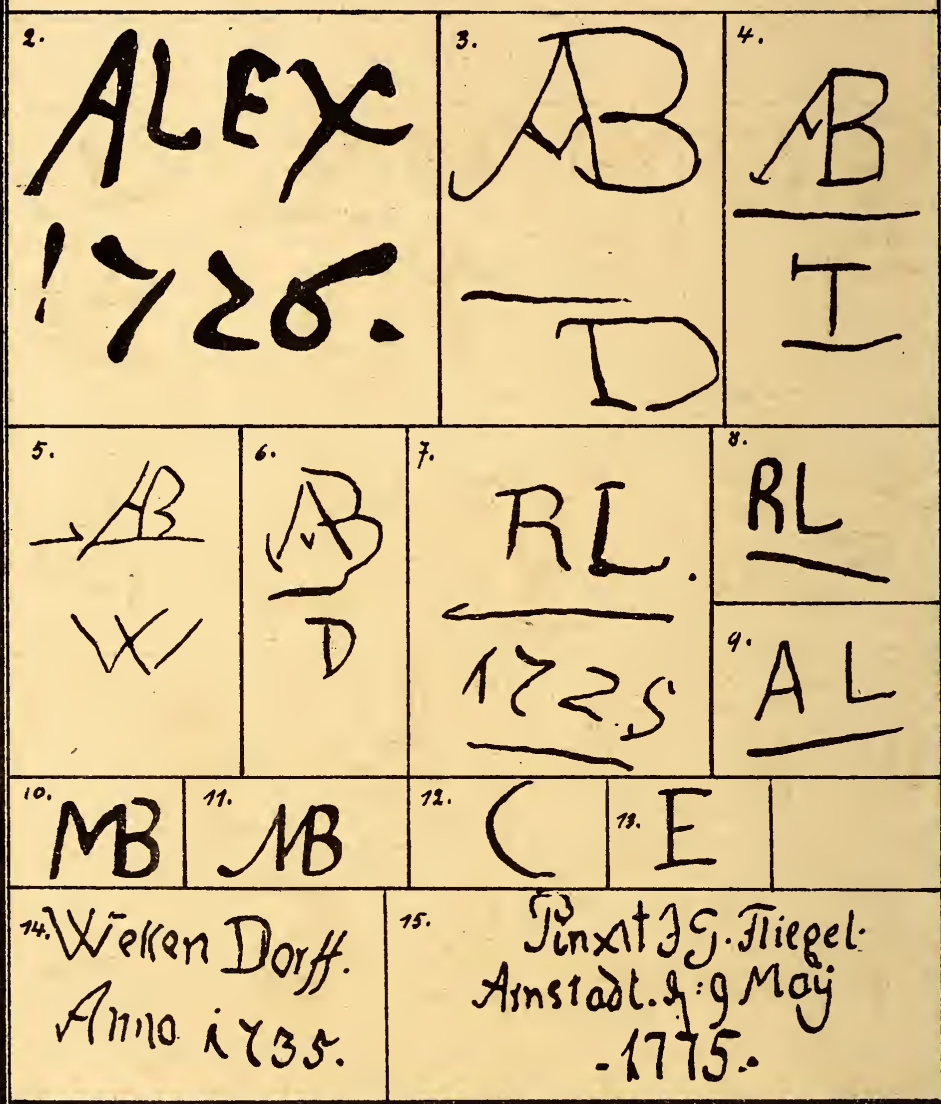

Erfurt

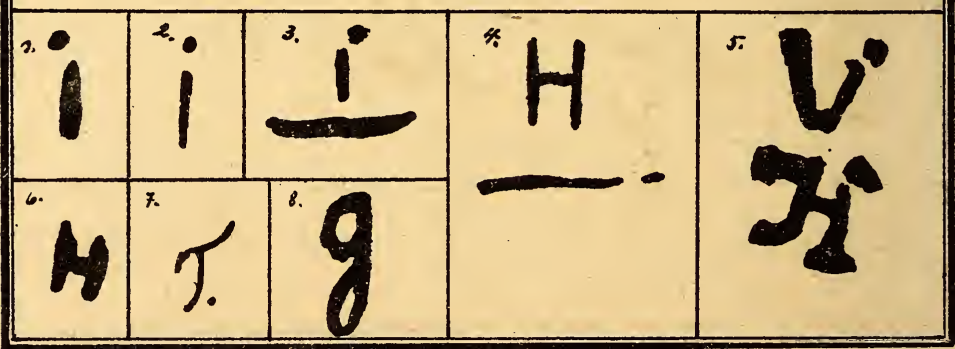




\section{Deutsche Fayencen}

Faĩence Allemande

(Erfurt)

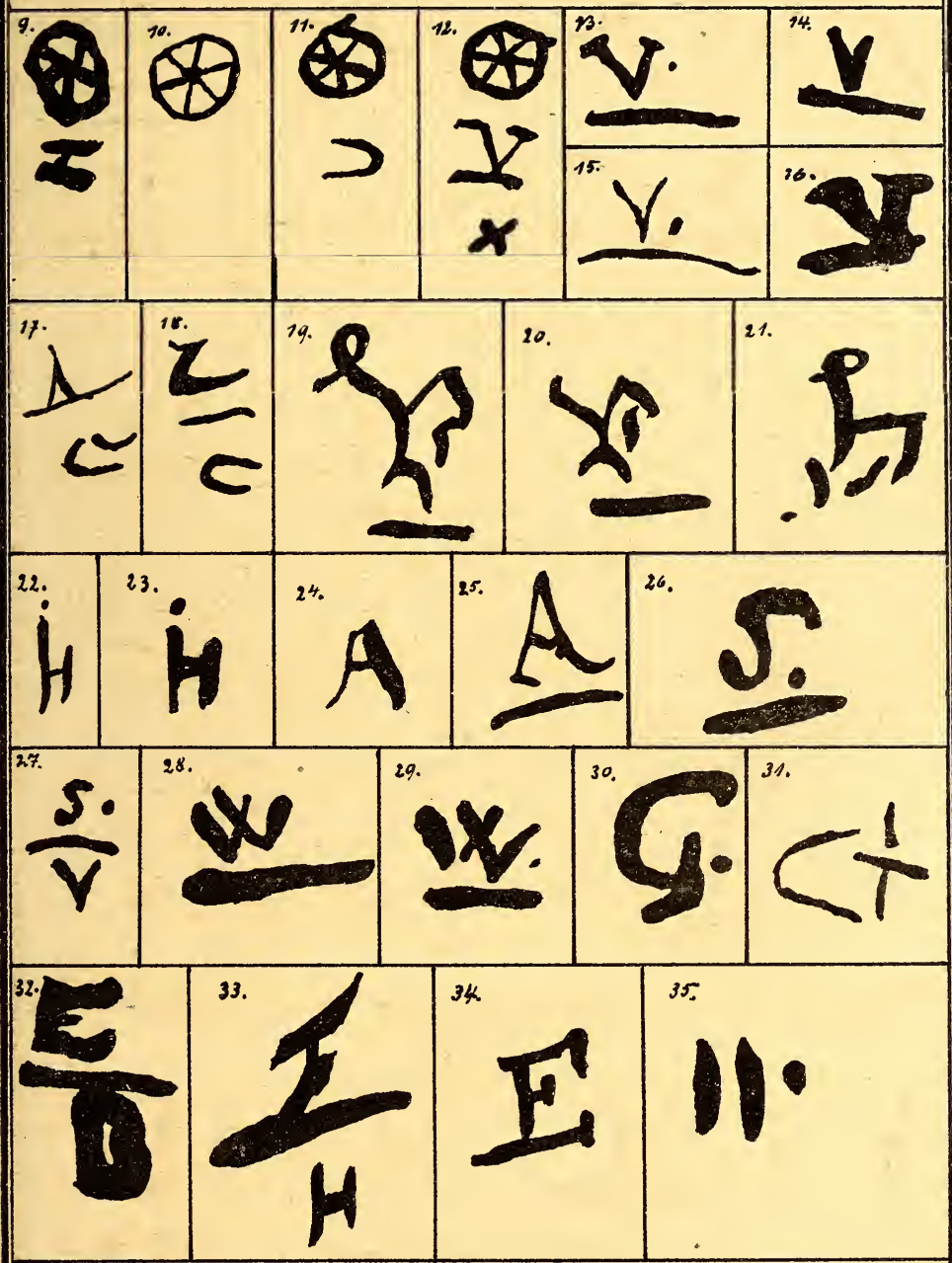




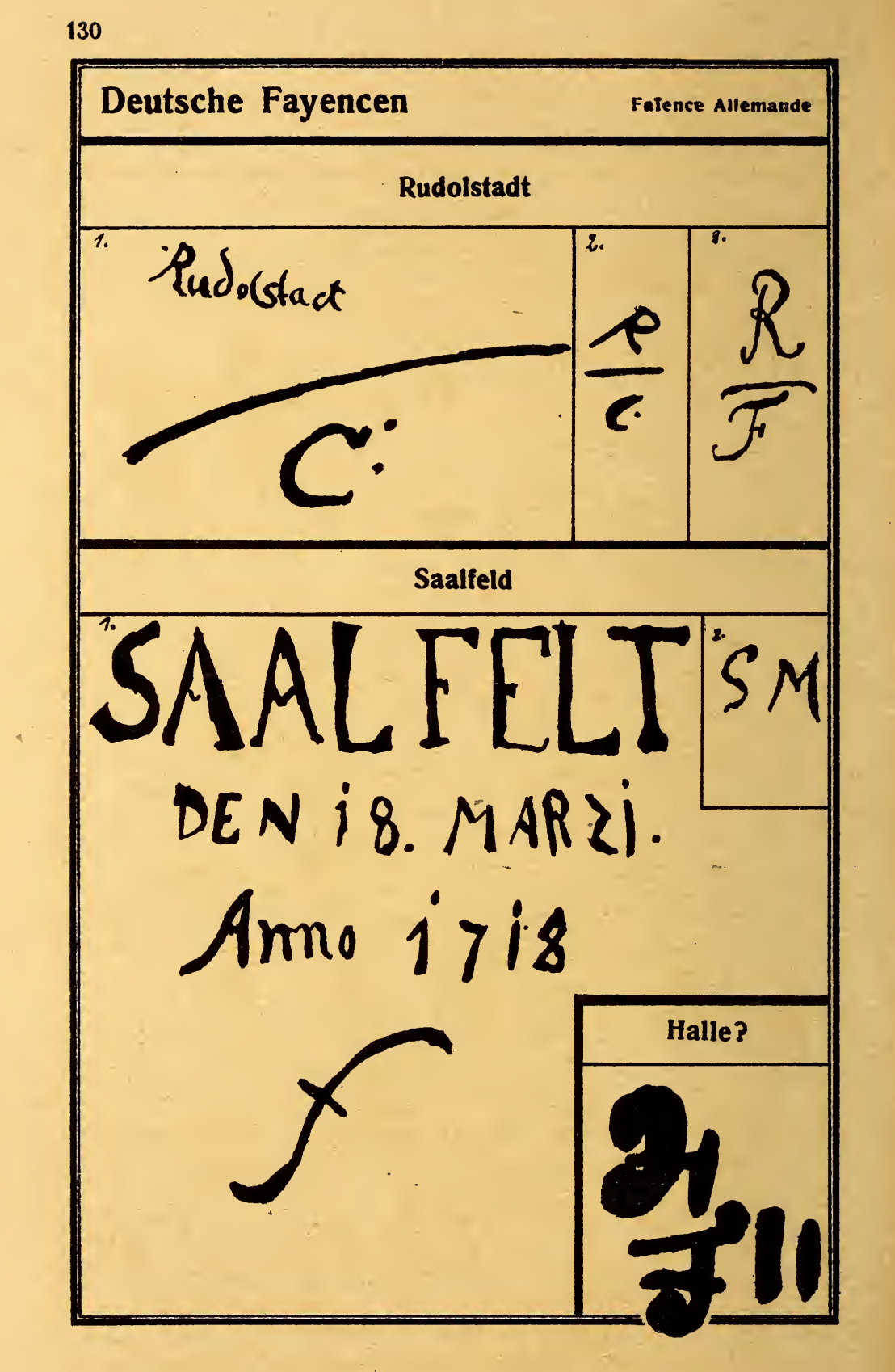




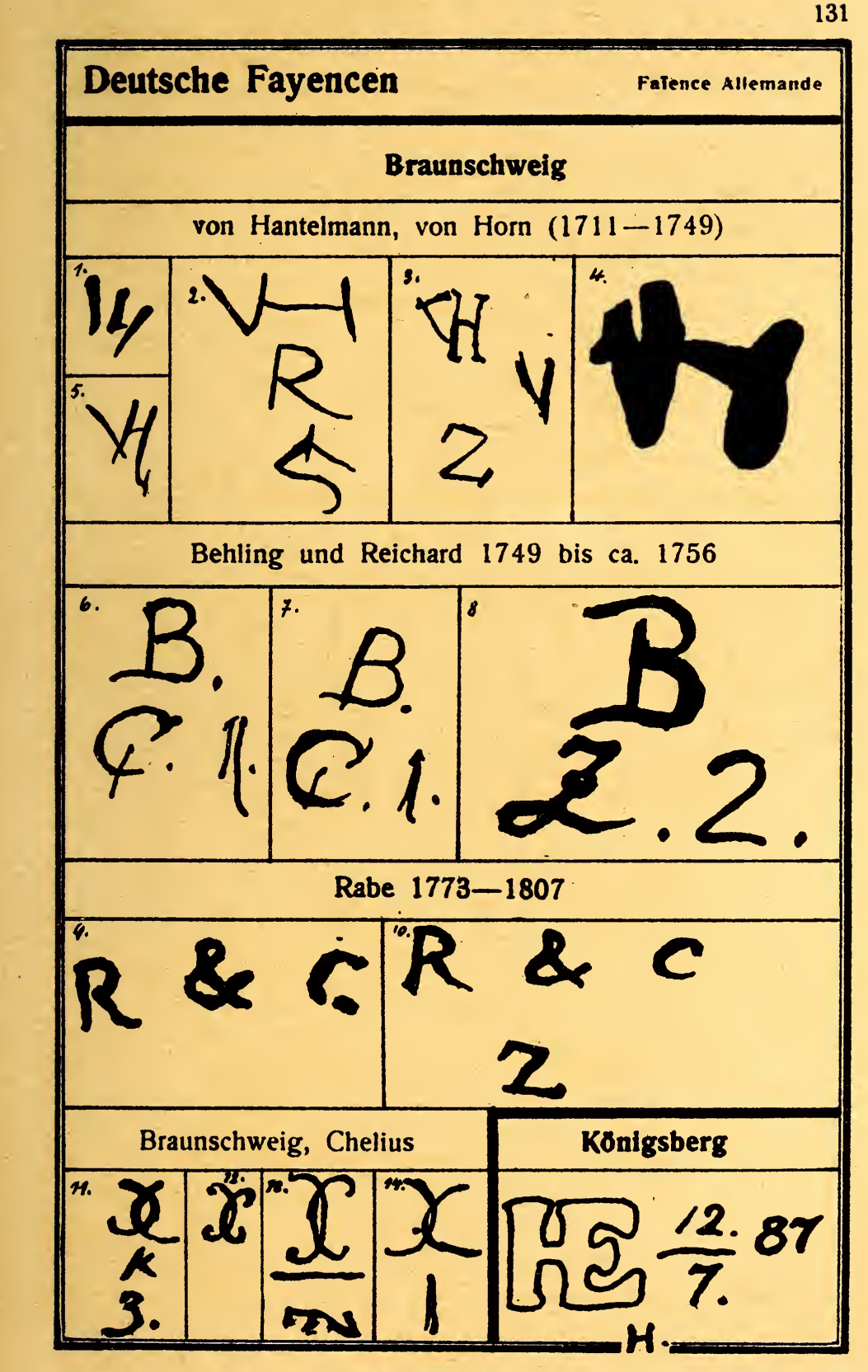




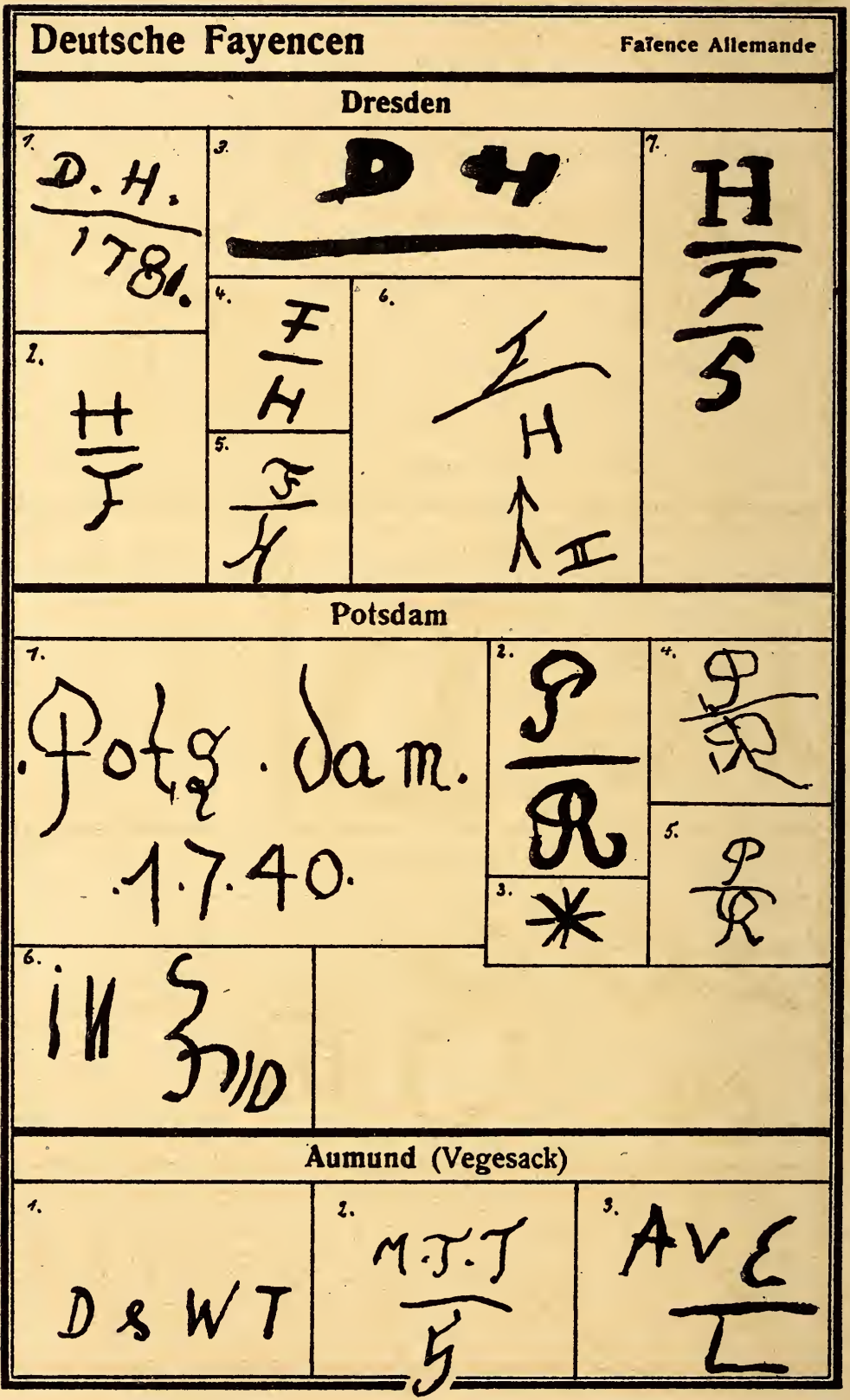


Deutsche Fayencen

Faience Allemande

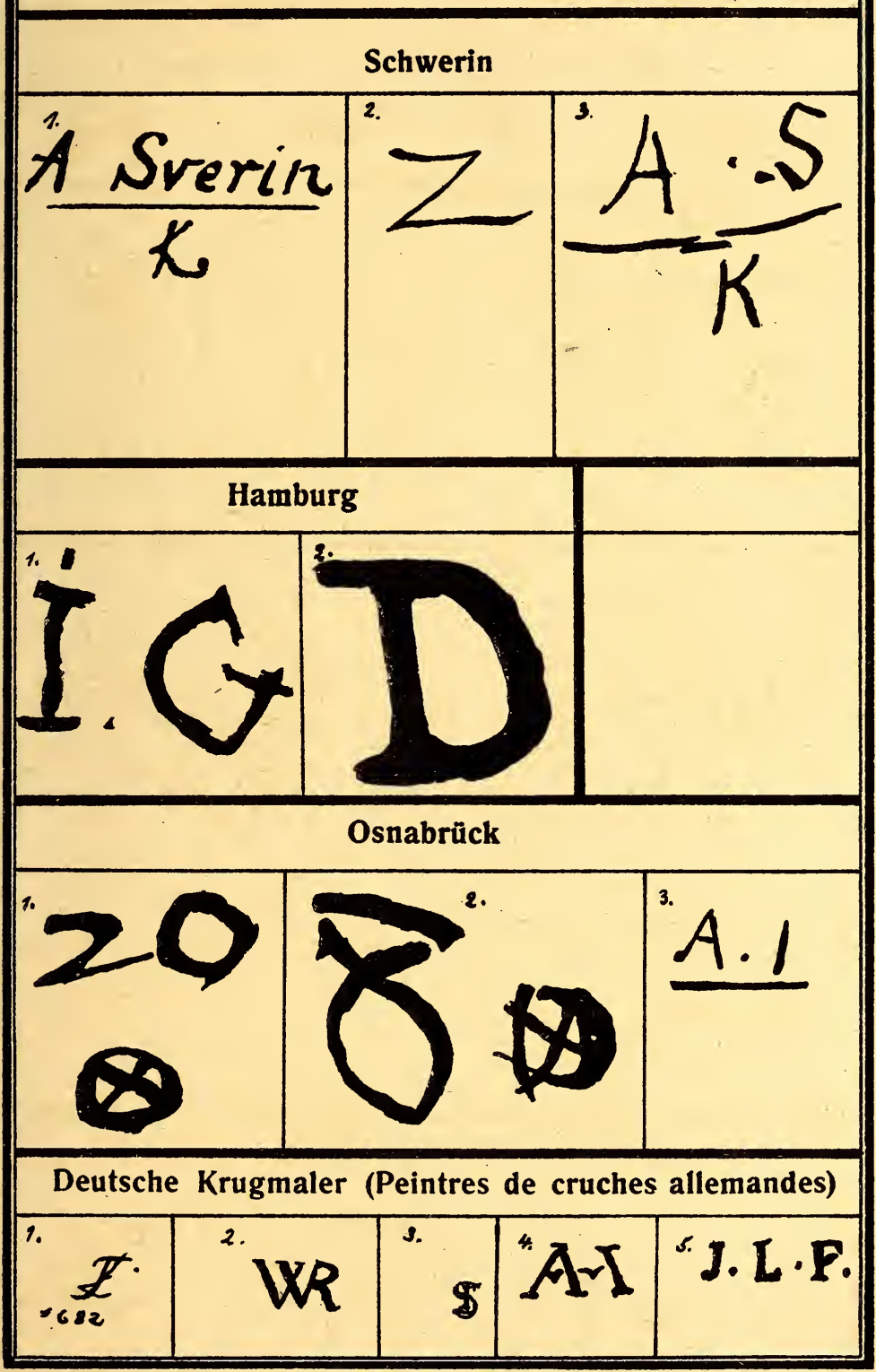




\section{Deutsche Fayencen Falence Allemande}

\section{Lesum}

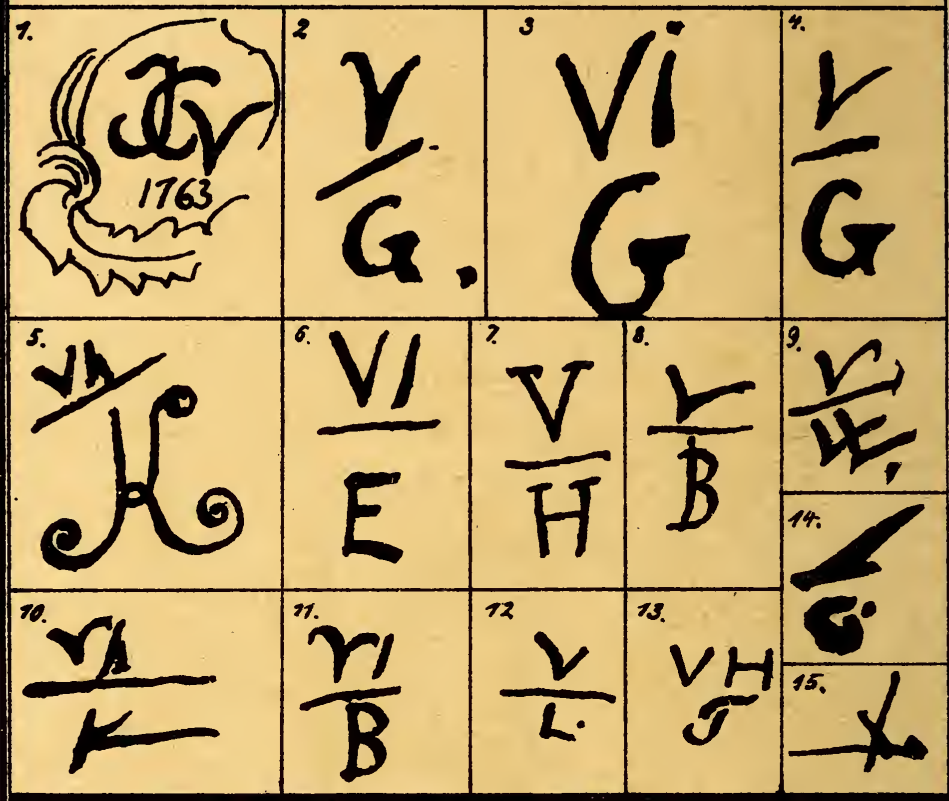

Schleswig

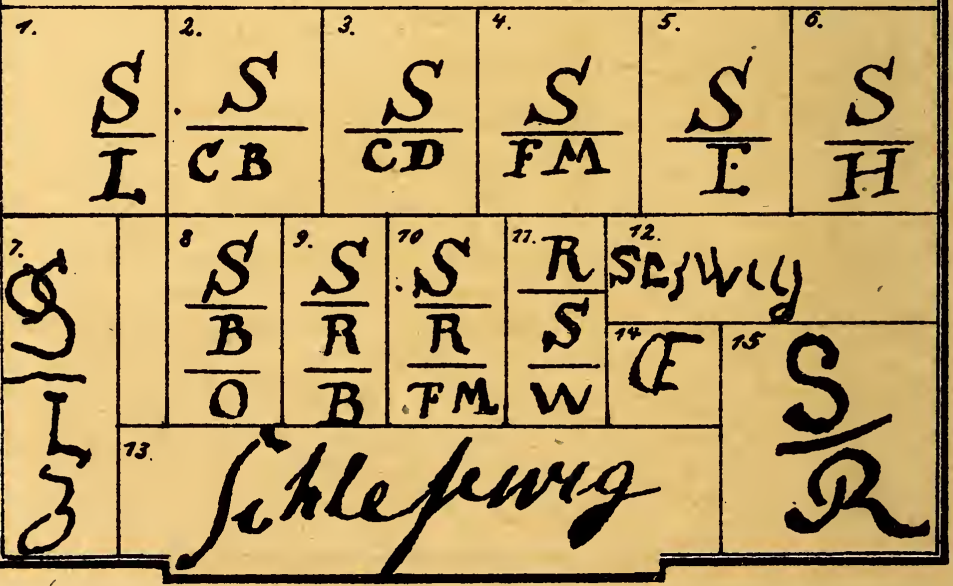




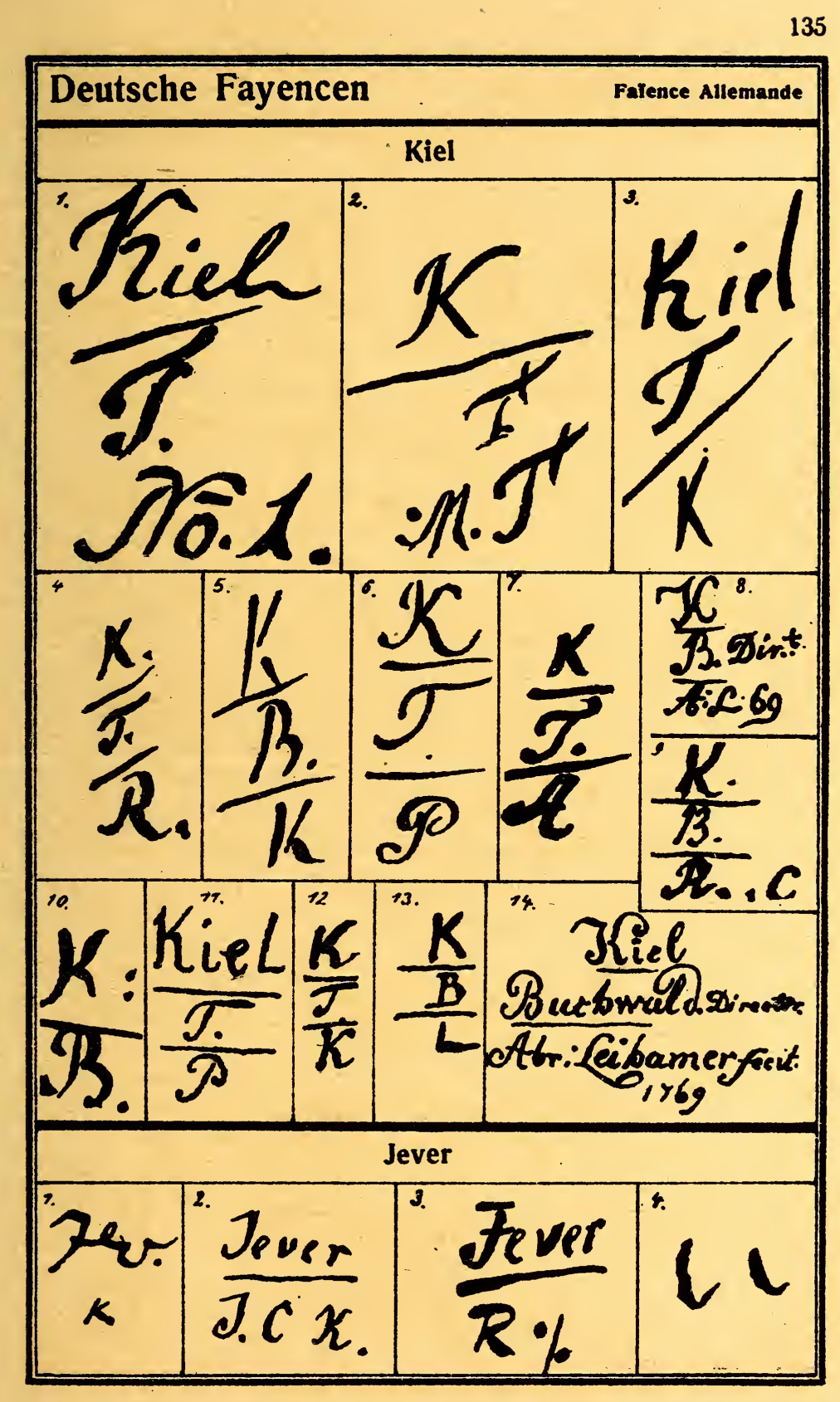




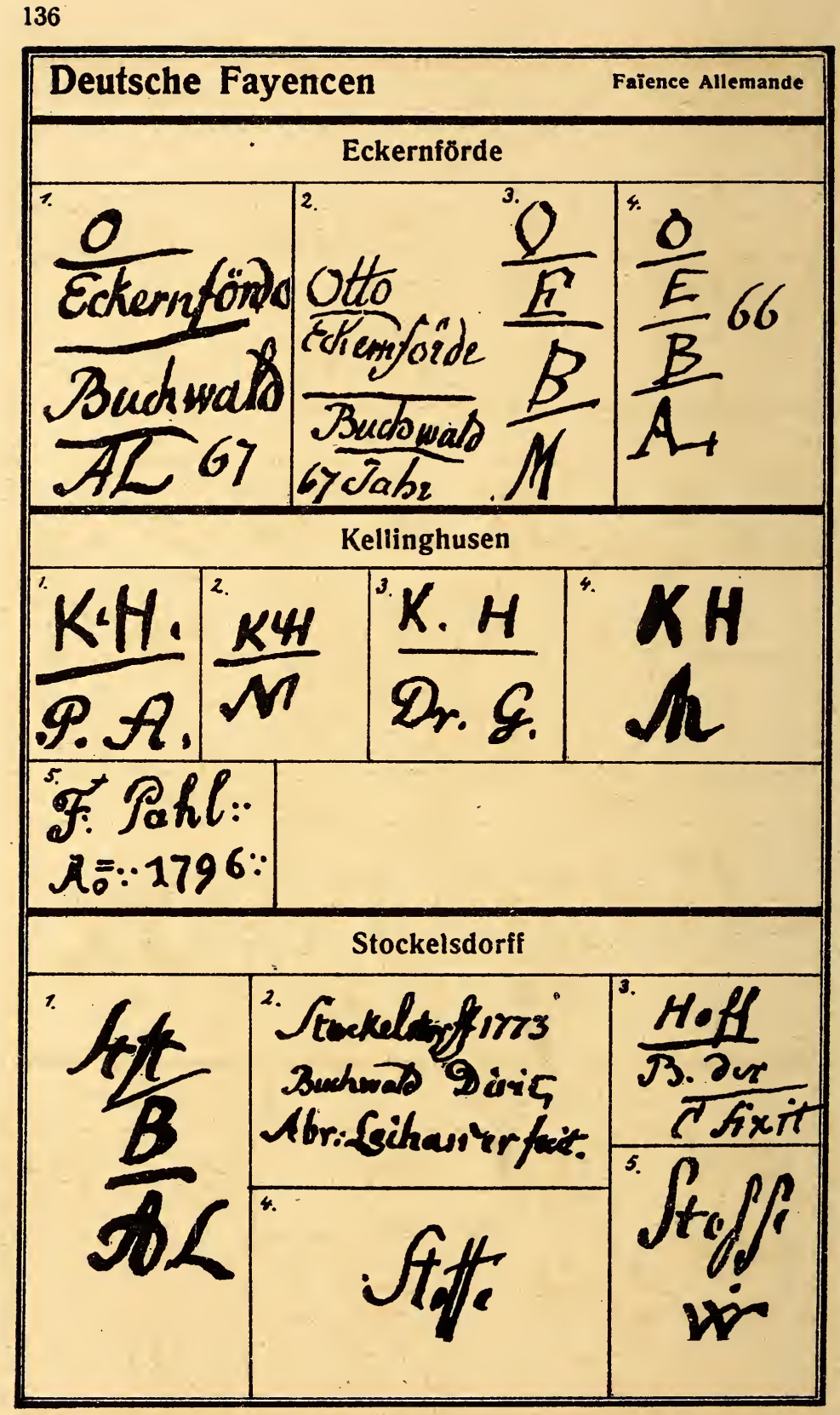




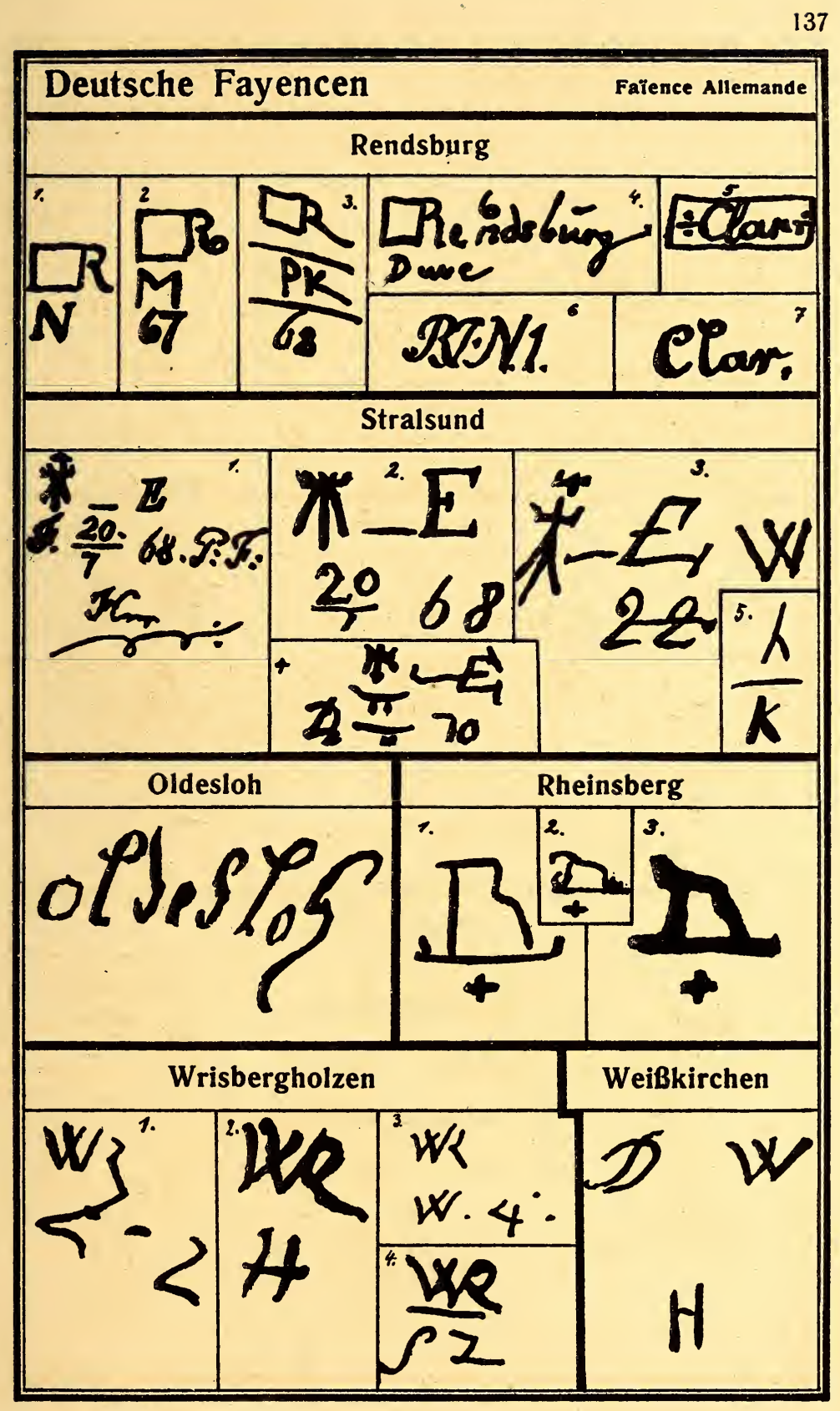




\section{Deutsche Fayencen}

Faience Allemande

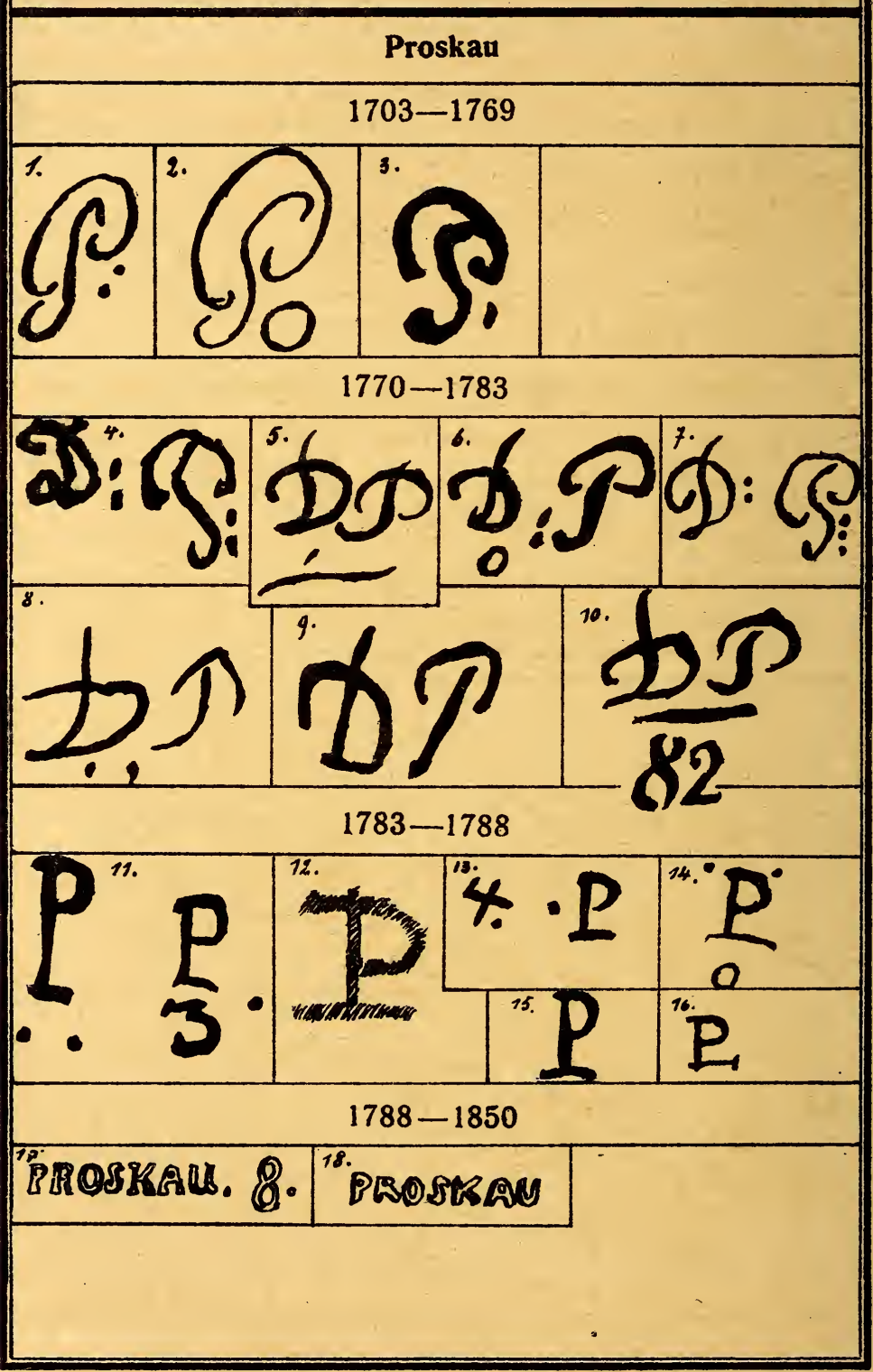


Deutsche rote Tonwaren

Bayreuth

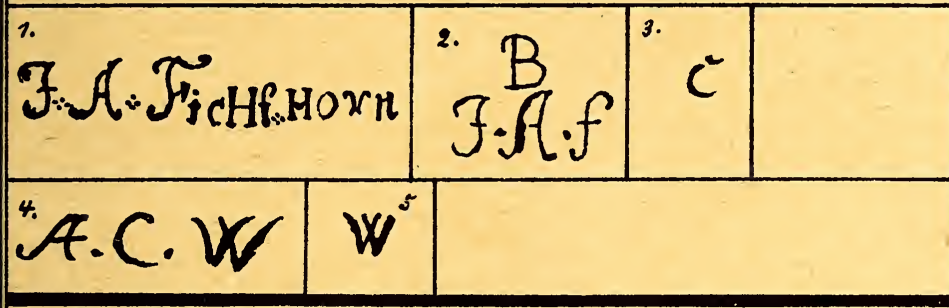

\begin{tabular}{|c|c|}
\hline \multicolumn{2}{|c|}{ Tetschen } \\
\hline $\begin{array}{c}\text { Tefschen } \\
\text { 5\&G. } \\
26\end{array}$ & 2. $5 \& 6$. \\
\hline
\end{tabular}

Hubertusburg (Steingut und Verwandtes) (Poterie etc.)

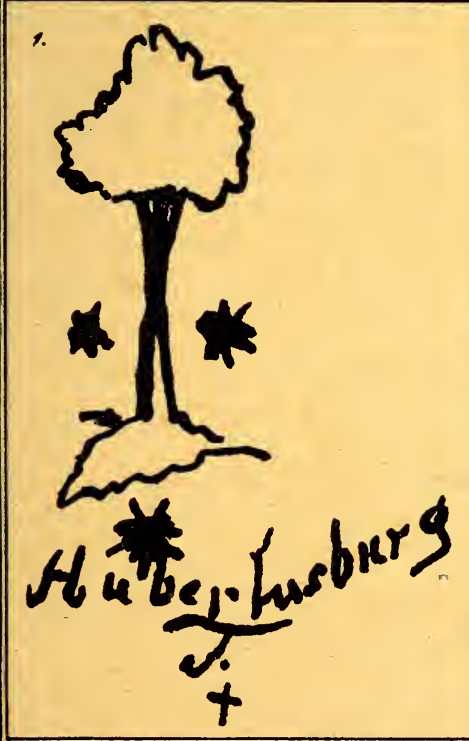

2.
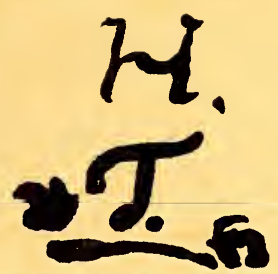

3.

K. S.St.F.

Mubertusburg

4. R.s.s.r.s. 


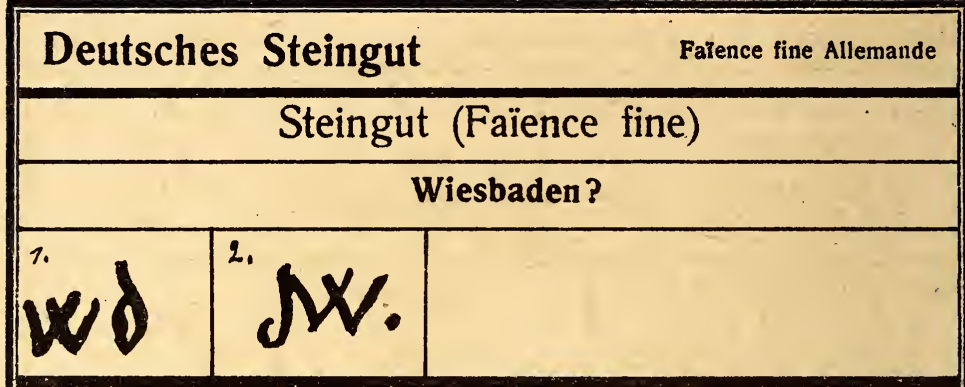




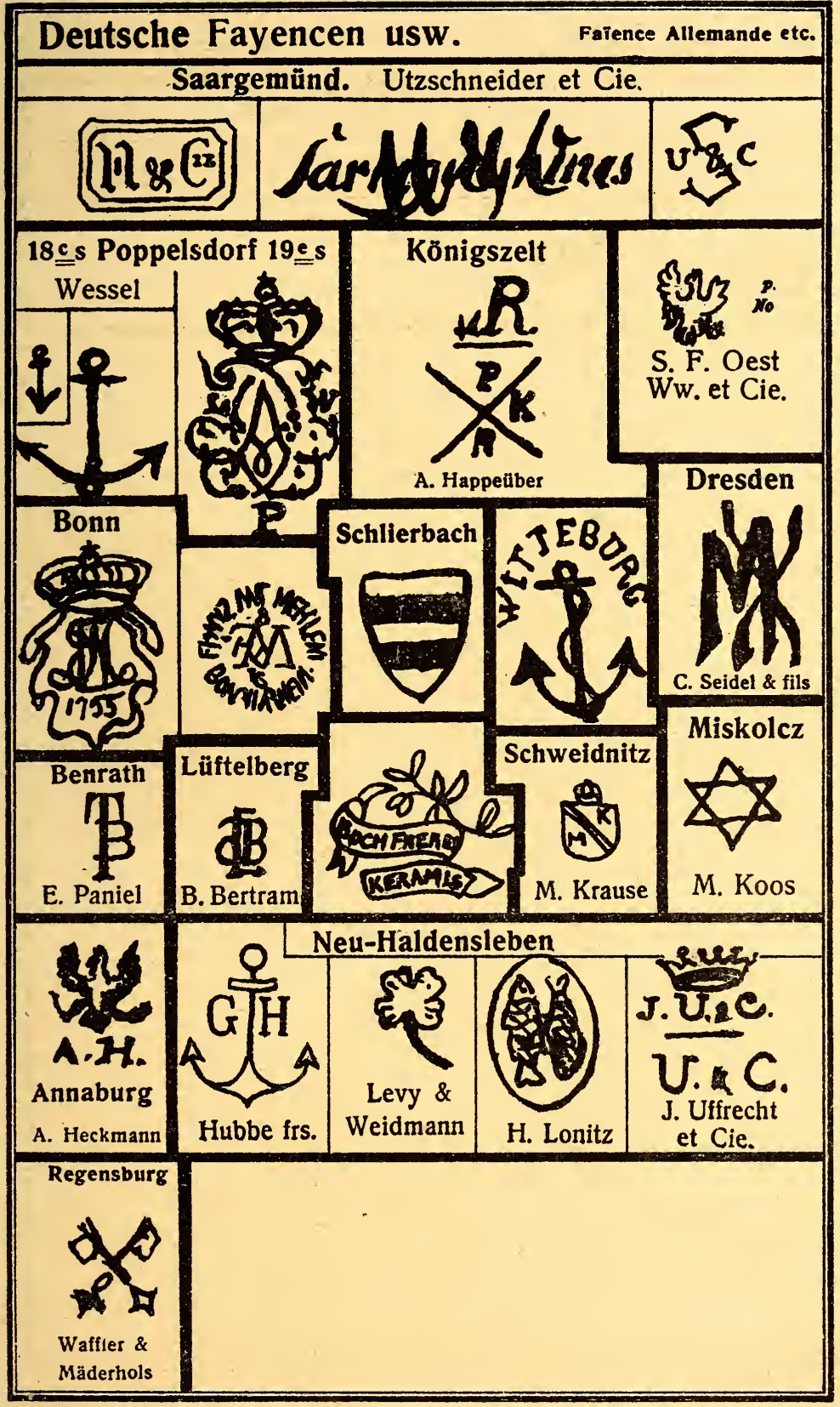




\section{Deutsches Steinzeug \\ Gres allemand}

Siegburg

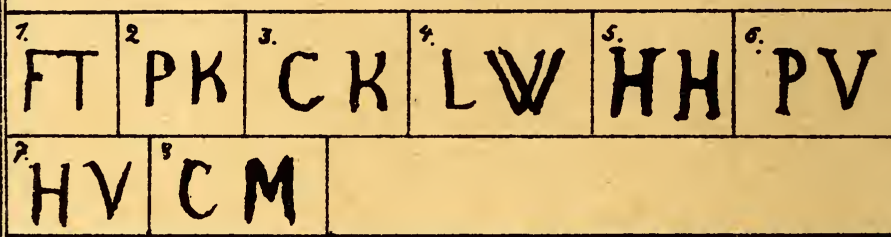

Raeren

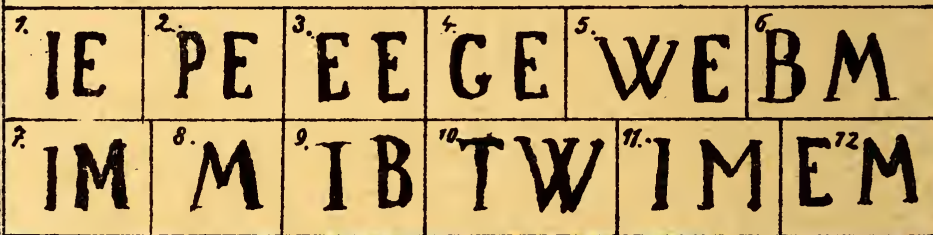

Höhr und Grenzhausen

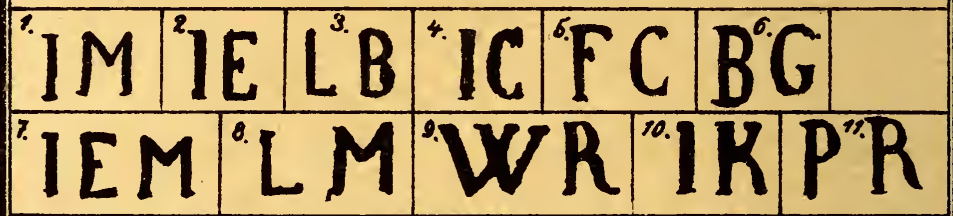

Dresden u. Meissen, Böttgersteinzeug (Grès de Böttger)

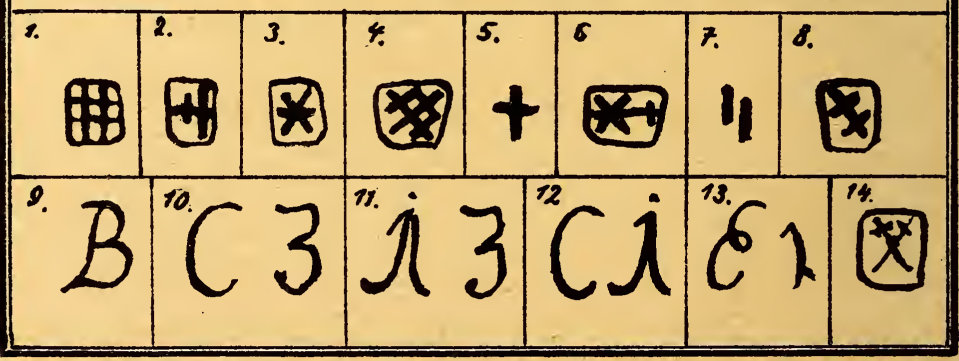




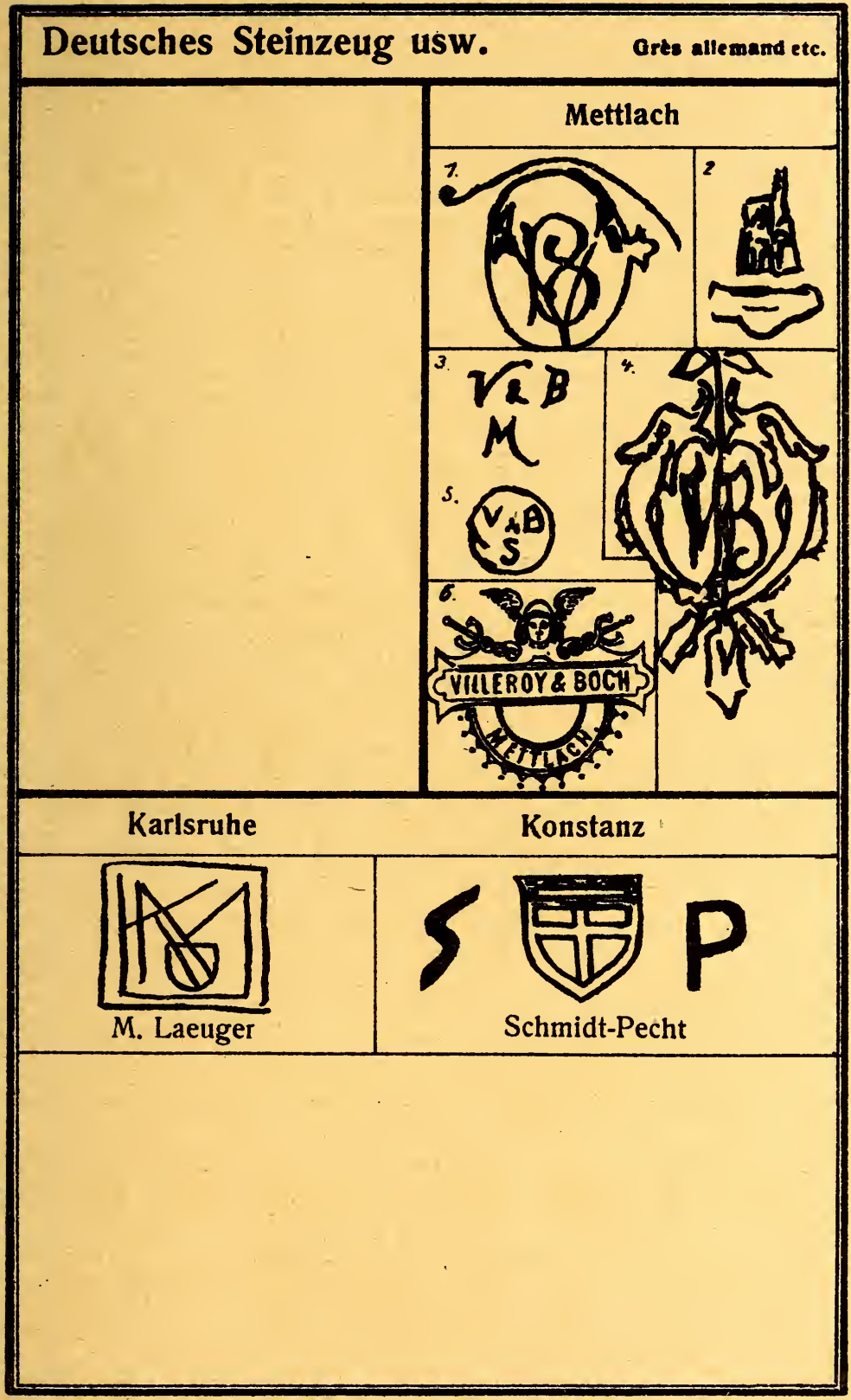


Österreichische Fayencen Faïence Autrichienne

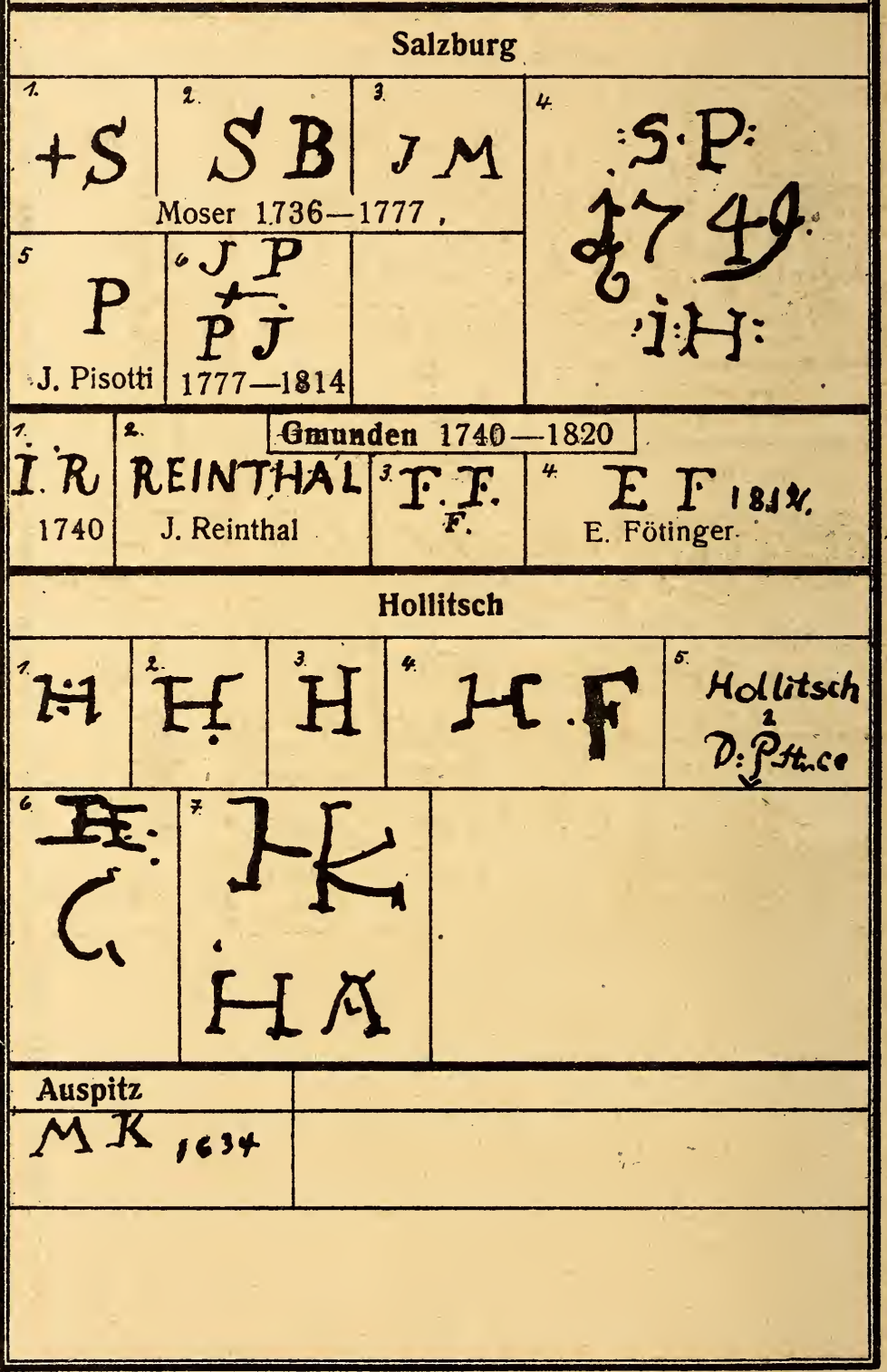




\section{Österreichische Fayencen \\ Faĩence Autrichlenne}

19. Jahrh. (19e siècle)

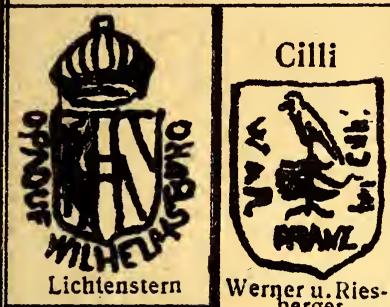

Nesselsdorf

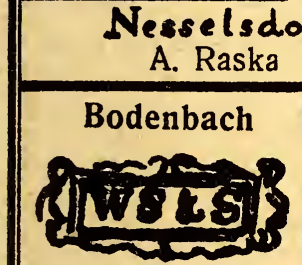

W. Schiller \& fils

$G, 55$

Gerbing \& Stephan erges

\begin{tabular}{|c|c|}
\hline $\begin{array}{l}\text { Liboje } \\
\text { Sthiites } \\
\text { Cill: } \\
\text { L R. Schütz }\end{array}$ & Schwaz \\
\hline $\begin{array}{c}\text { Krawska } \\
\text { Plank } \\
P\end{array}$ & Olomouczan \\
\hline osenau & $\begin{array}{l}\text { Hohenstein } \\
\text { B. B. }\end{array}$ \\
\hline
\end{tabular}

R OZSNYON
A. Dulvavsky jr

Altrohlau

Stınack ir Urbach frères J. Koretz

\begin{tabular}{l|l|l} 
Dux & Aussig & Probstau
\end{tabular}
C R.

Riese
Turn

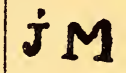

J. Maresch
BW.

Willner

frères
A. Klammerth

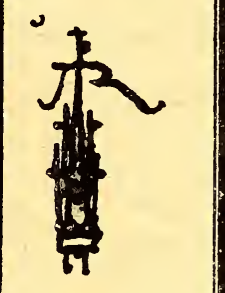

S. Repovecki 


\section{Holländische Fayencen}

\section{Delift}

Einzelne Fabriken (Manufactures diverses)

1. de metaale pot. 1639 .

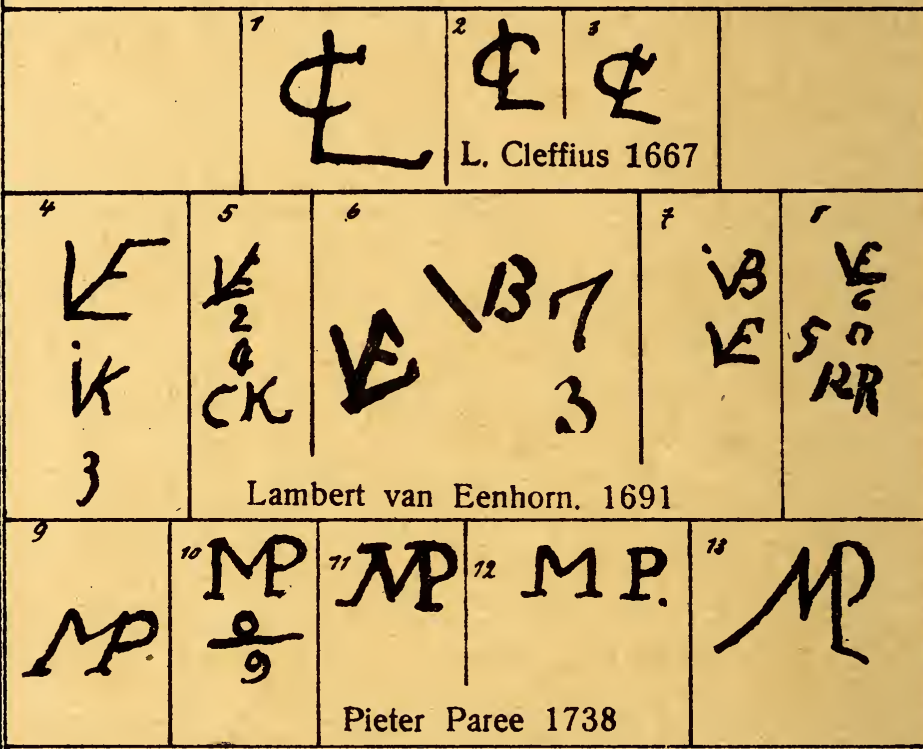

2. de griekse A. 1645

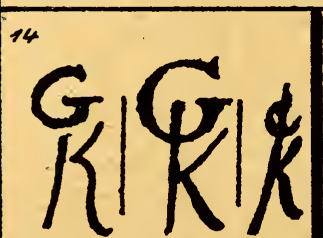

G. L. Kruyk 1645

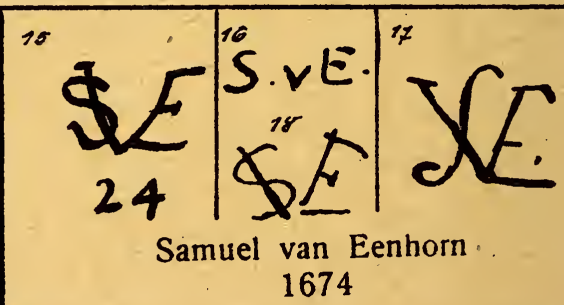




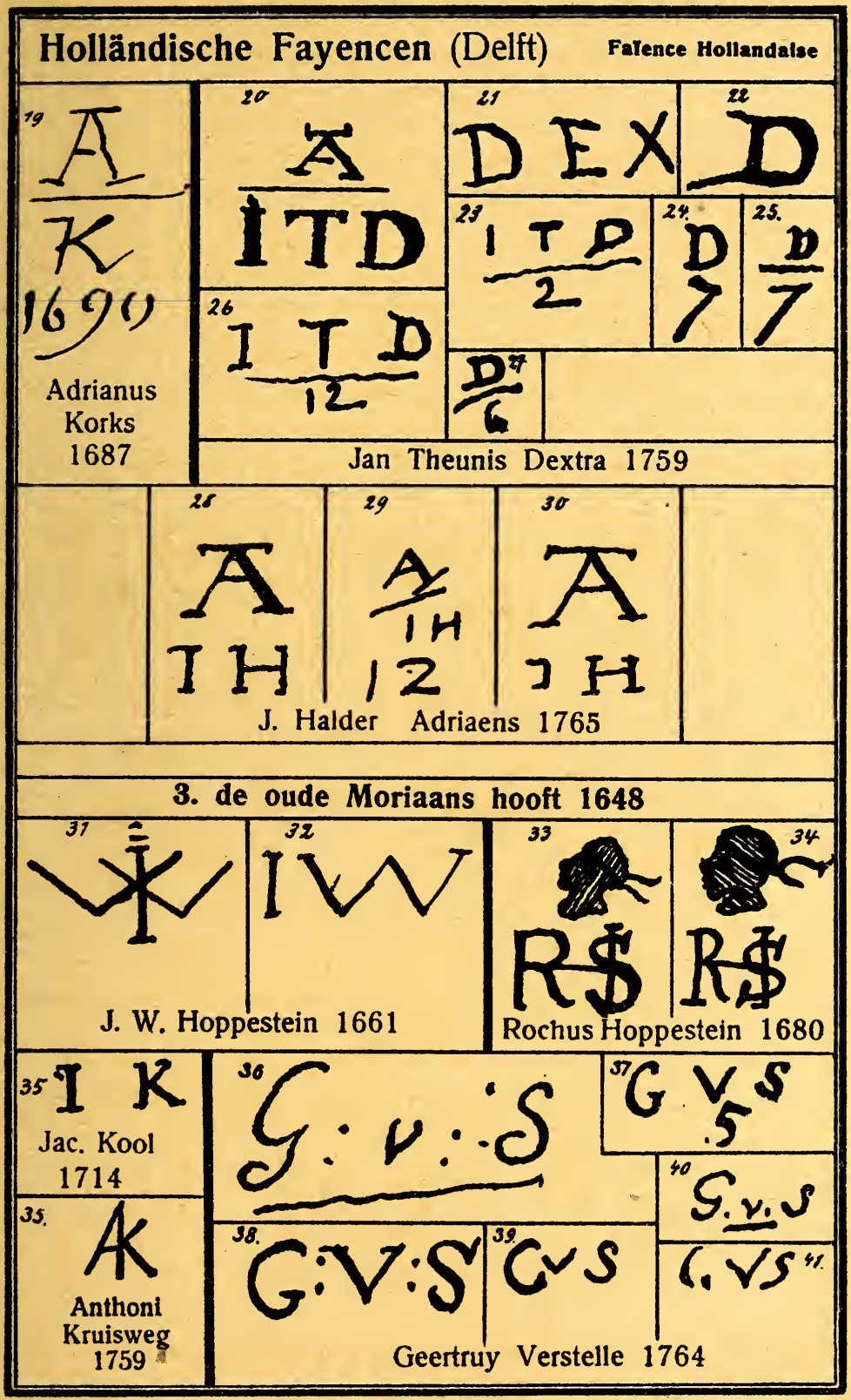




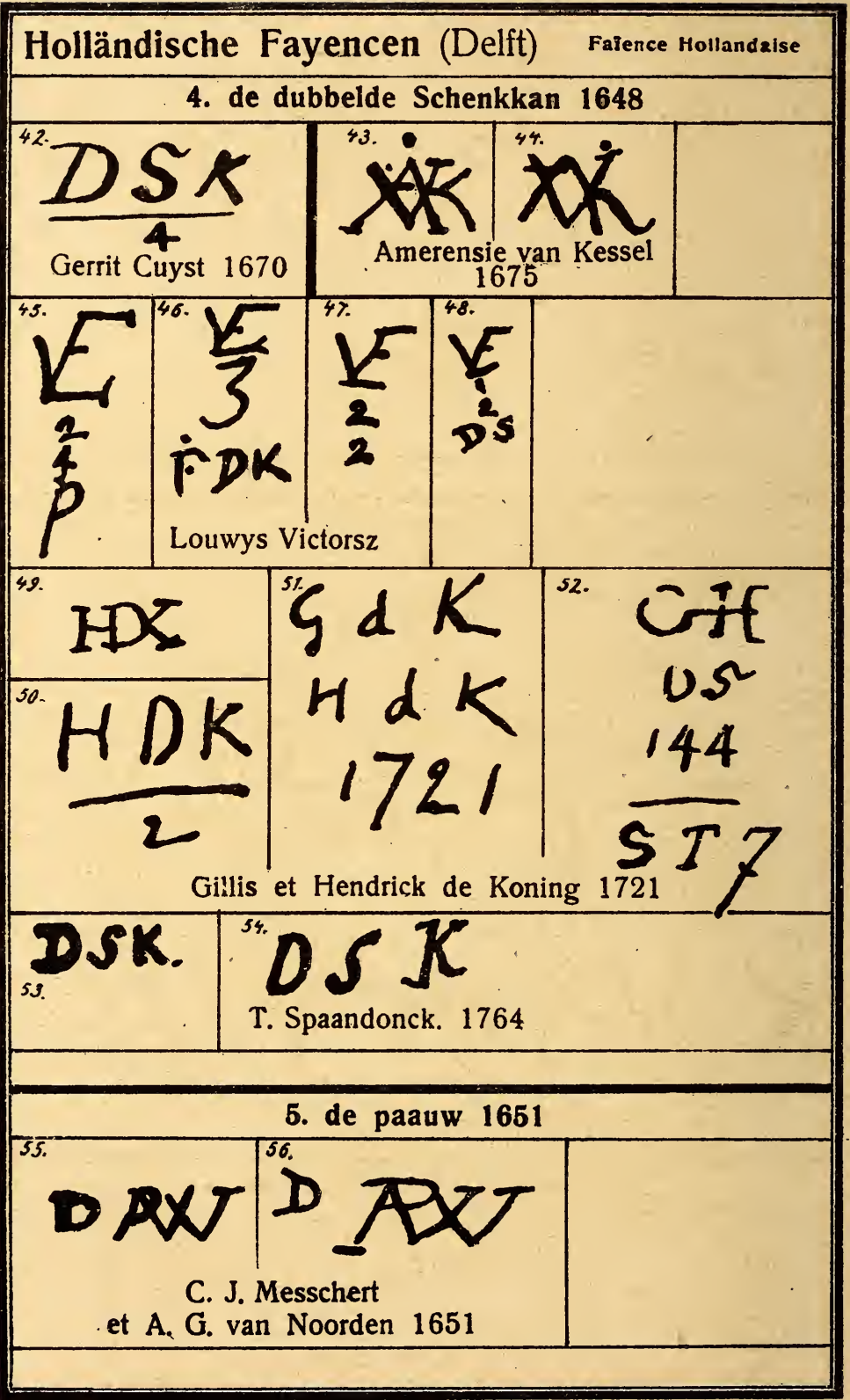




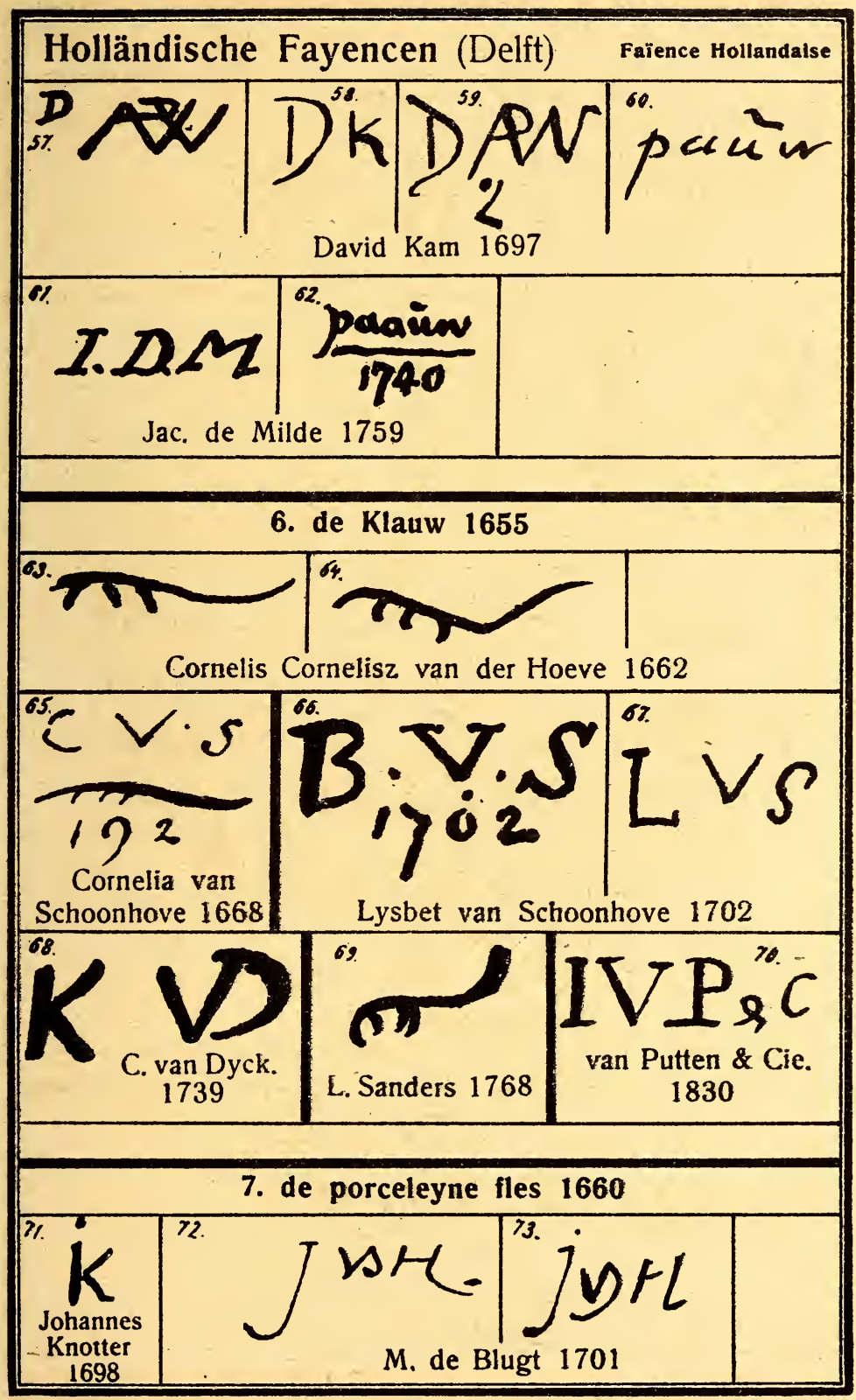




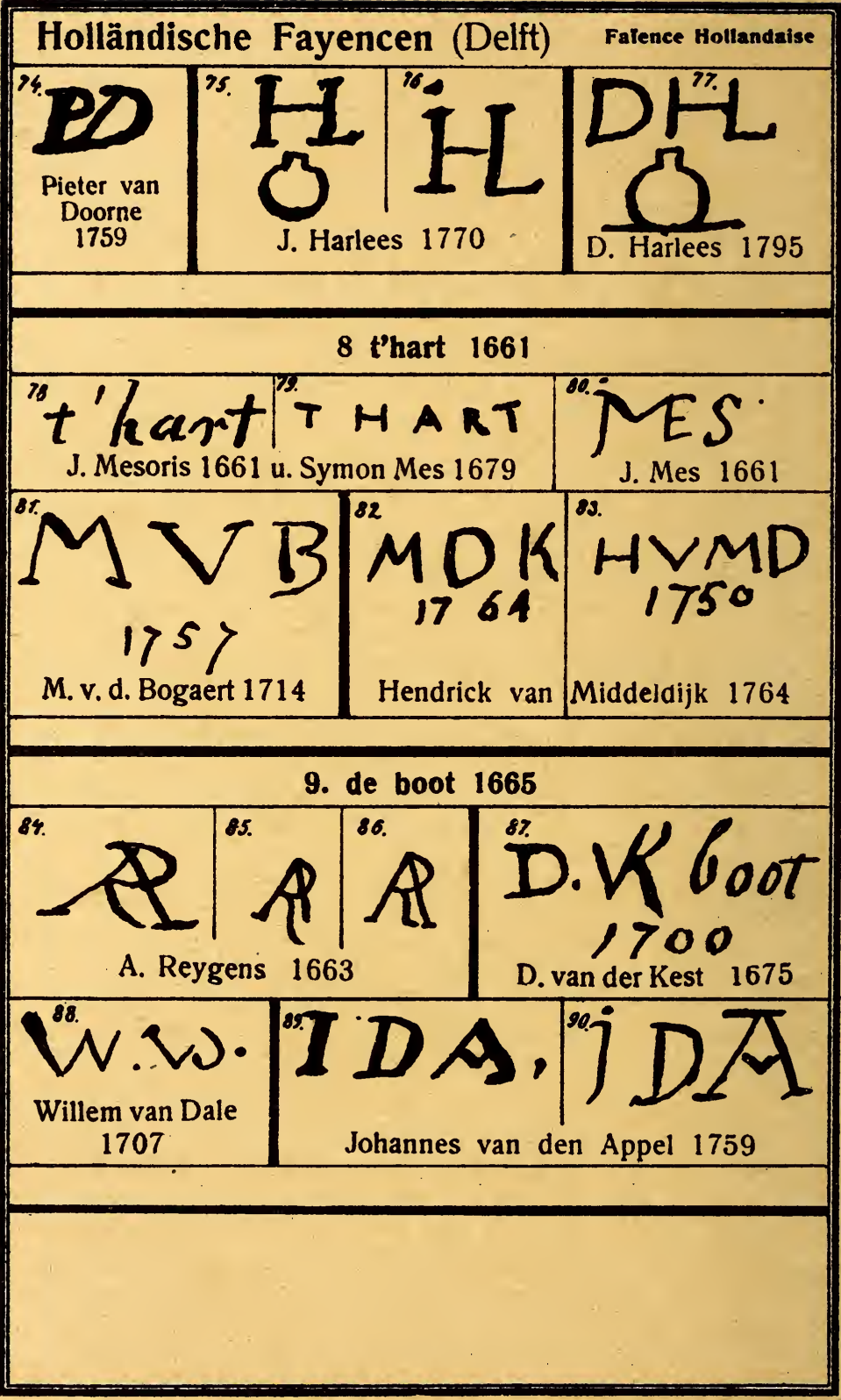




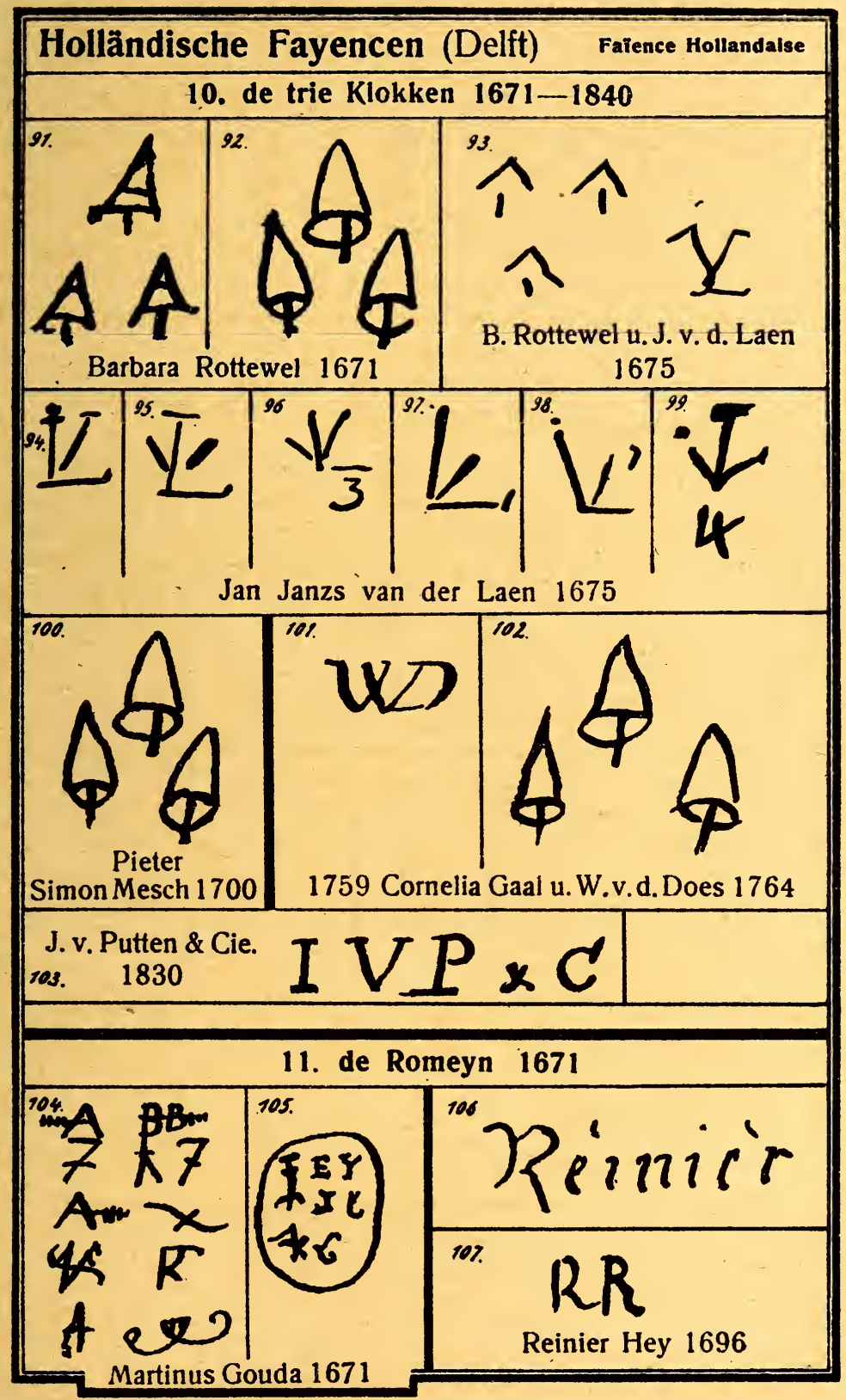




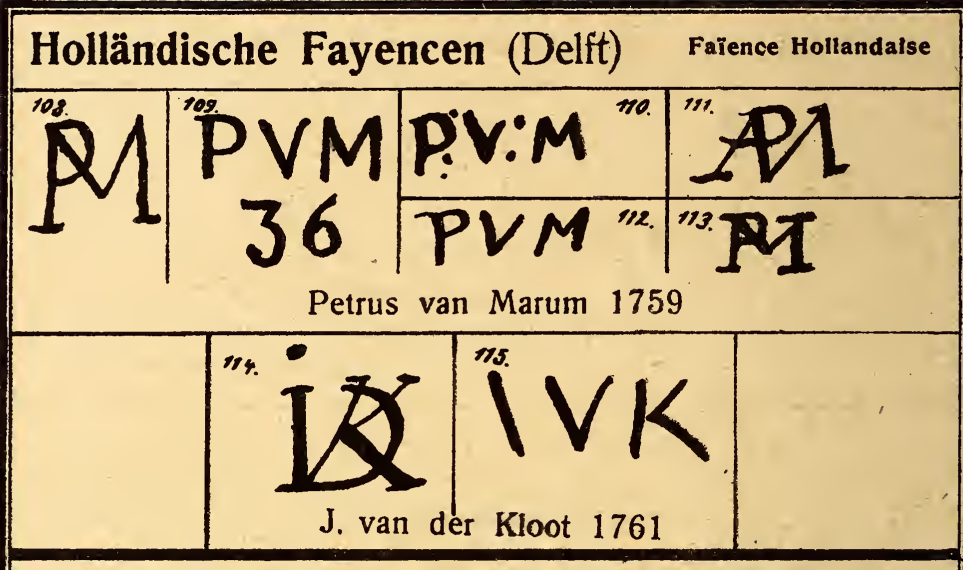

12. de trie porceleyne flessjes. 1672

\begin{tabular}{|l|c|c|c|c|}
\hline 116.118. & 119 \\
\hline \\
\hline
\end{tabular}

13. de drie astonne

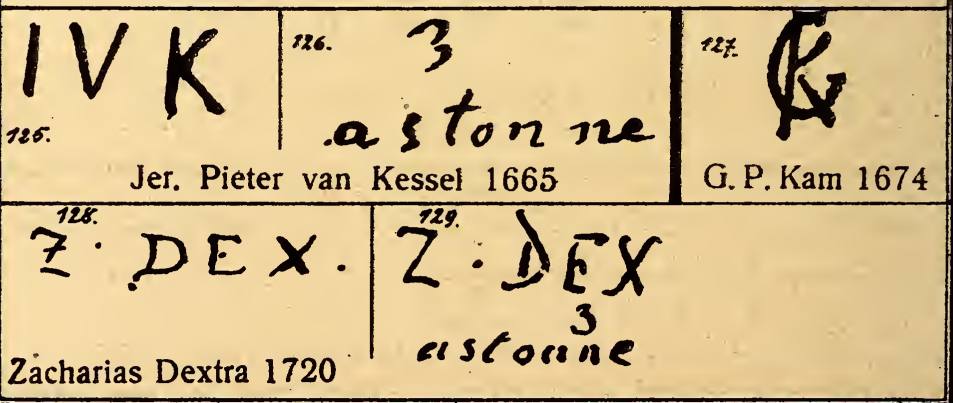




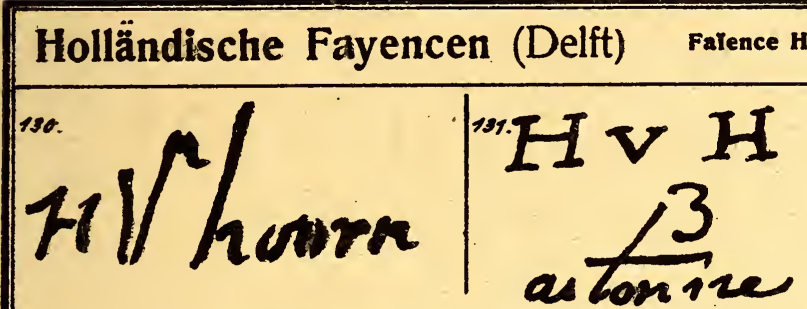

H. van Hoorn $1759^{\circ}$
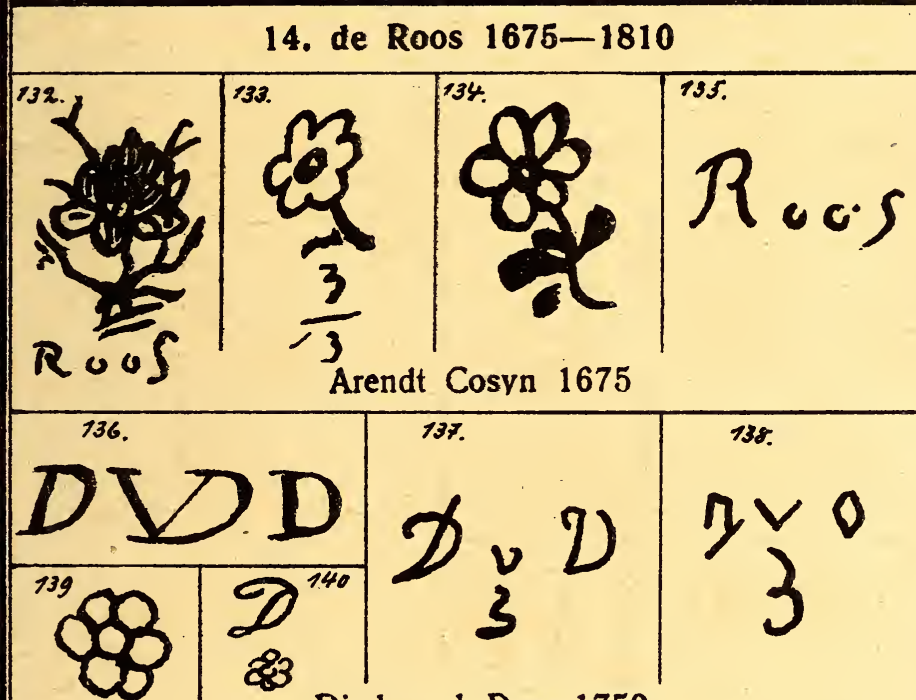

Dirck v. d. Does 1759

\begin{tabular}{|c|c|}
\hline $\begin{array}{l}18013 \\
H \vee D \text { B CoSi }\end{array}$ & 14 \\
\hline H. v. d. Bosch 1803 & J. $\mathbf{v}$ \\
\hline
\end{tabular}

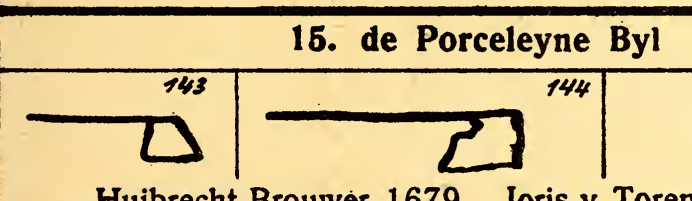

Huibrecht Brouwer 1679 Joris v. Torenburg 1697 

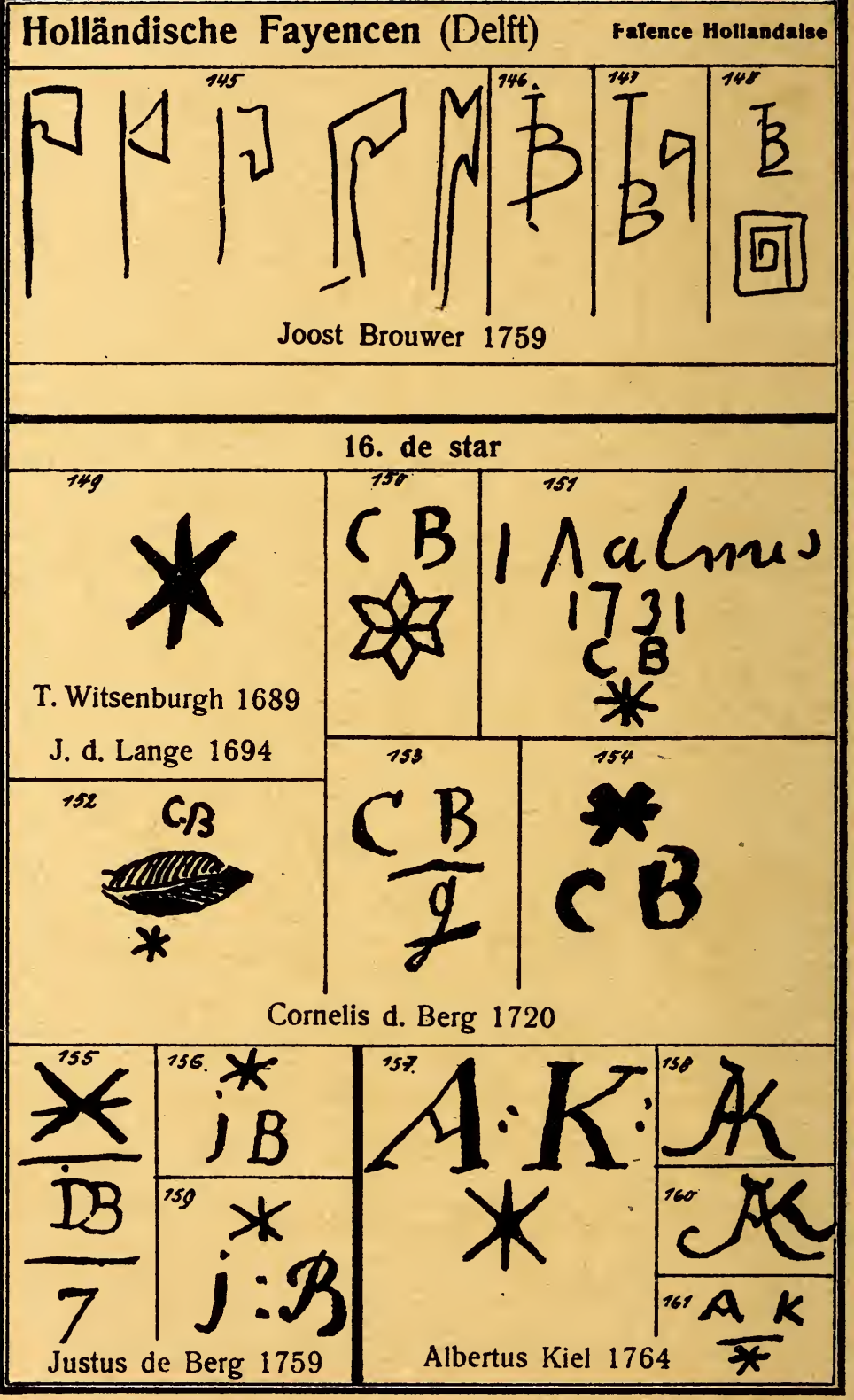


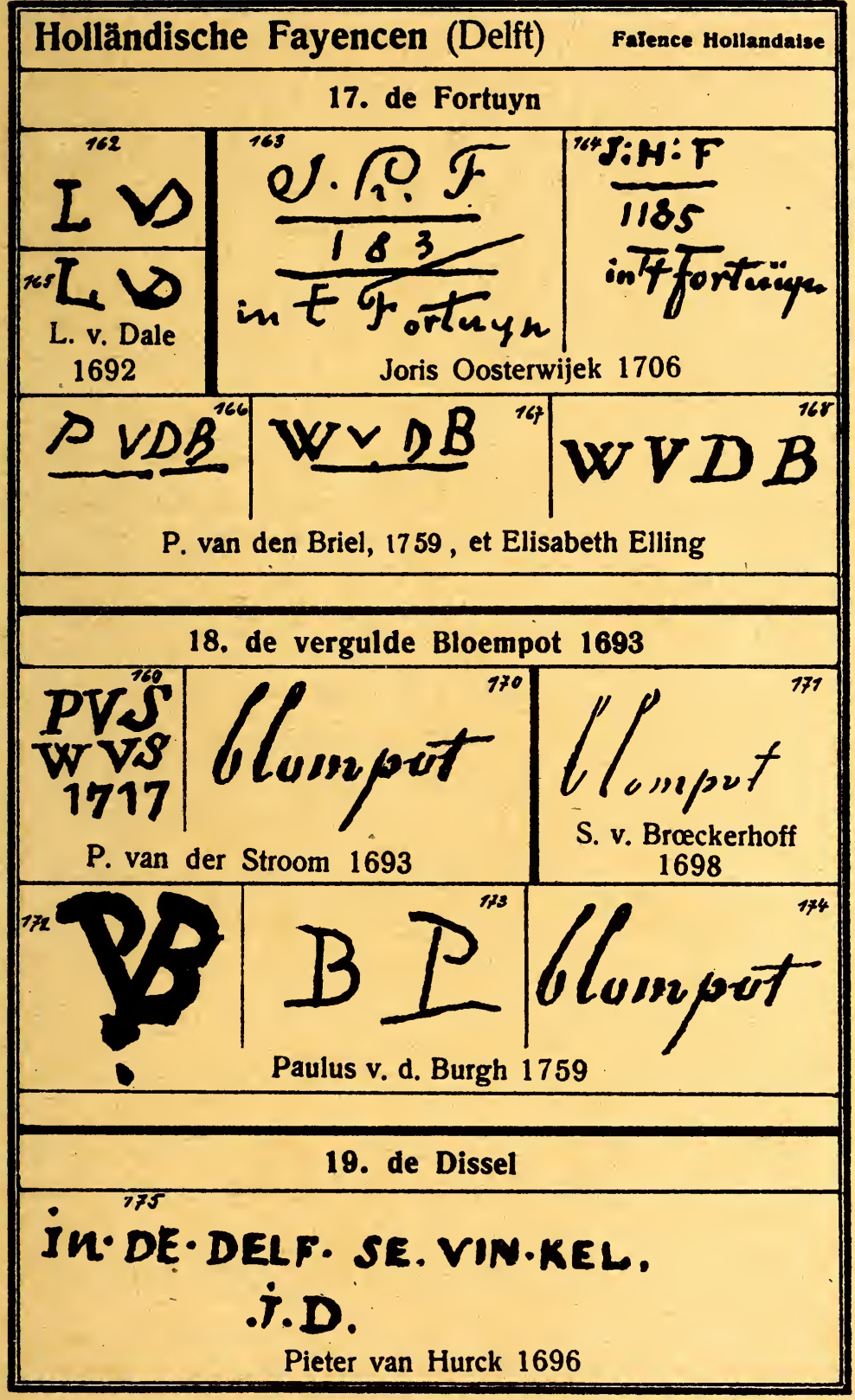




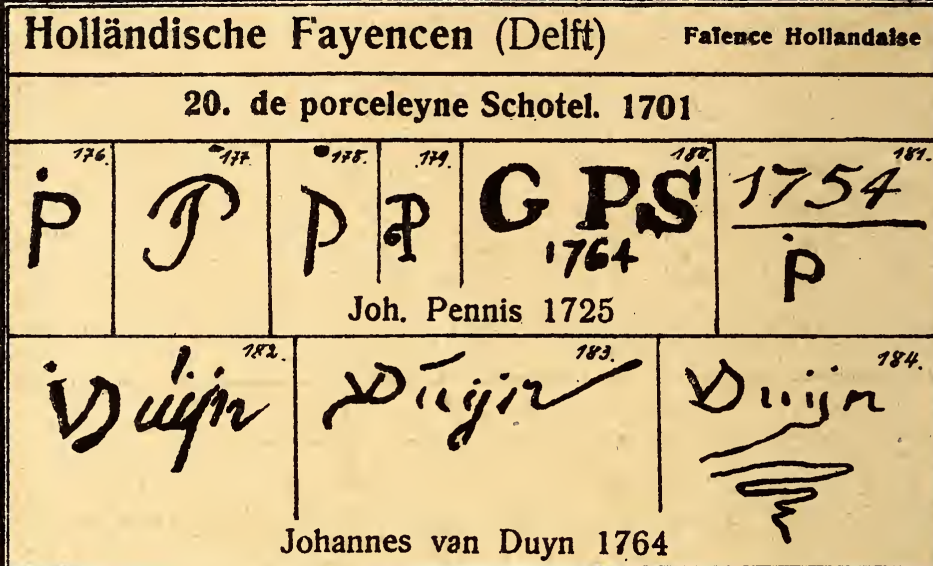

\begin{tabular}{|c|c|}
\hline \multicolumn{3}{|c|}{ 21. de vier helden van Roome } \\
\hline 185.
\end{tabular}

22. de jonge Moriaans hooft 1720

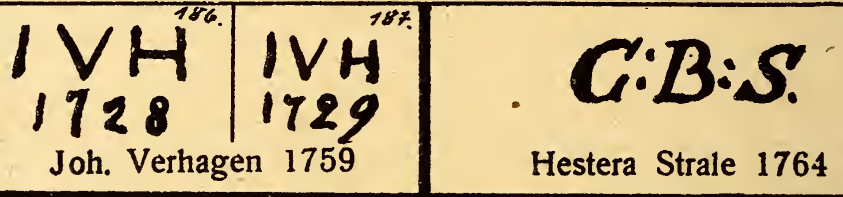

23. de twee wildemans

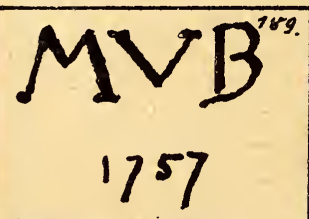

Matheus v. d. Bogaert 1714
$\mathbf{W} \boldsymbol{V B}$

Willem v. Beek 1764 
Holländische Fayencen (Delft) Faience Holiandaise

24. de twee schedpjes. 1759

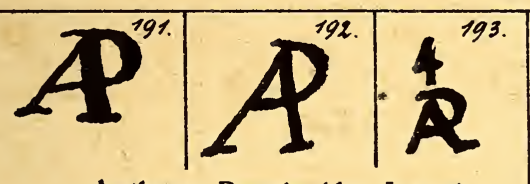

Anthony Pennis (der Junge) et Rachel Overgau 1759

25. de lampetkan 1759

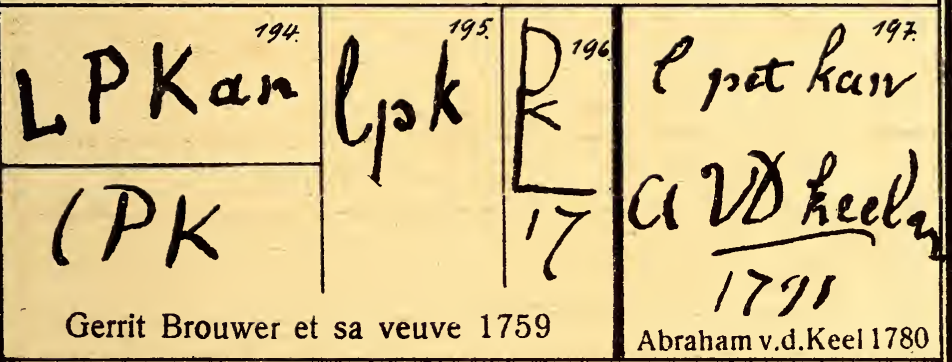

Einzelne Künstler (Artistes spéciaux)

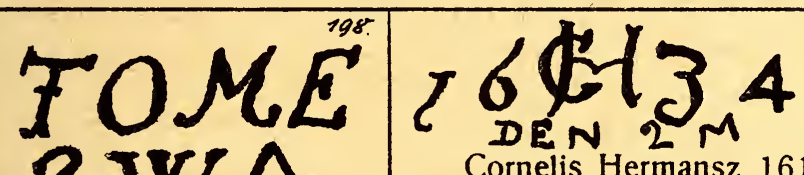

199.

ZWA

Thomes Jansz. 1599

Train $\frac{\sigma^{201}}{16}$

Isaac Junius 1640 


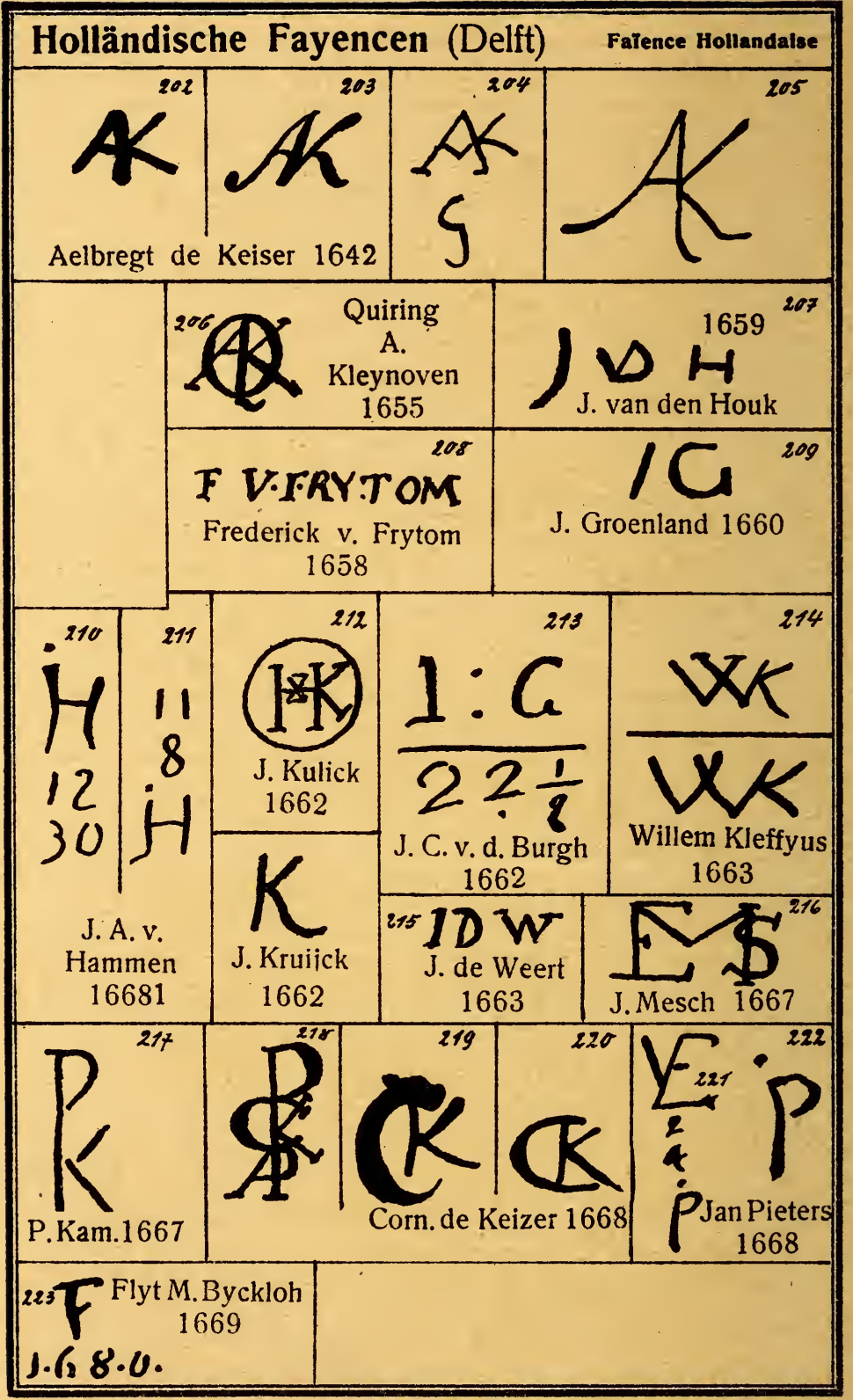




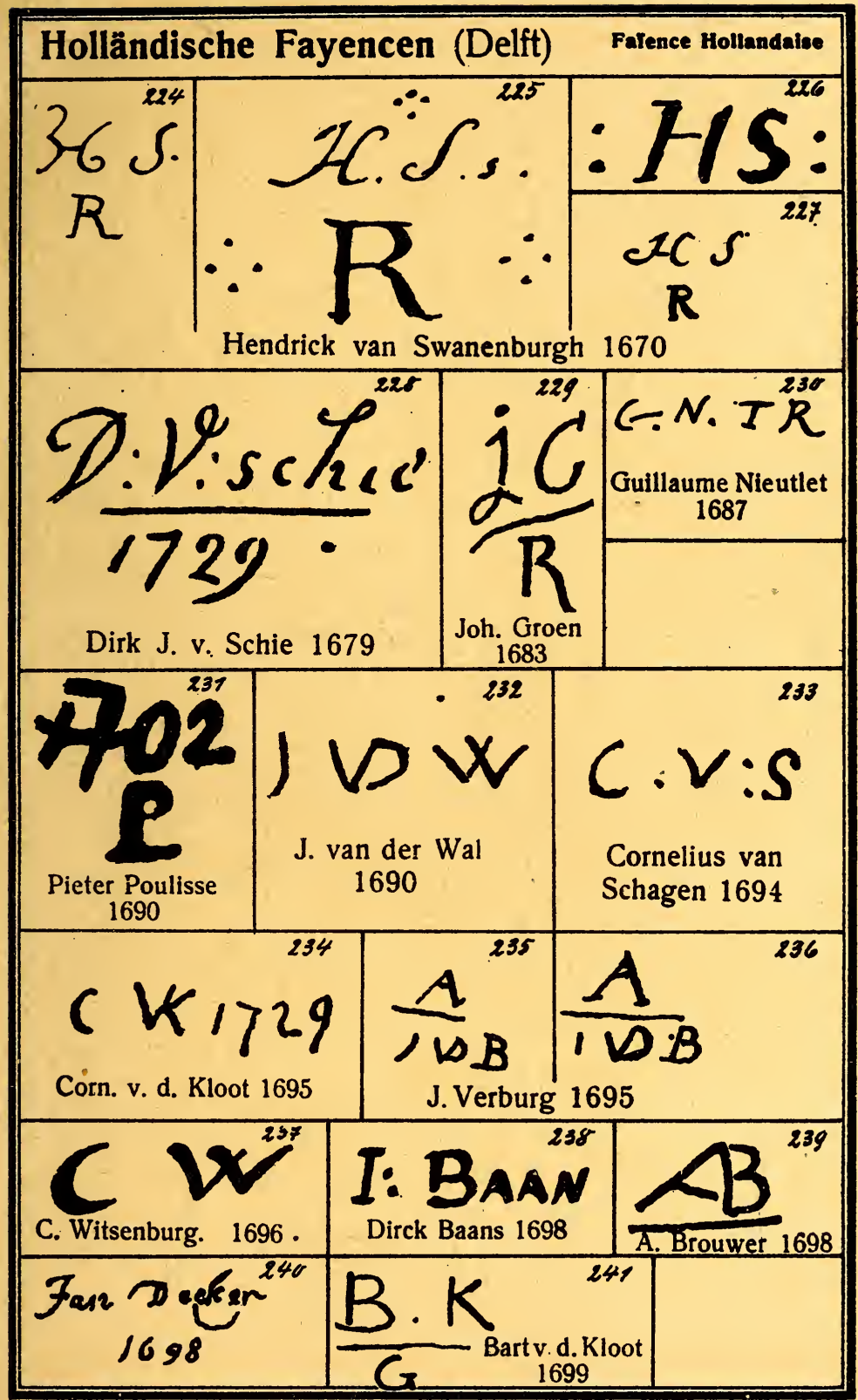


Holländische Fayencen (Delft) Farence Hollandalse
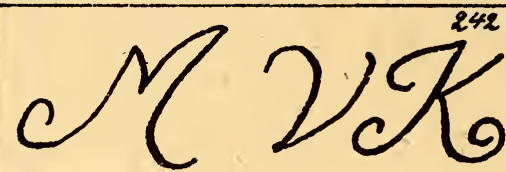

1720

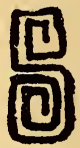

Michiel v. Kuick 1700

S. van der Sand 1705
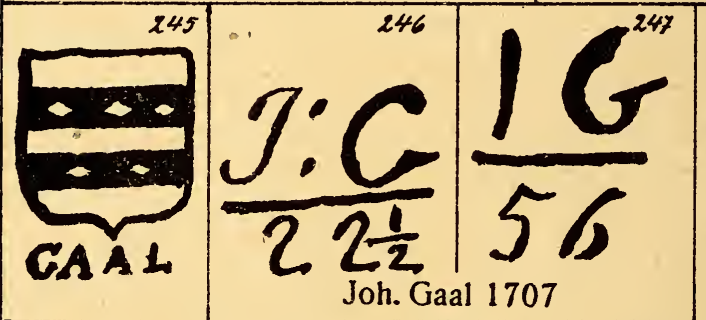

Jacob v. Houten 1701

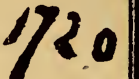

244 


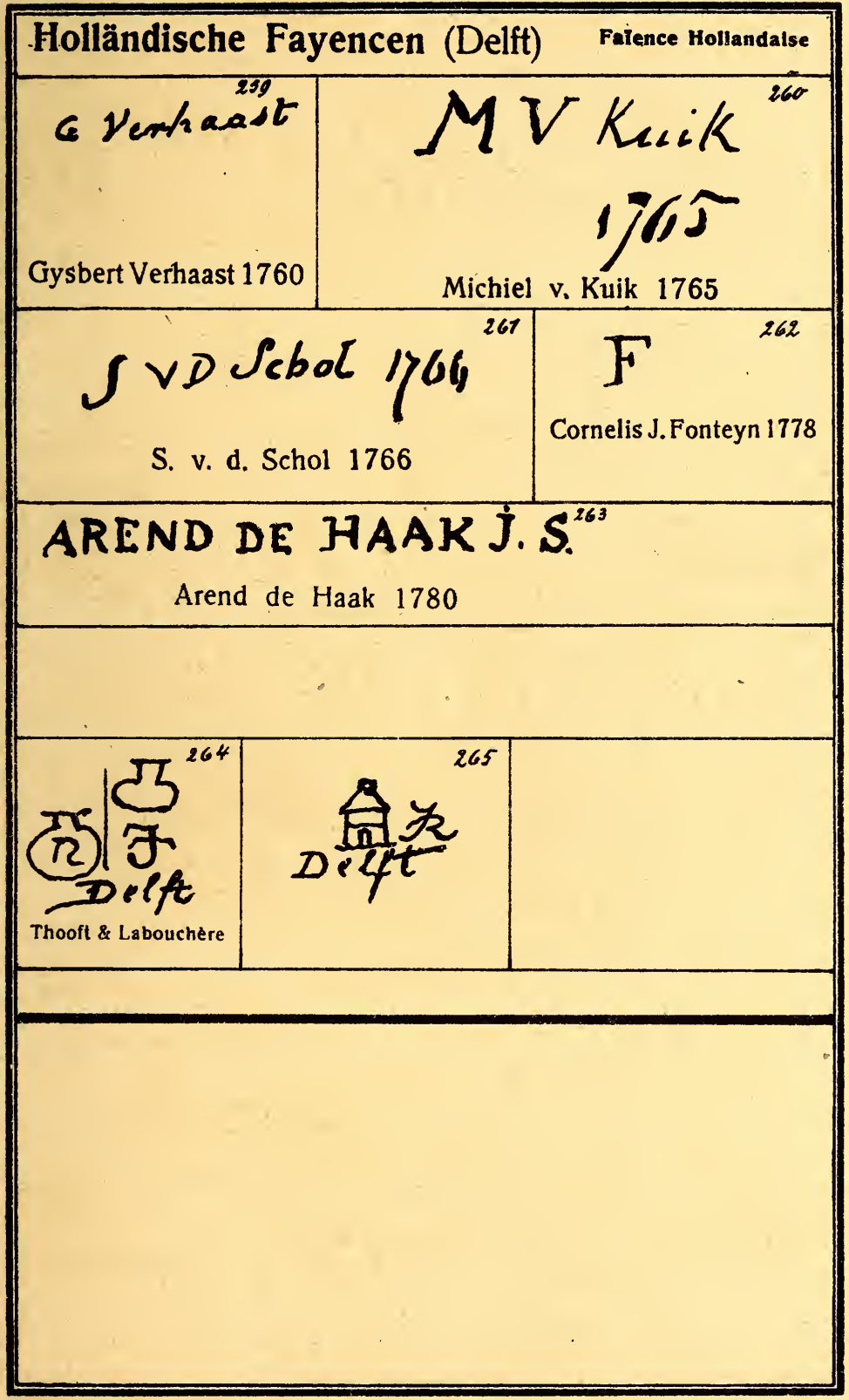




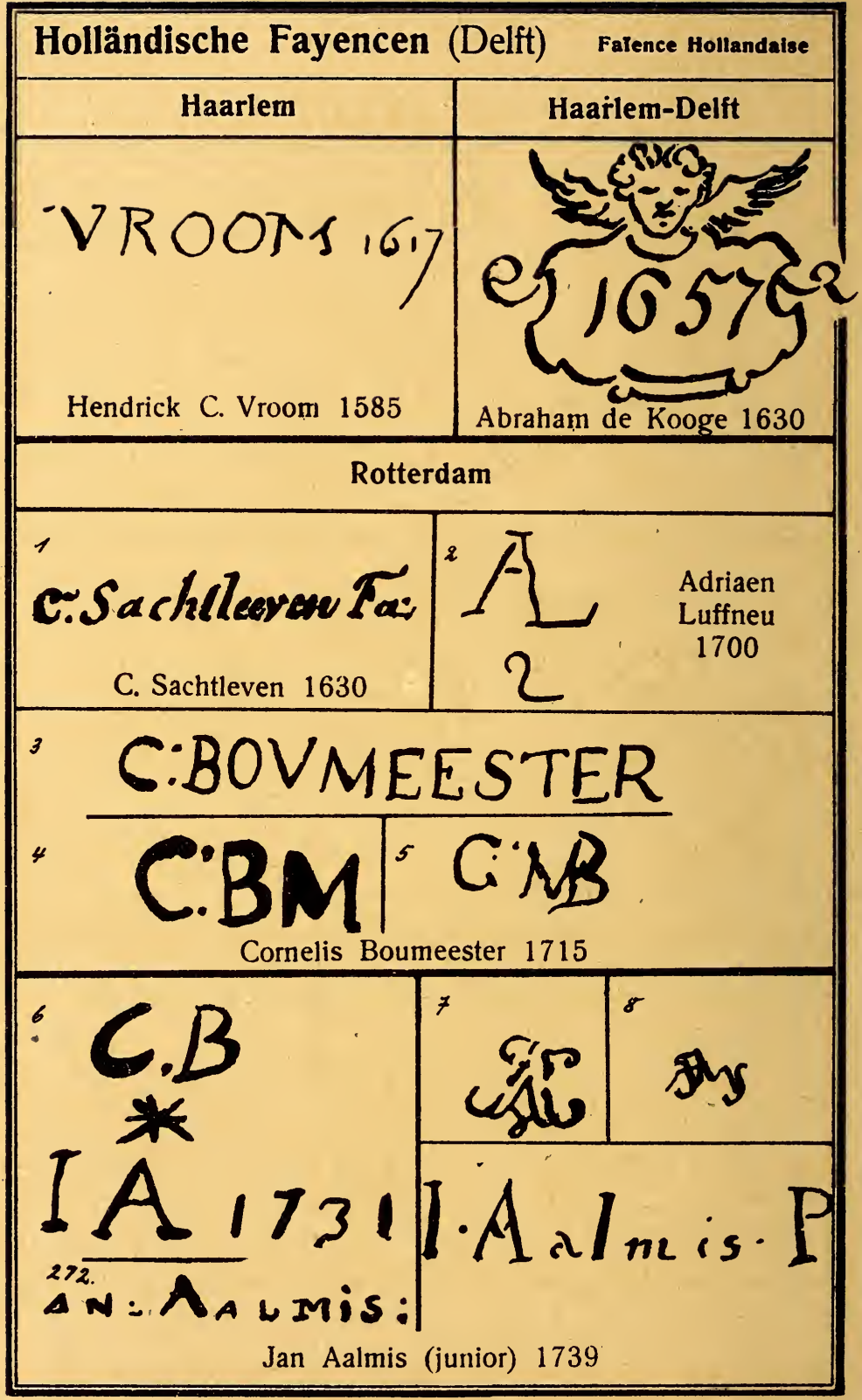




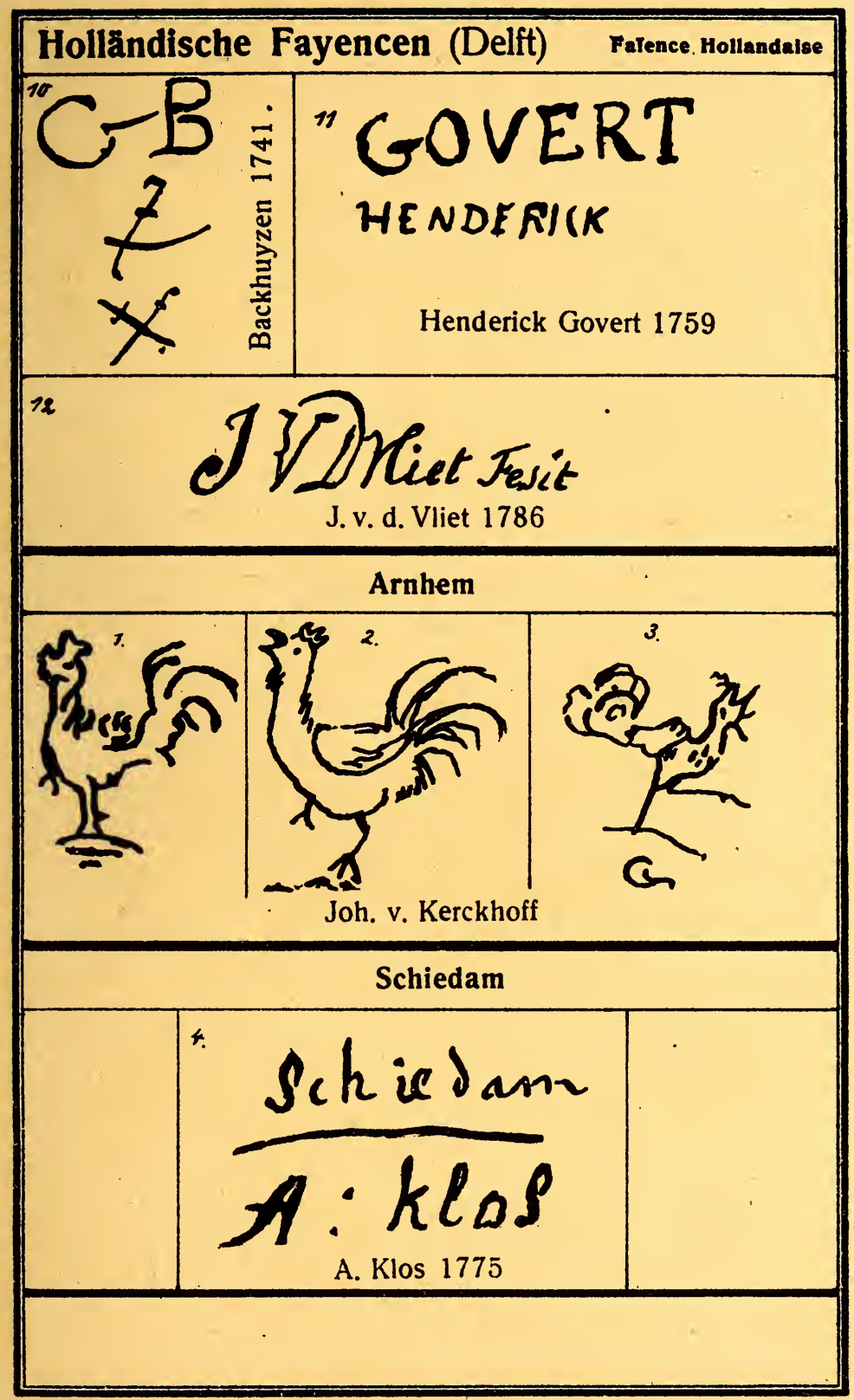


Holländische rote Tonwaren Poterle rouge Hollandaise

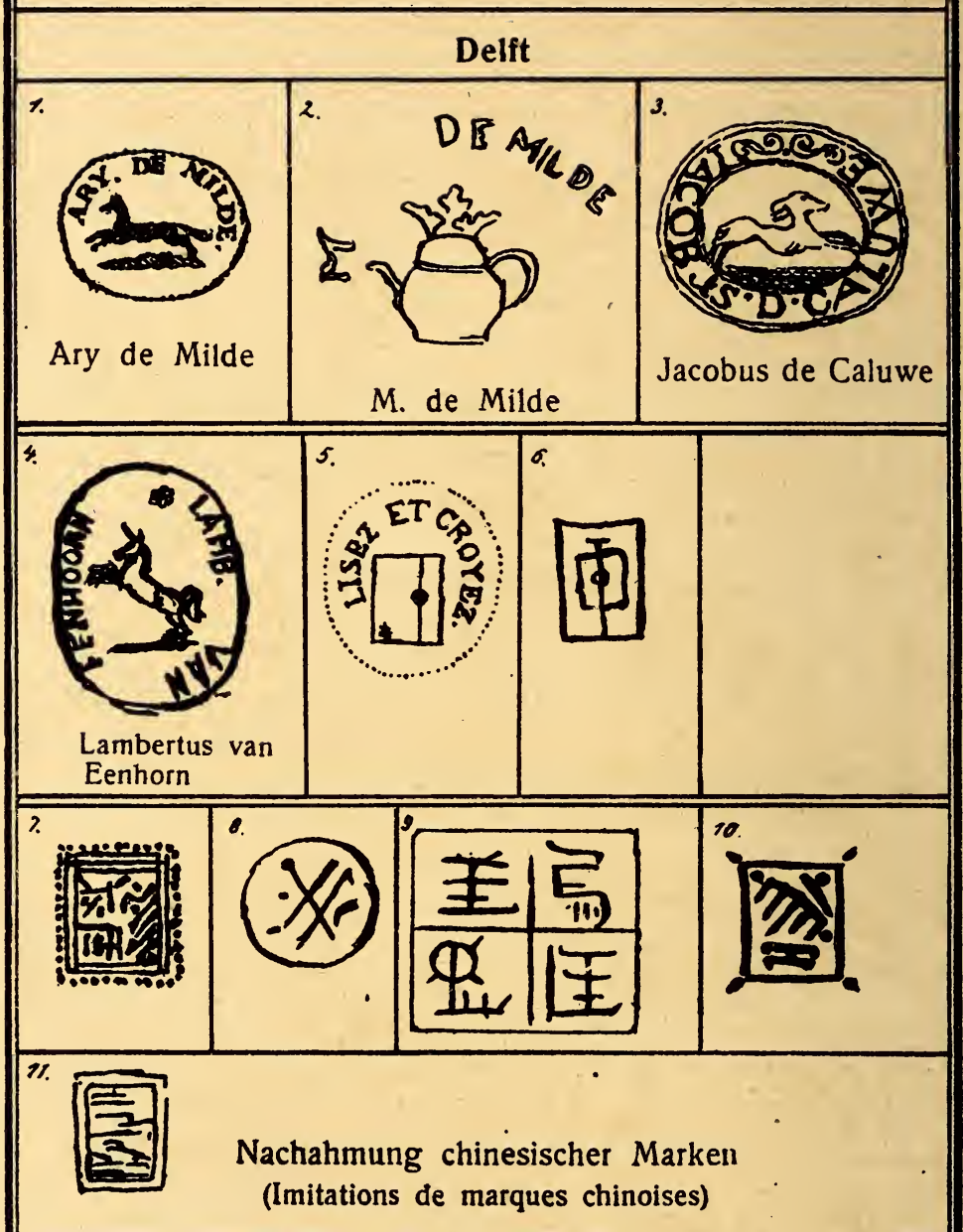

(Imitations de marques chinoises) 


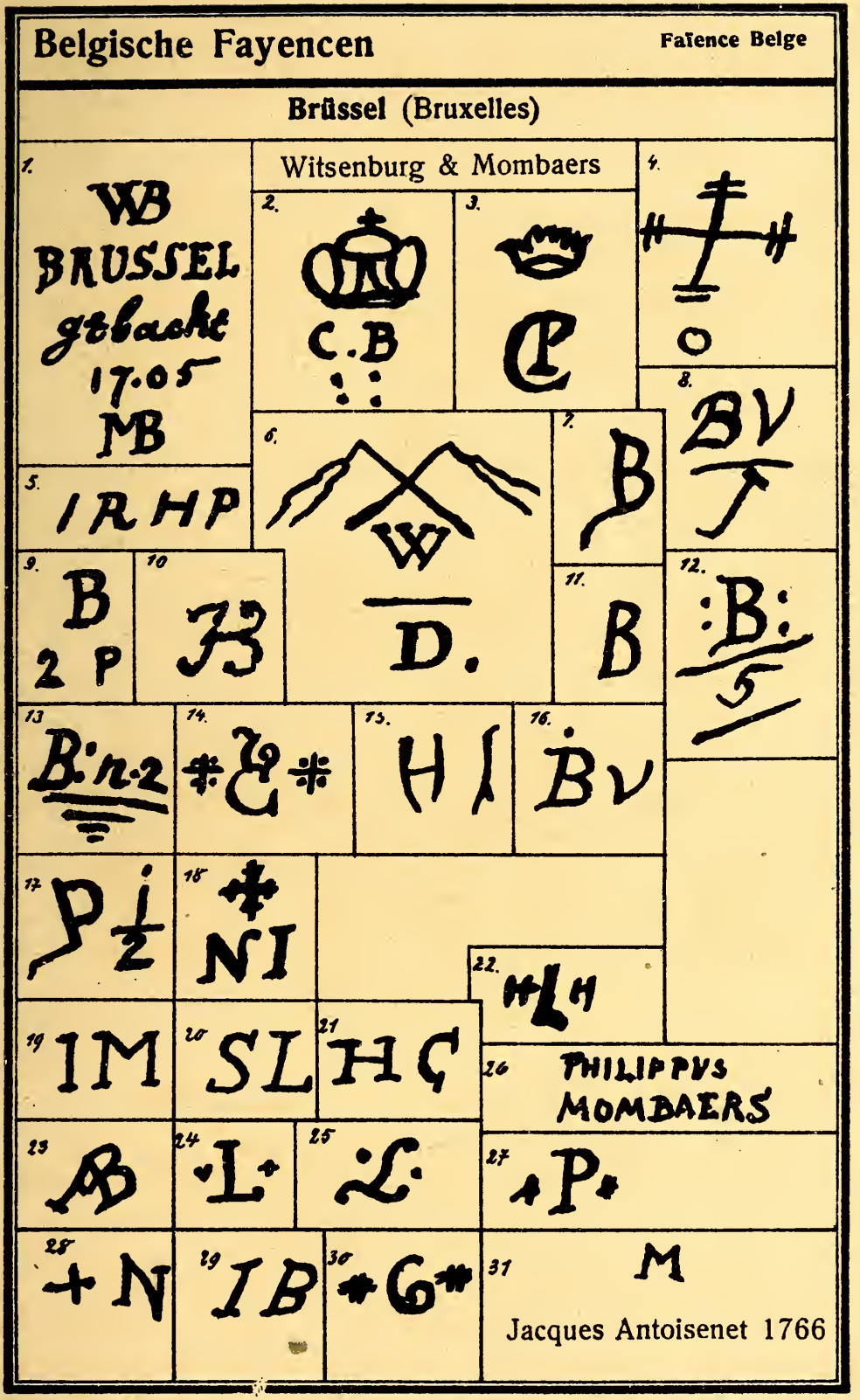




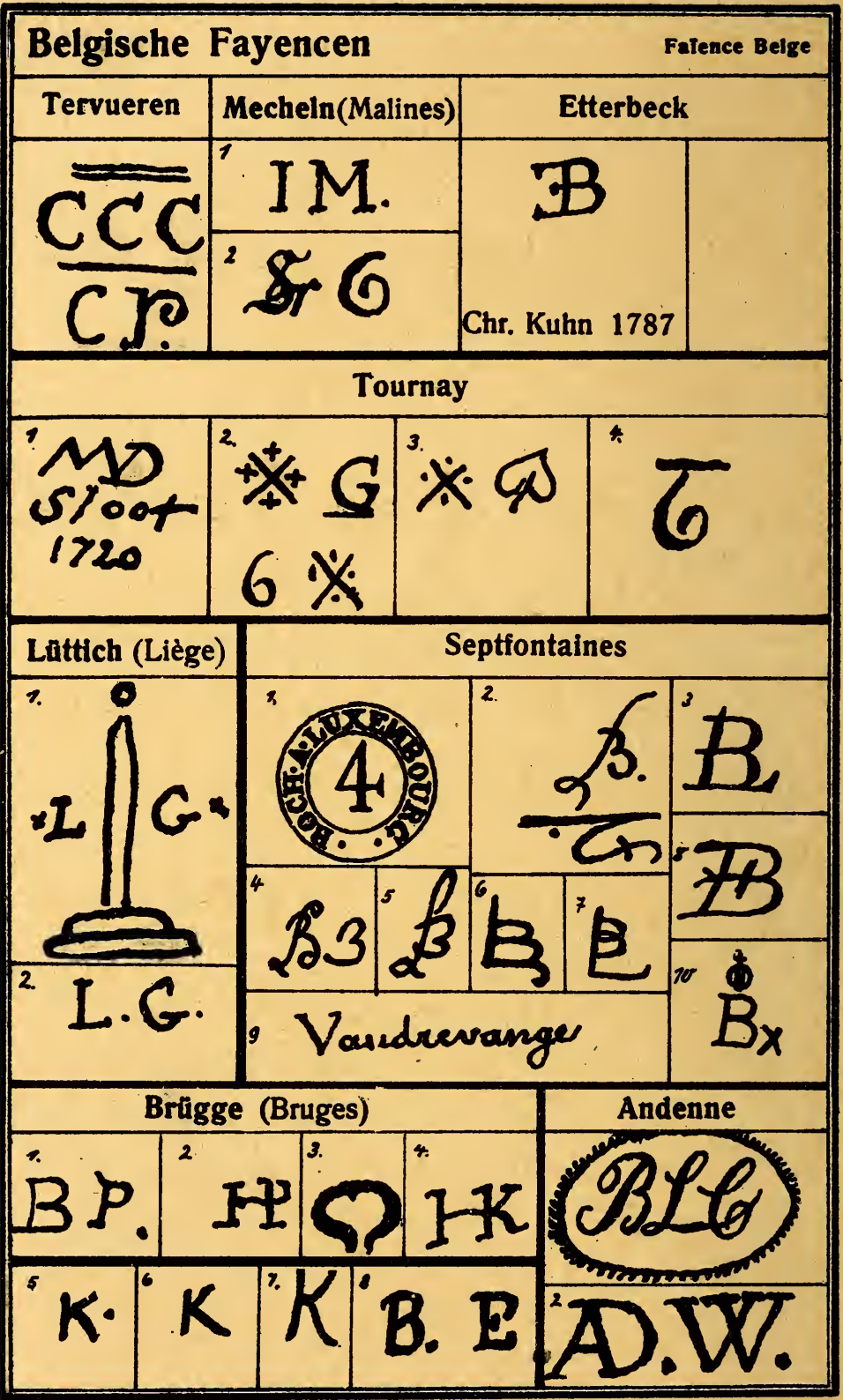


Schweizer Fayencen

Fatence Sulsse

Winterthur.

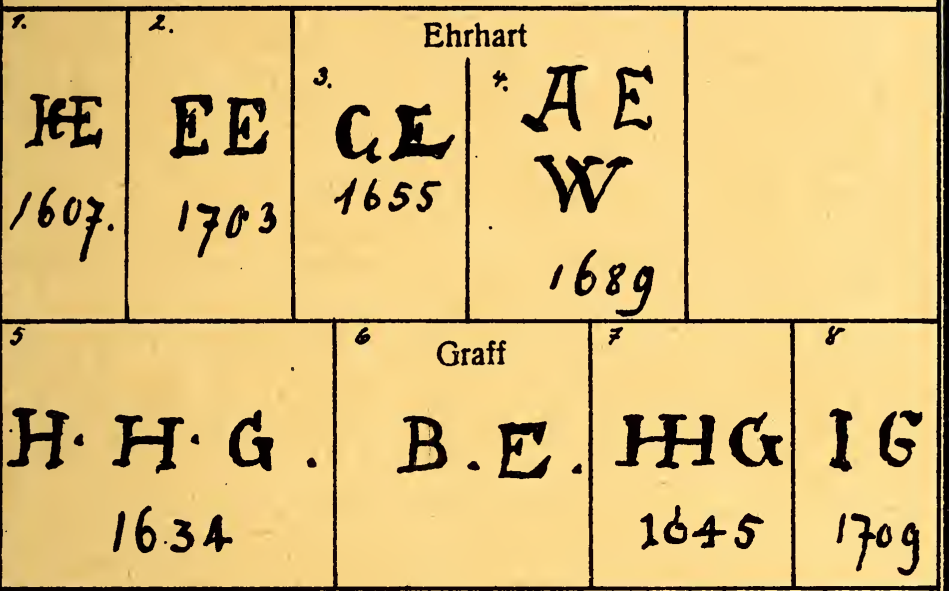

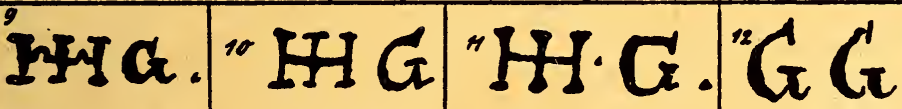
1678 1687

1688

\begin{tabular}{|c|c|c|c|}
\hline${ }^{73}$ & ${ }^{14} \mathrm{P} P$ & ${ }_{1636} \mathrm{DP}$ & ${ }_{1636} \mathrm{H} \cdot \mathrm{P}$ \\
\hline
\end{tabular}

CFEPD ${ }_{1660}^{A} A P$

David Pfaur Haffinem FP Hafnen ig in Winlerthror. 16g8 1716 
Schweizer Fayencen

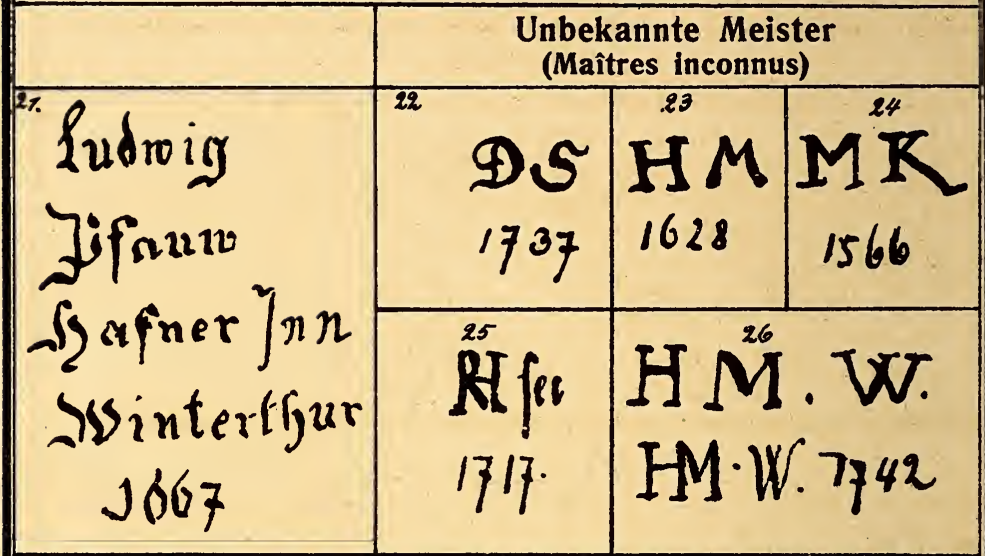

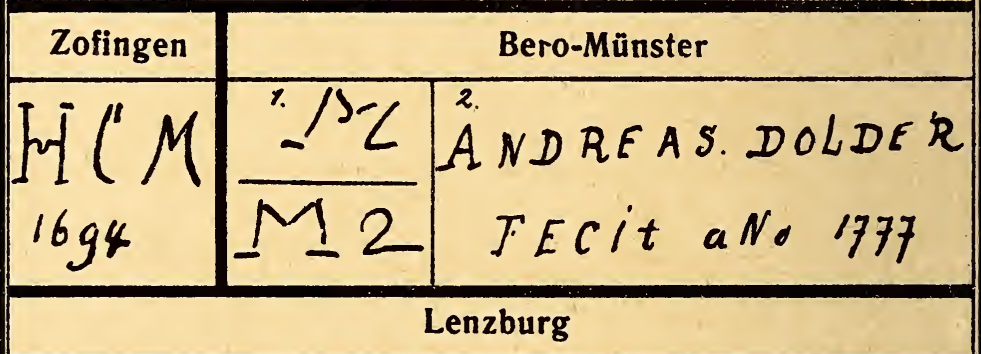

$H \cdot C K L V G \cdot H: M: H W::^{2} L B$ AIKLVG. BOR SOLAIM:M:

\section{Zürich}

Düringes i wo 7 ans Hrinsich thichel

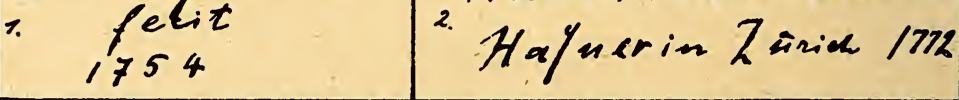

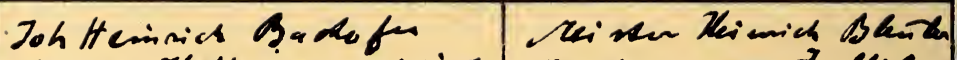

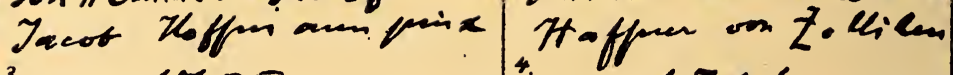
3. 17551766 


\section{Schweizer Fayencen}

Solothurn

슌제

$$
\text { की } 2 \text { 䗆 }
$$
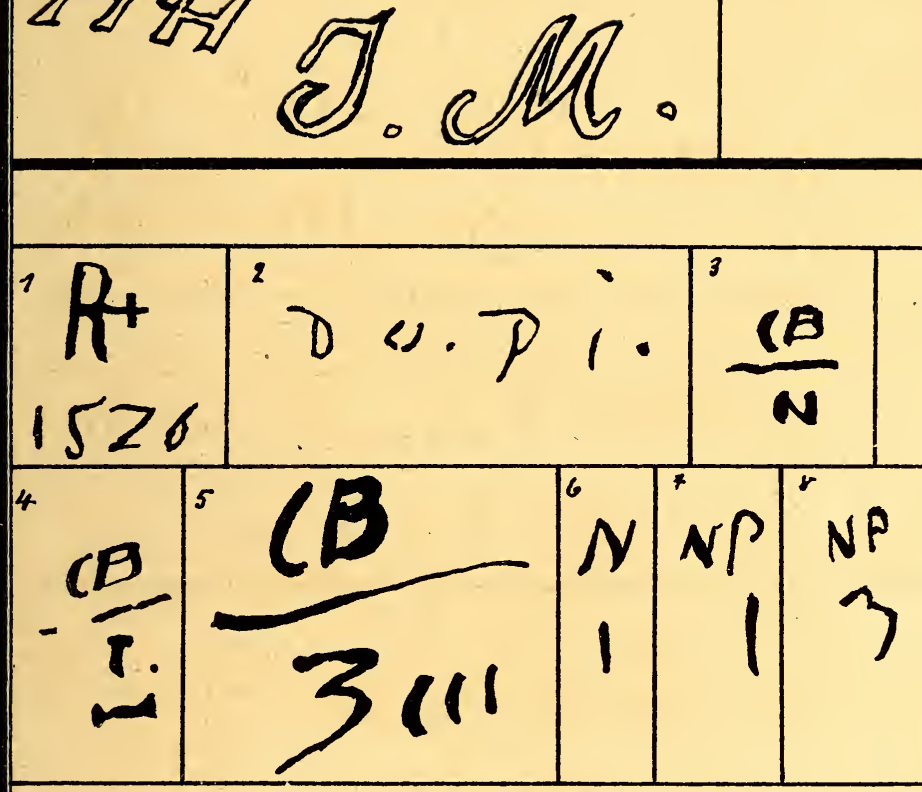
Englische Töpferwaren

Poterle Anglaice

Staffordshire

\begin{tabular}{|c|c|}
\hline RALPHTOII & RIEAPITOFN \\
\hline 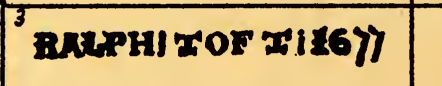 & \\
\hline 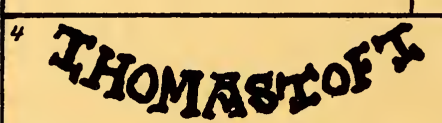 & THOFIASTORT \\
\hline
\end{tabular}

7

IOB!HEAMFI

-IH

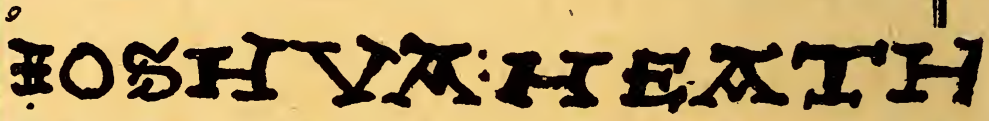

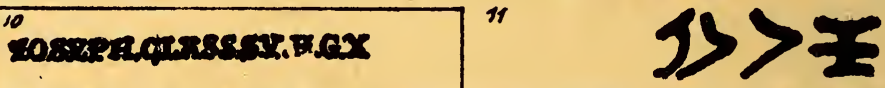

FOHN.WRIGH·J707

RAEPHITLRHOR

12

168

GEORGE:IYIYLOR 
EnglischeTöpferwaren (Staffordshire) Poterie Anglaase

\section{R:A:LTP:H: T:A:Y:L:O:R}

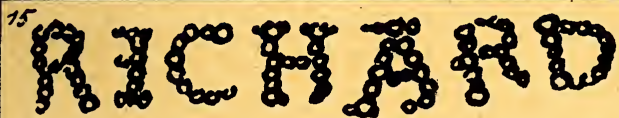

\&d

"IOHN:

\section{SIMPSON}

\section{5}

ROB TIC SIIX

$$
\text { ( }
$$

is WSLLZRMIWRIELI 5809

10

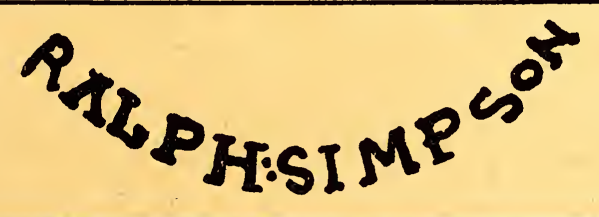


Englische Töpferwaren

Poterie Anglaise

Kent

Wrotham

*ROTHAM

EE X707 'T:Dr:

WROTHAM I>

*W: 7656HA1657

IL IL GR

$16121621 \quad 1650$

CR. 1659

IE 1681 NHII6G18:

IE: 169 ? 
Englische Töpferwaren(Derbyshire)Poterte Anglatse

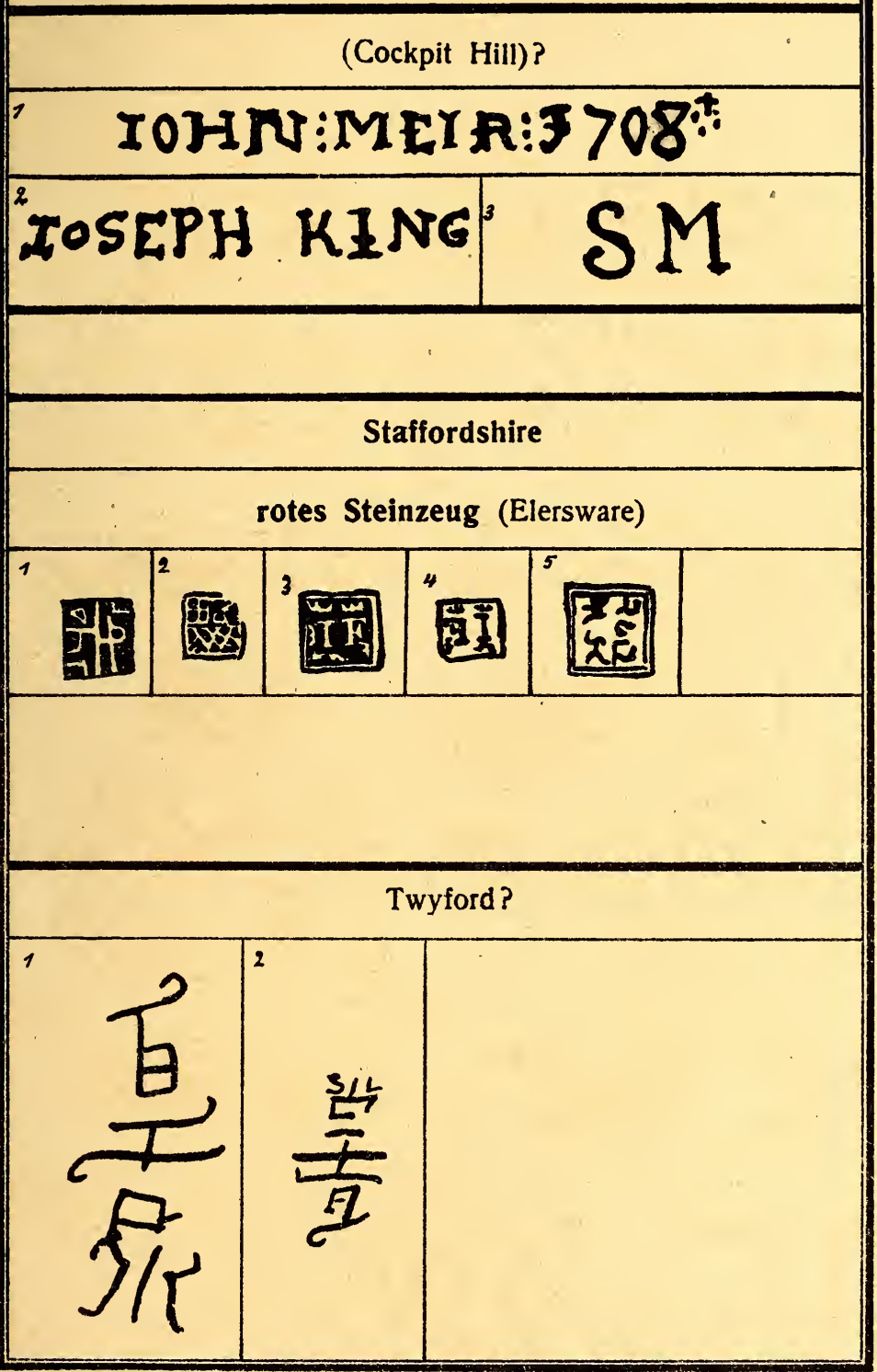


Englische Töpferwaren

Poterle Anglalse

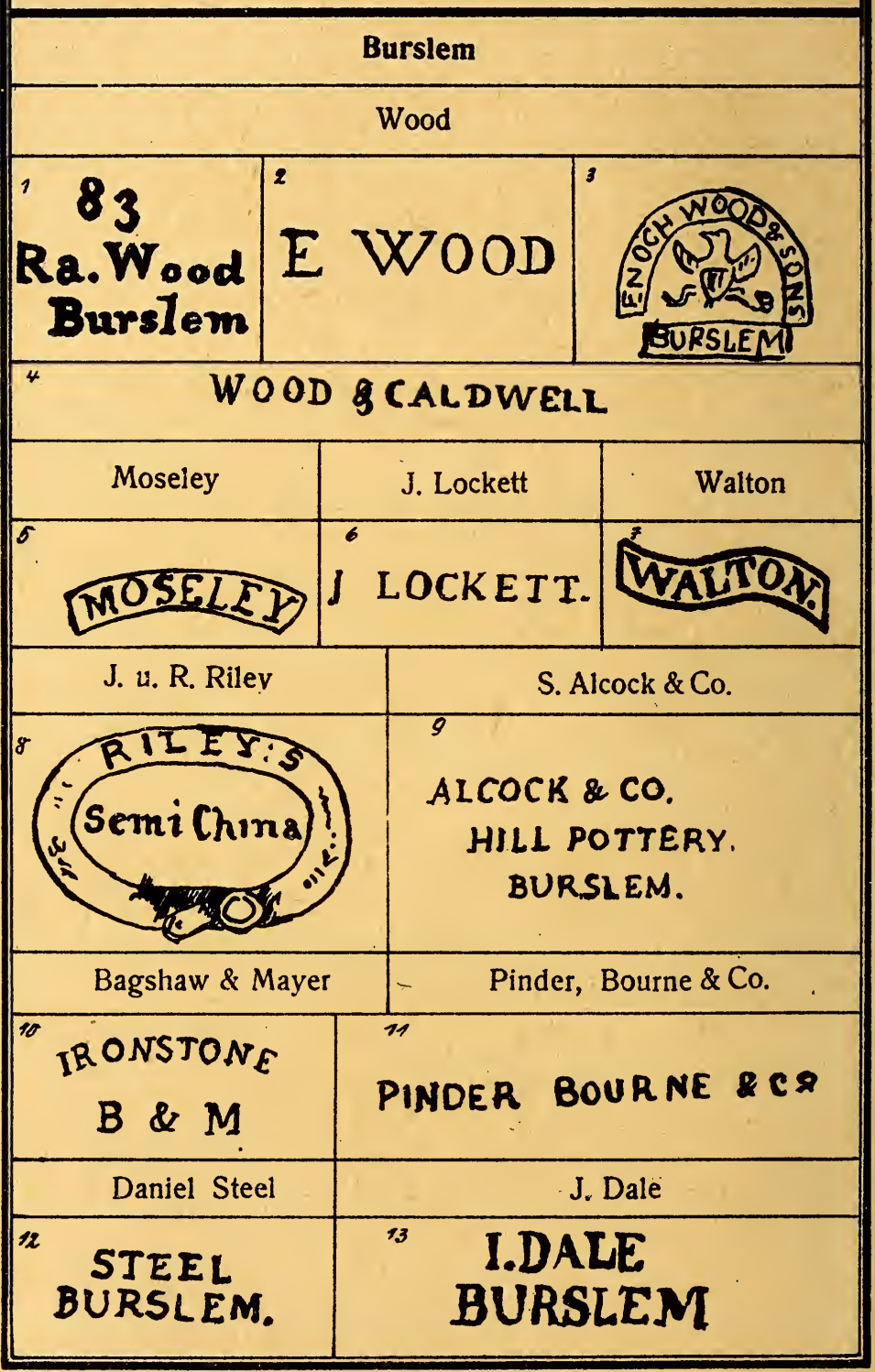




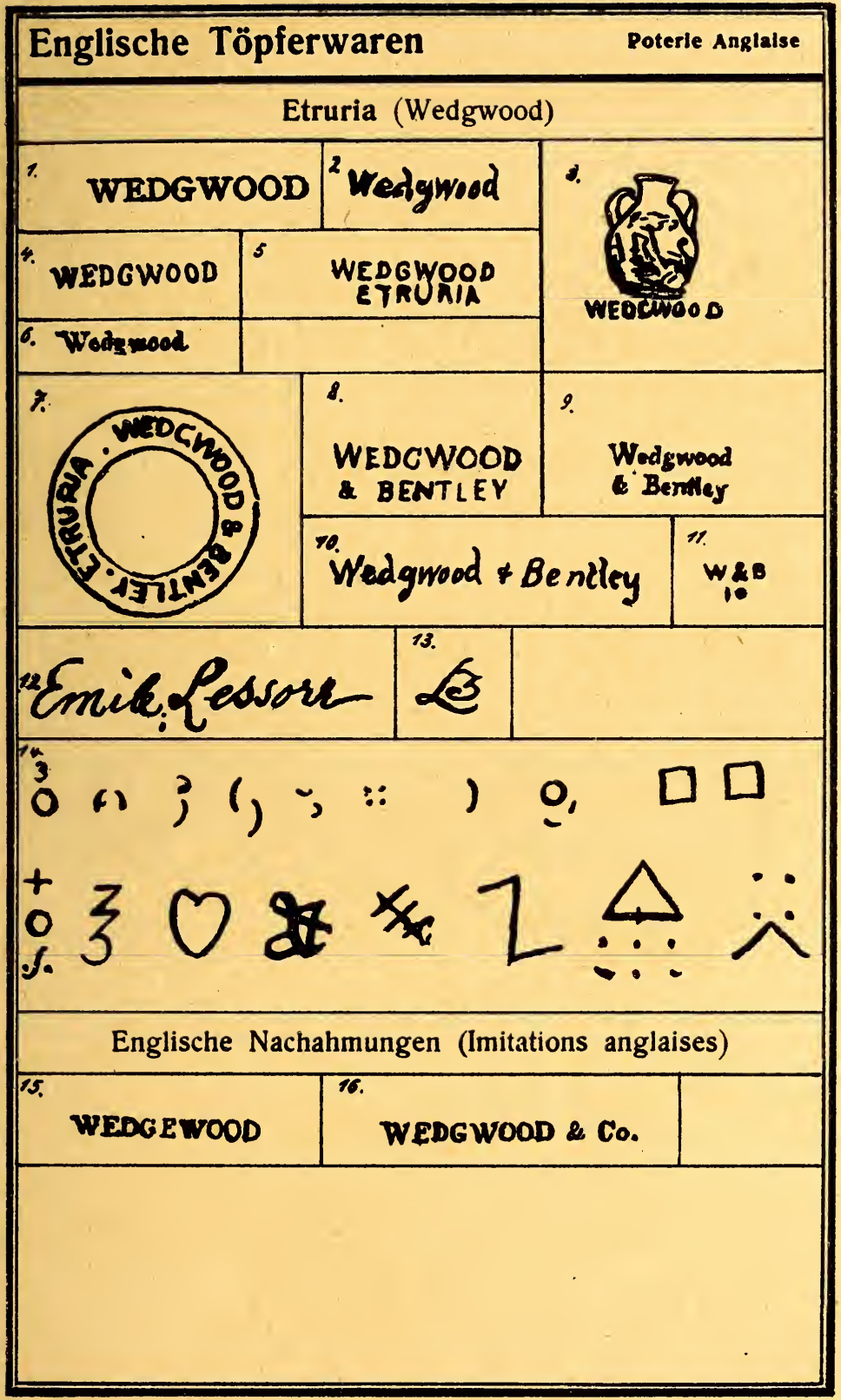




\section{Englische Töpferwaren}

\begin{tabular}{|c|c|c|c|}
\hline \multicolumn{4}{|c|}{ Tunstall } \\
\hline \multicolumn{4}{|c|}{ Adams } \\
\hline Adams \& Co. & W. ADAMS \& Co. & \multirow{3}{*}{$\begin{array}{l}\text { G. ADAMS } \\
\text { TUNSTALL }\end{array}$} & \multirow{3}{*}{$\begin{array}{l}\text { ADAMS } \\
\text { ESTBD } 1657 \\
\text { TUNSTALL } \\
\text { ENGCAMD }\end{array}$} \\
\hline 2 ADAMS \& Ca & \multirow{2}{*}{${ }^{\circ} \mathrm{A}$ ADAMS } & & \\
\hline 3 ADAMS & & & \\
\hline \multicolumn{2}{|c|}{ A. \& E. Keeling } & S. Child & Enoch Booth \\
\hline \multicolumn{2}{|c|}{${ }^{8}$ *.A \& E Keeling v } & CHILD. & Enoch Booth \\
\hline \multicolumn{4}{|l|}{ Ralph Hall } \\
\hline \multicolumn{4}{|l|}{$\bar{R} \overline{T_{A} Z_{2}}$} \\
\hline & Long & & \\
\hline
\end{tabular}
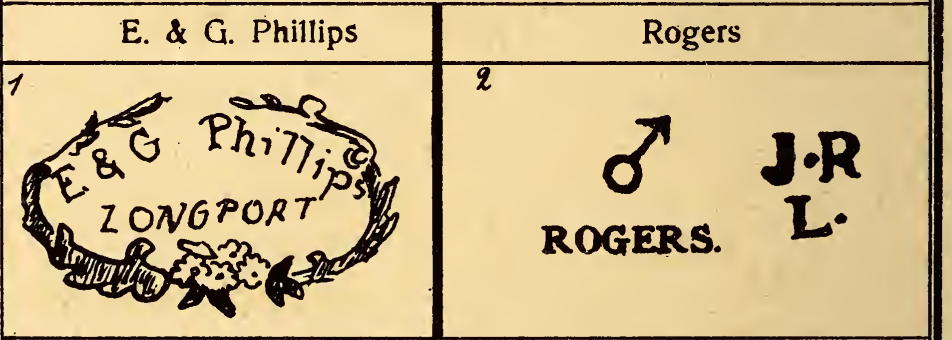

Davenport

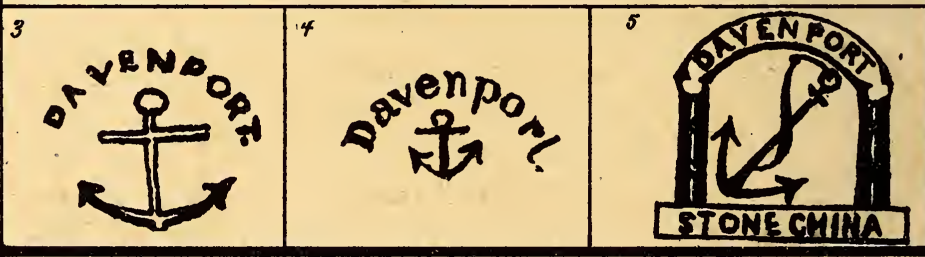

Cobridge

R. Daniel

R. DANIEL. 


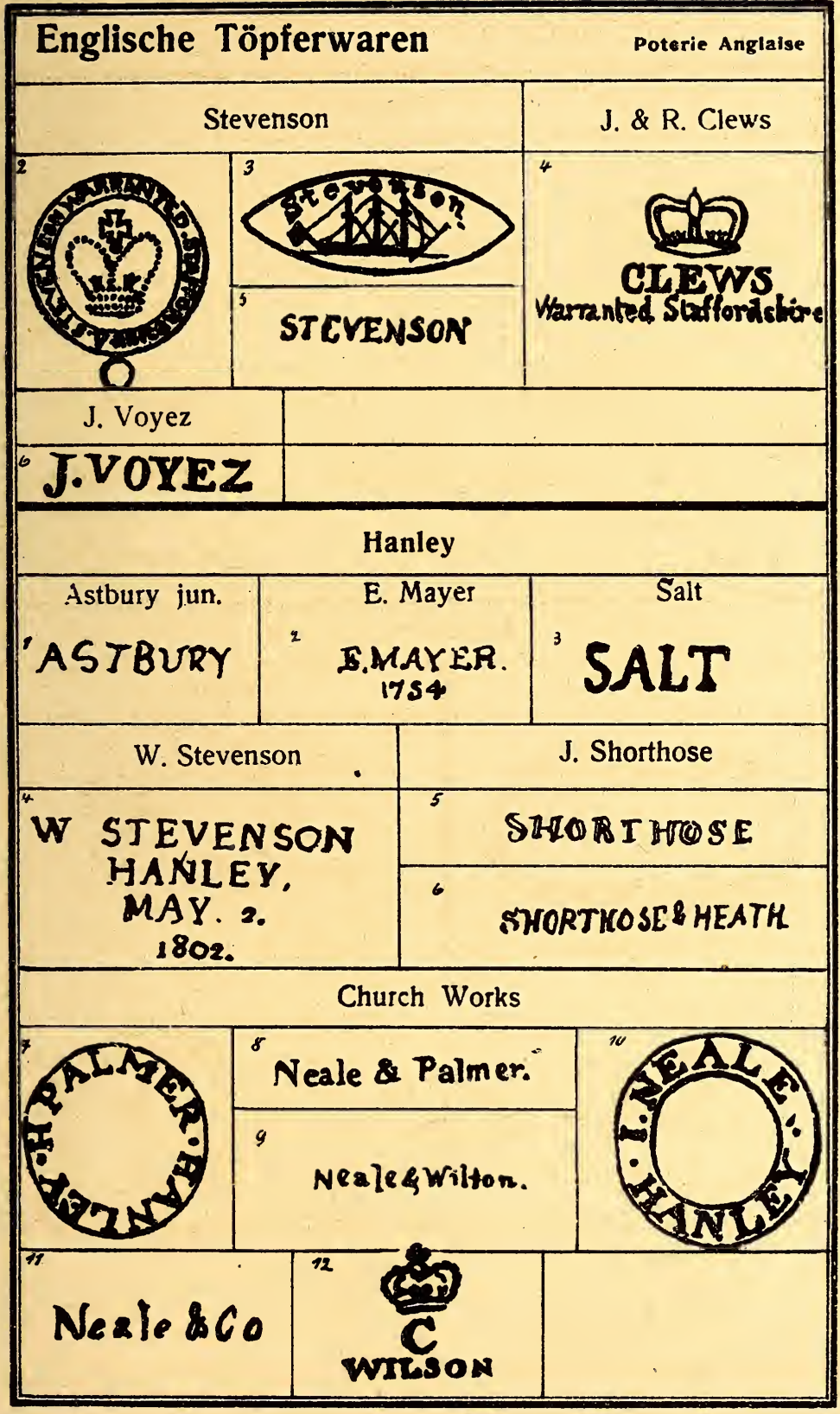




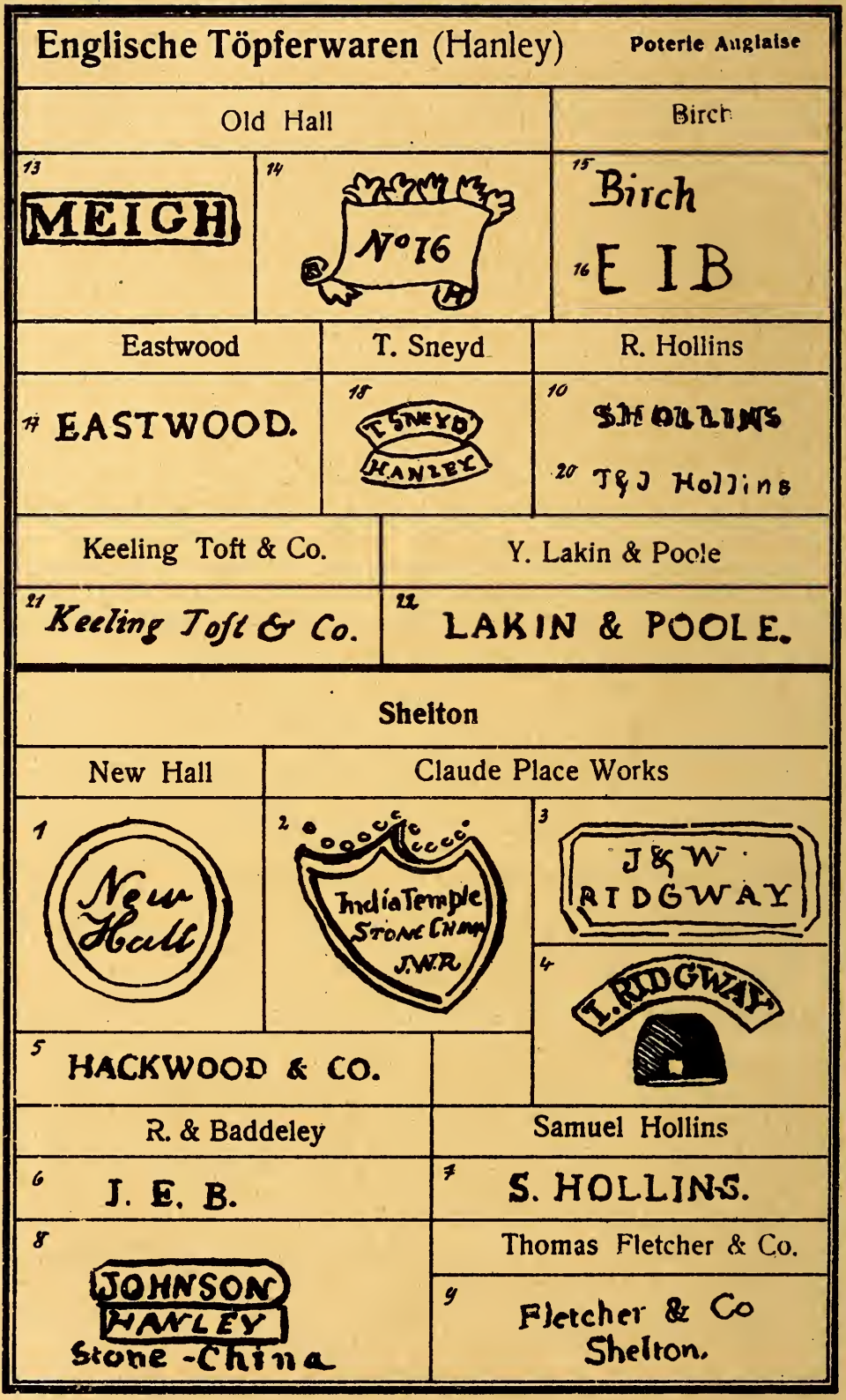




\section{Englische Töpferwaren}

Poterie Anglalse

Stoke upon Trent

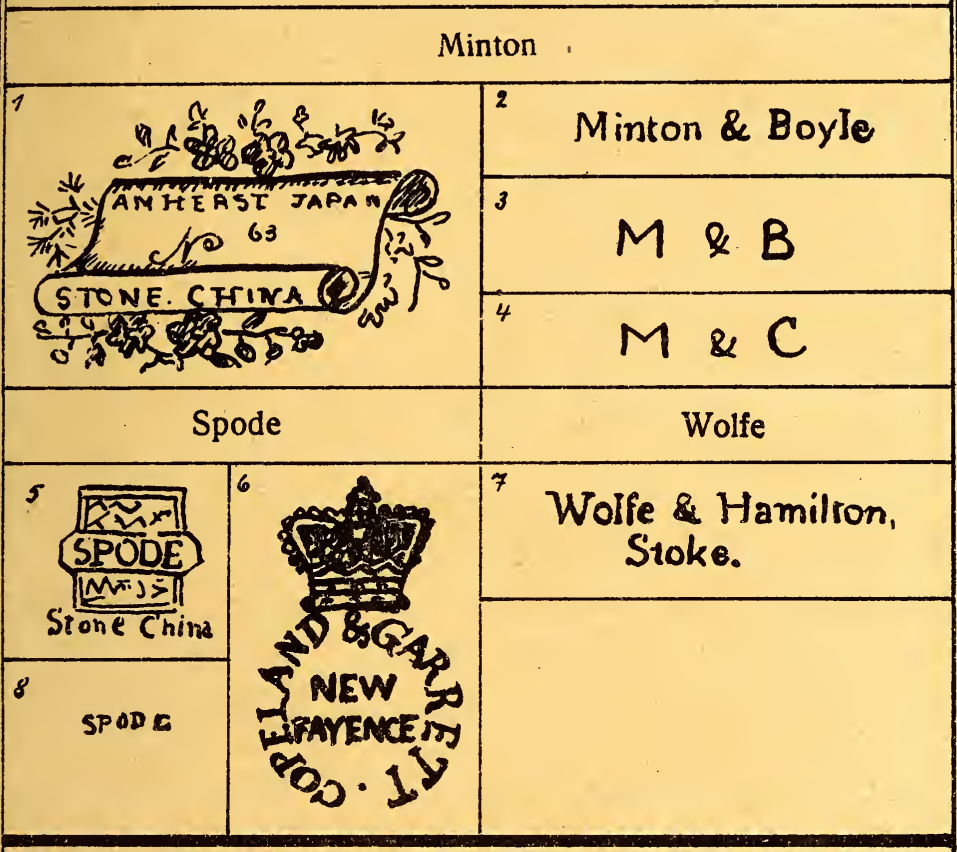

Fenton

\begin{tabular}{|c|c|c|}
\hline F. Pratt & R. M. Taylor & \\
\hline Pratt, & $\begin{array}{l}\text { FENTOW } \\
\text { STONE WORKS }\end{array}$ & S. GREENWOOD. \\
\hline \multicolumn{3}{|c|}{ Lane End (Longtog) } \\
\hline \multicolumn{2}{|c|}{ Mayer \& Newbold } & Bailey \& Batkin \\
\hline $18 N$ & $M a y^{\prime \prime} \& N e w b^{\alpha}$. & Bailey \& Batkin. \\
\hline \multicolumn{3}{|c|}{ J. Harley } \\
\hline FCARLE & $\mathscr{F}$ Har. & Lane end. \\
\hline
\end{tabular}


Englische Töpferwaren (Lane End) Poterie Anglaise

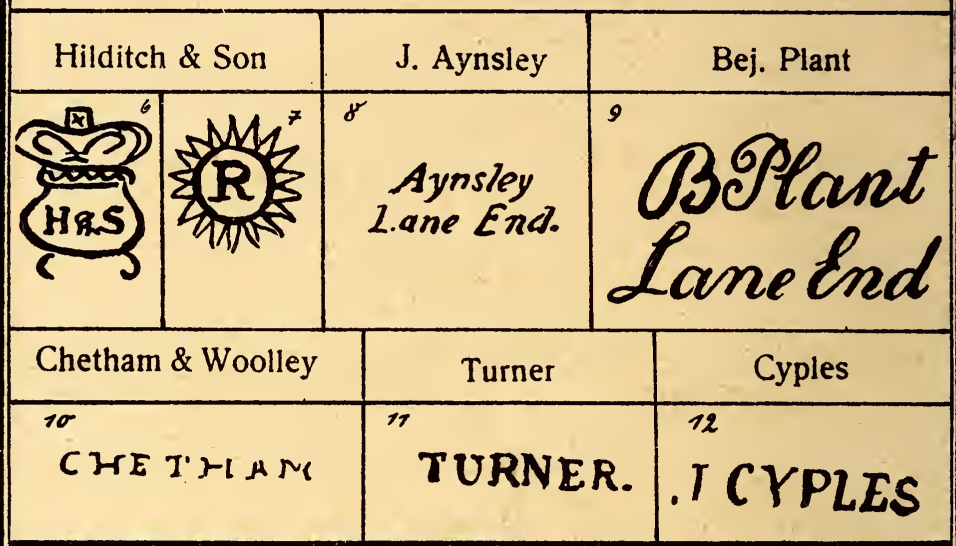

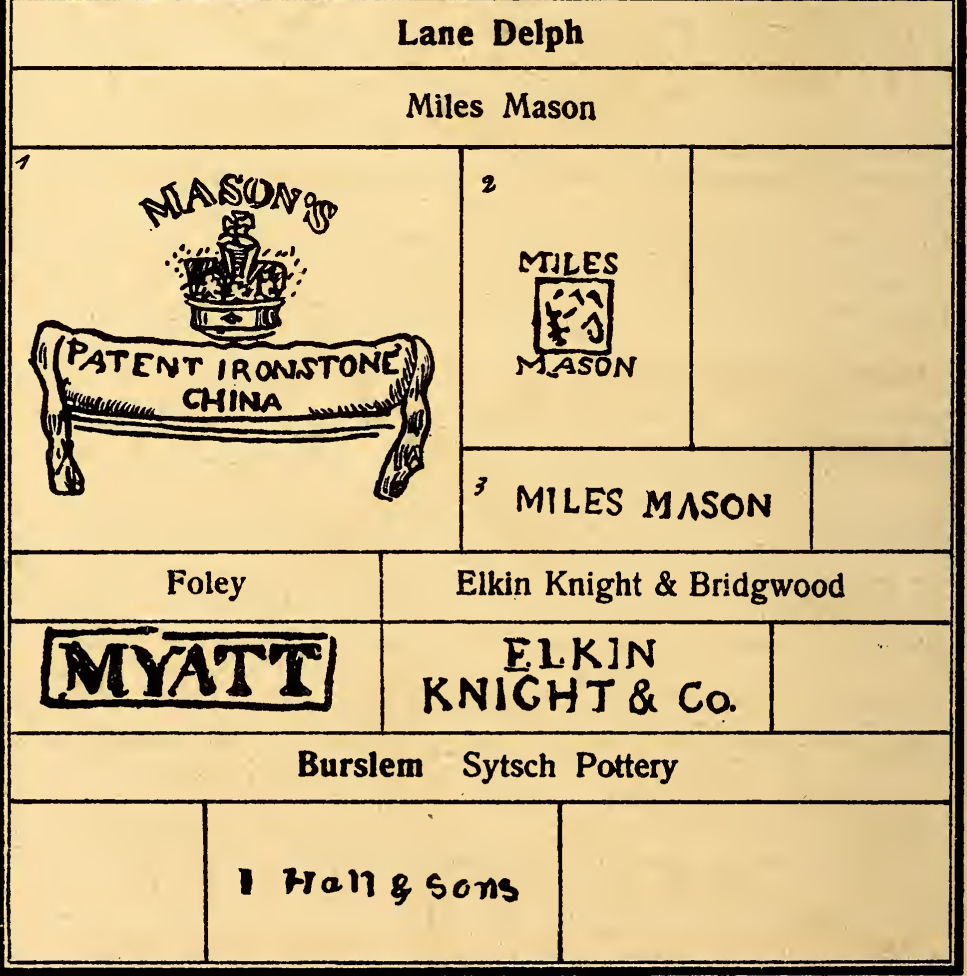




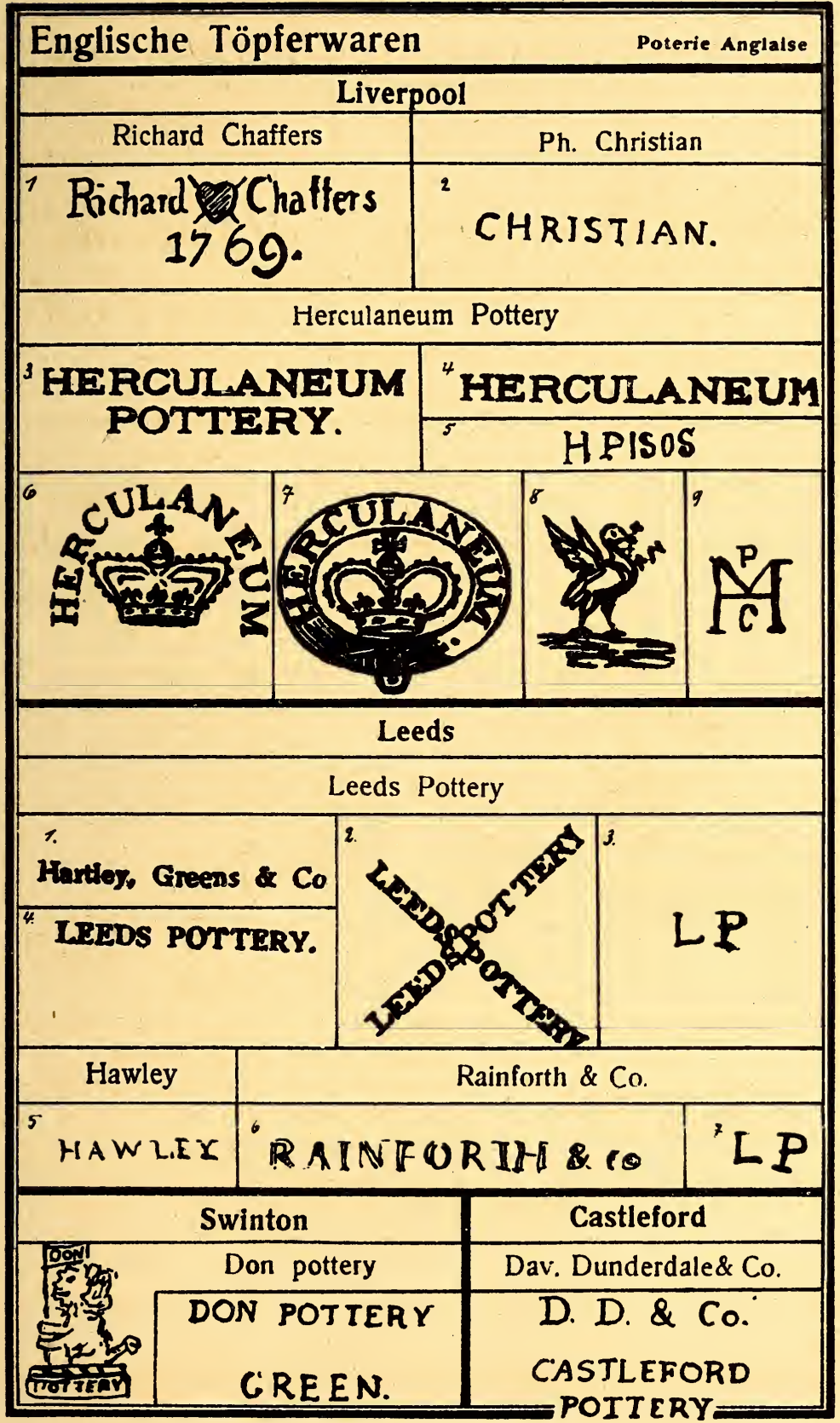




\section{Englische Töpferwaren}

Poterie Anglaise

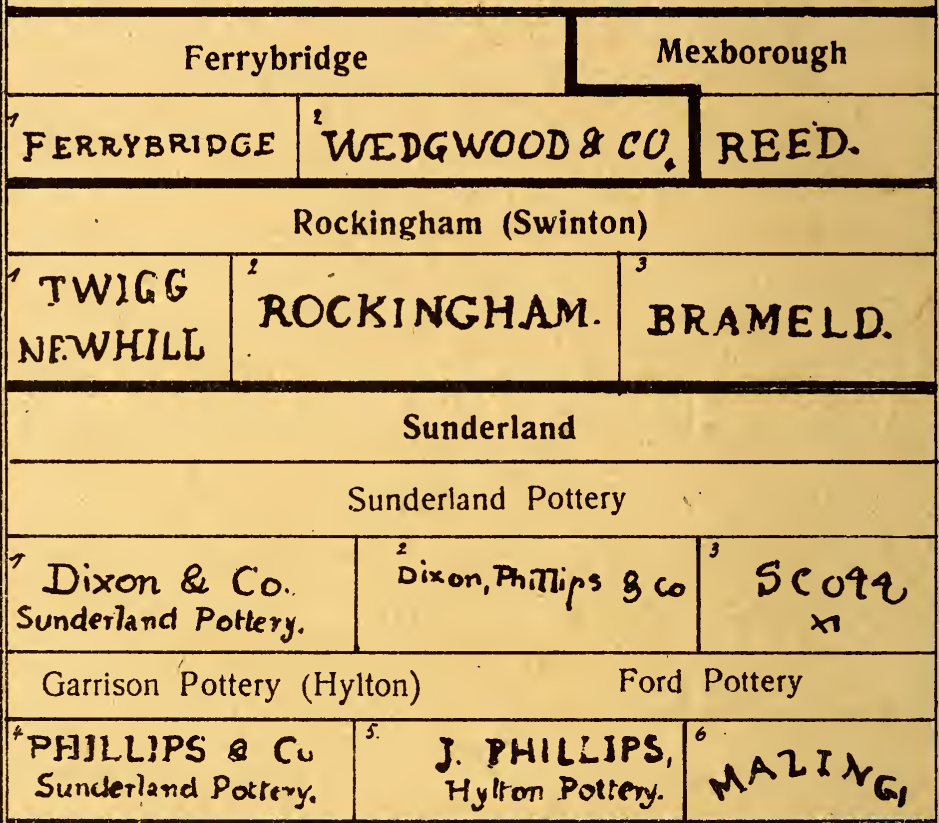

New Castle upon-Tyne

\begin{tabular}{|c|c|c|c|}
\hline T. Fell \& Co & \multicolumn{3}{|c|}{ Patterson \& Co } \\
\hline If $E \mathbb{I}$ & & FATTE KSON \& CO & $J \& P$ \\
\hline
\end{tabular}

Sewell \& Donkin \begin{tabular}{|c|c|c|}
\hline SEW ELI & A SEWELL \& DONKIN. \\
3 & S
\end{tabular} Southwick Wear Pottery

MOORE \& CO. SOUTHWICK. 
Englische Töpférwaren

Stockton on Tees (W. Smith \& Co.)

\begin{tabular}{|l|l|l|}
\hline STOCKTON & W. S. \& CO. & " \\
POTTERY. & QUEENS WARE. & WEDGEWOOD. \\
STOCKTON. & &
\end{tabular}

Bristol

Delftware

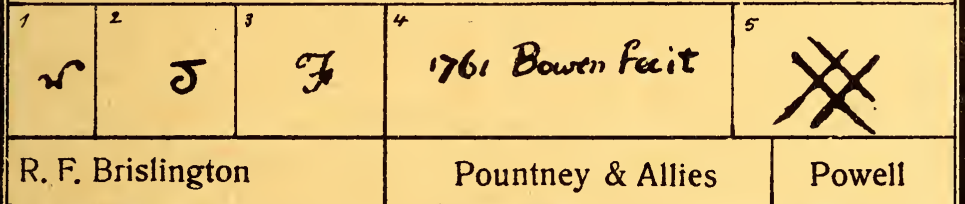

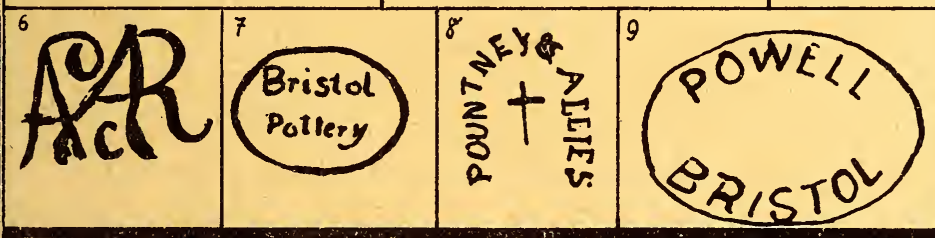

Lambeth

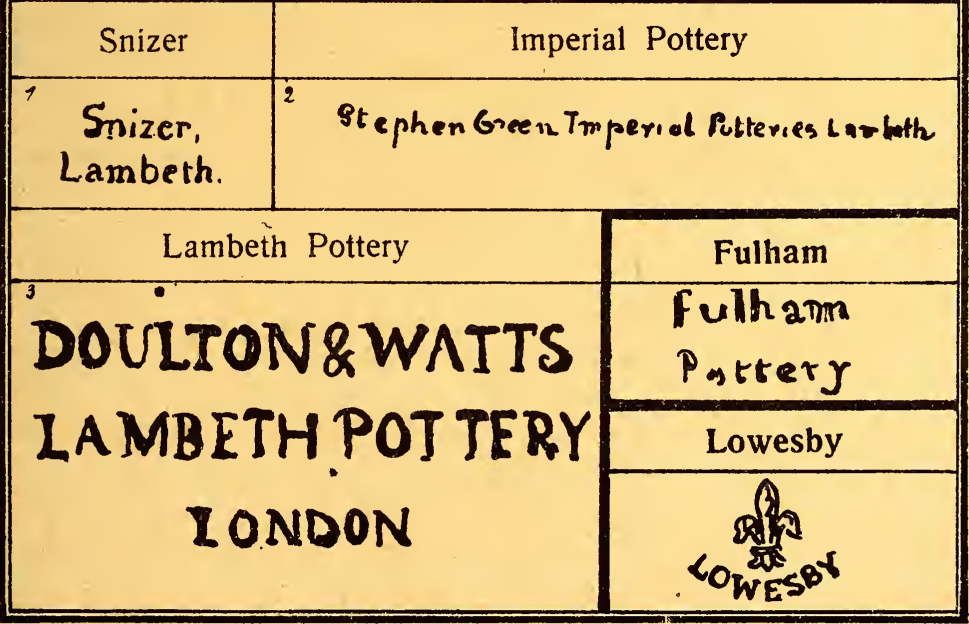


Englische Töpferwaren

Poterie Anglaise

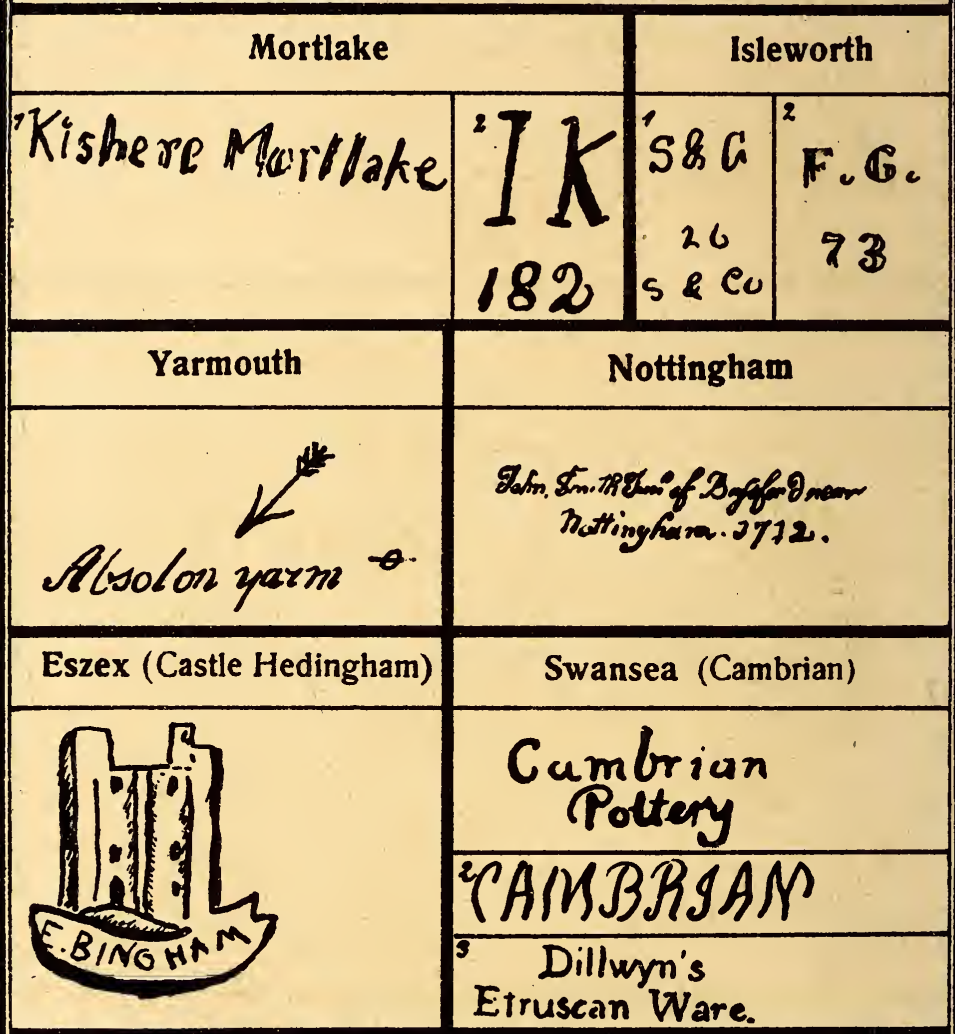

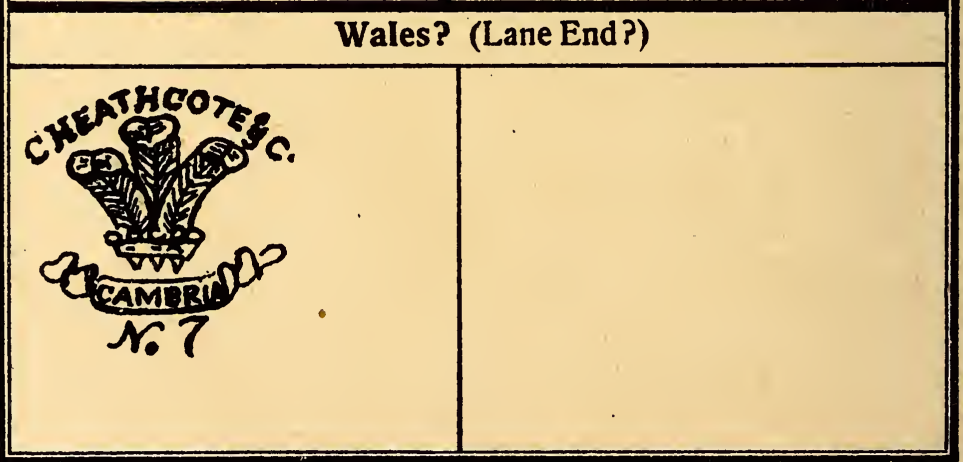




\section{Schottische Töpferwaren}

Poterie Ecossalse

\begin{tabular}{||l|l|l||}
\hline \multicolumn{3}{|c|}{ Porto bello } \\
\hline SCOTI & ${ }^{2}$ ScorT & \\
\hline Irische Töpferwaren & Poterie irlandaise \\
\hline & & \\
\hline
\end{tabular}




\section{Dänische Fayencen}

Faience Danoise

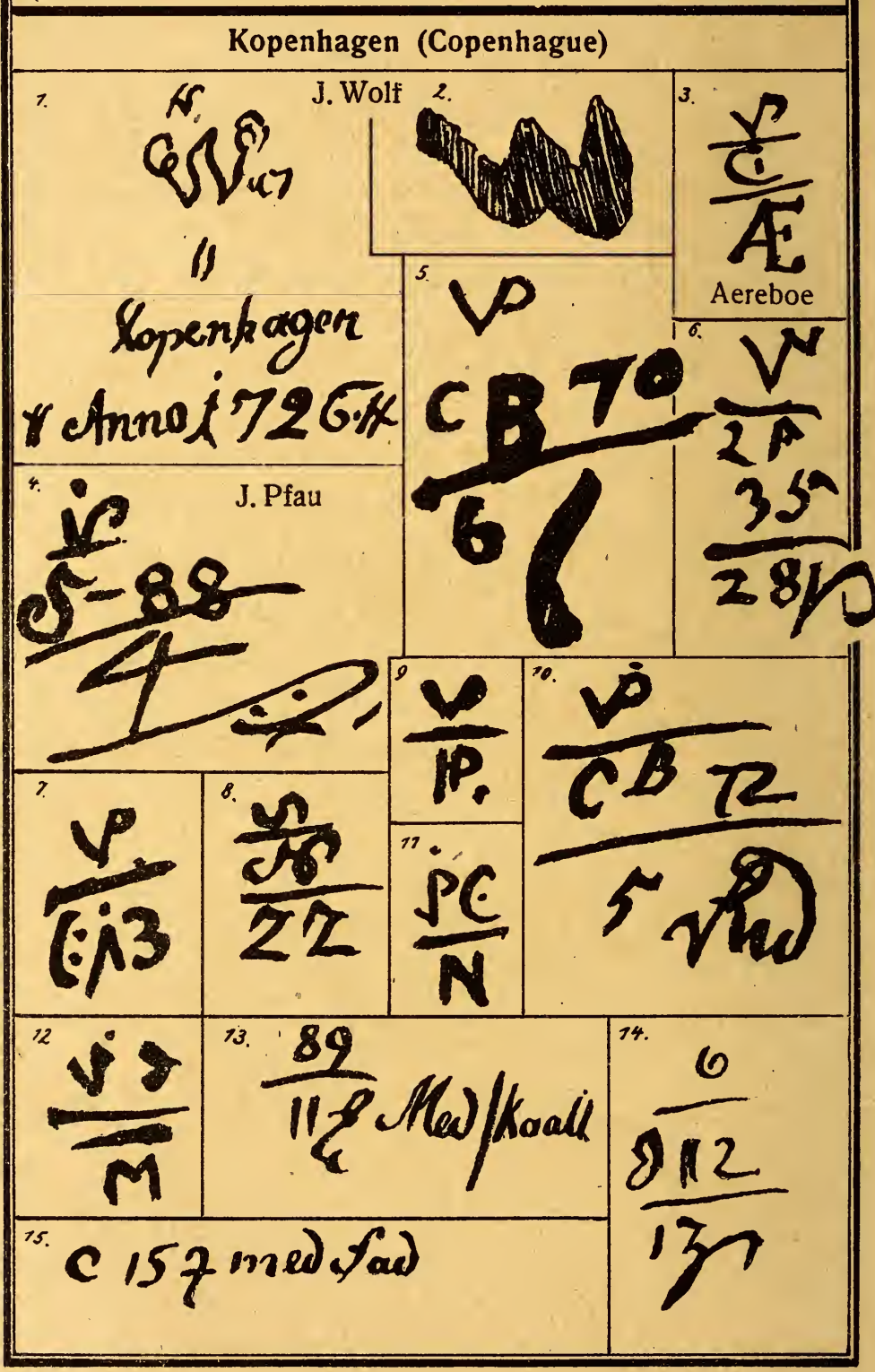




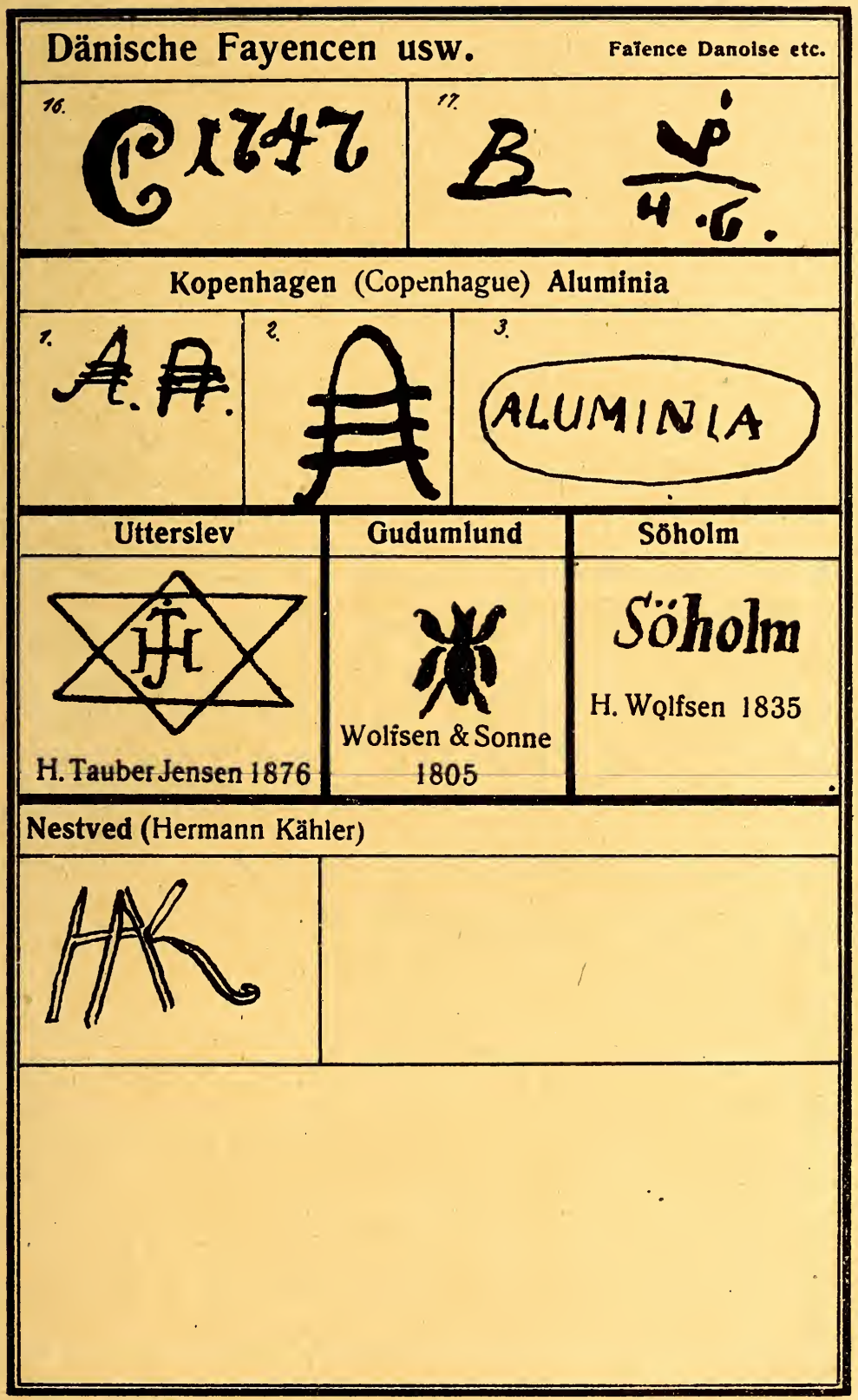




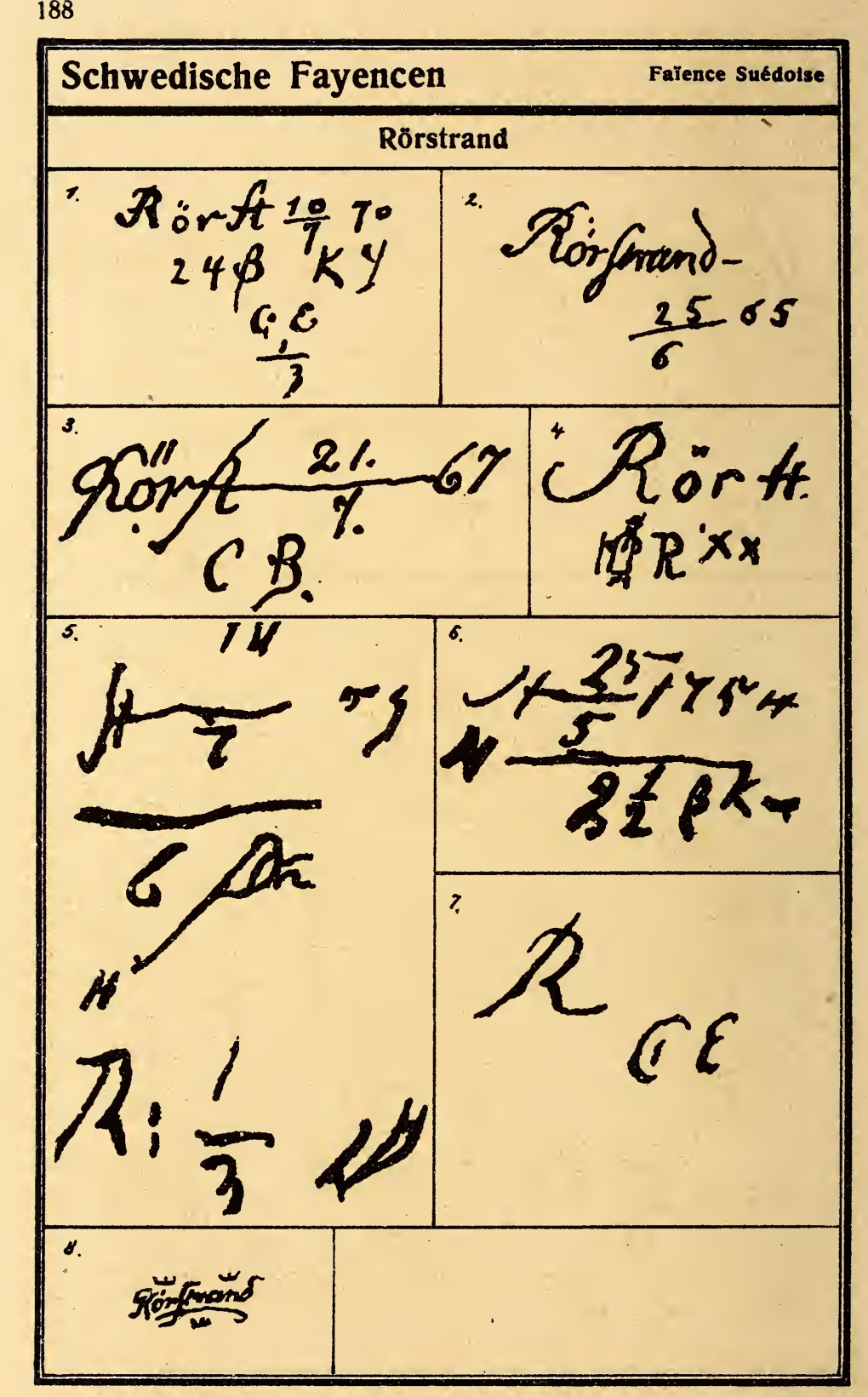




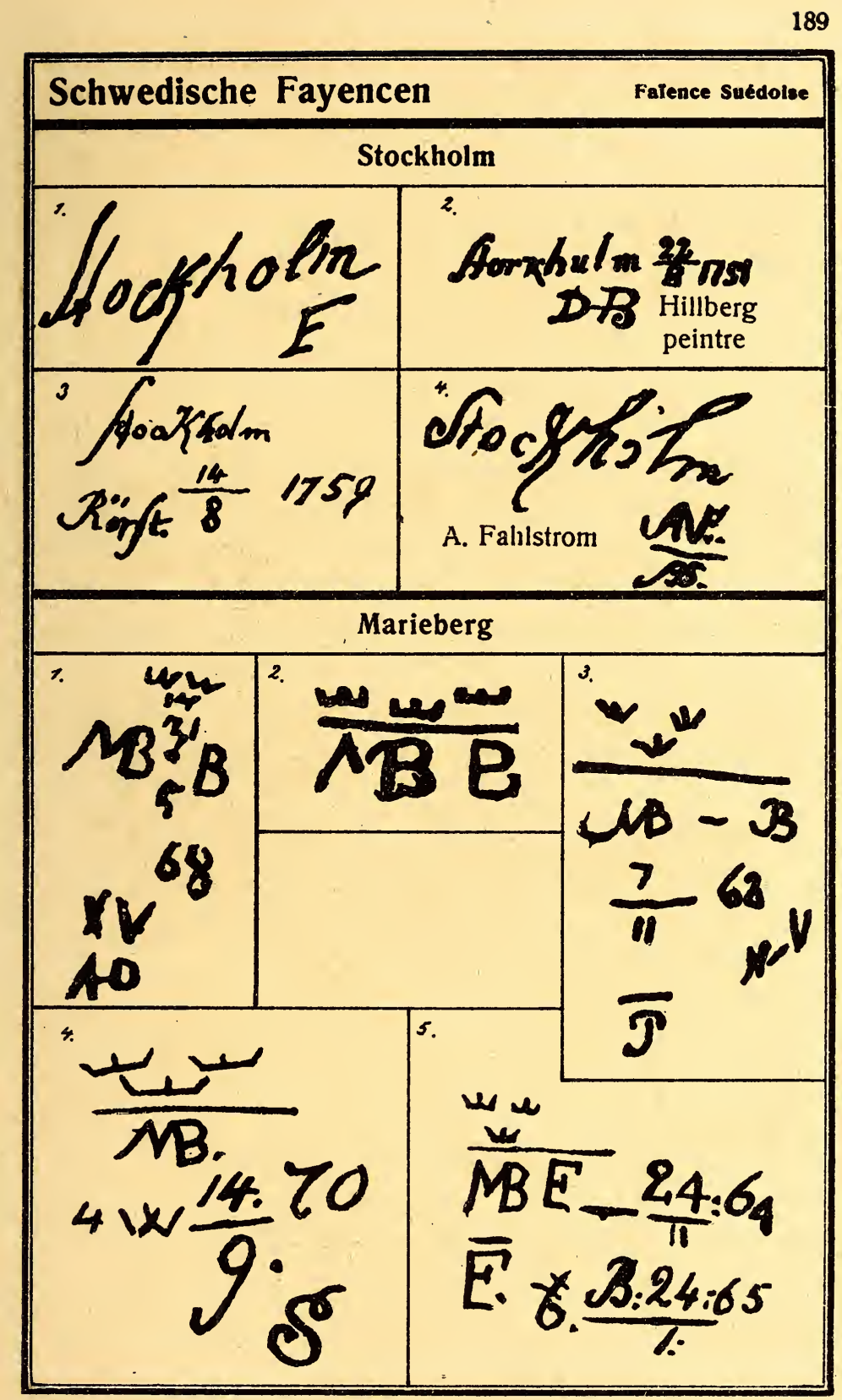




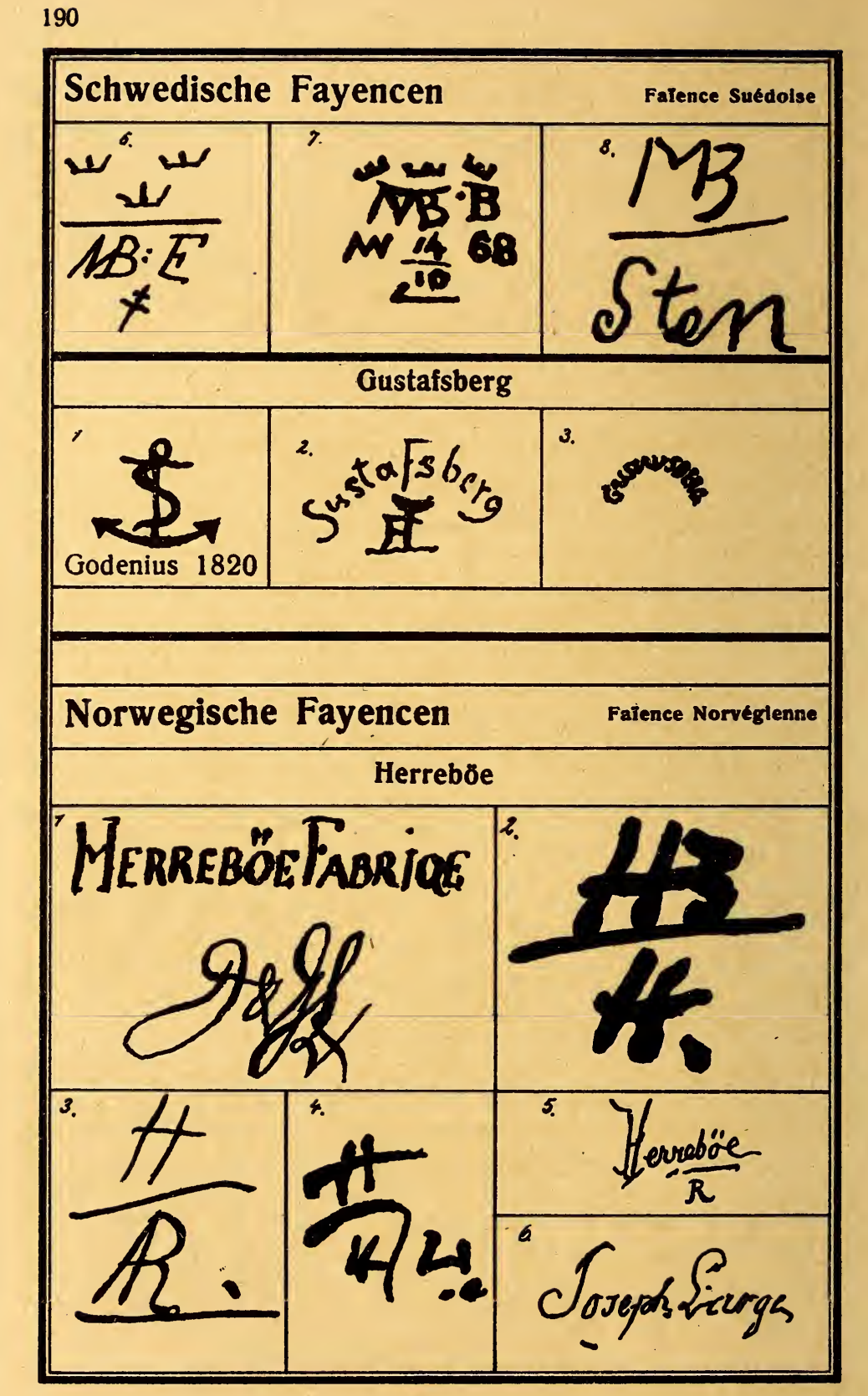




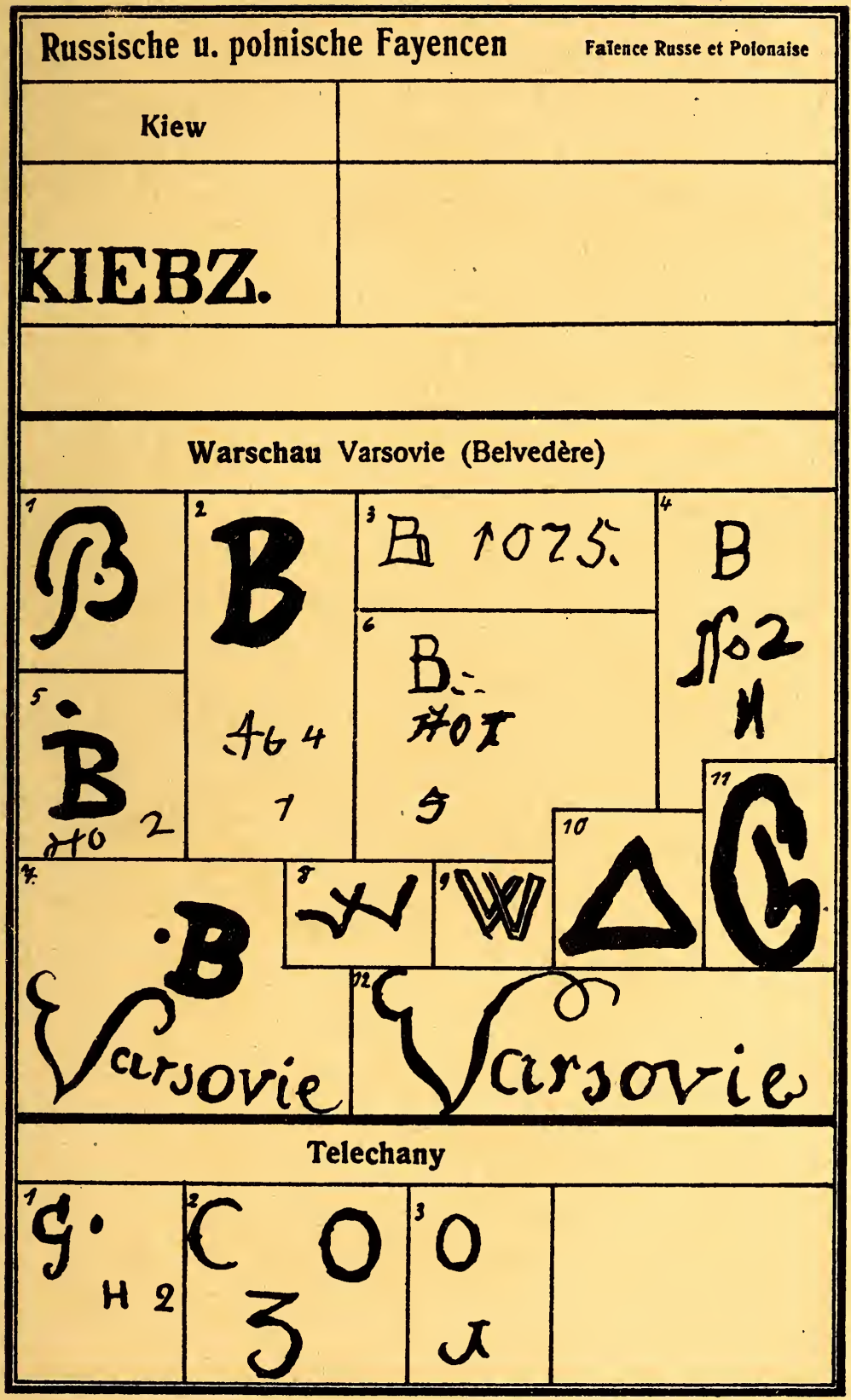




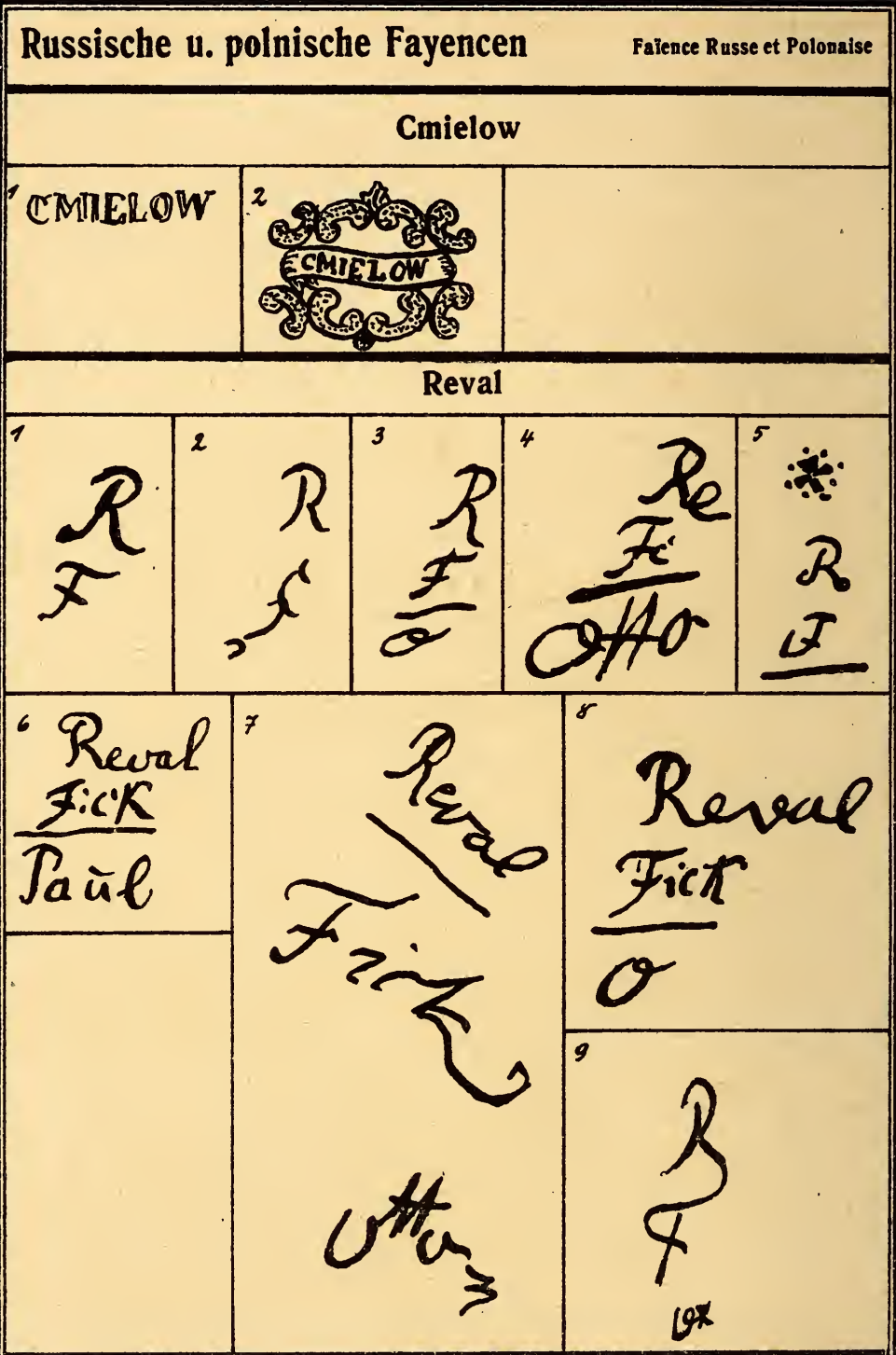




\section{II. \\ PORZELLAN \\ (PORCELAINES)}





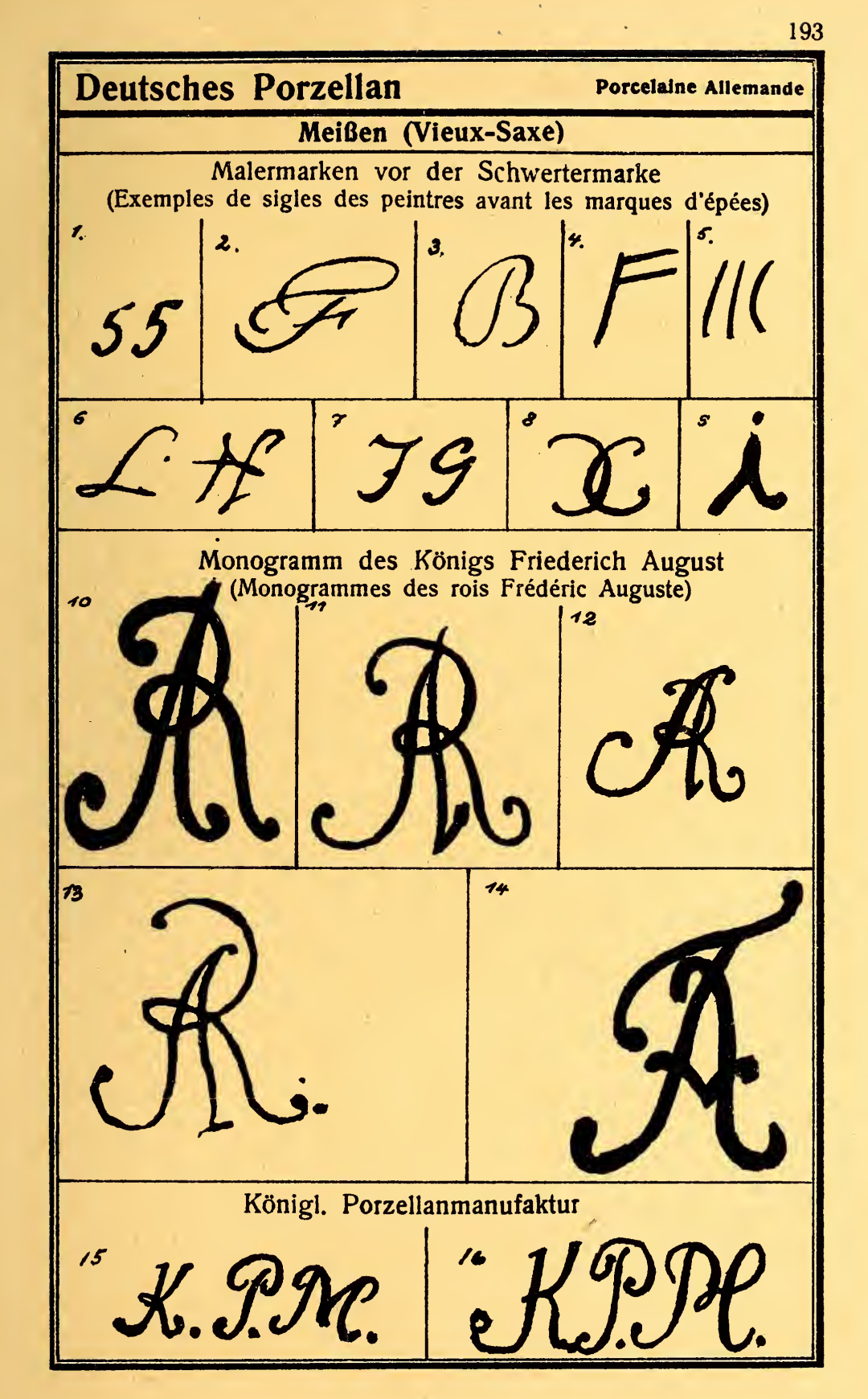




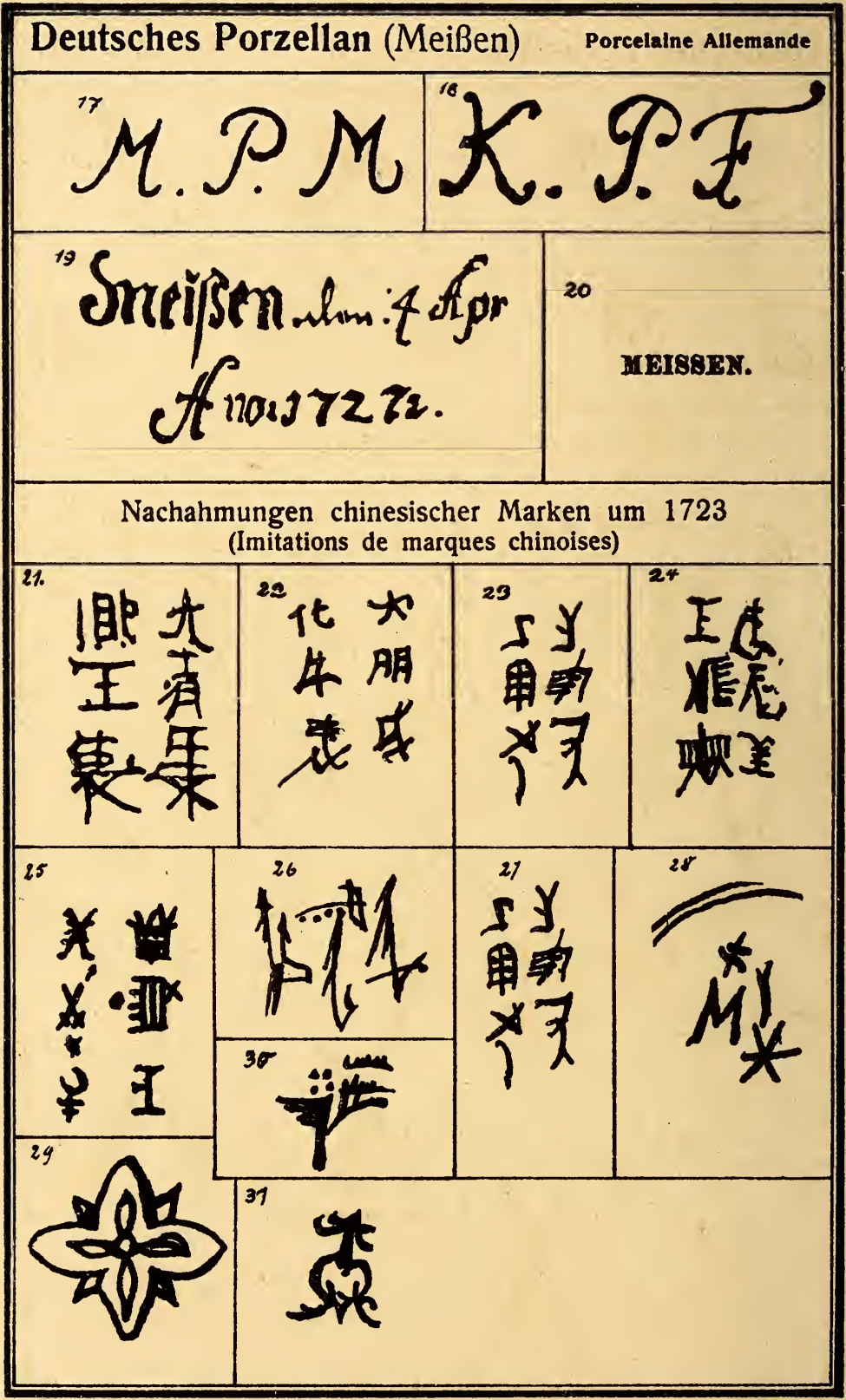




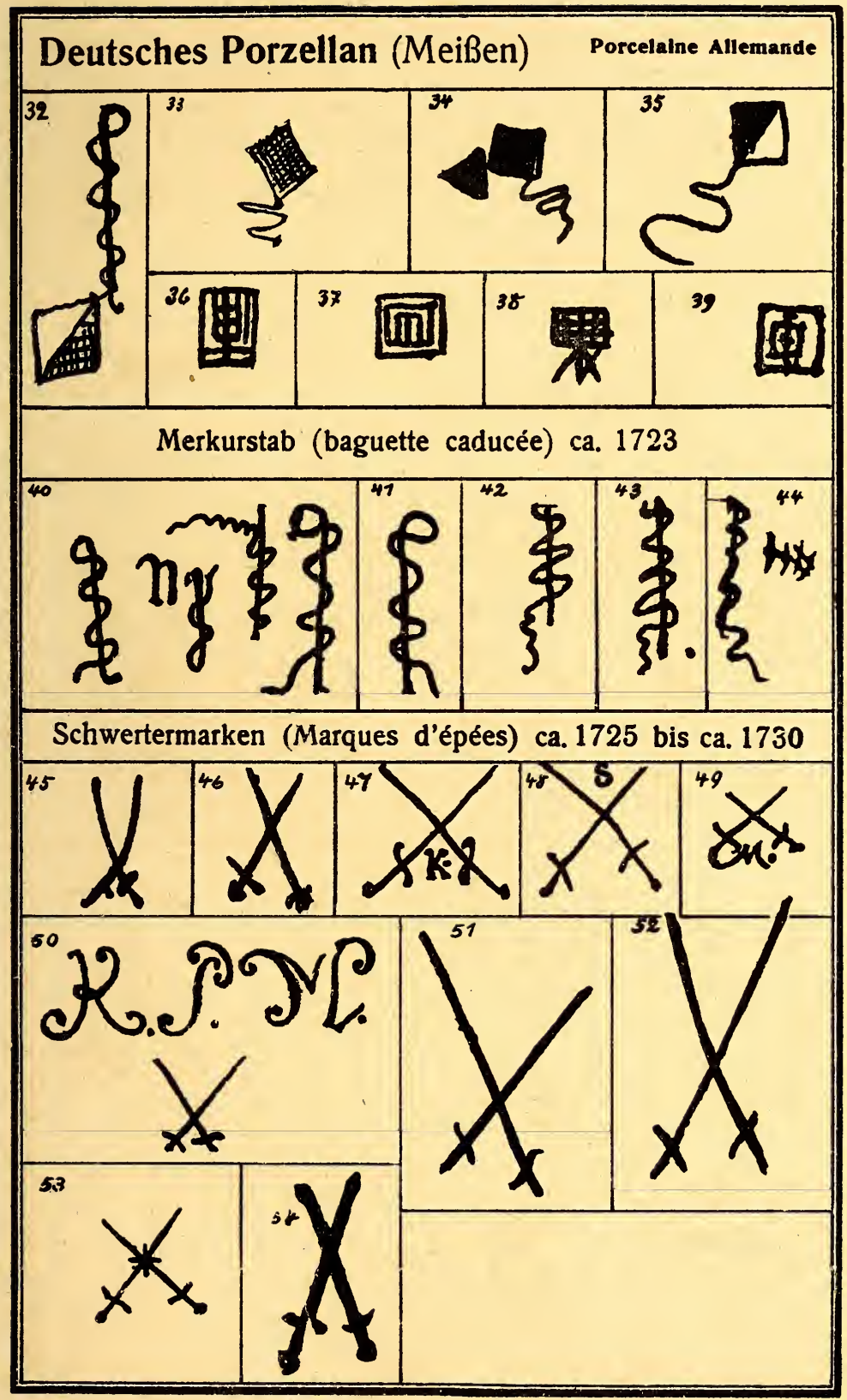




\section{Deutsches Porzellan (Meißen) Porcelaine Allemande}

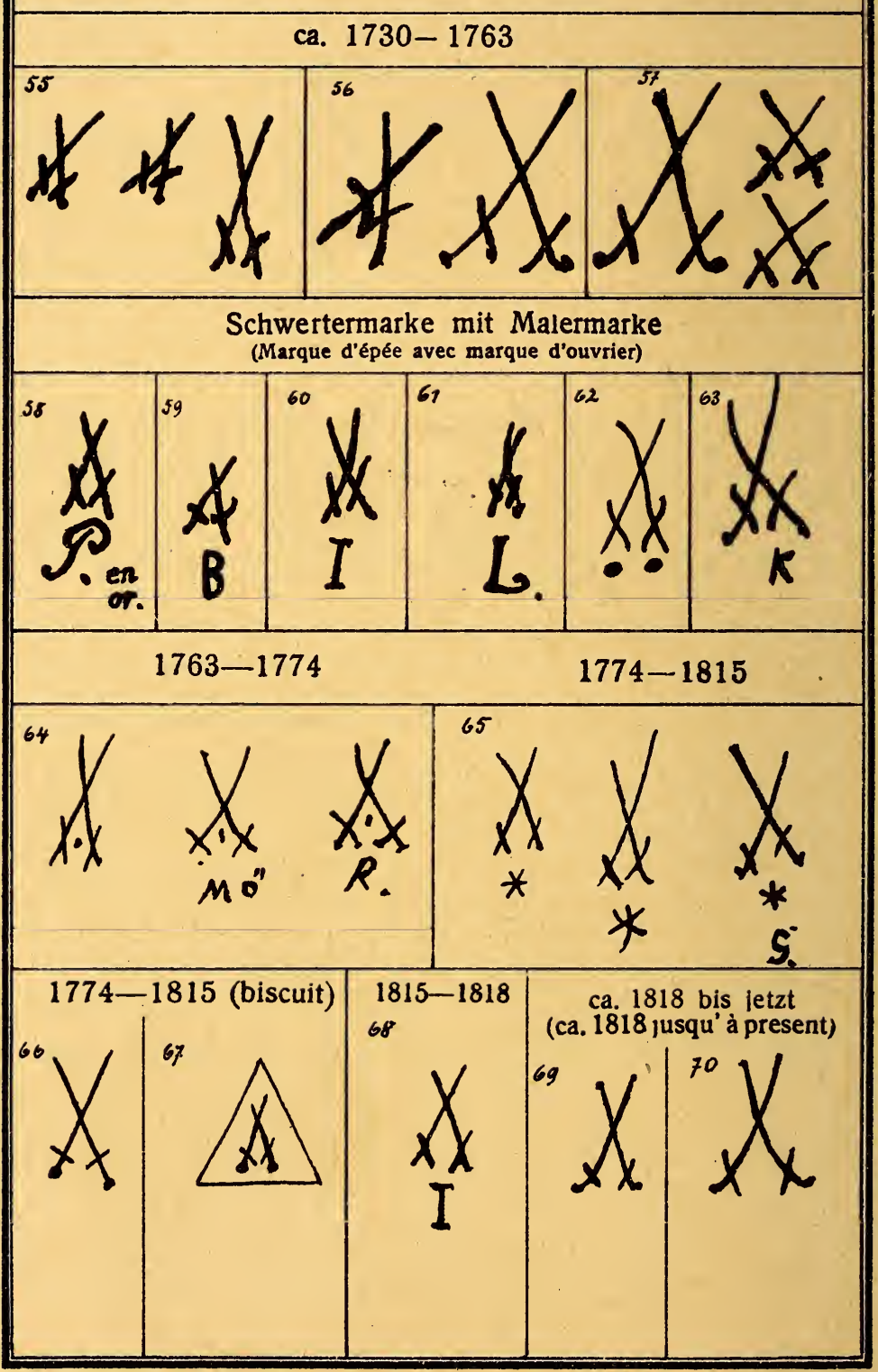




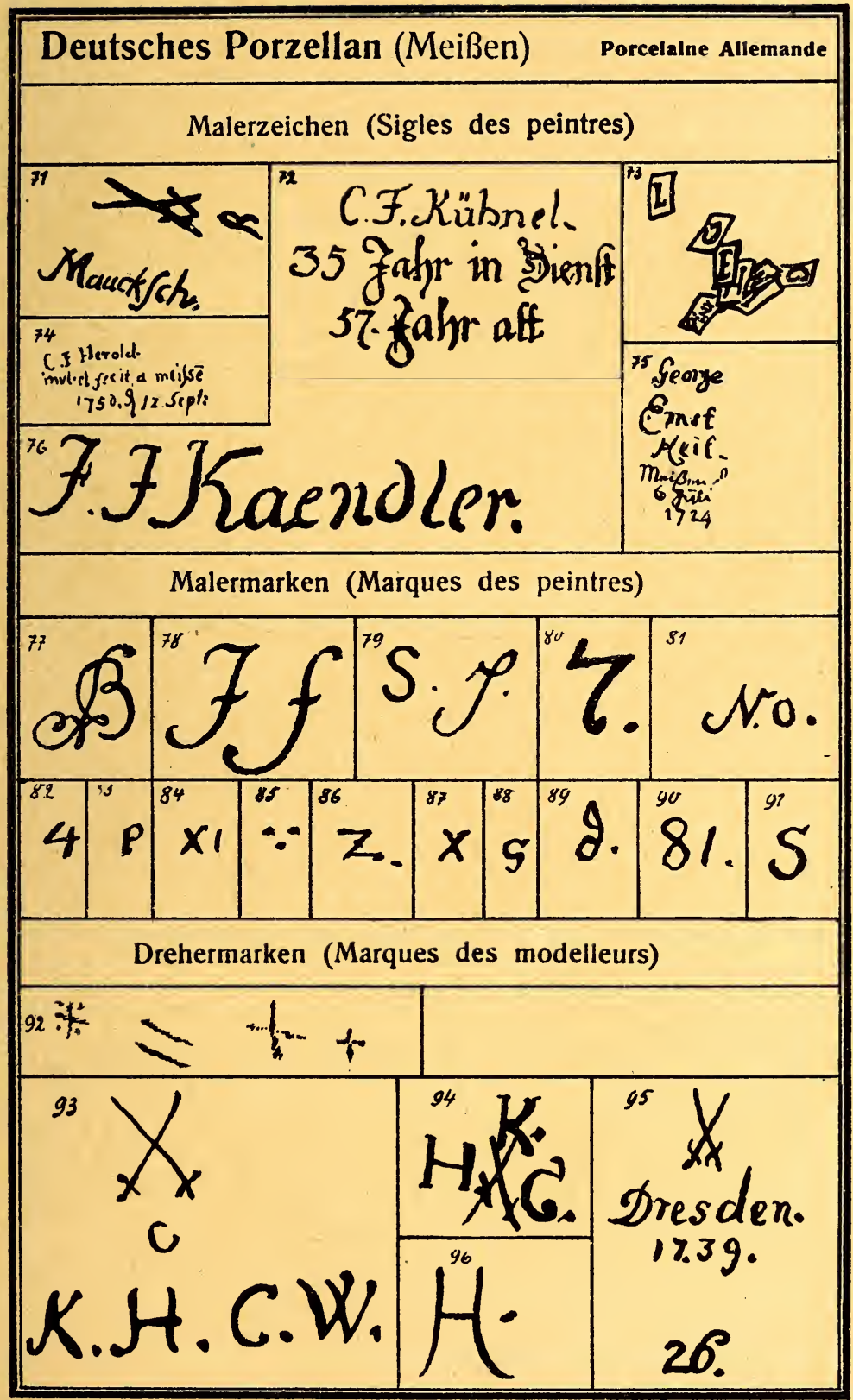




\section{Deutsches Porzellan}

Porcelaine Allemande

Bodenverzierungen (Ornaments au fond)

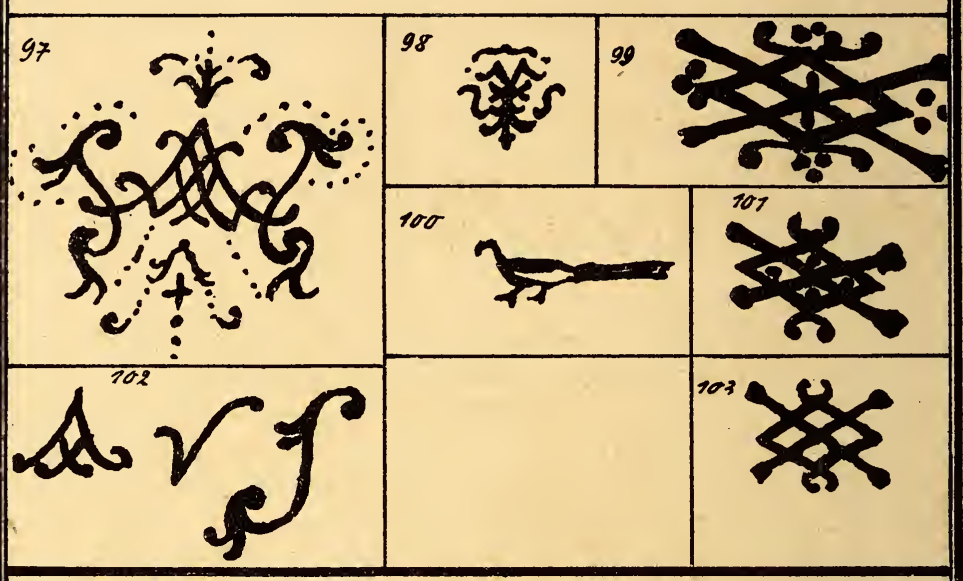




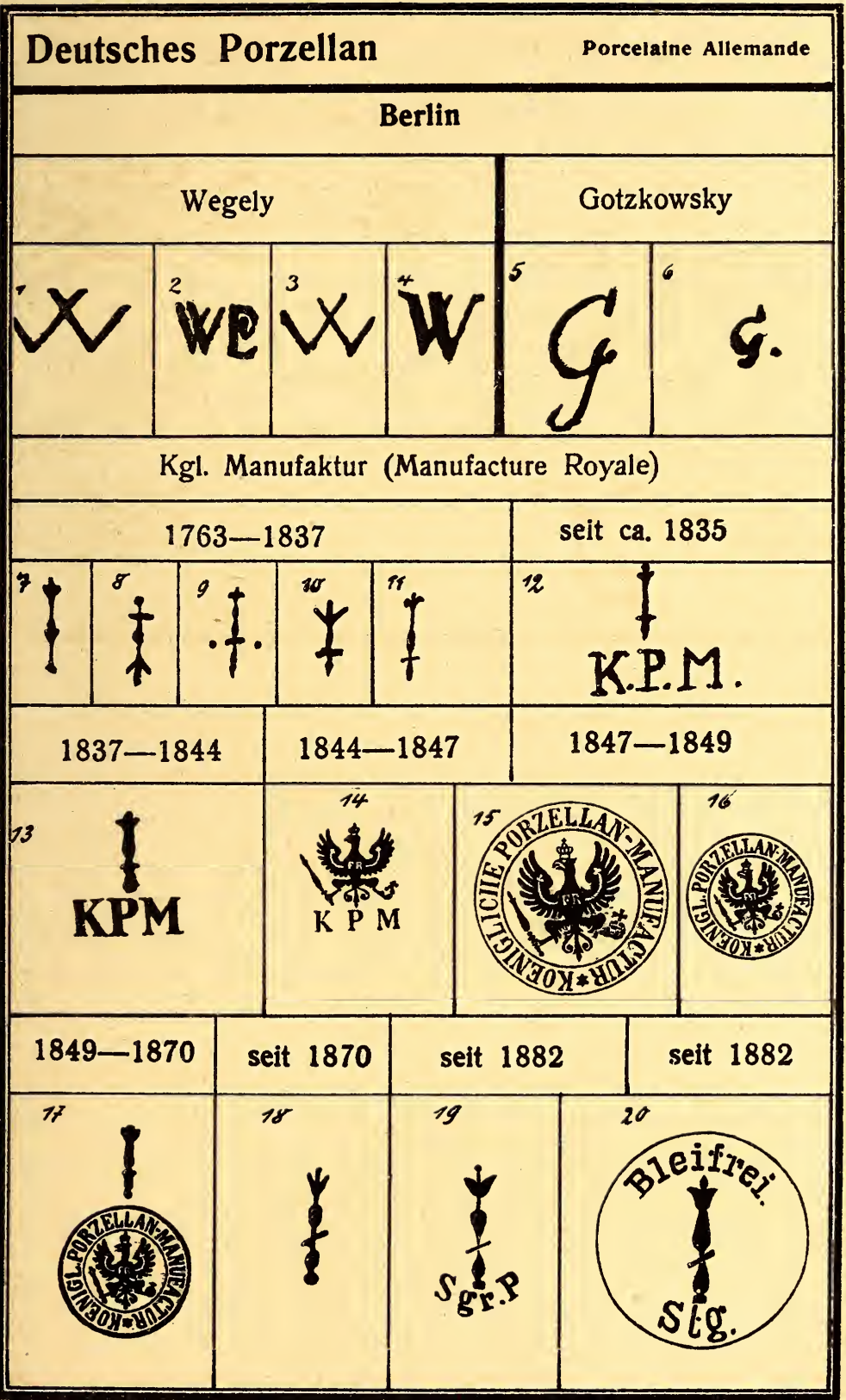




\section{Deutsches Porzellan (Berlin) Porcelaine Allemande}

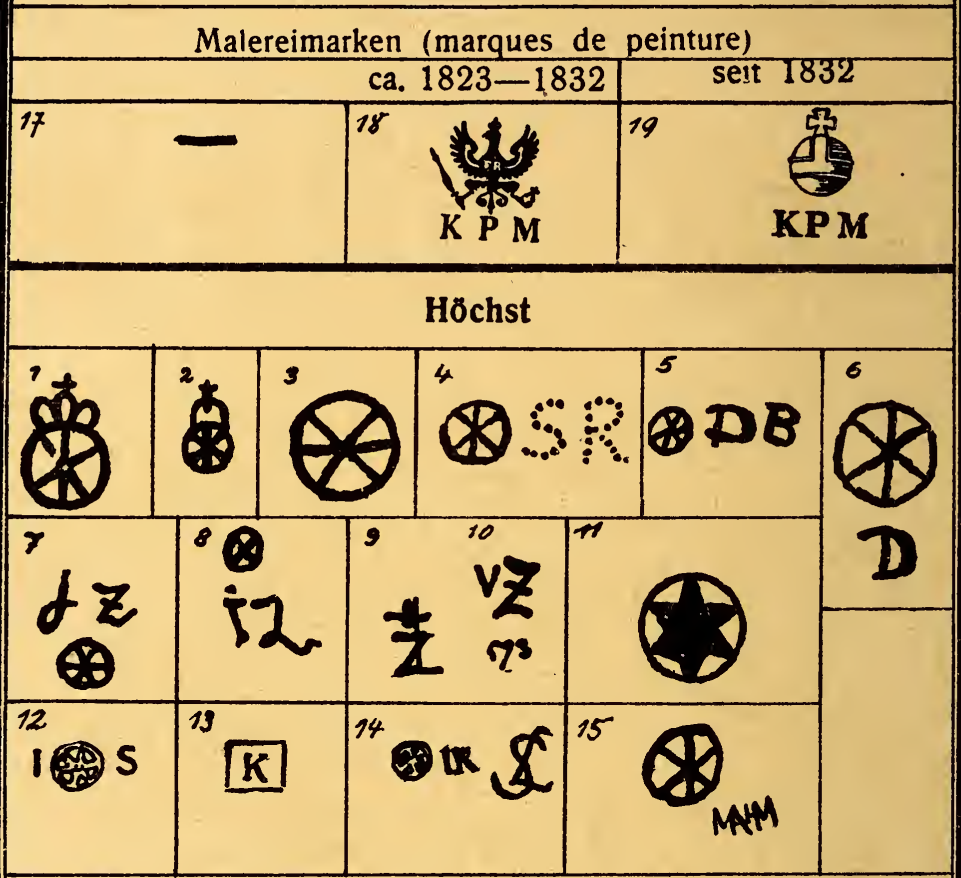

Fulda

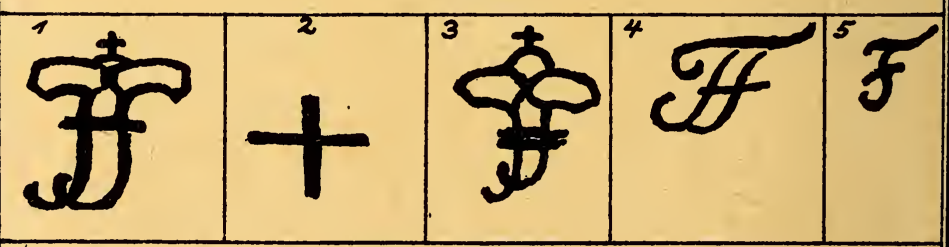




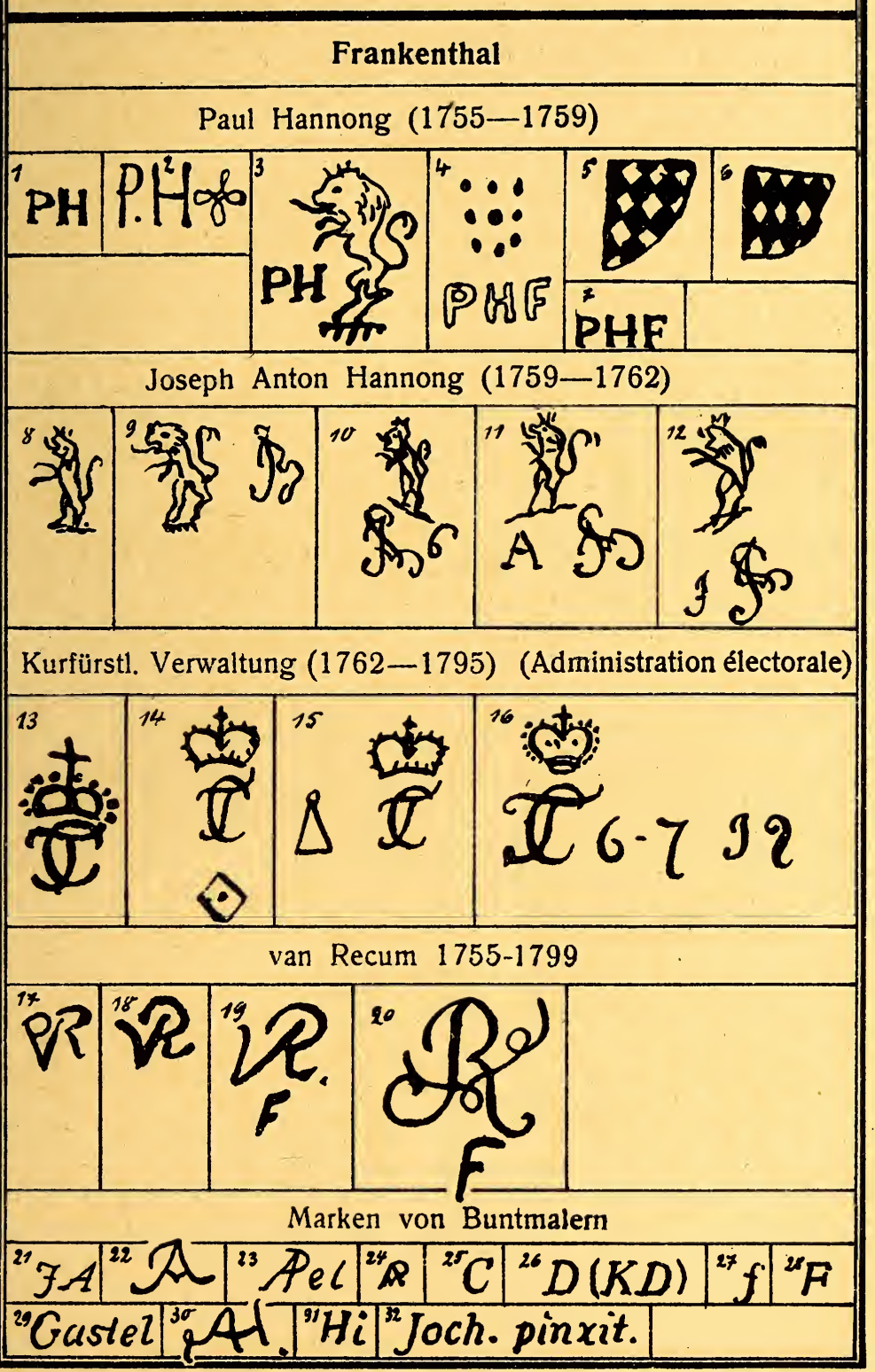


Deutsches Porzellan (Frankenthal) Porceralne Allemande $\left.K_{33}^{i}\right]^{3} H: K: P$ in: $\left.\right|^{35}$ Kilian pin. Michael Kloeckle Pinx. $\left.1783\right|^{37}$ G:FM:| HM $^{3 t^{2}}$ MM:: $:\left.^{38} \mathrm{LOCKEL}{ }^{39} \mathrm{Ma}\right|^{40}$ B.M.P. Pasnus pi

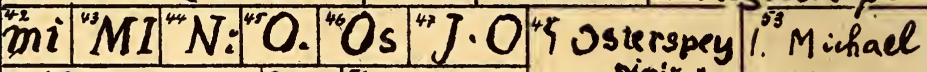

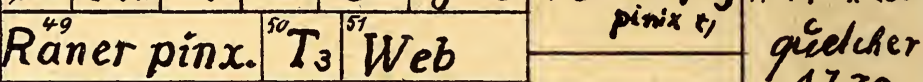
Wi ${ }^{\text {sz }}$ Winterstein pinx, 1704

Bossierer-u. Drehermarken (Marques des modelleurs et tourneurs) Clair ${ }^{55}: C{ }^{56}{ }^{57} 7$ barm Clmor

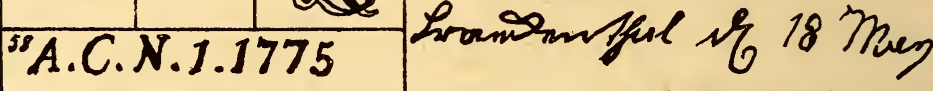

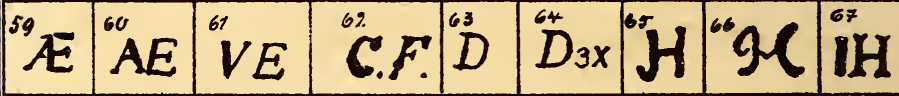
If $\left.4\right|^{69}$ Jacob Legrand 1786 [D

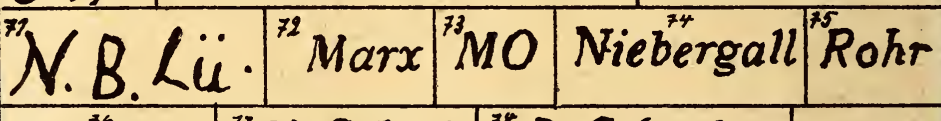
Schahter ${ }^{77} H$ Sch. $\left.2\right|^{77}$ J. Schoch ${ }^{79}$ J.S Saxnong $: 3$ ir6i

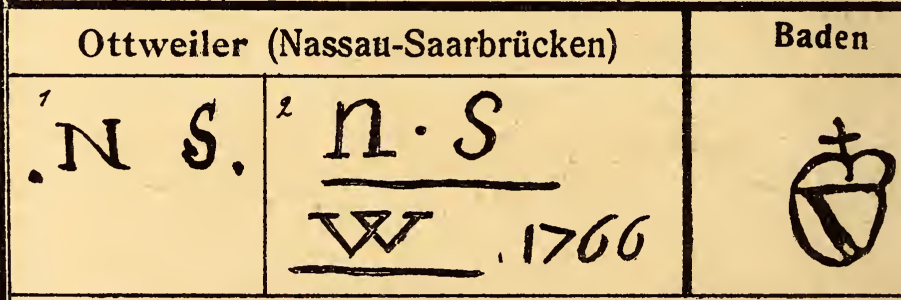


Deutsches Porzellan

Porcelaine Allemande

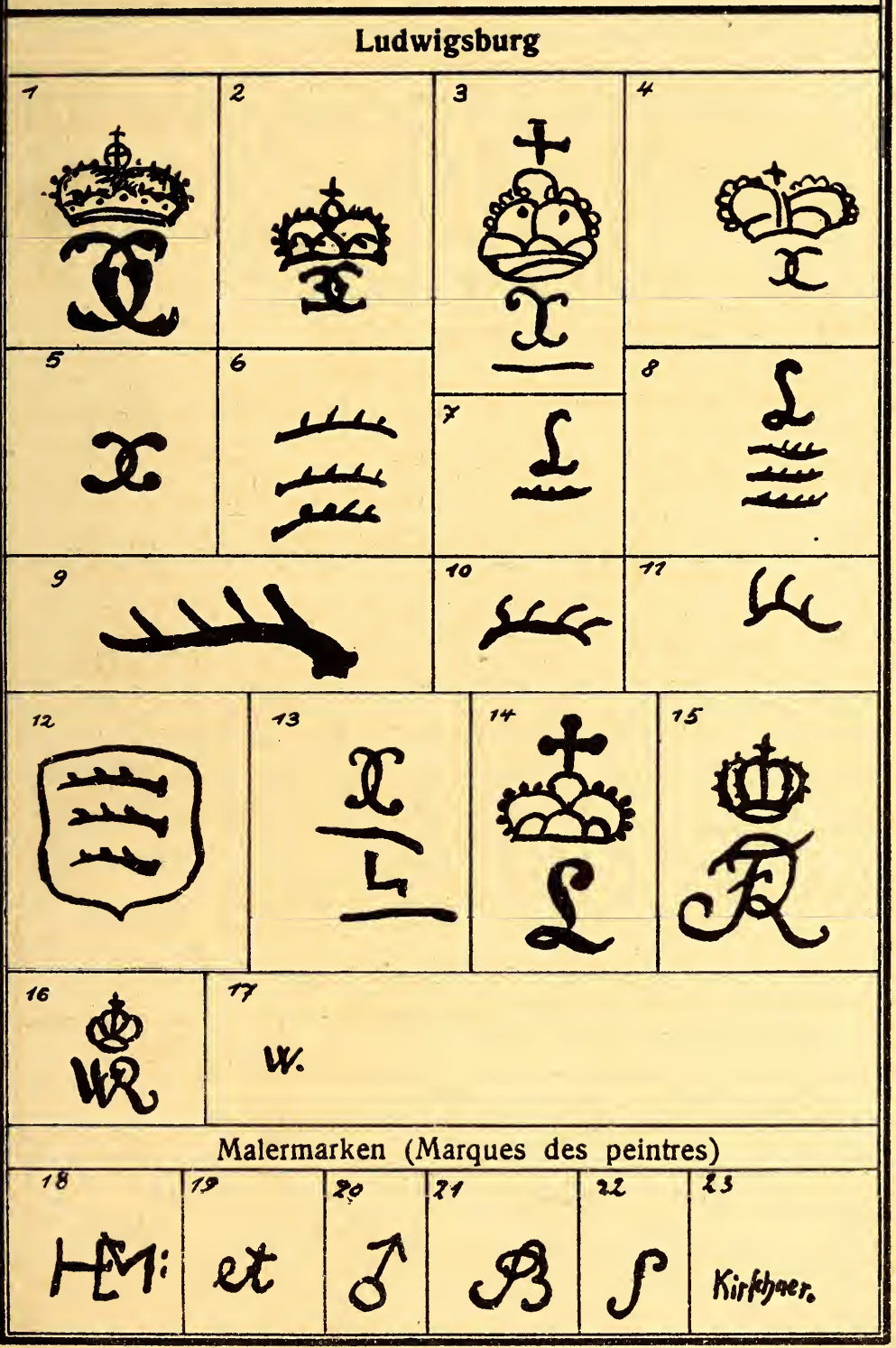




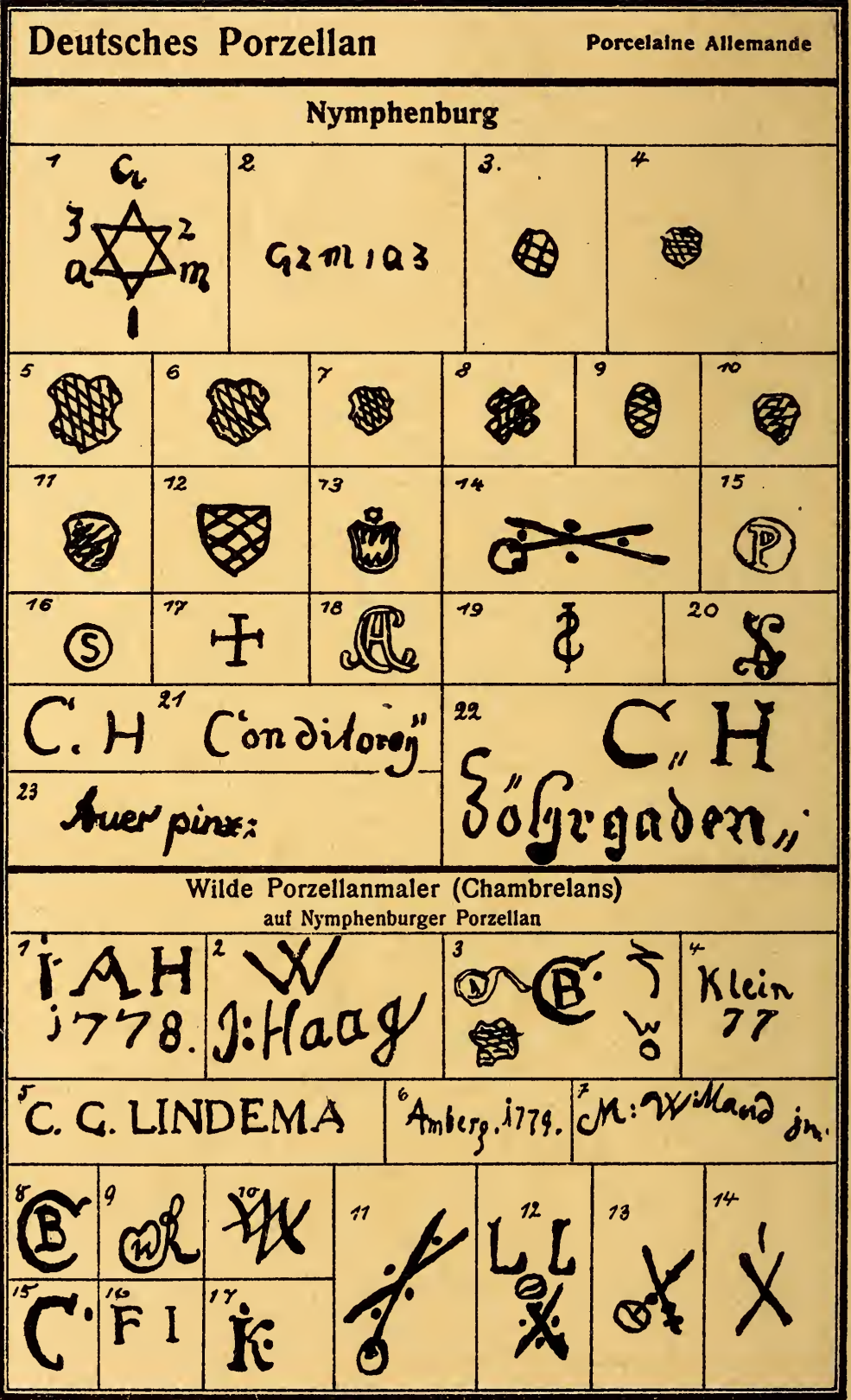




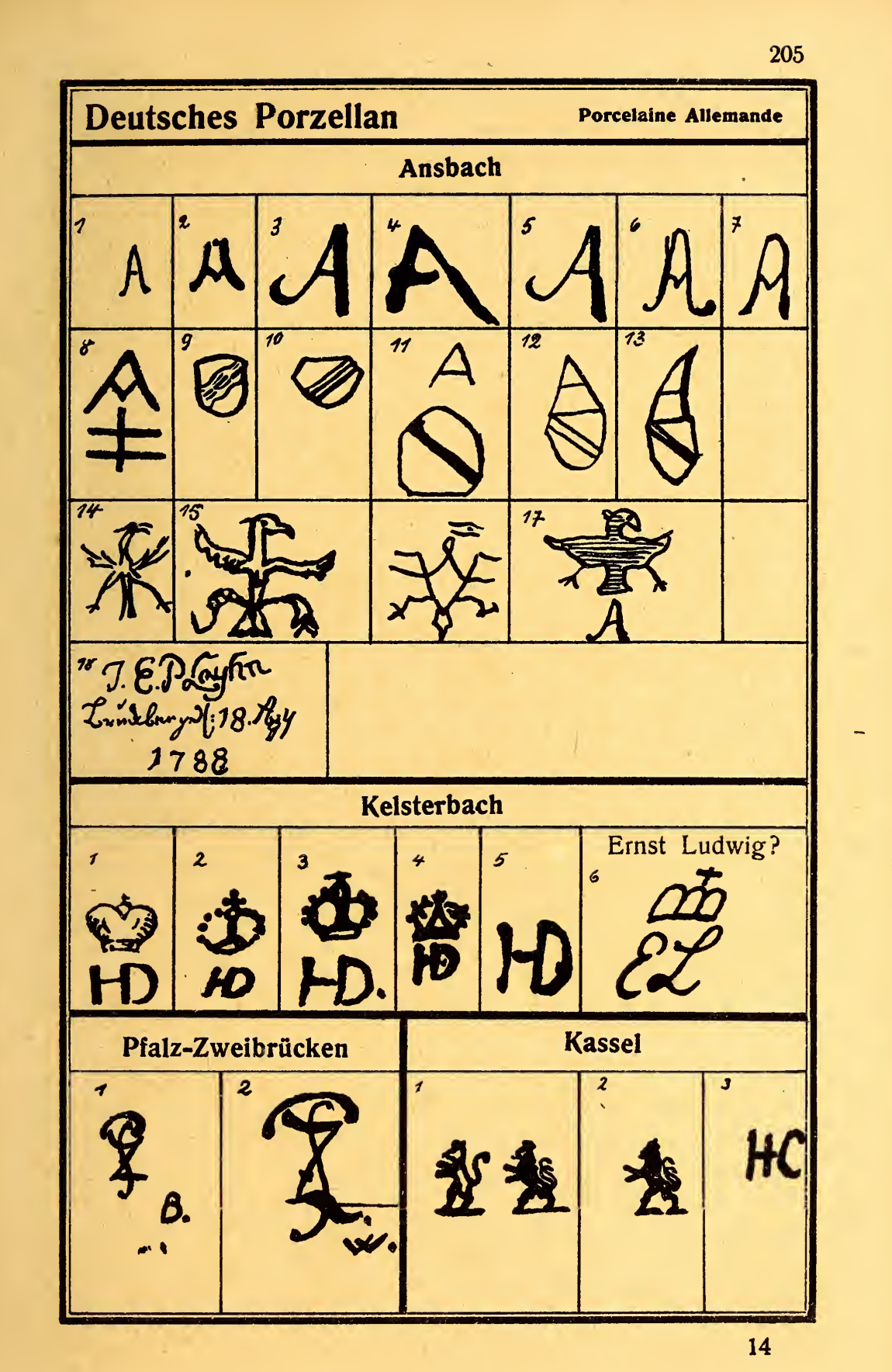




\section{Deutsches Porzellan Porcelalne Allemande}

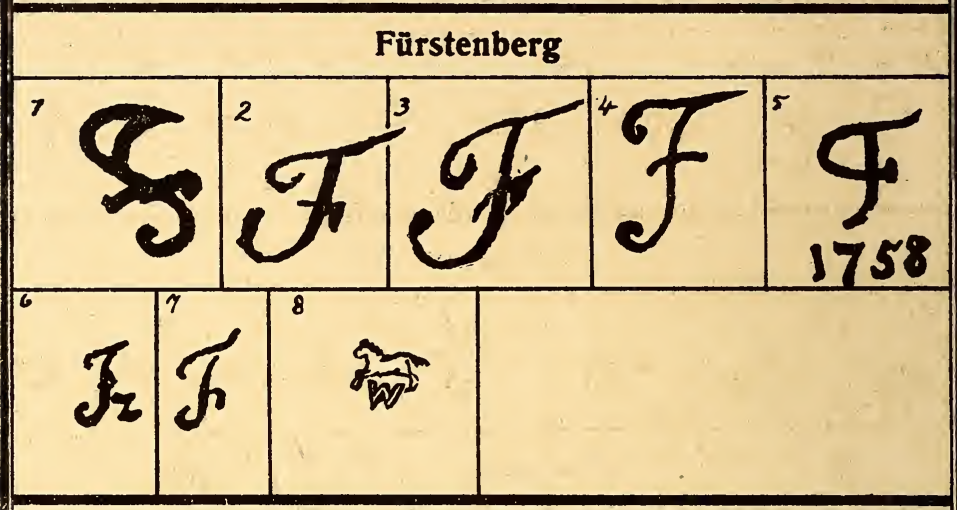

Volkstädt-Rudolstadt
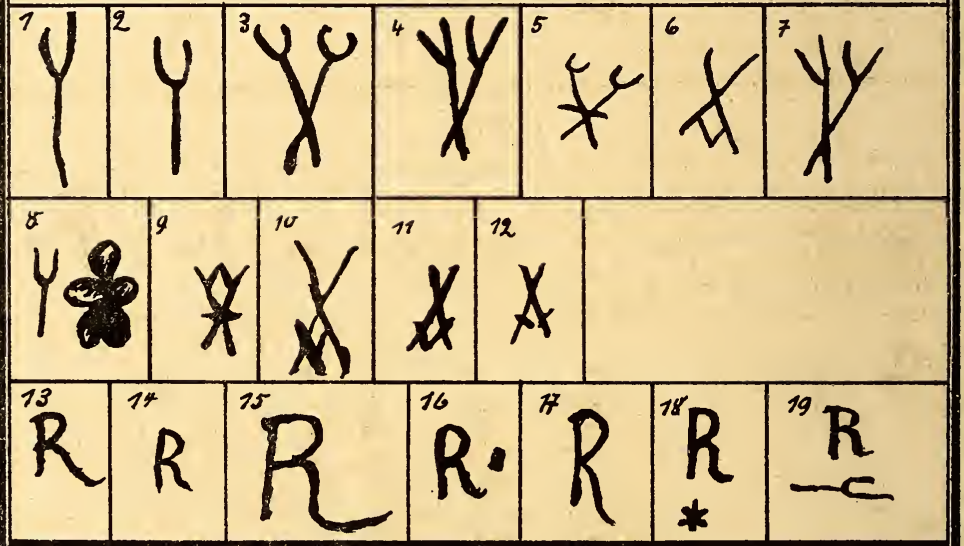

Kloster-Veilsdorf

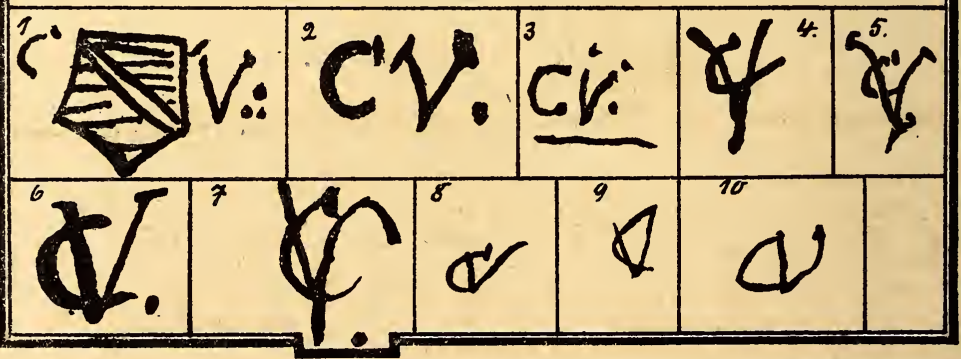


207

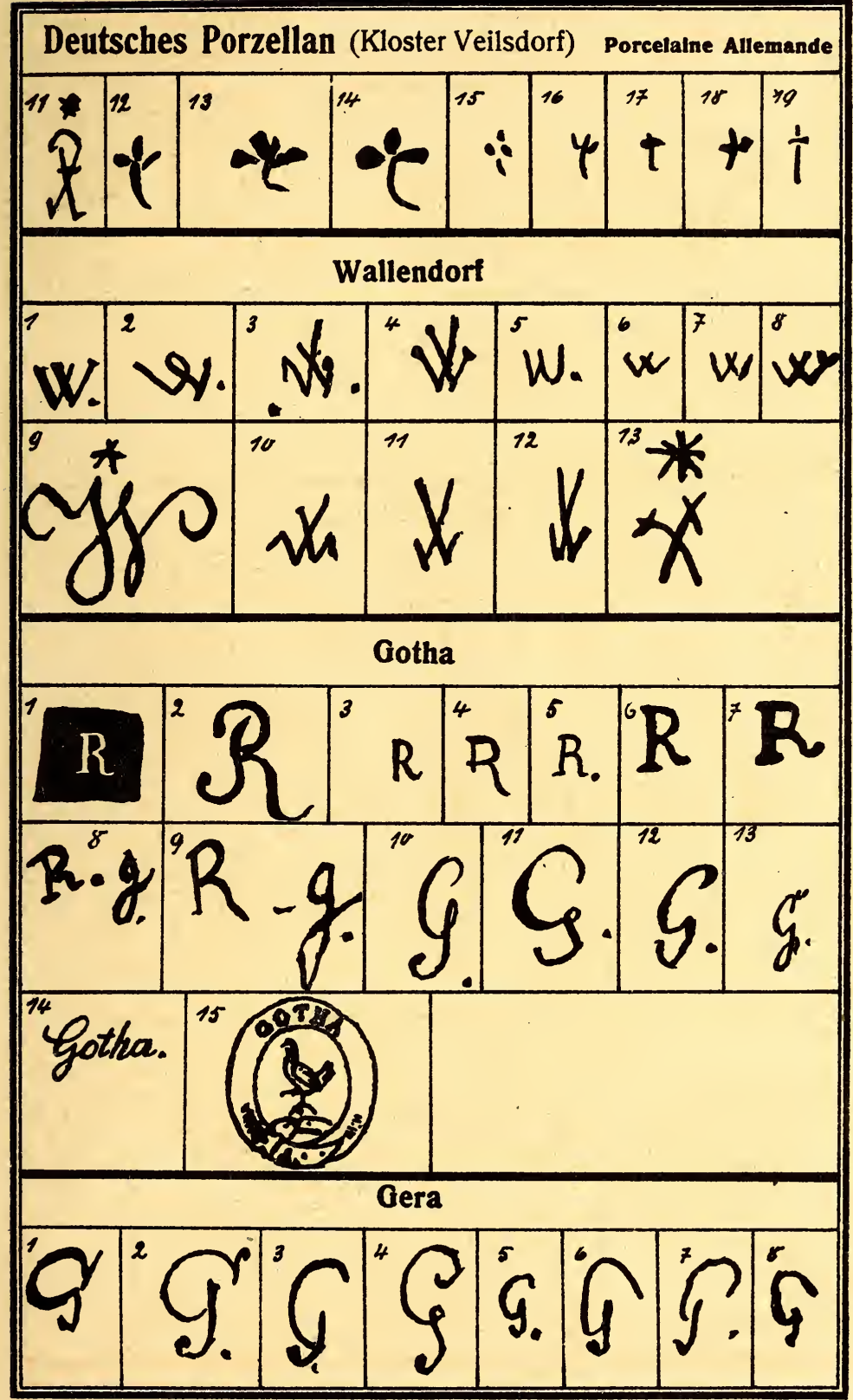


Deutsches Porzellan (Gera) Porcelarne Allemande

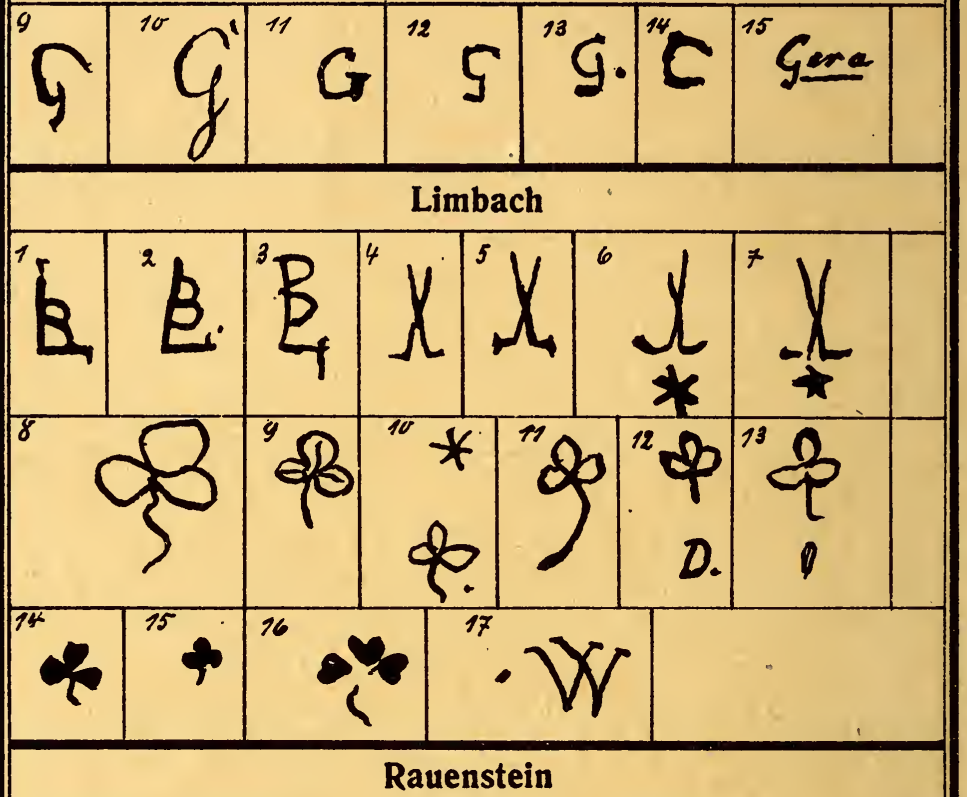

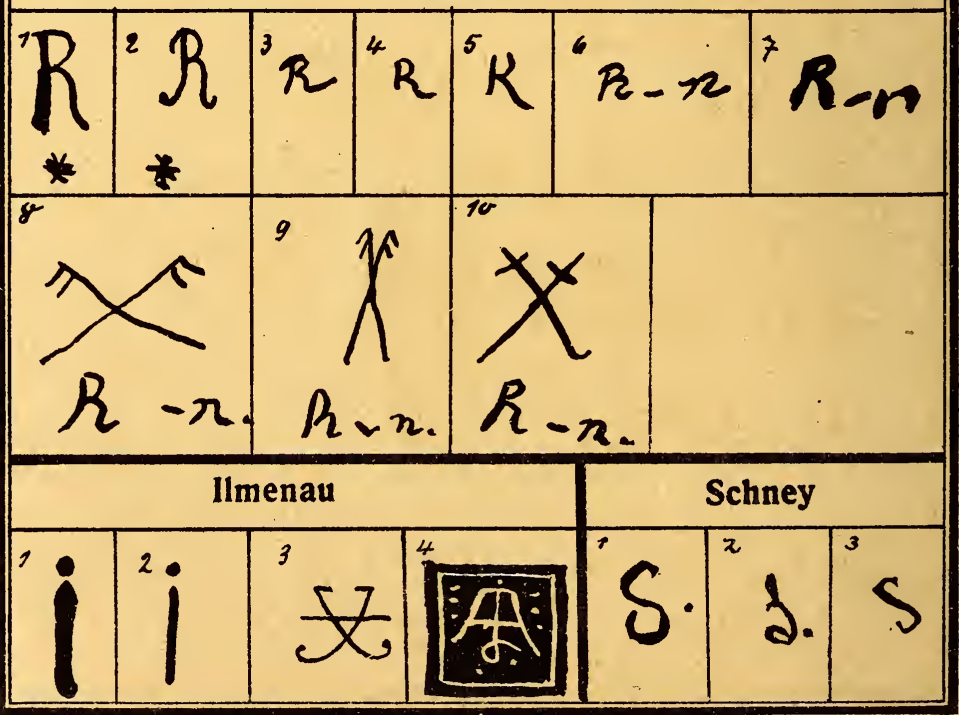




\section{Deutsches Porzellan}

Porcelalne Allemande

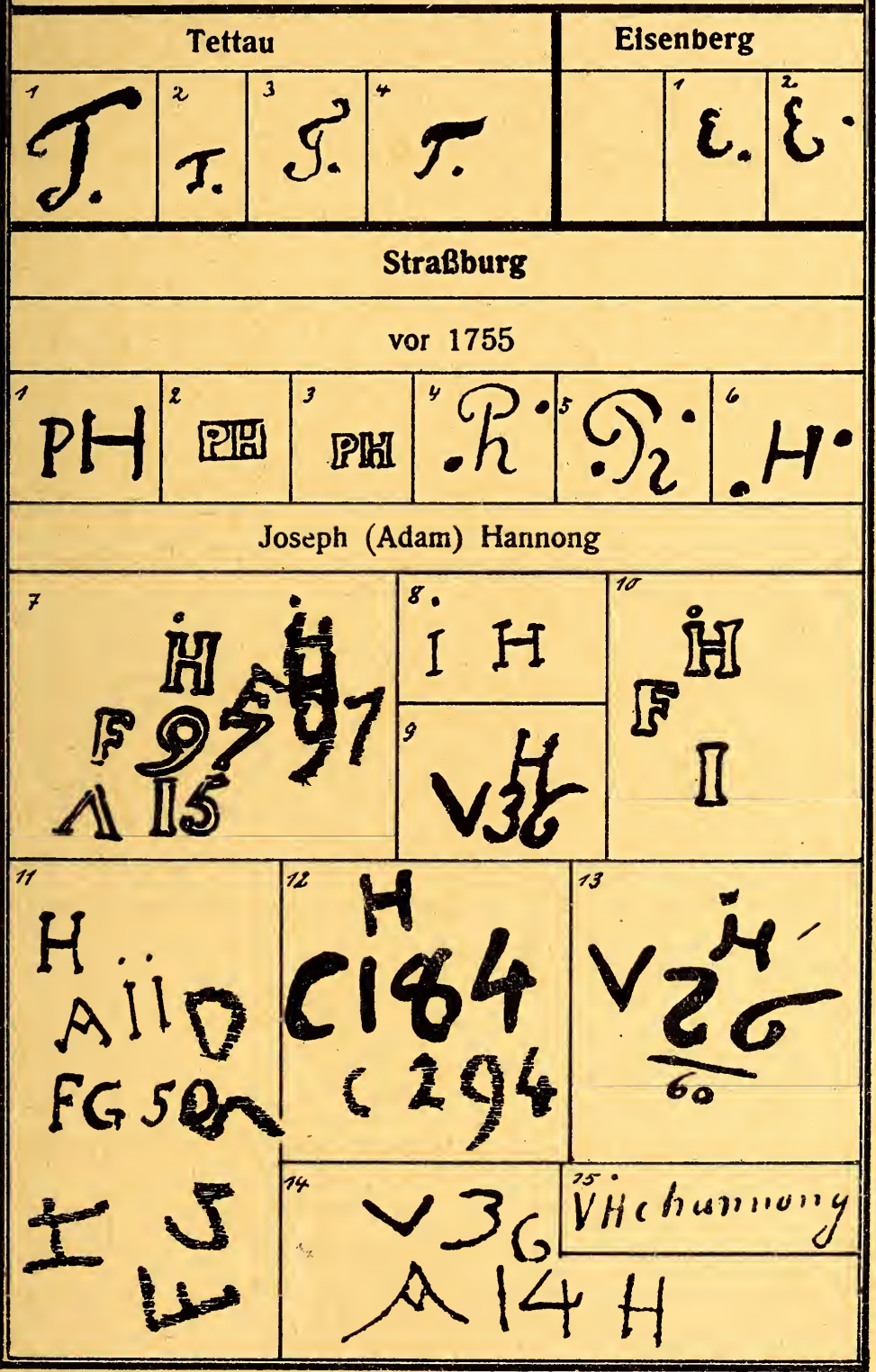




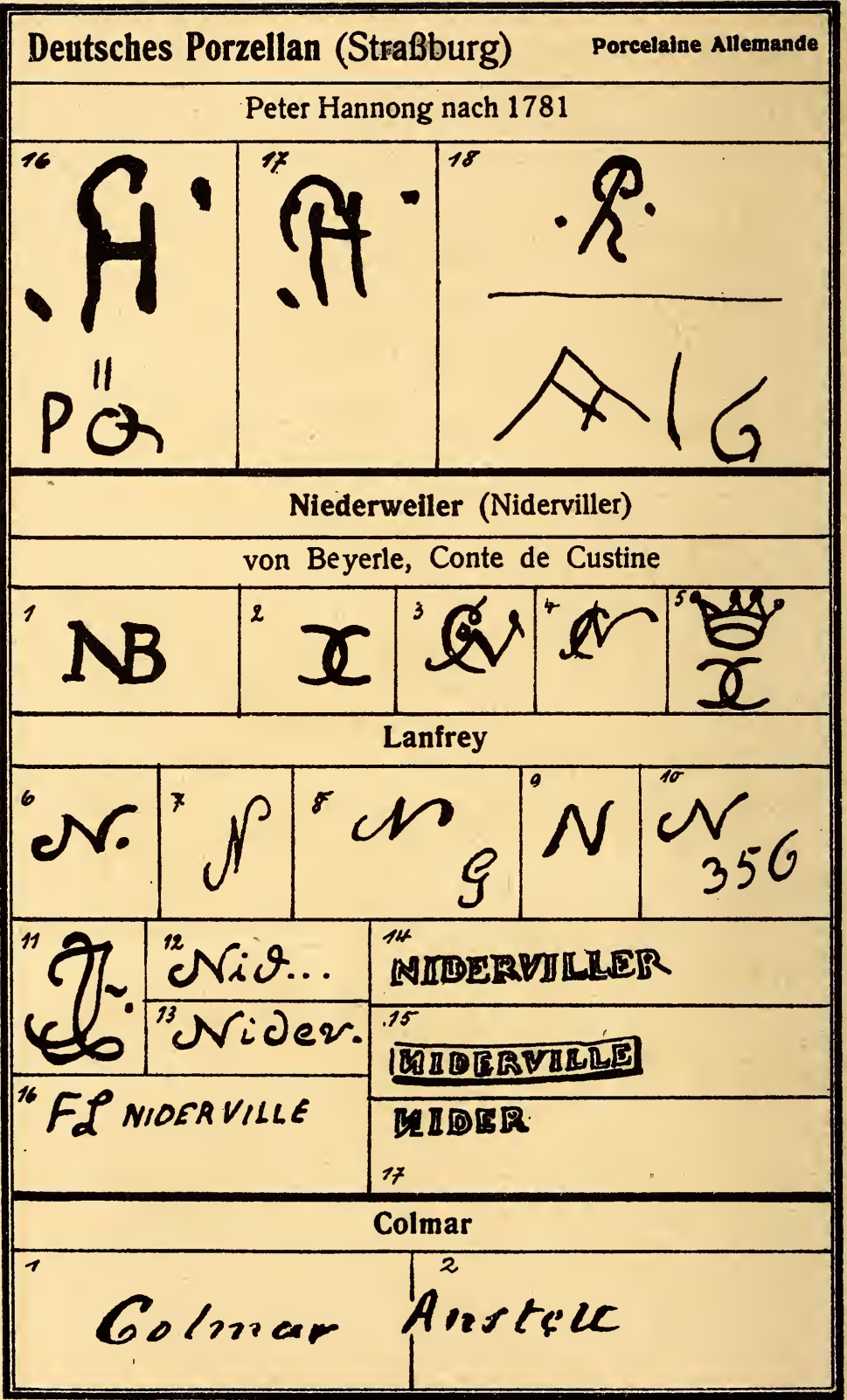


211

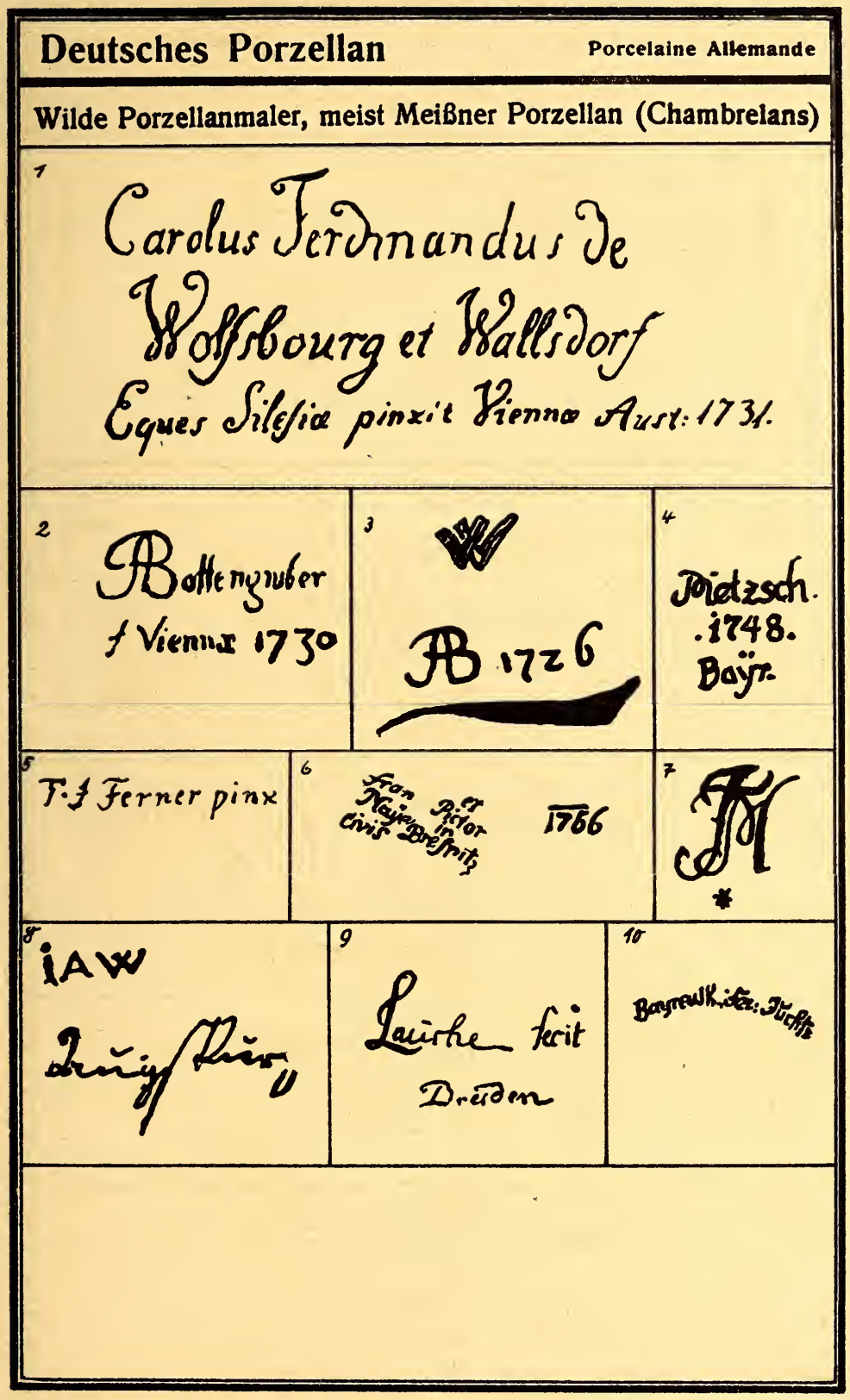




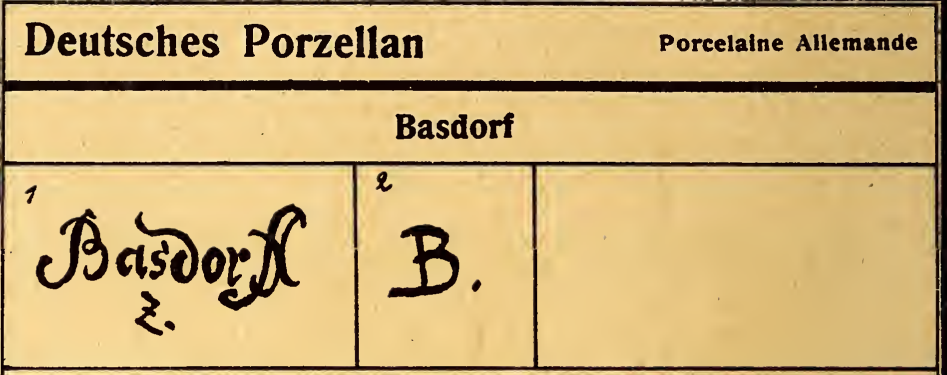

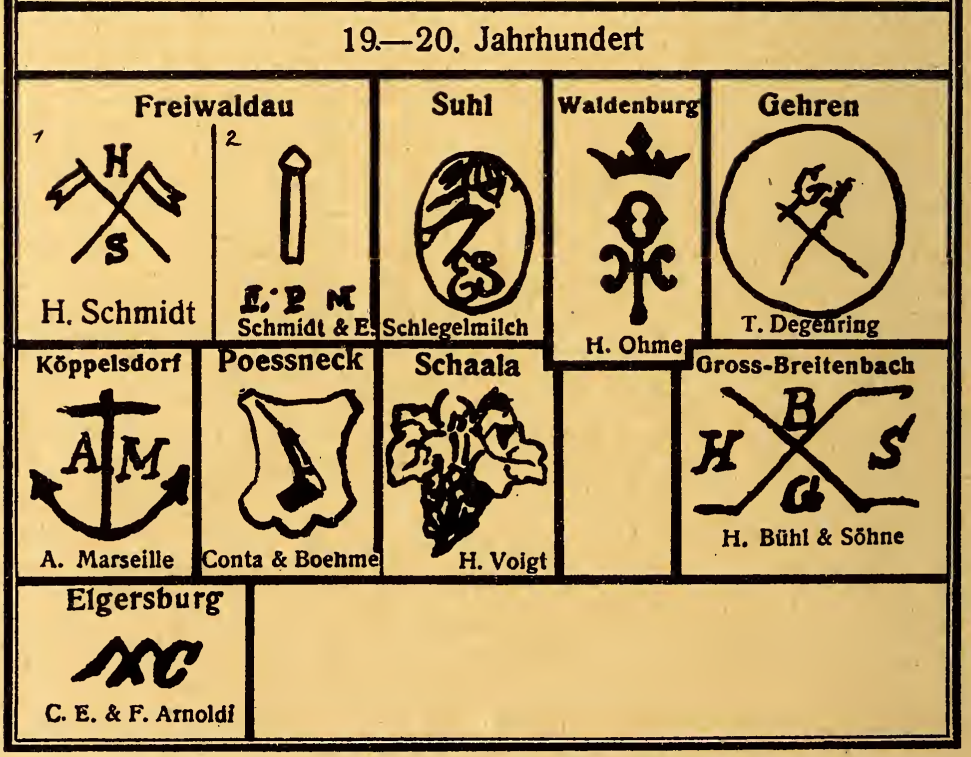


Deutsches Porzellan

Porcelaine Allemande

19. - 20 Jahrhundert

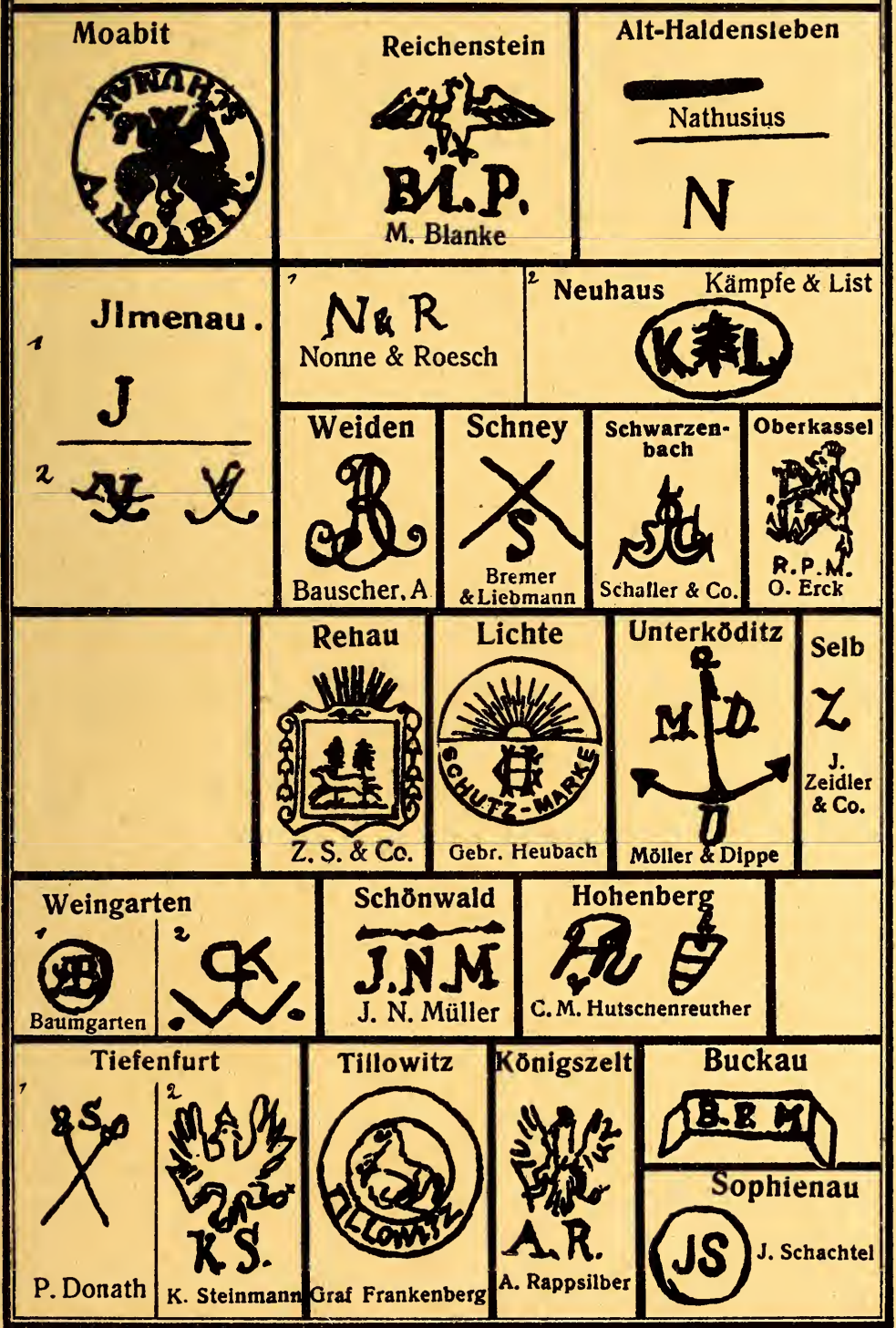




\section{Deutsches Porzellan}

Porcelaine Allemande

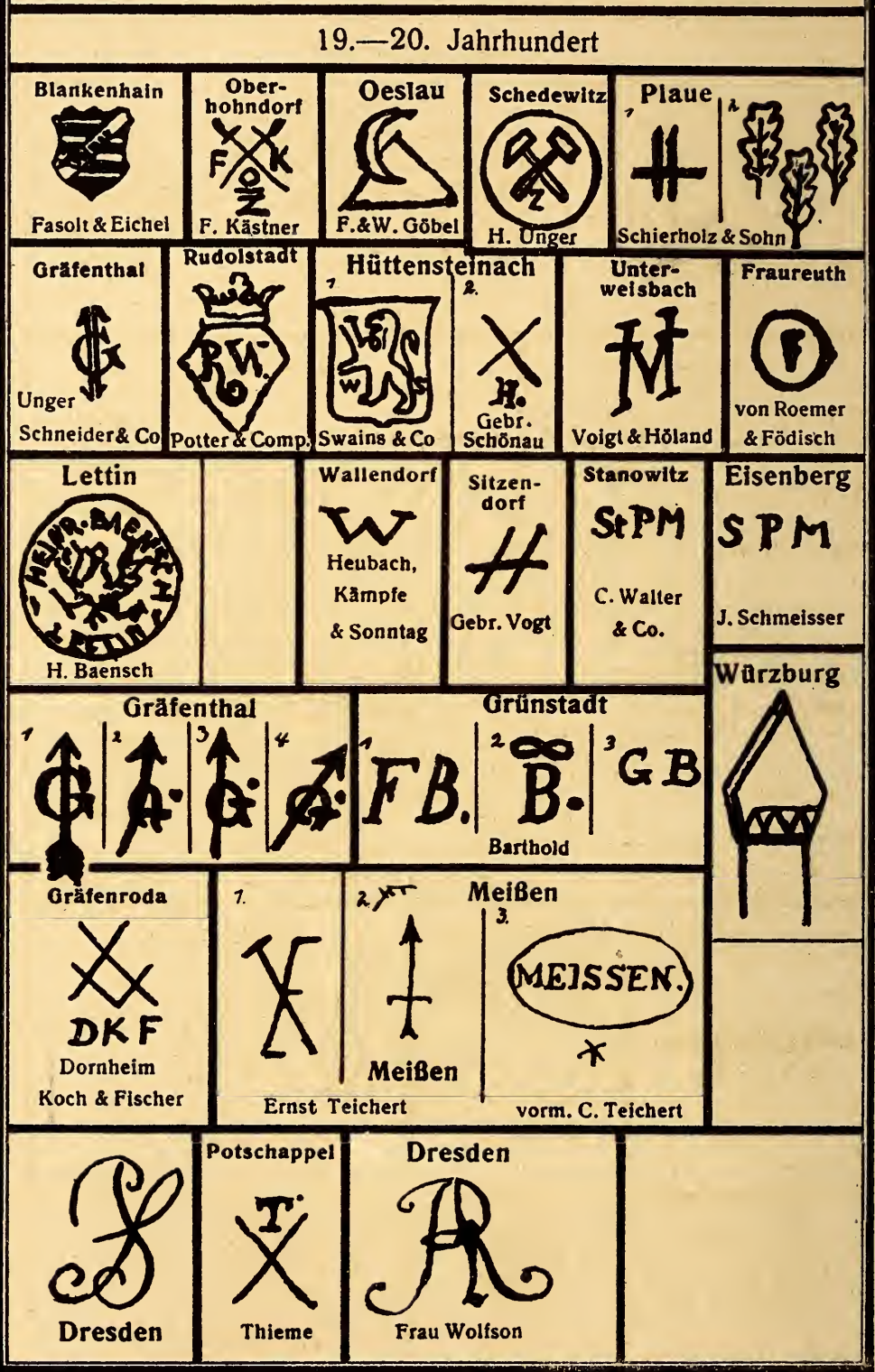




\section{Deutsches Porzellan}

Porcelaine Allemande

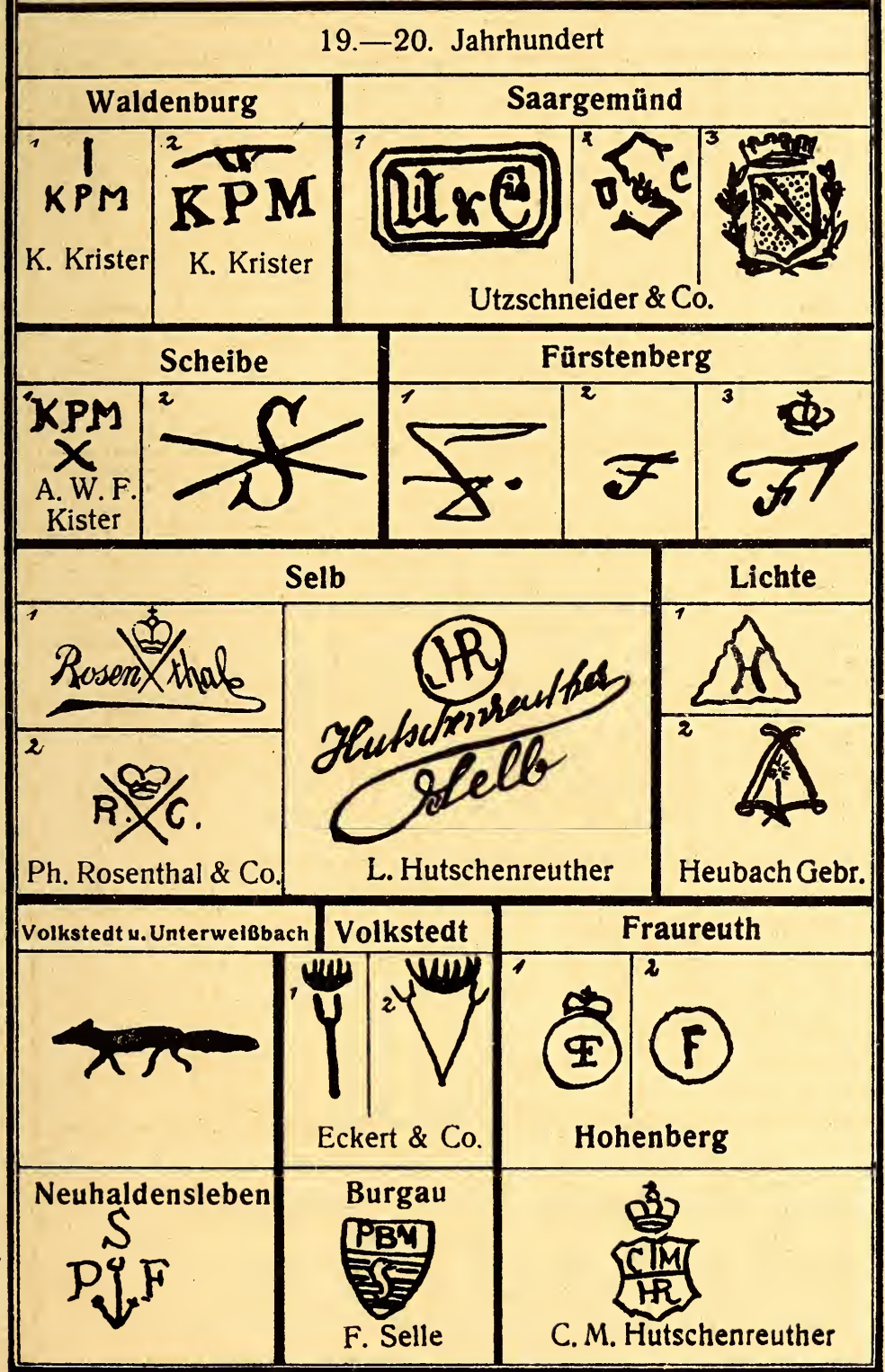




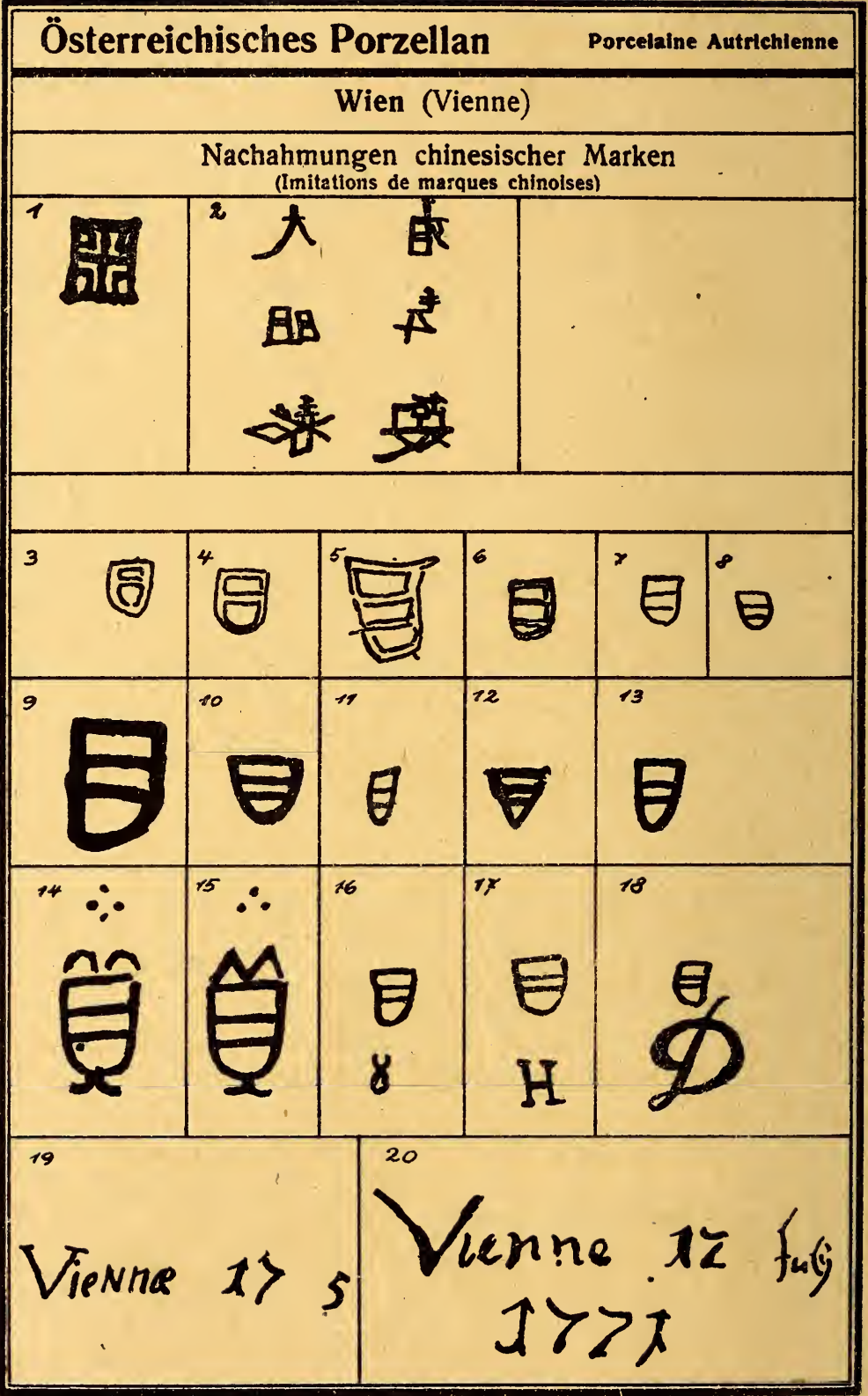


217

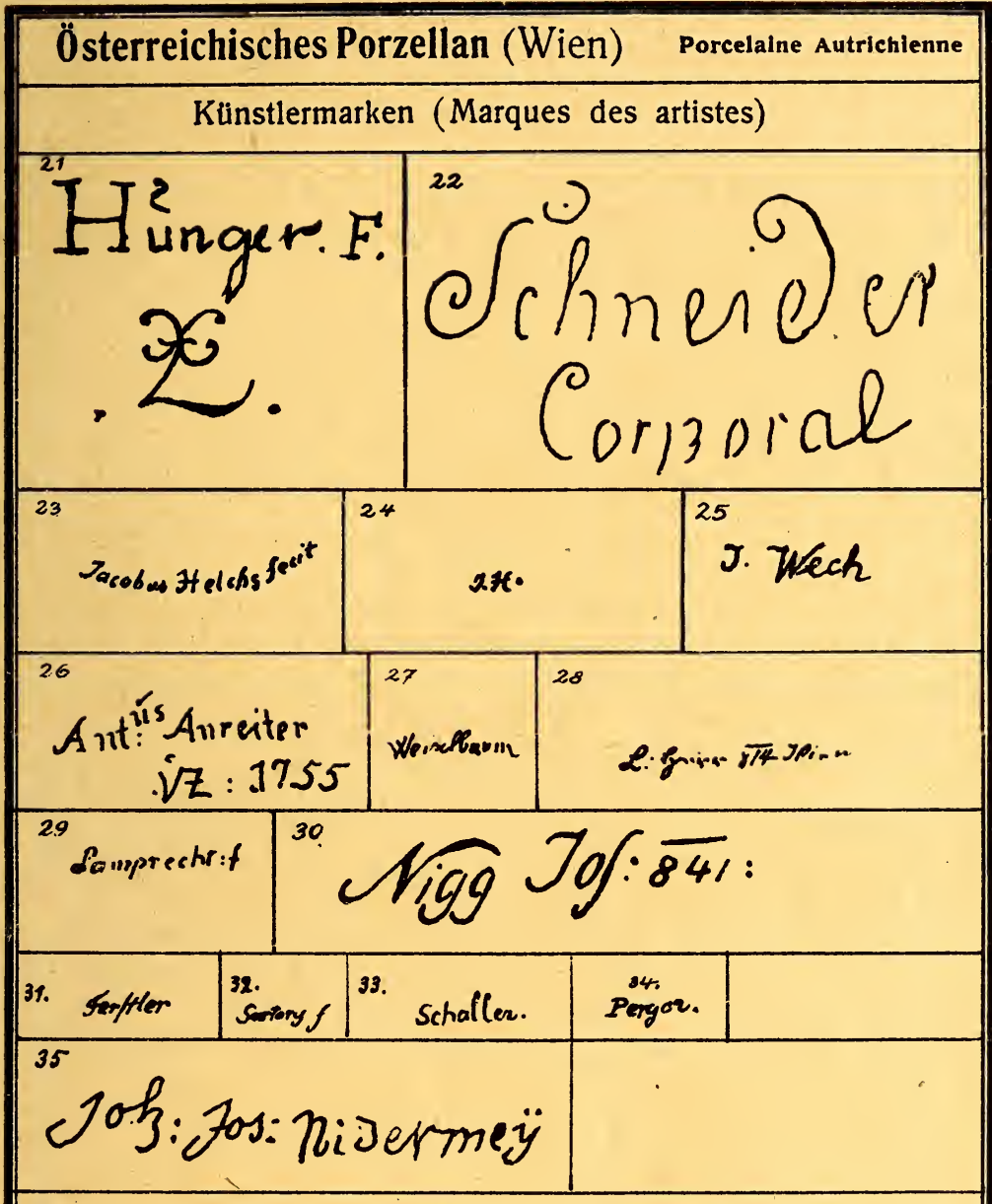

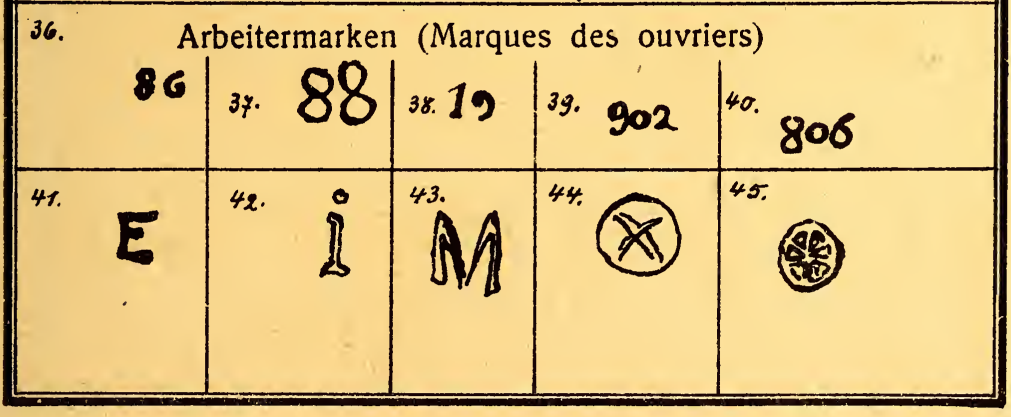




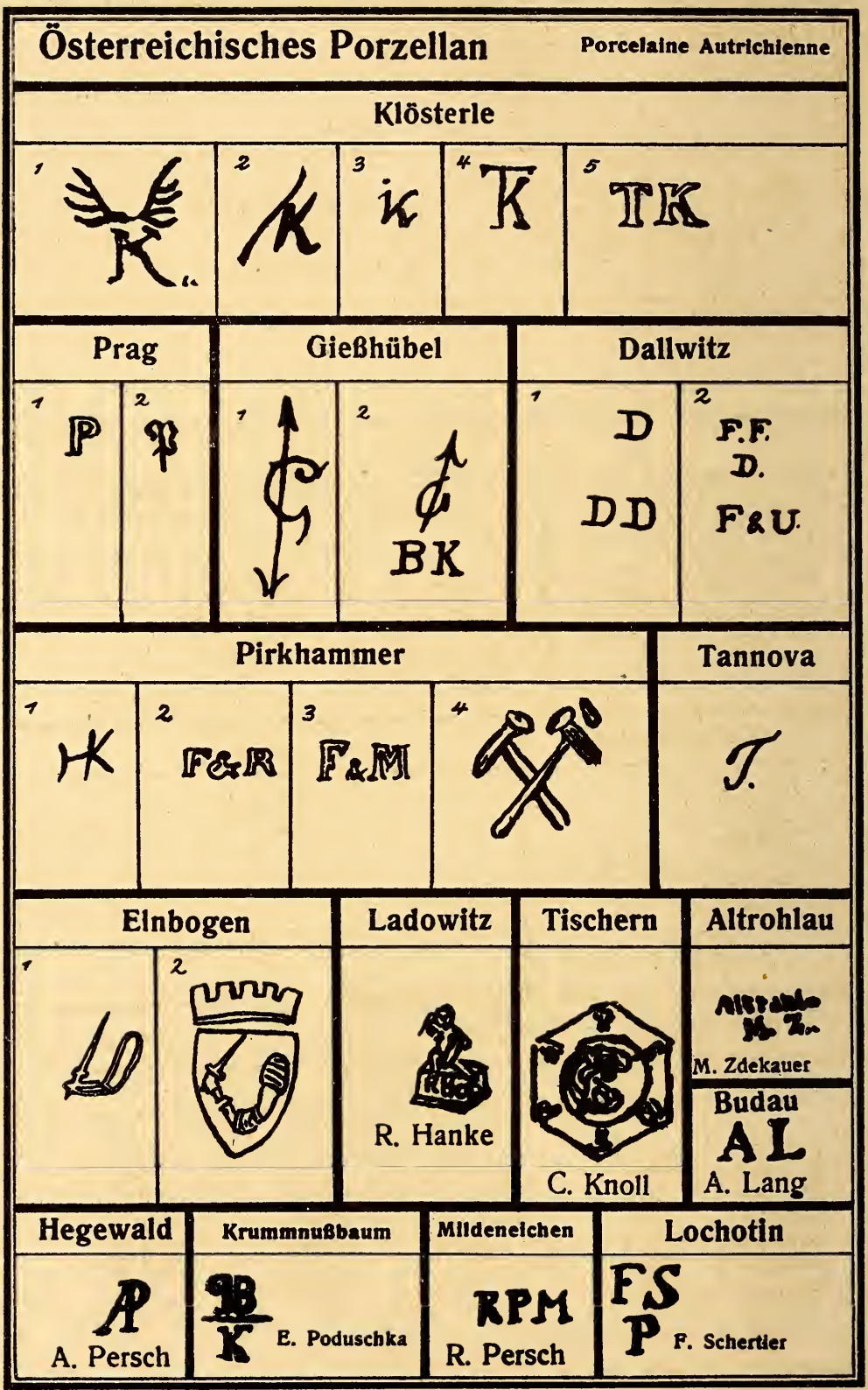




\section{Österreichisches Porzellan Porcelalne Autrchlenne}

\begin{tabular}{|c|c|c|}
\hline \multicolumn{3}{|c|}{ Schlaggenwald } \\
\hline S. $\left.\right|^{2} f$ & $\int^{3} \mathcal{H}$ & $\begin{array}{l}\text { LIPPERT\& HAAS } \\
\text { IN } \\
\text { SCHUGGENWALD }\end{array}$ \\
\hline
\end{tabular}

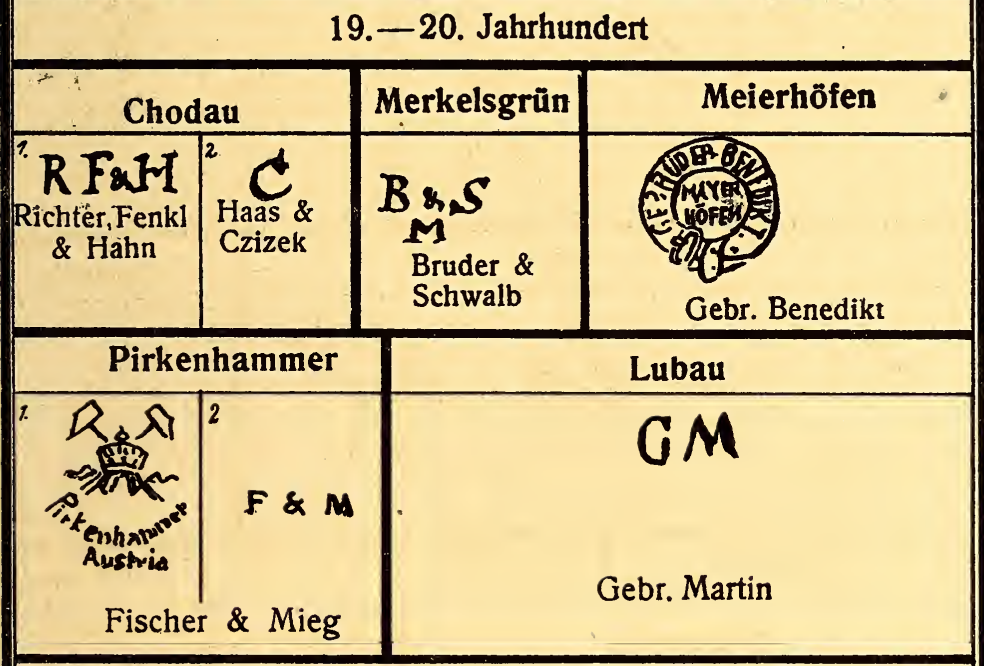

Ungarisches Porzellan Porcelaine Hongroise

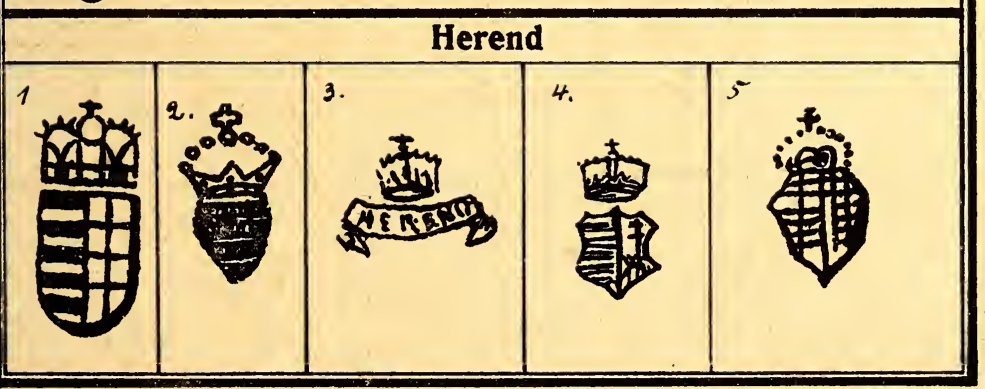




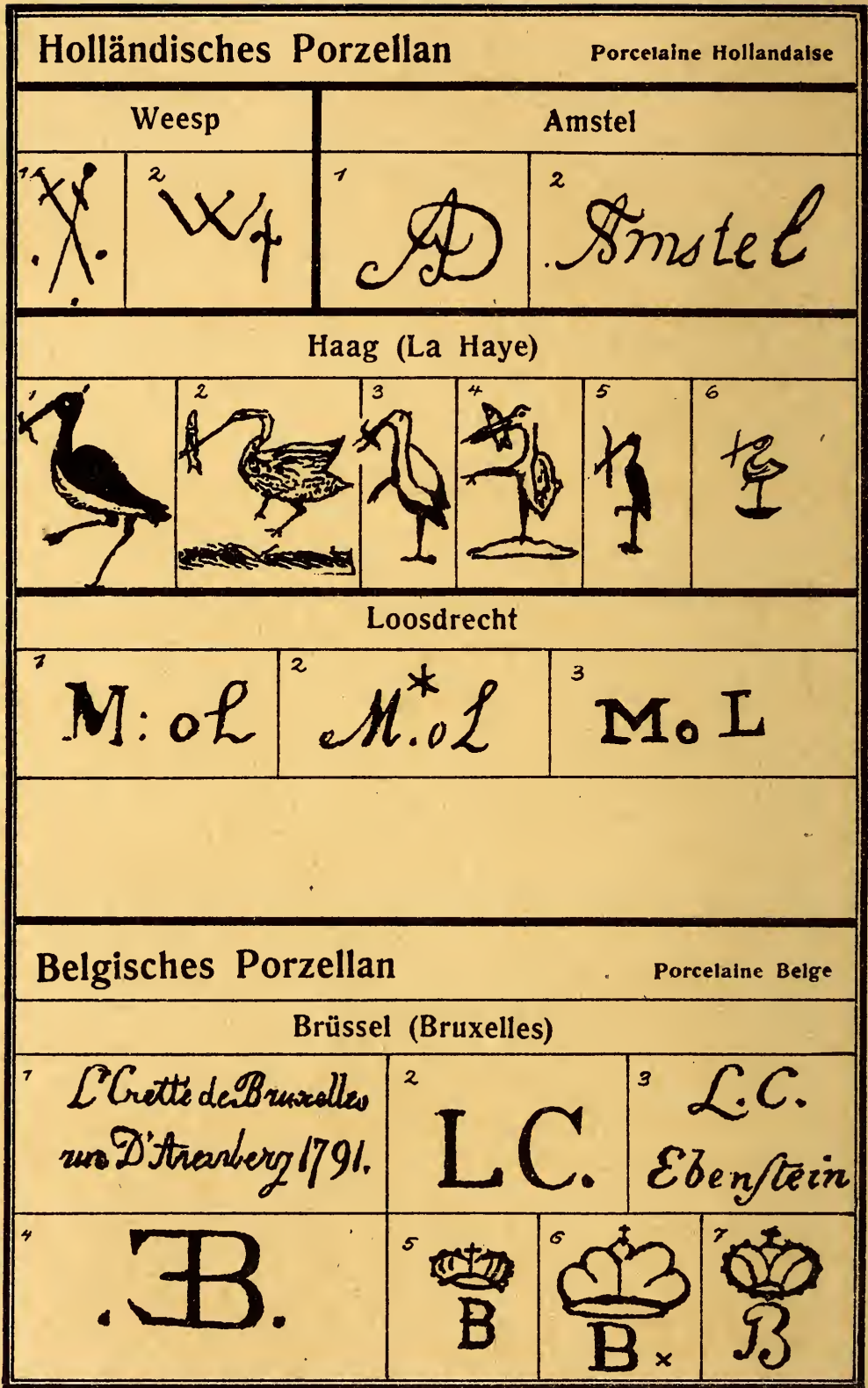




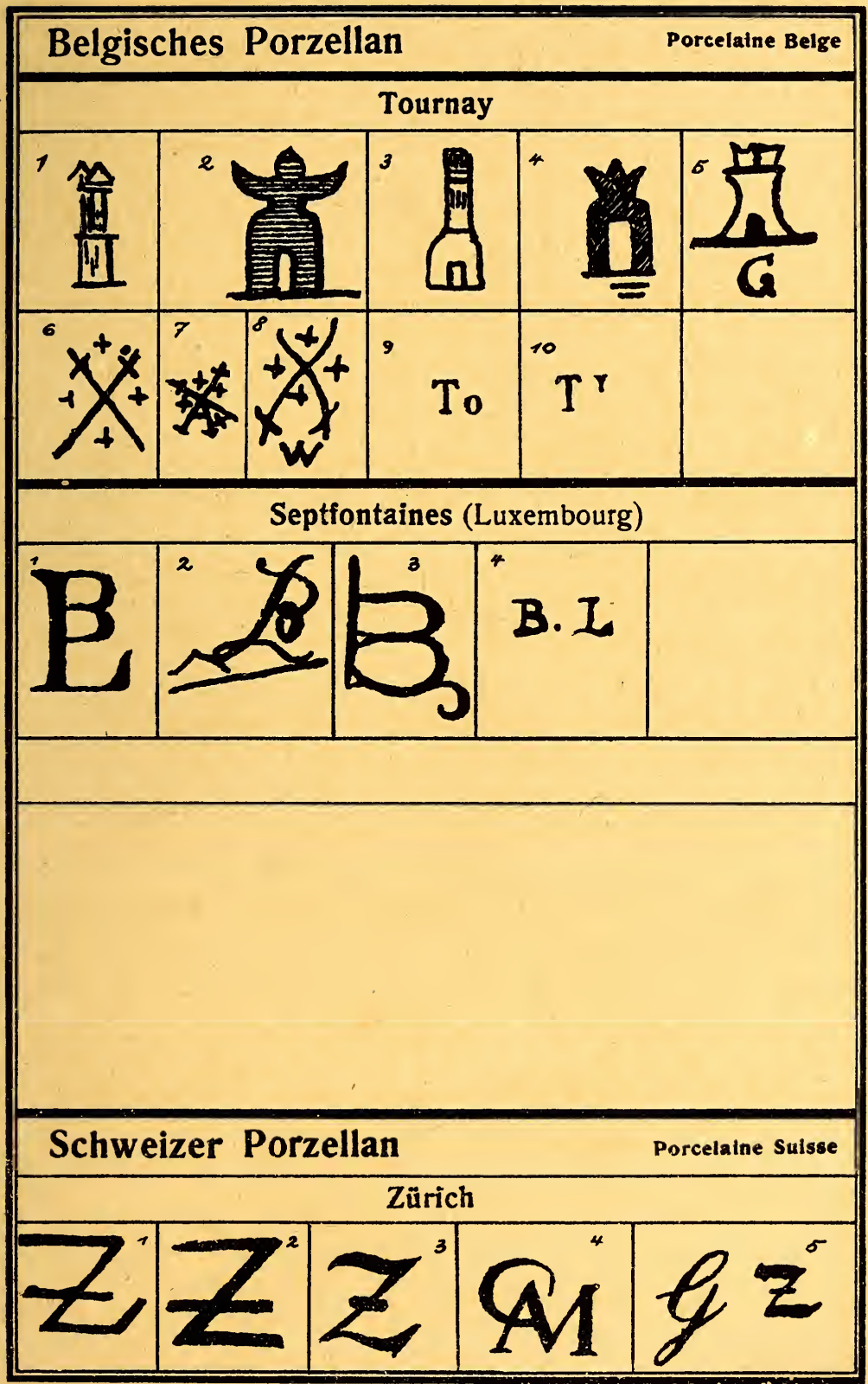




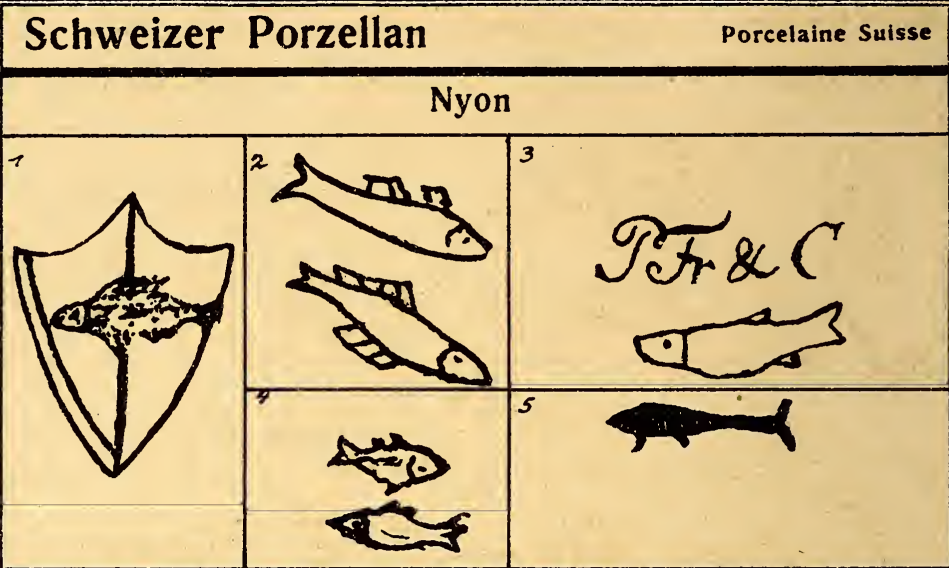

\section{Dänisches Porzellan}

Porcelaine Danoise

Kopenhagen (Copenhague)

Kgl. Porzellanmanufaktur (Manufacture royale)

Fournier $1760-1766$

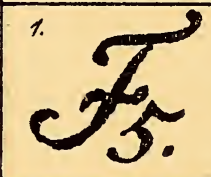

2 .

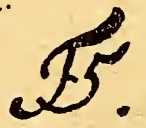

Franz Heinr. Müller $1773-1801$

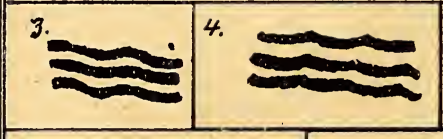
in Nis $\equiv+{ }^{*} \equiv$ $\longrightarrow$

". $\cong \mathrm{ML}$

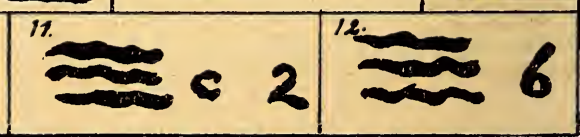




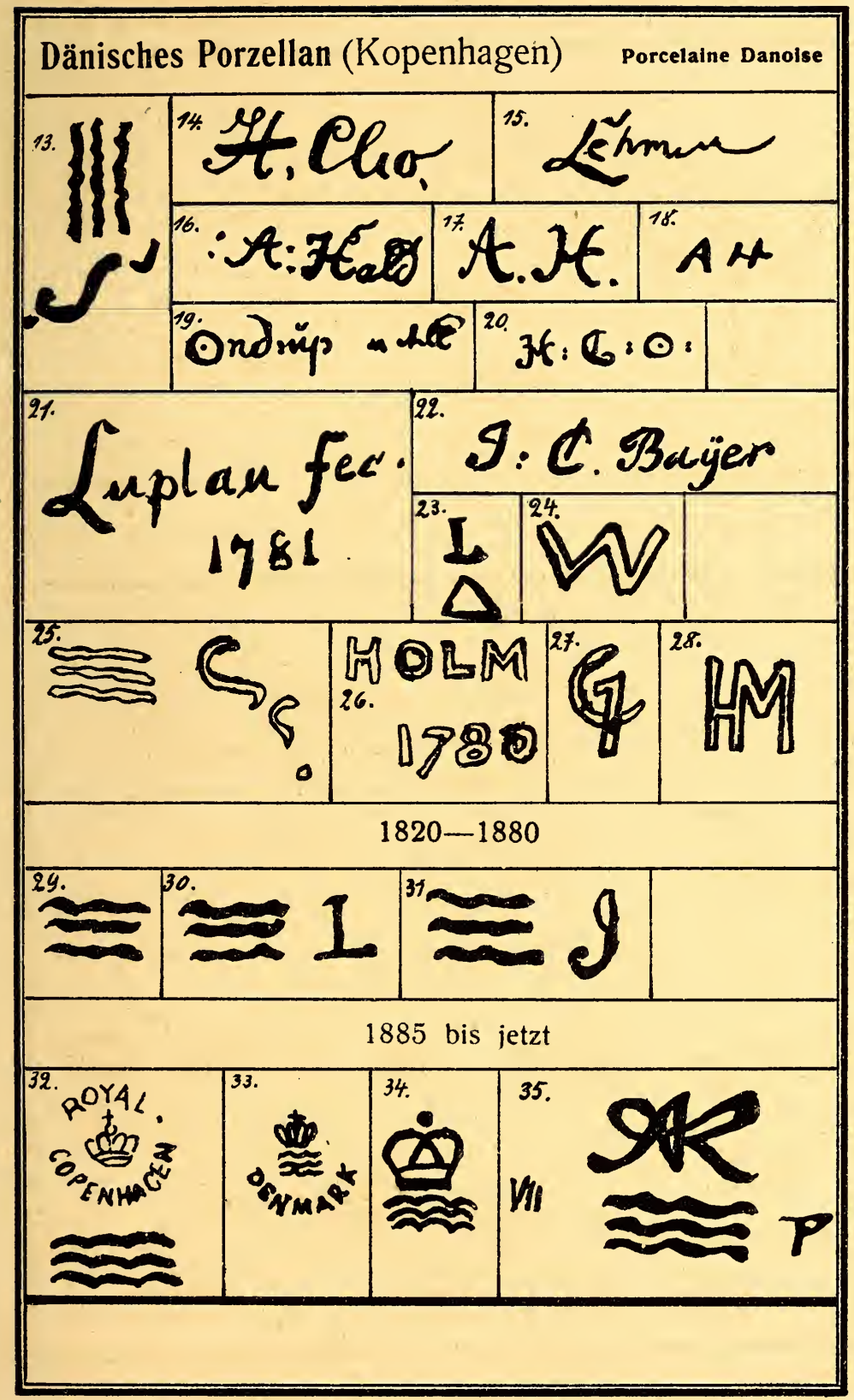




\section{Dänisches Porzellan}

\begin{tabular}{|c|c|c|}
\hline \multicolumn{3}{|c|}{ Kopenhagen (Bing \& Groendahl) } \\
\hline $\begin{array}{l}\text { SO \& GRQND } \\
\text { HOVEDDEPOU } \\
\text { HEBENHANN }\end{array}$ & $\begin{array}{c}2 \text { Danish Chino Worto } \\
\text { B. a } \\
\text { COPEMMAEEM } \\
\text { B \& G }\end{array}$ & 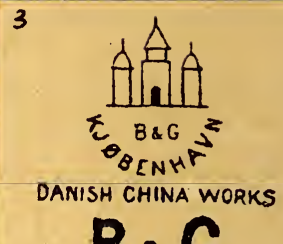 \\
\hline
\end{tabular}

Schwedisches Porzellan

Porcelaine Suédoise

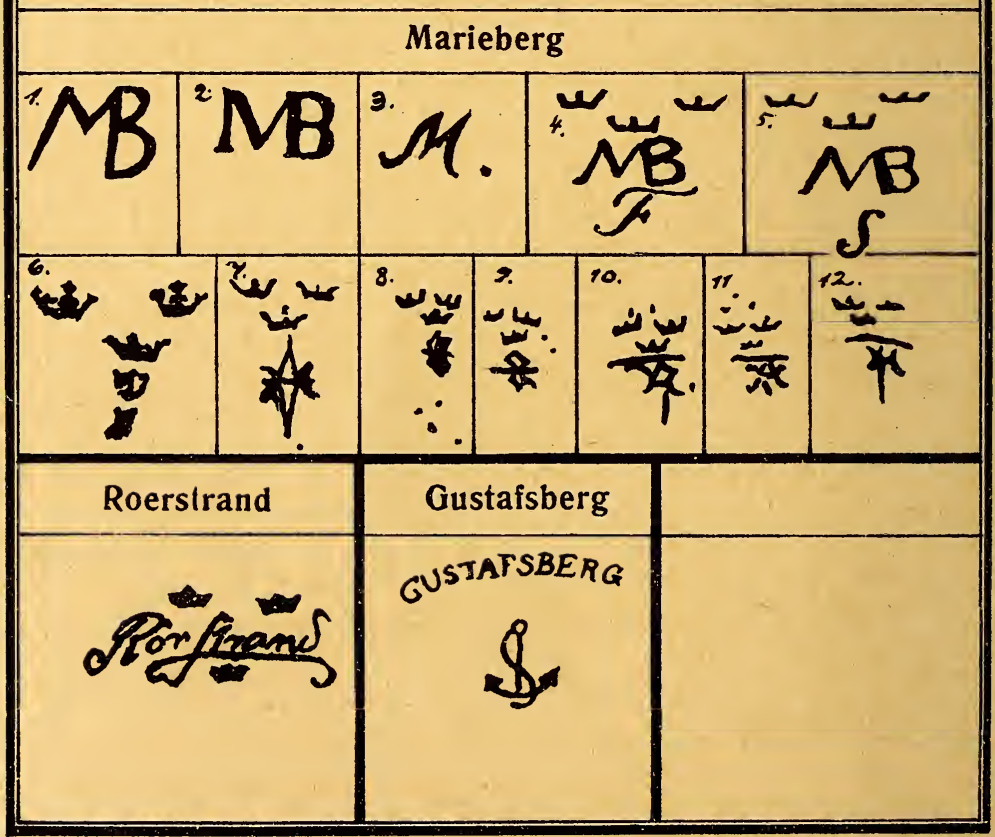




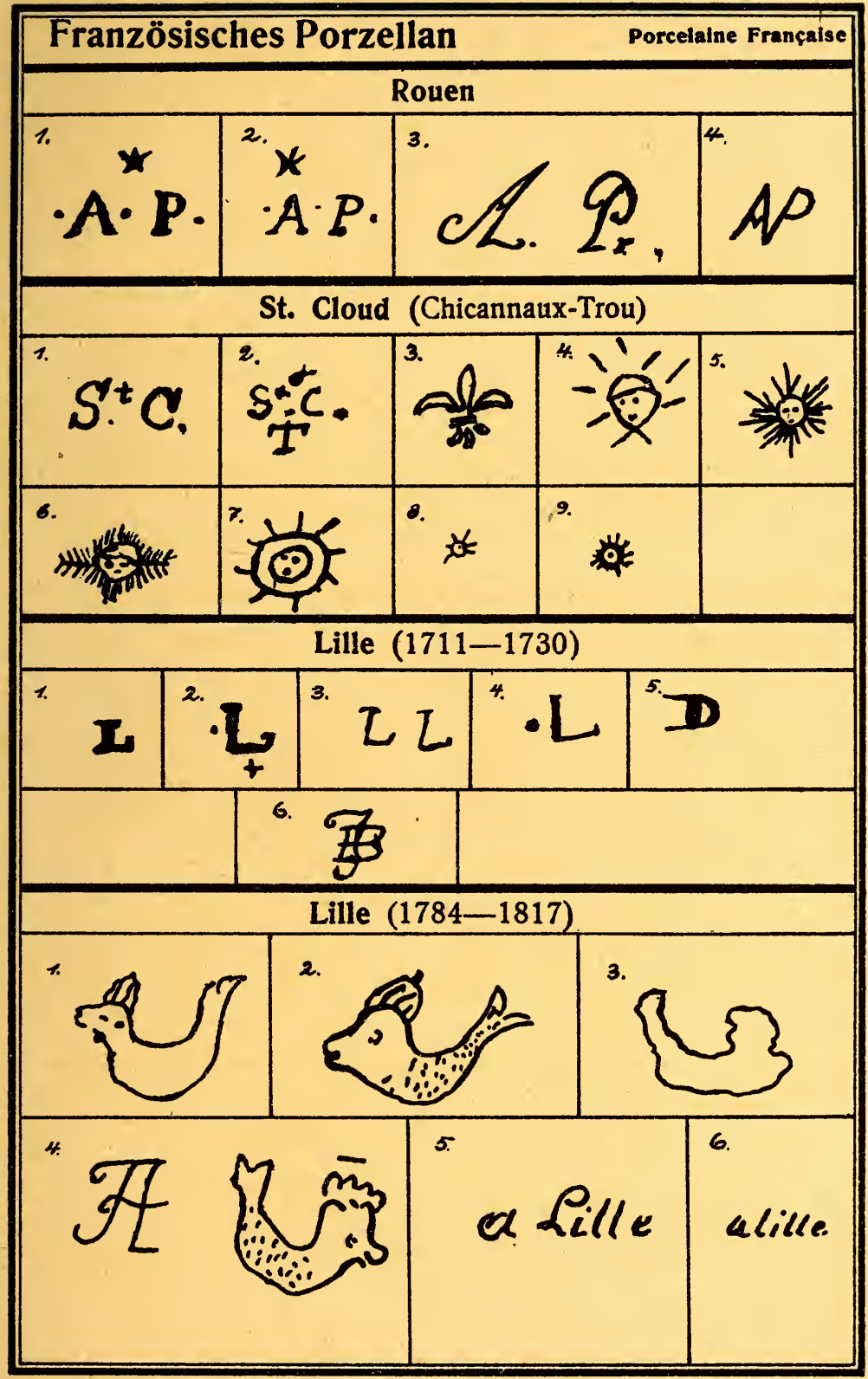




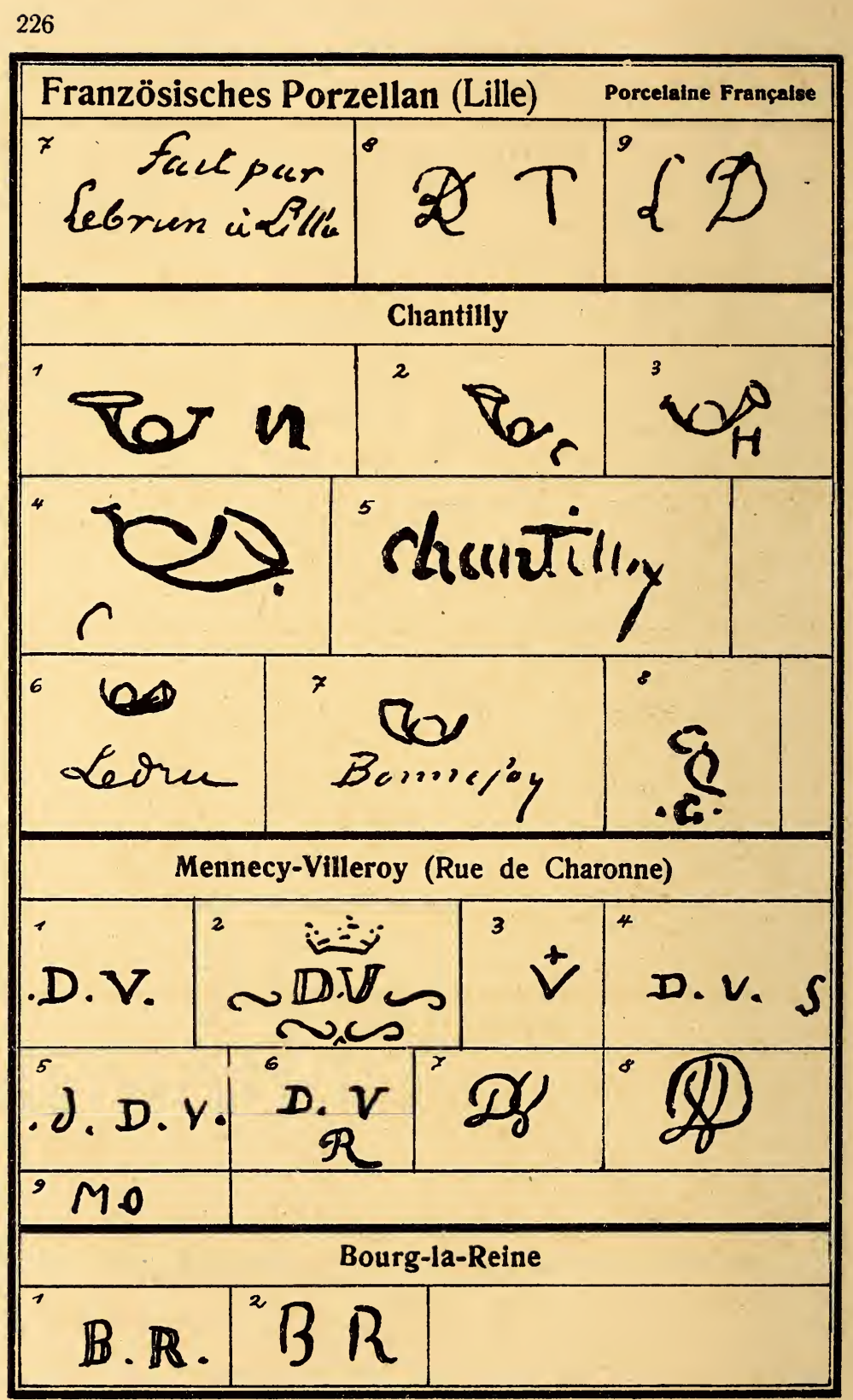




\section{Französisches Porzellan}

Porcelaine Françalse

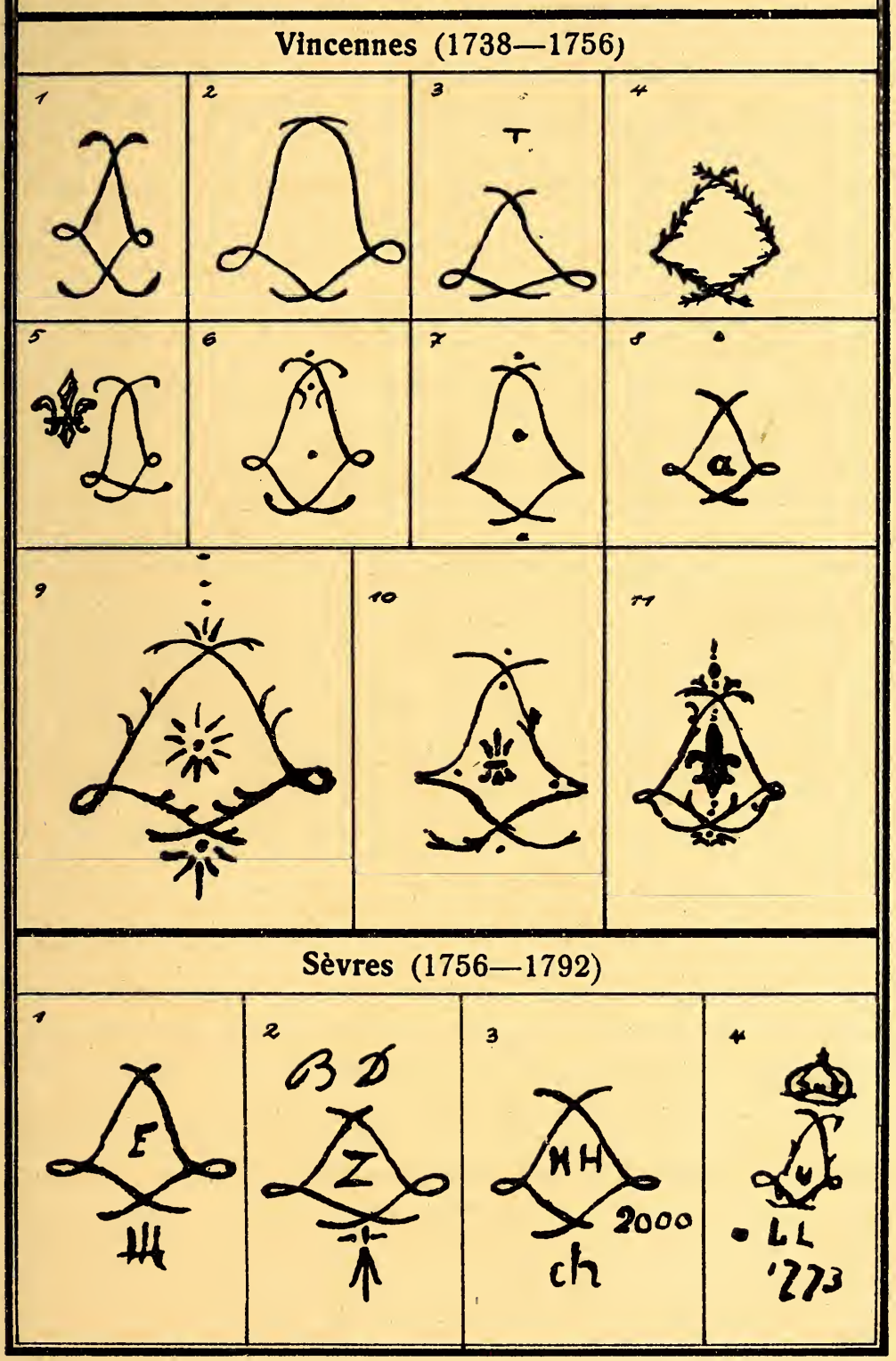




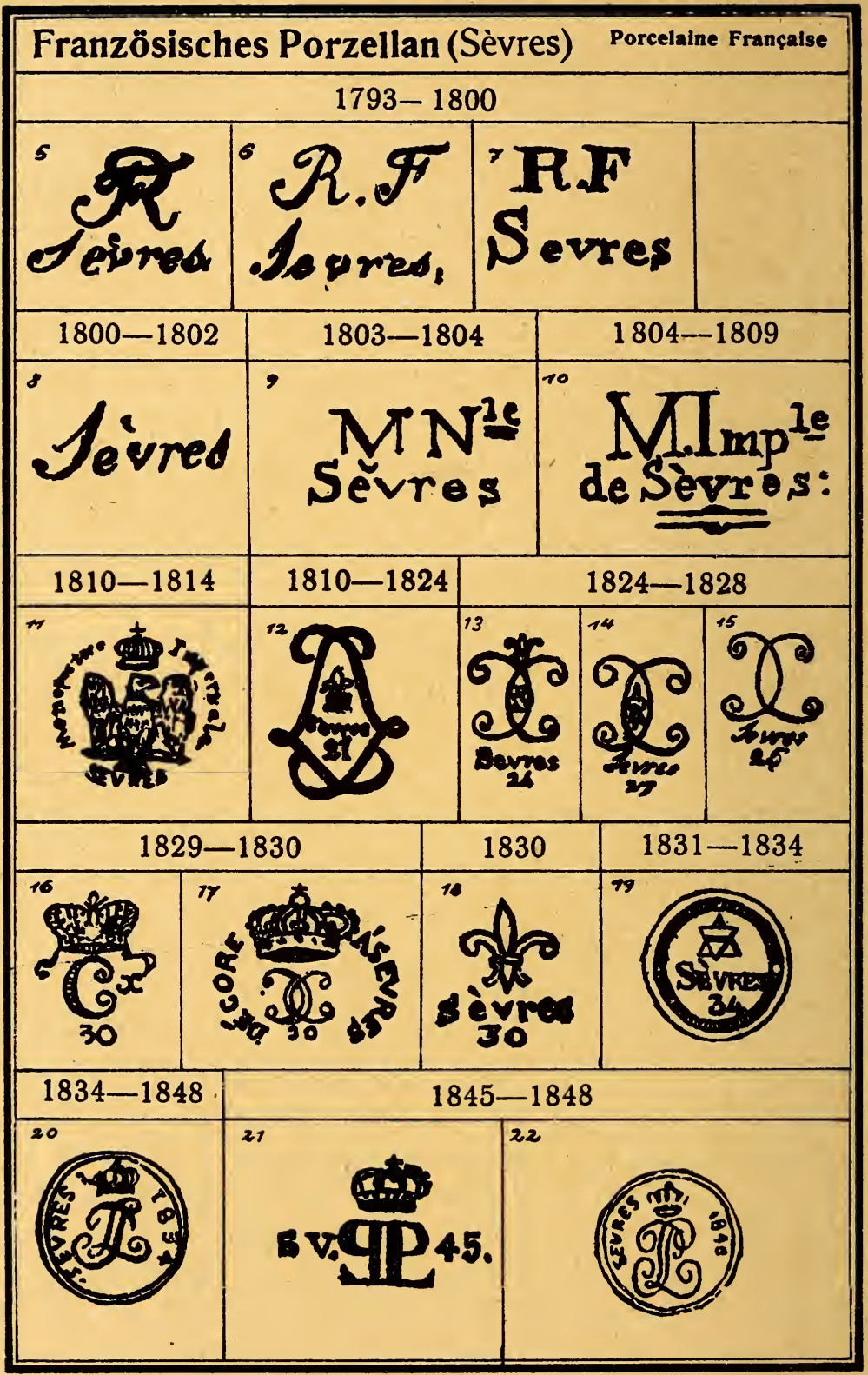


Französisches Porzellan(Sèvres) Porcelaine Françalse

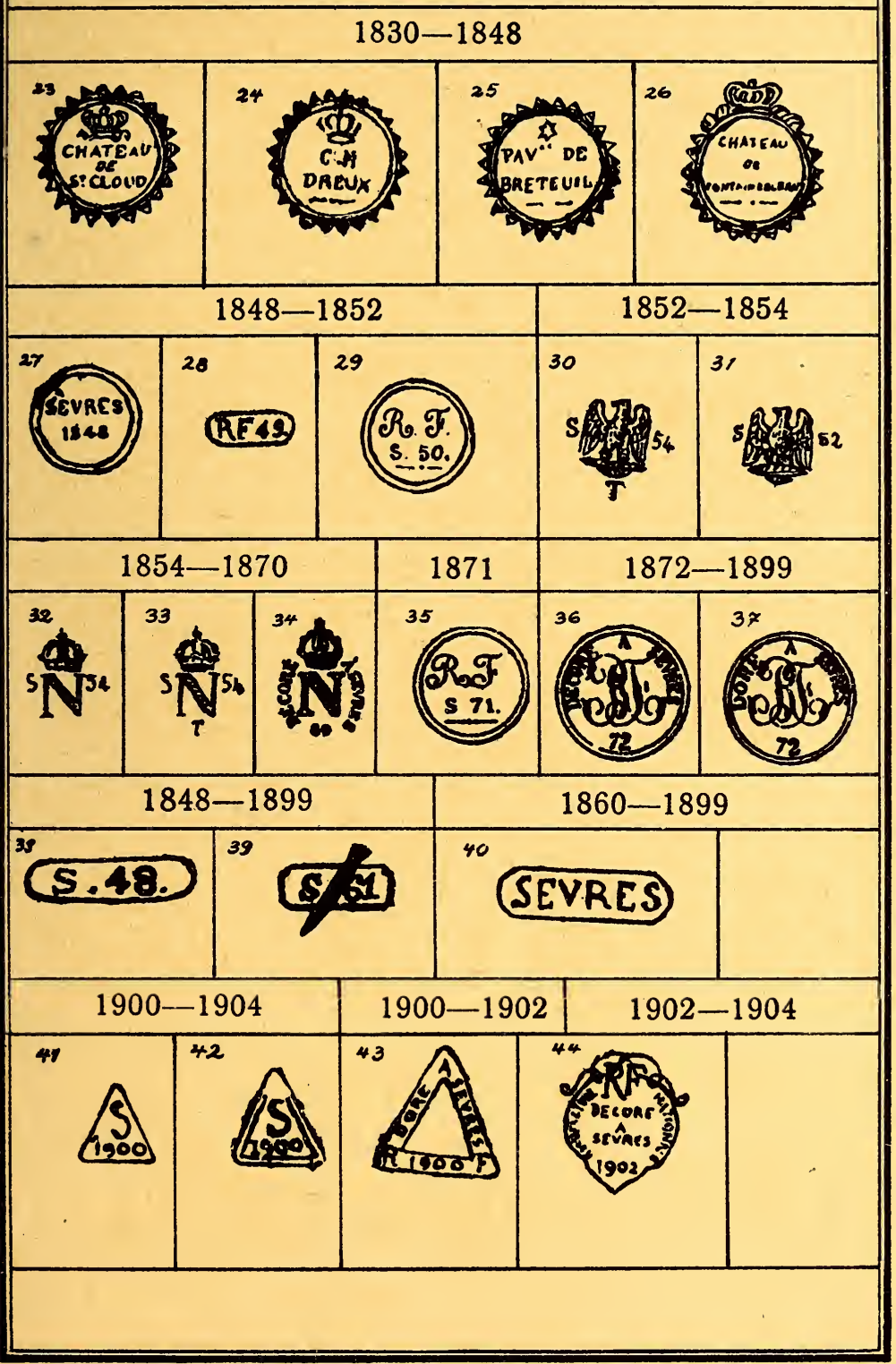


Franzősisches Porzellan (Sèvres) Porcelaine Francalse

Maler-, Dekorateur- u. Vergoldermarken von Sèvres Marques de pelntres, décorateurs et doreurs de Sèvres

\begin{tabular}{|c|c|c|c|}
\hline & 1753 & -1800 & \\
\hline$r$ & 73 & ${ }^{25} \Delta$ & ${ }^{3 x} j \cdot n$. \\
\hline 2 & ${ }^{14} \mathrm{~T}$ & ${ }^{26} \Delta$ & 38 \\
\hline 3 & ${ }^{15} \mathrm{Pe}$ & 27 & 39 \\
\hline $4 \quad A \operatorname{An}$ & 16 & 28 b. & $\infty$ \\
\hline 5 & $\Rightarrow$ & ${ }^{29} a$ & $\begin{array}{ll}41 & \\
& 0.12\end{array}$ \\
\hline $6 \quad$ Sy & 18 & $30 \%$ & 42 \\
\hline $7 \quad=$ & $19 \quad B$. & & ${ }^{43} C^{3}$ \\
\hline$\phi$ & 20 & ${ }^{32} C K$. & 4 \\
\hline 13 & $27 C_{n}$. & 33 & ${ }^{45} \mathrm{~K}$ \\
\hline 68 & $\begin{array}{ll}22 & \\
& \text { mand }\end{array}$ & ${ }^{34}$ c.p.p & ${ }^{*} \mathscr{L R}$ \\
\hline $\bar{\Sigma}$ & 23 & ${ }^{35} \mathrm{joc}$ & 47 \\
\hline 6. & 249 & ${ }^{36}$ 站 & \begin{tabular}{|ll}
$*$ & $\mathscr{X}$. \\
&
\end{tabular} \\
\hline
\end{tabular}


Französisches Porzellan (Sèvres) Porcelaine Francaise

\begin{tabular}{|c|c|c|c|}
\hline${ }^{49} \mathrm{DT}$ & ${ }^{64} G t$. & ${ }^{{ }^{9}} \mathcal{L}$ Gina & "مـ \\
\hline${ }^{50} 8$ & 65 & 80 & ${ }^{95} \mathscr{D}$ \\
\hline $\mathbf{F}$ & ${ }^{66} j h$. & ${ }^{81} \mathcal{C}_{\text {ou }} \mathrm{L}$ & ${ }^{96} \mathfrak{J P}^{\circ}$ \\
\hline $52 \quad 8$ & $\Rightarrow h c$. & ${ }^{82} f$ & ${ }^{97} \mathcal{L} .8$. \\
\hline 53 & ${ }^{68} \mathrm{YP}$ & ${ }^{\circ} \mathcal{3} \Omega$ & 98 \\
\hline$Y^{*}$ & ${ }^{69} \mathrm{FI}$. & ${ }^{84}{ }^{8}$ & $\begin{array}{ll}99 & \text { P.T. }\end{array}$ \\
\hline 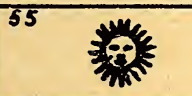 & $b^{\prime \prime}$ & ${ }^{85} \mathcal{S}$ & ${ }^{100} f$ \\
\hline frouf: $x$ & 2 & ${ }^{86} 9$ & ${ }^{105} \mathrm{po}^{\circ}$ \\
\hline 67 & & $x$ & $p . \%$ \\
\hline 5.8 & Syoulf & $m a M$ & P.IE. \\
\hline 59 & & $M$ & ${ }^{704} P_{.}$ \\
\hline${ }^{60} G_{d}$. & $\sqrt{75}$ & 90 & ${ }^{105}$ P.j. \\
\hline${ }^{61} \gamma{ }^{\prime} t$ & 76 & d & 8 \\
\hline${ }^{\circ 2} 8$ & chadL & 9 & HP. \\
\hline 63 & $\overline{78}$ & ${ }^{93} n q$ & ${ }^{108} A_{100}$ \\
\hline
\end{tabular}




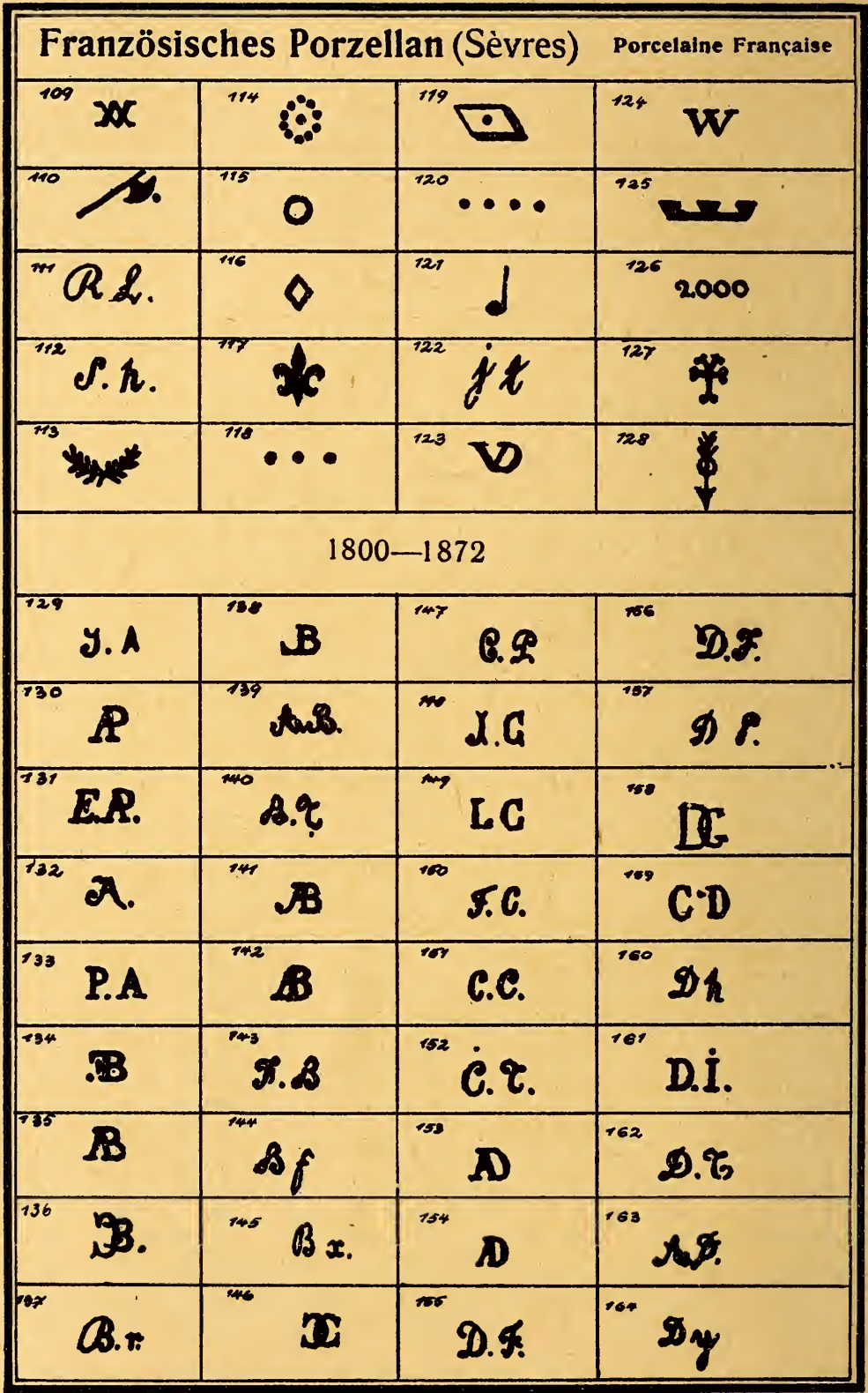




\begin{tabular}{|c|c|c|c|}
\hline \multicolumn{3}{|c|}{ Französisches Porzellan (Sèvres) } & \multirow{2}{*}{$\begin{array}{l}\text { Porcelaine Françalse } \\
210\end{array}$} \\
\hline${ }^{n 5} \mathrm{H}$ & $100 \quad 15$ & ${ }^{190}$ dibd & \\
\hline 86 & ${ }^{101} \mathrm{II}$ & ${ }^{190} \mathrm{AN}$ & $2 " 1$ \\
\hline 54 & ${ }^{10 x} \mathrm{Lg}$ & Por & 212 \\
\hline$v_{1}{ }_{3}$. & as & ${ }^{190} 8$ & 273 \\
\hline 160 & 38 & 29 & 284 \\
\hline A.G. & 206 & 200 & 215 \\
\hline g.g. & .2 & 201. & 216 \\
\hline 9.6.R & ${ }^{107} 29$ & ${ }^{202}$ IR & 217 \\
\hline D. 9. & P.es & 2 & 210 \\
\hline F.S. & 189 & $=0$ & 219 \\
\hline 72 & C.2.116 & 205 & 220 \\
\hline 71 & I91 & 200 & 229 \\
\hline$\pi$ & AMarinic & 207 & 222 \\
\hline${ }^{178}$ R. & ${ }^{193}$ dibe & 308 & 223 \\
\hline .e.3. & ${ }^{194} \mathrm{Cu}$ & 209 & 224 \\
\hline
\end{tabular}




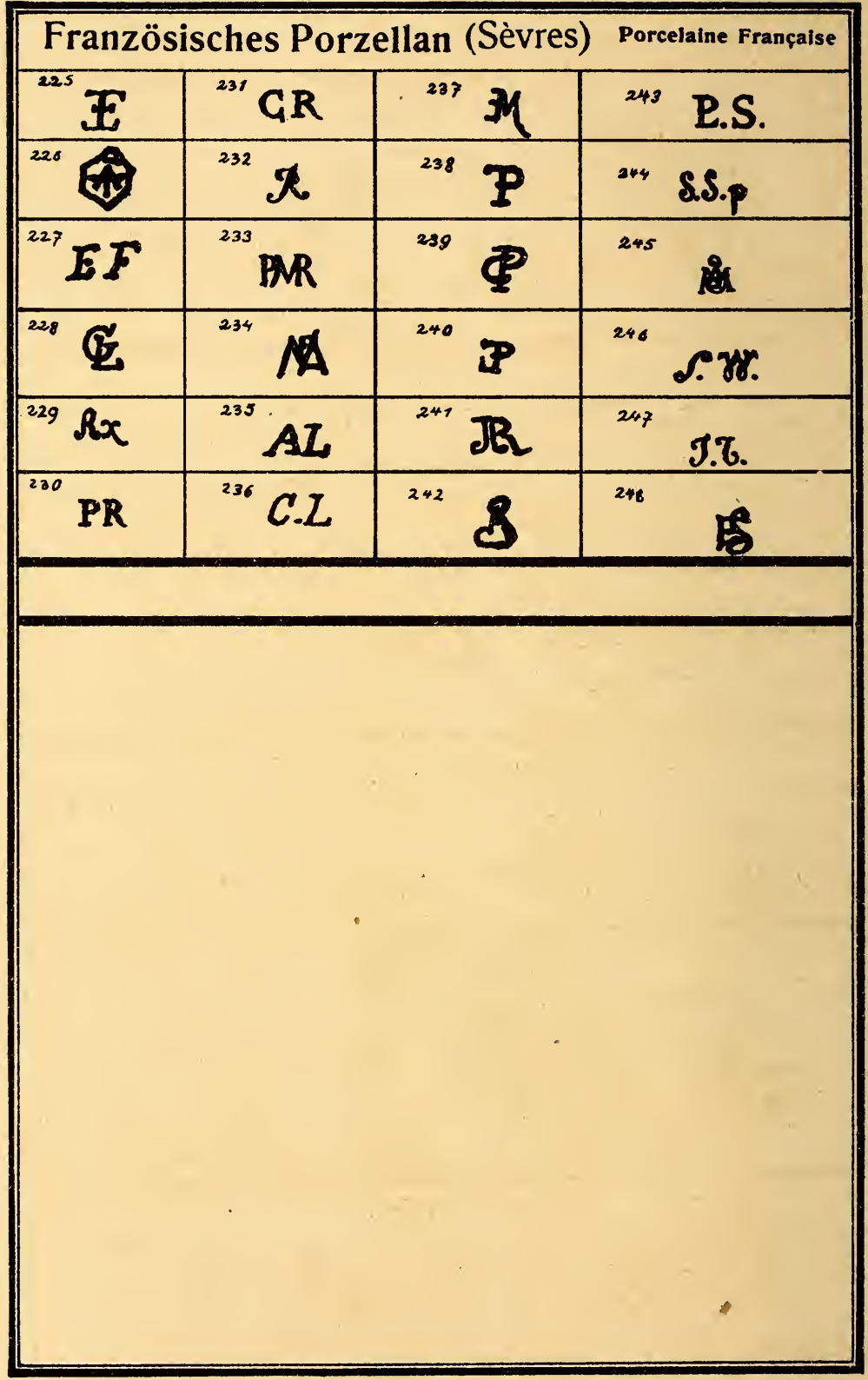




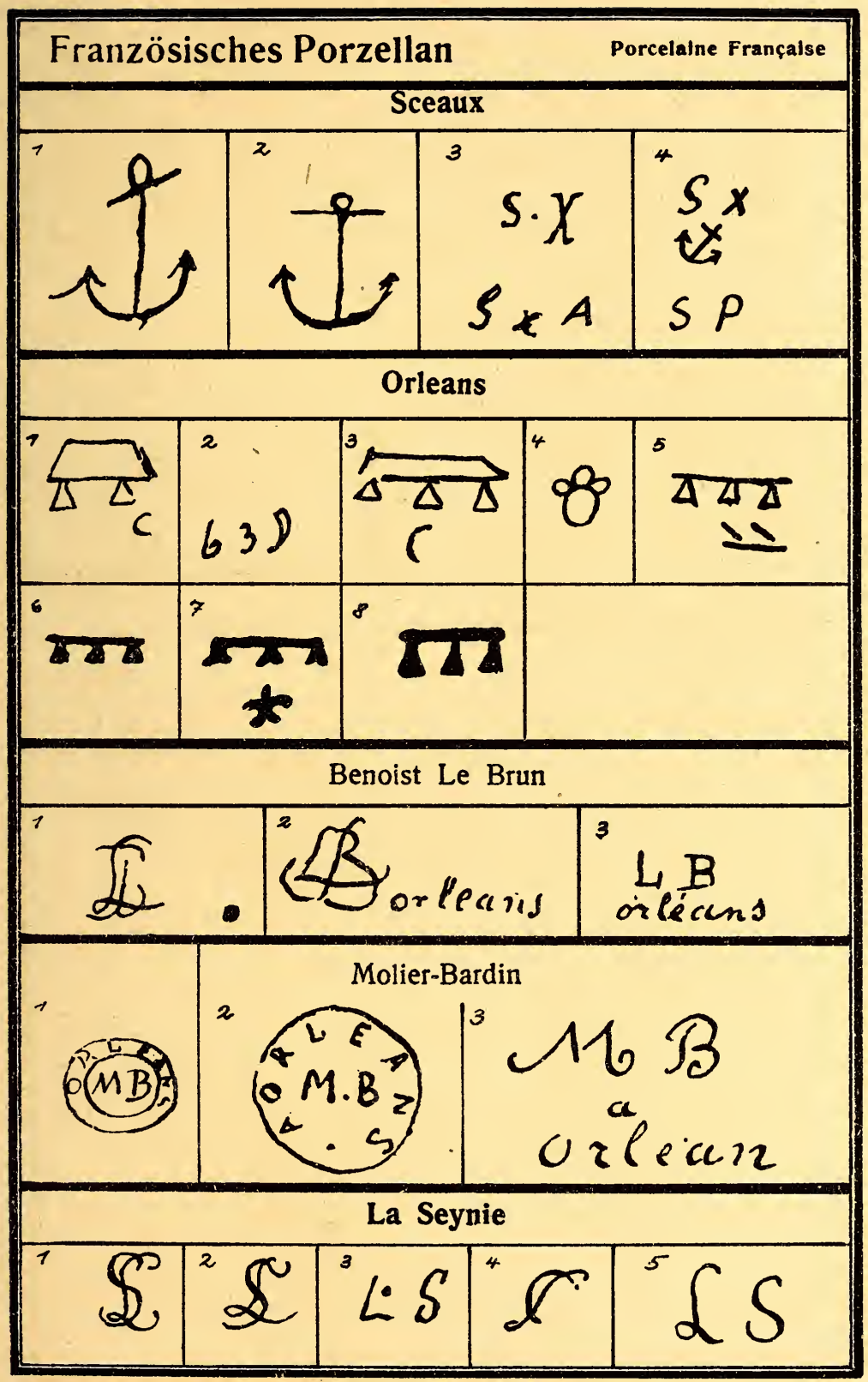




\section{Französisches Porzellan}

Porcelaine Française

Paris

\begin{tabular}{|c|c|c|}
\hline Ville d'évéque & Rue Taranne & Rue de la Roquette \\
\hline$C_{+} M$ & $\begin{array}{c}\text { DENONT } \\
\text { P.TRTHMP } \\
\text { PAR/S }\end{array}$ & \\
\hline
\end{tabular}

Faubourg Saint-Denis

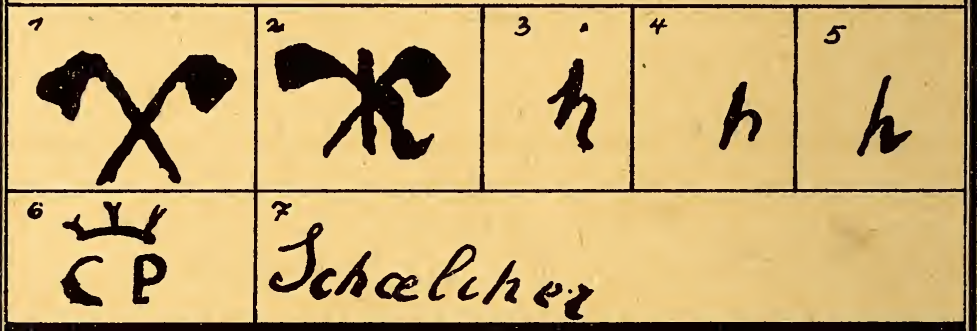

Rue Fontaine-au-Roy (la basse Courtille)

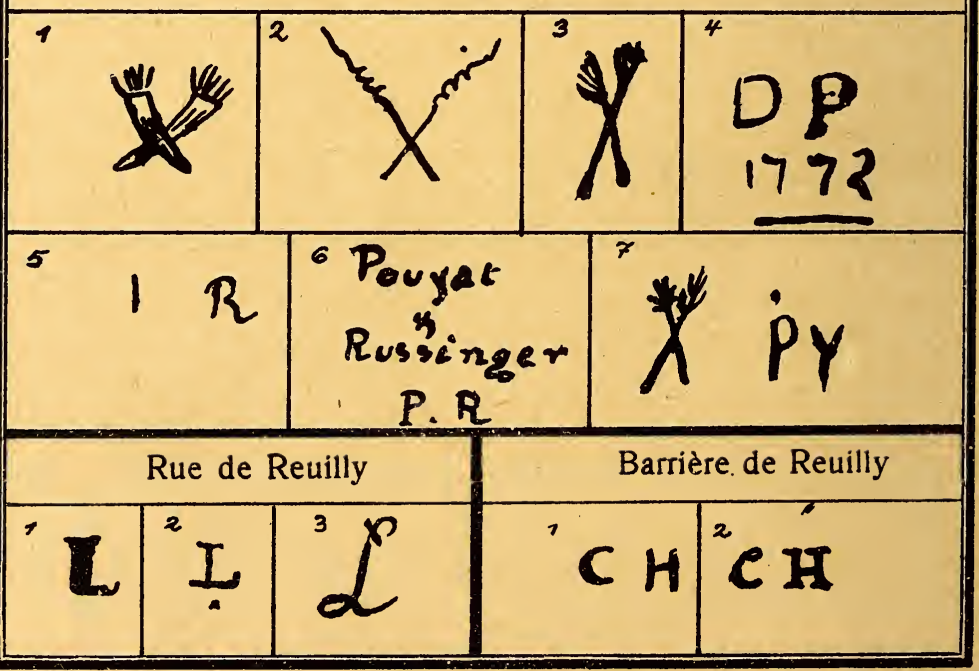




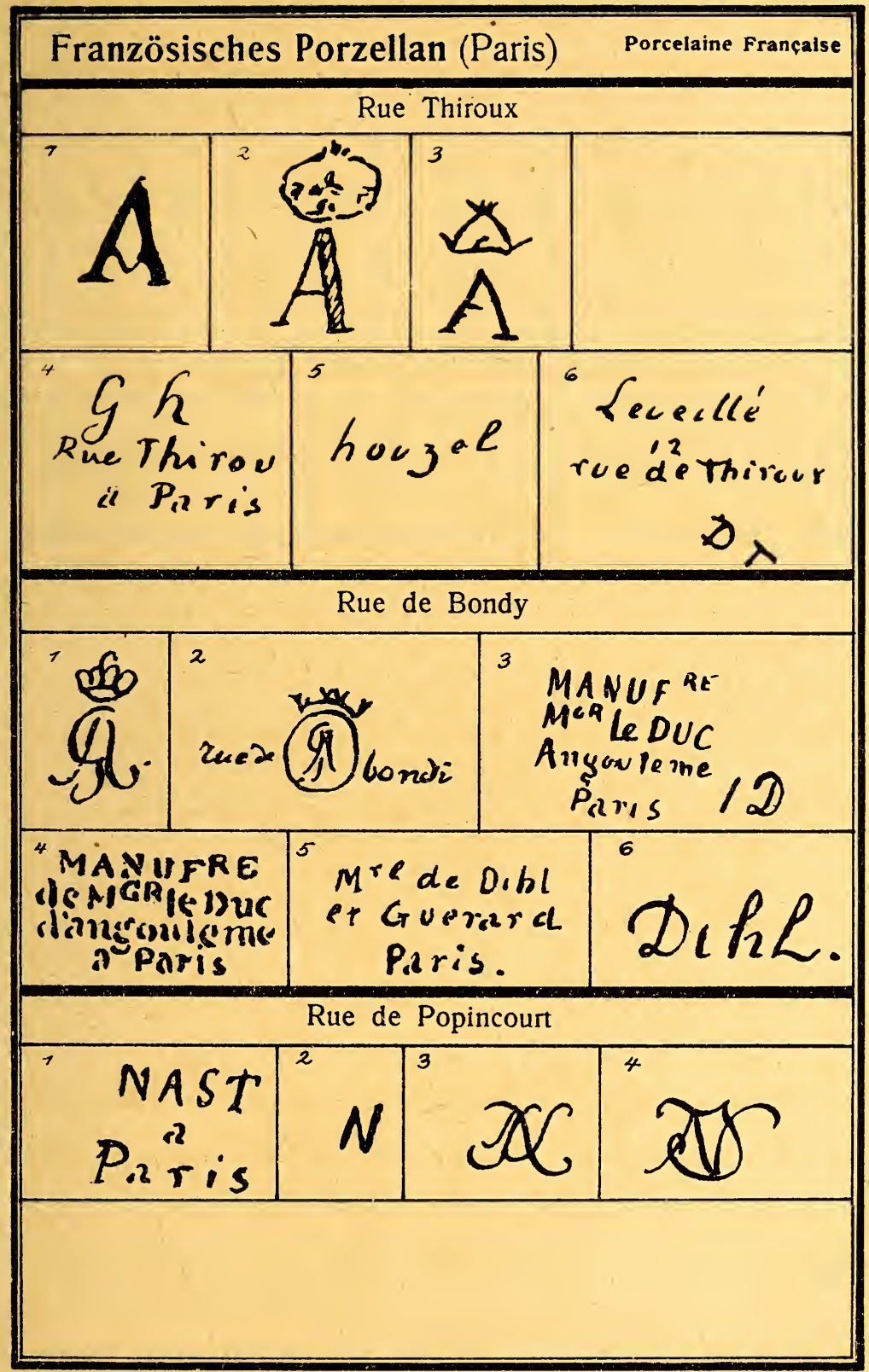




\section{Französisches Porzellan (Paris) Porcelaine Françalse}

Pont-aux-choux (Rue Amelot)

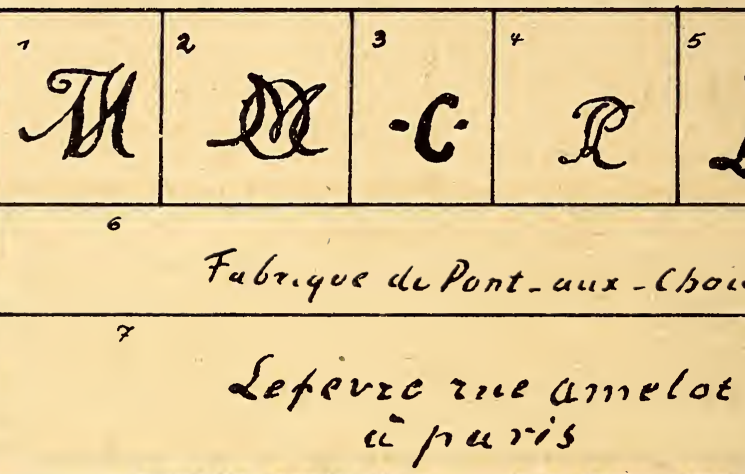

\begin{tabular}{|c|c|c|c|c|}
\hline \multicolumn{5}{|c|}{ Petit Carrousel } \\
\hline$c^{P}{ }_{0}$ & \multicolumn{2}{|c|}{$\begin{array}{l}C^{P} G \\
\text { MANUFACTURE } \\
\text { du Petit Cirocesel } \\
\text { a Paris }\end{array}$} & & \\
\hline \multicolumn{5}{|c|}{ Rue St.-Gilles } \\
\hline $\mathrm{dug}_{\mathrm{a}} \mathrm{O}_{\mathrm{CL}}$ & $P \cdot L_{A}{ }^{2}$ & $\begin{array}{l}\text { ACOTL } \\
\text { ARIS }\end{array}$ & $E d$ & $\begin{array}{l}\text { HONORE } \\
\text { AR,S }\end{array}$ \\
\hline$\$ C$ & 5 & $\overline{6}$ & & \\
\hline \multicolumn{5}{|c|}{ Rue de Crussol, Rue des trois bornes } \\
\hline $\begin{array}{c}B \\
\text { Potcer } \\
42\end{array}$ & $\begin{array}{c}\text { CH. } \\
\text { potter } \\
\text { a paris }\end{array}$ & $c_{9}^{\mathcal{B}} \mathrm{P}$ & $\begin{array}{c}4 \\
B \\
\text { Poiter } \\
2\end{array}$ & $E \cdot B$ \\
\hline
\end{tabular}




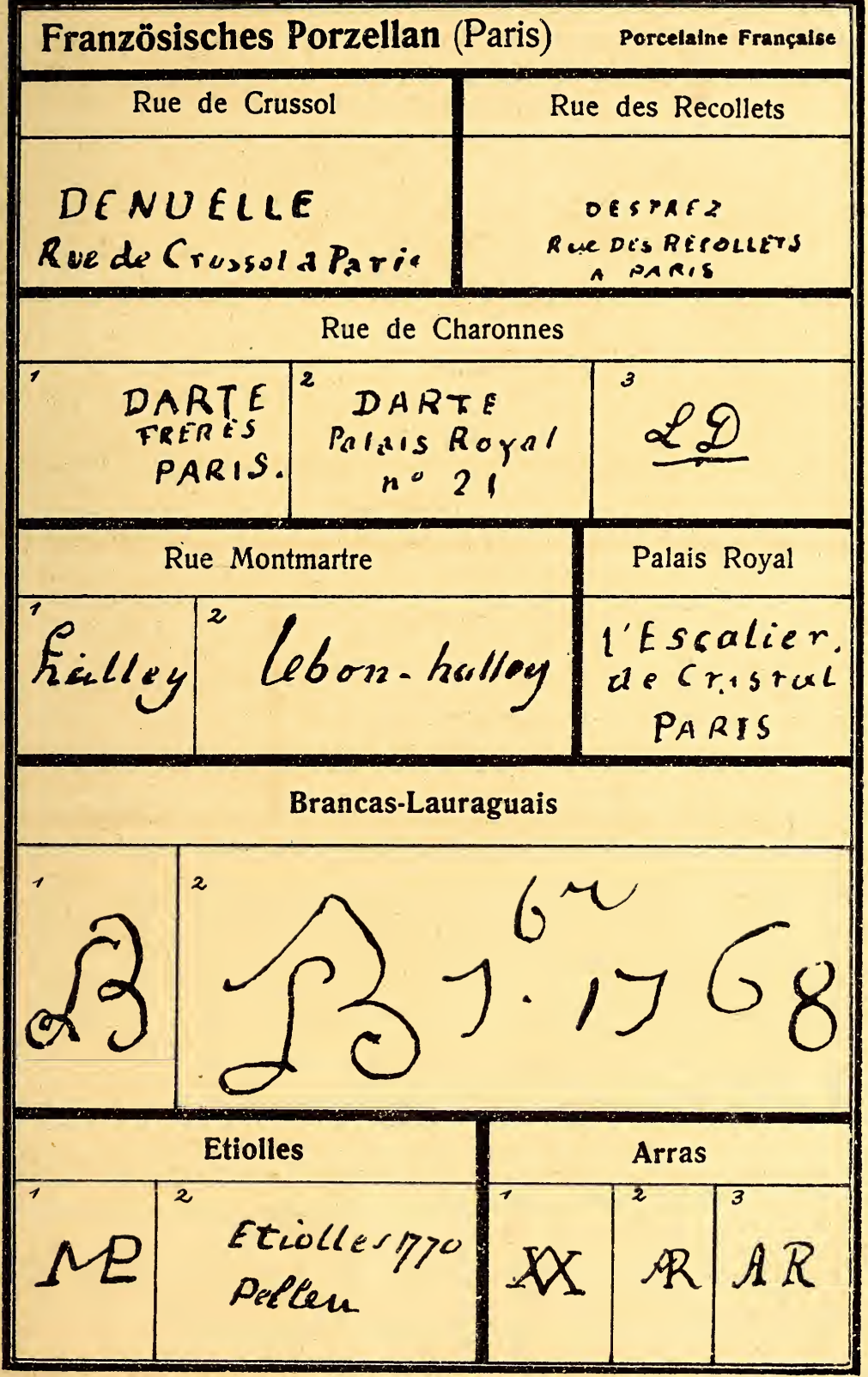


Französisches Porzellan

Porcelaine Françalse

\section{Clignancourt}
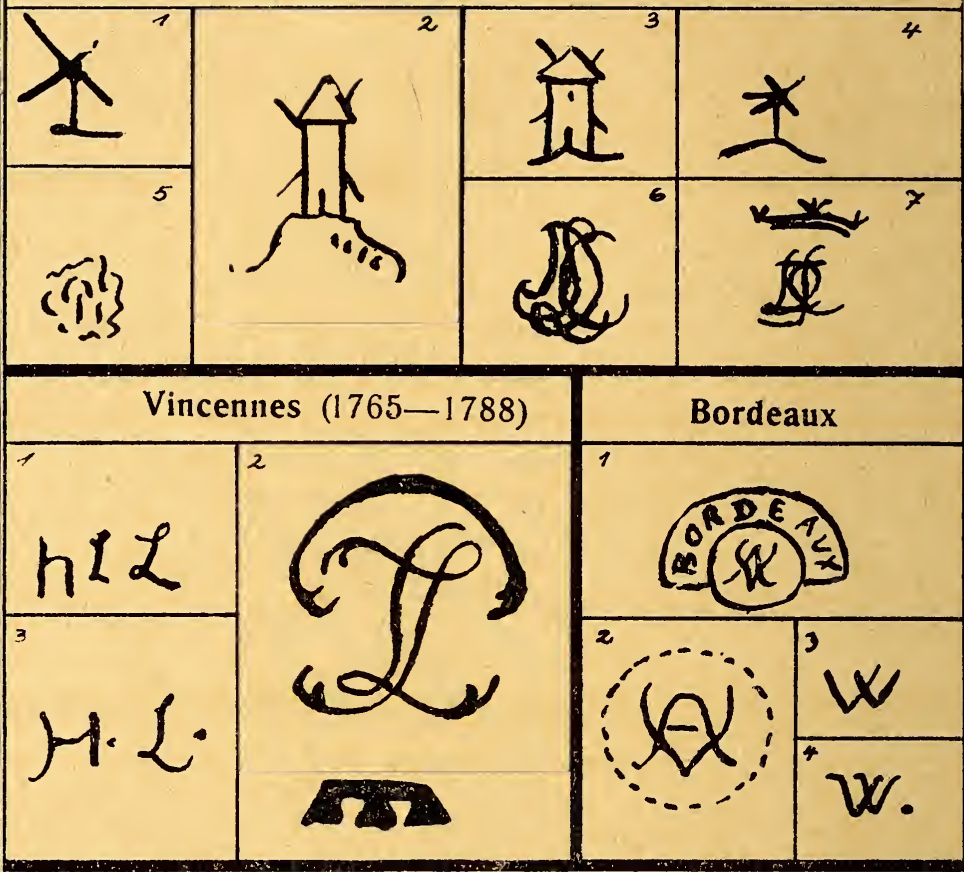

Lunéville

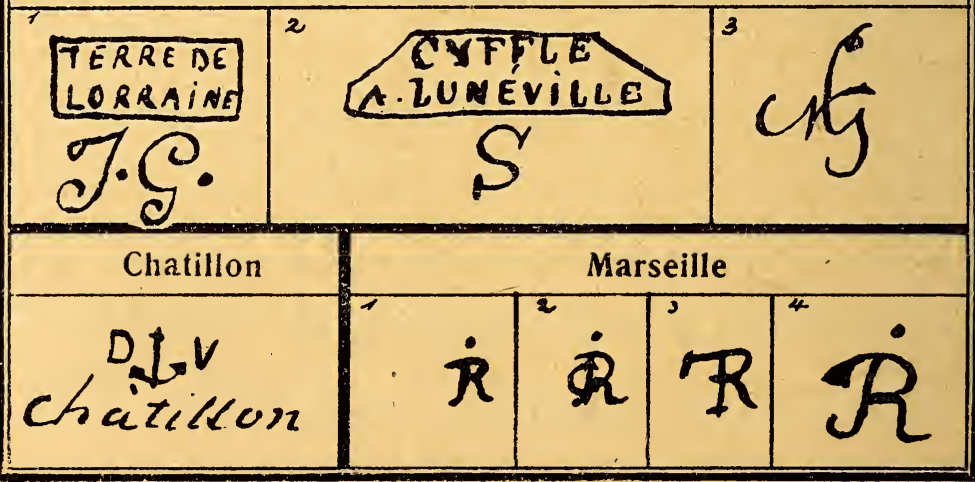


Französisches Porzellan

Porcelaine Française

Valenciennes

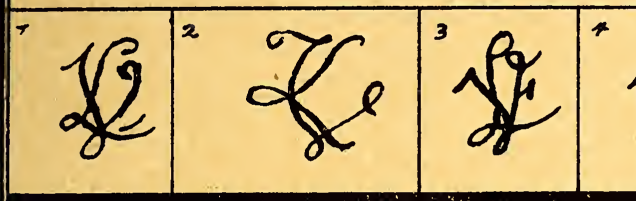

Bayeux

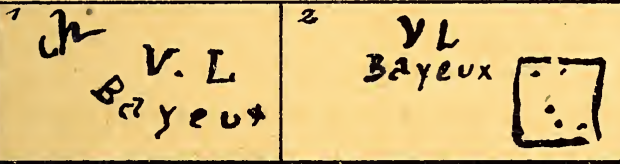

Boisette

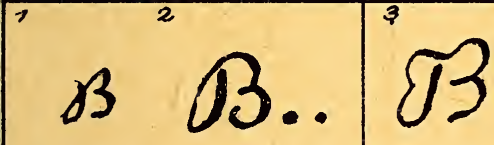

Saint-Maurice, Montreuil-Sous-Bois (Samson)

\section{Crepy-en-Valois}

crepy ${ }^{2}$ c.p.

Saint-Amand-les-Eaux

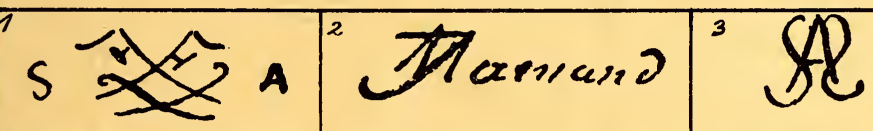


Englisches Porzellan

Porcelaine Anglaise

\section{Chelsea}

1750 bis ca. 1753
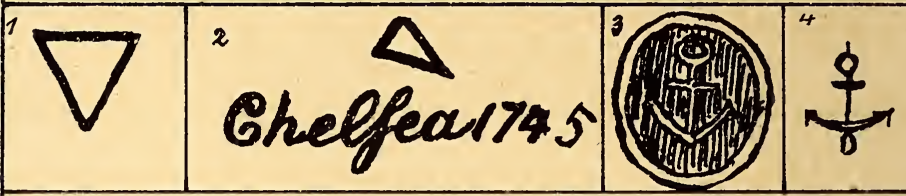

ca. $1753-1758$

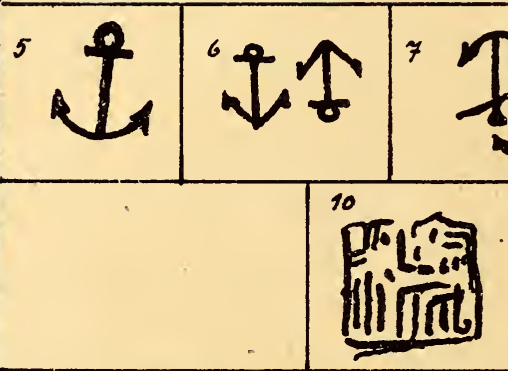

Chelsea-Derby $1770-1784$

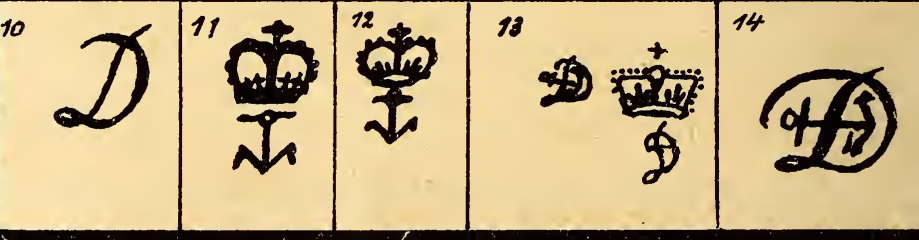

Derby

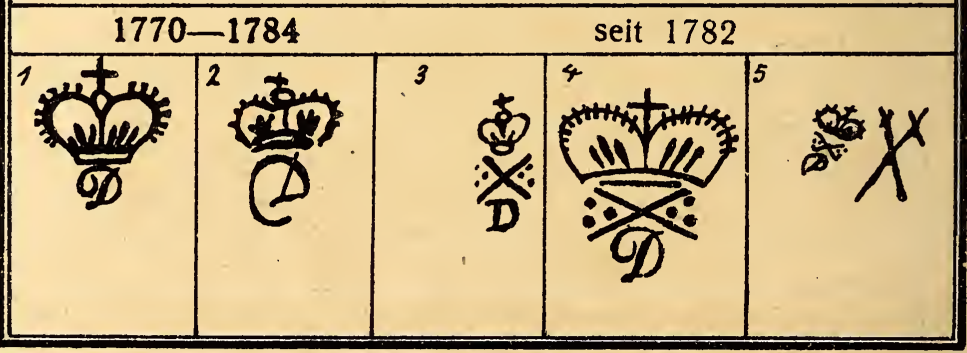


243

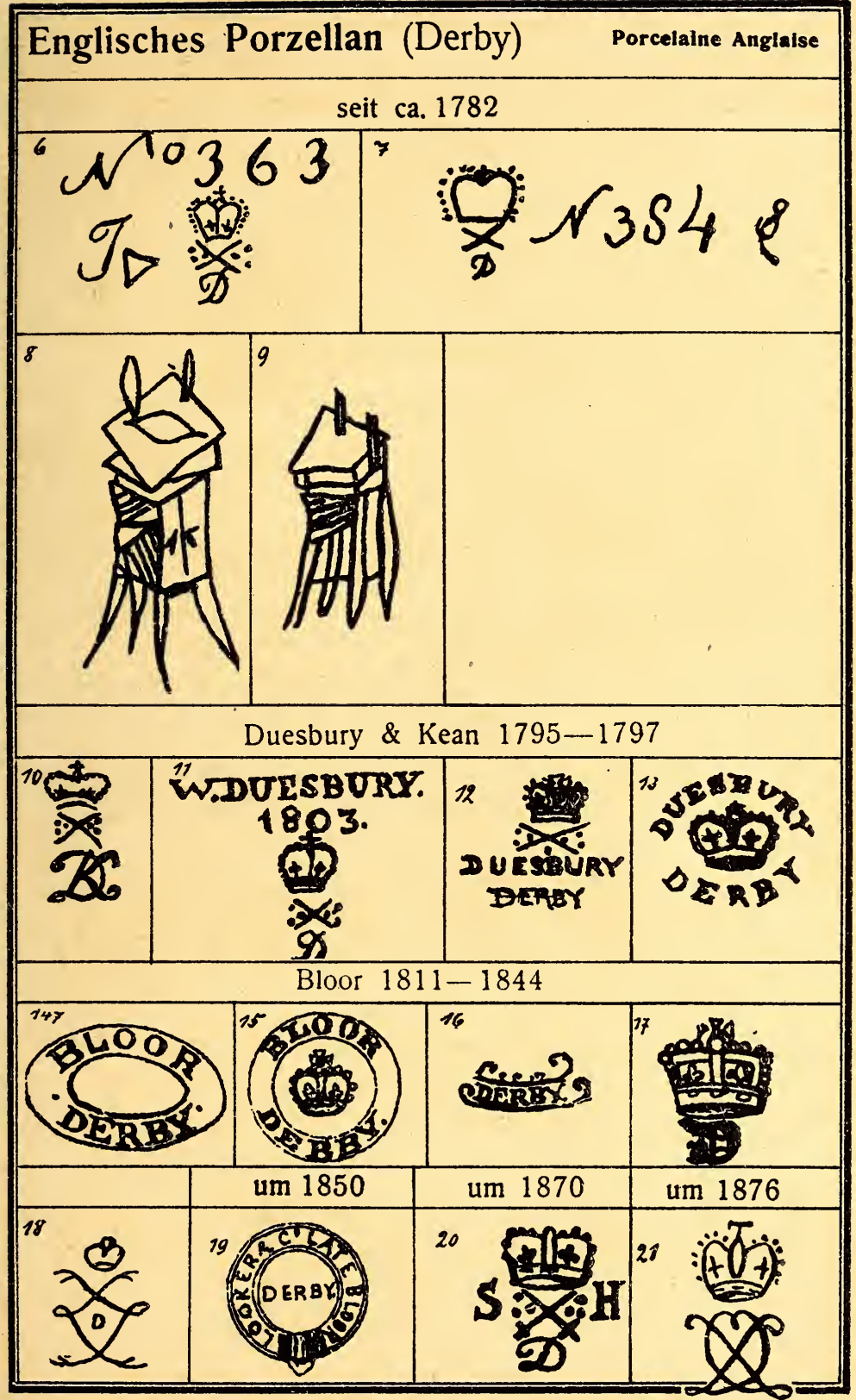




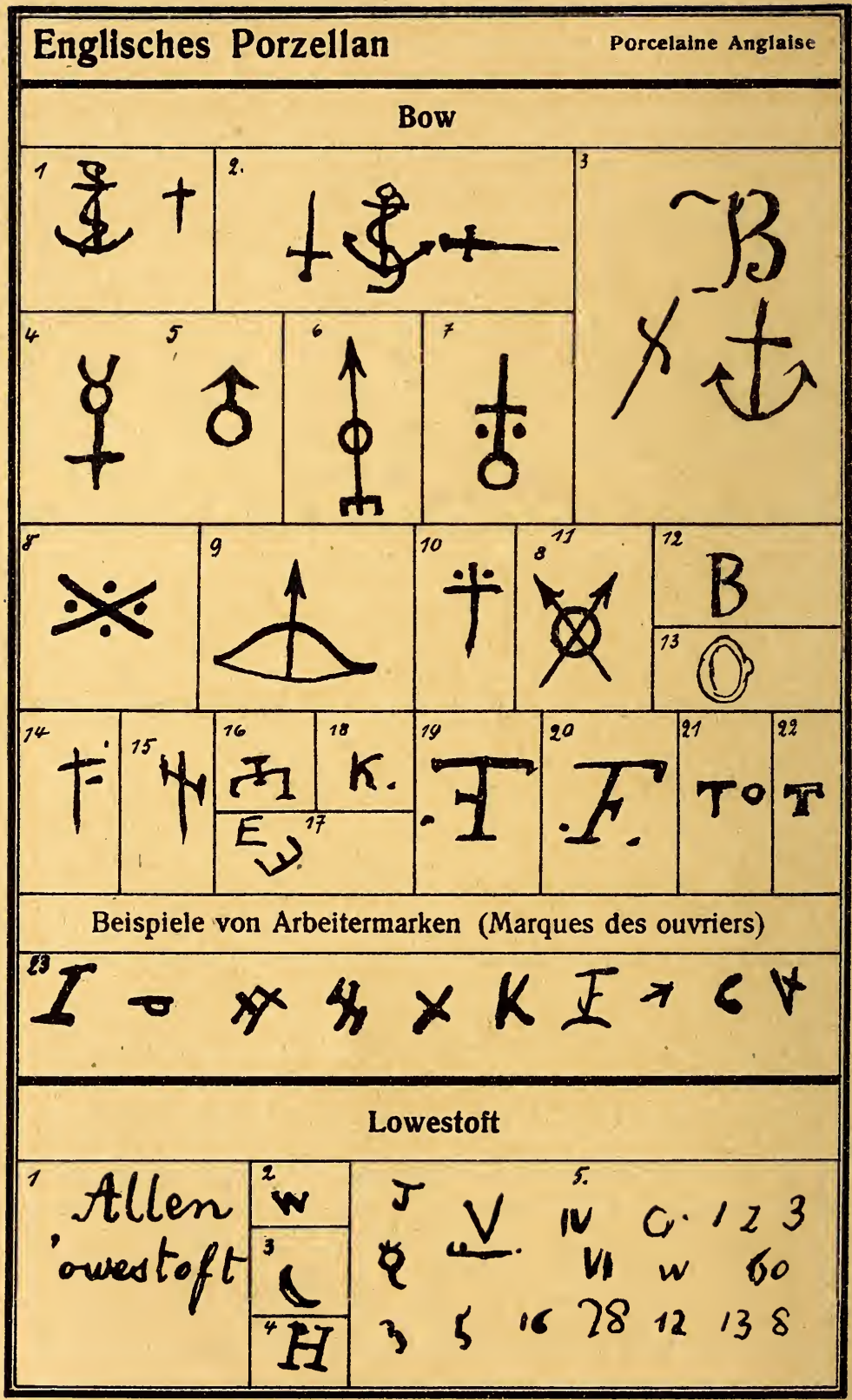




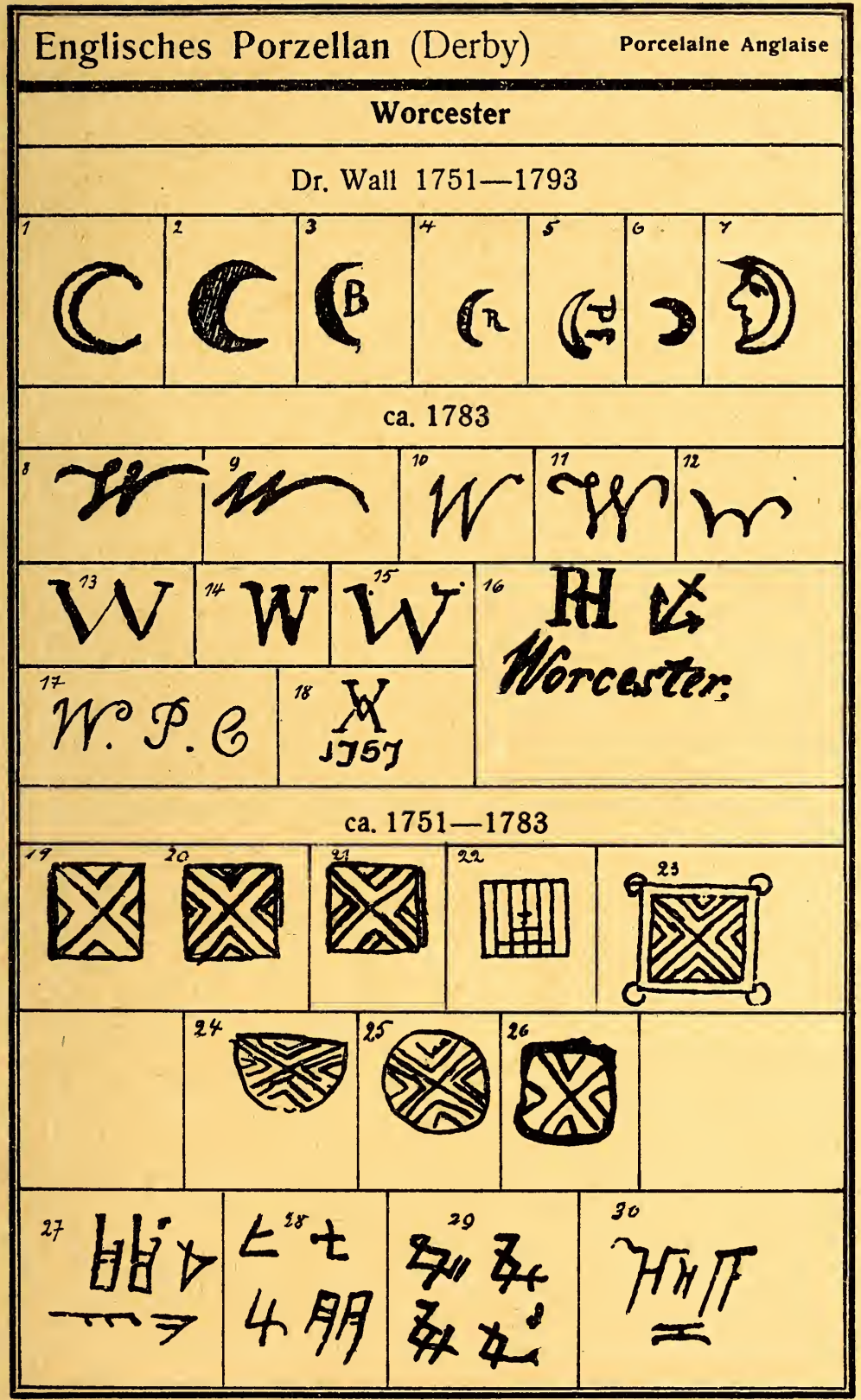




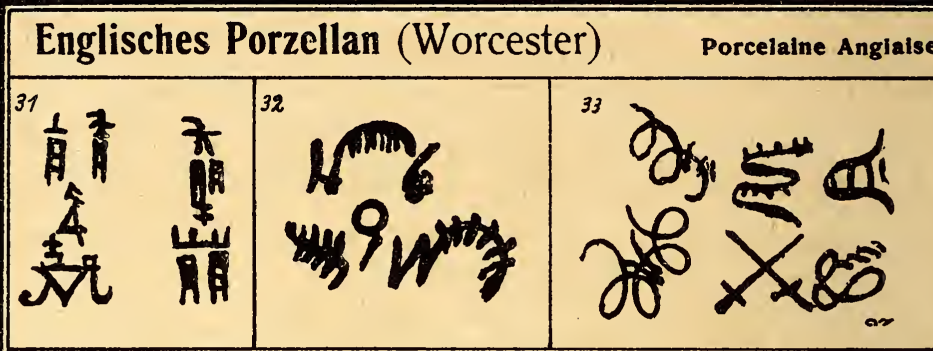

Nachbildungen Kontinentaler Marken (Imitations des marques du continent) ${ }^{34} \mathrm{q}^{35}$
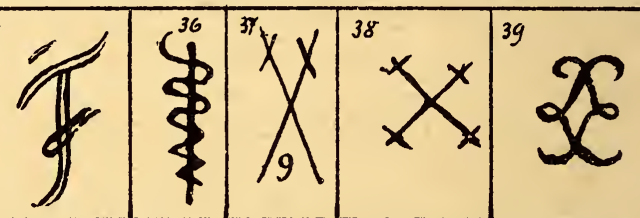

Arbeitermarken (Marques des ouvriers)

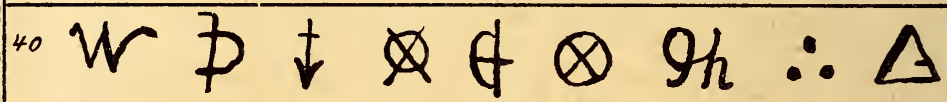
月义业入的的。 与 $\exists \odot \bar{\delta} \equiv 25$ 叶 - 以丈<xVE 然 $\rightarrow$ 业 EEH $\neq H \uparrow \rightarrow$ H A

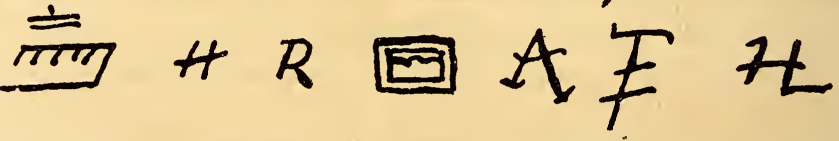




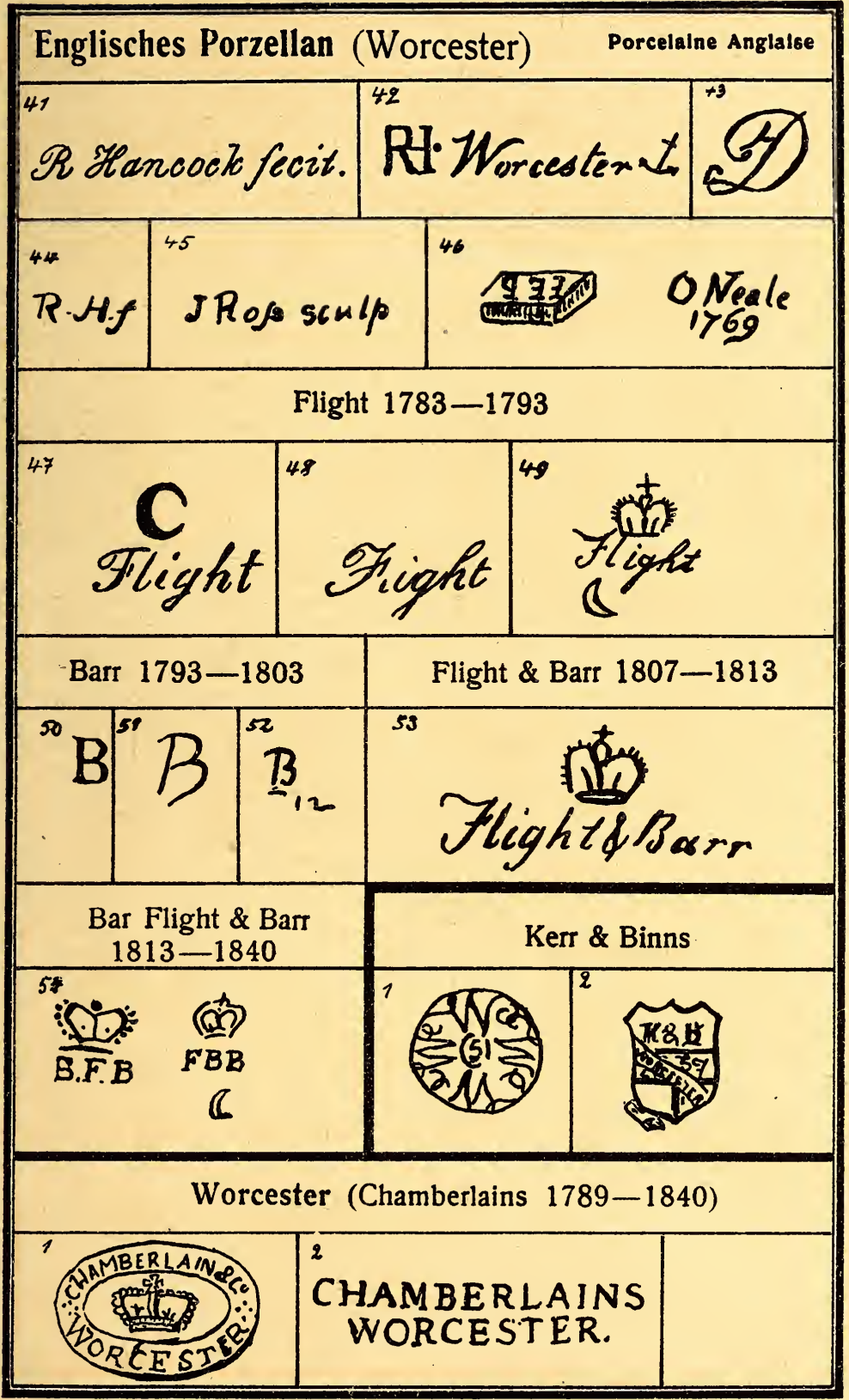




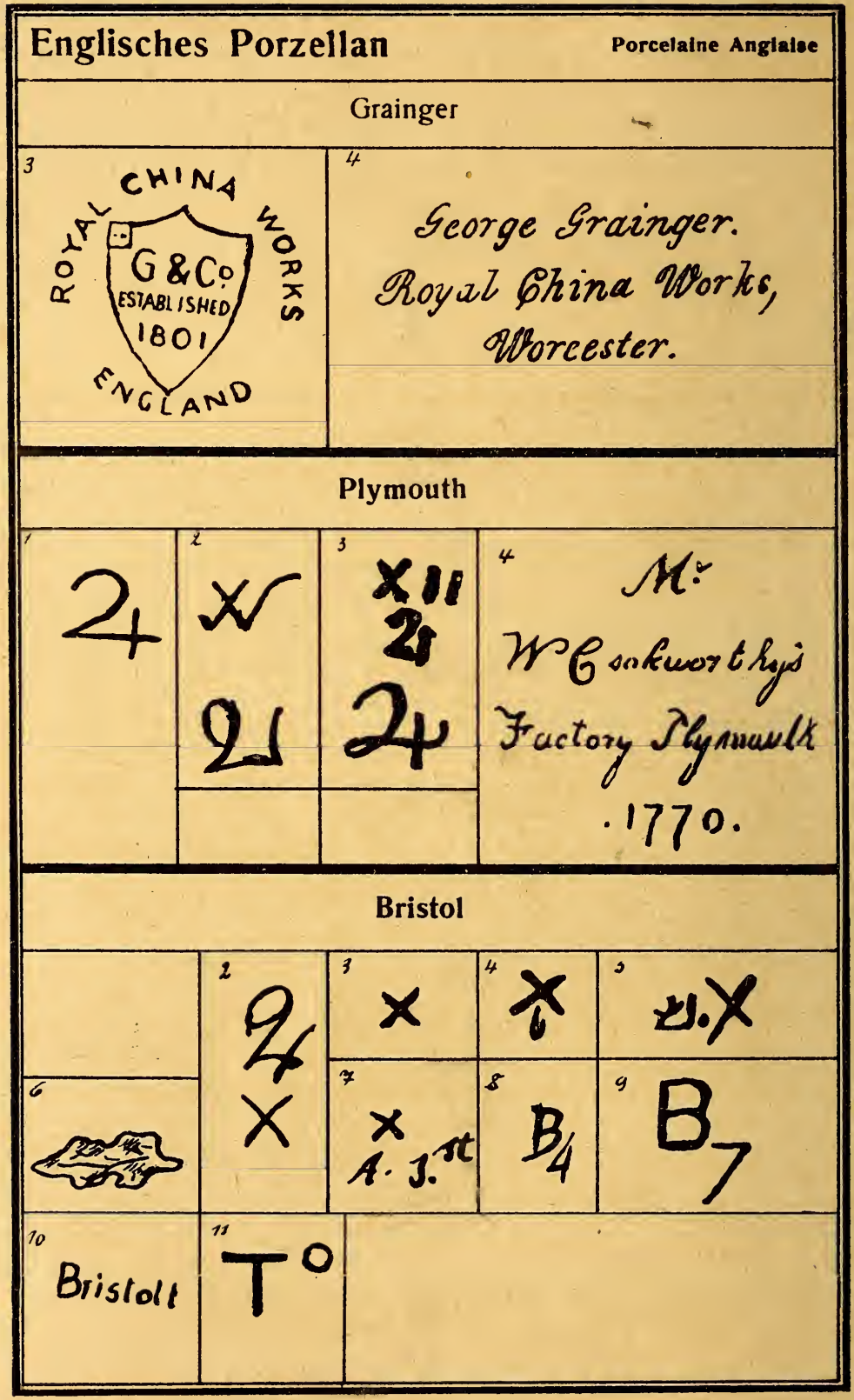




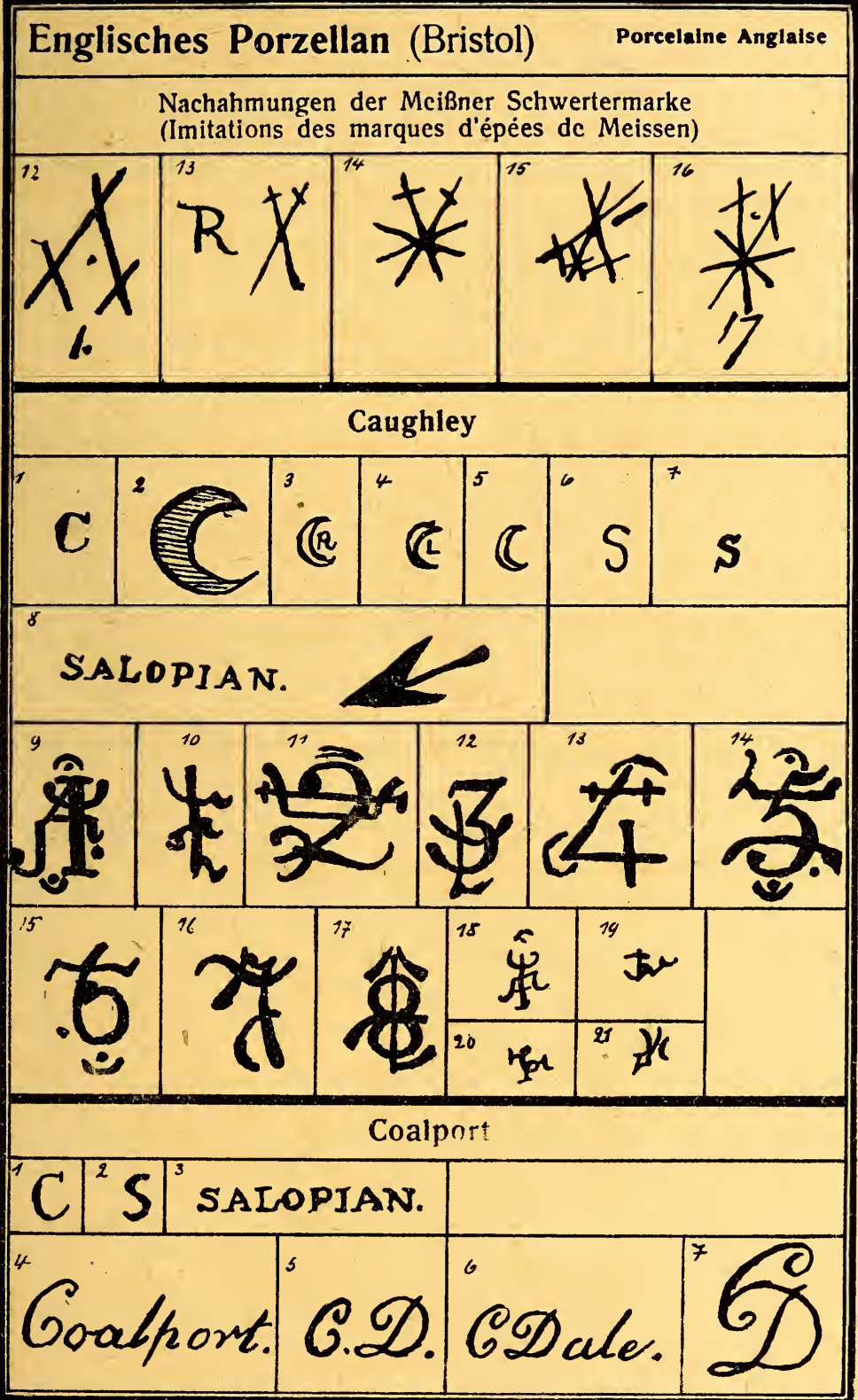


Englisches Porzellan (Coalport) Porcelaine Anglalse

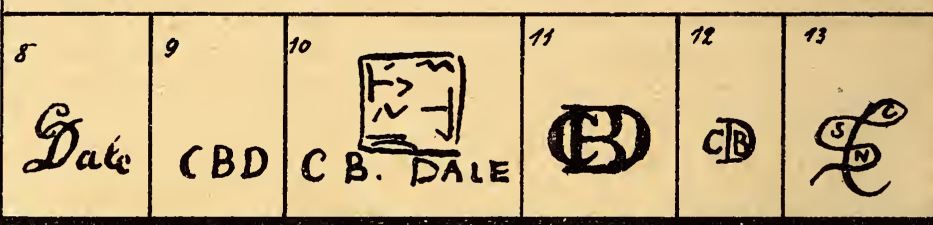

Longton Hall

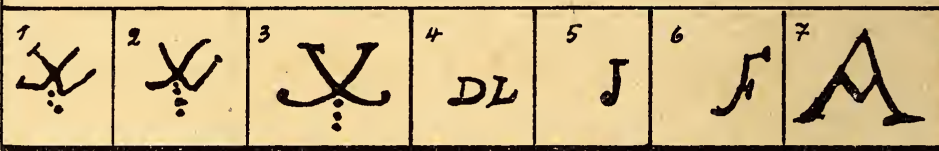

Shelton, New Hall

Isleworth

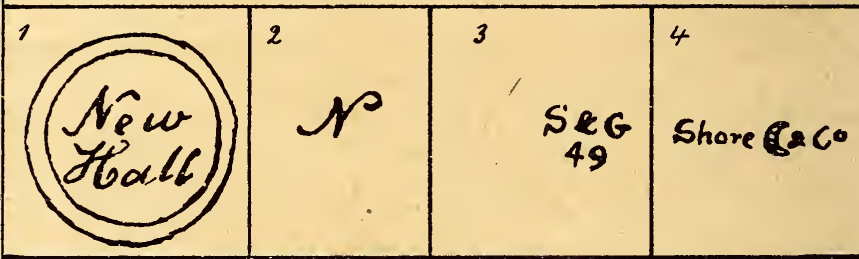

Nantgarw

"NANTGARW

'NANT GARW
C.W.

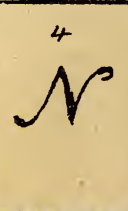

Swansea

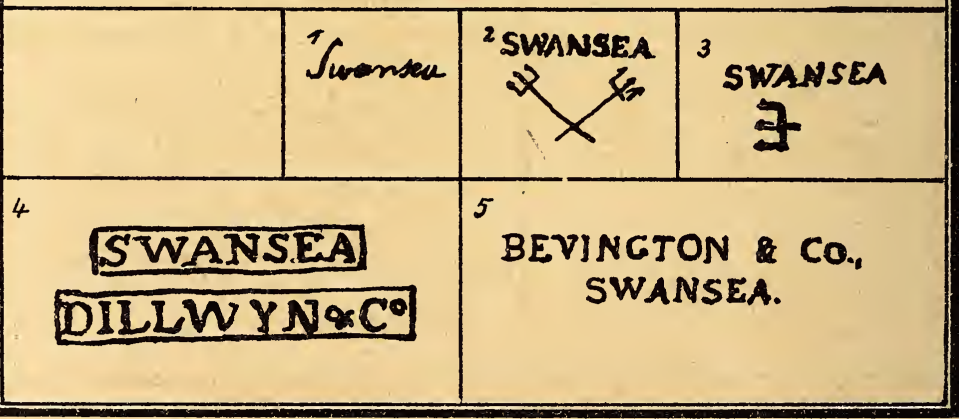


Englisches Porzellan

Porcelaine Anglaise

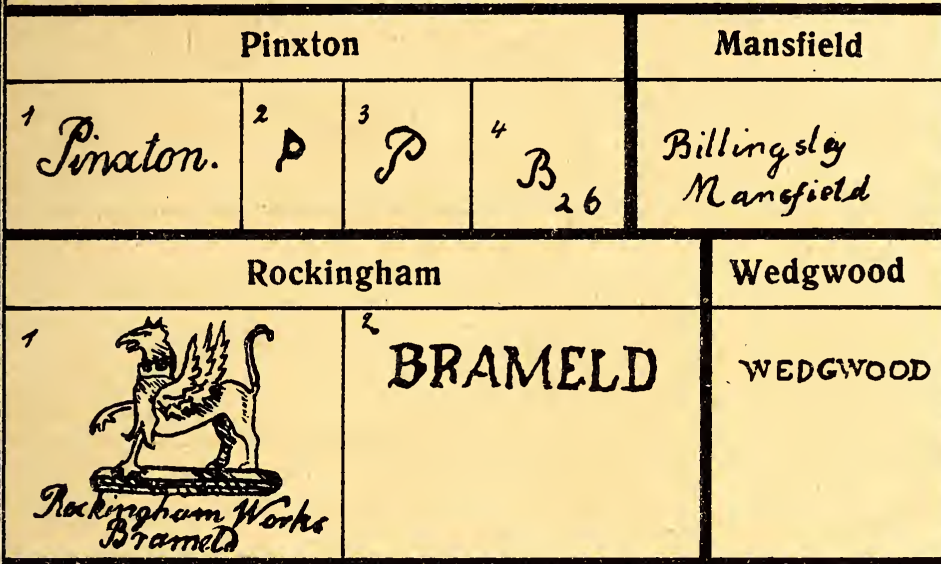

Stoke uper Trent Spode

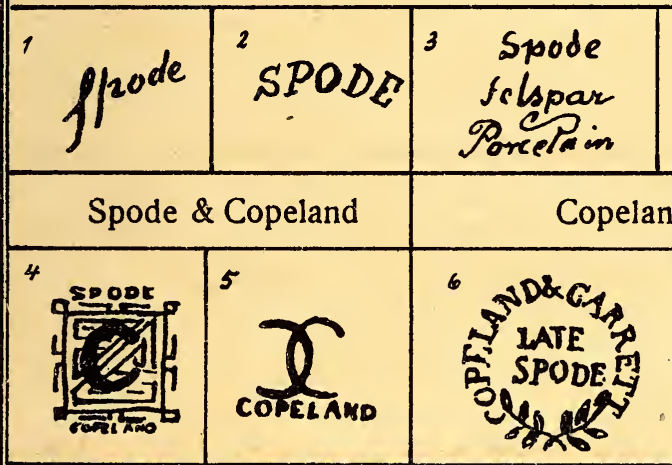

\begin{tabular}{|l|l|l|l|l|}
\hline \multicolumn{3}{|c|}{ Minton } & \multicolumn{3}{c|}{ Longport Davenport } \\
\hline & & & \\
\hline
\end{tabular}




\section{Irländisches Porzellan}

Porcelaine Irlandaise

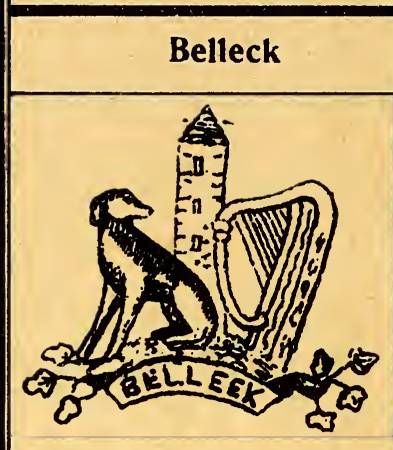

\section{Donovan}

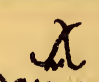

Donuraw 481

Donovan's Irish Menufaciure 
253

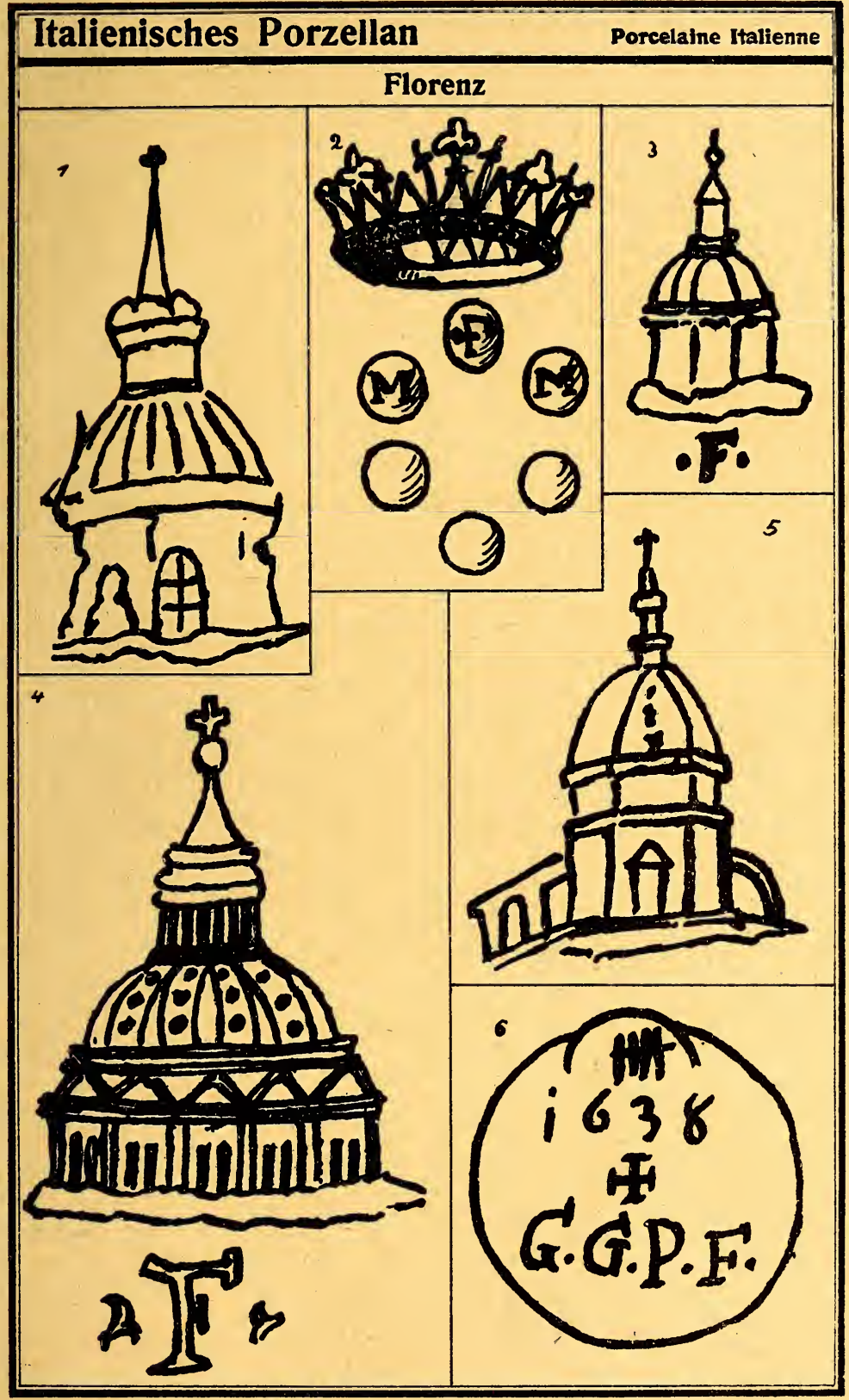




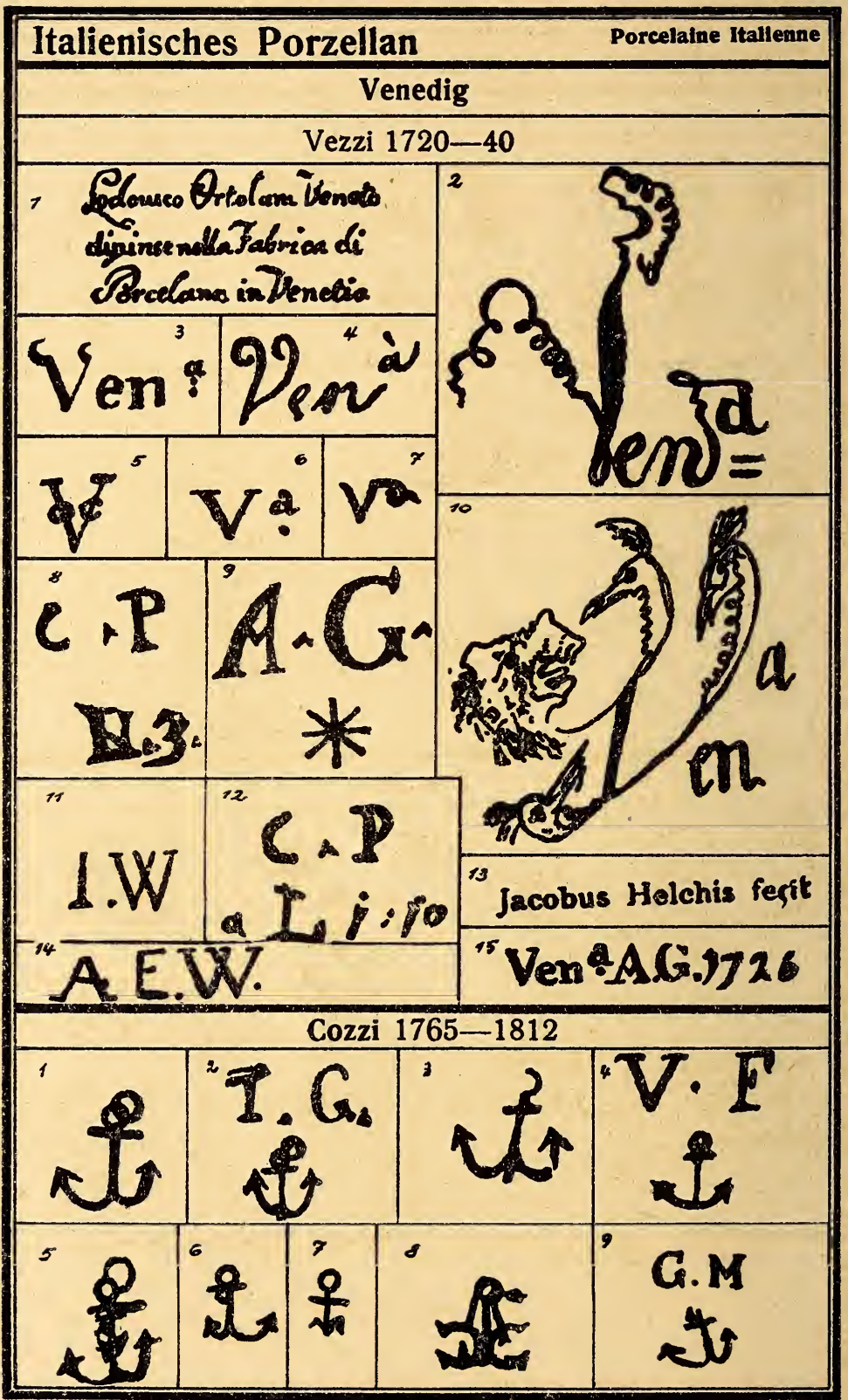




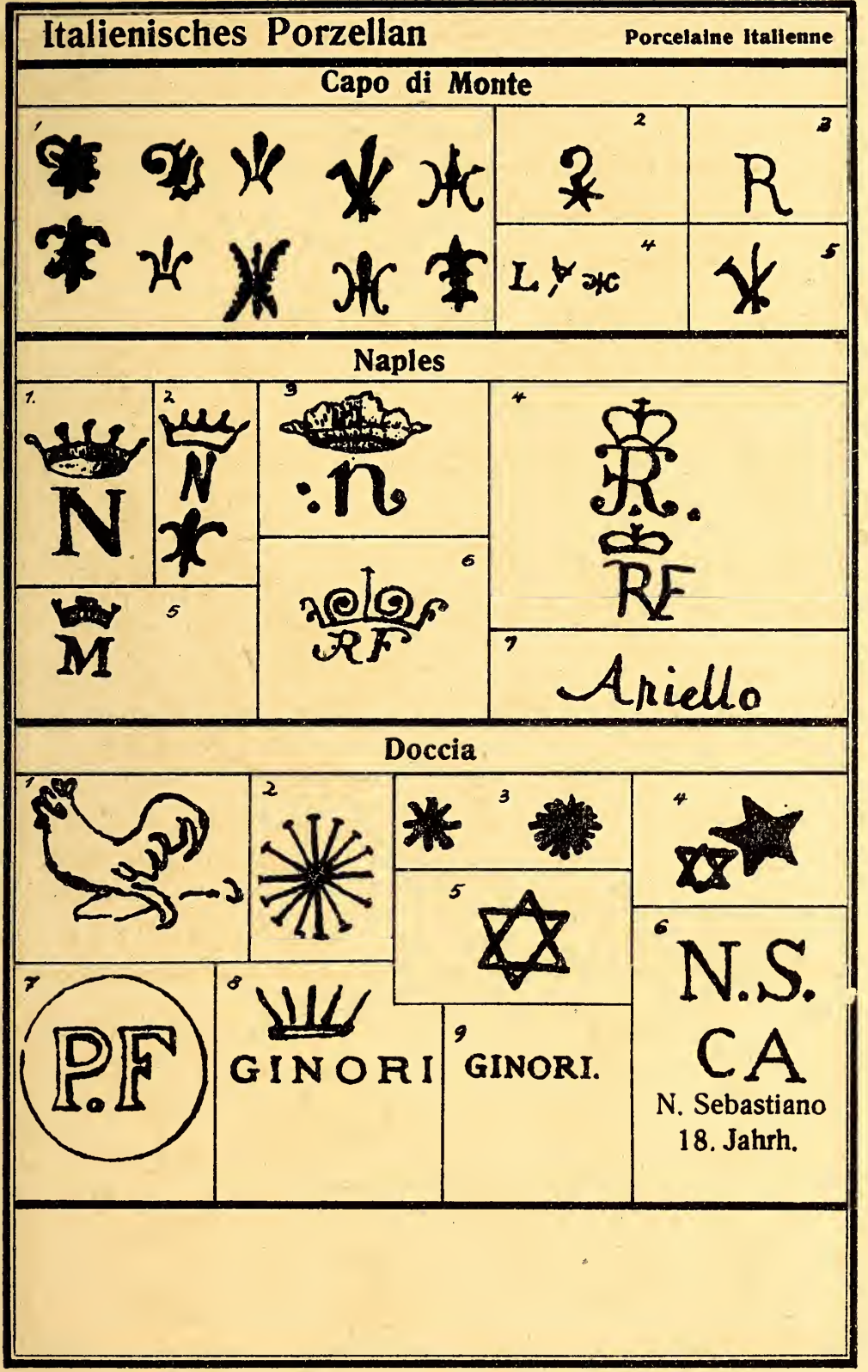


Italienisches Porzellan

Porcelaine Italienne

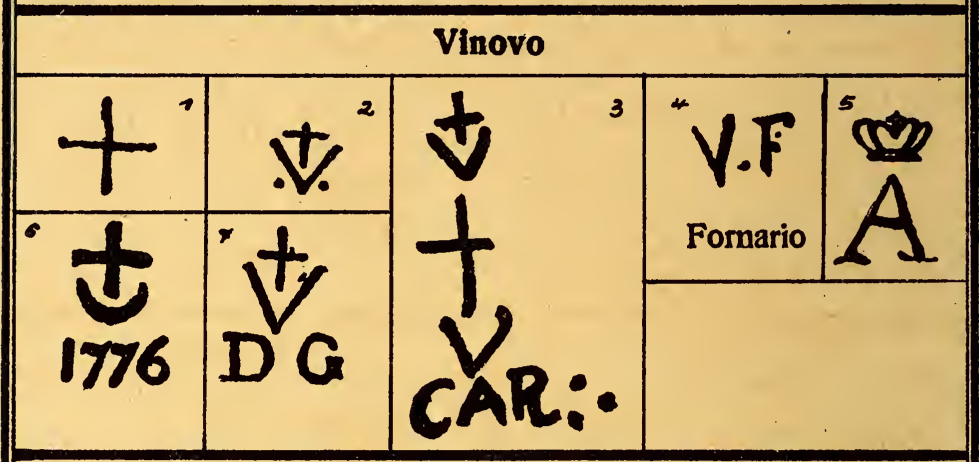
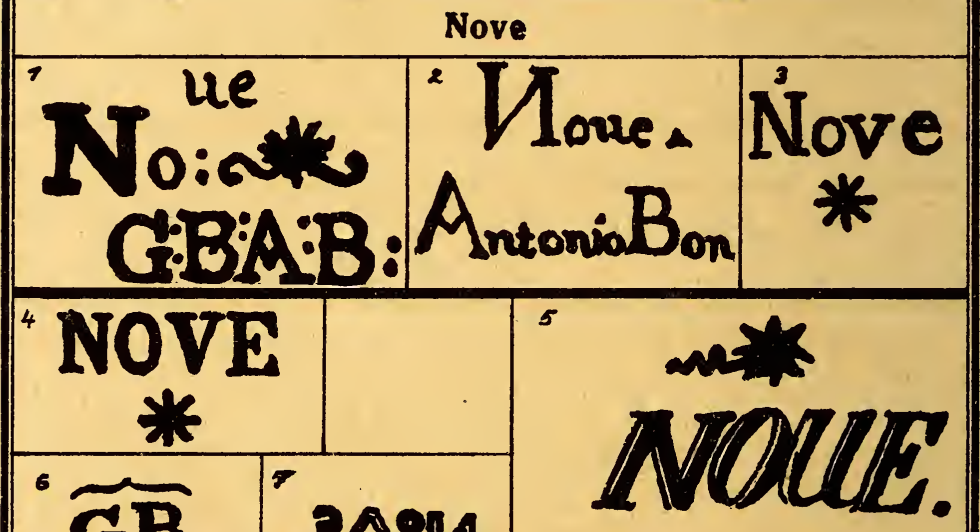

$\widetilde{\mathbf{G B}} 2 \times 0 \mathrm{H}$ NOLLS. NOVh Nove dianthomi poinstise

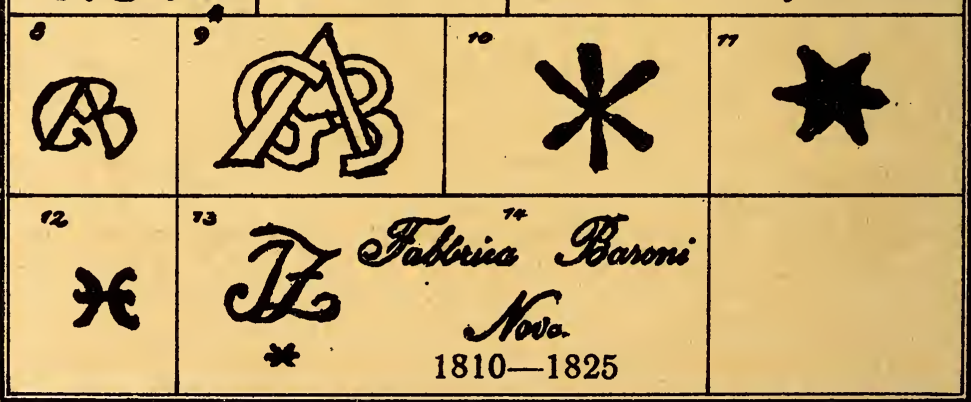


257

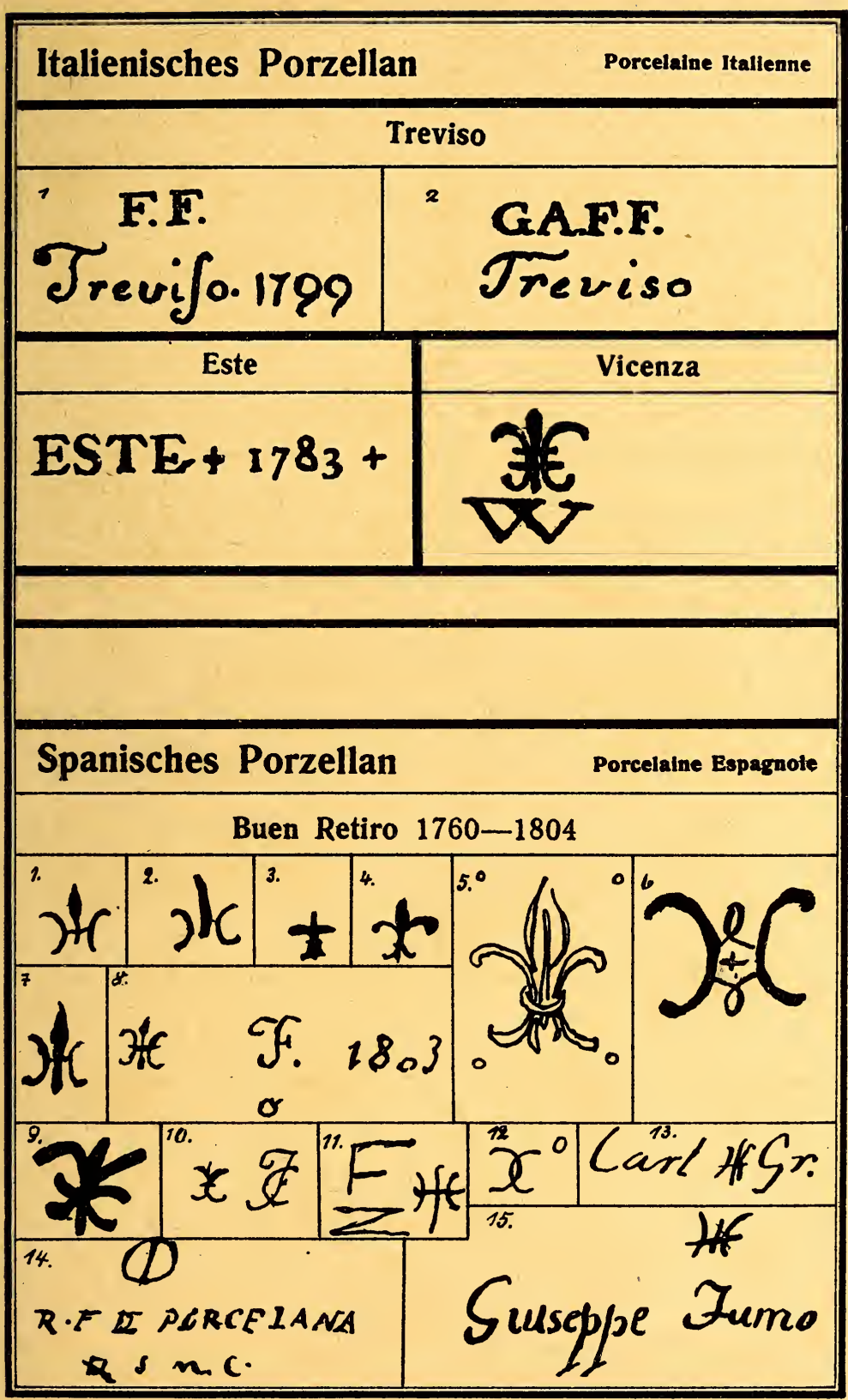




\section{Spanisches Porzellan}

Porcelaine Espagnole

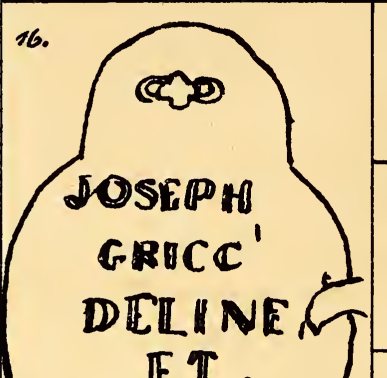

17. 7

7775

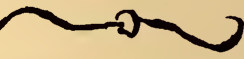

18.

SCOL

1 ता 3

it
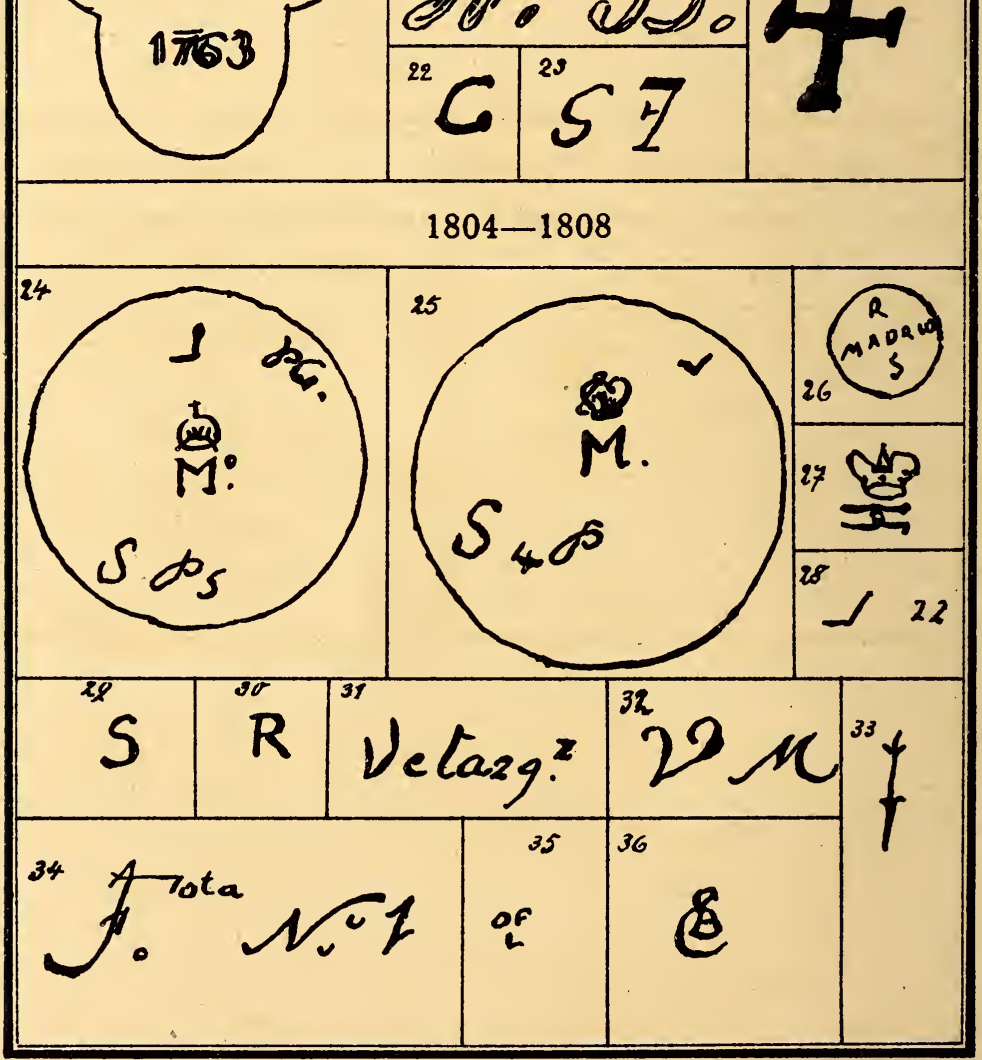


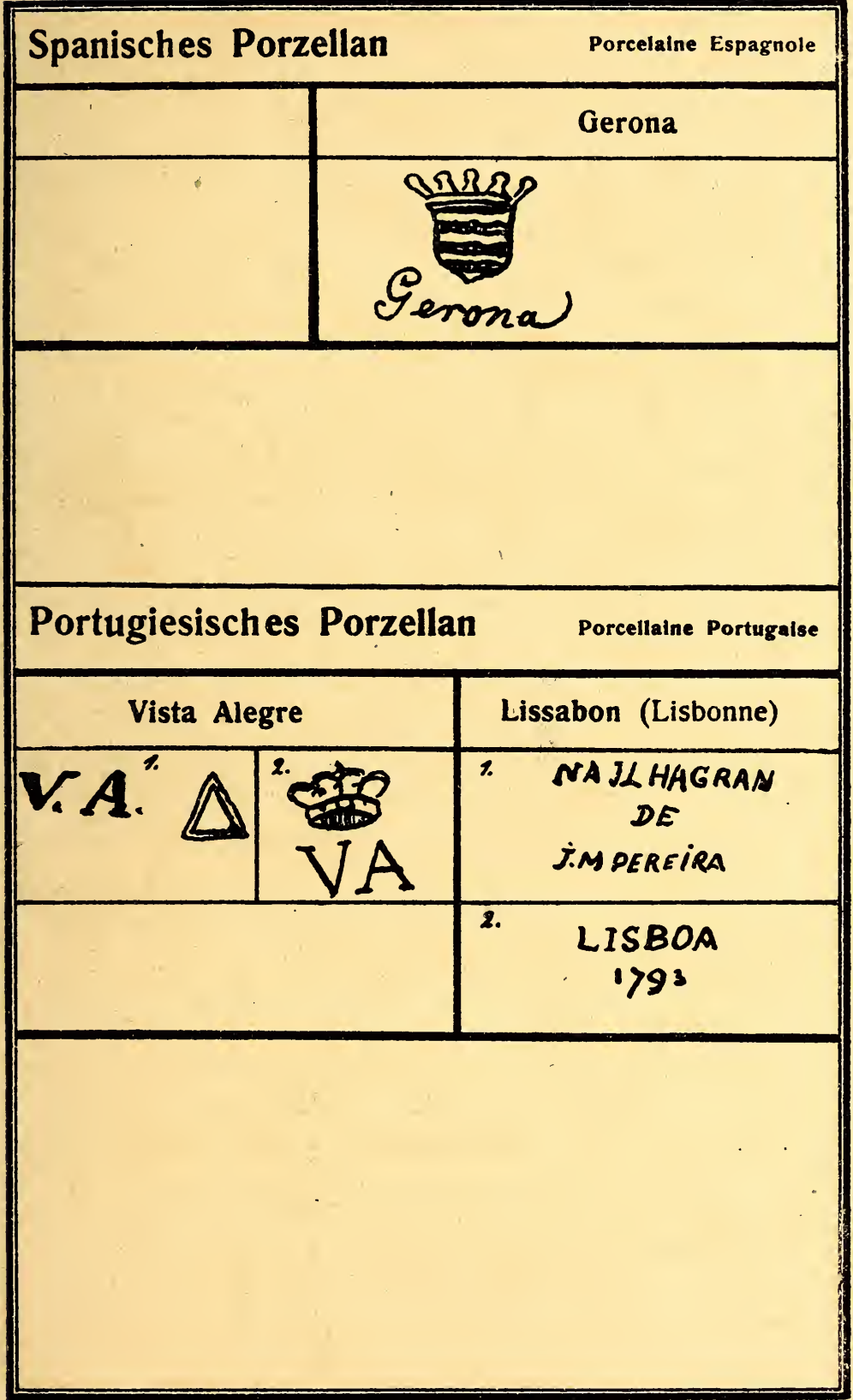



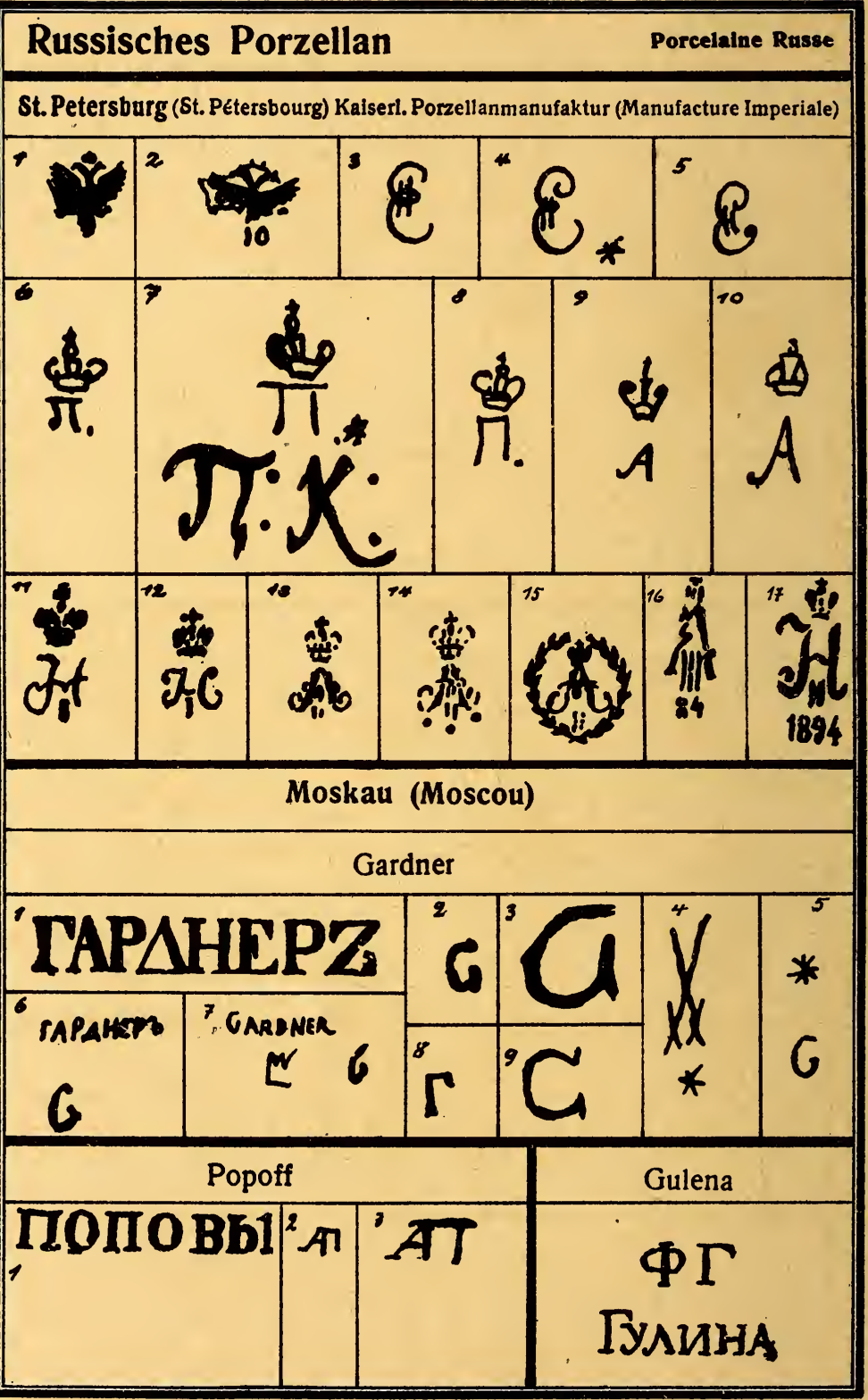
Russisches u. polnisches Porzellan Porcelane Russe el Polonaise

\section{Baranowka}

\begin{tabular}{|c|c|c|c|}
\hline 9 & ${ }^{2} *$ & 3 Borian oute. $^{*}$ & Karanowka \\
\hline 5 & & 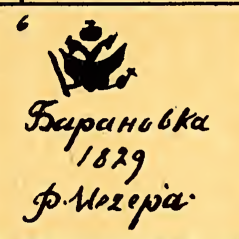 & Scercrionke \\
\hline & $M$ & $\begin{array}{c}\text { Paranauka } \\
\text { B }\end{array}$ & $826=$ \\
\hline
\end{tabular}

Korzec

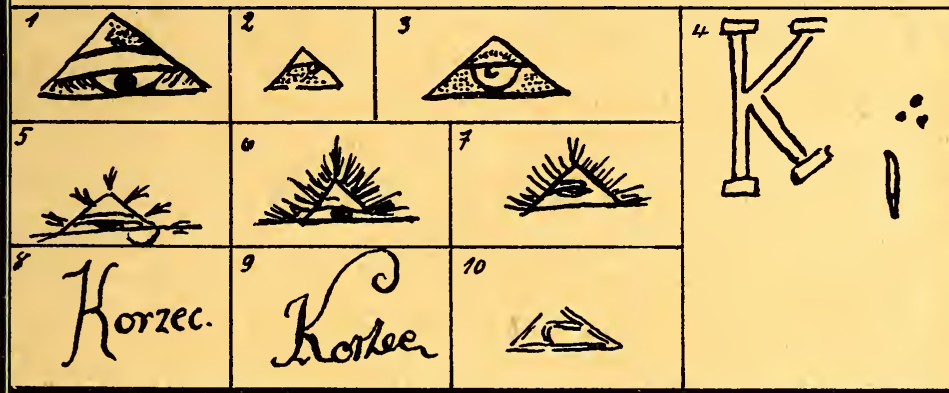

Proszowice

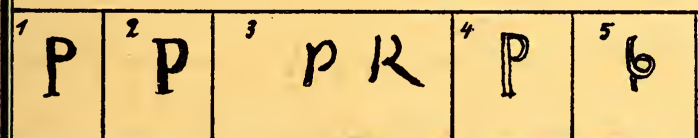


Russisches u. polnisches Porzellan Porcelaine Russe et Polonalse

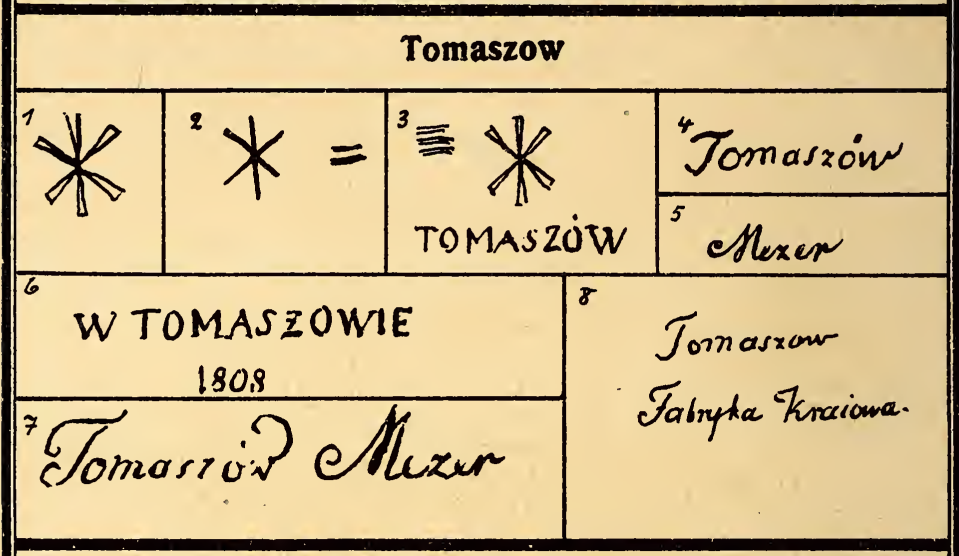


III.

\section{CHINESISCHE UND JAPANISCHE MARKEN}

(MARQUES CHINOISES ET JAPONAISES) 



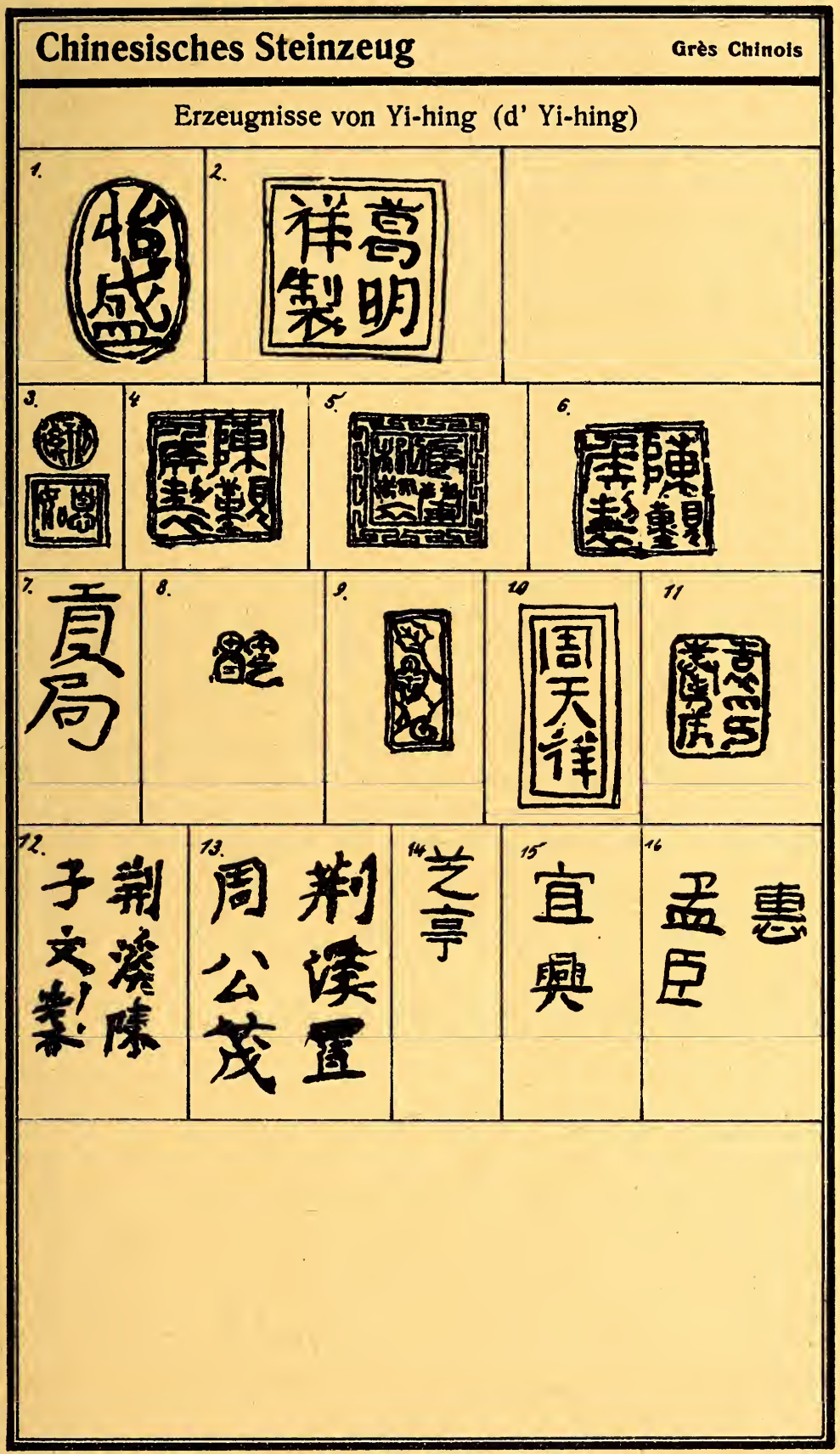




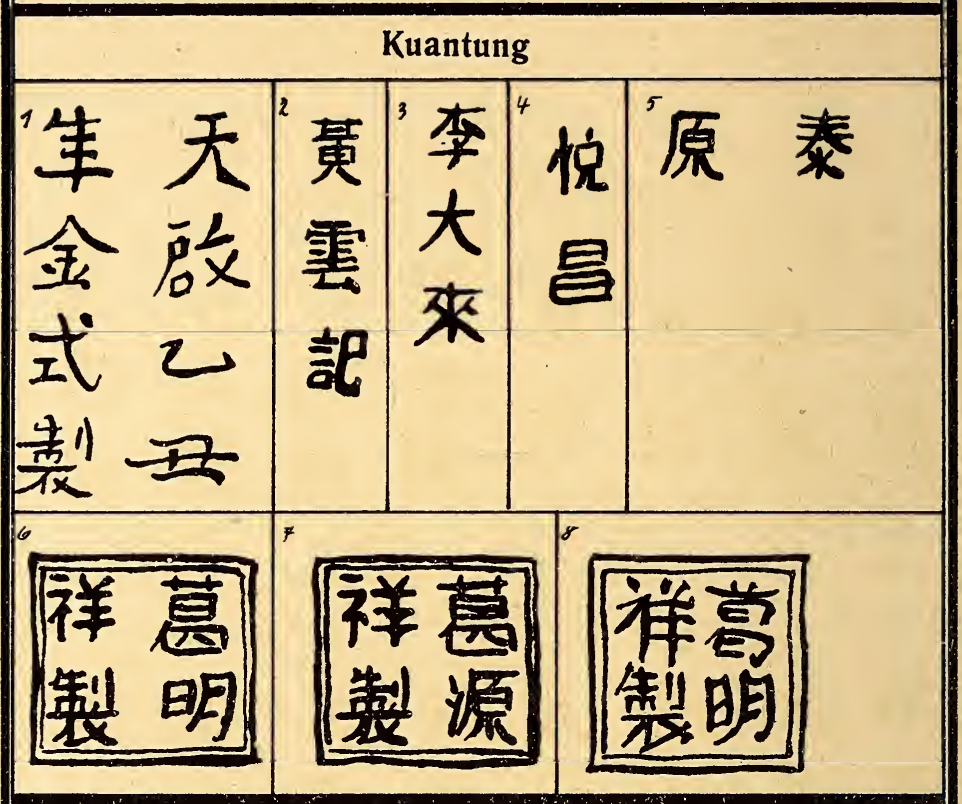

$$
\begin{aligned}
& \text { 陈 葛 } \\
& \text { 质 整 } \\
& \text { 垹 无 }
\end{aligned}
$$




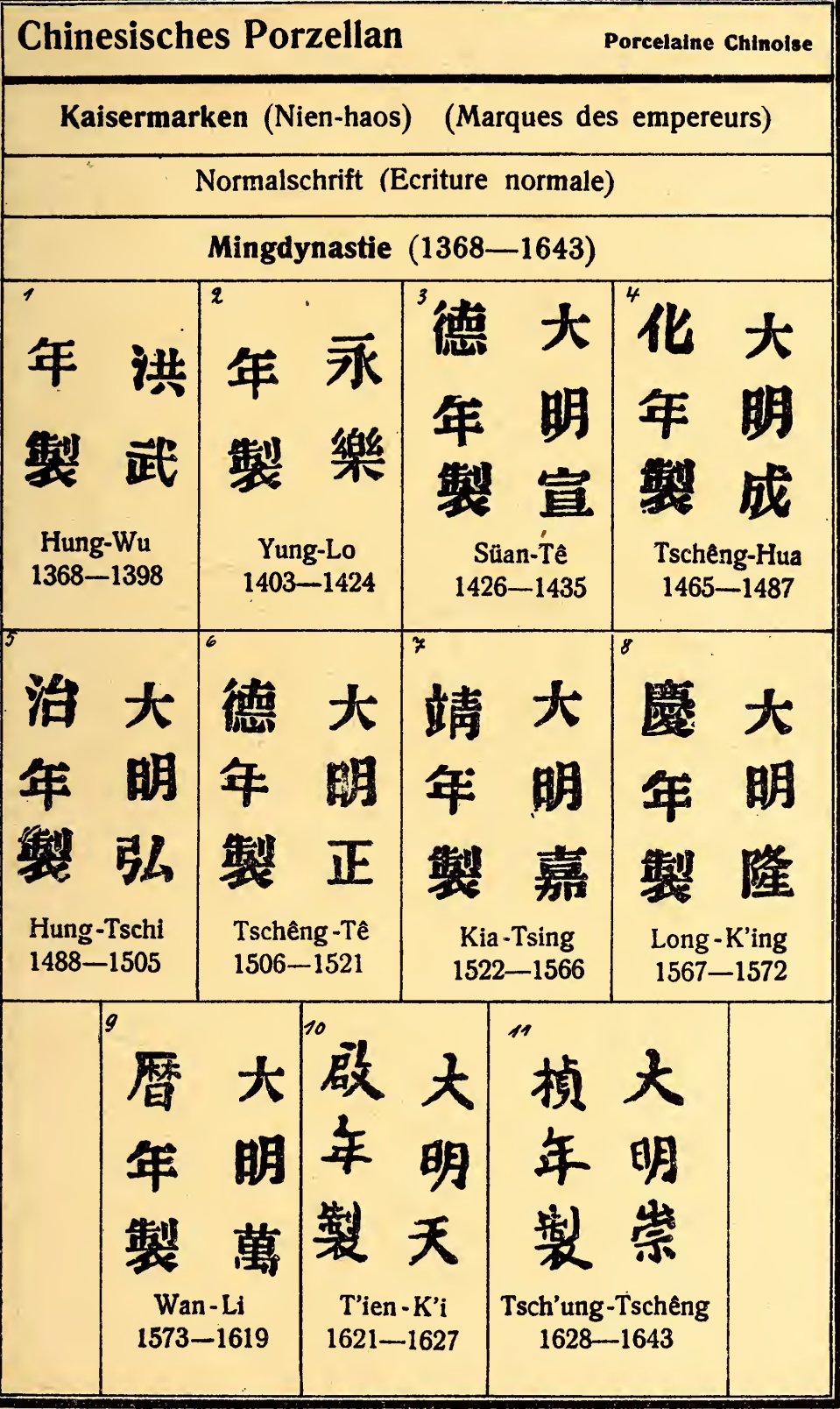


Kaisermarken (Nien-haos) (Marques des empereurs)

Normalschrift (Ecriture normale)

Ts'ingdynastie (1644-1712)

治大哭 $大{ }^{3}$ 正大 ${ }^{4}$ 隆大

年 淸 年 清 年 清 年 清

慗順製康製雄製乾

Schun-Tschi

1644-1661

5

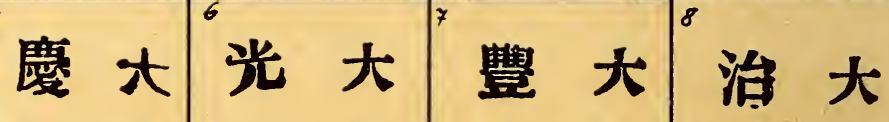

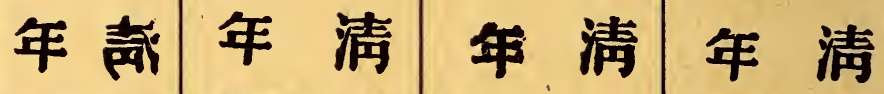

製嘉

Kia-K'ing

1796-1820
彆 道

Tao-Kuang $1821-1850$
Yung-Tschêng

Kien-Lung 1736-1795

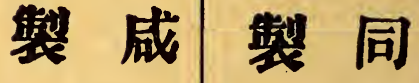

Hien-Fêng 1851-1861
T'ung - Tschi 1862-1874

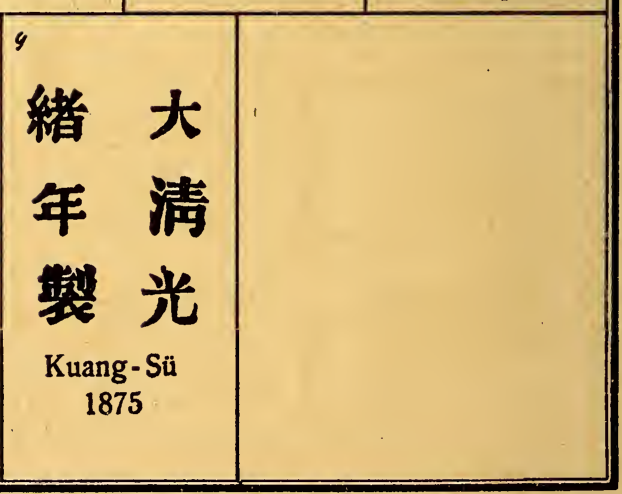


Kaisermarken (Nien-haos) (Marques des empereurs)

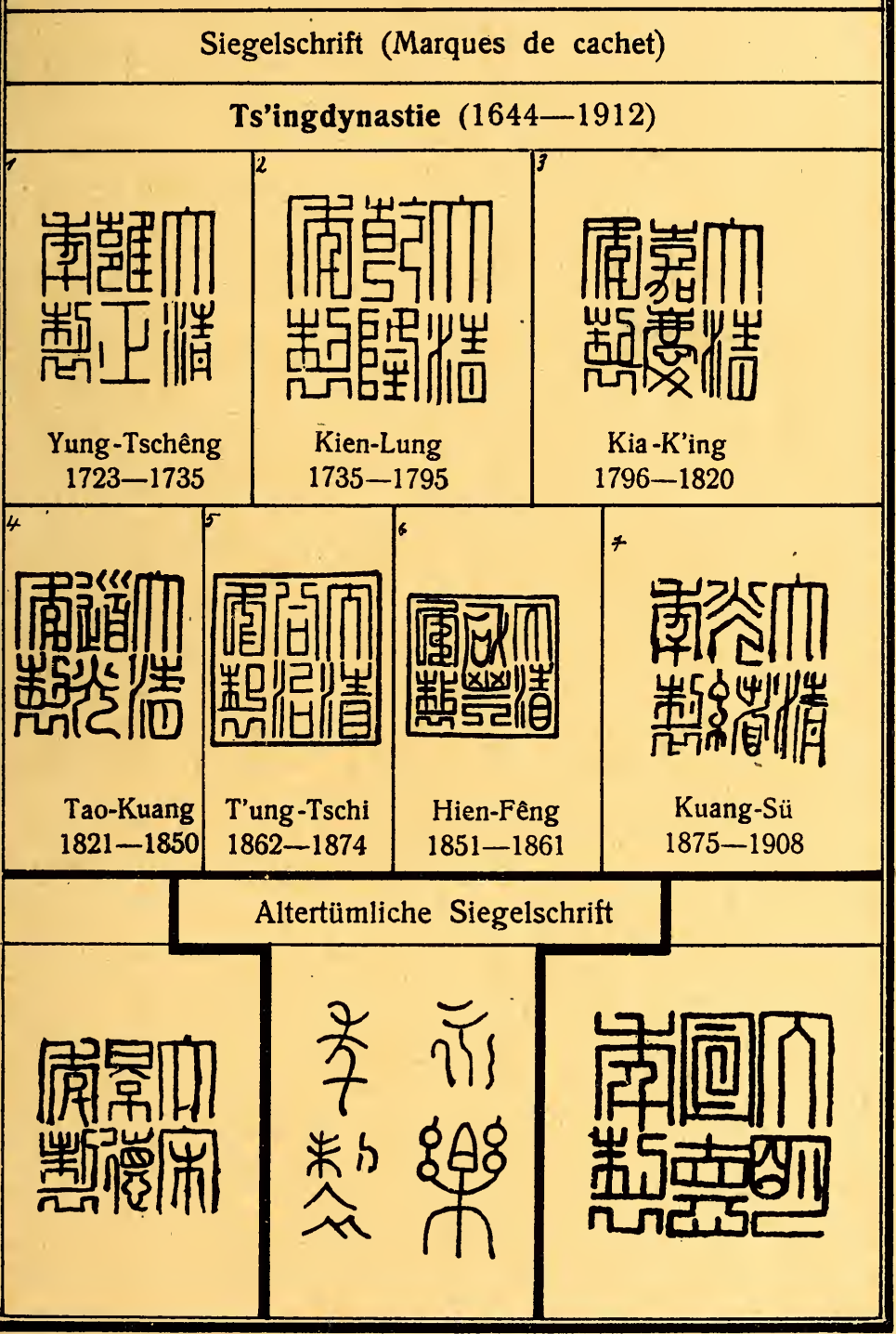




\section{Chinesisches Porzellan}

\section{Periodenmarken}

\begin{tabular}{|c|c|c|}
\hline Perio & Jahreszahl & Periodenzahl \\
\hline$\frac{b x}{3 x}$ & $\begin{array}{l}604,664,724 \ldots, 1684,1744 \text { usw. } \\
605,665,725 \ldots, 1685,1745 \text { usw. } \\
606,666,726 \ldots, 1686,1746 \text { usw. } \\
607,667,727 \ldots, 1687,1747 \text { usw. } \\
608,668,728 \ldots, 1688,1748 \text { usw. } \\
609,669,729 \ldots, 1689,1749 \text { usw. } \\
610,670,730 \ldots, 1690,1750 \text { usw. } \\
611,671,731 \ldots, 1691,1751 \text { usw. } \\
612,672,732 \ldots, 1692,1752 \text { usw. } \\
613,673,733 \ldots, 1693,1753 \text { usw. } \\
614,674,734 \ldots, 1694,1754 \text { usw. } \\
615,675,735 \ldots, 1695,1755 \text { usw. } \\
616,676,736 \ldots, 1696,1756 \text { usw. } \\
617,677,737 \ldots, 1697,1757 \text { usw. } \\
618,678,738 \ldots 1698,1758 \text { usw. } \\
619,679,739 \ldots, 1699,1759 \text { usw. } \\
620,680,740 \ldots, 1700,1760 \text { usw. } \\
621,681,741 \ldots, 1701,1761 \text { usw. } \\
622,682,742 \ldots 1702,1762 \text { usw. } \\
623,683,743 \ldots, 1703,1763 \text { usw. } \\
624,684,744 \ldots, 1704,1764 \text { usw. } \\
625,685,745 \ldots, 1705,1765 \text { usw. } \\
626,686,746 \ldots, 1706,1766 \text { usw. } \\
627,687,747 \ldots, 1707,1767 \text { usw. } \\
628,688,748 \ldots, 1708,1768 \text { usw. } \\
629,689,749 \ldots, 1709,1769 \text { usw. } \\
630,690,750 \ldots, 1710,1770 \text { usw. } \\
631,691,751 \ldots, 1711,1771 \text { usw. } \\
632,692,752 \ldots, 1712,1772 \text { usw. } \\
633,693,753 \ldots, 1713,1773 \text { usw. }\end{array}$ & 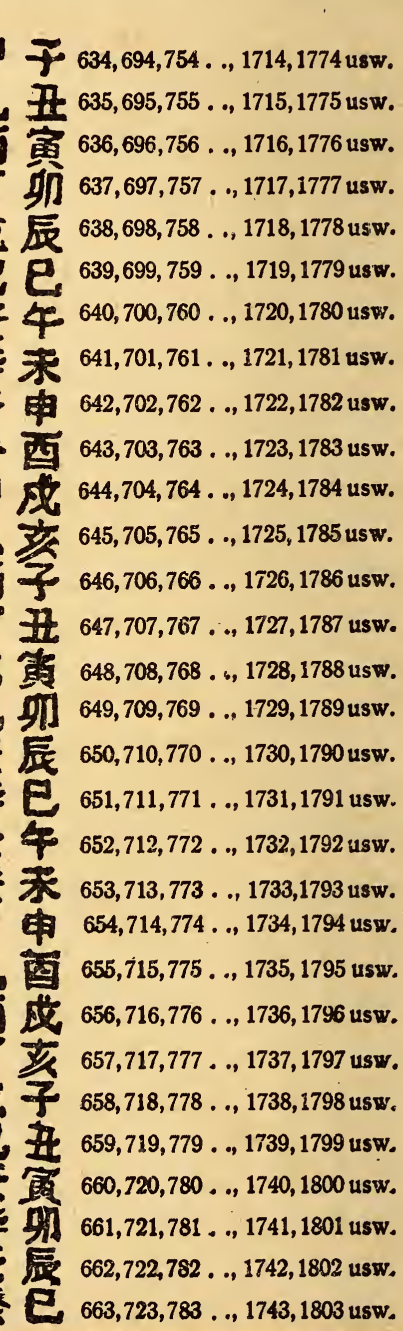 \\
\hline
\end{tabular}




\section{Chinesisches Porzellan}

Beispiele von Periodenmarken

\begin{tabular}{|c|c|c|}
\hline$\Rightarrow$ & 2 & \\
\hline 乎 & 等丙 & \\
\hline 年羊 & 制 & \\
\hline 製 开 & & \\
\hline
\end{tabular}

Marken bestimmter Zeiten

Sungdynastie $(960-1279$

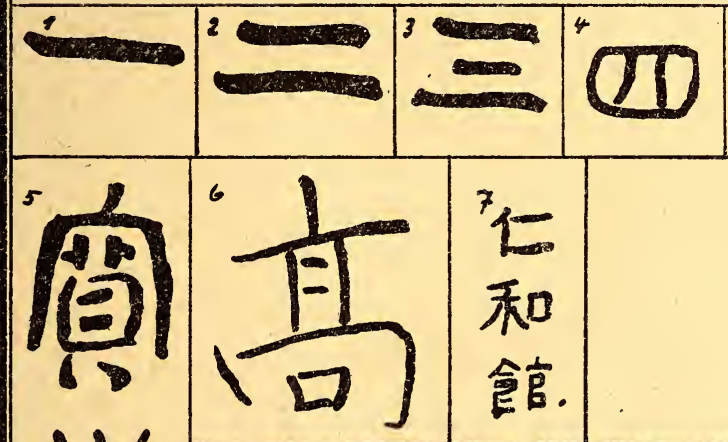

$1 \$ 1$

Yüandynastie (1280-1367)

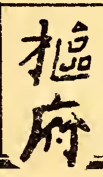


Chinesisches Porzellan

Porcelaine Chinoise

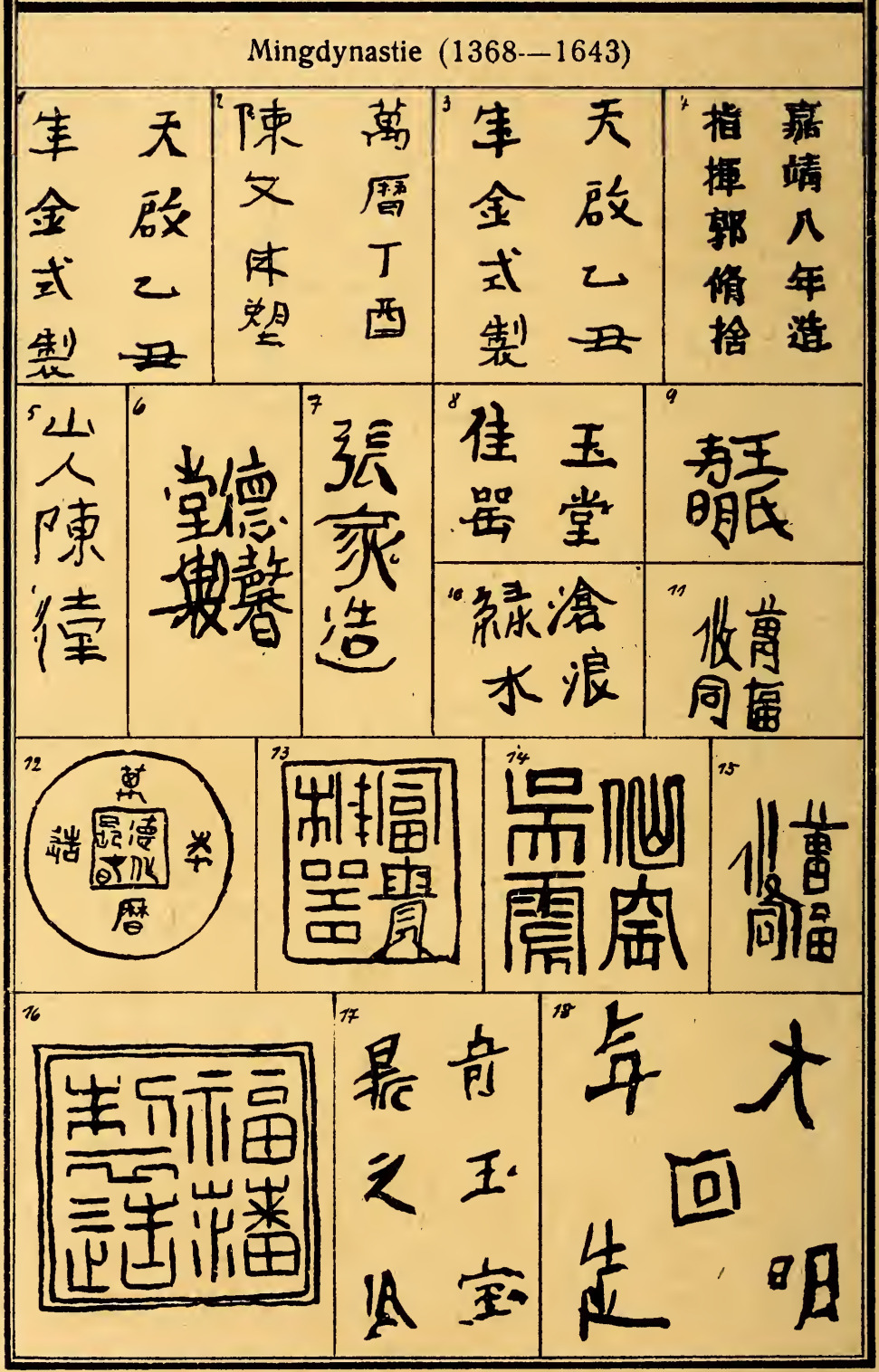




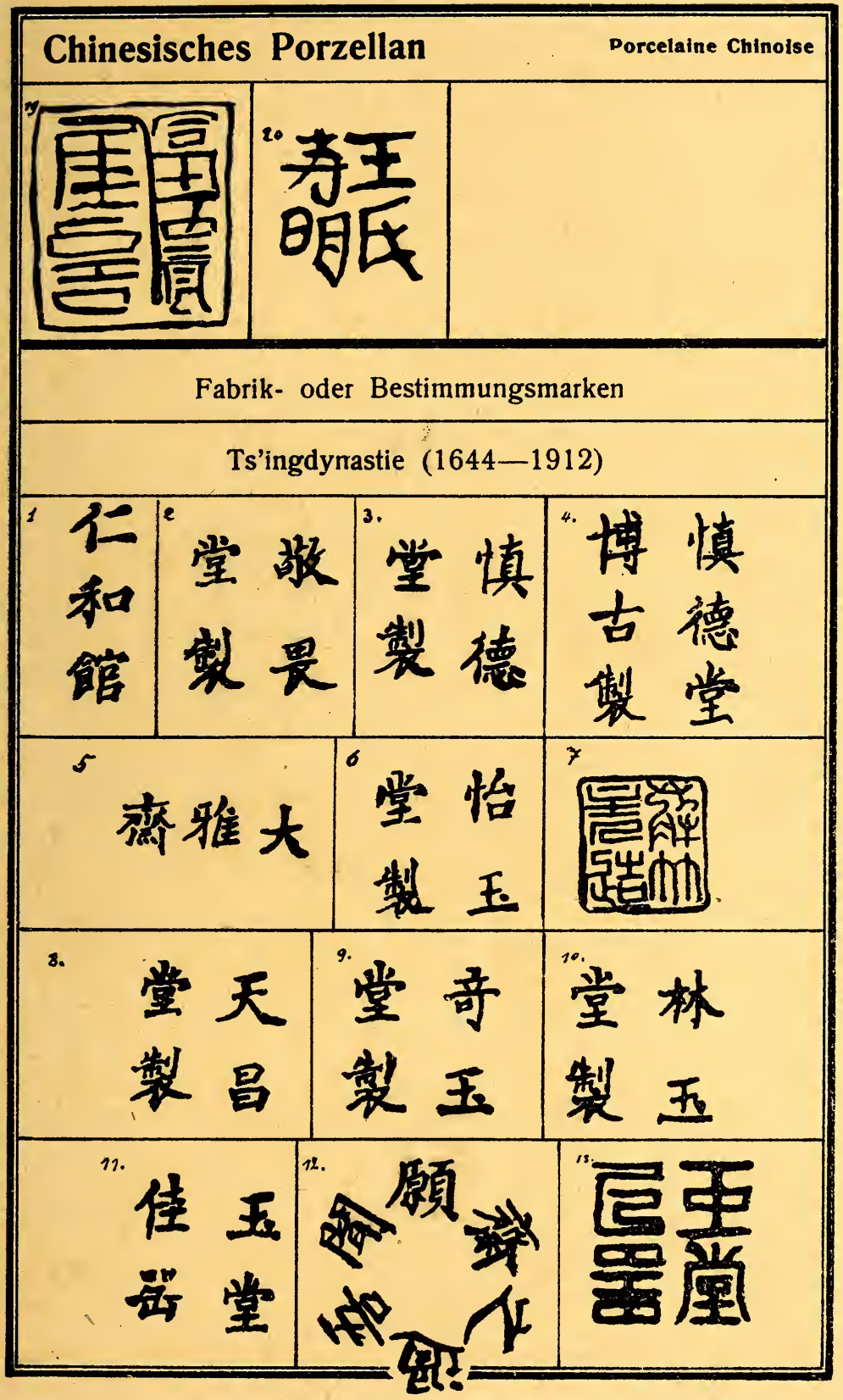




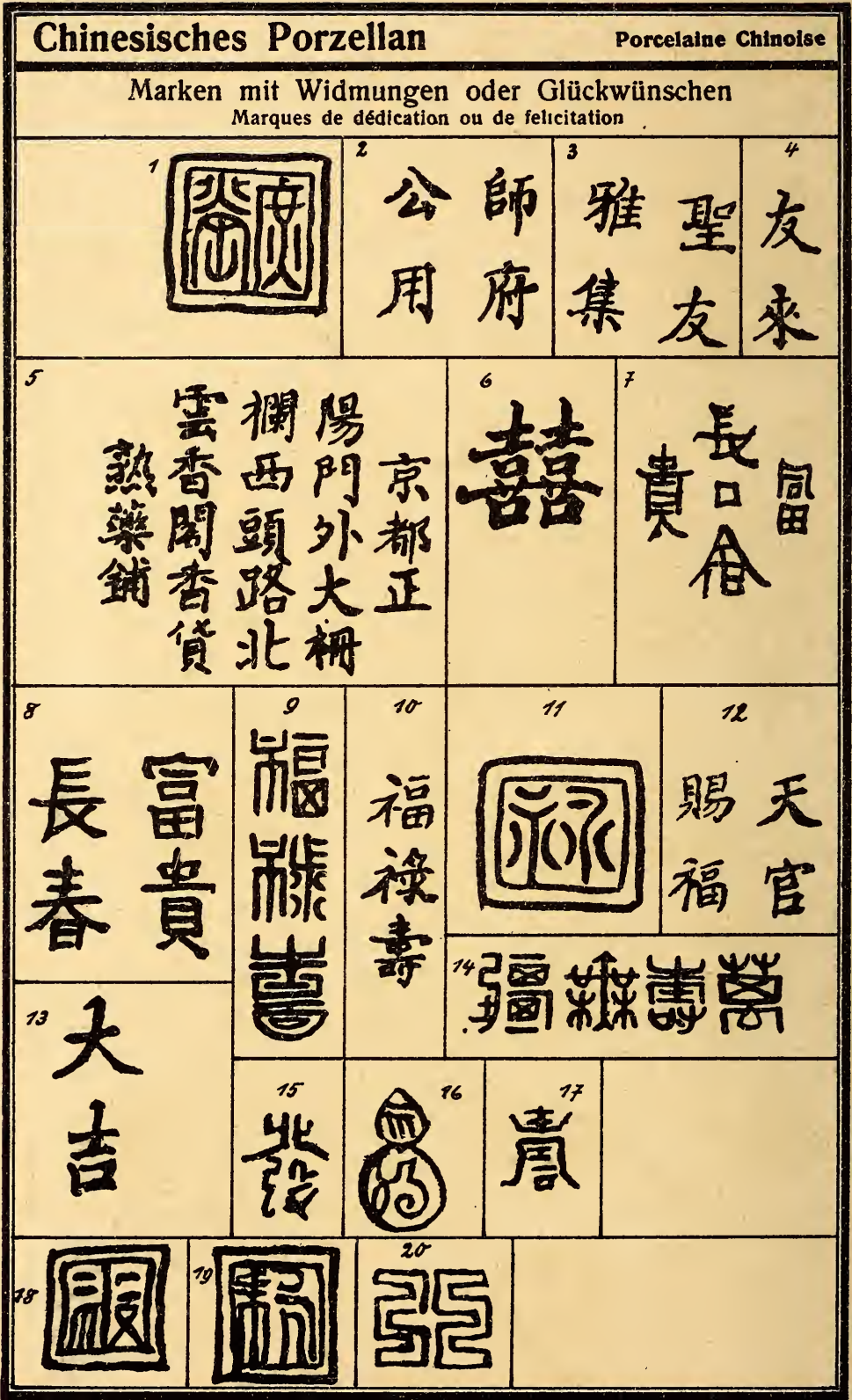




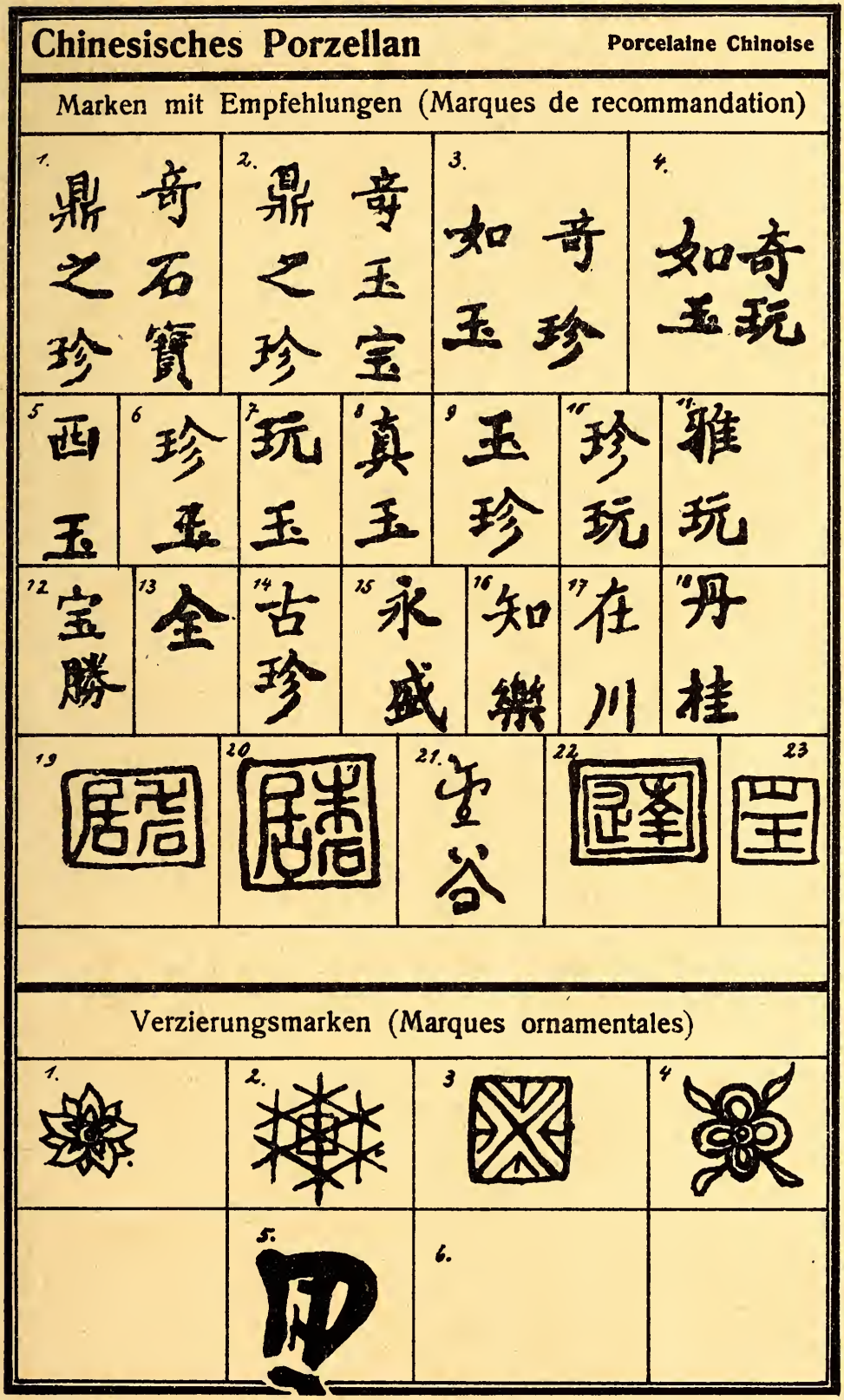




\section{Chinesisches Porzellan}

Porcelaine Chinoise

Künstlerbezeichnungen (Marques des artistes)

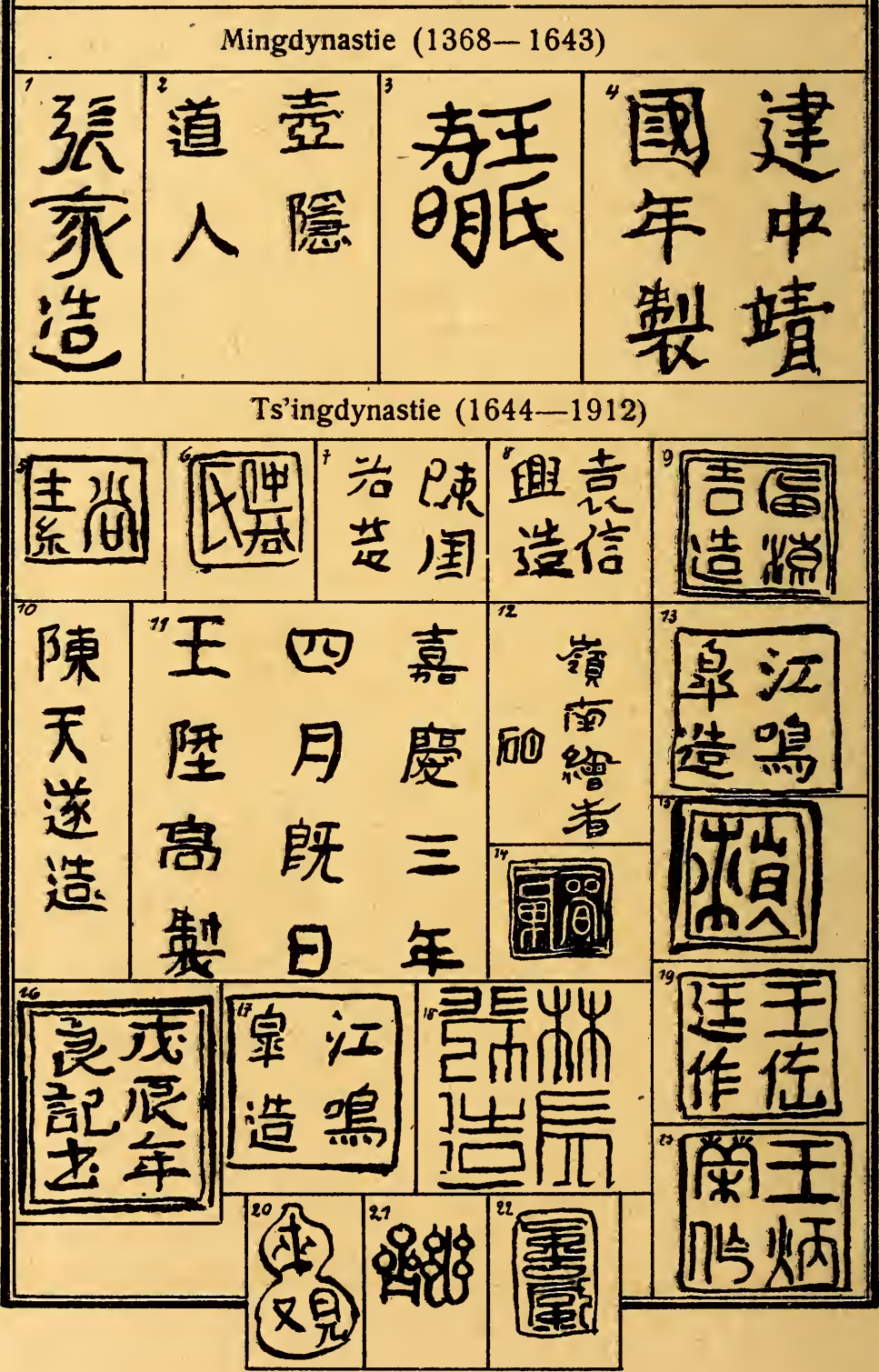




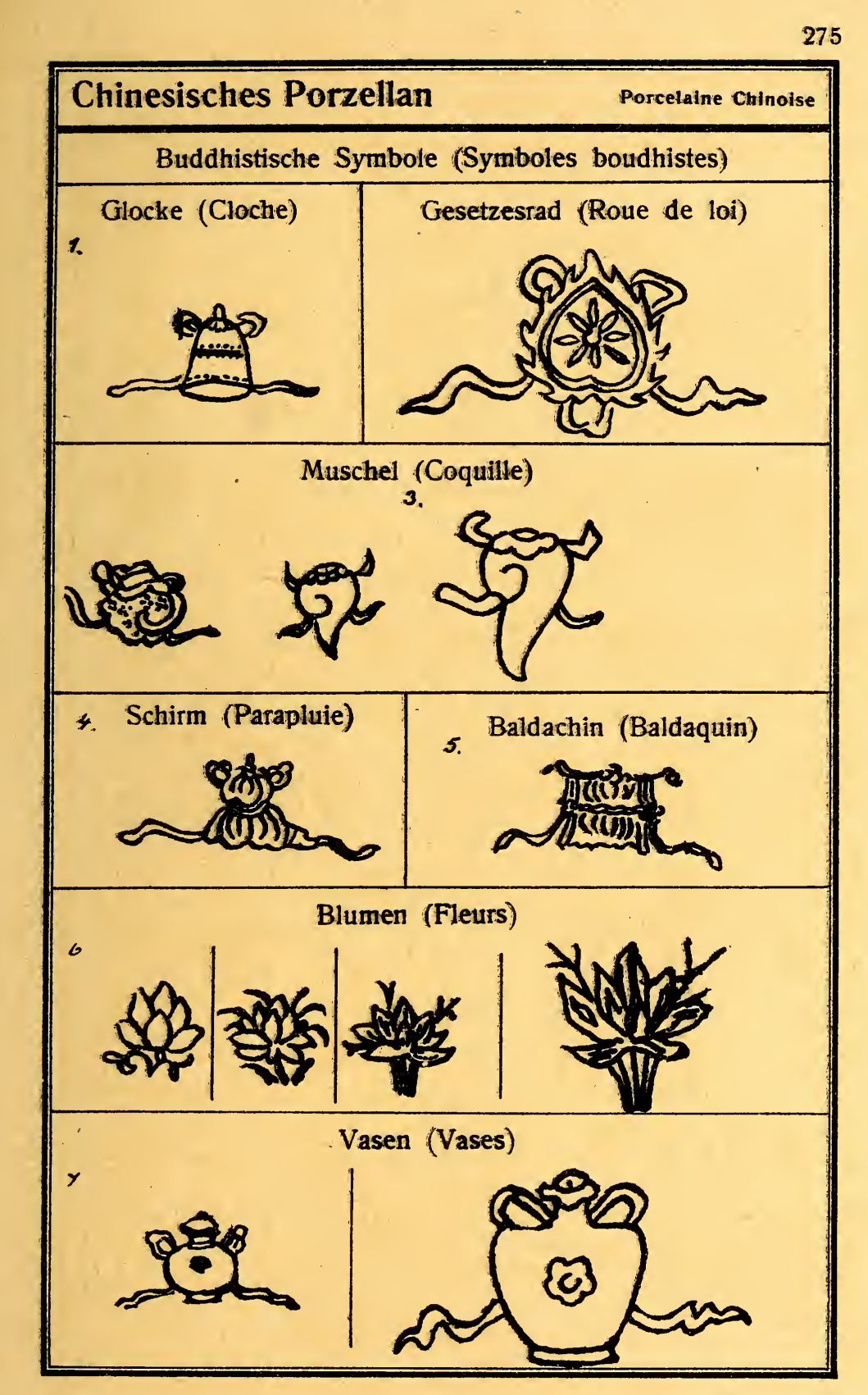



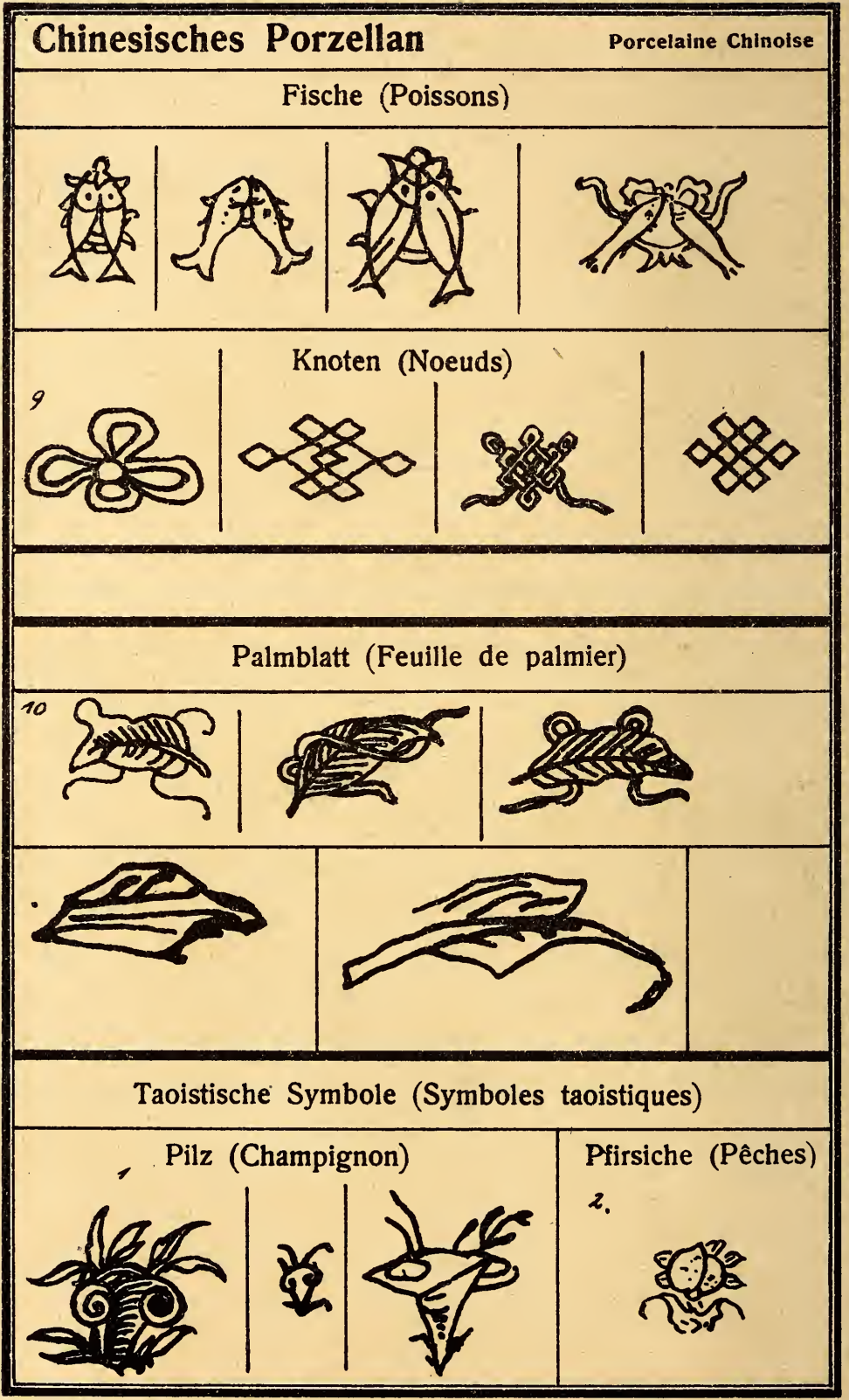


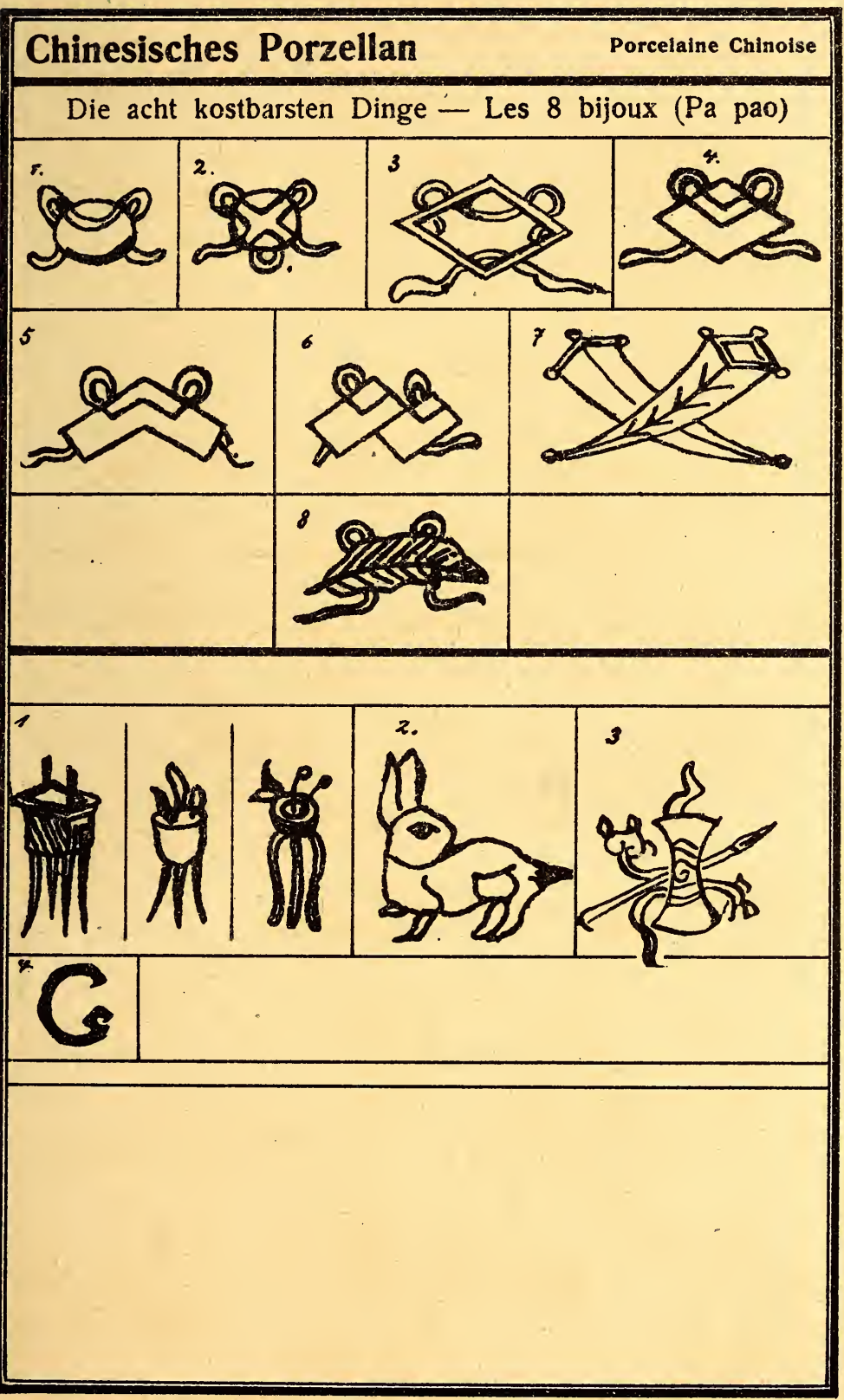




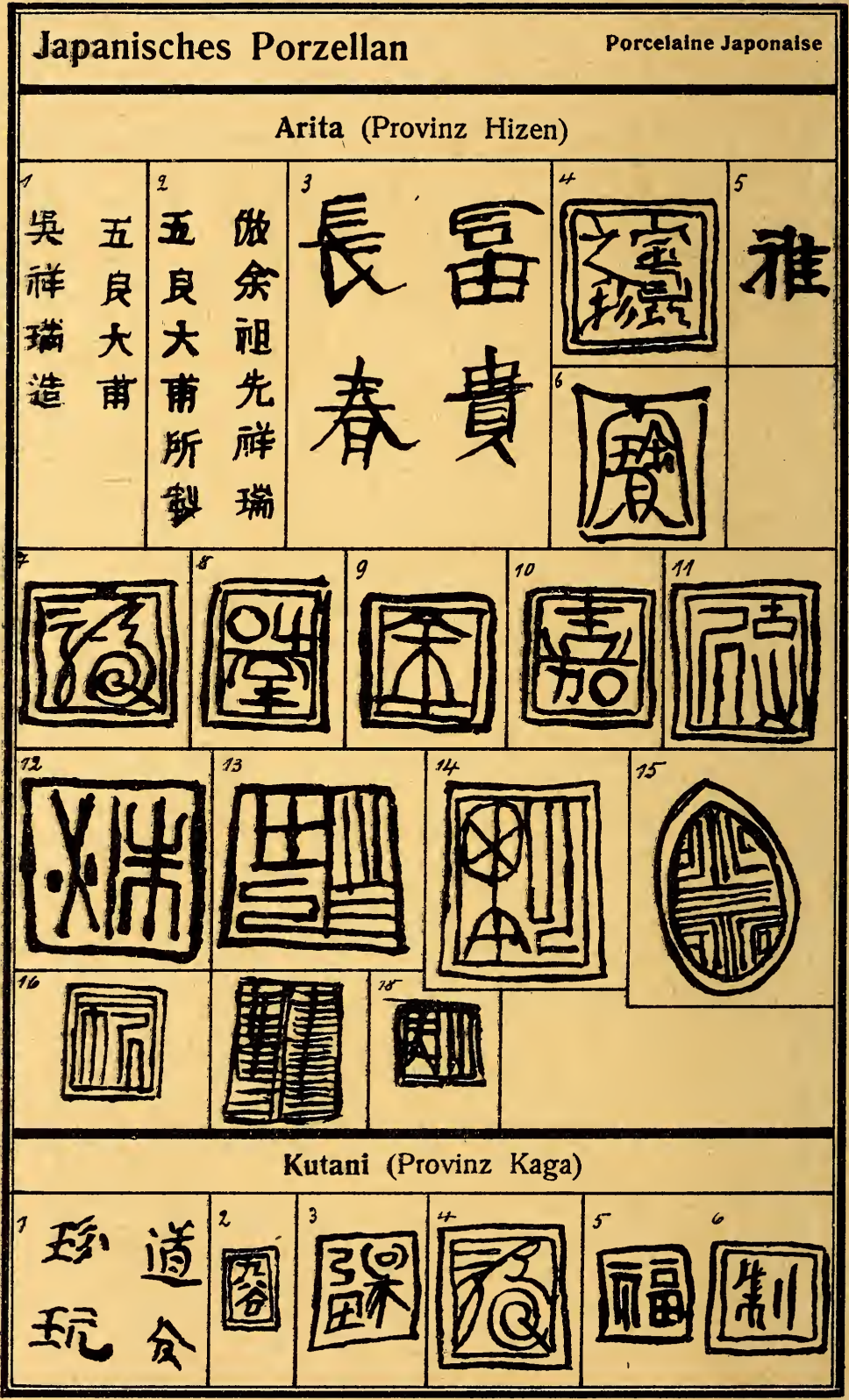




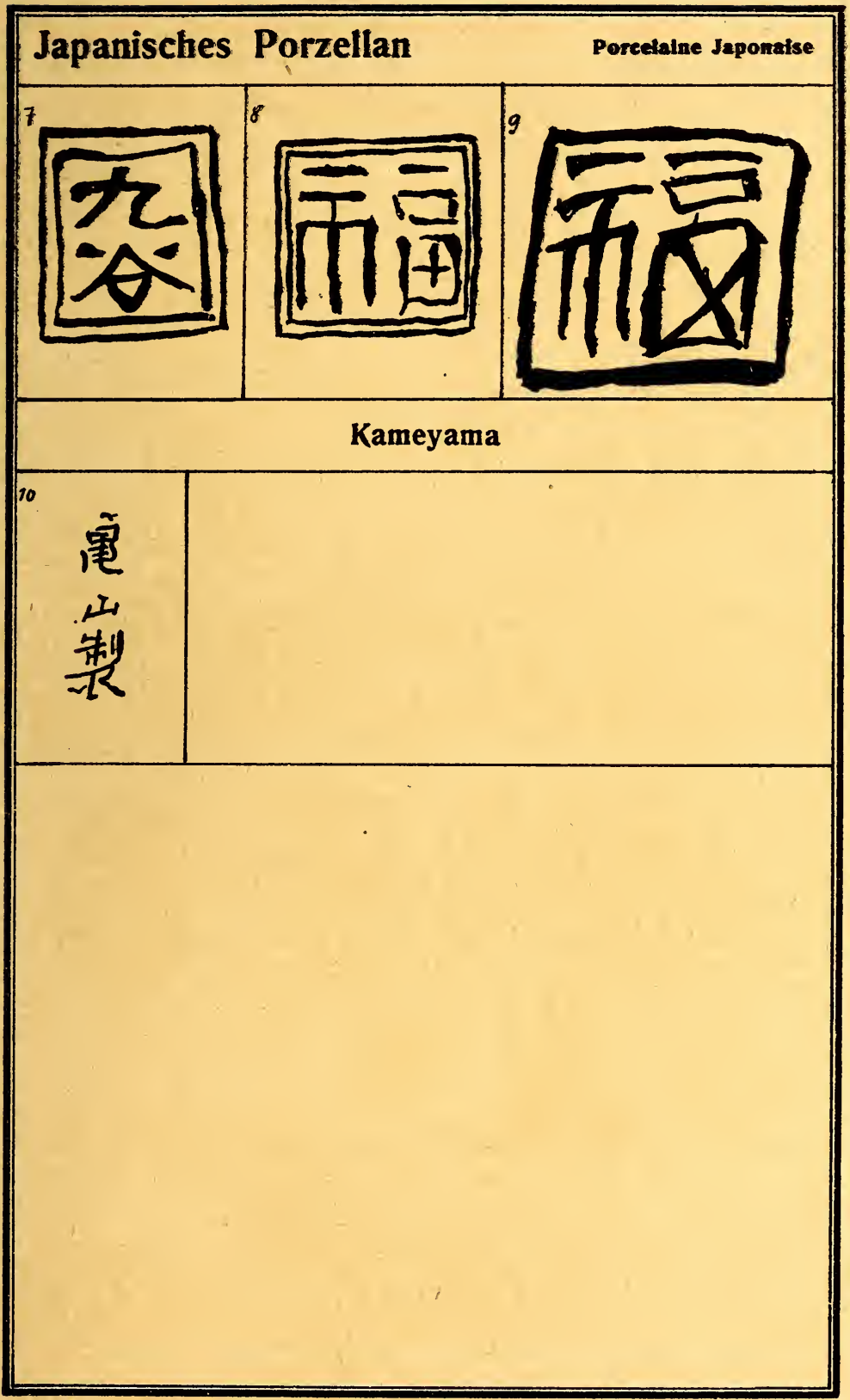




\section{Japanische Töpfereien}

Poterie Japonaise

\section{Provinz Hizen}

\begin{tabular}{|l|l|l|l|l|}
\hline \multicolumn{2}{|c|}{ Kameyama } & \multicolumn{2}{|c|}{ Bogasaki } & Shoto \\
\hline & & & \\
\hline & & & \\
\hline
\end{tabular}

Provinz Bizen

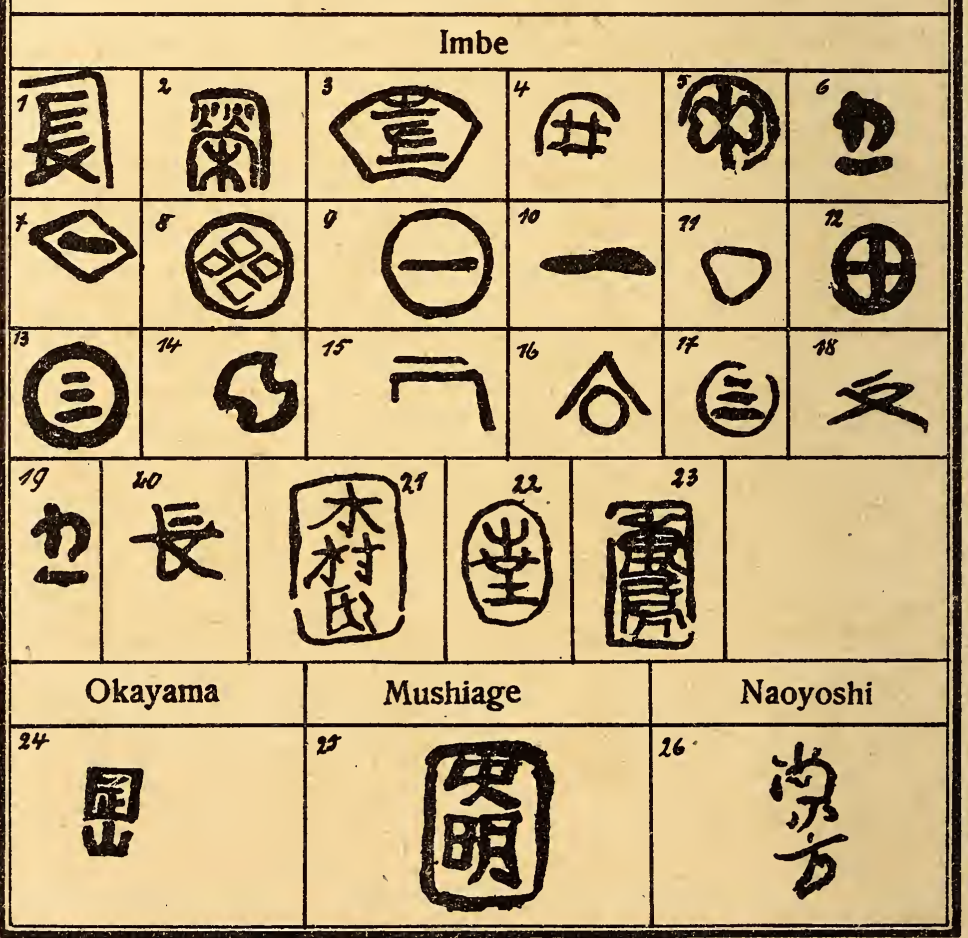




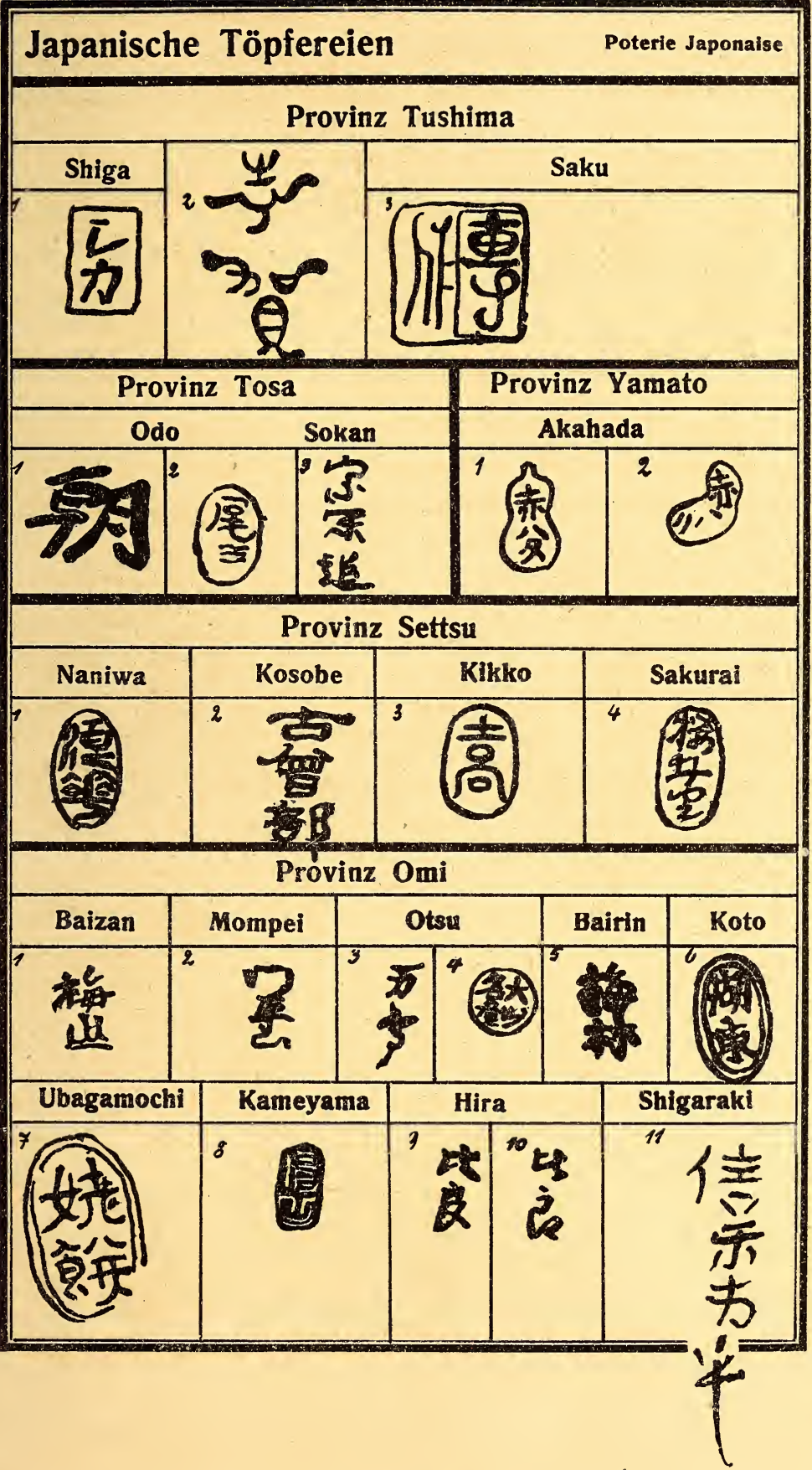


Japanische Töpfereien

Poterie Japomalse

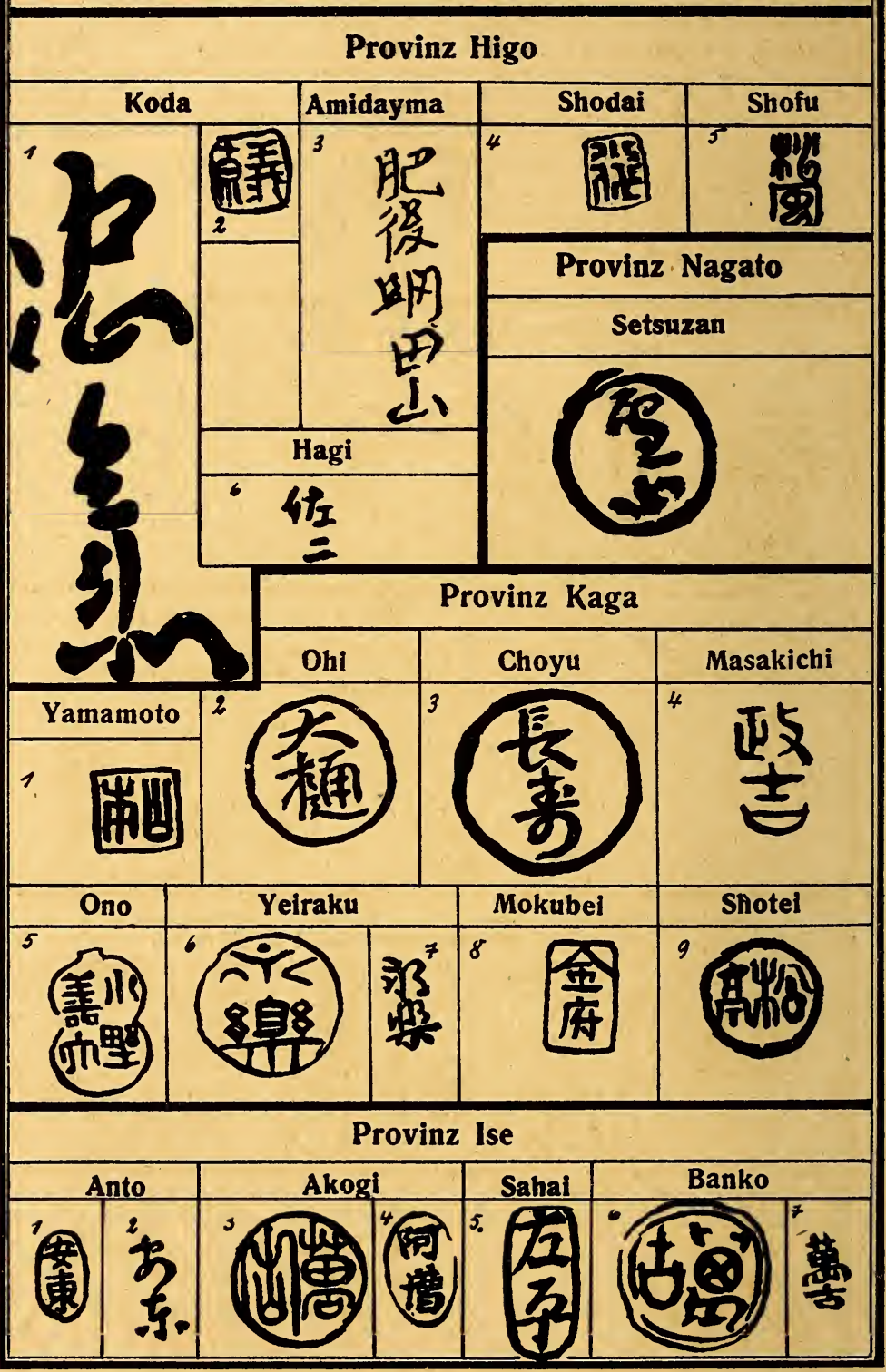




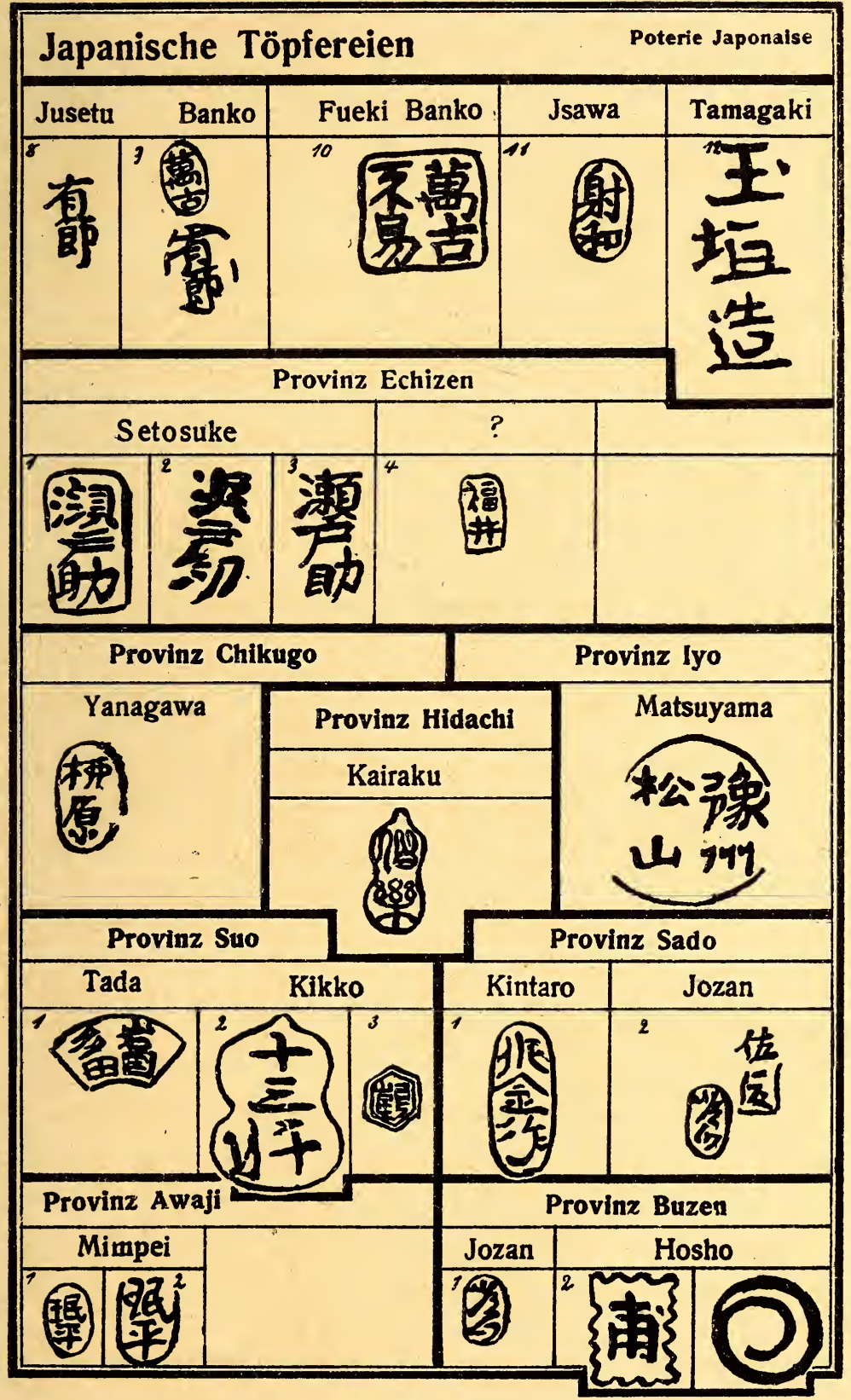




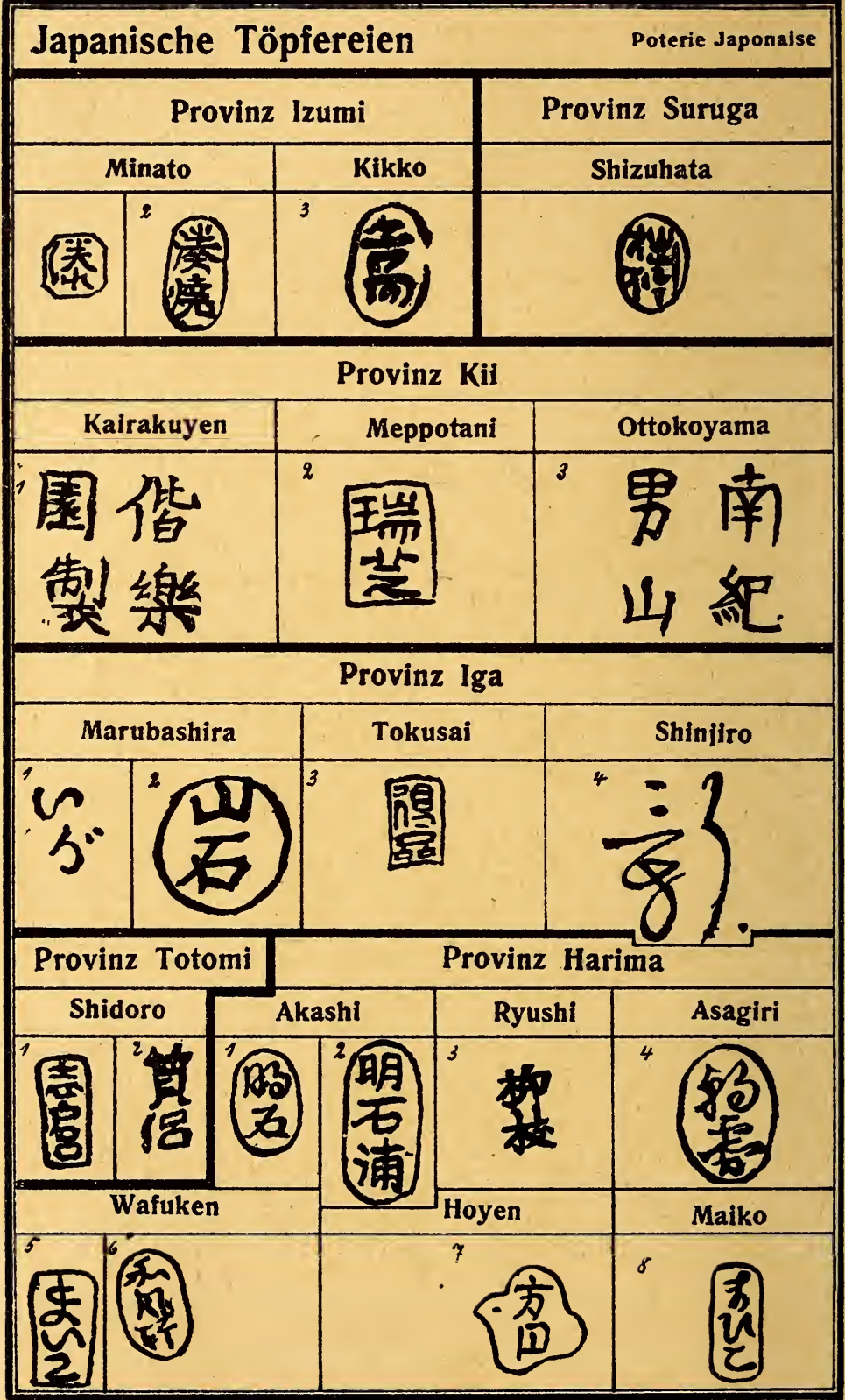




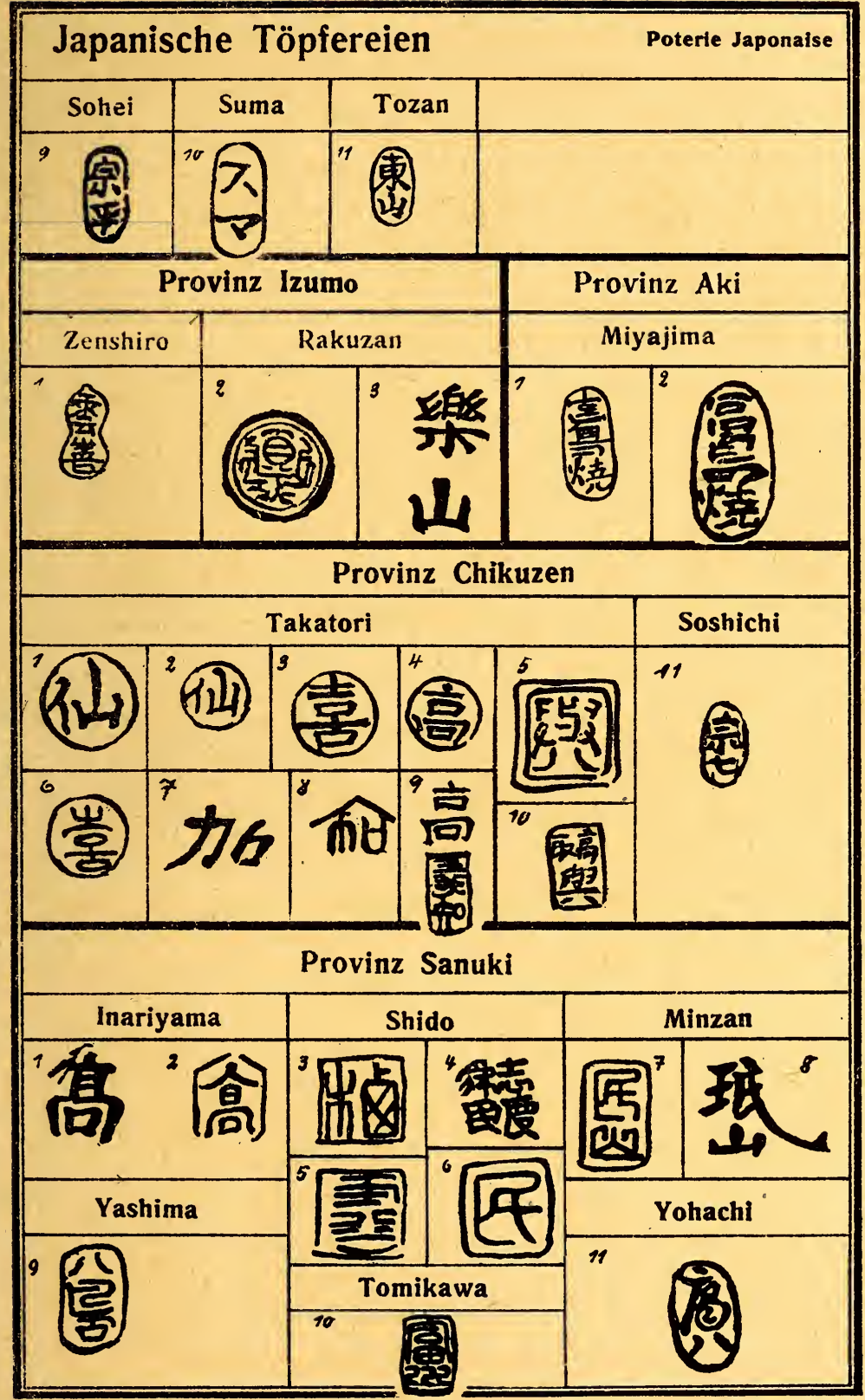


$280 \hat{~}$

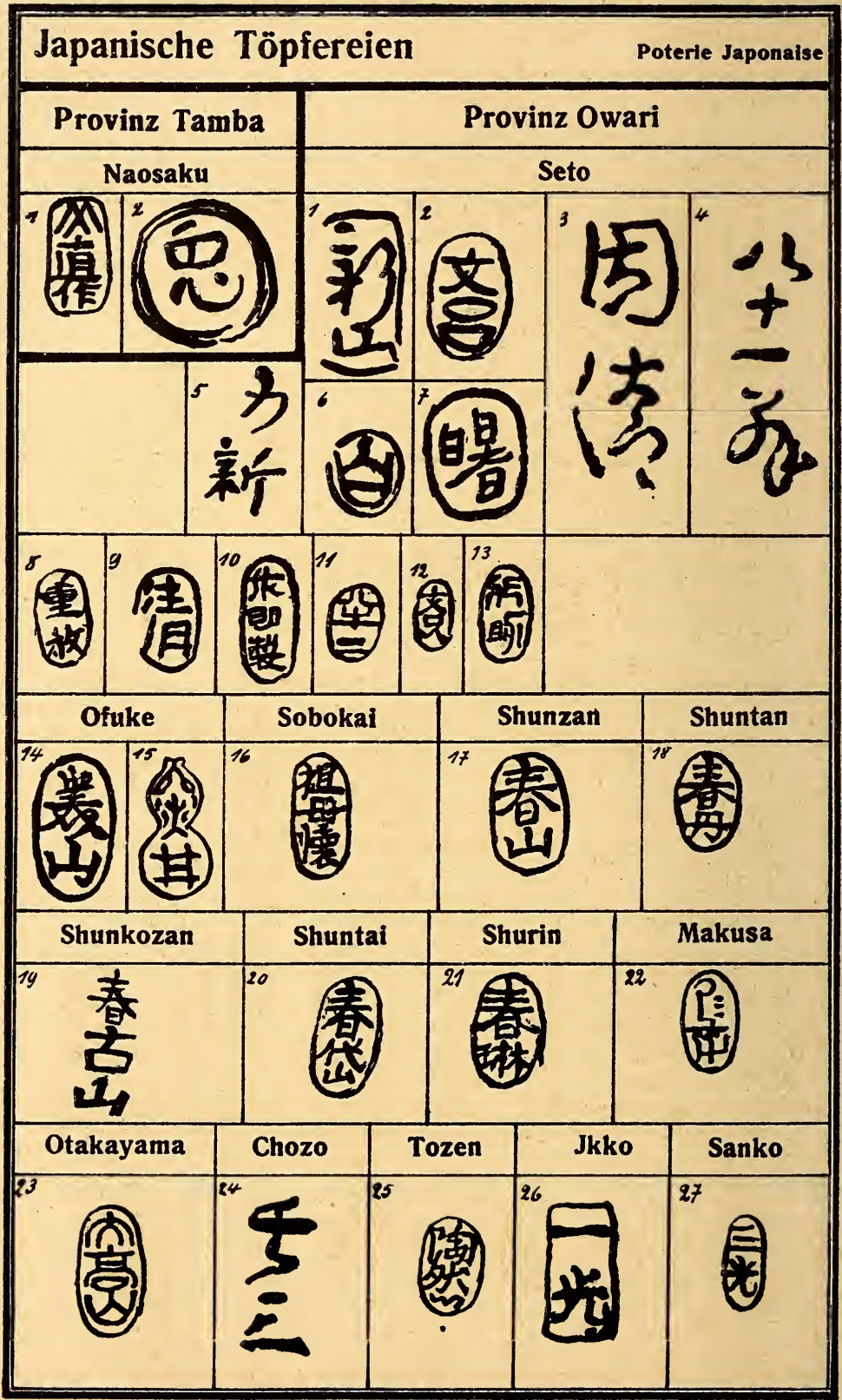




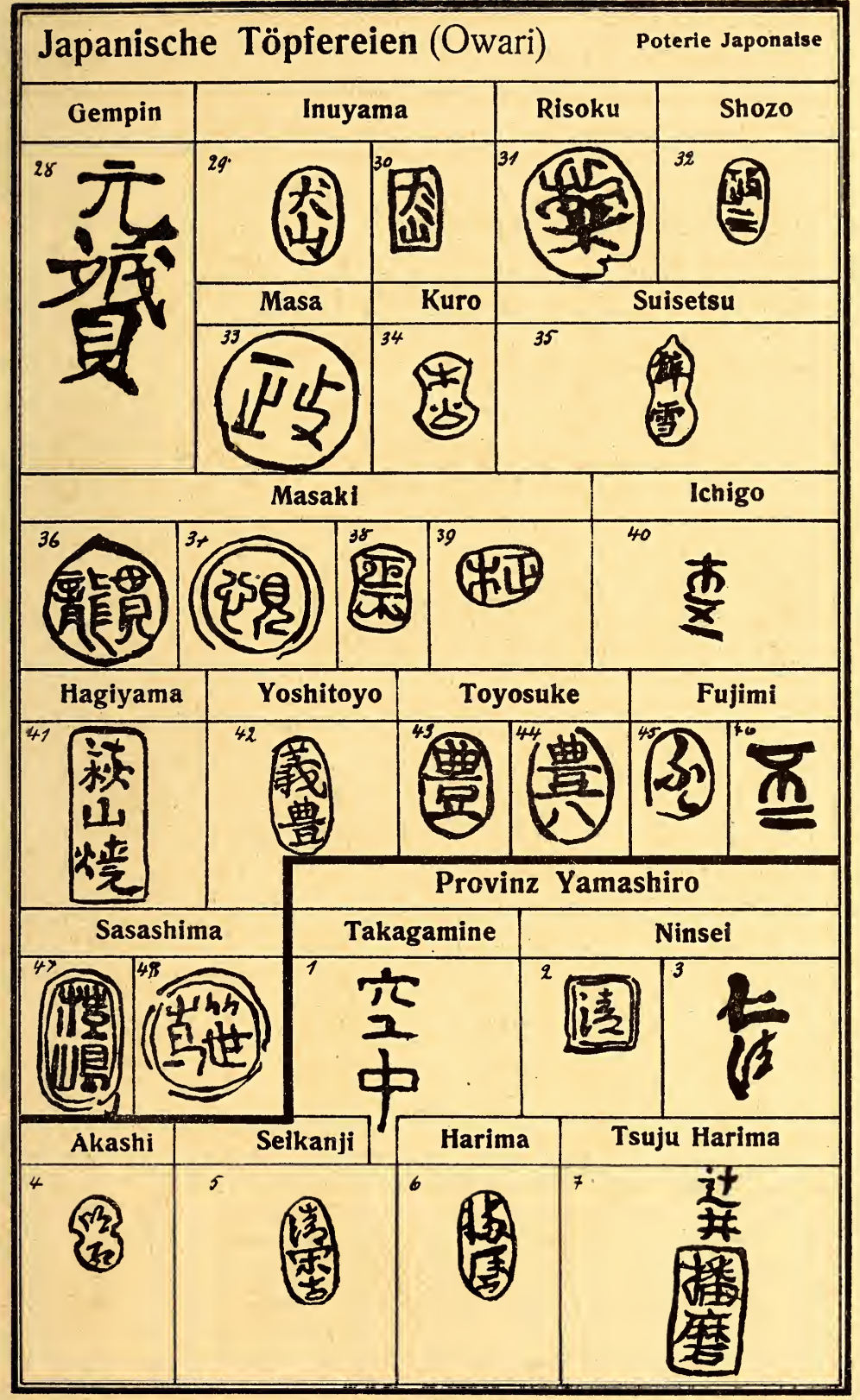




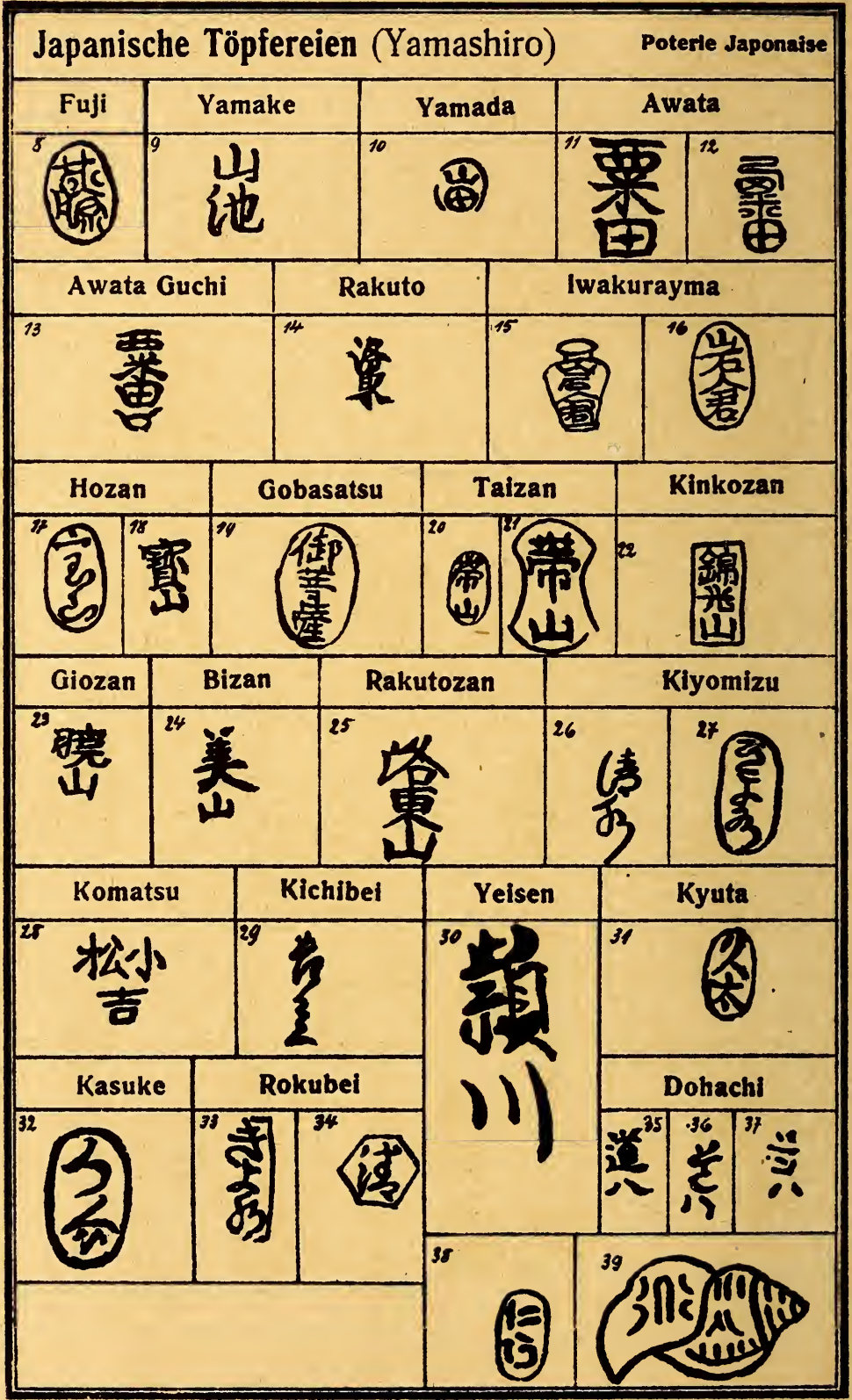




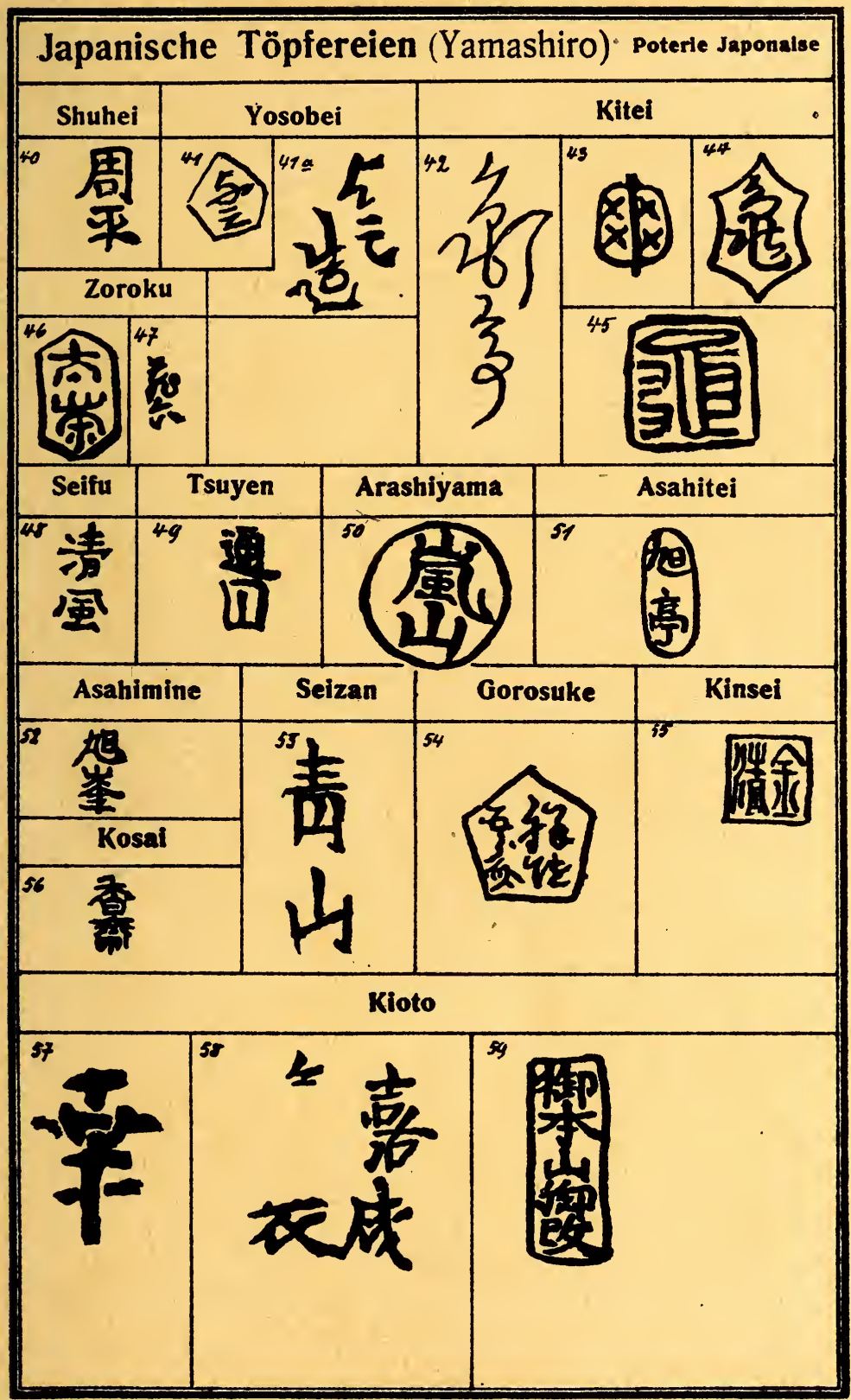




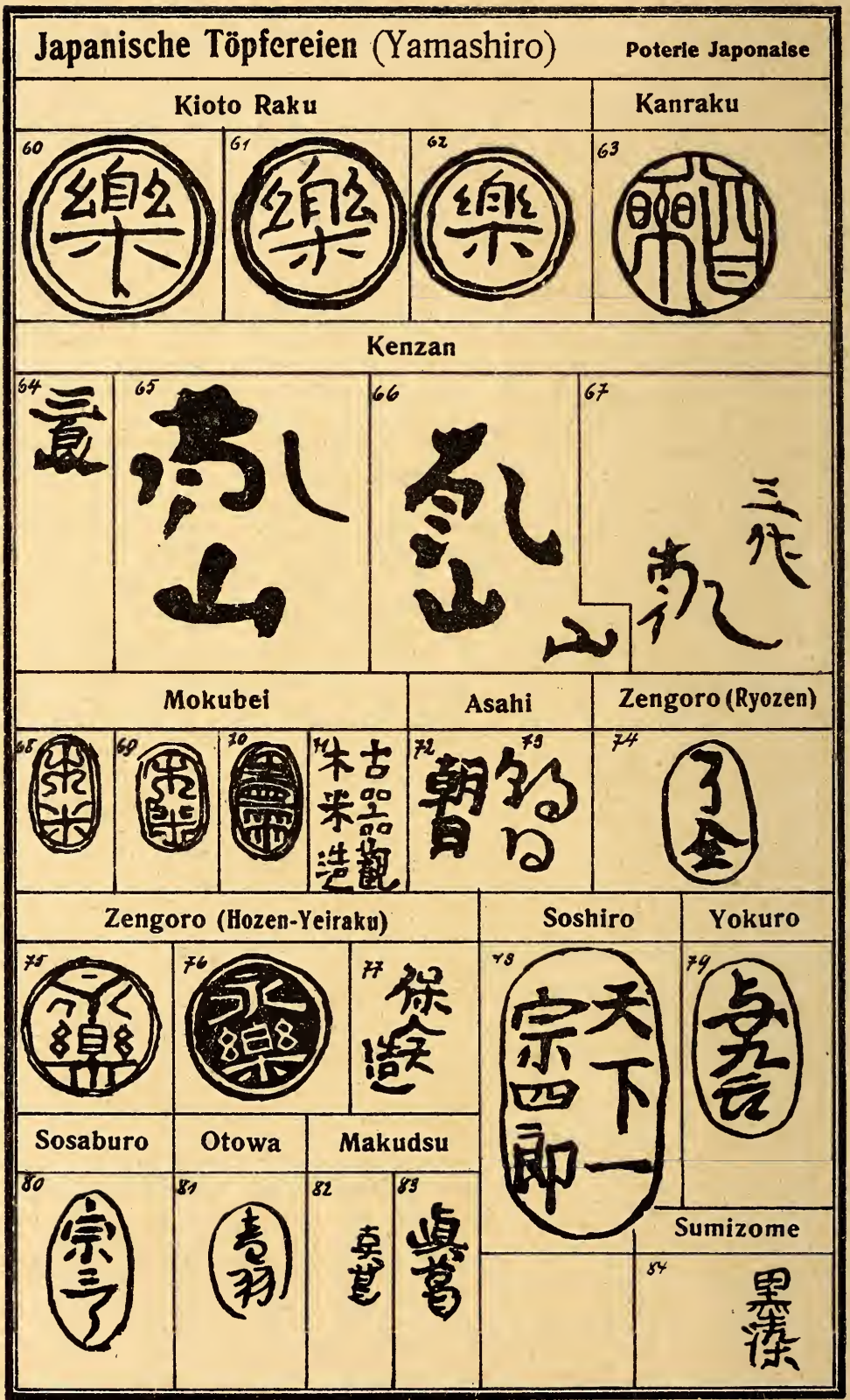




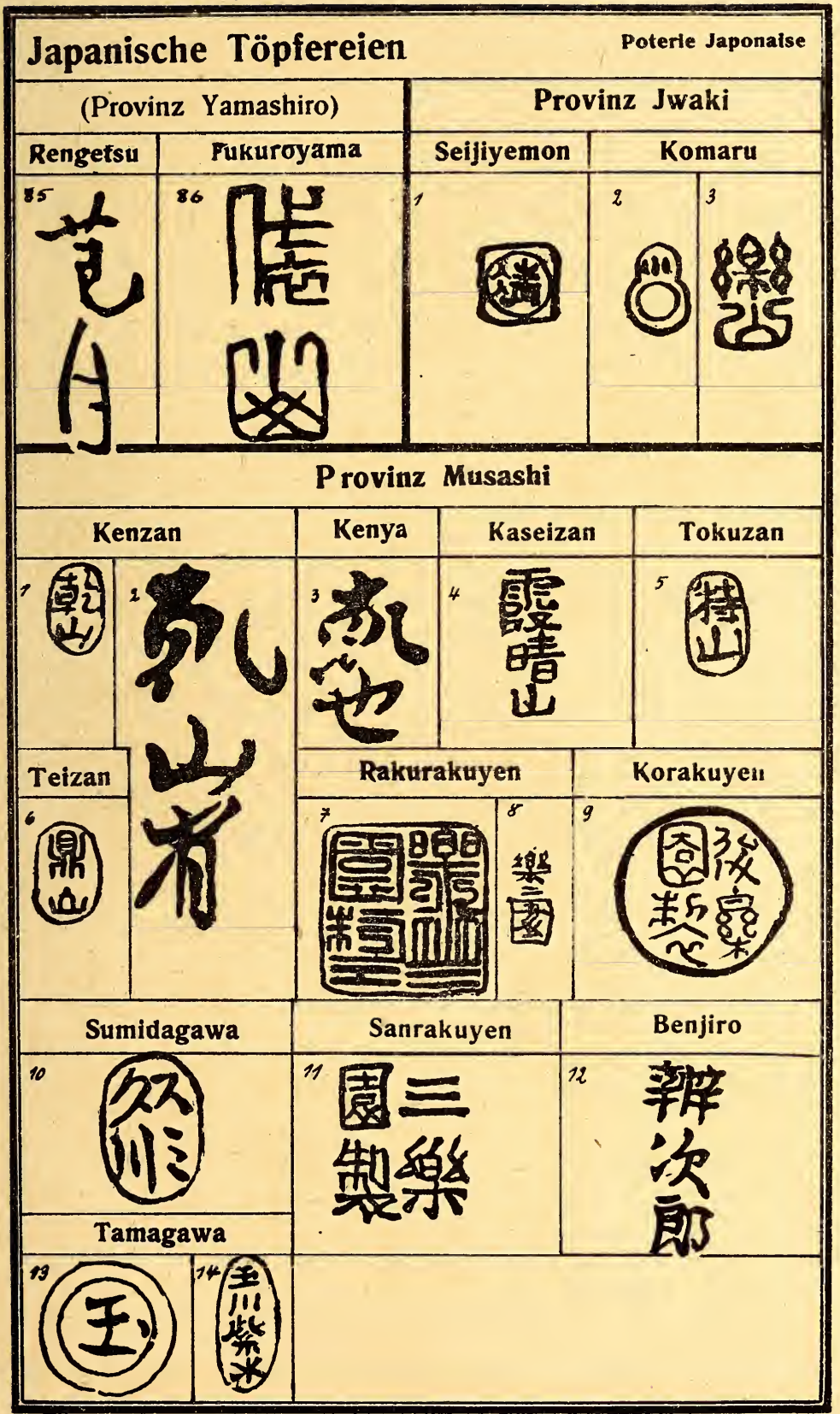




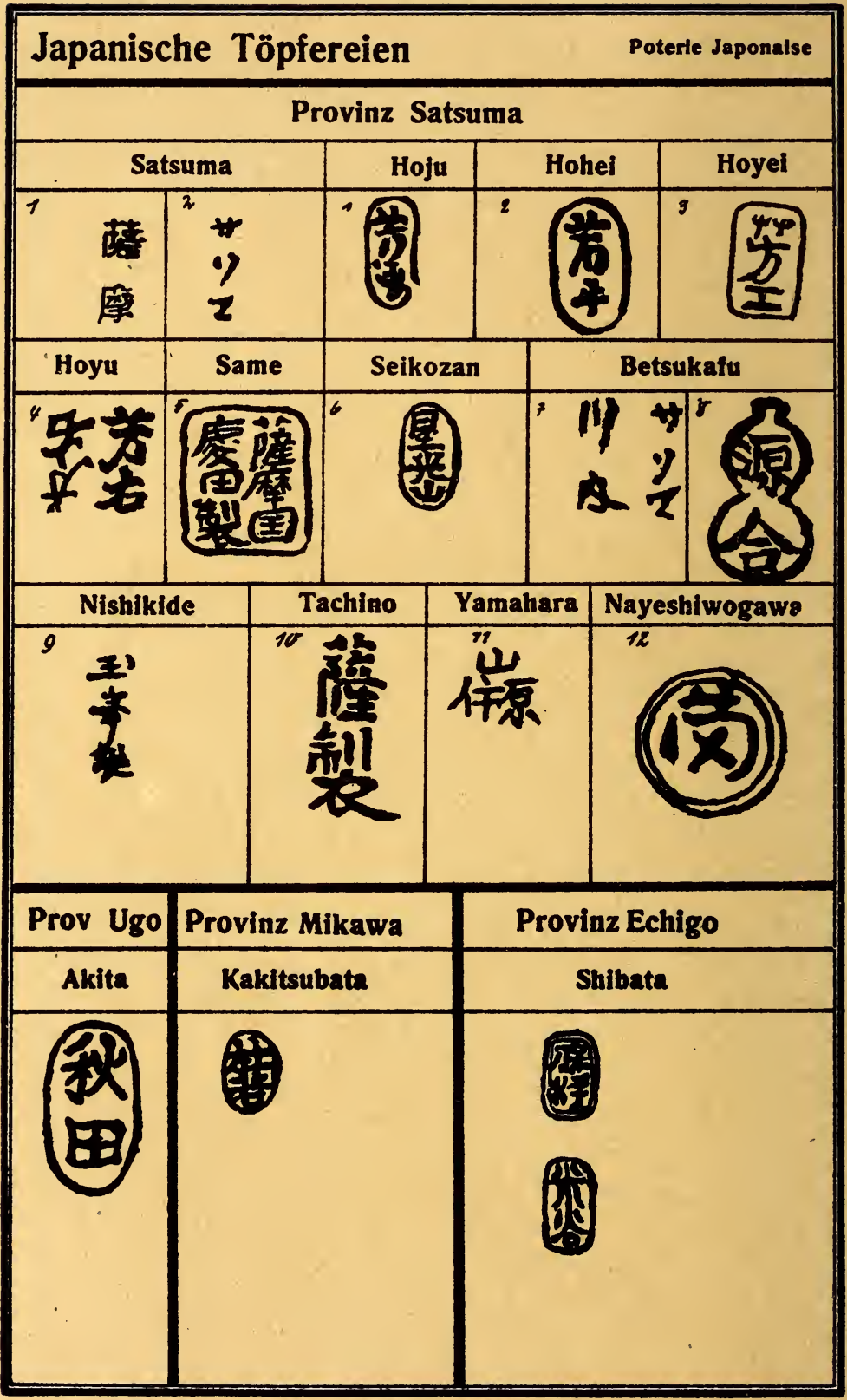




\section{Belege und Erläuterungen.}

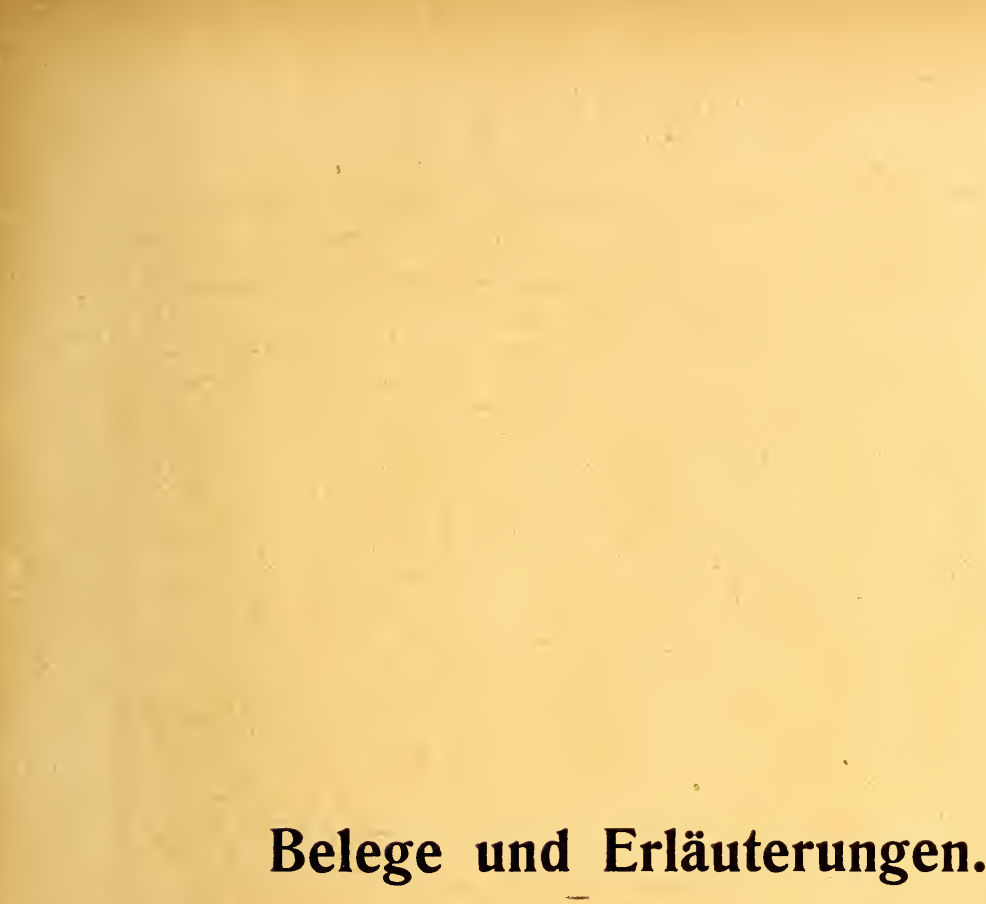





\section{Verzeichnis der Abkürzungen.}

Ch. u. Gr. = Chavagnac et Grollier (vgl. unten das Verzeichnis der Titel

Coll. = Collection.

g. M. = gewöhnliche Marke.

G. Mus. = Germanisches Museum.

H. M. = Hauptmarke.

Kgw. Mus. = Kunstgewerbe-Museum.

Mus. f. K. u. G. = Museum für Kunst und Gewerbe.

Mus. $=$ Museum.

O. = Ort.

P. S. = Porzellansammlung.

T. $=$ Töpfer.

\section{Titel der Werke,}

von denen im folgenden nur die Verfasser genannt sind.

Argnani, Le ceramiche e majoliche faentine. Faenza 1889.

Ballardini, G., Marche di fabbrica (Faenza, bollettino del Museo internazionale delle ceramiche. Fascicolo IV I9r3).

Berling, Das Meißner Porzellan und seine Geschichte. Leipzig I9oo.

Binns, The origin and the early history of the manufacture of porcelain at Worcester.

Brogniart, Traité des arts céramiques ou des poteries. Paris I 844 .

Bushell, Oriental ceramic Art. New York I899.

Chaffers, Marks and Monograms on Pottery and porcelain. London, I. Auflage I863, letzte Auflage 1908.

Chavagnac et Grollier, Histoire des manufactures françaises de porcelaine. Paris I906.

Davillier, Histoire des faïences et porcelaines de Moustiers, Marseille etc. Paris 1863 .

- Les origines de la Porcelaine en Europe. Paris et Londres I882.

Delange, Recueil des faïences italiennes des XV., XVI. et XVII. siècles etc. Paris, Delange I869. 


\section{$-302$}

Fortnum, Majolica. Oxford I896.

Forestie, Les anciennes Faïenceries de Montauban. Ardus 1876.

Folnesics und Braun, Geschichte der Wiener Porzellanmanufaktur. Wien I907.

Franks, Catalogue of a Collection of Oriental Porcelain and Pottery (Bethnal Green Branch Museum). London I878.

Garnier, Dictionnaire de la céramique. Paris.

Garnier (II), Catalogue de la collection Gasnault (Musée nationale Adrien Dubouché à Limoges). I 88I.

Genolini, Majoliche italiane, marche e monogramme. Milano I88I.

Graul und Kurzwelly, Altthüringer Porzellan. Leipzig I909.

Haward, Histoire de la Faïence de Delft. Paris I908.

Hobson = Burton a. Hobson, Handbook of Marks on Pottery and Porcelain II. Aufl. London I9I2.

Hodgkin, Examples of early English pottery. London I89r.

Hofmann, F. H., Das europäische Porzellan des Bayrischen Nationalmuseums. München I909.

Houzé, de l'Aulnoit. Essai sur les Faiences de Douai, ses Grès Anglais. Lille I 882 .

Jacquemart, Les merveilles de la céramique. Paris $\mathrm{I} 866$ - I 869 .

Jaennicke, Grundriß der Keramik. Stuttgart 1878-1879.

Jewitt, The ceramic art of Great Britain. London 1878 .

Kraus, Die Marken der Porzellanmanufaktur in Frankenthal 1756-I80o. Frankenthal 1899 .

Lill, G., Fayencemarken (Sonderabdruck aus den „Mitteilungen“ der Galerie Helbing). München, Helbing I9I4.

Malagola, Memoire storiche sulle majoliche di Faenza. Bologna I88o.

Marryat, Collections towards a history of Pottery and Porcelain. London I 850 .

Mély, La céramique italienne, marques et monogrammes. Paris.

Morse, Edw. S., Catalogue of the Morse Collection of Japanese Pottery (Museum of fine Arts Boston). Cambridge I8gr.

Panietnik Wystawy, Ceramiki i Szkla Polskies. Warszawa I9I3.

Passeri, Istoria delle pitture in majolica fatte in Pesaro etc. Venise I758.

Pazaurek, Keramik. (Nordböhmisches Gewerbemuseum.) Reichenberg I905.

Pottier, Histoire de la Faïence de Rouen. Rouen I870.

Ris-Paquot, Histoire générale de la Faïence ancienne française et étrangère. Amiens et Paris 1874 et 1876 .

Rondot, Céramique lyonnaise. Paris 1899.

Sauerlandt, M., Fabrikmarken und Malersignaturen der Thüringischen Fayencemanufakturen des XVIII. Jahrhunderts (Sonderabdruck der ThüringischSächsischen Zeitschrift für Geschichte und Kunst, II. Bd.).

Segange (Du Broc de Segange). La Faïence et les Faïenciers de Nevers. Nevers I 863 .

Vignola, Sulle Majoliche e Porcellane del Piemonte. Torino I878. 


\section{Fayence.}

Seite

\section{Florenz.} 3I-34: Bode. Die Anfänge der Majolikakunst in Toskana. Berlin I9II.

\section{Faenza.}

I: London, Vict. u. Alb. Mus., nach Ballardini: Caffagiolo? und nicht Name des Künstlers, sondern des Bestellers. 2: Sèvres, Mus., nach Ballardini: nicht Name des Künstlers, sonders des Bestellers. 3: London, Vict. u. Alb. Mus. 4: Pesaro, Mus.

3. 6: Bologna, S. Petronio.

Io: Chaffers (Coll. Narford). II: Limoges, Mus. I2: Mely (Pesaro). I3: London, Vict. u. Alb. Mus. I4: Fortnum (Coll. Basilewsky).

4. I6: Berlin, Kgw.-Mus. I7, I8: London, Brit. Mus. I9: London, Brit. Mus. 22: Genolini. 20, 21 : Fortnum. 25: Malagola. 26: Fortnum. 27: Chaffers. 28: Genolini. 30: Chaffers.

5. 35: London, Coll. Salting. 36: London, Vict. u. Alb. Mus. 37: Fortnum (Coll. Basilewsky). 38: Delange (Coll. d'Azeglio). 39: Fortnum, Coll. Castellani. 38, 39: Argnani. 40: Fortnum (40: Coll. Soulages). 4I: Paris, Louvre. 42: London, Vict. u. Alb. Mus. 43: London, Vict. u. Alb. Mus. 44: Chaffers, Coll. Falcke. 45: London, Vict. u. Alb. Mus. 46: London, Vict. u. Alb. Mus.

6. 47: Genolini. 48: London, Vict. u. Alb. Mus. 49: Berlin, Kgw.-Mus. 50: London, Brit. Mus. 5I: Fortnum (Delange). 53: London, Mus. of Geology. 54: Fortnum, Coll. Marryat. 55: Chaffers, Coll. Marryat. 56: Genolini, Collect. G. v. Rothschild. 57: Berlin, Kgw.-Mus. 58: Paris, Louvre. 59: Paris, Louvre.

7. 60: Oxford. 6I: Venedig, S. Sebastiano. 62: Fortnum. 63: London, Brit. Mus. 64: London, Brit. Mus. 65: Chaffers. 66: London, Coll. Salting. 67: London, Brit. Mus. 69: Fortnum. 70: Fortnum (Coll. Cajani). 71: London, Vict. u. Alb. Mus. 72: Fortnum. 73: London, Vict. u. Alb. Mus. 74: Fortnum (Delange). 75: Chaffers (Coll. Bernal).

8. 76: Genolini. 77: Berlin, Kgw.-Mus. 78: Berlin, Kgw.-Mus. 79: Sèvres, Mus., nach Ballardini: nicht Name des Künstlers, sondern des Bestellers. 80 : Sigmaringen, Mus. 8r: Coll. Bernal. 82: Venedig, S. Sebastiano. 83: Fortnum. 84: Chaffers. 85: London, Vict. u. Alb. Mus. 86: Chaffers (Coll. Campana). 87: Chaffers. 88: Coll. d'Azeglio. 89: Chaffers (Coll. Narford). 90: London, Coll. Salting. 9r: Coll. Soltikoff. 
9. 92: Fortnum (Delange). 93: Malagola. 94: London, Vict. u. Alb. Mus. 95: Chaffers (Venedig). 96: Coll. Castellani. 98: London, Brit. Mus. roo: Fortnum (Delange). ro3: Fortnum, Coll. Nesbitt. I04: Fortnum (Coll. Tellot de Dreux). 105: Argnani. ro6: Genolini. I07: Genolini.

I0. IIO: Malagola. III: Jaennicke. II3: Genolini. II4-II6: Fortnum Coll. Barker). II7: Fortnum. II 8: Louvre. II9: Chaffers (Coll. Uzielli). I20: Chaffers (Coll. Barker). I22: London, Vict. u. Alb. Mus. I23: Chaffers (Coll. Barker).

I I. I26: Fortnum (Urbani). I28: Faenza, Mus. d. Fabriche Ferniani. I29: Fortnum. I30: Fortnum (vielleicht Imola). I3I: Genolini, Limoges, Mus. 132: London, Vict. u. Alb. Mus.

Außerdem viele andere Marken bei Argnani: Le ceramiche e majoliche faentine, Faenza, I889 - Il Rinascimento delle ceramiche majolicate in Faenza. Faenza, 1898 .

I2. Verona.

I: London, Bracon Hall. 2: Kopenhagen, Kunstindụstrij museum.

\section{Gubbio.}

I: Chaffers (Coll. Falcke). 2: Mely. 3: Chaffers (Coll. Campana). 4: Geno-, lini. 5: Fortnum. 7: London, Vict. u. Alb. Mus. 8: Chaffers (Coll. Soulages). 9: London, Vict. u. Alb. Mus. Io: London, Vict. u. Alb. Mus. Ir: Chaffers (Coll. Solages). I2: London, Vict. u. Alb. Mus. I4: Fortnum (Coll. Barker). r6: Genolini.

I3. I7: Fortnum (Passeri). I8: Chaffers (Paris, Coll. Leroy-Laduric). I9: London, Vict. u. Alb. Mus. 20: London, Vict. u. Alb. Mus. 2I: London, Vict. u. Alb. Mus. 23: Chaffers (Coll. Barker). 24: Genolini. 25: Chaffers (Coll. Amhurst). 26: London, Coll. Salting. 27: I.ondon, Coll. Wallace.

I4. 28: Delange. 29: Delange (Rouen, Coll. Dutuit). 30: Genolini (Coll. Addington).

I5. 31: Genolini. 32: London, Vict. u. Alb. Mus. 33: Genolini. 34: Paris, Louvre. 35: Genolini. 36: Rom (Coll. Castellani). 37: Fortnum (Coll. Marnelli). 38: Fortnum. 39: London, Vict. u. Alb. Mus.

I6. 40: Fortnum (Coll. Barker). 4I: Bologna, Mus. de l'université. 42: Genolini. 44: Fortnum (Coll. Castellani). 46-48: Fortnum.

I7. 49: Fortnum (Coll. Cajani). 50: Paris, Louvre. 5I: London, Vict. u. Alb. Mus. 52: Sèvres. 53: Garnier. 55: Mely (Coll. A. de Rothschild). 56: Fortnum. 57: Genolini. 58: London, Vict. u. Alb. Mus. 59: Chaffers (Coll. Campana). 60: London, Vict. u. Alb. Mus. 6r: Oxford. 62: Chaffers. 63: Chaffers. 64: Delange (?). 66: Mely. 67: Chaffers.

I8. 68: Chaffers (Coll. Campana). 69: Paris, Louvre. 70: Fortnum (Coll. Spitzer). 7I: Fortnum (Coll. Castellani). 72: Fortnum (Coll. Castellani). 73: London, Brit. Mus. 74: Chaffers (Passeri). 75: Paris, Louvre. 76: Paris, Louvre. 77: Genolini. 79: Chaffers (Coll. Berney). 80: Chaffers (Coll. Berney). 8I: Fortnum (Coll. Falcke). 82: Paris, Louvre.

I 9. 83 : London, Brit. Mus. 85: Kopenhagen (Coll. Jacobsen). 86, 87: London, Brit. Mus. 88: Fortnum. 89: Pesaro, Mus. 90: Mely. 9r: Mely (Coll. A. de Rothschild). 92: London, Brit. Mus. 93: London, Vict. u. Alb. Mus. 
Seite

20 . (Coll. Narford). ror: London, Vict. u. Alb. Mus. ro3: Genolini. ro4: Chaffers (Coll. Barker). ro5: Paris, Louvre. 106: Chaffers. 107: Paris, Louvre. ro8: London, Vict. u. Alb. Mus. I09: Paris, Louvre. I Io: Paris, Louvre. I I I: Paris, Coll. Campana. II3: Fortnum (Coll. Gagliardi). II4: Fortnum. II5: Chaffers (Coll. Farrer).

\section{Caffagiolo.}

2I. I: London, Coll. Salting. 2: London, Vict. u. Alb. Mus. 3: Fortnum (Coll. G. de Rothschild). 4: Fortnum (Coll. Lockwood). 5, 6: Chaffers (Jacquemart). 7: Chaffers. 9: Paris, Louvre. ro: Fortnum. I2: Fortnum. r3: Mély (Bologna). I4: Chaffers (Jacquemart): Deruta? I6: Chaffers (Coll. Barker). I7: Jaennicke (Coll. Fountaine).

22. I8: Jaennicke (Coll. Davillier). I9: London, Alb. u. Vict. Mus. 20-22: Fortnum (Coll. Rothschild). 24: Paris, Louvre. 25: Delange (Coll. Fau). 26: Mély (Coll. A. de Rothschild). 27: Delange (Coll. d'Azeglio). 28: Fortnum (Coll. Basilewsky). 29: Fortnum. 30: London, Vict. u. Alb. Mus. (Fortnum: Faenza?). 31. London, Vict. u. Alb. Mus. 32: Paris, Louvre. 34: Oxford.

23. 36: Mely, Pesaro, Mus. (?). 37: Delange. 38: London, Vict u. Alb. Mus. 39: London, Vict. u. Alb. Mus. 40: Chaffers. 4I: Chaffers (Coll. Henderson). 44: London, Vict. u. Alb. Mus. 45: Oxford. 46: Chaffers (Coll. Hastings). 47: London, Vict. u. Alb. Mus.

\section{Pesaro.}

I: Delange (Demin). 2: Pesaro, Mus. 3: London, Vict. u. Alb. Mus. (Fortnum: Viterbo). 5: Bologna, Universitätsmuseum. 6: Brit. Mus. 7: Chaffers (Coll. d'Azeglio). 9: Chaffers (Passeri). ir: Pesaro, Mus.

25. I2: Chaffers (Passeri). I3: Limoges, Mus. I4: Pesaro, Mus. I5: Mely (Coll. A. de Rothschild). r6: Pesaro, Mus. I8: Neapel: Mus. de S. Martini. 21: Pesaro, Mus. 22: Fortnum (Coll. de Mely). 24: Limoges, Mus. 25: Pesaro, Mus. 26: Chaffers (Jacquemart): Pesaro (?). 27: Chaffers (Coll. M. Bruge). 29: Fortnum. 30: Mely (Coll. G. de Rothschild). 3r: Jacquemart.

26. 32: Fortnum (Coll. Castellani): Pesaro (?). 33: Haag, Mus. (Fortnum: Gubbio?). 34: Oxford. 35: Fortnum (Coll. Castellani): Pesaro (?). 36: I.ondon, Brit. Mus. 37: Fortnum (Coll. Castellani): Pesaro (?). 38: Garnier.

\section{Candiana.}

I: Genolini. 2: Genolini (Davillier). 3: Sèvres Mus.

\section{Monte Lupo.}

I: Paris, Mus. Cluny. 2: Genolini. 3: London, Vict. u. Alb. Mus. 4: Chaffers (Jacquemart). 5: London, Vict. u. Alb. Mus. 6: London, Vict. u. Alb. Mus.

\section{Loreto.}

\section{Urbino.}

Jaennicke.

I: Fortnum (Coll. Narford). 2: Genolini. 3: Paris, Louvre. 4: Chaffers 5: Chaffers.

28. 6: London, Brit. Mus. 7: Garnier. 8: Genolini. 9: Fortnum (Passeri). I I, I2: Genolini. I3, I4: Fortnum (Coll. Narford): Orazio Fontana? I5: Paris, Louvre.

Führer. 
Seite

29. Brit. Mus. 20: Florenz, Mus. Nazionale. 21: London, Brit. Mus. 22: London,
Brit. Mus. (Fortnum: Gubbio Diruta). 23: Paris, Louvre.

26: Fortnum (Coll. Basilewsky).

30. 27: Jaennicke (Loreto, Santa Casa). 28: Mely. 29: Jaennicke. 30: Genolini. 31: Chaffers. 32: Coll. Amhurst. 33: Mely. 34: Genolini. 36, 37: London, Vict. «. Alb. Mus. 38: London, Vict. u. Alb. Mus. 39: Paris, Louvre. 40: Genolini. 4I: Chaffers. 42: Genolini. 43: Fortnum (Coll. Narford). 44: London, Vict. u. Alb. Mus.

31. 45: Genolini. 46, 47: Mely. 48: Jaennicke. 50: Rom (Coll. Barberini). 5I: London, Vict. u. Alb. Mus. 52: Genolini. 53: Genolini. 54: Fortnum.

55: Genolini. 56: Genolini. 57: London, Vict. u. Alb. Mus.

32. 58: London, Brit. Mus. 59: London, Vict. u. Alb. Mus. 6o: Fortnum (Coll. Narford). 6r: Chaffers (Coll. S. de Rothschild). 62: Bologna, Universitätsmuseum. 63: London, Brit. Mus. 64: Chaffers (Coll. Fortnum). 65: Paris, Louvre. 67: Chaffers. 68: Chaffers (Coll. Evans Lombe). 69: Chaffers (Coll. Addington). 70: (gehört zu Nr. 68). 7I: London, Brit. Mus. 72: Venedig, Mus. Correr.

33. 73: Fortnum (Coll. J. de Rothschild, Paris). 75: Oxford. 77: Chaffers. 78: Paris, Louvre. 79: Mely. 80: London, Vict. u. Alb. Mus. 8I: Fortnum (Coll. Northwith). 82: Mely (Coll. Narford). 83: Chaffers (Coll. Berney).

34. 84: Garnier (Coll. Spitzer). 85: Fortnum (Coll. Foresi). 86: Pesaro, Mus. 88: London, Vict. u. Alb. Mus. 90: London, Vict. u. Alb. Mus. 9I: Fortnum. 92: Fortnum (Coll. Cajani). 93: Chaffers. 94: London, Vict. u. Alb. Mus. 95: Genolini. 97: London, Vict. u. Alb. Mus. 98: Chaffers (Coll. Delsette).

35. 99: Genolini. Ioo: Fortnum (Coll. Narford). Ior: Genulini. I02: Pavia. 103: Mely. ro4: Urbino, Mus. ro6: Chaffers (Coll. d'Azeglio). ro7: London, Brit. Mus. I09: Fortnum (Coll. d'Azeglio). Iro: Urbino Mus. II I: Gennlini. I12: Fortnum. II4: Fortnum (Coll. Narford). Ir 5: Chaffers (Coll. Napier). II6: Chaffers (Coll. d'Azeglio).

36. II7: London, Brit. Mus. I 8: Chaffers (Delange). II9: Paris, Louvre. I20: London, Vict. u. Alb. Mus. I22: London, Vict. u. Alb. Mus. 123: Chaffers (Coll. Falcke). 124: Cbaffers (Coll. Campana). 125: Pesaro, Mus. 127: Delange (Coll. Nieuwerkerke). 128: Fortnum (Coll. Cajani): Castel Durante? 129: Fortnum (Pisa, Coll. Toscanelli). I30: Jaennicke (Coll. Berney). I3I: Genolini. 133: Fortnum. 134: London, Brit. Mus. 135: Genolini. 135: London, Vict. u. Alb. Mus. 137: London, Brit. Mus. (Fortnum: Urbino?).

37. 138: Paris, Louvre. 139: London, Vict. u. Alb. Mus. 140: Fortnum (Coll. Narford). I4I, I42: Paris, Louvre. I43: Genolini. I44: Mus. Pesaro. I45: Neapel (Coll. Rey). I46: Mely. I47, I48: Urbino, Mus. I50: Paris, Louvre. I51: Mely.

Rimini.

I: Genolini. 2: Fortnum (Coll. Basilewsky). 3: London, Brit. Mus. 4: Mely. 5: Bologna, Mus. 6: Mely.

38. Castel Durante.

I: Fortnum (Coll. Hope). 2: Paris, Mus. Cluny. 3: Fortnum. 4: Mely (Pesaro, Mus.): 5: Bologna, Universitätsmuseum. 6: Chaffers (Coll. d'Azeglio). 
Sei te

7: Genolini (Coll. Genolini). 8: Fortnum (Coll. Castellani). 9: London, Brit. Mus.

39. II: Fortnum. 12: Pesaro, Mus. I3: Paris, Louvre. I4: Fortnum (Galerie Barberini). 15: Paris, Louvre. 16: Jaennicke (Coll. Neck). 17: Paris, Louvre. 18: London, Brit. Mus. 19: Mely (Pesaro, Mus.). 20: Genolini. 21: Pesaro, Mus. 23: Fortnum: Castel Durante? 24: London, Brit. Mus. 25: London, Brit. Mus., nach Ballardini: Castel Durante? 26, 27: London; Brit. Mus. 28, 29: London, Brit. Mus., nach Ballardini: Castel Durante? 30, 3I: London, Brit. Mus. 32: London, Brit. Mus., nach Ballardini: Castel Durante?

40. 33: London, Brit. Mus. 34: Jaennicke (Rom, Coll. Cajani). 35: Fortnum (Coll. Devidis). 36: London, Vict. u. Alb. Mus. 37: Chaffers. 39: Paris, Lourre. 40: Mely (Rom, S. Maria degli Angeli).

\section{Ravenna.}

I: Sèvres; Paris, Louvre. 2: Chaffers (Coll. Henderson).

4I. Treviso.

I: Chaffers (Coll. Addington). 3: Jacquemart.

\section{Fabriano.}

I: Garnier (Coll. Spitzer). 2: London, Vict. u. Alb. Mus.

\section{Padua.}

I: London, Brit. Mus. 2: Paris, Mus. Cluny. 3: Padua, Mus. 4: London, Mus. of Geology. 5: London, Vict. u. Alb. Mus.

\section{Ferrara.}

Este.

London, Vict. u. Alb. Mus.

Genolini (Coll. Reynold).

\section{Città di Castello.}

I: Genolini. 2: London, Vict. u. Alb. Mus.

\section{Deruta.}

I: Jaennicke (Coll. de Cambio). 2: London; Vict. u. Alb. Mus. 3: Paris, Louvre. 4, 5: London, Vict. u. Alb. Mus, 6: Jaennicke (Coll. Basilewsky). 7: Genolini. 8: Fortnum (Coll. Castellani). 9: London, Vict. u. Alb. Mus. Io: Paris, Louvre. II: London, Vict. u. Alb. Mus. I2: Paris, Louvre. I3: Gụbbio, Mus.

44. I5: Chaffers. I6: Chaffers (Perugi, Coll. Baglionia). I7: Chaffers. (Coll. Nieuwerkerke). 18: Genolini. 19: London, Vict. u. Alb. Mus. nach Ballardini: Faenza; 20: Paris, Mus. Cluny. 21, 23: Fortnum (Coll. Mely). 24: Fortnum. 26: Fortnum (Coll. S. de Rothschild). 27: Limoges, Mus. 28: Jaennicke (Coll. Addington).

45. Venedig.

I, 2: London, Vict: u. Alb. Mus. 4: London, Vict. u. Alb. Mus. 5, 6: Fortnum. 7: Chaffers (Coll. Bernal). 8: London, Brit. Mus. 9: Genolini. ro: Chaffers. II: Chaffers (Coll. Uzielli). 13: Chaffers (Coll. Roger de Beauvoir). 14: Berlin, Kgw. Mus. I5: Chaffers (Coll. Chaffers). I6: Limoges, Mus. (Fortnum: Porzellan?).

46. I7: London, Vict. u. Alb. Mus. I8: Fortnum (Coll. Fountaine). I9: Genolini (Coll. Fountaine). 20, 21: London; Vict. u. Alb. Mus. 22: Oxford, Mus. 


\section{- 308}

Seite

47.

24, 26: Mely. 27: Mely: Porzellan! 28: Fortnum (Genolini). 29: London, Brit. Mus. 30: London, Brit. Mus. 3r: Sèvres, Mus. 32: Chaffers (Genolini). 33: Fortnum (Genolini). 34: Limoges, Mus. 35: Fortnum (Genolini). 36: Genolini. 37: Stuttgart, Landesgewerbemuseum.

48. Forli.

I: Chaffers (Jacquemart). 2: Paris, Louvre, 4: Bologna, Universitätsmuseum. 5: Paris, Louvre. 6, 7: London, Vict. u. Alb. Mus. 8: London, Coll. Salting. 9: Ravenna, Bibliothek.

49. Io: Fortnum (Coll. Basilewsky). Ir: Forli, Bibliothek. I2: Fortnum (Coll. Basilewsky). I3: London, Coll. Salting.

\section{Siena.}

I: London, Vict. u. Alb. Mus. 2: London, Brit. Mus. 3: Paris, Louvre.

50. 6: London, Brit. Mus.

4: London, Vict. u. Alb. Mus. 5: Fortnum (Coll. A. de Rothschild).

San Quirico (Siena).

I, 2: London, Vict. u. Alb. Mus. 3: Fortnun.

\section{Perugia.}

I: Chaffers.

\section{Bagnorea.}

2: Fortnum London.

\section{Asolo.}

51. Rom.

Jaennicke (Coll. L.iesville). Genolini (Mely): Veredig?

I, 2: Fortnum. 3: Jaennicke (Coll. Lockwood).

\section{Bassano.}

I: London, Vict u. Alb. Mus. 2: Venedig, Mus. Correr. 3: Limoges, Mus. 4: Paris, Louvre. 5: Chaffers (Coll. Le Blanc). 6: Chaffers. 7, 8, 9: Fortnum.

52. Turin.

I: Chaffers (Coll. d'Azeglio). 2: Genolini. 3: Chaffers (Coll. d'Azeglio). 4: Chaffers: 5: Chaffers (Jacquemart). 6: Chaffers (Hull, Coll. Jackson). 7: Sèvres. 9: Chaffers (Coll. d'Azeglio). II: Fortnum. I 2: Chaffers (Coll. d'Azeglio). 15: Fortnum. 17: Genolini.

53. Pisa.

I: Mely (Coll. Castellani). 2: Garnier (Coll. A. de Rothschild).

\section{Nove.}

I: London, Vict. u. Alb. Mus. 2-4: Genolini. 5: Fortnum (Genolini). 6, 7, 8, 9: Genolini. ro: Fortnum (Genolini). Ir: Limoges, Mus. I2, I3: Genolini.

\section{Borgo S. Sepolchro.}

Jaennicke.

\section{Vinovo.}

I: Fortnum. 2: Garnier (II) (Limoges, Mus.). 3: Mely. 


\section{- 309}

Seite

\section{Lodi.}

I, 2, 3: Chaffers (Coll. Reynolds). 4: Sèvres. 5: Jacquemart. 6: Genolini(?). 7: Genolini (?).

\section{Viterbo.}

I: London, Vict. u. Alb. Mus. 2: Rom, Palazzo Barberini.

\section{Neapel.}

I, 2, 3, 4: Genolini. 5; Chaffers (Jacquenart). 6, 7: Limoges Mus. 8: Chaffers (Demmin). 9: Fortnum. Io: Chaffers (Jacquemart). I I: Genolini. I2: Chaffers (Brogniart). I3: Genolini. I4: Sèvres. I5: Chaffers. I6: Chaffers (Genolini). I7, 19, 20: Genolini. 21: Chaffers (Genolini). 22, 23: Genolini.

56. 26: Fortnum (Jaennicke: Castelli?).

\section{Palermo.}

\section{Castelli.}

I: Sèvres. 2: Fortnum (Coll. Castellani). 4, 5: Fortnum (Coll. Corona).

2: Chaffers. 3: Jaennicke (London, Coll. Neck). 4: Chaffers. 5: Sèvres, Mus.

57. 6: Chaffers (Coll. Hasting). 7: Chaffers. 8: Sèvres Mus. Ir: Chaffers (Coll. Diamond). I5: London, Vict. u. Alb. Mus. I6: Chaffers (Coll. Reynolds). I7, I8: Genolini. I9: Paris, Louvre. 21: Garnier. 22-26: Genolini. 27: Chaffers. 28: Berlin, Kgw. Mus. 29: Chaffers (Passeri). 30: Jaennicke (Coll. Neck). 3r: Garnier. 32: Fortnum.

\section{Savona.}

I, 2: Genolini. 3: Chaffers. 5: Chaffers (Coll. Mont Ferrand). 6: Fortnum. 7, 8: Chaffers. 9: Neapel, S. Martinu. Io: Genolini. 12, 13: Neapel, S. Martino. 14: Vignola. I5: Genolini. I6: Vignola. I7: Genolini. I9: Neapel, S. Martino. 20: Fortnum. 2I: Chaffers (Coll. Reynolds).

59. 22: Chaffers (Coll. Staniforth). 23, 25: Genolini. 26: Vignola. 27: Fortnum. 28: Genolini. 30: London, Vict. u. Alb. Mus. 3r: Neapel, S. Martino. 32: Pavia, Coll. Reale. 33-35: Chaffers (Paris, Coll. Dr. Belliol). 36, 37 : Genolini.

60. 38: Fortnum, Chaffers (Coll. d'Azeglio): Tuvin? 39: Chaffers (Coll. d'Azeglio): Turin? 40, 4I, 42, 43: Genolini. 45: Genolini. 46: Garnier. 47, 48, 49: Genclini. 50: Fortnum. 5I, 52: Genolini.

6I. 53: Chaffers. 54: Genolini. 55: Vignola. 56: Genolini. 57: Vignola 58: Garnier: B. Salomoni. 59: Genolini. 60: Vignola. 62-64: Genolini.

62. 65: Fortnum. 66, 68, 69: Genolini. 70: Vignola. 71, 72, 73: Genolini. 74: Vignola. 75: Garnier (II) (Mus. Limoges) 76, 77, 80: Genolini, 83: Garnier. 84: Chaffers (?). 85: Mely: Genua? 86: Genolini. 87: Genolini: Savona? Garnier: Cienua.

63. 89: Sèvres. 90: Garnier (II) (Limoges, Mus.). 91: Genolini. 92: Fortnum. 93: Garnier (II) (Limoges, Mus.). 94, 95, 96: Garnier (II) (Limoges, Mus.). 97: Chaffers (Coll. Reynolds). 98: Genolini. 99: Fortnum. roo: Genolini; Chaffers (Coll. Diamond): Ciemua?

\section{Mailand.}

I: Sèvres Mus. 2: Fortnum (Coll. Drake). 3-6: Genolini. 7: Jacquemart (Bordeaux, Mus.). 8: Genolini. 9: Mely. Io: Genolini. I2: Fortnum (Coll. Reynolds). 13: Chaffers (Coll. Reynolds). I4: Chaffers (Coll. d'Azeglio). 
Seite

65. Albissola.

\section{Pavia.}

I-3: Genolini.

I: Fortnum. 2: Fortnum (Coll. Brambilla, Pavia). 3: zu I. 4: Genolini. Genua.

66. Asciano.

2: Jaennicke (Turin, Coll. Devers). 3: Chaffers: Gienua?

I, 2: Fortnum.

Venedig.

4: Chaffers (Braunschweig. Mus.).

S. Cristoforo (bei Mailand): 29 . Jahrh. 3: Chaffers.

Mondovi.

I-3: Fortnum.

Doccia: 19. Jahrh.

67. Hispano-Maurisch.

I, 2: Jaennicke (Coll. Fortnum). 3: Jaennicke. 4: Chaffers (Coll. Amhurst). 5: Paris, Louvre.

Puente di Arzobispo.

Chaffers.

Talavera.

68. Alcora.

I: Jaennicke. 4, 5: Chaffers. 6: Jacquemart. 7: Jaennicke (Alcora?). 9: Garnier.

Sevilla.

I, 2: Hobson.

Manises.

Jaennicke (Coll. Reynolds).

Valencia.

Chaffers.

69. Miragaïa (Porto).

I, 2: Chaffers.

Lissabon.

I, 2: Chaffers.

70. Beauvais.

Paris.

I: Paris, Mus. Cluny. 2: Jacquemart.

Jaennicke (Coll. Tusseau).

St. Porchaire.

I: Jaennicke (Coll. J. v. Rothschild). 2: London, Vict. u. Alb. Mus.

Lisieux.

Ris-Paquot. 
Seite

Nachahmer Palissys.

I: Chaffers. 2: Paris, Louvre.

Lyon.

3: Garnier. 4: Lyon, Mus.

7r. Nantes.

Jaennicke.

\section{Nevers.}

I:: Sèvres. 2: Chaffers (Coll. Filloni). 3: Segange. 4: Jacquemart. 5: Sèvres. 6: Nevers, Mus. 7: Jacquemart. 8: Chaffers. 9: Chaffers (Coll. Pottier). ro: Jaennicke. II: Garnier (II) (Limoges, Mus.).

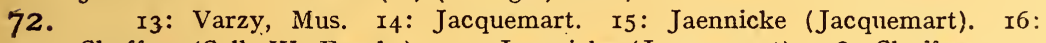
Chaffers (Coll. W. Franks). I7: Jaennicke (Jacquemart). 18: Chaffers. I9: Chaffers. 20: Jaennicke.

\section{St. Verain.}

Sèvres Mus.

\section{Rouen.}

I: Chaffers. 2: Jacquemart. 3: Rouen (Coll. Gouellain). 4: Jaennicke (Coll. de Lestanville). 5: Pottier. 6, 7: Pottier. 8: Chaffers (Coll. Bohn). Io: Jaennicke: Rouen? II: Pottier (Cull. Bellegarde). r2: Sèvres Mus. I3: Pottier. I4: Jaennicke (Rouen, Cull. d'Arboval). 15, r6: Pottier (Coll. Gouellain). I7: Rouen, Mus. I8: Jaennicke. I9-2I: Pottier (Coll. Baudry).

74. 22, 23: Pottier. 24: Pottier (Coll. Gouellain). 25: Pottier. 26: Jacquemart. 27: Pottier. 28: Jacquemart. 29: Pottier. 30-36: Ris-Paquot. 37: Pottier. 38: Ris-Paquot. 39: Pottier.

75. 4r: Pottier. 42, 43: Pottier. 44, 45: Pottier. 47: Pottier. 48-50: Pottier. 52: Jacquemart (?). 55: Rouen, Mus. 57: Pottier (Coll. Loifel). 59: Pottier. 60: Jacquenart. 6r: Jaenniske. 62: Pottier. 63, 65: Pottier. 67: Jacquemart, Pottier. 69: Pottier. 70: Jacquemart.

7r: Pottier (Coll. le Coupeur). 72: Ris-Paquot. 73: Jacquemart. 74: Pottier. 75, 76: Pottier. 77: Jacquemart. 8n: Pottier. 82: Rouen, Mus. 83 bis 88: Pottier. 89: Pottier (Coll. Maillet du Boullay). 90: Rouen, Mus. 91, 92: Pottier. 93, 94: Ris-Paquot. 95, 96: Pottier. 97: Mus. Rouen. 98, 99: Jacquemart.

77. IOO: Pottier: IOI, I02: Jacquemart. I03: Pottier. I04, I05: Jacquemart. ro6: Pottier (Coll. Gouellain). ro7: Chaffers. ro8: Ris-Paquot. Iro: Rouen, Mus. III, II2: Pottier. II3: Ris-Paquot. II4, II5: Pottier. II6: Ris-Paquot. II7: Ris-Paquot. II8: Jacquemart. II9: Pottier. I20: Ris-Paquot. I2I: Jacquemart (?). I22, I24: Jacquemart. I25, I26: Ris-Paquot. I24: Pottier. I29: Jaennicke. I30, I3I: Jacquemart. I32: Pottier. I33: Jacquemart.

78. 135, I36: Pottier. I37: Ris-Paquot. I38, I39: Jacquemart. I40-I42: Pottier. I43: Jaennicke. I45, I46-I48: Pottier. I49: Ris-Paquot. I50: Chaffers. I5I: Pottier. I52: Jacquemart. I53: London, Vict. u. Alb. Mus. I54: Pottier. I55-158: Jacquemart. I59: Ris-Paquot. I60: Jacquemart. I61-r63: Jacquemart. I64: Ris-Paquot. I65: Puttier. I66: Ris-Paquot. r67: Pottier. I69: Ris-Paquot. I70-I72: Pottier. 
79. I73: Ris-Paquot. 174: Ris-Paquot. I75: Jacquemart. I76: Pottier. I77, I78: Jacquemart. I80: Ris-Paquot. I8r: Jacquemart. I82: Pottier. I83: Ris-Paquot. I84, I85: Jacquemart. I86: Pottier. I87, I9I: Jacquemart. 188: Ris-Paquot. I89: Pottier (Coll. Delauney). I92: Jacquemart. I94: Ris-Paquot. 195: Jacquemart. I96: Jacquemart (?). r97: Pottier. r98: Jacquemart. 199: Pottier. 200: Jacquemart. 201 : Pottier. 202: Jacquemart. 203: Pottier. 204: Chaffers. 206: Ris-Paquot.

80. 207, 208: Pottier. 209: Chaffers. 210: Jacquemart. 2II, 213, 214: Pottier. 215: Ris-Paquot. 216: Pottier. 217: Ris-Paquot. 218: Ris-Paquot. 219: Jacquemart. 221: Pottier. 222, 223: Ris-Paquot. 22.4, 225: Jacquemart. 228: Pottier (Coll. Loifel). 229, 230: Pottier. 231, 232: Jacquemart. 233: Ris-Paquot. 235: Ris-Paquot. 236: Pottier. 237-239: Ris-Paquot. 240: Jacquemart.

8I. 24I: Rouen, Mus. 242: Ris-Paquot. 245: Jacquemart. 247: Ris-Paquot. 248: Pottier. 249-25I: Jacquemart. 252: Pottier. 254: Chaffers. 255: Ris-Paquot. 256: Jacquemart. 257: Pottier. 258, 259: Jacquemart. 260: Pottier. 26r: Jacquemart. 262, 263, 264: Ris-Paquot. 265-268: Pottier 269: Ris-Paquot. 270: Jacquemart. 27r: Pottier. 272: Jacquemart.

82. 273: Pottier. 274, 275: Ris-Paquot. 276: Jacquemart. 277: Pottier. 278, 279: Jacquemart. 280: Ris-Paquot. 281: Jacquemart. 282: Jacquemart. 283-285: Katalog d. Versteigerung Lana I, I909, Berlin, Lepke.

\section{Moustiers.}

I: Daviliier (Coll. Davillier). 2: Davillier. 3, 4: Chaffers. 5: Chaffers (Coll. Reynolds). 6-II: Davillier. I2: Sèvres, Mus. 13-I6: Davillier. I7: Sèvres Mus.

83. I 8-20, 22: Jaennicke. 23: Ris-Paquot. 25: Jaennicke. 26-29: Davillier. 30: Ris -Paquot. 31: Davillier. 32: Chaffers. 33-35: Davillier. 36: RisPaquot. 37-39: Davillier. 4I: Davillier. 42, 43: Ris-Paquot. 45: Davillier.

84. 46, 47, 48: Davillier. 49, 50: Sèvres Mus. 52: Ris-Paqu.t.

\section{Aire.}

\section{Vron.}

Sèvres Mus.

\section{Desure.}

2: Ris-Paquot.

85. I: Ris-Paquot. 2: Chaffers (Coll. Reynolds). 3-8: Ris-Paquot. 9, Io: Ris-Paquot.

Saint Paul.

Chaffers.

Saint-Omer.

\section{Douai.}

I-4: Ris-Paquot.

I-3: Houzé. 4: Houzé (Douai, Mus.). 5-8: Houzé (?). 9: Houzé (Coll. Houzé). ro: Houzé (Douai, Mus.).

\section{Lille.}

I: Jaennicke (Coll. Salin). 3, 4: Ris-Paquot. 5: Jacquemart (?). 6: RisPaquot. 8: Ris-Paquot. 9: Ris-Paquot. Io: Jaennicke. II: Ris-Paquot. 


\section{- $3 \mathrm{I} 3$}

Seite

12: Jaennicke. I3: Ris Paquot. I4: Sèvres Mus. I5: Ris-Paquot. I6: RisPaquot. I7-I9: Ris-Paquot.

87. 22: Garnier (Coll. Houdoy). 23: Ris-Paquot. 24: Ris-Paquot. 25: RisPaquot. 27, 28: Jaennicke. 3I-33: Ris-Paquot.

Samadet. Sèvres Mus.

88. St. Amand. I0: Chaffers. I I : Jaennicke. I3: Ris-Paquot. I7: Chaffers. I 8: Jaennicke 19: Ris-Paquot.

89. 23: Ris-Paquot, Jacquemart. 25: Ris-Paquot.

90. Valenciennes.

3: Lejeal, Recherches historiques sur la manufacture de faïence et do porcelaine de Valenciennes I868. 6: Ris-Paquot. 7: Jaennicke: Porzellan ? 8: Ris-Paquot.

91. Paris.

I-20: Jaennicke. 2I, 22, 23-26: Jaennicke. 27: Chaffers.

92. Sinceny.

I: Chaffers (Coll. Warmont). 2, 3: Ris-Paquot. 5: Sèvres Mus. 7: RisPaquot. 8: Chaffers. II: Jaennicke, Ris-Paquot. 9-I5, 20, 22: Ris-Paquot. 23: Sèvres Mus.

93. 27-34: Ris-Paquot.

\section{Val sous Meudon.}

I-3: Chaffers. 4: Jaennicke: Meudon?

\section{Creil.}

Chaffers.

\section{Cambray.}

Ris-Paquot.

\section{Vincennes.}

I2: Chaffers. 3: Jaennicke (Coll. Gasnault).

\section{St. Cloud.}

Chaffers.

\section{Dijon.}

I, 2: Marchant. Recherches sur la faïencerie de Dijon. Dijon r 885 (I : Coll. Morel-Retz).

\section{Aprey.}

2-5, 8: Ris-Paquot. 7, 9: Jaennicke. Io: Chaffers (Coll. Reynolds). II, I2: Ris-Paquot.

Lunéville.

I-3: Chaffers. 4: Jaennicke.

\section{Premières.}

I: Chaffers (Coll. Langford). 2-4: Chaffers.

\section{Arbois.}

Jaennicke. 
Meillonas.

I: Jaennicke. 2: Sèvres Mus. 3: Katalog d. Versteigerung Gasser (Mün chen, Helbing I9I2).

\section{Mennecy.}

2: Jaennicke.

95. Englefontaine.

Ris-Paquot.

Clermont.

Ris-Paquot.

Mathaux.

Garnier.

Ognes.

Garnier.

\section{Varages.}

I: Davillier. 3: London, Vict. u. Alb. Mus.

\section{St. Clement.}

Garnier.

Pourpres.

Sèvres Mus.

Bourges.

Toul.

Ris-Paquot.

Jaennicke.

Tavernes.

I: Sèvres Mus. 2: Garnier. 3: Jaennicke.

96. Marseille.

I: Davillier. 2-4: Chaffers. 5: Chaffers (Coll. Diamond), 6: Davillier. 7: Davillier (Sèvres Mus.). 8: Ris-Paquot. 9: Davillier. I0: Ris-Paquot. II: Chaffers. I2, I3: Ris-Paquot. I5: Chaffers (Coll. d'Azeglio). I6: Chaffers (Coll. Reynolds). I7: Ris-Paquot. I8: Ris-Paquot, Chaffers (Coll. Sauze). I9: Davillier (Coll. Davillier). 20: Ris-Paquot, Davillier (Coll. Davillier). 21: Chaffers (Coll. d'Azeglio). 22: Garnier. 23: Ris-Paquot.

\section{Montpellier.}

Chaffers.

Toulouse.

Chaffers (Coll. Reynolds).

Bordeaux.

2: Chaffers.

Poitiers.

I: Garnier. 2: Sèvres, Mus.

Mout Lomage.

2: Chaffers? 


\section{$-315$}

Seite

\section{Rennes.}

I, 2: Ris-Paquot. 3: Garnier.

\section{Bordeaux.}

I-3: Forestie, Les anciennes faĩenceries de Montauban. Ardus 1876.

Rubelles.

Jaennicke.

98. La Rochelle.

Rénac.

I: Musset. Les faïencieries Rochelaises I888 (Sèvres?). 4: Chaffers.

\section{Nîmes.}

Jaennicke (Jacquemart).

I: Sèvres, Mus. 2: Garnier (Coll. G. de Rothschild).

Ardus.

I-3: Forestie, les anciennes faïenceries de Montauban etc. I876.

Moulins.

I : Ris-Paquot. 2: Jaennicke (Moulins, Mus.). 3: Sèvres, Mus. 4: RisPaquot.

99. Quimper.

I: Garnier. 2: Chaffers. 3: Garnier. 4: Chaffers.

\section{Cleremont-Ferrand.}

Orléans.

Gien.

I: Garnier (Limoges, Mus.). 2: Jaennicke.

Ioo. Marans.

I: Jaennicke.

I: Ris-Paquot. 4: Sèvres, Mus. 5, 6: Ris-Paquot. 7: Garnier.

Sceaux.

I: Garnier. 2: Chaffers. 4: Garnier. 5: Garnier (Coll. Nattes-Villecomtal). 6: Jaennicke. 7: Garnier.

Bourg la Reine.

I, 2: Ris-Paquot. 3, 4: Sèvres, Mus.

Ior. Tour d'Aignes.

I, 2: Sèvres, Mus. 3: Jaennicke (Coll. Pascal). 4: Jaennicke.

\section{Tours.}

\section{Limoges.}

I: Sèvres, Mus. 2: Chaffers (?). 3-5: Chaffers.

\section{Apt.}

Limnges, Mus.

\section{Goult.}

Jaennicke.

I: Jaennicke. 2: Ris-Paquot. 
Seite

102. Lamarque.

Nancy

Mée.

Jaennicke.

Marzy.

Jaennicke.

Jaennicke.

Jaennicke.

Montereau.

Jaennicke.

S. Clement.

Jaennicke.

St. Jean de Maurienne. Jaennicke.

I03, I04. Straßburg.

I-50: nach Mitteilungen von Dr. Pollazek, Direktor des HohenloheMuseums in Straßburg. 2, 3: Balthasar Hannong? 4. Feltz. 5: Friedr. Loewen finck? 6: Gallen? 7-10: Höchster Herlemann oder Hannsmann? II-I3: Joh. Kugelmann? 21: Reith? 24: Samuel Lemourne? 26: Thetten? 27: Westermann oder Wachenfeld?

105. Niederweiler.

I-8: Nach Mitteilungen von Dr. Pollazek, Direktor des HohenloheMuseums in Straßburg.

\section{Bayreuth.}

I: Garnier. 2: Hamburg, Mus. f. K. u. G. 3, 4, 5: Hamburg, Mus. f. K. u. G. 6, 7: Würzburg, Mus. 8: Hamburg, Mus. f. K. u. G.

I07. Nürnberg.

-I7: Würzburg, Mus. I8: Mitteilungen der Galerie Helbing, Bd. I.

I, 2: Nürnberg, Germ. Mus. 4: Würzburg, Mus. 5: Hamburg, Mus. f. Kunst u. Gesch. 6: Würzburg, Luitpold-Mus. 7, 8: Würzburg, Kunsthandel (nach Stöhr). 9: Würzburg, Luitpold-Mus. Io, I I : Nürnberg, Landesgewerbeanstalt. I2, I3: Würzburg, Luitpold-Mus. I5: Leipzig, Kunstgewerbemus. 16: Hamburg, Mus. f. K. u. G. I7-20: Würzburg, Luitpold-Mus. 21 : Würzburg, im Kunsthandel (nach Stöhr). 22: Wiirzburg, Luitpold-Mus. 23: Nürnberg, Landesgewerbeanstalt. 24: Würzburg, Luitpold-Mus, 25: Hamburg, Mus. f. K. 11. G. 26: Jaennicke (Demin). 27: Landshut, Kreis-, Muster- u. Modellsammlung. 28-30: Würzburg, Luitpold Mus. 31 : Nürnberg, Germ. Mus.

I $08 . \quad 32$ : Stuttgart, Mus. Vaterländ. Altertümer. 33: Frankfurt, Histor. Mus. 34-36: Würzburg, Luitpold Mus. 37: Frankfurt, Hist. Mus. 38, 39: Würzburg, Luitpold Mus. 40: Nürnberg, Germ. Mus. 4I: Würzburg, im Kunsthandel (nach Stöhr). 42: Feuchtwangen, Volkskunstmuseum. 43, 44: Hamburg, Mus. f. K. u. G. 45: Sèvres, Mus. 46: Jaennicke (Coll. Demmin). 47: Niirnberg, Germ. Mus.

I 09. 48: Würzbuirg, im Kunsthandei (nach Stöhr). 49: Sèvres, Mus. 50: Jacquemart. 5I: Jaennicke, Frankfurt a. M. (Coll. Seibt). 52: Würzburg, 
Seite

im Kunsthandel (nach Stöhr). 53: Würzburg, Luitpold Mus. 54: Jaennicke (Coll. Reynolds). 55, 56: Würzburg, Luitpold Mus.

\section{I0-I I4. Hanau.}

I-ror: E. Zeh, Hanauer Fayence, Marburg I9r3. 6: Konr. Köhler? 7: Simon Herrmann? 8: J. Dönch? Ir: Abrah. Butz? I2: S. Weiss? I4: Joh. Helferich Auer? 15: Joh. Ad. Schmidt? i6: J. H. Auer? I8: Joh. Georg Duscheer? 21: Joh. Carle? 22: Joh. Georg Duscheer? 24: Joh. Dönch? 25: Heinr. Eissermann? 28: Joh. Helfrich Auer? 35: Simon Herrmann? 39: Jak. Schilles oder Joh. Schütz? 40: Joh. Dom. Gelberich? 44: Leonh. Weber? 54: (Dreher) J. P. Schütz? 57: Pet. Frickels? 58: Joh. Polts? 6o, 6r: Joh. Casp. Ripp? 6r: Wenz. Schreiber? 64, 65: Siemon? (Frankfurt?) 72: Wenz. Schreiber? 73: Joh. Wilh. Zörkel? 77: van Alphen. 79, 80: Hieronym. van Alphen (und Carl Christ. Fischer?). 81-84: Hieronym. van Alphen? 90: Joh. Leonh. Preiss? 94: (Dreher) Phil. Ant. Horn? 95, 96: Carl Heinr. Bläuer oder Joh. Berner oder Joh. Bonnet oder Hamburger? Ioo: Joh. Heinr. Hoffmann?

\section{Frankfurt.}

I-5: Zeh, Hanauer Fayence, Marburg x913. 6: Stöhr, Hanauer u. Frankfurter Fayencen (Cicerone IV).

I I 5. 7, 8: Stöhr, Hanauer u. Frankfurter Fayencen (Cicerone IV). 9: E. Zeh, Hanauer Fayence, Marburg I9I3. Io-Ir: Stöhr, Hanauer u. Frankfurter Fayencen (Cicerone IV). I2: E. Zeh, Hanauer Fayencen Marburg I9I3. I3 bis 19: Stöhr, Hanauer u. Frankfurter Fayencen (Cicerone IV).

II6. Ansbach.

I: Hamburg, Mus. f. K. u. G. 2: Würzburg, Luitpold-Mus. 3: Würzburg, im Kunsthandel (nach Stöhr). 4: Würzburg, im Privatbesitz (nach Stöhr). 5, 8: Würzburg, Luitpold-Mus. 9: Nürnberg, Germ. Mus. 1o, I I: Würzburg, Luitpold-Mus.

I I 7. I2: Würzburg, Luitpold-Mus. I3: Hamburg, Mus. f. K. u. G. I4: Würzburg, Luitpold-Mus.: Wolfgang Meyerhöfer, I724-1726. 15: Würzburg, Luitpold-Mus. I6: Braun, Über eine Gruppe von süddeutschen Fayencen mit Blaumalerei (Kunst u. Kǘnstler XII. I909). I7, r8: Würzburg, LuitpoldMus. I9 1. 22: (zusammengehörig) Würzburg, Luitpold-Mus., Braun, Über eine Gruppe von süddeutschen Fayencen (Kunst u. Künstler XII, I909). 20: Würzburg, Luitpold-Mus. 2r: Braun, Über eine Gruppe von süddeutschen Fayencen (Kunst u. Künstler XII, I909). 22 siehe 19, 23, 24: Würzburg, Luitpold-Mus. 25: Nürnberg, Germ. Mus. 26: Jacquemart (Paris, Coll. Pascal). 27: Würzburg, Luitpold-Mus.

\section{Schretzheim.}

r: Oldenburg, Slg. Riesebieter. 2: Jacquemart. 3: ähnlich in Würzburg, Luitpold-Mus.

\section{I8. Frankenthal.}

I: Garnier. 2, 3: Kraus.

\section{Ludwigsburg.}

I, 2: Würzburg, Luitpold-Mus. 
Seite

\section{Zerbst.}

I: Hannover, Kestner-Mus. 2: Hannover, Slg. Frau Senator Laporte: Zerbst, Langendorf (Maler Christian Langendorf). 3, 4, 5: Oldenburg, Slg. Riesebieter. 6: O. Riesebieter, Zerbster Fayence, Cicerone II : Johann Christian Langendorf (Maler ). 7: Mitteilungen der Galerie Helbing I. 8-ro: Oldenburg, Slg. Riesebieter. II, I2: Mitteilungen der Galerie Helbing I. I3: Stieda, Die keram. Industrie im Herzogtum Anhalt (Mitt. des Vereins tür Anhaltische Geschichte und Altertumskunde, Bd. X).

I 19. Mosbach.

I-4: Würzburg, Luitpold-Mus. ( 1 : seit 1818 ?; 2: List $1782-1786 ; 3$ : seit 1805). 5: Stöhr, Hanauer u. Frankfurter Fayencen (Cicerone IV, 4, 5: seit 1778). 7-ro: Mitteilungen der Galerie Helbins, München, Bd. I (ro: Täennich I774-I778, I779-I78I). II-I6: Würzburg.

Amberg.

I, 2: Würzburg, Mus.

\section{Friedberg.} Bd. I.

r: Hamburg, Mus. f. K. u. G. 2-4: Mitteilungen der Galerie Helbing,

I20. Offenbach.

I, 2: Würzbırg, Mus. 3: Braunschweig, Mus. vaterländ. Altertümer. 4: $\mathrm{W}$ ürzburg, im Kunsthandel (nach Stöhr). 5: Frankfurt, Histor. Mus. 6: Klingenberg, Privatbesitz (nach Stöhr).

\section{Donauwörth.}

Hamburg, Mus. f. K. u. G.

\section{Villingen.}

r: Wien, Hofburg. 2: Jaennicke (Peterkloster bei Burg).

\section{Oettingen.}

\section{I2I. Höchst.}

I: Wïrzburg, Luitpold-Mus. 2: Berlin, Kgw.-Mus.

I: g. M. 2, 3: Jacquemart. 4, 5: Jaennicke. 6: Stuttgart, Landesgewerbemus. 9: Jaennicke. ro, II: Katalog der Versteigerung, Lana I, Berlin, Lepke 1909, I. I2, 13: Braun, Die beiden Höchster Fayencemaler Friedrich Hess und Ignatius Hess (Cicerone V). I4: Würzburg, Luitpold-Mus.

Höchst-Damm: g. M.

Glienitz.

I-3: Oldenburg (Slg. Riesebieter).

\section{Groß-Stieten.}

Schlie, Alte mecklenburgische Fayencen aus der Zeit der Arcanisten (Kunstgewerbeblatt N. F. V.).

\section{Kelsterbach.}

r, 2: Drach, Die Fayence- und Porzellanfabrik zu Kelsterbach a. M. (Kunstgewerbeblatt II).

\section{Limbach.}

I, 2: Mitteilungen der Galerie Helbing I, München. 
I22. Flörsheim.

r-4: Würzburg, Luitpold-Mus. 5: München, National-Mus. 6: Würzburg, Luitpold-Mus. 7: Mainz, Histor. Mus. 9: nicht Flörsheim. ro: E. Heuser, Die alten Manufakturen ı ur Fayence und lür Steingut zu Flörsheim am Main (Cicerone II): Matthias Joseph Weingärtner.

Dirmstein.

Zais, Die bischöfl. Wormsische Fayencefabrik zu Dirmstein 1875 .

Schrattenhofen.

Hamburg, Mus. f. K. u. G.

123. Crailsheim.

r: Hamburg, Mus. f. K. u. G. 2, 3: Würzburg, Luitpold-Mus.

\section{Göggingen.}

2: Jacquemart. 3: Würzburg, Luitpold-Mus.

Göppingen.

r-4: Fr. Hofmann, Fayencen von Göppingen (Cicerone IV, S. 807).

\section{Durlach.}

I: Mitteilungen der Galerie Helbing, München, Bd. 1. 2: Wüirzburg, Luitpold-Mus. 3, 4: Gutmann, Die Kunsttöpferei des r8. Jahrhunderts im Großherzogtum Baden 1906 (3: Joh. Friedr. Gottlieb Lösver; 4: Joh. Jak. Keim). 5: Gutmann, Die Kunsttöpferei des r8. Jahrhunderts im Großherzogtum Baden r9o5. 6: A. Stöhr, Ein Frühwerk der Durlacher Favencefabrik (Cicerone II). 8: Gutmann, Die Kunsttöpferei im Großherzogtum Baden I9o6.

Fulda.

3: Oldenburg, Slg. Riesebieter. 4, 5: Hamburg, Mus. f. K. u. G. 6, 7 : Würzburg, Luitpold-Mus.

125. Künnersberg.

I: g. M. 2: Hamburg, Mus. f. K. u. G. 3, 4: Würzburg, Luitpold-Mus. 6: Versteigerung Gasser. München. Helbing rgr2. 7-rr: Braun, Úber eine Gruppe süddeutscherFayencen mit Blaumalerei (Kunst u. Kunsthandwerk XII), nach Stöhr: Künnersberg??

Cassel.

I: meist in anderer Stilisierung. 2: H. C.: häufige Marke. 3: häufige Marke. 4: Cassel ?? 6: Hamburg, Mus. f. K. u. G. Cassel? 7. Fulda?

Münden.

I: Brinckmann, Mündener Fayence (Kunstgewerbeblatt Bd. I). 2: nach Angaben von Herrn Staatsanwalt Riesebieter in Oldenburg. 3, 4, 9: Brinckmann, Mïndener Fayence (Kunstgewerbeblatt Bd. I).

Magdeburg.

126. Gera.

Hamburg, Mus. f. K. u. G.

Coburg.

I-3: Sauerlandt, Fabrikmarken.

I: R. Schmidt, Fayencen auf der Veste Coburg (Cicerone. II, S. 667). 2: Oldenburg, Slg. Riesebieter. 3: Schmidt, Fayencen auf der Veste Coburg (Cicerone II, S. 667). 4: Oldenburg, Slg. Riesebieter. 


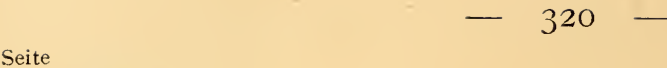

\section{Bernburg.}

I, 2: Sauerlandt, Fabrikmarken. 3, 4: Sauerlandt, Bernburger Fayencen (Cicerone II).

\section{I27. Abtsbessingen.}

I-5: Sauerlandt, Die Fayencemanufaktur von Abtsbessingen (Cicerone III). 6: Sauerlandt, Fabrikmarken. 7, 8: Sauerlandt, Die Fayencemanufaktur von Abtsbessingen (Cicerone III).

\section{Dorotheenthal (Arnstadt).}

I: Sauerlandt, Die Fayencemanufaktur im Dorotheenthal bei Arnstadt (Cicerone II).

I28. 2: Sauerlandt, Die Fayencemanufaktur im Dorotheenthal bei Arnstadt. (Cicerone II). 3-I5: M. Sauerlandt, Dorotheenthaler Fayence (Cicerone IV).

\section{Erfurt.}

I-8: O. Riesebieter, Erfurter Fayencen (Cicerone V).

I29. 9-35: Sauerlandt, Erfurter Fayencen (Cicerone V).

I30. Rudolstadt.

I: Slg. d. Freiherrn von Riedesel-Eisenbach auf Schloß Eisenbach in Hessen. 2, 3: Sauerlandt, Fabrikmarken.

Saalfeld.

I: Sauerlandt, Fabrikmarken. 2: Sauerlandt, Fabrikmarken: Saalfeld?

\section{Halle?}

Sauerlandt, Fabrikmarken.

\section{I3I. Braunschweig.}

I-Io: Mitteilungen der Galerie Helbing, Bd. I.

\section{Braunschweig, Chelius.}

Ir: Scherer, Die Chelysche Fayencefabrik zu Braunschweig (Quellen u. Forschungen zur Braunschweigischen Geschichte, Bd. V'I, I9I4). I2-I4: Oldenburg, Slg. Riesebieter.

\section{Königsberg.}

Brinckmann, Beiträge zur Geschichte der Töpferkunst in Deutschland. I: Königsberg. 2: Durlach in Baden (Jahrbuch der Hamburgischen Wissenschaftlichen Anstalten XIII).

\section{I32. Dresden.}

I: Dresden, P. S.: Dresden, C. G. Hörisch. 2-6: Oldenburg, Slg. Riesebieter (vgl. Cicerone V).

Potsdam.

I: Berlin, Kgw.-Mus. 2: O. Riesebieter, Die Fayencefabriken zu Berlin und Potsdam (Cicerone IV). 3: Hamburg, Mus. f. K. u. G. 4, 5: O. Riesebieter, Die Fayencefabriken zu Berlin und Potsdam (Cicerone IV). 6: nach Angaben von Herrn Staatsanwalt Riesebieter, Oldenburg.

Aumund (Vegesack).

I: Oldenburg (Slg. Riesebieter). 2: Hamburg, Mus. f. K. u. G. 3: Oldenburg (Slg. Riesebieter). 
I33. Schwerin.

I, 2: Pabst, Schweriner Fayencen (Kunstgewerbeblatt I). 3: Hamburg, Mus. f. K. u. G.

\section{Hamburg.}

I: Hamburg, Mus. f. K. u. G. 2: Katalog der Versteigerung. Lana I. Berlin r9o9. I.

\section{Osnabrück.}

I-3: Riesebieter, Die Fayencefabrik in Osnabrück (Cicerone IV).

\section{Deutsche Krugmaler.}

I, 2: Hamburg, Mus. f. K. u. G. 3: Nürnberg, German. Museum: Johannes Schaper. 4: Jaennicke. 5: München, National-Museum.

\section{34. Lesum.}

I-2, 4-II: Focke, Fayencefabrik von Vegesack und Lesum (Kunstgewerbeblatt III). I2: Hamburg, Mus. f. K. u. G. I4, I5: Mitteilungen der Galerie Helbing, München, Bd. I.

\section{Schleswig.}

\section{I35. Kiel.}

7, I2, I4: Hamburg, M. f. K. u. G.

I-7: Hamburg, Mus. f. K. u. G. 8, 9: Jacquemart. Io: Hamburg, Mus. f. K. u. G. II: Chaffers. I2, I3: Jacquemart. I4: Chaffers (Slg. Reynolds): ähnlich: Hamburg, Mus. f. K. u. G.

Jever.

I-4: Riesebieter, Beiträge zur Gesch. d.' Fayence-Fabrikation in Jeverland und Ostfriesland (Schriften des Oldenburger Vereins für Altertumskunde und Landesgeschichte, Bd. XXXIII).

I36. Eckernförde.

I-4: Hamburg, Mus. f. K. u. G.

\section{Kellinghusen.}

I-4: Hamburg, Mus. f. K. u. G.

\section{Stockelsdorf.}

I37. Rendsburg.

I-5: Hamburg, Mus. f. K. u. G.

Stralsund.

I, 2: Sèvres Mus. 3: Jaennicke (Slg. Gasnault). 4: Garnier. 5: Oldenburg, Mus.: Stralsund?

\section{Oldesloe.}

Hamburg, Mus. f. K. u. G.

\section{Rheinsberg.}

Ähnlich: Berlin, Kgw.-Mus.

\section{Wrisbergholzen.}

Riesebieter, Fayencefabrik zu Wrisbergholzen (Cicerone III).

Führer. 
Seite

\section{Weißkirchen.}

Hamburg, Mus. f. K. u. G.

\section{I38. Proskau.}

I-r8: Hintze, Die Proskauer Fayence- und Steingutfabrik (Schlesiens Vorzeit in Wort u. Bild, Bd. IV) (seit $\mathrm{I}_{783}$ nach Weißkirchen in Mähren verlegt, seit Anfäng des r9. Jahrhunderts Steingutfabrik).

I39. Bayreuth (braune glasierte Tonwaren).

I, 2: Hamburg, Mus. f. K. u. G.: J. A. Fichthorn. 3: München, Nat. Mus.: Johann Clarner? 4: Stuttgart, Landesgewerbemuseum. 5: München, Nat. Mus.: Clemens Wanderer?

Tetschen (rote Tonwaren). berg.

I: Gotha, Herzogl. Mus. 2: Dresden, im Besitz des Herrn Grafen Harden-

\section{Hubertusburg.}

I: Berling, Die Fayence- und Steingutfabrik Hubertusburg. 2: W. Roch, Hubertusburger Steinzeug (Cicerone I). 3: Dresden, P. S. 4: Berling, Die Fayence- und Steingutfabrik Hubertusburg.

I40. Wiesbaden (Steingut?).

\section{Siegburg.}

I: Hamburg, Slg. Blohm. 2: Wiesbaden, Museum.

Alle Marken nach Falke, Das rheinische Steinzeug. Berlin-Schöneberg 1908.

I: Monogrammist F.T. 2: Peter Knütgen. 3: Christian Knütgen. 4: Monogrammist L. W. 5: Hans Hilgers.

\section{Raeren.}

Alle Marken nach Falke, Das rheinische Steinzeug. Berlin-Schöneberg 1908.

I: Jan Emens. 2: Peter Emens. 4: Monogrammist G. E. 6: Baldem Mennicken. 7: Jan Mennicken und andere Meister. 8: Jan Baldem Mennicken: 9: Jan Baldem Mennicken? ro: Tilman Wolt? Ir: Johann Mennicken. I2: Edmonds Mennicken (auch auf Westerwälder Steinzeug).

Höhr und Grenzhausen. Nach Falke a. a. O.

r: Johann Mennicken u. andere. 3: Lenhardt Blum? 7: Johann Edmond Mennicken. 8: Leonhardt Mennicken. 9: Wilhelm Remy? ro: Johannes Kalb. Dresden und Meißen (rotgebrann es Steinzeug).

I-14: Dresden, P. S. Zimmermann. Die Erfindung und Frühzeit des Meißner Porzellans. Berlin 1908.

I44. Salzburg.

4: Katalog der Auktion Lanna I. Berlin 1909.

\section{Hollitsch.}

Kunst u. Kunsthandwerk.

6, 7: Katalog der Auktion Lanna I. Berlin rgog.

\section{Auspitz.}

Jaennicke.

I46-I62. Delft, Haarlem, Rotterdam.

I-276: Havard, La céramique hollandaise, Amsterdam r9o9. 


\section{$-323$}

Seite

163. Arnheim.

Havard.

Schiedam.

Havard.

164. Delft (Rote Tonwaren).

165. Brüssel.

I-4: Dresden, P. S. 5: Havard. 6-II: Dresden, P. S

I7: Schretzheim? 27: Garnier.

I66. Tervueren.

Garnier.

Mecheln (Malines).

Jaennicke.

Tournay.

2: Garnier. 3: Garnier.

Lüttich (Liège).

I, 2: Garnier.

Septfontaines.

Brügge.

I: Jaennicke. 2: Garnier: ähnlich. 5: Jaennicke?

Andenne.

I, 2: Jaennicke.

167, 168. Schweiz.

Nach Mitteilung von Dr. Lenz, Direktor des Züricher Landesmuseums.

Fast alle: Zürich, Landesmuseum.

169. Solothurn.

Lill, Fayencemarken (Mitteilung der Galerie Helbing I).

Unbekannte Fabrikationsorte in der Schweiz.

2: Stuttgart, Landesgewerbemus. 3-8: Lill, Fayencemarken (Mitteilungen der Galerie Helbing I).

I70. Staffordshire.

I, 2: Hodgkin. 3: Chaffers (Coll. Reynolds). 4, 5: London (Mus. of Geology). 6: Hodgkin (Coll. Solon). 7: Hodgkin (Coll. Westwood). 9: London, Mus. of Geology. ro: Chaffers (Coll. Hodgkin). Ir: Hodgkin (Burslem, Institutc Wegdwood). I2: London, Brit. Mus. I3: Hodgkin (Coll. Solon).

I71. I4: Chaffers (Coll. Sheldon). I5: Liverpool, Mus. I6, I7: Hodgkin. I8: Hodgkin (Coll. Hodgkin), 19: London, Brit. Mus.

I72. Kent (Wrotham).

I: Hodgkin (Coll. Lord d'Isle et Dudley). 2: Hodgkin (Coll. Willet). 4: London, Brit. Mus. 5: Hodgkin (Maidstone Mus.). 6: Liverpool, Mus. 7: London, Mus. of Geology. 8, 9: London, Brit. Mus. Io: London, Mus. of Geology. I I, I2: Hodgkin.

I73. I: Hodgkin (Coll. Nottingham). 2, 3: London, Brit. Mus. 
Staffordshire, rotes Steinzeug (Elersware).

I: Hobson. 2, 3: (Brit. Mus.) A. Guide to the English Pottery and Porcelain. 4, 5: Hobson.

Twyford.

I: (Brit. Mus.) A. Guide to the English Pottery and Porcelain. 2: Hobson.

I74. Burslem.

I-I3: Chaffers.

I75. Etruria (Wedgwood).

I-9: Rathbone, Old Wedgwood, London I898. Io, Ir: Meteyard, The Wedgwood Handbook, London I875. I2, I3: Rathbone, Old Wegdwood, London 1898. I4: Meteyard, The Wedgwood Handbook: Arbeitermarken, außerdem viele andere. I5, I6: Jewitt, The Wedgwoods, London I865 (vgl. Tafel I83).

I76. Tunstall.

I-II: Chaffers.

Longport.

Chaffers.

Cobridge.

I77. I-6: Chaffers.

Hanley.

I-I 2: Chaffers.

I 78. I3: Chaffers. I4: Hobson. I5-22: Chaffers.

Shelton.

I-9: Chaffers.

I79. Stocke upon Trent. Minton.

I-4: Hobson. 5-8: Chaffers.

Fenton.

I-3: Chaffers.

Lane End (Longton).

I-5: Chaffers.

I80. 6-12: Chaffers.

Lane Delph.

I-3: Chaffers.

Foley: Chaffers.

Elkin, Knight \& Bridgwood: Chaffers.

Hall \& Sons: Chaffers.

Burslem.

Chaffers.

I8I. Liverpool.

I-9: Chaffers.

Leeds.

I $\rightarrow$ 7: Chaffers.

Swinton.

I-4: Chaffers. 
Seite

Castleford.

r-3: Chaffers.

182. Ferrybridge.

r, 2: Chaffers.

Mexborough.

Chaffers.

Rockingham.

I-3: Chaffers.

Sunderland.

I-6: Chaffers.

New Castle upon Tyne.

I-6: Chaffers.

Southwick.

Chaffers.

183. Stockton on Tees.

$\mathrm{r}-3$ : Chaffers.

Bristol.

I-4: Hobson. 5: Chaffeıs 6, 7: Hobson. 8, 9: Chaffers.

Lambeth.

r-3: Chaffers.

Fulham.

Chaffers.

184. Lowesby.

Chaffers.

Mortlake.

r, 2: Chaffers.

Isleworth.

r, 2: Chaffers.

Yarmouth.

Chaffers.

Nottingham.

Chaffers.

Essex.

Chaffers.

Swansea.

I-3: Chaffers.

Wales? (Lane End?)

Chaffers:

185. Porto bello.

r, 2: Chaffers.

Dublin.

I, 2: Chaffers. 
I86. Kopenhagen.

4: Nach Mitteilung von Architekt Frohne, Kopenhagen. 5: Hamburg, M. f. K. u. G. 6-8: Nach Mitteilung von Architekt Frohne, Kopenhagen. Io: Hamburg, M. f. K. u. G. 13, I5: Nach Mitteilung von Architekt Frohne, Kopenhagen.

I87. I6: Nach Mitteilung von Architekt Frohne, Kopenhagen. I7: Hamburg, M. f. K. u. G.

I7: Hamburg, M. f. K. u. G.

Kopenhagen, Aluminia.

I88. Roerstrand.

I: Jacquemart. 2: Jaennicke. 3: Hamburg, M. f. K. u. G. 4-7: Strảle Rörstrands, Historia och Tilverkningar I726-1850. Stockholm r879. 8: g. M.: "modern.

I89. Stockholm.

I: Garnier. 2: Sèvres Mus. 3, 4: Jaennicke.

Marieberg.

I: Stråle, Mariebergs Historia och Tillverkningar I758-r788. Stockholm I880. 2: Chaffers. 3: Hamburg, M. f. K. u. G. 4, 5: Jaennicke.

I90. 7: Chaffers. 8: Stråle (vgl. Nr. r).

Gustafsberg.

I: Chaffers.

Herreböe.

I91. Kiew.

I-6: Grosch, Herreböe-Fay̌encen. Kristiania rgor.

I: Chaffers.

Warschau.

Panietnik Wystawy.

Telechany.

192. Cmielow.

Panietnik Wystawy.

Reval.

Panietnik Wystawy.

Zoege Manteuffel, eine Fayencefabrik des 18 . Jahrhunderts in Reval (Cicerone Bd. VI). 


\section{Porzellan.}

Seite

193. Meißen.

Um I720 bis um I725:

I-9: Dresden, P. S.: außerdem viele ähnliche.

Von um 1725 an:

Io-I3: Dresden, P. S.: Namenszug König August (II.) des Starken (I694-I733), Kurfürsten von Sachsen, Königs von Polen, seltener: König III. von Polen (I733-I763). I4: Dresden, P. S.: wahrscheinlich: Namenszug des Kurfürsten Friedrich August III. (I733-I763) vor seiner Wahl zum König, von Polen I733.

Von um 1725 an und noch in den nächsten Jahren.

15: Berling, kgl. Porzellanmanufaktur. I6: siehe I5.

I94. I7: h. M.: Meißner Porzellanmanufaktur. I8: Dresden, P. S.: Königl. Porzellanmanufaktur. I9: Dresden, P. S. 20: Dresden, Kgw.-Mus. 21 : Dresden, P. S. 22: Berlin, Slg. von Dallwitz. 23, 24: Dresden, Kgw.-Mus. 25: Berling (Dresden, ehem. Slg. Fischer). 26-30: Dresden, P. S. 3r: Berling (Dresden, ehem. Slg. Fischer).

I 95. 32: Berling (Dresden, ehem. Slg. Fischer). 33-35: Dresden, P. S. 36: Berling (Slg. Graf Vitztum). 37, 38, 39: Dresden, P. S. 40: h. M. 4I-44: Dresden, P. S. .45-54: Dresden, P. S.

I96. 55-57: wichtigste und so gut wie einzige Fabrikmarke dieser Zeit (ganz selten auch Schwerter mit zwei Parierstangen). 58-63: dieselbe Marke mit Beispielen von Malermarken. 64: Beispiele der sogenannten ,Punktmarke" der nach ihr benannten ,Punktzeit". 65: Beispiele der ,Schwertermarke mit Stern" (sogenannte ,Marcolinizeit", in der der Graf Marcolini der Manufaktur vorstand). 66-68: Dresden, P. S. 69, 70: einzige Fabrikmarke dieser Zeit.

Häufig findet sich bei der Schwertermarke ein Strich durchgeschliffen. Dies geschah (wahrscheinlich von der Zeit um I 760 ) an), um das betreffende Stuick Porzellan als Ausschuß, als nicht ganz geglücktes zu bezeichnen (in unserer Zeit daneben auch oft zwei durchgeschliffene Striche, für noch geringwertigeren Ausschuß).

I97. 7г: Dresden, Kgw.-Mus. 72: London, Bethnalgreen Mus. (Slg. Franks). 73: Dresden, Kgw.-Mus. 74, 75: London, Bethnalgreen Mus. (Slg. Franks). 76: Dresden, Kgw.-Mus. 77-81: Dresden, P. S. 82-91: Dresden, P. S. 92 : Dresden, Kgw.-Mus. 93: London, Bethnalgreen Mus. (Slg. Franks). 94: Dresden, Kgl. Hofsilberkammer. 95: London, Bethnalgreen Mus. (Slg. Franks). 96: Hamburg, Mus. f. K. u. G. 
I98. 97, 98: Schwerin (Slg. d. Großherzogl. Palais). 99: Dresden, P. S., tälschlich „Koselmarke' genannt. Ino: Karlsruhe, Zähringer Mus. Ior, Iо3: Dresden, P. S., fälschlich „Koselmarke" genannt.

I99. Berlin.

I: g. M. 2, 3: Chaffers.

Wegely-Periode (I7.50-I7.57).

5: Chaffers. 6: g. M.

Gotzkowsky-Periode (I76I-I763).

Königliche Porzellanmanufaktur ( 1763 bis jetzt).

7-20: Nach der von der Kgl. Porzellanmanufaktur zu Charlottenburg

200 . herausgegebenen Markentafel. I9: Serer-Porzellan (seit I882). 20: Steingut.

I7-I9: Nach der von der Kgl. Porzellanmanufaktur herausgegebenen Markentafel.

\section{Höchst.}

I-3: Hauptmarke. 5, 6: Nachbildungen von Höchster Figuren des I8. Jahrh. in Fayense v. Damm. II: Chaffers. I2, I3: Hofmann. I4, I5: London, Bethnagreen Mus. (Slg. Franks).

\section{Fulda.}

20I. Frankenthal.

I-5: h. M. (2: frühe Periode).

I-7: Fr. Hofmann, Frankentha.er Porzellan, I9II. 8-I7: Joh. Kraus, Die Marken (Fabrikzeichen) der Porzellanmanufaktur in Frankenthal, I 899. 14: Hofmann, Frankenthaler Porzellan: Peter von Recum, I795. I8-20: Kraus, Die Marken (Fabrikzeichen) der Porzellanmanufaktur in Frankenthal, I899. 2I-32: Hofmann, Frankenthaler Porzellan, I9II.

202. 33-79: Hofmann, Frankenthaler Porzellan, I9Ir. Nassau-Saarbrücken.

I, 2: Hamburg, Mus. f. K. u. G.

\section{Baden.}

Heuser, Eine Markgräfl. Badische Porzellanmanufaktur (Mannheimer Geschichtsblätter, Ed. X).

\section{Ludwigsburg.}

I-23: Wanner-Brandt, Album der Erzeugnisse der ehemaligen Württembergischen Manufaktur Alt-Ludwigsburg, Stuttgart. I-5: I8. Jahrh. bis I806. I5: seit I806. I6: seit I8I8.

\section{Nymphenburg.}

I-20: München, Bayrisches Nationalmuseum (vgl. F. H. Hofmann, Das europäische Porzellan d. Bayr. Nationalmuseums, X. Bd., München I909): I, 2: ca. I747-I760; 3, 4: ca. I756; 5, 6: ca. I756-I765; 7: I766-I780; 8, 9: um I800; I0: um I800; II: ca. I810-I850; I2: um I800; I3: um I840; 14: 18. Jahrhundert, auf für den Export nach der Türkei bestimmten Tassen; 18: Adam Clair, seit I799 in Nymphenburg; 19: Modelleur Schmaus; 20: Porzellanmaler Ludreig Sebbers; 21, 22: London, Bethnalgreen Mus. (Slg. Franks); 23: Stuttgart, Landesgewerbemuseum.

\section{Wilde Porzellanmaler.}

I-I4: Fr. Hofmann, Die.,,Pfuscherei“" in Nymphenburg (Cicerone V). 
Seite

205. Ansbach.

I: Hofmann. 3: Marryat. 4: Chaffers. 5-7: Marryat. 8. Chaffers. 9: Hofmann. II: Jaennicke. I2: Brüning. I3: Marryat. I4: Brüning. I5, I6: Chaffers. I7: Marryat. I8: Hofmann.

Kelsterbach.

2-5: v. Drach, Die Porzellan- u. Fayencefabrik zu Kelsterbach a. M. (Bayr. Gewerbezeitschrift I89I).

Pfalz-Zweibrücken.

Kassel.

I, 2: Heuser, Die Pfalz-Zweibrückener Pozellanmanufaktur, I904.

g. M.

206. Fürstenberg.

I: Chaffers (Brogniart): Fürstenberg? 2, 3: g. M. 4: London, Vict. u. Alb. Mus. 8: London, Bethnalgreen Mus. (Slg. Franks) meist auf Biskuitporzellanen ( $W=$ Wegener, $G=$ Günter, $J=$ Jürgens).

Volkstädt-Rudolstadt.

I-I7: Graul u. Kurzwelly. I8: Eisenach, Thüringer Museum. I9: Rudolstadt, Gewerbeverein.

Kloster-Veilsdorf.

I-ro: Graul u. Kurzwelly.

207. II-I9: Graul u. Kurzwelly.

Wallendorf.

I-I3: Graul u. Kurzwelly.

Gotha.

I-I5: Graul u. Kurzwelly.

Gera.

I-8: Graul u. Kurzwelly.

208. 9-I5: Graul u. Kurzwelly.

Limbach.

I-I7: Graul u. Kurzwelly.

Rauenstein.

I-Io: Graul u. Kurzwelly.

Ilmenau.

I-3: Graul u. Kurzwelly. 4: Hamburg, Mus. f. K. u. G.: Ilmenau.

Schney.

I-3: Eisenach, Thüringer Museum.

209. Tettau.

I-4: Eisenach, Thüringer Museum.

Eisenberg.

I, 2: Eisenach, Thüringer Museum.

Straßburg.

Nach Mitteilungen von Dir. Pollazek, Direktor des Hohenlohe-Mus. in Straßburg.

210. Nach Mitteilungen von Dir. Pollazek. 
Seite $-330-$
Niederweiler.
I-I6: Nach Mitteilungen von Dir. Pollazek, Direktor des Hohenlohe-
Mus. in Straßburg. I4: Steingut.

2I I. Wilde Porzellanmaler.

I: Wien, Slg. Figdor. 2: Wien, Österr. Mus. f. Kunst u. Industrie. 3: London, Bethnalgreen Mus. (Slg. Franks: A. Bottengruber). 4: London, Bethnalgreen Mus. (Slg. Franks). 5: Dresden, P. S. 6: ehem. Slg. Lanna (in Prag). 7: Stuttgart, Landesgewerbemuseum: J. Metzsch, Bayreuth. 8: London, Bethnalgreen Mus. (Slg. Franks: Auffenwerth). 9, ro: London, Bethnalgreen Mus. (Slg. Franks).

\section{Basdorf.}

216. Wien.

I: Hamburg, Mus. f. K. u. G 2: Darmstadt, Slg. Dr. Ostermann.

I-20: Følnesics u. Braun, Geschichte d. k. k. Wiener Porzellanmanufaktur. Wien 1907.

2I7. 2I-34: Folnesics u. Braun. 35: Braun, Signierte Porzellangruppen von Niedermeyer (Kunst u. Künstler, Bd. XIII, I9ro). 36-45: Folnesics u. Braun, Geschichte der k. k. Wiener Porzellanmanufaktur, Wien I907, daselbst weitere Arbeitermarken, Malerzeichen usw.

\section{I 8. Klösterle.}

Prag.

I-5: Pazaurek.

I, 2: Pazaurek.

\section{Gießhübel.}

I, 2: Pazaurek.

Dallwitz.

I, 2: Pazaurek.

Pirkhammer.

I-4: Pazaurek.

Tannova.

Pazaurek.

Elnbogen.

I, 2: Pazaurek.

219. Schlaggenwald.

220. Weesp.

I-4: Pazaurek.

I, 2: Havard.

Amstel.

\section{Haag.}

I: Havard. 2: Chaffers.

I: Chaffers (Slg. Langford). 2: London, Vict. u. Alb. Mus. 4: Slg. Chaffers. 5, 6: Havard.

\section{Loosdrecht.}

I: Chaffers (Slg. Rücker). 2, 3: Havard. 
Seite

\section{Brüssel.}

I: London, Bethnalgreen Mus. (Slg. Franks. 2: Chaffers (Slg. Staniforth). 3: Chaffers. 4: Chaffers (Slg. Reynold). 5: Chaffers (Slg. Willett). 6: Chaffers.

221. Tournay.

I-3: Jaennicke. 4: Chaffers (Slg. Staniforth). 5: London, Bethnalgreen Mus. (Slg. Franks). 6: Chaffers (Slg. Huth). 7: Chaffers (Slg. Crowe). 9, ro: Chaffers (?).

\section{Septfontaines.}

I: Jaennicke. 2: Chaffers. 4: Jaennicke (?).

\section{Zürich.}

I-3: Chaffers. 4: Chaffers: Zürich? (Monogramme von Pierre Mulhouser.)

222. Nyon. 5: Chaffers (?).

\section{2, 4: Hauptmarken.}

\section{Kopenhagen.}

Alle Marken nach A. Hayden, Royal Copenhagen Porcelain. London I9I2.

I, 2: König Frederik. V. 3-15: Fabrikmarken mit Maler-, Bildhauerund Modelleurmarken. 14: Hans Clio. 15: Maler P. H. P. Lehmann. 16-18: Bildhauer Andreas Hald. 19, 20: Maler Hans Christopher Ondrup. 21: Bildhauer Anton Luplau. 22: Maler Joh. Christoph Bayer. 25: Bildhauer Jacob Schmidt. 26: Bildhauer Jesper Johansen Holm. 28: Modelleur Hans Meehl. 30: Maler L. Lyngbe. 3r: Maler Jensen. 35: Arnold Krog, künstlerischer Direktor seit $\mathrm{r} 885$.

\section{Bing \& Groendahl.}

Nach Mitteilung von Architekt Frohne in Kopenhagen. I: r88o-r898.

\section{Marieberg.}

I: Jaennicke. 2: London, S. K. M. 3: Jaennicke. 4: Chaffers (Coll. Huth): Decorateur Frantzen? 5: Chaffers (Coll. Huth): Directeur Sten? 6: London, S. K. M. 7: Chaffers (Coll. Marryat). 8 ff.: Stråle.

\section{Roerstrand.} modern.

\section{Gustafsberg.}

225. Rouen.

g. h. modern.

I: Ch. u. Gr. (Slg. Chavagnac). 2-3: Sèvres (Slg. Grollier). 3: Ch. u. Gr. (Slg. le Breton).

St. Cloud. I, 2: Ch. u. Gr. 3: Sèvres (Slg. Grollier): r696. 4: Ch. u. Gr.

\section{Lille (I7II-I730).}

I: Ch. u. Gr. (Slg. Chavagnac). 2: Sèvres (Slg. Grollier). 3: Ch. u. Gr. (Sgl. Jacquemart). 4, 5: Sèvres (Slg. Grollier).

Lille (I 784 - $\left.88_{7}\right)$.

I: Ch. u. Gr. (Slg. Charles). 2: Ch. u. Gr. (Slg. Delaherche). 3: Ch. u. Gr.

(Slg. Harbaville de Boulogne). 4: Sèvres. 5, 6: Sèvres (Slg. Grollier). 


\section{Chantilly.}

I, 2, 3: Ch. u. Gr. (Slg. Chavagnac). 4: Ch. u. Gr. 5: Ch. u. Gr. (Slg. Chavagnac). 6: Ch. u. Gr. (Slg. Chasles). 7: Ch. u. Gr. 8: Sèvres (Slg. Grollier). Rue de Charonne.

I: Sèvres (Slg. Grollier). 2, 3: Ch. u. Gr. (Slg. Chavagnac). 4: Sèvres (Slg. Grollier). 5: Sèvres. 6: Ch. u. Gr. (Slg. Darblay). 7: Sèvres. 8: Sèvres (Slg. Grollier).

\section{Bourg-la-Reine.}

I: Sèvres (Slg. Grollier). 2: Ch. u. Gr. (Slg. Chavagnac).

\section{Vincennes.}

I-II: Ch. u. Gr.

227-234. Sèvres.

Sämtliche Marken nach: Chavagnac u. Grollier, Histoire des Manufactures Françaises de Porcelaine. Paris I9o6 und Vogt, La Porcelaine. Paris.

23-26: Marken aüf den für die königlichen Schlösser bestimmten Stücken.

27-44: Die Zahlen je nach dem Jahr der Anfertigung. 38-42: Marken für

Biskuitporzellan. 39: Durch Balken entwertete Marken für die A usschußware.

\section{0-234. Maler- und Vergoldermarken von Sèvres.}

I. Periode. (I753-I80o.)

I. Aloncle, Vögel, Tiere, Attribute.

2. Antheaume, Landschaften und Tiere.

3. Armand, Vögel, Blumen usw.

4. Asselin, Porträts, Miniaturen usw.

5. Aubert der Altere, Blumen.

6. Bailly (Sohn), Blumen.

7. Bardet, Blumen.

8. Barré, Streublumen.

9. Barrat, Blumenzweige, Buketts.

ro. Baudoin, Verzierungen, Borten usw.

Ir. Becquet, Blumen usw.

12. Bertrand, Streublumen.

13. Bienfait, Vergoldung.

I4. Binet, Streublumen.

15. Binet (Frau, geb. Sophie Chanou), Blumen.

I6. Boucher, Blumen, Zweige usw.

I7. Bouchet, Landschaften, Figuren, Verzierungen.

I8. Bouillat, Blumen, Landschaften.
I9. Boulanger, Streublumen.

20. Boulanger (Sohn), Schäferszenen, Kinder.

21. Bulidon, Streublumen.

22. Bunel (Frau, geb. Manon Buteux), Blumen.

23. Buteux (Vater), Blumen, Attribute usw.

24. Buteux (älterer Sohn), Streublumen usw.

25. Buteux (jüngever Sohn), Schäferszenen, Kinder.

26. Capelle, Verschiedene Borten.

27. Carrin, Streublumen.

28. Carrier (Carrié), Blumen.

29. Castel, Landschaften, Jagden, Vögel.

3o. Caton, Schäferszenen, Kinder, Porträts.

3I. Catrice, Blumen, Streublumen:

32. Chabry, Miniaturbilder, Schäferszenen.

33. Chanou (Frau, geb. Julie Durosey), Blumen.

34. Chapuis (der Altere), Blumen, Vögel usw. 
35. Chapuis (der Jüngere), Streublumen.

36. Chauvaux (Vater), Vergoldung.

37. Chauvaux (Sohn), Streublumen, Vergoldung.

38. Chevalier, Blumen, Buketts usw.

39. Choisy, de, Blumen, Arabesken.

40. Chulot, Attribute, Blumen, Arabesken.

4I. Commelin, Streublumen, Zweige, Girlanden.

42. Cornaille, Blumen, Streublumen.

43. Couturier, Vergoldung.

44. Dieu, Chinesischer Geschmack, chinesische Blumen, Vergoldung.

45. Dodin, Figuren, Personen, Porträts.

46. Drand, Chinesischer Geschmack, Vergoldung.

47. Dubois, Blumen, Girlanden usw.

48. Dusolle, Streublumen.

49. Dutanda, Streublumen, . Girlanden.

50. Evans, Vögel, Schmetterlinge, Landschaften.

5r. Falot, Arabesken, Vögel, Schmetterlinge.

52. Fontaine, Attribute, Miniaturbilder.

53. Fontelliau, Vergoldung usw.

54. Fouré, Blumen, Buketts usw.

55. Fritsch, Figuren, Kindergestalten usw.

56. Fumez, Streublumen, Vergolder.

57. Gauthier, Landschaften und Tiere.

58. Genest, Gestalten und Genreszenen.

59. Genin, Blumen, Girlanden, Borten.

6o. Gérard, Schäferszenen, Miniaturbilder.

6r. Gérard (Frau, geb. Vautrin), Blumen.

62. Girard, Arabesken, chinesischer Geschmack usw.

63. Gomery, Blumen und Vögel.

64. Gremont, Girlanden und Buketts.
65. Grison, Vergoldung.

66. Henrion, Girlanden, Streubiumen.

67. Héricourt, Girlanden, Streublumen.

68. Hilken, Figuren, Schäferszenen usw.

69. Houry, Blumen usw.

7o. Huny, Blumen, Streublumen usw.

7r. Joyau, Streublumen usw.

72. Jubin, Vergoldung.

73. La Roche, Blumen, Girlanden, Attribute.

74. Le Bel (der Altere), Gestalten und Blumen.

75. Le Bel (der Jüngere), Girlanden, Buketts usw.

76. Léandre, Schäferszenen, Miniaturbilder.

77. Lecot, Chinageschmack usw.

78. Ledoux, Landschaften und Vögel.

79. Le Guay, Vergoldung.

8o. Le Guay, Miniaturbilder, Kinder, Chinesischer Geschmack.

8r. Levé (Vater), Blumen, Vögel, Arabesken.

82. Levé, Félix, Blumen, Chinageschmack.

83. Maqueret (Frau, geb. Rachel Bouillat), Blumen.

84. Massy, Blumenarrangements, Girlanden.

85. Mérault (der Ältere), Verschiedene Bordüren, Blumen.

86. Mérault (der Jüngere), Blumen, Vergolder.

87. Micaud, Blumen, Buketts, Uhren.

88. Michel, Streublumen.

89. Moiron, Streublumen.

9o. Monginot, Blumen, Buketts.

9r. Morin, Militärische und Marineszenen.

92. Mutel, Landschaften.

93. Nicquet, Streublumen usw.

94. Noel, Blumen, Ornamente.

95. Nouailhier (Frau, geb. Sophie Durosey), Blumen.

96. Parpette, Philippe, Blumen, Streublumen. 
97. Parpette (Frl.), Blumen.

98. Pajou, Figuren.

99. Petit, Blumen.

roo. Pfeiffer, Streublumen.

ror. Pierre (der Altere), Blumen, Streublumen.

102. Pierre (der Jüngere), Buketts; Girlanden.

ro3. Philippine (der Ältere), Schäterszenen, Kinder usw.

I04. Pithou (der Altere), Porträts, Historische Szenen.

105. Pithou (der Jüngere), Figuren, Blumen und Ornamente.

ro6. Pouillot, Streublumen.

ro7. Prévost, Vergoldung.

I08. Raux, Streublumen.

rog. Rocher, Figuren, Miniaturen usw.

I Io. Rosset, Landschaften usw.

Ir r. Roussel, Streublumen.

II2. Schradre, Vögel, Landschaften.

I13. Sisson (Simpson oder Sisson), Blumen, Girlanden, Gruppenbilder.

I 14. Sisux (der Altere), Streublumen, Girlanden.

I15. Sioux (der Jüngere), Blumen und Girlanden.

I 16. Tabary, Vögel usw.

II7. Taillandier, Streublumen, Buketts.

I 8 . Tandart, Blumengruppen, Girlanden.

Ir9. Tardy, Streublumen usw.

120. Théodore, Vergoldung.

I2 I. Thevenet (Vater), Blumen, Uhren, Gruppen.

I22. Thevenet (Sohn), Ornamente, Borten usw.

I23. Vandé, Vergoldung, Blumen.

124. Vavasseur, Arabesken.

I25. Vieillard, Attribute, Ornamente.

I26. Vincent (der Altere), Vergoldung.

127. Xrowet (Xhouuet), Arabesken, Blumen usw.

I28. Yvarnel, Landschaften, Vögel.
II. Periode. Seit $1800-1874$.

I29. André, Jules, Landschaften.

I30. Apoil, Figuren, Personen usw.

I31. Apoil (Frau), Figuren.

132. Archelais, Dekorateur (Pâtes sur pâtes-Malerei).

I33. Avisse, Saul, Dekorateur.

134. Barbin, Franc., Ornamente.

135. Barré, Blumen.

I36. Barriat, Figuren.

137. Béranger, Ant., Figuren.

I38. Blanchard, L., Dekorateur.

139. Blanchard, Alex., Ornamentenmaler.

I40. Boitel, Ch., Vergoldung.

I4I. Bonnuit, Dekorateur.

I42. Boullemier, Ant., Vergoldung.

143. Boullemier, Fr. (der Altere), Vergoldung.

I44. Boullemier, H. (Sohn), Vergoldung.

I45. Buteux, Eug., Blumen.

146. Cabau, Blumen.

147. Capronnier, Vergoldung.

148. Célos, Ornamentenmaler (Pâtes sur pâtes).

I49: Charpentier, Dekorateur.

150. Charrin (Frl. Fanny), Figuren, Personen, Porträts.

I5 I. Constant, Vergoldung.

I52. Constantin, Figuren.

153. Dammouse, Figuren und Ornamente (Pâtes sur pâtes).

154. David, Alex., Dekorateur.

I55. Davignon, Landschaften.

156. Delafosse, Figuren.

157. Desperais, Verzierungen.

158. Derichsweiler, Dekorateur.

r59. Develly, Charles, Landschaften und Genreszenen.

r6o. Deutsch, Verzierungen.

r6r. Didier, Verzierungen.

162. Drouet, Blumen.

163. Ducluseau (Frau), Figuren, Personen, Porträts.

I64. Durosey, Vergoldung. 
165. Faraguet (Frau), Figuren, Personen usw.

r66. Ficquenet, Blumen und Verzierungen (Pâtes sur pâtes).

167. Fontaine, J., Blumen.

I68. Fragonard, Figuren, Genreszenen usw.

I69. Ganeau (Sohn), Vergoldung.

I7o. Gély, Ornamentenmaler (pâtes sur pâtes).

I7x. Georget, Figuren, Porträts usw.

172. Gobert, Figuren in Email- und sur pâtes-Manier.

I73. Godin, L., V., Vergolaung.

r74. Goupil, Figuren.

175. Guillemain, Dekorateur.

176. Hallion, Eugène, Landschaften.

I77. Hallion, François, Vergolder und Dekorateur.

178. Huard, Ornamente verschiedener Art.

I79. Humbert, Figuren.

I80. Julienne, Eugène, Ornamente im Renaissancestil.

I 8I. Lambert, Blumen.

182. Langlace, Landschaften.

183. Latache Vergoldung.

I 84. Le Bel (der Jüngere), Landschaften.

185. Legay, Ornamentenmaler (Pâtes sur pâtes).

r86. Le Guay (Et. Charl.), Figuren, Porträts und andere Stoffe.

I 87. Legrand, Vergoldung.

I88. Leroy, Eugène, Vergoldung.

189. Martinet, Blumen.

I90. Maussion (Frl. von), Figuren.

I9I. Mérigot, Ornamente usw.

192. Meyer, Alfred, Figuren usw.

193. Micaud, Vergoldung.

194. Milet (Felix Optat), Dekorateur (Fayence- und Pâtes sur pâtesArbeiten).

195. Moreau, L., Vergoldung.

196. Moriot, N., Figuren usw.

197. Parpette, Frl., Blumen.

198. Philippine, Blumen und Ornamente.
199. Pline, Vergolder und Dekorateur.

20o. Poupart (Achille), Landschaften.

20r. Regnier, Ferd., Figuren verschiedener Stoffe.

202. Regnier, Hyacinthe, Figuren usw.

203. Réjoux, Emile, Dekorateur.

204. Renard, Emile, Dekorateur.

205. Richard, Emile, Blumen.

206. Troyon, J., Ornamente, goldungen.

207. Walter, Blumen.

209. Bonnuit, Vergoldungen, Ornamente.

2ro. Belet, E., Blumen, Vögel.

2 I r. Belet, $A$., Ornamente.

2I 2. Bieuville, Ornamente.

2I3. Brecy, Ornamente.

2I4. Briffaut, Ornamente.

2I5. Richard, E., Blumen.

2 6 . Richard, Fr., Vergoldungen, Blumen.

217. Richard, J., Ornamente.

218. Richard, P., Vergoldungen, Ornamente usw.

219. Riocreux, J., Landschaften.

220. Drouet, E., Figuren, Ornamente.

221. Bulot, E., Blumen, Vögel.

222. Courcy, A., Figuren.

224. Doat, T., Kleine plastische Arbeiten.

225. Escallier, Marie, Blumen.

226. Fournier, A., Ornamente.

227. Froment, E., Figuren, Genre.

228. Gébleux, G., Ornamente.

229. Riocreux, D., Blumen.

230. Robert, Pierre, Landschaften.

23I. Robert, Frau, Blumen und Landschaften.

232. Robert, J. Fr., Landschaften.

233. Roussel, P. M., Figuren.

234. Maugendre, Ch., Plastische Arbeiten.

235. Ligné, D., Ornamente.

236. Lucas, Ch., Ornamente, M०delleur.

237. Moriot, Frl., Figuren, Genre. 


\section{$-336$}

238. Paillet, F., Ornamente, Figuren.

239. Pihan, Ornamente.

240. Porchon, Ornamente.

24I. Roger, Th., Plastische Ornamente.

242. Sandoz, A., Plastische Arbeiten.

243. Schilt, L., Blumen.

244. Sinsson (Simpson), Blumen.

245. Solon, Plastische Arbeiten.

246. Swebach, Landschaften, Genre.

247. Trager, J., Blumen, Vögel.

248. Sieffert, L', Figuren, Genre.

\section{Mit vollständigem Namen zeich-} neten

Baldisseroni, Figuren.

Brunel, Figuren.

Bulot, Blumen.

Cool (Frau von), Figuren.

Courcy, Fréd. de, Figuren und Emailarbeiten.

Degault, Figuren.

Froment, Figuren.
Gallois, Frau, geb. Durand, Figuren. Garneray, Landschaften.

Goddé, Dekorateur, Emailarbeiten und Reliefs.

Hamon, Figuren.

Jaccob (Jaccober), Blumen und Früchte.

Jacquotot (Frau M. Victoire), Figuren, Personen, Porträts.

Jadelot, Frau, Figuren.

Langlois, Polyclès, Landschaften.

Laurent (Frau Pauline), Figuren, Personen usw.

Lessore, Figuren.

Meycr-Heyne, Figuren und Ornamente auf Glasur.

Parant, Figuren.

Philip, J. B. C., Dekorateur auf Glasur.

Schilt, Abel, Figuren, Personen, Porträts.

Solon, Frl., Figuren, Personen.

Treverret, Frl. von, Figuren.

Van Os, Blumen und Früchte.

Van Marck, Landschaften.

\section{Sceaux.}

I: Ch. u. Gr. (Slg. Gasnault). 2: Ch. et Gr. (Slg. Chavagnac). 3: Ch. u. Gr. (Slg. Chavagnac). 4: Ris-Paquot. 5: Chaffers.

\section{Orleans.}

Frittenporzellan. I, 2: Sèvres, Mus. (Slg. Grollier). 3: Sèvres, Mus. 4: Sèvres, Mus. (Slg. Grollier). 5: Sèvres, Mus. (Slg. Gasnault). Hartporzellan. 6: Ch. u. Gr. (Slg. Jacquemart). 7: Sèvres, Mus. (Slg. Grollier). 8: Sèvres, Mus. Marken von Benoist le Brun.

I, 2: Orleans, Mus. 3: Ch. u. Gr.

Marken von Molinier-Bardin.

I: Orleans, Mus. 2: Orleans (Slg. Gasnault). 3: Orleans, Mus.

\section{La Seynie.}

I: Ch. u. Gr. (Slg. Jacquemart). 2, 3: Sèvres, Mus. 4, 5: Ch. u. Gr. (Slg. Gasnault).

236. Paris. La Ville d'évèque.

Ch. u. Gr.

Rue Taranne.

Sèvres, Mus. (Slg. Grollier).

Rue de la Roquette.

I, 2: Sèvres, Mus. (Slg. Grollier). 
Seite

\section{Faubourg Saint-Denis.}

Marken von Hannong.

I, 2 : Ch. u. Gr. 3: Sèvres, Mus. (Slg. Grollier). 4: Ch. u. Gr. (Slg. Gasnault). 5: Sèvres, Mus.

Marken der Patrone der Fabrik.

6, 7: Sèvres, Mus. (Slg. Grollier).

Rue Fontaine-au-Roy.

Marken von Locré oder von Russinger.

I, 2: Ch. u. Gr. 3, 4: Sèvres, Mus. (Slg. Grollier).

Marken von Russinger $u$. Pouyat.

5: Sèvres, Mus. (Slg. Grollier). 6: Chaffers. 7: Limoges, Mus.

Rue de Reuilly.

I-3: Sèvres, Mus. (Slg. Grollier).

Barrière de Reuilly.

I: Sèvres, Mus. 2: Sèvres, Mus. (Slg. Grollier).

237. Rue Thiroux.

I-3: Sèvres, Mus. (Slg. Grollier).

Marken von Leboeuf.

Marken der Nachfolger Leboeufs.

I : Sèvres, Mus. (Slg. Grollier). 2: Ris-Paquot. 3: Ch. u. Gr.

Rue de Bondy.

I-3: Sèvres, Mus. (Slg. Grollier). 4: Ch. u. Gr. (Slg. Protat). 5: Ch. u. Gr. (Slg. Jacquemart). 6: Sèvres, Mus.

Rue de Popincourt.

I, 2: Ch. u. Gr. (Slg. Nast). 3, 4: Sèvres, Mus. (Slg. Grollier).

38. Pont-aux-choux.

I-5: Sèvres, Mus. (Slg. Grollier). 6: Chaffers. 7: Sèvres, Mus.

\section{Petit-Carrousel.}

I: Ch. u. Gr. 2: Sèvres, Mus. (Slg. Grollier).

Rue St. Gilles.

I-3: Sèvres, Mus. (Slg. Grollier). 4: Sèvres, Mus. 5: Ris-Paquot -

Sèvres, Mus. (Slg. Grollier).

Rue de Crussol (1789-1807).

I-4: Sèvres, Mus. (Slg. Grollier). 5: Chaffers.

239. Rue de Crussol (I80o).

Sèvres, Mus. (Slg. Grollier).

Rue des Recollets.

Ch. u. Gr.

Rue des Charonnes.

I: Sèvres, Mus. 2: Sèvres, Mus. (Slg. Grollier). 3: Ch. u. Gr.

Rue Montmartre.

I, 2: Sèvres, Mus. (Slg. Grollier).

Palais Royal.

Ch. u. Gr.

Führer. 
Seite

\section{Brancas-Lauraguais (Brancas, Graf von Lauraguais).}

I, 2: Sèvres, Mus.

\section{Clignancourt.}

240. I: Ch. u. Gr. (Slg. Chavagnac). 2: Sèvres, Mús. (Slg. Grollier). 3: London, Bethnalgreen Mus. (Slg. Franks). 4: Sèvres, Mus.

Marken der Patrone der Fabrik.

5: Sèvres, Mus. (Slg. Grollier). 6: London, Bethnalgreen Mus. (Slg. Franks). 7: Sèvres, Mus.

\section{Vincennes.}

I: London, Bethnalgreen Mus. (Slg. Franks). 2: Sèvres, Mus. (Slg. Grollier). 3: Ch. u. Gr. (Slg. Chavagnac).

\section{Bordeaux.}

I, 2: Sèvres, Mus. 3, 4: Sèvres, Mus. (Slg. Grollier).

\section{Lunéville.}

I: Nancy, Mus. Lorrain. 2: Ch. u. Gr. 3: Nancy, Mus. Lorrain.

\section{Chatillon.} Sèvres, Mus.

Marseille.

24I. Valenciennes.

I: Ch. u. Gr. (Slg. Chavagnac). 2-4: Sèvres, Mus. (Slg. Grollier).

I-4: Sèvres, Mus. (Slg. Grollier). 5: Ch. u. Gr.

\section{Bayeux.}

\section{Caen.}

I, 2: Sèvres, Mus.

Sèvres, Mus.

\section{Boisette.}

I, 2: Sèvres, Mus. (Slg. Grollier). 3: Sèvres, Mus.

\section{Fontainebleau.}

I, 2: Sèvres, Mus. (Slg. Grollier).

\section{Saint Maurice.}

I: Sèvres, Mus. 2: Ch. u. Gr.

\section{Crépy-en-Valois.}

I, 2: Ch. u. Gr.

Saint-Amand-les-Eaux.

I: Sèvres, Mus. (Slg. Grollier). 2, 3: Sèvres, Mus.

Weitere Marken der französischen Porzellanfabriken bei Chavagnac et $M$. de Grollier. Histoire des Manufactures françaises de Porcelaine. Paris 1906.

\section{Chelsea.}

I-3: Chaffers. 4: Hobson. 5-8: Chaffers. 9, Io: Hobson.

\section{Chelsea-Derby.}

I0, II: Chaffers. I2: Jewitt. I3, I4: Chaffers. 
Seite

Derby.

I: Jewitt. 2: Hobson. 3: Jewitt. 4: Chaffers. 5. Hobson.

243. 6, 7: Hobson: Nummern der Muster oder Arbeiter. 8, 9: Jaennicke. Io: Hobson. II-I7: Chaffers. I8: Hobson: auf Sèvres-Nachahmungen. I9: Chaffers. 20: Chaffers: Stevenson u. S. Hancock. 21: Chaffers: Royal Crown Derby Porcelainworks in Osmaston, Rd. 1876 .

244. Bow.

I : Hobson. 2-II: Chaffers. I2: Hobson. I3: Hobson. I4-I8: Hobson. I9: Chaffers: Maler Thomas Fry. 21, 22: Hobson: Former Tebo? 23: Chaffers.

\section{Lowestoft.}

I: Chaffers. 2-4: Hobson: Nachahmingen von Worcester-Marken. 5: Hobson: Arbeitermarken.

\section{Worcester.}

I-5: Chaffers. 6: Hobson. 7: Chaffers. I0-I3: Chaffers. I5, I6: Chaffers. I7: Hobson: Worcester Porcelain Company. 18: Hobson. 19-30: Chaffers: Nachbildungen chinesischer Marken.

246. 31-33: Chaffers: Nachbildungen chinesischer Marken. 34, 36, 37: Chaffers: Nachahmung Meißner Marken. 35: Chaffers: Nachahmung der Marke von Fürstenberg. 38: Hobson: Nachahmung der Marke von Tournay. 39: Chaffers: Nachahmung der Sèresmarke. 40: Chaffers: daneben viele andere (siehe Chaffers und Hobson).

247. 4I: Chaffers. 42: Chaffers: Richard Holdship? 43: Chaffers: John Donaldson. 44: Hobson: $R$. Hancock (auf bedruckten Porzellanen). 45, 46: Hobson: auf bedruckten Porzellanen. 46: Hobson. 47-51: Chaffers. 52: Hubson: Bar. 53: Chaffers. 54, 55: Kerr \& Binns: (Chamberlains) Hobson. I, 2: Chaffers.

Worcester (Chamberlains).

$248 . \quad 3,4$ : Chaffers.

\section{Plymouth.}

\section{Bristol.}

I-4: Chaffers.

2: Chaffers: I772-I774. 3: Hobson. 4, 5: Chaffers. 6-8: Hobson. 9: Chaffers. Io: Hobson. II: Chaffers: Former Tebo.

249. I2: Jewitt. I3-I6: Chaffers.

\section{Caughley.}

I: Hobson. 2-5: Chaffers. 6: Chaffers: Salopian. 7: Hobson. 8, 8 a: Chaffers. 9-I7: Chaffers. I8-2x: Hobson.

\section{Coalport.}

250. I-3: Hobson. 4-6: Chaffers. 7: Chaffers: Colebrook Dale (in dem Coalport liegt). 8-Io: Hobson: siehe 7. II: Chaffers: siehe 7. I2: Hobson: siehe 7. I3: Hobson: seit I860 Monogramm C. S. u. C. (= Caughley), S (= Swansea), $N$ (= Nantgarw).

\section{Longton Hall.}

I-3: Chaffers: Lister \& Longton. 4-6: Hobson. 7: Chaffers. 


\section{Shelton, New Hall.}

I: Chaffers. 2: Hobson.

\section{Isleworth.}

3: Hobson: Shore \& Goulding ( 1760 bis ca. I 80o). 4: Hobson.

Nantgarw.

I-2: Chaffers. 3: Chaffers: $C W=$ China works? 4: Hobson.

Swansea.

25I. Pinxton.

I-3: Hobson. 4, 5: Chaffers. Hobson.

I: (Brit. Mus.) A Guide to the British Pottery and Porcelain. 4-2:

\section{Mansfield.} bemalte.

Hobson: Marke eines Malers, I80I, der Porzellane verschiedener Fa rik@:

\section{Rockingham.}

I-2: Chaffers: wach 1800 .

Wedgwood.

(Brit. Mus.) A Guide to the British Pottery and Porcelain.

Stoke upon Trent.

I-3: Hobson.

$$
\text { Spode } 1797-1833 \text {. }
$$

Spode $u$. Copeland.

4: Hobson. 5: Hobson: seit I833 C. alleiniger Inhaber.

Minton.

Copeland u. Garett.

6: Hobson: $1835-1847$.

r: Chaffers. 2: Chaffers: seit $185 \mathrm{I}$.

Longton, Davenport.

252. Belleck.

I, 2: Chaffers.

Chaffers.

\section{Donovan.}

I, 2: Hobson: Donovan, aus Poolbeg Street Dublin, bemalte Porzellane von Minton und anderen Fabriken.

\section{Florenz.}

2: Davillier (Slg. G. de Rothschild). 3: Davillier (Slg. Leroux). 4: Davillicr (Slg. G. de Rothschild). 5: Davillier. 6: Davillier (Slg. G. de Rothschild).

254. Venedig.

I, 2: London, Bethnalgreen Mus. (Slg. Franks). 3: Jaennicke. 4: Jacquemart. 6: Chaffers (Slg. Reynolds). 8: Chaffers (Slg. Davillier). 9: Chaffers. I0: Chaffers. Ir: Chaffers: Venedig? 12: Chaffers (Davillier). 13: Jaennicke. 14: Chaffers (Slg. Preston): Venedig ? 15: Chaffers (Slg. d'Azeglio).

I: Chaffers. 2, 3: Jaennicke. 9: Chaffers: Maler Giovanni Marconi. 
Seite

255. Capo di Monte.

I: Chaffers. 2: Jacquemart.

Neapel.

I, 3: Chaffers. 4: London, Bethnalgreen Mus. (Slg. Franks): König Ferdinand. 6: Chaffers: König Ferdinand. 7: Chaffers.

Doccia.

I, 2: Chaffers (Slg. d'Azeglio). 3: Chaffers (Slg. Bohn u. Bethnalgreen Mus. Slg. Franks). 4-6: Chaffers. 7: Chaffers (Slg. d'Azeglio). 8: Jaennicke. 9: Chaffers.

256. Vinovo.

I: London, Bethnalgreen Mus. (Slg. Franks). 2: Chaffers. 3, 4: Chaffers (Slg. Davillier). 6, 7: London, Bethnalgreen Mus. (Slg. Franks).

Nove.

I: Fortnum, London, Vict. u. Alb. Mus. 2: Jaennicke. 3, 4: London, Vict. u. Alb. Mus. 5, 6: Chaffers. 7: Chaffers (Slg. Gladstone). 8, 9: Chaffers (Slg. Davillier): Giov. Ant. Antonibon. Io, Ir: Chaffers. I2: Chaffers (Slg. Verschoyle). 13: Chaffers: Venedig. I4: Chaffers (Slg. Chaffers).

Treviso. Este.

I: London, Bethnalgreen Mus. (Slg. Franks). 2: Chaffers.

Vincenza.

Chaffers (Slg. Schreiber).

\section{Chaffers: Vicenza?}

257. Buen Retiro.

I-I5: M. Pérez-Villamil. Artes é industrias del Buen Retiro. Madrid I904. 258. I6-33: M. Pérez-Villamil. Artes é industrias del Buen Retiro. Madrid 259. Gerona.

Chaffers.

Vista Allegre.

2, 3: Chaffers.

260. St. Petersburg.

Moskau.

I-I7: La manufacture impériale à St. Petersbourg 1909 .

I: Panietnik Wystawy.

261. Baranowka.

Panietnik Wystawy.

Korzec.

Panietnik Wystawy.

Proszowice.

262. Tomaszow.

Panietnik Wystawy.

Panietnik Wystawy. 


\section{Chinesisches Steinzeug.}

I-I3: Dresden, P. S. I4: Hobson: Chiting = Sesam-Pavillon. I5: Chaffers: (Töpfer) Ching-Yüan-yu. I6: Chaffers: (Töpfer) Mengch'en und Hui. I: Chaffers: T'ien Ch' $i$ i ch'ou nien chin shih chi = angefertigt von Chinshih in $i$ ch'ou-Jahre von T'ien Ch' $i(d$. $h$. I625). 2: Chaffers: Huang yün chi = registriert von Huang-yün. 3: Chaffers: Li Ta-lai (Töpfer, um I800). 5: Chaffers: (Töpfer) Yüeh-ch'ang. 6: Chaffers: Ko ming hsiang chih = angefertigt von Ko Ming-hsiang. 7: Chaffers: Ko yüan hsiang chih = angefertigt von Ko Yüan-hsiang. 8: Hobson: Ko Ming hsiang chih = angefertigt von Ko Ming-hsiang.

9: Chaffers: Wan li ting yu ch'en wen ching su - Ch'en Wen-ching bildete es in Ton in dem ting-yu-Jahre von Wan-Li (d, h. I597).

\section{Chinesisches Porzellan.}

\section{5-267. Kaisermarken (Nien-haos).}

Zimmermann, Chinesisches Porzellan, seine Geschichte und Technik. Leipzig I9I3.

Seit der Zeit der Ming-Dynastie (1368-1643) bis in die unsrige hinein wird es vielfach Sitte, die Porzellane (meist am Boden, seltener an versteckten Teilen der Wandungen) mit Marken zu versehen, die den Namen des chinesischen Kaisers enthalten, unter dem sie hergestellt worden sind. Diese Marken bestehen fast immer aus sechs, seltener aus vier chinesischen Schriftzeichen, die für gewöhnlich in zwei Kolonnen senkrecht, viel seltener in wagerechter Richtung angeordnet sind. Sie sind von rechts-oben nach unten, dann weiter von links-oben nach unten zu lesen und lauten $z$. B. für Marke Tafel 265, Nr. 3:

$\begin{array}{cc}4 & \text { I } \\ \text { Tê } & \text { ta } \\ 5 & 2 \\ \text { nien } & \text { Ming } \\ 6 & 3 \\ \text { tschih } & \text { Süan }\end{array}$

ta Ming Süan Tê nien tschih, d. h.; groß Ming Süan Tê Periode gemacht $=$ hergestellt in der Süan-Tê-Periode der großen Ming(dynastie).

Bewegt sich die Marke in wagerechter Linie, dann ist sie in obiger Weise von rechts nach links zu lesen, enthält sie nur vier Schriftzeichen, dann fehlen immer die beiden ersten.

Um aus diesen Marken die Zeit eines Stückes, die eine solche trägt, zu bestimmen, braucht man demnach nur die Bedeutung des 3. und 4. (resp. I. und 2.) Schriftzeichens festzustellen.

267. Mit der Ts'ing-Dynastie (I644-I9I2) kommen neben den oben angegebenen Marken noch Marken in Siegelschrift auf, die in gleicher Weise $\mathrm{zu}$ lesen sind wie jene.

Alle Marken dieser Art vor dieser Zeit (z. B. die die Regierungszeit des Mingskaisers Süan-Tê enthaltene Marke auf Tafel 267 , rechts unten) gehören 
Seite

nicht der Zeit, die sie vorgeben, an; sie sind, einer sehr verbreiteten chinesischen Unsitte folgend, erst auf spätere, d. h. Porzellane der Ts'ing-Dynastie gesetzt worden.

Unten, Mitte: Marke des Kaisers Yung-Lo (I403-I424) in altertümlicher Schrift (London, Brit. Mus.), aber nicht aus der Zeit.

Keine der Kaisermarken gibt volle Sicherheit, daß das mit einer solchen versehene Stück Porzellan wirklich aus der Zeit, die die betreffende Kaisermarke angibt, noch auch überhaupt aus China stammt. $\mathrm{Zu}$ allen Zeiten sind in China ältere Kaisermarken auf spätere Stücke gesetzt worden. Daneben sind sie sehr viel in Japan (siehe Seite 346), ja auch in Europa nachgemacht worden (siehe Tafel I94, 216, 245 u. 246).

268. Periodenmarken.

Außer den Kaisermarken kommen auf den chinesischen keramischen Erzeugnissen auch bisweilen die sogenannten Periodenmarken vor, so benannt, weil sie als Datierung ein Jahr jener Perioden von jedesmal sechzig Jahren enthalten, nach denen der Chinese seit dem Jahre 2637 v. Chr. G. seine Zeit zu berechnen pflegt. Diese Jahre werden immer (siehe Tafel 268) durch zwei Schriftzeichen angegeben, die zwischen den übrigen Schriftzeichen der betreffenden Marken (vgl. Tafel 269, Nr. I-3) stehen. Zur Datierung können diese Marken naturgemäß nur dienen, wenn man schon aus dem Stil der sie tragenden Gegenstände die ungefähre Zeit ihrer Entstehung festzustellen vermag.

269. Beispiele bestimmter Marken.

I: Franks: Yew Sin-chow nien chi $=$ in dem wiederkehrenden SinchowJahre hergestellt. 2: Chaffers: Ping hsï nien chi=angefertigt im ping-hsüJahre (d. h. I886 oder 1826 oder 1766 usw.). 3: Chaffers: Ta ming ch'eng hua yüan nien $i$ yu $=i$ yu (d.h.erstes) Jahr der Tschêng-Hu-(Periode) (I465-I487) der großen Ming-(Dynastie).

\section{Marken bestimmter Zeiten.}

Sungdynastie.

I-4: Chaffers: Schriftzeichen für die Zahlen I-4, neben denen sich auch die diesen folgenden finden.

3: Hobson: Pao yung = kostbar für den Gebrauch. 6: Hobson: Kao $=$ hoch.

7: Hobson: Jen ho kuan = Haus der wohlwollenden Harmonie.

Yüandynastie.

Hobson: Shu-fu = kaiserlicher Palast.

Mingdynastie.

270. I: Hobson: T'ien ch'i $i$ ch'ou nien chin shih = angefertigt von Chin-shit. im i-ch'ou-Jahre des (Kaisers) 'T'ien-K'i (d.h. I625). 2: Hobson: Wan li ting yu chen wen ching $s u=C h$ 'en Wen ching bildete es in dem tin-yu-Jahre des Kaisers Wan-Li (d.h. I597). 3 vgl. 2, 4: Hobson: Chia ching pa nien tsao chih hui kuo hsiu she = angefertigt im 8. Jahre des (Kaisers) Kia-Tsing, d. h. 
Seite

I 529. Der Anführer Kuo Hsiu-she. 5: Hobson: Shan jen ch'en wei $=$ der Eremit Ch'en-wei (I7. Jahrh.?). 6: Hobson: Te hsing t'ang = angefertigt für die Halle der wohlriechenden Tugend (I573-I620). 7: Hobson: Chang chia tsao = angefertigt von Chang-chia (18. Jahrh.). 8: Bushell: Ÿ̈ t'ang chia ch'i = schönes Gefäß aus der Jade-Halle (um I600). 9: Hobson: Wang shih ch'ih ming = Herr Wang Ch'ih-ming (späte Mingzeit). Io: Hobson Ts'ang lang lii shiu = grünes Wasser des grenzenlosen Ozeans ( 16 . Jahrh.). II: Hobson: Wan fu yu t'ung = möge unendliches Glück allen deinen Angelegenheiten zuteil werden! (I6. Jahrh.). I2: Hobson: in der Mitte: Te hua ch'ang ch'un = Tugend, Bildung und dauernder Frühling; ringsum: Wan-Li (I573-I6I9). I3: Hobson: Fu kuei chia ch' $i=$ schöne Vase für den Reichen und Ehrenhaften (I6. Jahrh.). I4: Bushell: Yï t'ang chia ch'i= schönes Gefä $\beta$ aus der Jadehalle. I5: Dresden, P. S.: 16. Jahrh. I6: Chaffers: $F u$ fan chih tsao $=$ angefertigt an den Grenzen (der Provinz) Fukien (I6. Jahrh.). I7, I8: Dresden, P. S.

27I. I: Dresden, P. S. 2: Hobson: Wang shih ch'ih ming = Herr Wang Ch'ihming.

Fabrik- oder Bestimmungsmarken (Hallmarken).

Ts'ingdynastie.

Uber diese Marken herrscht im einzelnen noch keine volle Klarheit: sie bezeichnen teils Werkstätten, teils Läden von Porzellanhändlern, teils aber auch die Orte, für die die Porzellane bestimmt waren, so z. B. die Halle eines Mandarinen, den Pavillon eines Kaisers u. dgl. m.

Die hier gegebenen Marken können nur als einzelne charakteristische Beispiele angesehen werden:

I: Bushell: Haus des Wohlwollens und der Harmonie. 2: Bushell: gemacht in der Halle der höchsten Ehrfurcht (Zeit des Kaisers Kien-Lung, I735 bis 1795?). 3: Bushell: gemacht in der Halle der Shun-tih (Tugendübung). [Zeit des Kaisers Tao-Kuang I82I-I850?]. 4: Franks: Alt gemacht in der Shun-tih-(Tugendübung) Halle (Zeit des Kaisers Tao-Kuang I82I-I850?). 5:-Bushell: Wohnstätte großer Kultur. 6: Bushell: gemacht in der geschmeidigen Jadehalle' (Zeit des Kaisers K'ang-Hi I662-I722?). 7: Bushell: gemacht in der (oder für die) Halle des. Hsieh-Bambus. 8: Bushell: gemacht in der Halle des vom Himmel gesandten Glücks. 9: Franks: gemacht in der Halle Ki-yuh (des seltenen Jade). Io: Franks: gemacht in der Lin-yuh (reiche Jade), Halle. I I: Bushell: schönes Gefäß der Jadehalle. I2: Bushell: Die Halle, wo ich von meinen Ubertretungen hören möchte: I3: Bushell: wie Nr. II.

\section{Marken mit Widmungen oder Glückwünschen.}

Die hier gegebenen Marken sind wieder nur Beispiele von vielen.

I: Bushell: gemacht für Shun-ch'ang (Universität der Hanlin Jouen in Peking). 2: Franks: Für den öffentlichen Gebrauch in der Generalshalle. 3: Bushell: für den feinen Kreis verehrter Freunde. 4: Bushell: für kommende Freunde. 5: Bushell: Wolkige Dufthalle (ein Laden für wohlriechend gemachte Waren und präparierte Drogen am westlichen Ende von Ta-shan-lan, an der Nordseite der Straße, außerhalb des großen Südtors der Stadt). 6: Bushell: zweifache Freude! 7: Franks: Langes Leben, Reichtum und Ehre! 8: Bushell: Reichtum, Ehre und dauernder Frühling! 9, I0: Bushell: Glückseligkeit, Rang, langes Leben! I I: Bushell: Rang! I2: Bushell: Mögen die Lenker des Himmels 
Seite

Glück bringen! I 3: Bushell: Großes Glück! I4: Bushell: Myriaden von Jahren, niemals endend! I5: Franks: glücklich! I6: Franks: sichtbares Glück! I7: Franks: langes Leben! I8: Franks. I9: Franks: sichtbares Glïck. 20: Franks: Glück (?).

Weitere Marken dieser Art bei Franks, Catalogue of a collection of Oriental Porcelain and Pottery. London I879, Bushell, Oriental ceramic Art. New York I897. Burton und Hobson, Marks on pottery and Porcelain. I9I2.

\section{Marken mit Empfehlungen.}

Die hier angegebenen Marken sind wieder nur Beispiele unter vielen.

Sie empfehlen und preisen die Porzellane, auf denen sie sich befinden.

I: Bushell: Edelstein unter köstlichen Gefäßen aus seltenem Gestein. 2: Bushell: ein Edelstein unter köstlichen Gefäßen aus seltenem Jade. 3: Bushell: ein Edelstein selten wie Jade. 4: Bushell: ein Flitter selten wie Jade. 5: Bushell: westlicher Jade. 6: Bushell: köstlicher Jade. 7: Bushell: Flitter-Jade. 8: Bushell: echter Jade. 9: Bushell: Jade-Juwel. Io: Bushell: köstlicher Flitter. I I : Bushell: künstlerischer Flitter. 12: Bushell: von einzigem Wert. 13: Bushell: vollkommen. I4: Bushell: alter Edelstein. 15: Bushell: innen glücklich. I6, I7: Bushell: Ich weiß, daß sie sich in Wasser freuen. I 8: Bushell: Roter duftender Olea. 19: Bushell: Zufluchtsort von Myriaden von Felsen. 20: Bushell: Zufluchtsort des roten Felsens. 21: Bushell: Goldenes Tal. 22: Bushell: Marke links: Ta (Name des Künstlers); Marke rechts: kostbar (?).

Weitere Marken dieser Art bei Franks, Catalogue of a collection of Oriental Porcelain and Pottery. London 1879; Bushell, Oriental ceramic Art. New York 1897 und Burton und Hobson, Marks on pottery and porcelain. I9I2.

\section{Verzierungsmarken.}

Sehr häufig in dieser oder verwandter Form vorkommende, lediglich zur Verzierung des Bodens der Porzellane dienende Marken, die aber auch auf europäischen Porzellanen des I 8. Jahrhunderts (vgl. Tafel I94, I95, 243 u. 245) vielfach nachgeahmt worden sind.

I: Bushell: stilisierte Lotosblume (vgl. Tafel $275 \mathrm{Nr} .6$ ). 2, 3: Bushell. 4: Franks: Knoten (vgl. Tafel $276 \mathrm{Nr}$. 9).

5: Iranks: Bedeutung unbekannt (auf für Siam angefertigten Porzellanen). 6: Hobson II: Nachahmung eines europäischen Buchstabens.

\section{Künstlerbezeichnungen.}

I: Chaffers: 'Chang chia tsao $=$ hergestellt von Chang-chia. 2: Chaffers: $H u$ yin tao yên $=$ der in einem Topf verborgene Taoist (Hao Shih-chiu) um 160o. 5: Hobson III: Wang shih ming (späte Mingzeit). 4: im Kunsthandel: Chen Tsung Tsin Kuo nien Chi $=$ Im Chang-Tsung (erste Jahr des Kaisers Wan-Li d. i. 1573 von Tsin Kuo gemacht). 5: Chaffers: Shang su. 6: Hobson: Chêng ku shih = Familie des Chêng-ku (18. Jahrh.). 7: Hobson: Ch'ên-kuochih (I662-I722). 8: Hobson II: Yüan Sin-hsing tsao (I9. Jahrh.?). 9: Kung-liang chi tsao (um I700). Iо: Ch'en t'ien sui tao = angefertigt von Chen T'ien-sui (1662-I722). II: Chaffers: Chia ch'ing san nien ssu ming chi jiih wang sheng kao chih = angefertigt durch Wang Sheng-kao am Ende des 4. Monats des 3. Jahres des Kaisers Kia-K'ing (d. i. 1798). I2: Hobson: Ling nan hui 
Seite

che (= des 5. Monats des dritten Jahres von Kia-K'ing, d. h. I798; Lingnan = Kantongemälde) und Pai shih (Name eines Malers). I3: Hobson: Chang Ming kao tsao = hergestellt von Chang Ming-kao (I662-I722). I4: Hobson: Chao-chin? Anfang d. I8. Jahrh. I5: Hobson: Lai? (I662-I722). I6: Chaffers: $W u$ shen nien liang chi shu $=$ Gemälde von Liang-chi in the wu shen year (d. h. I808). I7: Chaffers: Chiang ming kao tsao=angefertigt von Kuang Liang-chi Ende des I7. Jahrh. I8: Chaffers: Lin ch'ang fa tsao = angefertigt von Lin Ch'ang-fa, Anfang des I9. Jahrh. I9: Chaffers: Wang pu t'ing tso = angefertigt von Wan Pu-t'ing um I800. 20: Hobson: Lai Knan (I7. Jahrh.). 2I: Hobson: Yu-chai (um I725). 22: Hobson: Li-chih (I8. Jahrh.). 23: Chaffers: Wang pu t'ing tso = angefertigt von Wang Pu-t'ing, um. I80o.

275-277. Die auf diesen Tafeln wiedergegebenen Marken finden sich sehr häufig in dieser oder ähnlicher Gestalt als Verzierungen des Bodens der Porzellane.

277. I: Räuchergefäß. 2: Der hl. Hase des Mondes, des Symbols des langen Lebens. 3: Franks: Pinsel, Tusche und das Szepter des langen Lebens, bedeutend: mögen die Dinge fest bleiben, wie du es wïnschest.

\section{Porzellan.}

\section{Japan.}

Die Marken des älteren japanischen Porzellans lehnen sich eng an die des chinesischen an. Vielfach finden sich unmittelbar Kopien der ,Kaisermarken" desselben, besonders von dem aus dem Ende der Mingzeit (vgl. Tafel 265), im übrigen Marken mit Empfehlungen, Widmungen usw. (vgl. Tafel 270-273). Künstlerbezeichnungen sind in älterer Zeit auf japanischem Porzellan noch selten. Hier können nur-Beispiele der zahllosen, meist ziemlich bedeutungslosen japanischen Porzellanmarken gegeben werden.

\section{Arita (Provinz Hizen).}

I: Hobson: Gorodayu Go Shonsui tsukuru = angefertigt von Gorodayú Go Shonsui tsukuru. 2: Hobson: Narau waga zosen Shonsui Gorodayu tokoro sei $=$ angefertigt in Nachahmung meines Vorfahren Shonsui Gorodayu. 3: Dresden, P. S. 4: Hobson: Hô tei no takara $=$ ein Edelstein unter kostbaren Gefäßen. 5: Dresden: P. S. 6: Hobson: Hô $=$ kostbar. 7: Dresden, P. S.: $F u k u=$ Glück. 8: Dresden, P. S.: Ka=Glück. 9: Dresden, P. S.: Kui = Gold. ro: Dresden, P.S.: $K a=$ Glück. Ir-I8: Dresden, P.S.

\section{Kutani (Provinz Kaga).}

I: Hobson: Dosuke chin gwan = kostbarer Tand von Dosuke. 2: Chaffers: Kutani. 3: Dresden, P. S. 4: Hobson: Fuku=Glück. 5: Hobson: Fuku = Glück. 6: Hobson: Sei = gemacht.

279. 7: Dresden, P. S.: Kutani. 8: Chaffers: Fuku=Glück. 9: Dresden, P. S.: $F u k u=$ Glück.

\section{Kameyama.}

Chaffers: Kameyama tsukura = zu Kameyama gemacht. 
Seite

280. Japanische Töpfereien.

Die Zahl der auf japanischen Töpfereien vorkommenden Marken ist Legion, wie auch die Zahl der Herstellungsorte dieser Produkte ungemein groß ist. Es können daher hier nur besonders wichtige und charakteristische Beispiele gegeben werden. Die größte Sammlung von Marken auf japanischen Töpfereien findet sich in Edw. S. Morse, Catalogue of the Morse collection of Japanese pottery. Cambridge I9or. Diese Marken geben entweder den Namen des Fabrikationsortes oder des Herstellers an, im letzteren Falle oft auch seinen Künstlernamen, der bisweilen mehrfach gewechselt wurde. Geschrieben sind sie meist mit chinesischen Buchstaben.

Provinz Hizen.

I-3: Kameyama (E). 4: Bogasaki (O). Shoto. 5: Shohaku.

\section{Provinz Bizen.}

I: Cho. 2: Imbe: Yei. 3: Tera? 6: Ka-ichi, um I68o. 7: Kaku-ichi. 8: Yotsune. 9: Maru-ichi. 10: Ichi. 12: Maru-ji. 13: Marusan. 16: Yamamaru. I7: Maru-san. I9: Ka-ichi, um I680. 20: Cho. 21: Kimura-nji (I780 bis I830). 22: Riku (I780-1830). 23: To-i. 24: Okayama (O). 25: Mushiage $(O)$. Hobson: Naoyoshi $(0)$.

\section{Provinz Tushima.}

I, 2: Shiga (O). 3: Sen Saku (O).

\section{Provinz Tosa.}

I: Asa. 2: Odo. 3: Sokan tsukuru.

\section{Provinz Yamato.}

I, 2: Akahada (O).

\section{Provinz Settsu.}

I: Naniwa (O). 2: Kosobe. 3: Kikko (T). 4: Sakurai (O) no Sato.

\section{Provinz Omi.}

I: Baizan. 2: Mompeizan (Mompei, O). 3: Otsu (O) Meisan. 4: Yorodzu tei. 5: Bairin (= Pflaumenblüte). 6: Koto. 7: Ubagamochi. 8: Kameyama. II: Hobson: Shigaraki Uichu (I7. Jahrh.).

\section{Provinz Higo.}

I: Chubei, 2: Higo. 3: Hobson: A Higo Amidayama. 4: Shodai (Shodaijama, $O$ ).

\section{Provinz Nagato.}

\section{6: Saji. 7: Setsuzan.}

\section{Provinz Kaga.}

I: Yamamoto (T). 2: Ohi. 3: Choju. 4: Masakichi (T). 5: Ono Zenroku. 6, 7: Yeiraku (T). 8: Kinju. 9: Shotei.

\section{Provinz Ise.}

283. $\quad$ I, 2: Anto. 3: Banko (T). 4:Akogi (O). 5: Sahei (T). 6, 7: Banko (T). II: Isawa $(O)$. I2: Tamagaki (O) tsukuru. 
Seite

\section{Provinz Echizen.}

I-3: Setosuke. 4: Fukui.

Provinz Chikugo.

Yanagawa (O).

Provinz Hidachi.

Kairaku.

Provinz Iyo.

Yoshu Matsuyama.

Provinz Suo.

I: Iwakuni Tada (O). 2: Iusan-ken.

Provinz Sado.

I: Sa Kin saku (Kintaro, O). 2: Sado Iozan (T).

Provinz Awajo.

I. 2: Mimpei (T).

Provinz Buzen.

I : Iozan (T). 2: Ho.

284. Provinz Izumi.

I: Minato. 2: Mina!o yaki. 3: Kikko (T).

Provinz Suruga.

Shizuhata (O).

Provinz Kii.

I: Kairakuyen (O). 2: Zuishi. 3: Nanki Otokoyama (O).

Provinz Iga.

I: Iga. 2: Iga (O) Iwa. 3: Tokuhin. 4: Shinziro (T).

Provinz Totomi.

I, 2: Shidoro $(0)$.

\section{Provinz Harima.}

I: Akashi. 2: Akashi-ura. 3: Ryushi. 4: Asagiri. 5, 6: Wafuken, Maiko. 7: Höyen (T). 8: Maiko.

285. 9: Sohei (T). Iо: Suma (O). II: Tozan (Name des Bergs, von dem der Ton kam).

Provinz Izumo.

I: Unzen (von T. Zenshiro). 2: Raku. 3: Rakuzan (Name eines Teehauses bei der Töpferei).

Provinz Aki.

I, 2: Miyajima (O) yaki.

\section{Provinz Chikuzen.}

I, 2: Sen. 3: Ki. 4: Hobson: Taka (Takatori, O). 5: Yo. 6. Ki. 7: Ka. 8: Yama Ka. 9: Taka (Takatori O) Yoshiwa. Io: Takatori yo. II: Hobson: Soshichi (T) (i. Hakata I827). 


\section{Provinz Sanuki.}

I, 2: Taka (Takamatsu, O). 3, 5: Nishiki (Kasugi?). 4: Shido (O) Shunmin. 5 siehe 3, 6: Min. 7, 8: Minzan (T). 9: Yashima. ro: Tomikawa. I I: Yohachi $(T)$.

\section{Provinz Tamba.}

I: $\operatorname{Naosaku}(T)$. 2: To.

\section{Provinz Owari.}

I: Shinzan. 2: Bunro. 3: Densho. "4: Hachi-ju-ichi-o. 5: Rikishin. 6: Yamaguchi. 7: Akebono. 8: Shigenori. 9: Sei. ro: Sakusuke sei. I r: Hanji. 12: Bunshi. I3: Bakesuke. 14:Bizan. I5:Fuke. r6: Sobokai. I7: Shunzan (T). 18: Shuntan (T). 19: Shunkozan. 20: Shuntai (T). 21: Shurin. 22: Makusa. 23: Atakayama. 24: Choza (T). 25: Tozen (T).

287. 26: Ikko (T). 27: Sanko (T). 28: Gempin (T). 29, 30: Inuyama. 31: R. (Risokuan T). 32: Shozo. 33: Masa (T). 34: Matsu. 35: Suisetsu. 36: Kanriu. 37: Kenshin. 38: Masaki (T). 39: Masa. 40: Ichigo. 41: Hagiyama yaki. 42: Yoshitoyo. 43: Toyo (Toyosuke O). 44: Toyohachi. 45, 46: Fuji (Fujimi, T). 47, 48: Sasahima.

\section{Provinz Yamashiro.}

I: Kuchu. 2, 3: Ninsei (Töpfer Ende d. I7. Jahrh.). 4: Akashi. 5: Seikanji. 6: Harima (T). 7:Tsuja Harima (T). 8:Fuji. 9: Yamake. ro: Yamada. I I, I2: Awata (Distrikt). I3: Awata Guchi. I4: Rakuto. I5, r6: Iwakura. 17, 18: Hozan (T). 19: Gobasatsu. 20: Taizan (T). 22: Kinkozan (T). 23: Giozan. 24: Bizan (T). 25: Rakutozan (Name für das östliche Kioto). 26: Kiomizu (Distrikt). 28: Komatsu (O) kichi. 29: Kichibei (T). 30: Yeisen (T). 31: Kyuta (T). 32: Kasuke (T). 33: Kiomizu (T). 34: Sei. 35: Dohachi ( $T$ ). 36, 37: Dohachi sei. 38: Nin-am. 39: Dohachi (T).

289. 40: Shuhei (T). 4I: Yoso (Yosobei T). 42: Kitei (T). 44, 45: Ki. 46: Taisa. 47: Zoroku. 48: Seifu (T). 49: Tsuyen (T). 50: Arashiyama (O). 51: Asahitei. 52: Asashimine. 53: Seizan. 54: Shonsui. Gorosuke (T). 55: Kinsei. 56: Kosai. 57: Ko. 58: Kasei nensei. 59: Gohonzan onaratame.

290. 6o-62: Raku (= Gluick). 63: Kanraku. 64: Saburo. 65-67:Kenzan (T). 67: Hobson: Sandai Kenzan (T, Anf. d. 19. Jahrh.). 68-70: Mokubei (T). 7I: Kokikwan Mokubei (T) tsukuru. 72, 73: Asahi. 74: Ryozen (T). 75: Yeiraku (T). 76: Hobson: Yeiraku (T). 77: Kakin Shiriu. 78: Tenka-ichi Soshiro (T). 79: Yokuro. 80: Sosaburo (T). 81: Otawa. 82, 83: Makudsu (T). 84: Sumizone.

29I. 85: Rengetsu (Töpferei). 86: Fukuroyama.

\section{Provinz Iwaki.}

I: Sei (Seiziyemon T). 2: Komaru (T). 3: Rakuzan.

\section{Provinz Musashi.}

I: Kenzan ( $T$ ). 2: Hobson: Kenzan ( $T$ ) sho (Kenzan schrieb dies). 3: Kenya. 4: Kaseizan. 5: Tokuzan. 6: Teizan (T). 7: Rakurakuyen (O) sei. 9: Korakuyen (O) sei. Io: Sumidagawa (O). II: Sanrakuyen sei. I2: Benjiro (T). I3: Tama, Tamagawa-shisui. 
Seite

292. Provinz Satsuma.

I, 2: Hobson: Satsuma. r: Huju (T). 2: Hohei. 3:Hoyei (T). 4: Hoyu (T). 5: Satsuma no Kuni Keiden sei. 6: Seikozan. 7: Satsuma Kawauchi. 8: Gengo. 9: Gioku Ho sei (Gioku Hozan) T. Io: Satsu sei. II: Yamahara tsukuru. 12: Naye.

Provinz Ugo.

Akita (O).

Provinz Mikawa.

Kakitsubata.

Provinz Echigo.

Hoshina, Beikoku. 


\section{Alphabetisches Verzeichnis}

der in vorstehenden Marken vorkommenden einzelnen Buchstaben.

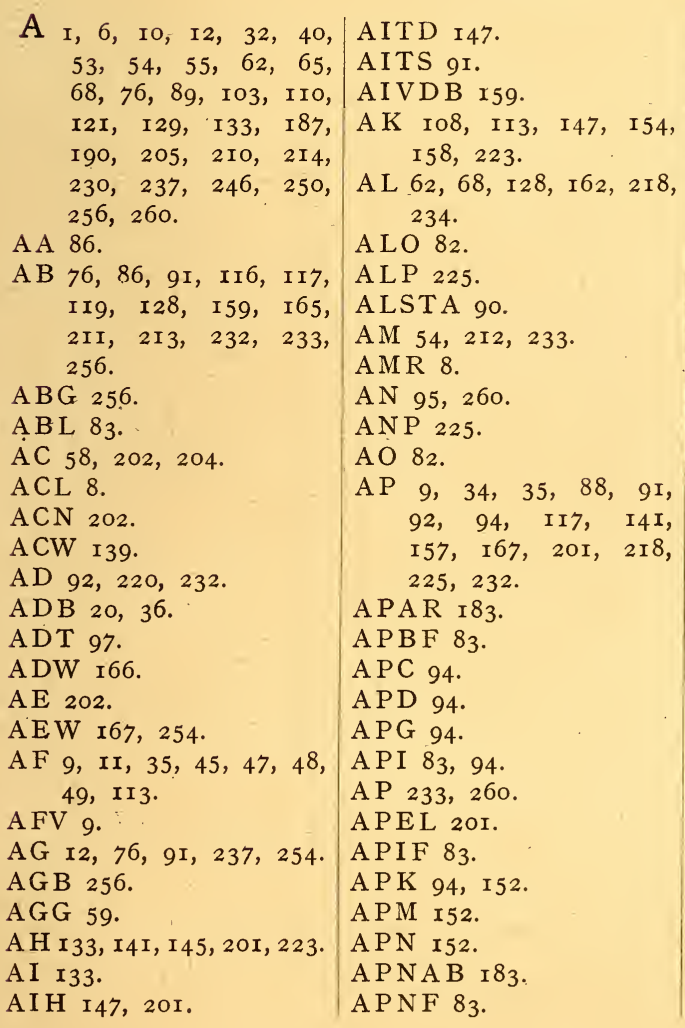

A P P 94.

APR 94.

A P V 94.

A Q K I 58.

AR 9, 40, 76, 94, I50, I93, 2I3, 2I4, 239.

A RP I50.

AS I8, 68, 88, I1о, I33, 237.

ASK I33.

ASS 24I.

ASTA 90.

A T 260.

$\mathrm{AV}$ Ior, II3, I6o, 239, $240,245$.

AVAVR 160.

AVBC 70.

$A V E$ 9, 132.

A VEV 9.

AVF II3.

$A V K$ I 48 .

AW 76 .

AX 234.

AZ 125 .

B $5,24,39,43,44,48$, $55,60,70,7 x, \quad 76$ bis $79,81,82,83$, $85,86,89,92,93$, $96,97,99$, го0, 107, IIO, II4, II7, I25, I26, I3I, I66, I 9 I, I96, 203, 2I2, 2I4, 


$$
\begin{aligned}
& \text { 220, 230, 233, 239, B V 9I, I 43, I65. } \\
& 24 \mathrm{I}, 244,245,247, \text { B V P.I } 87 . \\
& 248,25 \mathrm{I}, 26 \mathrm{I} \text {. }
\end{aligned}
$$

BA $5,16,61$.

BAM I3.

BAMG I 6 .

B B 76, I45, I66, 241 .

BBA 80 .

BC $39,55,61,76,77$, I10, I 3 I.

$\mathrm{BCP} 238$.

$\mathrm{BD} 75,227,230$.

BDSR I 4 .

BE 83, I66, 167.

BELO 82 .

В F $77,79,86$, го6, г 26, 232.

B F B 247.

B F B B 86.

BFS 106.

B FVF 30.

B G 55, 66, I $42,224$.

B H 76, 100, 103.

BHM I 6 .

BIAF I 39 .

B I B 77; 79 .

BID 75 .

B K I00, I05, I24, 218.

B KG I 59.

$\mathrm{BL} 63,73,78$, II 7, I 57 , I $66,188,221,232$.

B LB I4I.

BLC I 66.

BLO 82, 83 .

$\mathrm{BM} 6,55$, I 42, I 72 , 213.

BMP 202.

$\mathrm{BN}$ 105.

$\mathrm{BNP} 213$.

BO $5,97$.

B P 77, I06, I55, I 66.

B P F ro6.

B R I00, 226, 230, 232.

BRA $85,97$.

BS $47,61,186$.

BSM 219, 223.

BT I, 92 .

B U 145 .
BVT 165.

BW I 45 .

BX 105, 166.

BY 230.

$\mathrm{BZ}$ I3I.

BZIB 78 .

C

C $32,47,56,58,62,78$, I I0, - I 28-I 30, I 39, $157,187,201,203$, 204, 210, 219, 223. $228,230,232,238$, $244,245,249,257$.

CA 78,95 .

CAT 4 I.

CATR 44, 74, 78, II9, I26, I65.

С В 74, 78, I19, I26, I 54, I 59, 204, 232, 258.

CBD 250 .

CBG 154 .

CBIA 162.

CBL I 69.

CBM 86, I62.

CBN 169.

CC $33,47,56,58,62$, I Io, $203,210,228,232$, $25 \mathrm{I}, 257,258$.

CCCCP I 66.

CCL 203.

CD $38,232,249$.

CD L 44 .

CF 47, 49, 65, II9, 202, $233,258$.

CFC 70.

CFF 5 I.

CGP 57.

$\mathrm{CH} 78,95, \mathrm{r} 57,204,230$, $236,238$.

CHON 26.

CI 142.

CIA 91 .

CIB 10, 77, 78 .

CIR 240 .

C K I 42,158 .
$\mathrm{CL}_{2}, 34,36,86, \mathrm{II}_{8}, \mathrm{I}_{4} 6$.

CLL 86.

CLNO 29 .

CM 37,56, I 22, I 42,221 , 230, 236, 26r.

CMA 9.

CMB I62.

CMHR 215.

CN II, I22, 210.

CNO 29, I22, 210.

CNTR.I59.

CO 68, 78, 85, 93, I9I.

COP 85 .

CP 39, II9, I65, 230, 232,

C P K 157.

CPLI 254.

CPM II9, I8I.

CPP 55.

C R I 45.

CRC 47.

CRI II6.

CRLP 35.

CS 78, 96, 235.

CT II9, 201, 232.

CV 37, 44, 206.

CVK 159 .

CVS I 49, I 59 .

CW 159.

CX 78.

$\mathrm{CZ} \mathrm{I}_{42}$.

D I6, 19, 20, 36, 44, $78,85,87,89,90$, 92, 93, 98, IIO, III, I 1 , I25, I 27, I 33, I47, 197, 200, 202, $216,218,225,230$, $242,243,246,247$.

DA 78 .

DAPW I 48, I 49.

D B 7I, 78, 87, I54, 200. DBE 87 .

DCR 85 .

DD 25, 78, 218 .

DD \& Co I 81 .

DE 233.

DER 85 . 


\section{3}

\begin{tabular}{|c|c|c|}
\hline 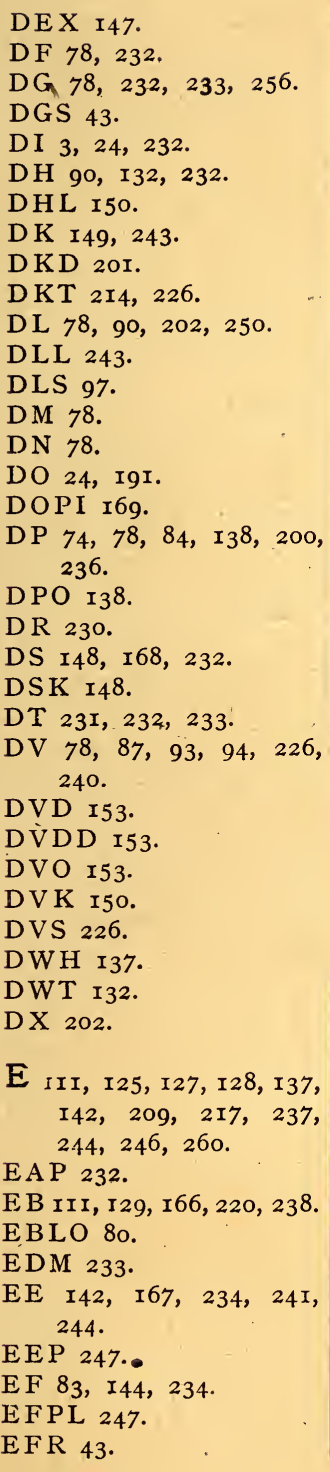 & 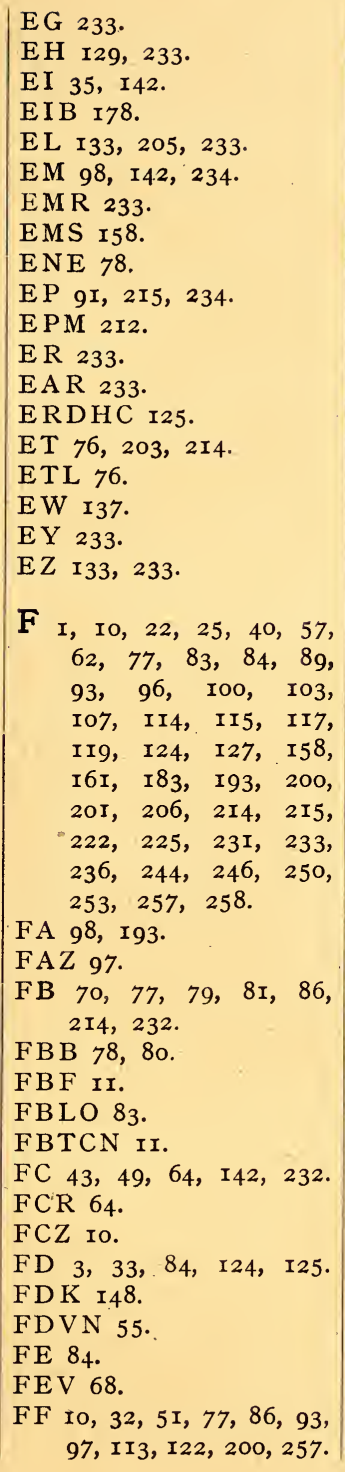 & 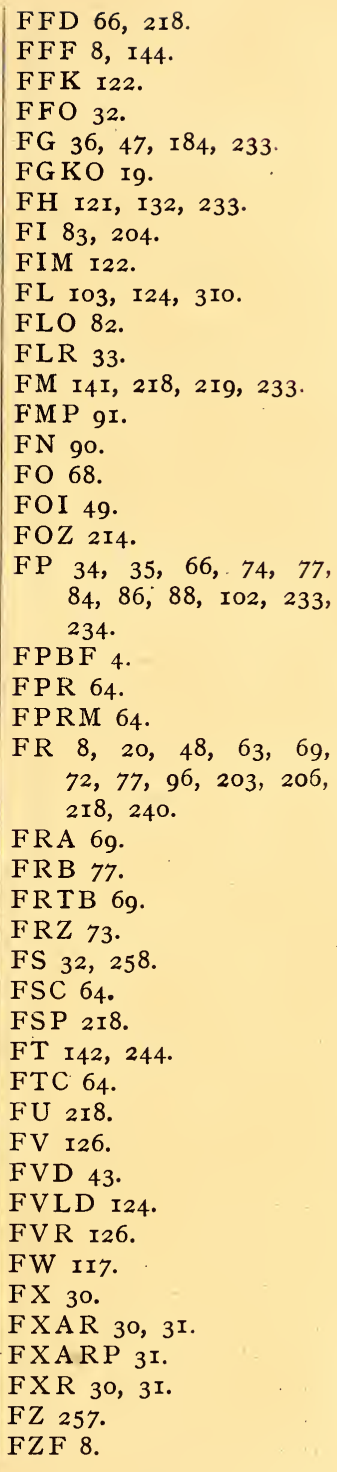 \\
\hline
\end{tabular}

Führer. 
G $12, \quad 17,20,22,25, \quad \mathrm{GO} 79$ $55,58,63,65,77$, GOBR 233. $79,80,83,84,89,95$, GOMC III. 103, 107, 121, 126, GP 9, 26. I28, I29, I45, I65, GP P 42. I66, I9I, I96, I99, GPS I56.

$207,208,212,213$, GR 46, 52, 60, 66, 106, $214,218,221,226$, $23 \mathrm{I}, 244,258,26 \mathrm{o}$, 277.

GA $77,78,80$.

GAB $53,256$.

GAF 96.

GAFF 257.

GAG 59.

GAL II.

GAOF 65.

GAR 73, 77.

G B - 53, 6I, 73, 77, 93, $\mathrm{I} 63,2 \mathrm{I} 4,256$.

GBAB 53,256 .

GBF 36.

GBK 2 I 8 .

GBM I62.

GBS 156 .

GC 77, 93 .

GD 75, 77, 231 .

GDF 37 .

G.DG 97.

GE I26, I42, I67.

GF $27,37,66,96,258$.

GG 55, 77, I67, 233.

GGM 77 .

GGPF 253 .

GH 92, I4I, I48, 237.

GHA 77.

GHM 202.

GI 49 .

GIN 77.

GK 108, 146, 152 .

GL II, 49, 77, 79, 234.

GLO 82 .

GLP 35, 36.

GM 36, 79, 219, 254.

GMD 79.

GMR 108.

GN I7, 79.

(iNTR I59.
$\mathrm{HH} 39, \quad 79, \quad 1_{4}, \quad \mathrm{I}_{42}$, 227.

$\mathrm{HHCO} 20$.

HHG 167.

HHH rgo.

HHIM I69.

HI 79, I65, I72, 201.

$\mathrm{HICO} 20$.

HK I03, I44, I58, I66, $187,201,218$

GRID 79 .

GS 39, 5I, 58, 76 .

G \& St I45.

GT $23 \mathrm{I}$.

GUD (GUT) 73 .

$\mathrm{GV}$ r2, 44, 6r.

GVS 147 .

GW 79.

GZ 22 I.

GZMIAZ 204.

H $19,39,75,79,84$, $85,89,91,93,99$,

Iо3, I04, Iо7, IIO,

III, II4, II5, II7,

II 8 , II9, I28, I29,

I44, I97, 202, 209, 213-216, 23I, 236, $244,246,25 \mathrm{I}, 260$.

HA 80, III, I 44.

HAR 190 .

HB 7I， 79，9I，92，99, II 4, I 52, I90, 233.

$\mathrm{HBH}$ I9o.

HBSG $2 I 2$.

HC 79, IOI, I25, I65, 205, $23 \mathrm{I}, 238$.

HCE 167.

HCM I68.

HCO 79, 223.

HD III, I2I, 205, 233.

HDG III.

HDK I48.

HE 53, I07, I3I, I44.

HEM 203.

HF 55,57, 130, 132, 144 , 233.

HFI 209.

HG 65,80 .

\section{HKG 197.}

HKHA 144.

HKI 103.

HL I25, I5O, 240, 246.

HLH I 65.

HLL 240 .

HM 79, I03, III, I68, 202, $223,240$.

HMW I68.

HN II3.

HP 9I, I25, I66, I67, 231.

HPM 214 .

HR 79, 215.

HS 72, $120,125,159,180$, 212.

$\mathrm{HSCH} 202$.

HSR I59.

HT 79, I39.

HTI 187 .

HTO 32.

HV 79, I42, 209.

$\mathrm{HVH} I 53$.

HVMD 150.

HW 125.

I $8,20,70,84,89,90$, 95, IO7, IIO, III, I28, I6I, I83, I93, $208,217,223,231$, 244,250 .

IA $55,201,232$.

IA G 69 .

IAH 204.

IA I II 4 .

IAL 225.

I AM 108.

IA P 94.

IATS 9 I. 


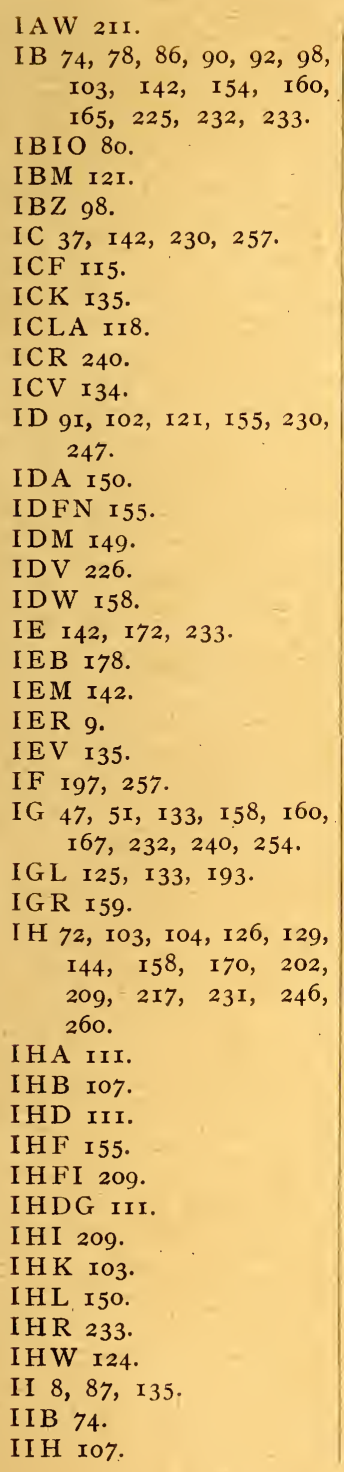

IIM I 45 .

I K I42, I45, I 47, I 49 , 152,184 .

IL I9, 94, I72, 210,240 . I L F 87, I33.

ILS 92.

I M 54, I03, I42, I65, I66, 2 II, 238.

I MBH 123 .

I N 55, 80, 230, 237.

IN F 66 .

INM I8I, 213.

I NZND I 32 .

IO 202.

IP 49, 9I, I44, I 56, I 82, 23I, $24 \mathrm{I}$.

I PS 70.

I PY 236.

I R 9, 96, I44, I6I, 234, $-236,240$.

I RH P I65.

I RL I76.

I RLO 83 .

IS 36,7 I, 85, III, I33, 200, 213 .

IT 23I, 234.

ITD 147.

I UC I4I.

IV I24, 209.

IVDH I49, 158 .

IVDK 152.

IVDW 159.

IVFL I 49.

IVH $149,156,160$.

IV K I52, 156.

IVLR 80.

IVP 149.

IVP \& C I49, I5I, I53.

IW I40, I47, I72, 254.

IZ 53, IO2, I2I, 200, 256.

$\mathrm{K}_{25}$, 105, 108, I10, II4, II 5, I I 8, I 2 I, I 27, I35, I37, I58, I66, 195, 196, 200, 202, 204, 218, 230, 244, $26 \mathrm{x}$.

KB I25, 135, 247.
KB K 135 .

KBL I35.

KBN 125 .

KD Ioo.

KFF 5 r.

KG 94 .

$\mathrm{KH}$ II $5, \mathrm{I}_{3} 6$.

KHC $105,197$.

KHCW 197.

KHPA 136.

$\mathrm{KI} 2 \mathrm{O} 2$.

$\mathrm{KL} 2 \mathrm{I} 3$.

$\mathrm{KLO} 83$

KO II 8.

KPF 194.

KPM I36, 193， 194， 195, I99, 200, 2 I 5 .

KR II 5 .

$\mathrm{KS} 213$.

KSStF I 39.

KT II2.

KTK I35.

KT P 135.

KTR I35.

KVD $I 49$.

$\mathrm{KZ}$ I27.

L I0, 20-22, 24, 36, 37, $62,80,90,91,97$, IOI-IO3, I2I, I 24 , I25, I65, I75, I96, $203,223,230,236$, 24I, 249, 255, 258, 260.

I.A 2I, 80, 92 .

LAC 8 .

LAR 8o.

LB 7, I42, I54, I66, I68, I75, $208,22 \mathrm{I}, 23 \mathrm{I}$ bis 233, 235.

LC $9,35,75,92,1_{4} 6,220$.

LD I, 80, 226, 232, 239.

LE 231.

LF 36,80 .

LFM 107.

LG I66, 23I, 233.

LGCE 233.

LGP 56 . 


\section{6}

LH 219, 233.

LI 80 .

LILC 92.

LK I37.

LL 46, 62, 93, 204, 225, 227, 23I, 246, 250.

LLO 243.

LLT I02.

LM 80, 92, I 42 .

LMS 258.

LO 22, 82, 83, 97, 220.

LOA 83 .

LOG 82 .

LOIC 82.

LOO 83 .

LOP 82,83 .

LOPL 82 .

LOS 82,240 .

LOSC 82 .

LOSt. 83 .

LP I, 55, I8I, 238, 258.

LPK 157.

LPM 212.

LPN 212.

L R 97, 231.

LS 92, 235.

LSD 231.

LSO 240.

LUU 80.

LV 37, 125.

LVD 44, 155 .

LVS I 49.

LW III, I42.

LWG II2.

LW P 167.

LZ I2I, I75.

I

7-9, 13, 17, 19, 22, $43,64,68,73,75,8$ o, $87,90,95,97,99$, гоo, I07, II2, II 8, I25, I27, I42, I62, I65, I68, I95, 2I7, 224, 23I, 234, 25I, 252, 258.

MA 26, 66, 75, 202.

MAB 5 I.

MAIM 20.
MAM 65.

MAXAR 35 .

$\operatorname{MAXX} 35$.

MB 76, 87, 9I, II9, I28, I56, I79, I89, I90, 224, 230, 235.

M B B I 89 .

MBE I89, 190 .

MC 55, II 2, I79, 2I 2, 233.

MCA 83.

MCG 83 .

MD 80, I66, 213.

MD K 150 .

MDL 19.

ME 234 .

MF 80, 107.

MFF 7 .

MFM 253.

MFS 35.

$M G$ I2, I3, I5-I7, 80.

MGO I2, I3, I7, I 8 .

MGV 12 .

MI 5, 54, 66, I03, 202.

MIMS 79.

MIW I22.

M K I07, I44, I68.

M KF I4I.

ML 143.

MLO 82.

MLP 239.

MM 66, 93.

MN 179 .

MO 80, 202, 226, 234.

MOL 220.

MP $66,69,75, \mathrm{I}_{46}$.

MPC 181.

MPL 239.

MPM 194.

MR I00, II6, II7, 233.

MS 80, 107.

MSP 258.

MTT 132.

MV 76.

MV B $150,156$.

$\mathrm{MVK} 160$.

MX 66, I4I.

MZ II8, 218.
N I, I7, 22, 25, 37-39, $32,42,55,71,73,80$, $85,89,90,105,107$, I65, I69, I 88, I93, 202, 210, 213, 226, 229, 230, 237, 250, 255,260 .

NA 87,89 .

$\mathrm{NAB} 5 \mathrm{r}$.

NB 105, 107, 108, 210.

NBL 97.

NBLU 202.

NC I7, 83.

$\mathrm{N} \&$ Co $\mathrm{I} 4 \mathrm{I}$.

NCOL 29.

ND 258.

$\mathrm{NF}$ 9, 42, 75, 8I.

NG 59, I4I, 2 IO, 240.

NH 80.

NI I2, I65.

NIB 75 .

N K 260.

$\mathrm{NM}$ I03.

NO I7, 53, 231 .

NOS 29.

NP 63, I69.

NQ 23 I.

NR 8, 213.

NS 202.

NSCA 255.

NSW 202.

NV 107.

NW 204.

NX 7.

NY 237.

O II, 22, 35, 37, 99, II2, II3, I65, I9I, 202, $232,244$.

OA 25 .

OAPP 34 .

OB 100.

OE 134 .

OЕ B 136 .

OEBA 136 .

OEBAL I36.

OF 29.

OFF 120. 
OFL 258.

OFV 29, 36 .

OL 28, 82, 243.

OM 233, 238.

ON 25, II6.

OP 100.

OPP 82 .

OS $28,116,202,246$.

OSLZ I34.

OSW Ir6.

OT 36 .

OTOR 29.

OY 82.

$P$ I, I 8, 21-23, 26, 39, $44,67,70,74,75$, $8 \mathrm{I}, 82,84-86,88$, 90, 91, 93, 103, 107, II2-II4, I2I, I29, I38, 144, I56, I65, 196, 197, 201, 204, $218,230,251,260$, $26 \mathrm{I}$.

PA 3, 75, 77, 79, 80, 232.

PABF 83 .

PANF 83.

PAP 94 .

PAR 75.

PAT 76 .

PB 60, 73, 80, 185, 203.

PBC 98.

PBK 218.

PBM 215.

PC 57, 72, 75, 77, 81, 92, $93,99,138$.

PCG 238.

$P C N$ I86.

PCO 81.

PCP 57, 74.

PCS rog.

PD 75, 8I, 93, 150.

PE 142.

PF 84, 88, II2, 245, 255.

PG 39, 42, 8I, 231 .

$\mathrm{PH}$ I03, II8, 201, 209, 2 Io.

PHF 201.

PI 75, 144.
PIA 3.

PIL 240.

PK 142, I52, I58, 260.

PKCAP 158.

PL 55, 86, II 2, I38, I59, 228, 23I, 233, 238, 25I, 26I.

PL II3.

PLC 93.

PLPL 228.

PLS 234.

PM 82, II2, I52, 23 I.

PMC I8I.

PMR 234.

PN 8r.

PO 82, II6, 138 .

PP 2I, 24, 34, 79, 8I, II2.

P PC 74.

PR 32, 49, 77, 85, 88, 94, 132, 142, 234, 261 .

PS I, 23, 72, 80, 88, 93, 214.

PT 231.

PV 142.

PVDB 155.

PVDS 160.

PVK 90.

PVM 152.

PVR 201.

PVS 155.

PWB 155 .

PX 8o.

PY 236.

PZ 205.

PZB 86.

Q 97, 214.

QZ 99 .

R. 9; 10, 20, 22, 39, 47, $66,69,8 \mathrm{I}, 90,93$, 96, 98, гог, 103, 107, I18, I26, 137, I69, I80, I 88 , I92, 206, $207,208,214,233$, 234, 242, 245, 246, $249,255,258$.
RA 8I.

RAR 8I.

RB 231.

RBF II.

RC I30, I3I, 215.

RCE 188.

RCG 47.

RD 8I.

RF 130, 192, 228, 229, 255.

RFH 219, 247.

RFO I92.

RG 20, 22, 207.

RH 39, 84, I68, 245, 247.

RHF 247.

RINI 137.

RIS 87.

RL I28, 232.

RLO 83 .

RM I2, 137.

$\mathrm{RN} 137,208$.

RO 8I.

ROERH I 88.

ROM 12.

RPKR I4I.

RPM I4I, 218.

RR I5I.

RS $85,258$.

RSI 147.

RSW I34.

RV 90.

RVA 40.

RX 96.

S I, I8, 20, 21, 32, 37, $39,43,45,58,60$,

$62,75,89,90,92$,

94, I00, 103, I07,

I08, II2, II3, I25,

I29, I34, I44, I60,

197, 203, 204, 208,

2I3, 2I5, 219, 223,

$229,231,234,236$,

240, 24I, 249, 258.

SA $48,88,24$ I.

SAGS 9, 59 .

SAR 76.

SAS 76 . 


\section{$35^{8}$}

SB 39, 76, 144 .

SBO I34.

SC 9I, I08, II2, 225, 230.

SCA 43.

SCB 134 .

SCD $\mathrm{r} 34$.

SCE 8.

SCNC 250.

SCT $93,225$.

SCY 92.

$\mathrm{SD} 231$.

SE I34.

SEEA 24I.

SFC 26.

SFM I 34 .

SG $56,75, \mathrm{I} 39, \mathrm{I} 84,250$.

SGIB 45 .

SGP 57.

SgrP 199.

$\mathrm{SH} 96,134,232$.

$\mathrm{S} \& \mathrm{H}$ I45.

SHD 243.

SI IIO, II2, I97, 23I, 247.

SIG 53.

SK 231 .

SL 62, 68, 83, I03, I34, I $65,200$.

SLO 82,83 . .

$\mathrm{SM} 5 \mathrm{I}$, IO2, II 2, II 3 , I30, 172.

$\mathrm{SN} 258$.

SNB I07.

SNC 250 .

SO 215 .

SOA 89 .

SOP 84 .

SP 23, 75, I00, II2, I43, 144.

SPF $2 I 5$.

SPM 214 .

SR 39，50，60， I34，200.

SRB J 34 .

SRFM I34.

SSM. 25I, 252.

SSP 234.

ST II, II7, I88, 204.

St F F 136 .

Stg 199.
StPM 182.

$\mathrm{SV}$ II3, I29.

SVE 146.

SW II 5, 234.

$\mathrm{SX}$ 92, 103, 235.

SXSP 235.

T 7, 52, 81, 90, 95, 103, II3, II7, II9, I23, I 28, I65, I66, I 83 , 202, 206, 209, 214, $218,230,233,244$.

TB 7, 69, 81, I4I, I 54 .

TEL 76 .

TF 25.

TG 52 .

TH III.

THD 9 I.

THR 233.

THX III.

TK 2 I 8 .

T.L $9 \mathrm{I}$.

TLO 82.

TO 36,22 I, 244, 248.

TOMESWA 157.

TR 52, 72, 102, 240.

TS 23, II7, I74, 204.

TW $\mathrm{I} 42$.

TY 221.

$\mathrm{U}_{\mathrm{C}} 2 \mathrm{I} 5$.

$\mathrm{U} \& \mathrm{C}$ I4I, 215.

USC 215 .

$\mathrm{V}_{\mathrm{I} 2}, 24,25,54,6 \mathrm{I}, 69$, $8 \mathrm{r}, 90,95$, I28, 129, I $60,226,23 \mathrm{I}, 244$, $246,256$.

VA 49, II3, I6o, 254, 259. VAH 209.

VB $82,90,134,145,146$.

VBM 143 .

VBS 143 .

VC 68 , 129.

VCAE $\mathrm{r} 86$.

VCAR 256.

VD 75, 232.

VDG 54 .
VDOF 28.

VDX 44 .

VE I46, I48, I58, 202.

VENA 254 .

VEP 158.

VF $254,256$.

VFFE 84 .

VG $8 \mathrm{I}, \mathrm{r} 34$.

VH I2I, I28, I3I, I 34.

VHK I34.

VHT 134.

VI 8I, I 28 .

VIB $\mathrm{I} 34$.

VIE I34.

VIG I34.

VIH $\quad I_{28} 8, \quad 134$.

VIK I34.

VL 76, I34, I5I, $24 \mathrm{I}$.

VLE $24 I$.

VLI 76 .

VLN 76.

VM 214, 258 .

VN I20, I 86.

VOC 254 .

VOFD 28.

VOFF 28 .

VP 47, 8I, 96, I45, I 86

VPC 186.

VPCAE I86.

V PCB 186.

VPF 84.

VPGAE 186 .

VR 8I, 201 .

VRAF 9.

VRAP 9.

VRP 35.

VRT 201.

VS 186.

VTBQ 8.

VTM 186.

VV 95, I02, 232, 239, 245.

VW 134 .

$\mathrm{VZ} 3,200$.

$W_{47,73,82,85,90,99}$, I03, I08, II0, II 3 , II7, II 8, I23, I29, 


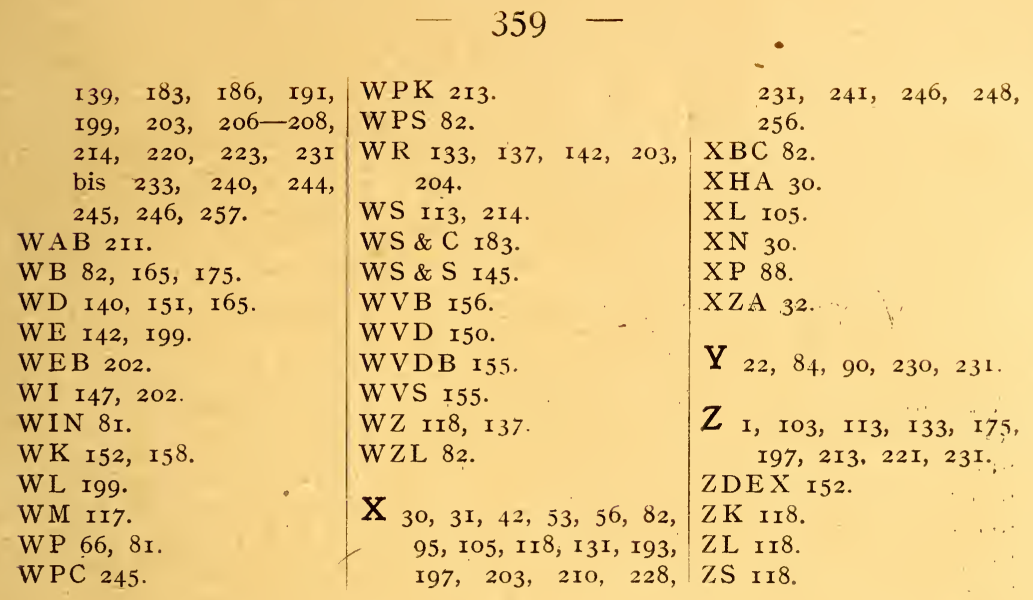




\section{Verzeichnis der Fabrikationsorte.}

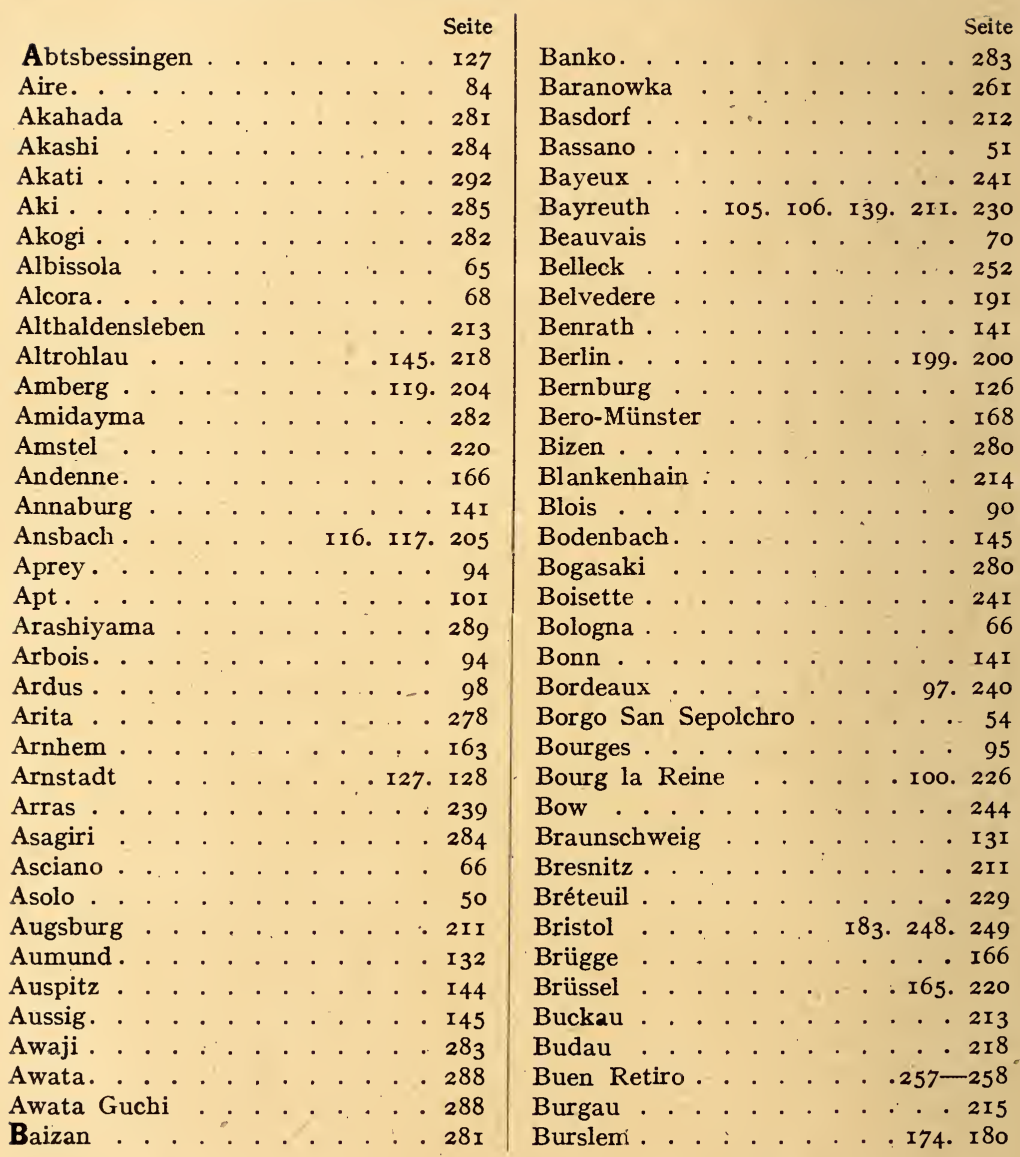


Caen .. . . . . . . . . 24I

Caffagiolo . . . . 21-23. 305

Cambray .. . . . . . . . . 93

Cambrian . . . . . . . . . 184

Candiana . . . . . . . . . 26

Capo di Monte. . . . . . . 255

Cassel . . . . . . . . 125. 205

Castel Durante. . 38-40. 306. 307

Càstelli .. . . . . 56. 57. 309

Castello, Città di. . . . . . . . 42

Castleford . . . . . . . . . . I8I

Castle Hedingham . . . . . . . 184

Caughley . . . . . . : . 249. 339

Chantilly . . . . . . . .. . . 226

Chatillon . . . . . . . . 240

Chelsea . . . . . . . . . . . 242

Chikugo . . . . . . . . . 283

Chikuzen . . . . . . . . . 285

Chodau . . . . . . . . . 2I9

Choisy le Roy. . . . . . . . . 99

Cilli. . . . . . . . . . . . I45

Città di Castello . . . . . . . 42

Clermont . . . . . . . . . . 95

Clermont Ferrand . . . . . . . 99

Clignancourt . . .. . . . . . . 240

Cmielow . . . . . . . . . . . 192

Coalport. .. . . . 249. 250. 339

Cobridge. . . . . . . . . . I76. I77

Coburg . . . . . . . . . I 26

Cockpit Hill . . . . . . . . . I73

Coimbra . . . . . . . . . . . . 69

Colebroke Dale. . . . . . . . . 339

Colmar . . . . . . . . . . 2 I0

Creil . . . . . . . . . . . 93

Crailsheim . . . . . . . . . . . I 23

Crépy-en-Valois . . . . . . . 24I

Dallwitz . . . . . . . . 2 I 8

Damm (Höchst) . . . . I I2I. 328

Delft . . . . . . . I46-I6r. 164

Derby. . . . . . . . . 242. 243

Deruta . . . . . 43. 44. 307

Desvre .. . . . . . 84. 85

Dijon . . . . . . . . . . . . . 93

Dirmstein . . . . . . . . . . . I22

Diruta siehe Deruta.

Doccia . . . . . . . 66. 255

Donauwörth . . . . . . . I20

Donovan . . . . . . . . . 252

Dorotheenthal . . . . . . I27. 128

Douai . . . . . . . . . . . . . 85

Dresden . I32. I4I. I42. I97. 2 II. 2 I4

Dreux. . . . . . . . . . 220

Dublin . . . . . . . . . 185

Durlach . . . . . . . . . . 124

Dux . . . . . . . . . . $\mathrm{I} 45$

Echigo . . . . . . . . . . 292

Echizen . . . . . . . . . 283

Eckernförde . . . . . . . . . . 136

Eisenberg . . . . . . . 209. 214

Elgersburg. . . . . . . . $2 \mathrm{I} 2$

Elnbogen . . . . . . . . . . 218

Englefontaine . . . . . . . . . 95

Epinal . . . . . . . . . . . 85

Erfurt . . . . . . . I28. I29

Essex . . . . . . . . . . . 184

Este . . . . . . . . . 42. 257

Etiolles . . . . . . . . . . . . 239

Etruria (Wedgwood) . . . I75. 25I

Etterbeck . . . . . . . . . . I66

Fabriano . . . . . . . . . $4 \mathrm{I}$

Faenza . . . . . . 2-II. 303

Fenton . . . . . . I79. I80

Ferrara . . . . . . . . . 42

Ferrybridge . . . . . . . . 182

Floersheim. . . . . . . . . 122

Florenz . . . . . . . I. 2. 253

Fontainebleau . . . . 229. 24I

Forli . . . . . . . . . . . . 48

Frankenthal . . . . II8. 201. 202

Frankfurt a. M. . . . II4. II5. 3I7

Fraureuth . . . . . .2I4. 2I5

Freiwaldau . . . . . . . 2I2

Friedberg . . . . . . . . . . II9

Fünfkirchen . . . . . . . . . I45

Fürstenberg . . . . 206. 215. 329

Fujimi . . . . . . . . . . 287

Fukien . . . . . . . . . . 344

Fulda. . . . . . I24. 200. 319

Fullham . . . . . .... . 183

Gehren . . . . . . . . . 212

Genua. . . . . .65. 309. 310 


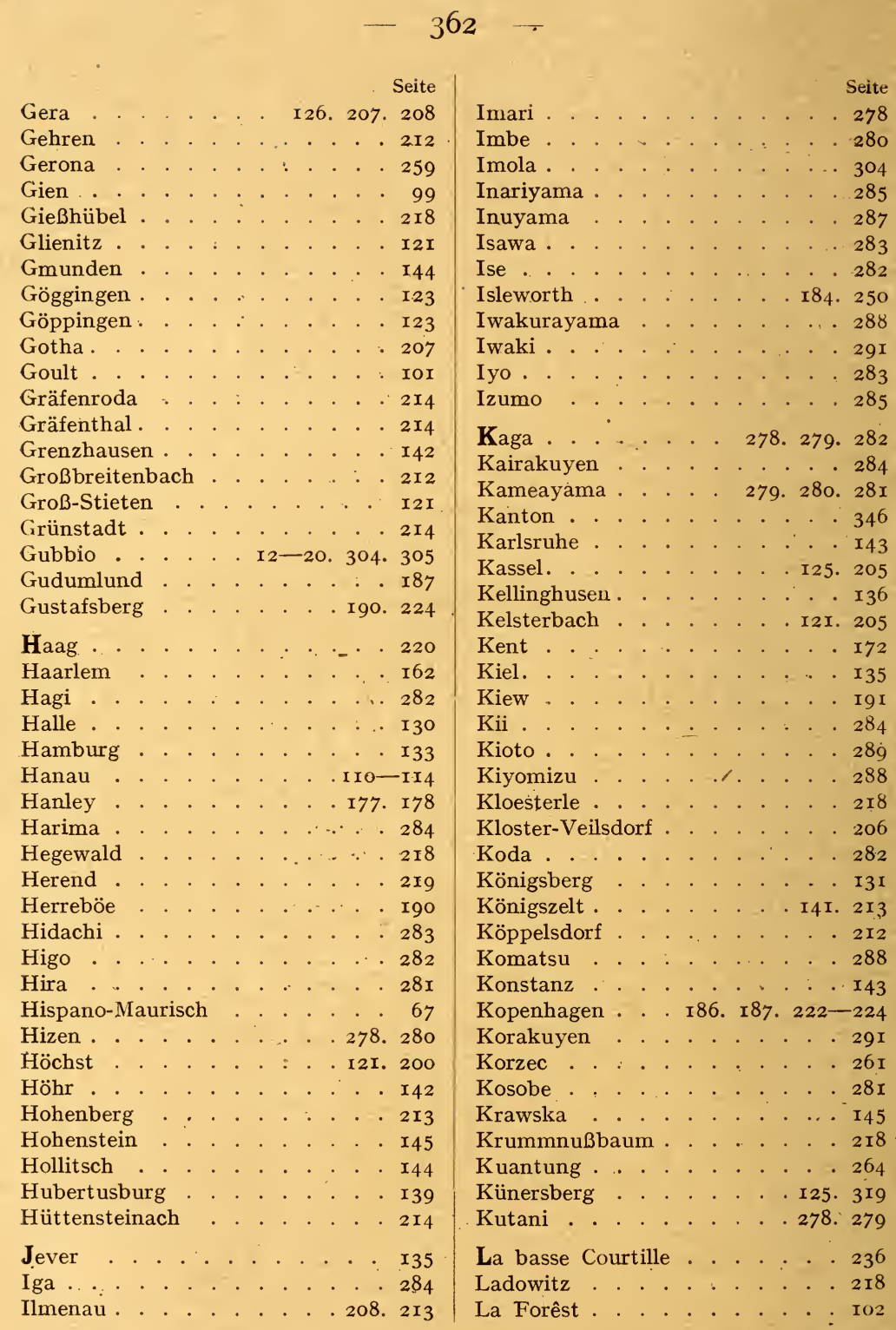




\begin{tabular}{|c|c|c|c|c|c|c|c|c|c|c|c|c|c|c|}
\hline all & & & & & & & & $\begin{array}{r}\text { Seite } \\
\text { I02 }\end{array}$ & Marienne siehe & $\mathrm{St}$ & & ean & & \\
\hline in & & & & & & & & I 83 & 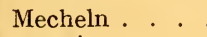 & & & & & I6 \\
\hline ane Delph & & & & & & & & 180 & & & & & & \\
\hline ane End & & & & & & . & & I 84 & rhöfen & . & & & & \\
\hline a Rochelle & & & & & & & & 98 & as & & & & & \\
\hline E & & & & & & & . & 235 & I42. 193 & & 15 & $.2 \mathrm{I}$ & 11.214 .327 & \\
\hline eeds & & & & & & & & I $8 I$ & $\cdot$ & . . & & & 94. & \\
\hline urg & & & & & & & & I 68 & ni . & . & & & . & \\
\hline & & & & & & & & $I_{3}$ & rün & 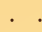 & & & . & 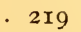 \\
\hline & & & & 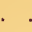 & & & . & $2 \mathrm{I}$ &. & . & & & . & \\
\hline & & & & & & & 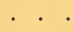 & I 45 & 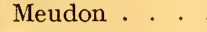 & . & & . & 93. & \\
\hline & & & & & & & .213. & . 2 & agh. & . & & & ${ }^{\circ}$ & \\
\hline & & & & & & & 36. 87. & 2 & 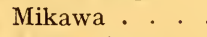 & & & & . & \\
\hline & & & & & 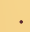 & & . $\mathrm{I} 2 \mathrm{I}$. & . 2 & hell. & 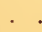 & & & . & 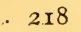 \\
\hline & & & & & 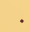 & & . . . & . $I$ & . . & . & & & . & \\
\hline & & & & & . & & . 69. & . 259 & . . . & . & & . & . . . . . & \\
\hline & & & & & & & . . . & 70 & $\therefore$ & . & & & . . . & \\
\hline & & & & & & & . . . & - $18 \mathrm{I}$ & 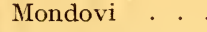 & & & & . & \\
\hline & & & & & " & & ... . & - 218 & age . & 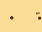 & & & . & \\
\hline & & & & & . & & . . . & 54 & . . & . & & . & . . . & \\
\hline & & & & & & & .176 . & . $25 \mathrm{I}$ & 1. . & . & & & . . . . & \\
\hline n & & & & . & & &. & - $I$ & . . & .. & & &.. & \\
\hline $\mathrm{Hal}$ & & . & & & 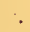 & & $\therefore$. & - 2 & er. & . & & . & . & \\
\hline & & : & & & & & . & - 220 & . & 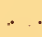 & & & . & 24 \\
\hline & & & & & & & 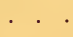 & 26 & . & - & & & . & 18 \\
\hline Te & & d & & . & . & & . & - 240 & . & & & & . & \\
\hline & & & . & . & . & & 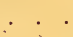 & I\& & . & . & & & . & \\
\hline & & & & & & & & 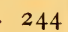 & & & & & . & \\
\hline & & & & & . & & & 2 & . & & & & . & \\
\hline & & . & . & $\therefore$ & . & . & $\therefore 18$. & . 2 & . & & & & . & \\
\hline & & 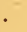 & & & & & . . . & - $I$ & & & & & . & \\
\hline & & & : & & & . & . . . & - $I C$ & ige & & & & . . . & \\
\hline & & & & & & . & . 94. & . 240 & & & & & & \\
\hline & $\therefore$ & & & & & . & & $22 \mathrm{I}$ & & & & & 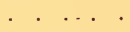 & \\
\hline & & & . & & & & & 70 & . & & & & 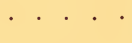 & \\
\hline & & & & & & & & & & & & & & \\
\hline & & & & • & & & & & . & & & & .250 & \\
\hline & & & & & & &. & 68 & ogawa & & & & $\begin{array}{l}.250 . \\
. \quad . \quad .\end{array}$ & 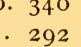 \\
\hline & & & . & & & & & 5 & & & & & . 55.56 . & 6. 25 \\
\hline & & & & & & & . & . $I$ & & & & & . . . . . & \\
\hline & & & & & & & . 189 . & 2 & nsleber & & & & . . . I4 Ir. & r. \\
\hline & & & & & & & . 96. & & & & & & & \\
\hline & & & & & & & . & . 2 & & & & & & \\
\hline & & & & & & & & $x$ & & & & & & \\
\hline & & & & & & & & & 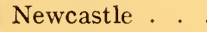 & & & & & \\
\hline na & & & & & & & & & Tew Hall & & & & & \\
\hline
\end{tabular}


Seite

Seite

Niederweiler . . . . . . . 105. 210

Nîmes . . . . . . . . . . . . 98

Nottingham . . . . . . . . . 184

Nove . . . . . . . . . 53. 256

Nürnberg . . . . . . . .107-109

Nymphenburg . : . . . . . . . 204

Nyon . . . . . . . . . . 222

Oberhohndorf . . . . . . . . . 2 I4

Oberkassel . . . . . . . . . 2 I3

Odo . . . . . . . . . . . $28 \mathrm{I}$

Oeslau . . . . . . . . . . . 214

Oettingen . . . . . . . . . . I20

Offenbach . . . . . . . . . . I20

Ognes . . . . . . . . . . . . . 95

Ohi . . . . . . . . . . . 282

Okayama . . . . . . . . 280

Oldesloh . . . . . . . . . . . I37

Olomouczan . . . . . . . . I45

Omi. . . . . . . . . . . . $28 \mathrm{I}$

Orléans . . . . . . . . . 99. 235

Osmaston . . . . . . . . . . 339

Osnabrück . . . . . . . . . . I33

Otokoyama . . . . . . . . 284

Otsu . . . . . . . . . . 28 I

Owari . . . . . . . . 286

Padua . . . . . . . . . . . 42

Palais Royal. . . . . . . . . 239

Palermo . . . . . . . . . . 256

Paris . . . . . 70. 91. $236-239$

Pavia . . . . . . . . . 65

Perugia . . . . . . . . . . 50

Pesaro . . . . . 24-26. 305

Pfalz-Zweibrücken . . . . . . 205

Pinxton . . . . . . . . . . . . 251

Pirkhammer . . . . . . 218. 219

Pisa. . . . . . . . . . . . 53

Plaue . . . . . . . . . . 214

Plymouth . . . . . . . . . 248

Pößneck . . . . . . . . . . . $2 \mathrm{I2}$

Poitiers . . . . . . . . . . 97

Pont-aux-choux . . . . . 238

Poppelsdorf . . . . . . . . . . I4I

Porto . . . . . . . . . . . . . 69

Portobello . . . . . . . . . . 185

Potschappel . . . . . . . . . 214

Potsdam. . . . . . . . . . . . I32

Poupres . . . . . . . . . . 95

Prag . . . . . . . . . 218

Premières . . . . . . . . . . 94

Probstau . . . . . . . . . . $\mathbf{I} 45$

Proskau . . . . . . . . . . $\mathrm{r}_{3} 8$

Proszowice . . . . . . . . $26 \mathrm{I}$

Quimper . . . . . . . . . . 99

Raeren . . . . . . . . : . I42

Raku . . . . . . . . . . . . . 290

Rakurakuyen . . . . . . . . . 29 I

Rakutozan . . . . . . . . . : 288

Rato . . . . . . . . . . . 69

Rauenstein . . . . . . . . . 208

Ravenna . . . . . . . . . . . 40

Regensburg . . . . . . . . . I4I

Rehau. . . . . . . . . . $2 I_{3}$

Reichenstein . . . . . . . . . $2 I_{3}$

Rénac. . . . . . . . . . . . 98

Rendsburg . . . . . . . . . . . I37

Rennes . ... . . . . . . . . 97

Reval . . . . . . . . . . . . . 192

Rheinsberg . . . . . . . . . 137

Rimini . . . . . . . . . . 37

Rockingham . . . . . . I82. $25 \mathrm{I}$

Roerstrand . . . . . . . I88. 224

Rom . . . . . . . . . . . . 5 I

Rosenau. . . . . . . . . . I45

Rotterdam . . . . . . . . 162

Rouen. . . . . . . 73-82. 225

Rouy . . . . . . . . . . . 93

Rubelles... . . . . . 97. 102

Rudolstadt . . . . I30. 206. 2 I4

Rue Amlot . . . . .. . . . . . 238

Rue de Bondy. . . . . . . . . 337

Rue de Charonnes . . . . . . . 239

Rue de Crussol . . . . . 238. 239

Rue de trois bornes . . . . . 238

Rue du Fontaine-au-Roy . . . . 236

Rue St. Gilles . . . . . . . 238

Rue de Popincourt . . . . . . . 237

Rue de Recollets. . . . . . . . 239

Rue de la Roquette . . . . . 236

Rue de Reuilly . . . . . . . 236

Rue Montmartre. . . . . . . 239 


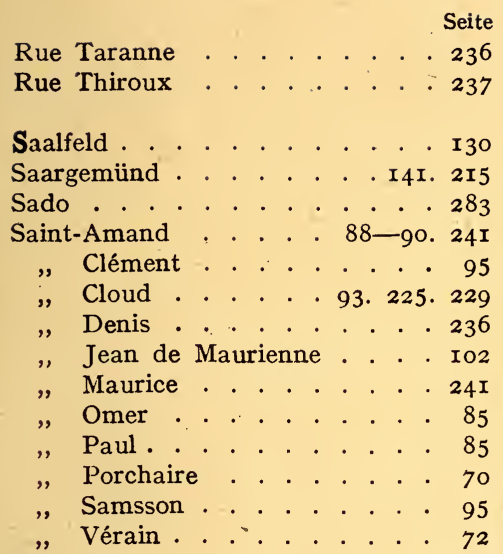

Saku . . . . . . . . $28 \mathrm{I}$

Sakurai . . . . . . . . 28I

Salopian. . . . . . . 249. 339

Salzburg . . . . . . . . I44

Samadet . . . . . . . . . 87

San Christoforo . . . . . . . 66

, Quirico......... 50

Sanrakuyen . . . . . . . 29I

Sanuki . . . . . . . . . 285

Sasashima . . . . . . . . 287

Satsuma . . . . . . . . . 292

Savona . . . . . . 58-63

Sceaux . . . . . . . . 100. 235

Schaala . . . . . . . . . 212

Schedewitz. . . . . . . . 214

Scheibe . . . . . . . . . 215

Shidoro . . . . . . . . 284

Schiedam . . . . . . . . 163

Schlaggenwald ...... . . . 2 I9

Schleswig . . . . . . . . . . I34

Schlierbach . . . . . . . . I4I

Schney .. . . . . . 208. 213

Schönwald . . . . . . . . 2I 3

Schrattenhofen . . . . . . . . I22

Schretzheim . . . . . . . . II7. 323

Schwarzenbach . . . . . . 213

Schwaz . . . . . . . . . . 145

Schweidnitz . . . . . . . . I4I

Schwerin . . . . . . . . I 133

Seikanji . . . . . . . . 287

Septfontaines . . . . . . I66. 22 I

Seto . . . . . . . . 286

Settsu. . . . . . . . $28 \mathrm{I}$

Sevilla . . . . . . . . 68

Sèvres . . . 227-234. 315. 339

Shelton . . . . . . I78. 250

Shibata . . . . . . . . 292

Shido . . . . . . . . . 285

Shiga . . . . . . . . 28I

Shigaraki . . . . . . . 28I

Shizuhata . . . . . . . 284

Shodai . . . . . . . 282

Shohaku . . . . . . . 280

Shoto . . . . . . . . 280

Shu-fu . . . . . . . . . 343

Siegburg . . . . . . . . $\mathbf{I}_{42}$

Siena . . . . . . . 49. 50

Sinceny . . . . . . . . 92. 93

Sitzendorf . . . . . . . . 2 I4

Sobokai . . . . . . . . . 286

Söholm . . . . . . . . . . 187

Solothurn . . . . . . . . . . . I69

Sophienau . . . . . . . . . 213

Southwick . . . . . . . . . . 182

Staffordshire... . I70. I7I. I73

Stanowitz . . . . . . . . . . 214

Stockelsdorf . . . . . . . . 136

Stockholm . . . . . . . . . I 189

Stockton on Tees . . . . . . 183

Stoke upon Trent . . . . I79. 25I

St. Petersburg . . . . . . . . 260

Stralsund . . . . . . . . . . 137

Straßburg . . . I03. I04. 209._210

Subl . . . . . . . . . $2 \mathrm{I} 2$

Suma . . . . . . . . . . 285

Sumidagawa . . . . . . . . . $29 \mathrm{I}$

Sunderland . . . . . . . 182

Suo . . . . . . . . . . . 283

Swansea . . . . . . I 84.250 .339

Swinton . . . . . . I81. I82

Tachino . . . . . . . 292

Tada . . . . . . . . . 283

Takatori. . . . . . . . 285

Talavera. . . . . . . . . 67

Tamagaki . . . . . . . . 283 


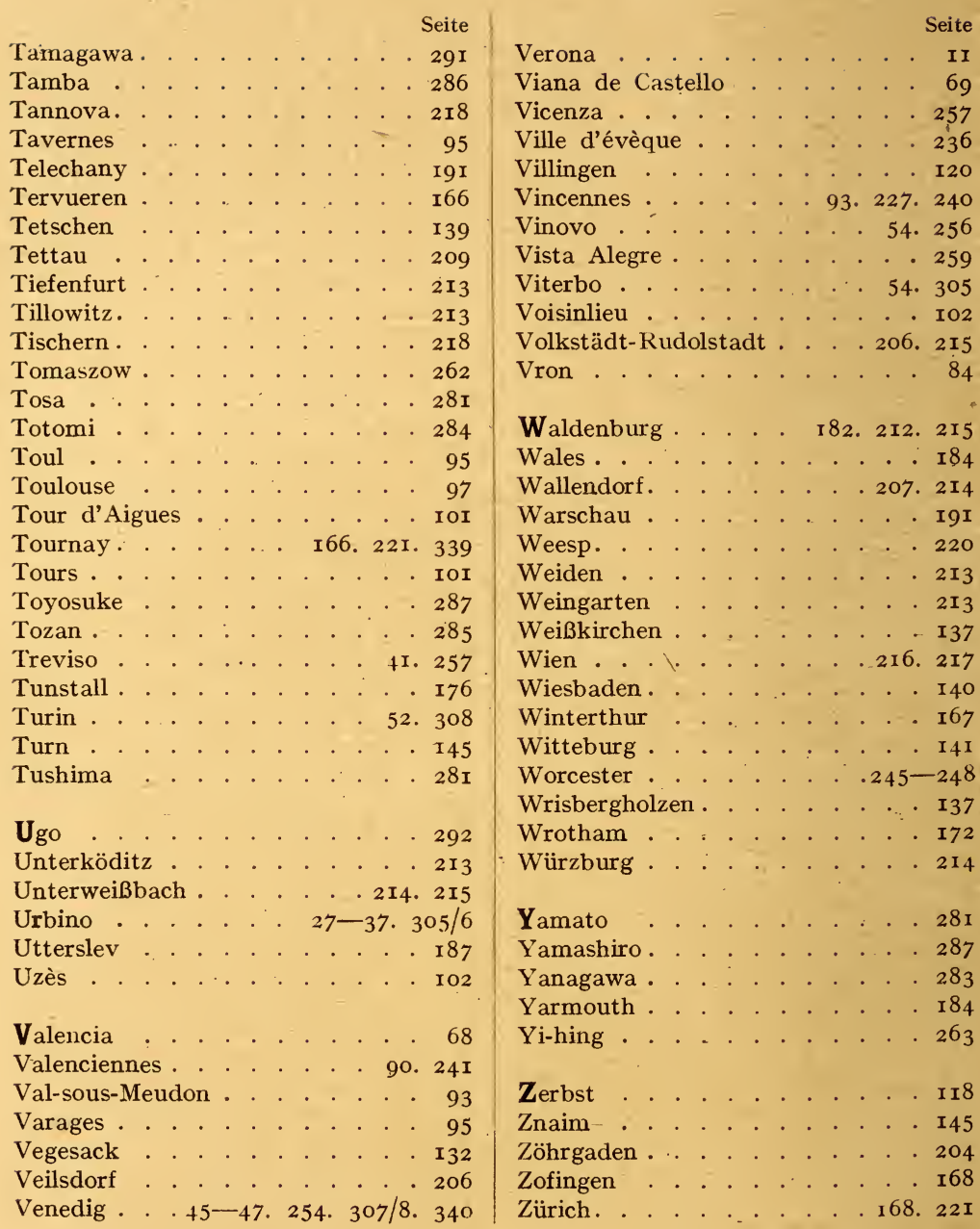




\section{Verzeichnis der Künstler, Maler, Dekorateure usw.}

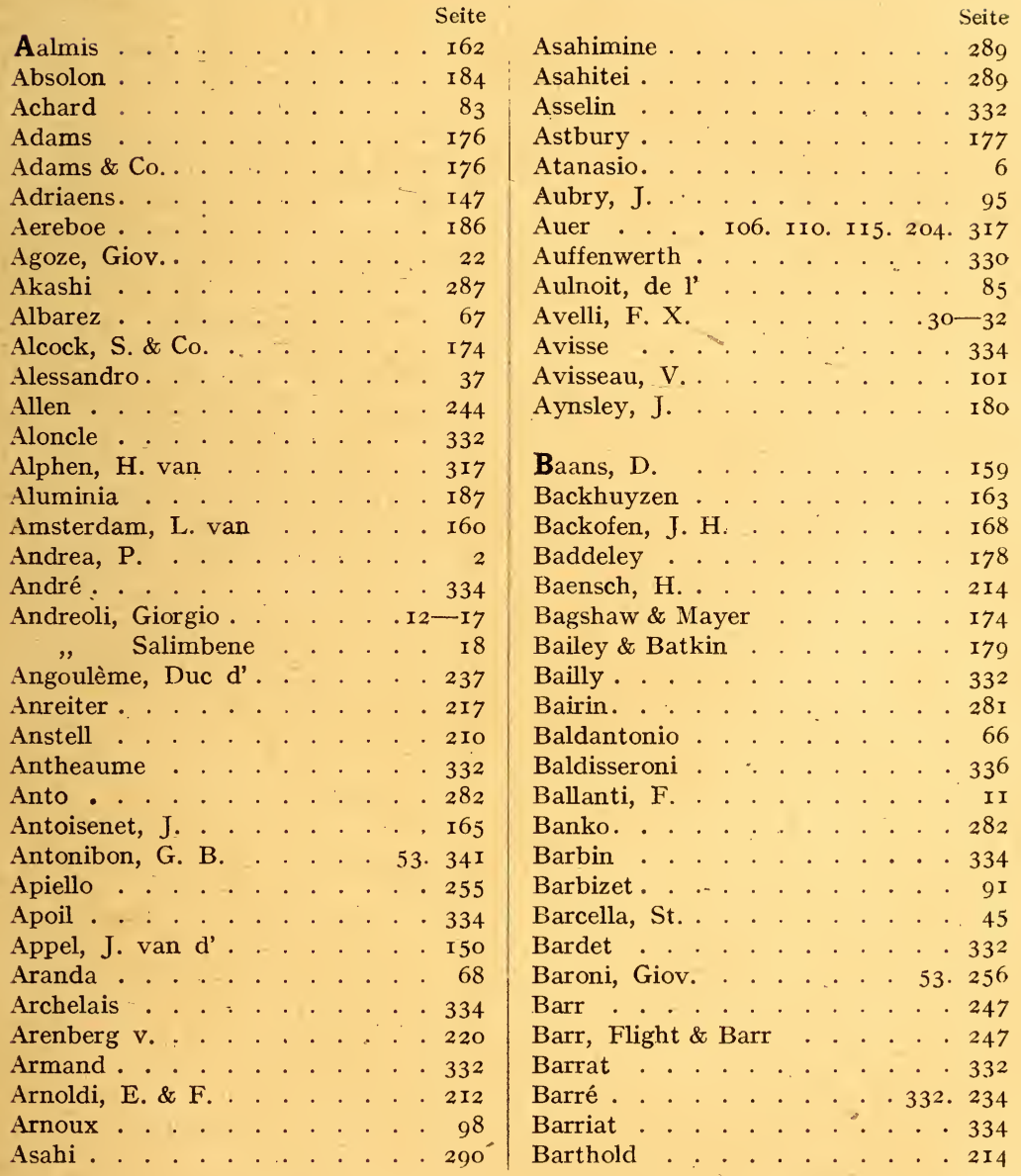




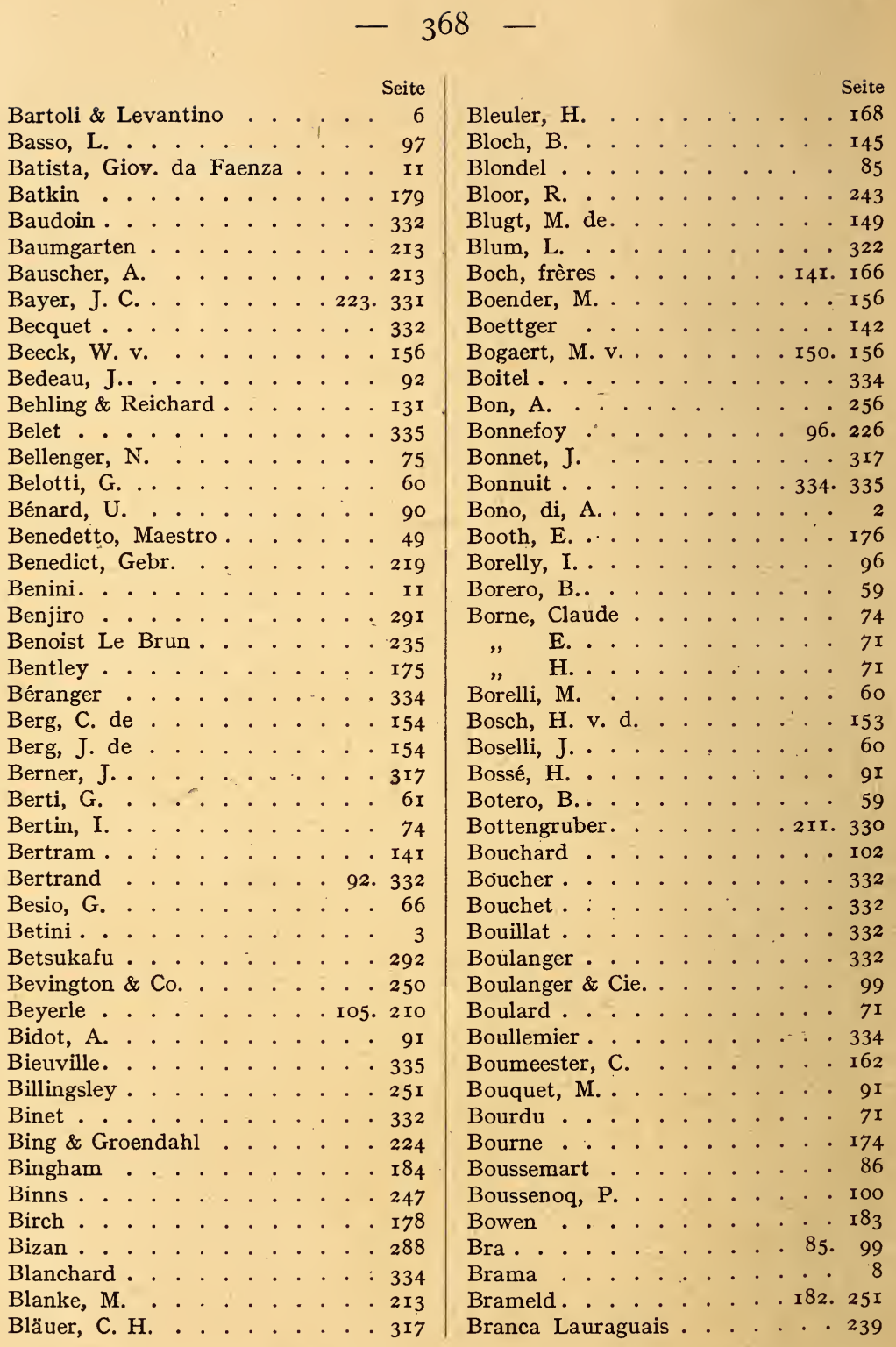



55

" A.......... . . 56

Brecy . . . . . . . . 335

Bremer \& Liebmann . . . . . 213

Briel, P. van d. . . . . . . I 155

Briffaut . . . . . .... . 335

Briot, F. . . . . . . 70

Briou, E. . . . . . . . . . . 72

Briqueville, J. . . . . . . . . 98

Brislington, R. F. . . . . . . I83

Broeckerhoff, S. v. . . . . I55. I60

Brouwer, A. . . . . . . . . 159 $" \quad$ G. . . . . . . . . I57

$" \quad$ H. . . . . . . I52. I53

" J. . . . . . . . . . I54

Bruder \& Schwalb . . . . . . 2 I9

Brunel . . . . . . . . . . 336

Buchwald . . . . . . I35. I36

Buehl \& Sohn . . . . . . . . 2 I 2

Bulidon . . . . . . . . . . 332

Bulot. . . . . . . 335. 336

Bunel . . . . . . . . . . 332

Burch, P. v. d. . . . . . . . 155

Burdig . . . . . . . . . . 97

Burgh, J. C. v. d. . . . . . 158

Buteux . . . . . . 332. 334

Butz, A. . . . . . . . 3I7

Byckloh, F. M.. . . . . . . 158

Cabau ... . . . . . . 334

Cadwell . . . . . . . . . . . . I74

Callegari \& Casati . . . . . . 25

Caluwe, J. d. . . . . . . I64

Cambiasi, L. . . . . . . . . 35

Campani, F. M.... . . . 50

Cantu . . . . . . . . 65

Capelle . . . . . . . . 332

Capronnier . . . . . . . . . . 334

Cari, C. . . . . . . . . 33

Carl . . . . . . . . . 257

Carle, J.. . . . . . . . . 317

Carrier . . . . . . . . 332

Carrin. . . . . . . . 332

Cartus. . . . . . . . . . .97

Casati . . . . . . . . 25

Caselli, G. . . . . . . . . . 44

Castel . . . . . . . . 332

Führer.
Seite

Castelli, Rocco di ..... . 57

Caton . . . . . . . . . 332

Catrice . . .. . . . . . . 332

Cauldon Place ... . . . . . 178

Caussy, P. . . . . . . . 75. 99

Caux, F. . . . . . . . 84

Cefale, J. . . . . . . . . . 56

Célos . . . . . . . . . . 334

Cerf, le . . . . . . . . 92

Chabry . . . . . . . . . . 332

Chaffers, R. . . . . . . . I8I

Chamberlains . . . . . . 247

Chang-chia. . . . . . 344. 345

Chang Ming-kao . . . . . 346

Chanou ...... . . . 332

Chaoch'in . . . . . . . 346

Chapelle, P. . . . . . . . . . 73

Chapuis . . . . . . . . . 333

Charpentier . . . . . . . . 334

Charrin ... . . . . . . 334

Chauvaux . . . . . . . . 333

Cheathcote \& Co. . . . . . . . . II4

Chelius . . . . . . . . I3I

Chely . . . . . . . . . . . I2I

Ch'en ku shih . . . . . . . 346

Ch'en-kuo-chih . . . . . . . . . 346

Ch'en Tien sui . . . . . . . . . 346

Ch'en-wei . . . . . . . . . . 343

Ch'en Wen ching ... . . . 343

Chetham \& Woolley . . . . . . 180

Chevalier . . . . . . . . . 333

Chicannaux-Trou . . . . . . 225

Child, S. . . . . . . . I76

Chin-shih . . . . . . . . 343

Chiodo ....... . . 63

Choisy de........ . . 333

Choju . . . . . . . . . 282

Chollet . . . . . . . . . . . 98

Chozo . . . . . . . . 286

Christian, Ph. . . . . . . I $8 \mathrm{I}$

Chulot . . . . . . . . 333

Church . . . . . . . . I77

Clair, A.. . . . . . 202. 328

Clar . . . . . . . . . I 37

Clarner . . . . . . . . $32 \mathrm{I}$

Claude Place . . . . . . I78 
Seite

Cleer . . . . . . . . 202

Cleffius, L. . . . . . . ${ }^{4} 6$

Clericy, F. . . . . . . . . . 64

Clerissy, A. . . . . . . . . . 96

Clews, J. \& R. . . . . . . . . I77

Clio, H. . . . . . . 223. 33 I

Clyom . . . . . . . . . . 72

Coccorese, C.. . . . . . . 56

Collesano . . . . . . . . . . 56

Combe, L. . . . . . . . . . 70

Commelin . . . . . . . . . 333

Cono Lazerone. . . . . . . . 56

Conrade . . . . . . . . 7I

Constant . . . . . . . . . . . 334

Conta \& Boehme . . . . . . . . 212

Cookworthy, W. . . . . . . 248

Cool . . . . . . . . . 336

Copeland . . . . . . . . . $25 \mathrm{I}$

Copeland \& Garett . . 179. 25I. 340

Cornaille. . . . . . . . . . 333

Cornu, L. . . . . . . . . . 75

Cosyn, A. . . . . . . . . I 53

Courcy . . . . . . . 335. 336

Couturier . . . . . . . . 333

Cozzi . . . . . . . . . 254

Cretté . . . . . . . . 220

Crevani, F. . . . . . . . 54

Croce ......... . 59

Crosa . . . . . . . . . 26

Custine . . . . . . I05. 210

Custode, I. . . . . . . . 7I

Cuyst, G. . . . . . . . . $\mathrm{I} 48$

Cyfflé. . . . . . . 94. 240

Cyples. . . . . . . . . . . 180

Dagoty . . . . . . . . 238

Dale, C. . . . . . . 249. 250

" C. B. . . . . . . 250

" J. . . . . . . . . . . . I74

" L. van. . . . . . . . I55

"-W. van ....... . 150

Dammann, M. . . . . . 85

Dammouse. . . . . . . . 334

Daniel, R. . . . . . . . . I76

Darté . . . . . . . . 239

Daussy, A. . . . . . . . 92

Davenport . . . . . I76. 25 I

David . . . . . . . . . 334

Davignon . . . . . . . . . . 334

Deck, Th. . . . . . . . . $9 \mathrm{r}$

Decker, J. . . . . . . . . . . r 159

Degault . . . . . . . . . . 336

Degenring, F. . . . . . . 2 I2

Delafosse . . . . . . . . 334

Delartier . . . . . . . . 102

Del Vecchio . . . . . . 55

Demont . . . . . . . 236

D'Entoine . . . . . . . . . 95

Denuelle . . . . . . . . . 239

Derichsweiler. . . . . . . . 334

Desperais . . . . . . . . . 334

Desprez . . . . . . . . . . 239

Deutsch . . . . . . . . . 334

Develly . . . . . . . . 334

Devers, I. . . . . . . . 9 9

Dextra, I. T. . . . . . . . I47

Dextra Z. ......... I52

Didier. . . . . . . . . . . 334

Diel, G. . . . . . . . . . II9

Dien ... . . . . . . . 333

Dieul .. . . . . . . . . . 74

Dihl \& Guerard . . . . . . . . 237

Dillwyn \& Co. . . . . . . . I84. 250

Diomede, Durantino . . . . $5 \mathrm{I}$

Disdier, M. S. . . . . . . 68

Dixon \& Co. . . . . . . . . . 182

Dixon, Philipps \& Co.. . . . . 180

Doat . . . . . . . . . 335

Dodin . . . . . . . . . . . 333

Dönch, J. . . . . . . . . . 317

Does, D. v. d. . . . . . . . . $\mathrm{r} 53$

Does, W. v. d. . : . . . . . . . I5I

Dohachi . . . . . . . . 288

Doiz, F. M. . . . . . . 33. 36

Dolder, A. . . . . . . . . 168

Domenego da Venetia . . . . 66

Donaldson, J. . . . . . . . 339

Donath, P. . . . . . . . . . 213

Donovan . . . . . . 185.252

Don Pottery. . . . . . . . r $8 \mathrm{r}$

Doorne, P. von . . . . . . . I50

Dorez, B. . . . . . . . . 87

, F. L. . . . . . . . . . 90

" N. A.. . . . . . . 89 


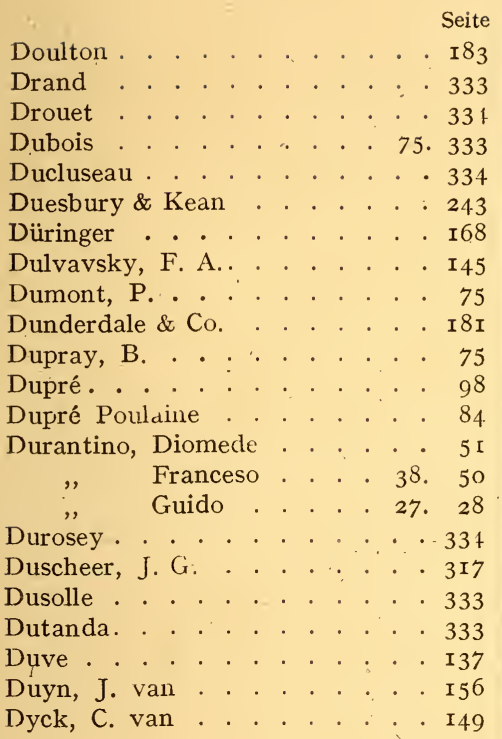

Eastwood . . . . . I78

Ebenstein . . . . . . . 220

Eckert \& Co. . . . . . . . . . 215

Eenhorn, van, L.. . . . . I46. I64 "S. . . . . . $\mathrm{I} 46$

Ehrhart . . . . . . . . I I67

Eissermann, H. . . . . . . 3I7

Elb . . . . . . . . . . 95

Elers . . . . . . . : . . I73

Elkin Knight \& Bridgwood . . . I80

Elling . . . . . . . . I55

Emens, J. . . . . . . . 322

" P. . . . . . . 322

Entoine . . . . . . . . 95

Erck, O. . . . . . . . 2 I3

Escallier . . . . . . . . . 335

Evans. . . . . . . 333

Faenza, Giov. B. da . . . . I I

Fahlstrom, A. . . . . . . : 189

Falot . . . . . . . . 333

Faraguet . . . . . . 335

Farini, A. . . . . . . . II
Fasolt \& Eichel ...... Seite

Fau. . . . . . . . . I08

Faubourg, St. Denis . . . . 236

Fauchier. . . . . . . 96

Faucon, F. . . . . . . . . 97

Fauquez, P. J. . . . . . 88

Fazolt \& Eichel . . . . . . . . $2 I_{4}$

Februrier . . . . . . . . 86

Fell \& Co. . . . . . . . . . I82

Feltz . . . . . . . . 316

Féritte . . . . . . . 97

Féraud . . . . . . . 84

Ferdinand, König . . . . . . 341

Ferner . . . . . . . 2 II

Ferniani . . . . . . . . II

Ferrat . . . . . . . . 94

Ferstler . . . . . . . . 217

Fiamingo . . . . . . . . . . . 33

Fick .. . . . . . . . . 192

Fichthorn, J. A. . . . . . 139. 322

Fi quenet . . . . . . . . . 335

Fischer, C. C. . . . . . . . 3I7

Fischer \& Mieg . . . . . . . . . 2 I9

Fletscher \& Co.. . . . . . . . . I778

Fliegel, I. G. . . . . . . II9. I 28

Flight . . . . . . . . . . 247

Flight \& Barr . . . . . . . . . 247

Foetinger . . . . . . . . I44

Folco . . . . . . . . . 62

Fontaine . . . . . . 333. 335

Fontana, Flaminio . . . . . 32

" Guido . . . . . 27. 28

Orazio . . . $=8.29 .306$

Fontelliau . . . . . . . . . 333

Fonteyn, C. J. . . . . . I6I

Fornario. . . . . . . . 256

Fossé . . . . . . . . . 74

Fouquay, N. . . . . . . 8 I

Fouqué . . . . . . . . 884

Fouquez, Arnoux \& Cie.. . . 98

Fouré . . . . . . . . 333

Fournier . . . . . . . . . 335

Fournier, P. . . . . 83. 222

Fränkel \& Schreck . . . . : . . Iо6

Fragonard . . . . . . . . . . 335

Francesco, Durantino . . 38 . 50

Urbini . . . . 43

$$
24 *
$$




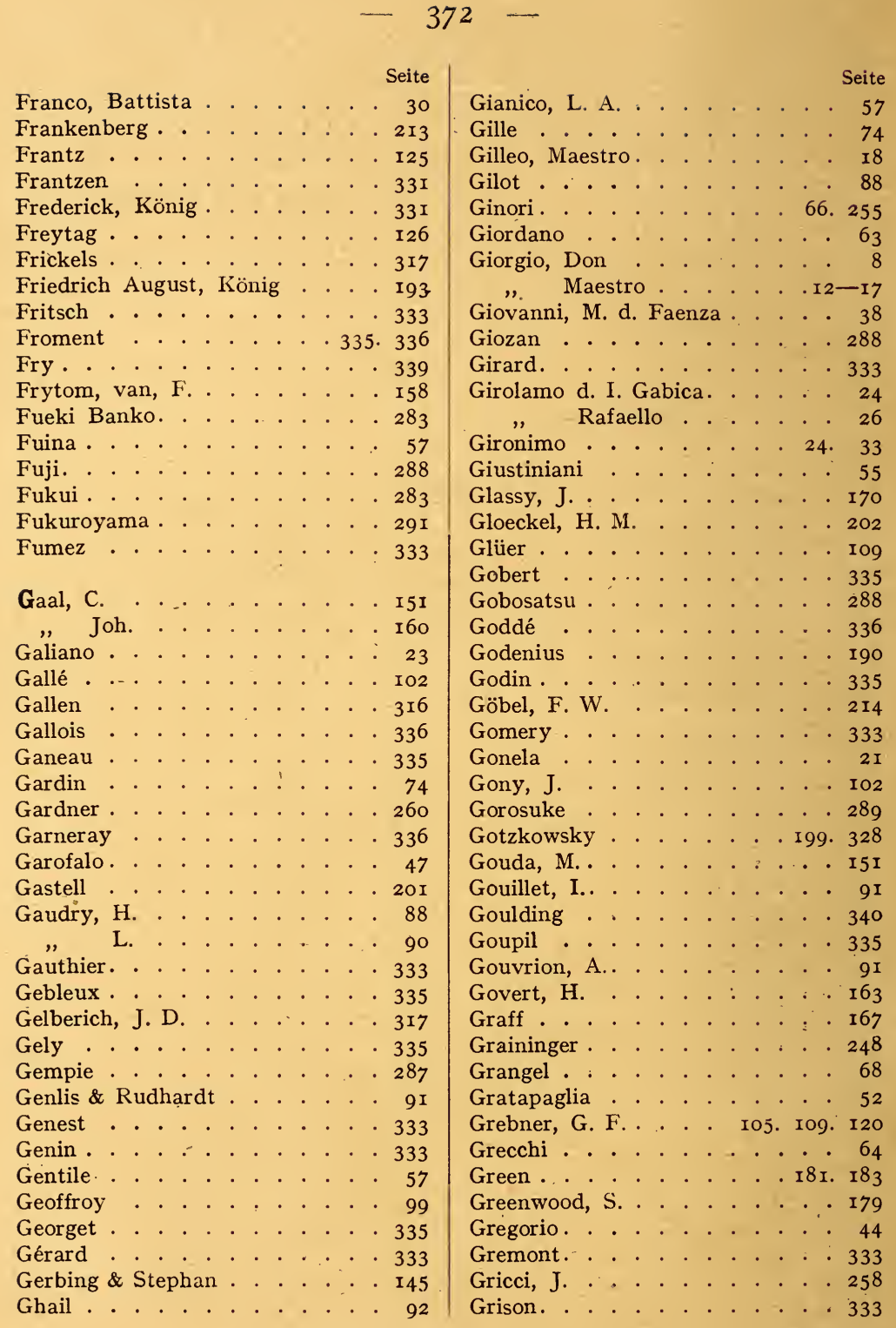




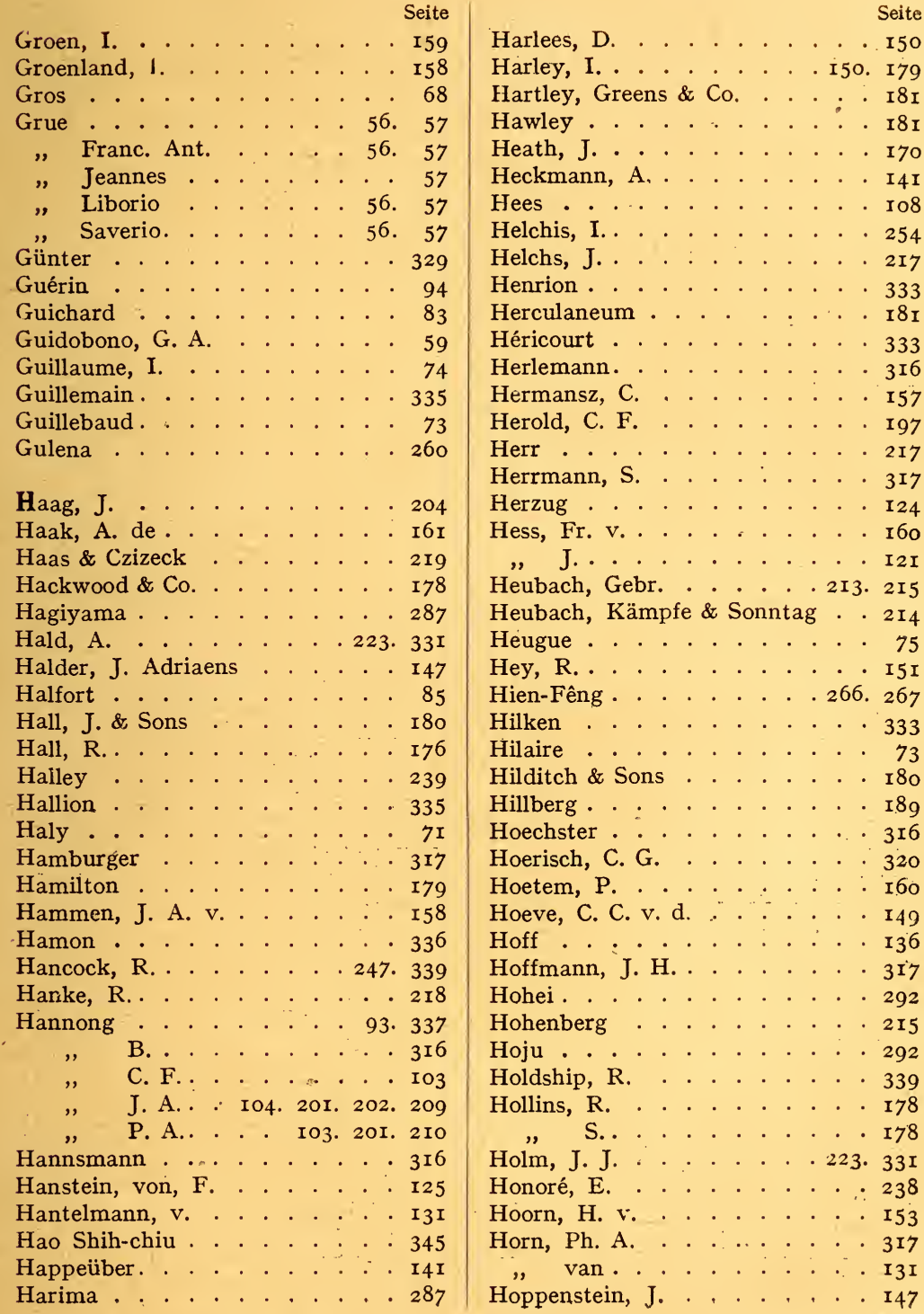




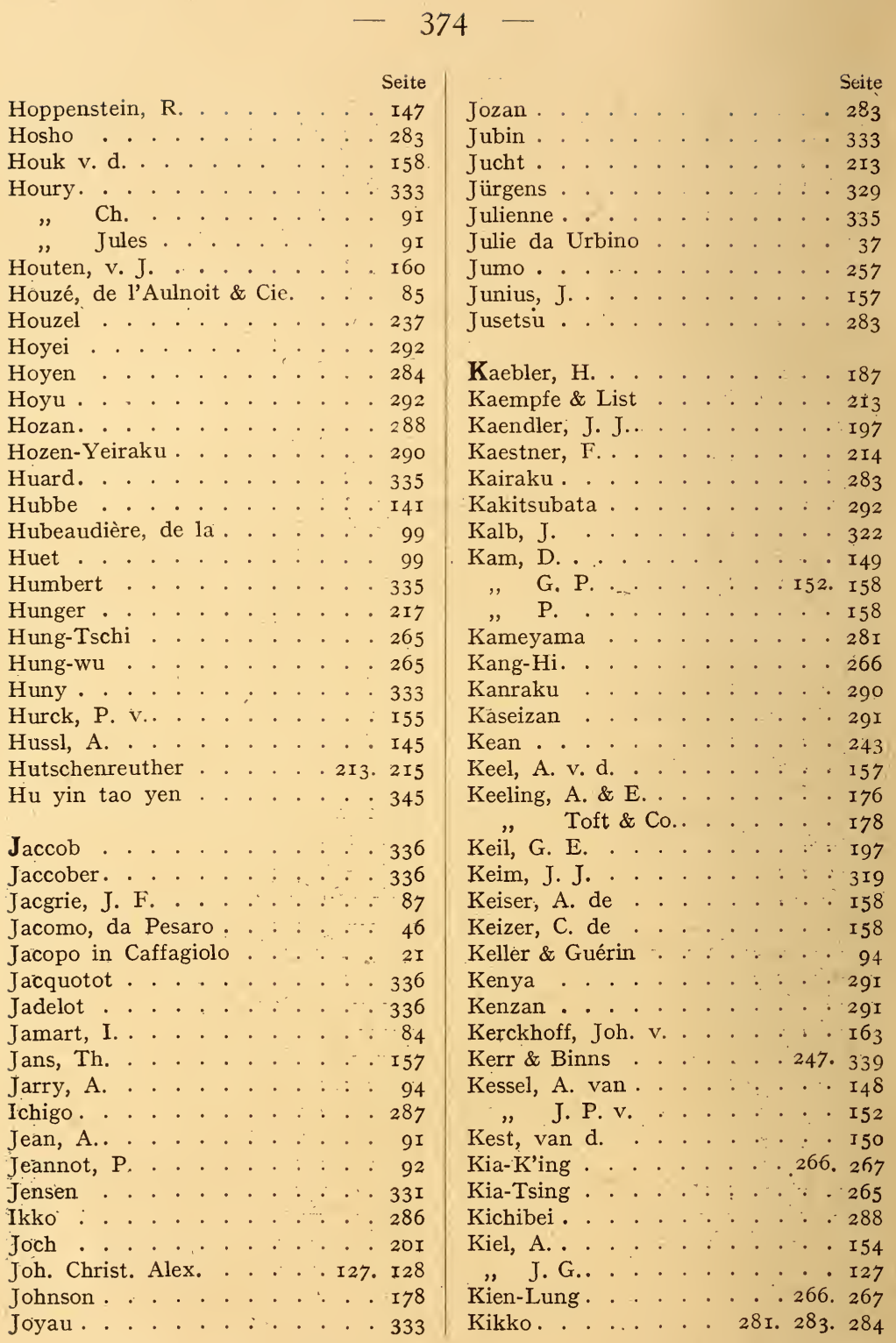




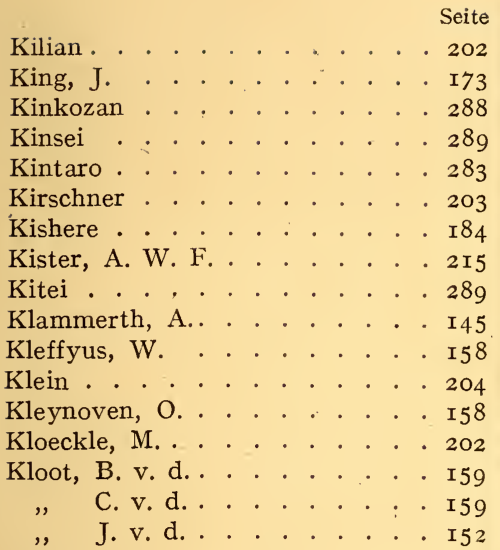

Klos, A. . . . . . . . I63

Klug, H. C. . . . . . . . . I68

Knöller . . . . . . . . . . I05

Knoll, C. . . . . . . . . 218

Knotter, J. . . . . . . . . . . I49

Koch \& Fischer . . . . . . . . 2 I4

Köhler, K. . . . . . . . . 3I7

Komaru . . . . . . . . . 29I

Koning, G. \& H. de . . . . I I 48

Kooge, A. de . . . . . . . . . I62

Kool, I. . . . . . . . . I47. I52

, W. . . . . . . . . . I I52

Koos, M. . . . . . . . I4I

Kordenbusch, G. . . . . . . I08

Koretz, I. . . . . . . . . . . I45

Korks, A. . . . . . . . . I47

Kosel, Gräfin . . . . . . 328

Koto . . . . . . . . . 28I

Krause, M. . . . . . . I4I

Kraut, H. . . . . . . . I2O

Krister, C. . . . . . . 2 2 I5

Krog, A. . . . . . . . . 33I

Kruísweg, A. . . . . . . . I I47

Kruyk, G. L. . . . . . . I46

" I. . . . . . . . . . I 58

Kuang Lian-chi . . . . . 346

Kuang-Sü . . . . . . 266. 267

Kugelmann, J. . . . . . . . 316

Kühnel, C. F. . . . . . . . . I97

Kuhn, C. . . . . . . . I66

Kuick, M. v. . . . . . . I60, I6I

Kulick, I. . . . . . . . . . . . I 58

Kung-liang . . . . . . . . 346

Kuo Hsiu-she . . . . . . . . . 343

Kuro . . . . . . . . . . . 287

Kyuta. . .. . . . . . 288

Laen, J. v. d. . . . . . . . I5I

Laeuger . . . . . . . . . . . . I43

Lahens \& Rateau . . . . . . . . 97

Lai . . . . . . . . . . 346

Lai Kuan . . . . . . . . . 346

Lakin \& Poole . . . . . . . . . I78

Lambert . . . . . . . . . 335

Lamotte. . . . . . . . . 92

Lamprecht . . . . . . . 2I7

Landais, M. . . . . . . . IOI

Lanfrey . . . . . . . 105. 2 I0

Lang, A. . . . . . . 2I 2

Lange, J. d. . . . . . . . I54

Langendorf, Chr. . . . . . 318

Langlace . . . . . . . 335

Langlois . . . . . . . . . . 336

Lapierre \& Quinquiry . . . . . . 97

Large, J. . . . . . . . . . . . 190

La Roche .. . . . . . . 333

Latasche ....... . . . 335

Lauche . . . . . . . . 2 II

Laude. . . . . . . . . 2II

Laurent . . . . . . . 336

Laurjorais . . . . . . . I02

Lazerone Cono . . . . . . . . 56

Léandre . . . . . . . . 333

Le Bel . . . . . . 333. 335

" \& Thibaut . . . . . I02

Lebon-halley. . . . . . . . 239

Lebrun . . . . . . . . 226

" B. . . . . . . 235

Lecerf, I. . . . . . . . . 92

Lecot . . . . . . . . . . 333

Ledoux . . . . . . . . 333

Ledru . . . . . . . . . 226

Lefèvre . . . . . . . . 238

Legay . . . . . . . . . 335

Legrand . . . . . . 202. 335

Le Guay . . . . . . 333. 335

Lehmann, P. H. P. . . 223. 33I 


\section{$-\quad 376$}

Seite

Leigh \& Co. . . . . . . . . . . 85

Leihamer . . . . . . . . . 136

Lemourne, S. . . . . . . . 316

Léonhard, v. Amsterdam . . . . 134

Leopold . . . . . . . . . . . . 94

Leroy . . . . . . . . . . . 335

Lessore . . . . . . . . . 336

E. . . . . . 91. I75

Lestrade, D. . . . . . . . . . 97

Letellier . . . . . . . . . . . . 76

Levantino . . . . . . . 62. 63

Levavasseur . . . . . . 73. 76

Levé . . . . . . . . . . . . 333

Leveillé . . . . . . . . . 237

Levoulant . . . . . . . . . . . 97

Levy \& Weidmann . . . . . . : I4I

Leyhn, J. E. P. . . . . . . 205

Liang-chi . . . . . . . 346

Liaute, L. . . . . . . . . . IOI

$\mathrm{Li}$-chih . . . . . . . . 346

Lichtenstern . . . . . . . . . . 145

Ligné . . . . . . . . . . 335

Lindemann . . . . . . . . . . 204

Ling Ch'a-fa. . . . . . . . 346

Lippert \& Haas . . . . . . . . 219

Lister \& Longton . . . . . . . . 339

Locker \& Co.. . . . . . . . ... 243

Lockett, J. . . . . . . . . I74

Locré, M. . . . . . . . . 337

Lodovico, Maestro . . . . . 45

Loehnig . . . . . . : . . . 197

I.oewenfinck . . . . : : . . 3 I6

Loewer, C. . . . . . . . . . . 124

„G. . . . . . 124. 319

Long-King . . . . . . . . . 265

Lonitz, H. . . . . . . . . . I4I

Lucas . . . . . . . . . .335

Luffneu, A. . . . . . . . . 162

Luplau, A. . . . . . 223. 33I

Lyngbe, L. . . . . . . . . 33I

Magnus . . . . . . . . . 202

Maiko . . . . . . . . . 284

Majorelle . . . . . . . . . 102

Makudsu . . . . . . . . ...290

Makusa . . . . . . . . . 286

Malériat . . . . . . 92

Maling ........... 182

Mallet. . . . . . . . . . 74

Manara, B. . . . . . . 6

Manardi . . . . . . . . . 5I

Mand, M. . . . . . . . . . 204

Mandele, H. v. d. . . . . . . 153

Maqueret . . . . . . . . 333

Marcenaro . . . . . . . . 59

Marck, van . . . . . . . 336

Marcolini . . . . . . . 327

Marconi, G. . . . . 53. 256. 340

Maresch . . . . . . . . I 45

Marforio, S. . . . . . . . . 38

Maria, Giov. . . . . . . 38

Marini, D. . . . . . . . . 45

Marino, Giammario . . . . . 36

Marinoni, S. . . . . . . . 5 I

Marseille, A. . . . . . . . 212

Marsollet . . . . . . . . . . 74

Martin, Gebr. . . . . . . 219

Martinet . . . . . . . . 335

Marum, P. van. . . . . . . 152

Marx . . . . . . . . 202

" L. F. . . . . . . . . 108

Masa . . . . . . . ... 287

Masaki . . . . . . . . . 287

Masakichi .. . . . .... . 282

Mason, M. . . . . . . . . . 180

Masquelier . . . . . . . . . . 87

Massarello . . . . . . . . . ... 69

Masselli, T. . . . . ...... 42

Massy . . . . . . . . . . 333

Maucksch . . . . . . ... I97

Maugard. . . . . . . . .. 75

Maugendre . . . . . . . . 335

Maussion . . . . . . . . 335

Mauzin . . . . . . . . . IO2

Mayer . . . . . . . . . . . I74

„E. . . . . . . . I77

" Fr. .........2II

" \& Newbold . . . . . . . I79

Medskaall . . . . . .. . 186

Meehl, H. . . . . . . . . . . 33I

Meigh . . . . . . . . . . . I78

Meir, J. . . . . . . . . . . I73

, R.. . . . . . .... I7I

Mennicken, B. . . . . . 322 


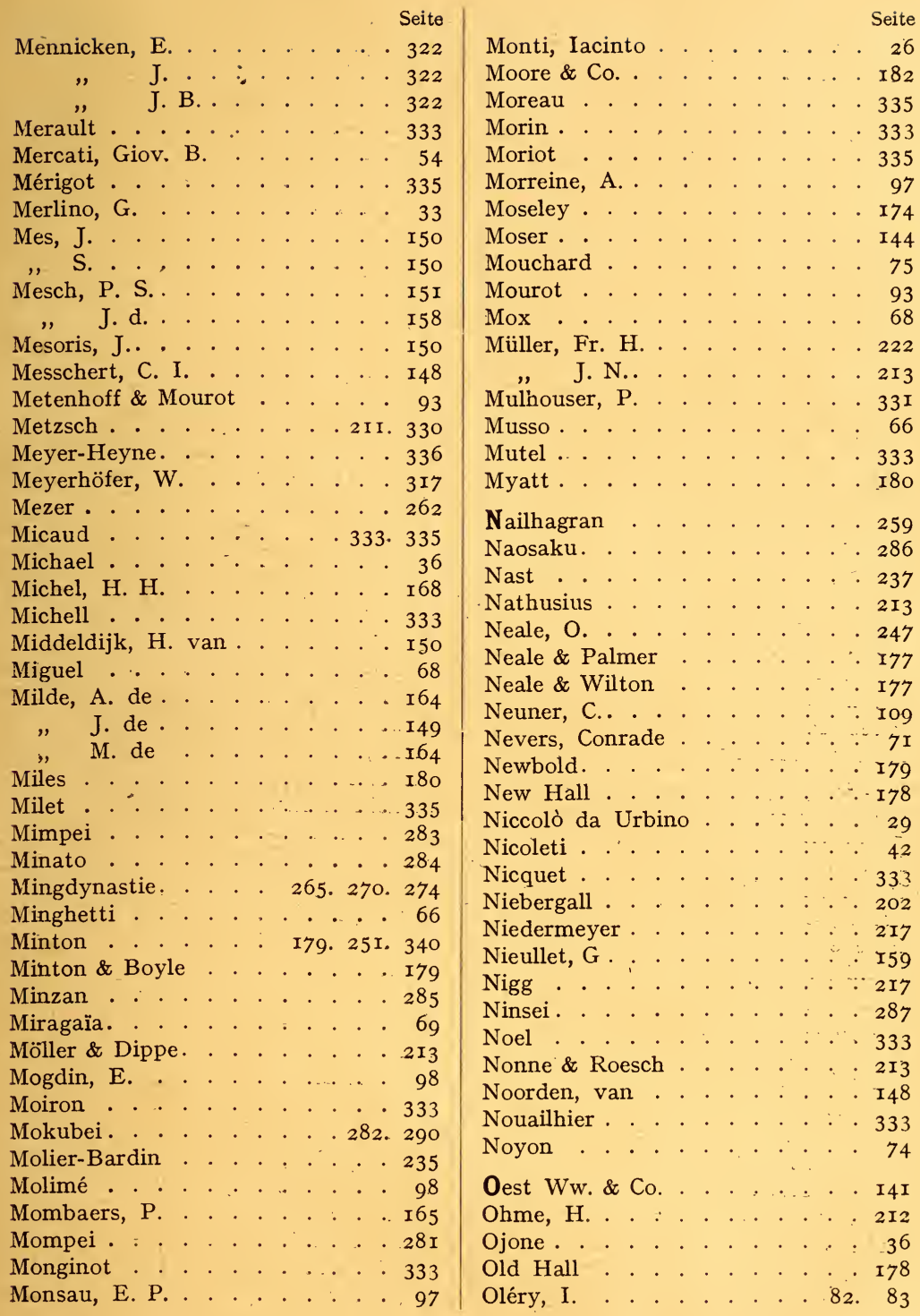




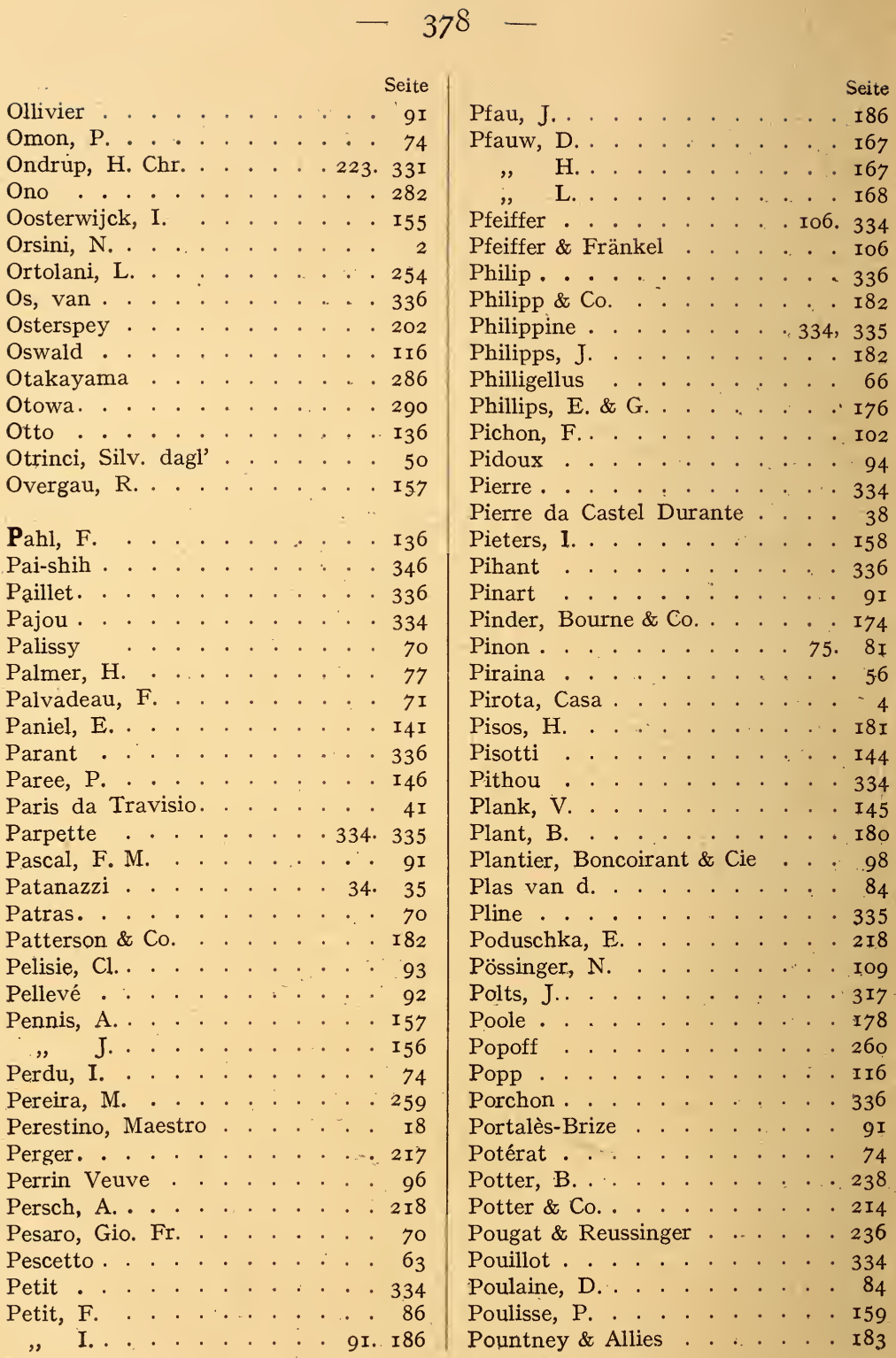




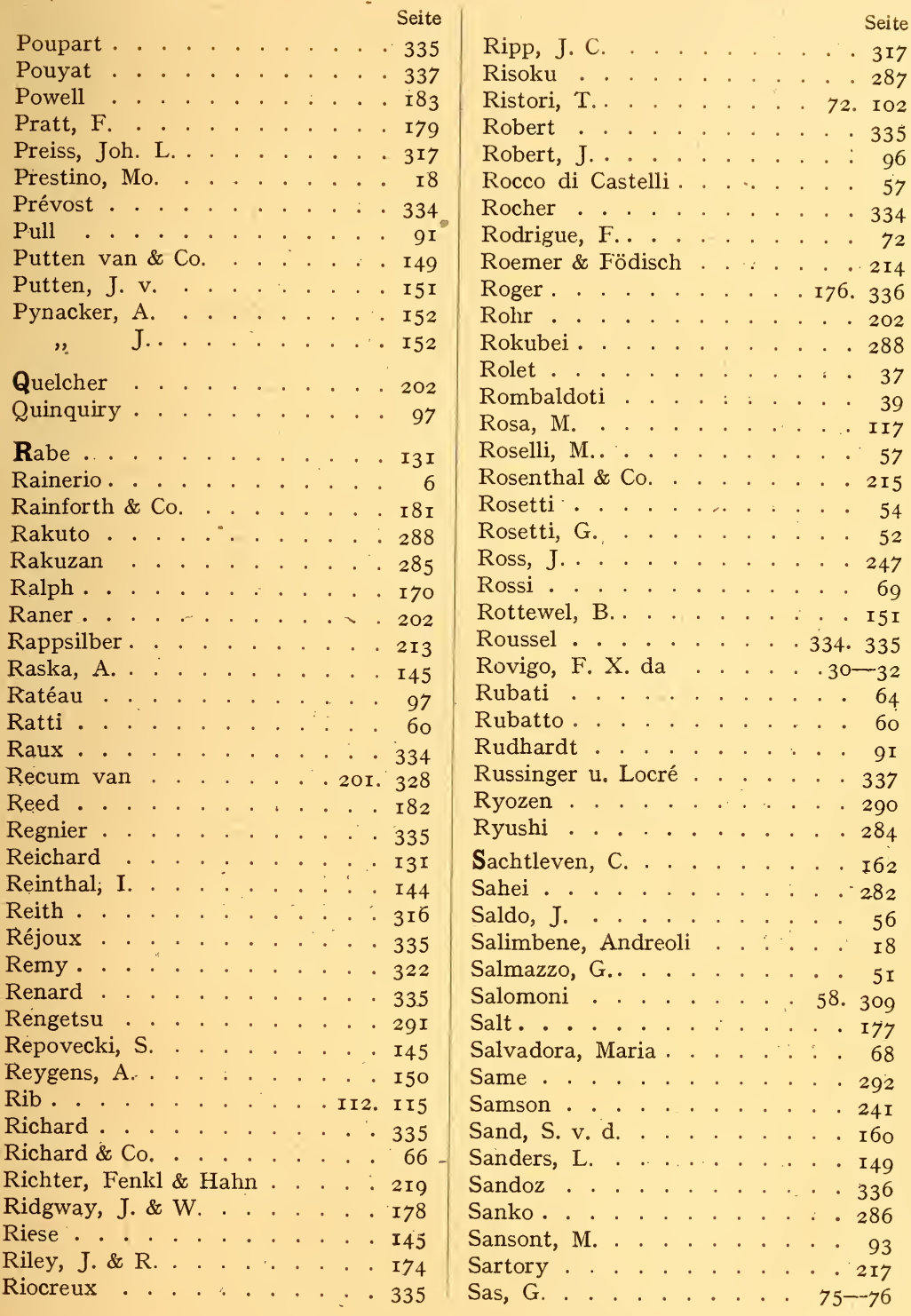


Savino, G. P. . . . . . . . . 5 I

Savy . . . . . . . . . . . 96

Schachtel, J. . . . . . . 2 213

Schagen, C. v. . . . . . . . . I59

Schahler. . . . . . . . 202

Schaller . . . . . . . 217

$$
\text { \& Co. ...... . . . } 213
$$

Schertler, F. . . . . . . . . $2 \mathrm{I} 8$

Schie, D. J. v. . . . . . . . . . 159

Schierholz \& Sohn . . . . : . . 214

Schiller \& Sohn. . . . . . . . . 145

Schilles, J.. . . . . . . . 317

Schilt . . . . . . . . . 336

Schipper, C. . . . . . . I57

Schlegelmilch, E. . . . . . 212

Schmaus. . . . . . . . 328

Schmeisser, J. . . . . . . . $2 \mathrm{I}_{4}$

Schmidt . . . . . . . . I 20

" H. . . . . . . . . $2 \mathrm{I} 2$

" J..... . . . . . 33 I

" J. A. ...... . . 316

" -Pecht....... . I43

Schneider . . . . . . . . 2I7

" \& Co. . . . . . : : : 214

Schoch . . . . . . 202

Schölcher . . . . . . . 236

Schönau . . . . . . . . $2 \mathrm{II}_{4}$

Schol, S. v. d. . . . . . . . I I6I

Schoonhove, C. v. . . . . . 149

, L. v. . . . . : 149

Schradre. . . . . . . . 334

Schreck . . . . . . 106

Schreiber . . . . . . . . 3I7

Schütz, J. . . . . . . . . . 3I7

L. R. . . . . . : . 145

Schumann . . . . . . . . $2 \mathrm{I}_{3}$

Schwab, C. P. . . . . . : 109

Scott ........ . I82. 185

Sebastiano, N. ...... 255

Sebbers . . . . . . . . 328

Seger . . . . . . . . . 328

Seidel . . . . . . . . . . I4I

Seifu . . . . . . . . . . 289

Seigne, I. . . . . . . . . . . 7 I

Seijiyemon . . . . . . 29I

Seikozan. . . . . . . . . . 292

Seizan . . . . . . . 289

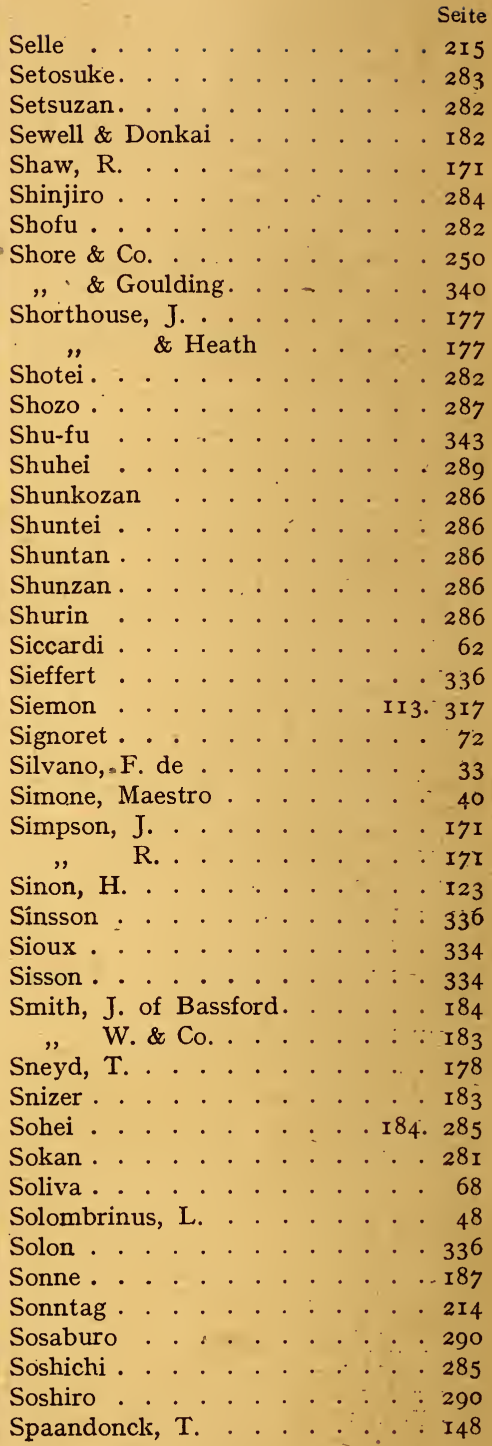


Seite

Spode ... . . . . . I79. 25I. 340

" \& Copeland . . . . 25I. 340

Stebner . . . . . . . . . . 109

Steel, D. . . . . . . . . . . . I74

Steinmann, K. . . . . . . . . 213

Sten . . . . . . . . . . . 33 I

Stevenson . . . . . . . . . I77. 339

Stoße . . . . . . . . . . 136

Strale, H. . . . . . . ... . . . I56

Ströbel . . ..... . . . . . . I09

Stroom, P. van d. . . . . I55. I60

Strnack, jr. . . . . . . . . . . I45

Sturgeon, W. . . . . . . . 82

Süan-Tê . . . . . . . . . . 265

Sungdynastie. . . . . . . . 269

Suitsetsu . . . . . . . . . 287

Sumizome . . . . . . . . . 290

Swaine \& Co. . . . . . . . . 2 I4

Swanenburgh, H. V. . . . . . I 59

Swebach .. . . . . . 336

Sytsch . . . . . . . . . 180

Tabary . . . . . . . . . 334

Taennich . . . . . . . 318

Taillandier . . . . . . . . 334

Taizan . . . . . . . . 288

Takagamine . . . . . . . . 287

Tandart ... . . . . . . . . 334

Tao-kuang . . . . . . . . 266. 267

Tardy . . . . . . . . . . . 334

Tauber, G. M. . . . . . . . 109

H., Jensen . . . . . . . I87

Taylor, G. . . . . . . . . . . I70

, R. M. . . . . . . . . I I79

, R........... . I7I

Tebo . . . . . . . . . . 339

Teichert, C. . . . . . . . . $2 I_{4}$

, E. . . . . . . . . . $2 \mathrm{I} 4$

Teizan . . . . . . . . . 29I

Terchi, Ant. . . . . . . . $5 \mathrm{I}$

" B. . . . . . . 50. 5 I

Theodore . . . . . . . . 334

Thetten . . . . . . . . 316

Thevenet . . . . . . . . 334

Thieme. . . . . . . . . . $2 \mathrm{I}_{4}$

Thooft \& Labouchère . . . . . I6I

Tien-K'i . . . . . . . . . 265
Tilman Wolff ... . . . . . . 322

Timimotea, J. . . . . . 49. 5 I

Toft, R. . . . . . . . I I7O

" T........... . I70

Tokusai . .. . . . . . . . 284

Tokuzan . . . . . . . . 29 I

Tomikawa . . . . . . . . . 285

Torenburg, J. van . . . . . . I53

Toselli. . . . . . . . . . . 9 I

Tozen . . . . . . . . . 286

Traeger . . . . . . . . 336

Treverret . . . . . . . 336

Troyon . . . . . . . . 335

Trud (Truvo?), M. . . . . . 56

Tschêng-Hua . . . . . . . 265

Tṣchêng-Tê . . . . . . . 265

Tsch'ung-Tschêng. : . . . . . . 265

Ts'ingdynastie . . 266. 267. 271. 274

Tsin Kuo . . . . . . . . . 346

Tsuju Harima . . . . . . . . . 287

Tsuyen . . . . . . . . . . 289

T'ung-Tschi . . . . . 266. 267

Turner . . . . . . . . . . 180

Turnor, Ralph . . . . . . . I70

Twyford . . . . . . . . . . I73

Twigg . . . . . . . . . . $\mathrm{I} 82$

Uffrecht \& Co. . . . . . . . . I4I

Unger, H. . . . . . . . . . . . 214

Urbach . . . . . . . . . . 145

Urbini, Francesco . . . . . : . 43

Urbino, Niccolò da . . . . . . 29

Utzschneider \& Co. . . . . . I4I. 2 I 5

Valaressi, Z. . . . . . . . . . II

Valente . . . . . . : . $\quad \cdots 66 \mathrm{I}$

Vallet, M. . . . . . . . . 76

Vandé. . . . . . . . . 334

Van Marck . . . . . . . . 336

Van Os . . . . . . . . . 336

Vaudrevange . . . . . . . . 166

Vavasseur . . . . . . . . 334

" Le . . . . . 73. 76

Vechio, del . . . . . . . . 55

Velasquez . . . . . . . . . 258

Verburg, J. . ... . . . . . . . 159

Verhaast, G. . . . . . . . . I6I

Verhagen, J. . . . . . . . I56 


\begin{tabular}{|c|c|c|c|c|c|c|c|c|}
\hline & & & & & Seite & & & Se \\
\hline e, G. . & & & & 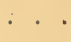 & I 147 & Willner & & 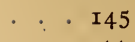 \\
\hline$\cdot$ & & & & & 254 & Wilson . . & . & 73 \\
\hline sz, L. & & & & & $\mathrm{I} 48$ & Winterstein, W. & & $\because$ \\
\hline illard. & & & & & 334 & Witsenburg, C. . & & . \\
\hline \& Cie.. & & & & & 97 & T.. & & . \\
\hline a $\cdot \cdot$ & & & & & $68-$ & \& Mombaer & & 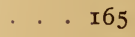 \\
\hline y \& Boch & & & & & . 143 & Wolf \& Hamilton. & - & . \\
\hline t... & & & & & . 334 & T..... & . & 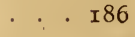 \\
\hline N. . & & & & & $7 \mathrm{I}$ & I'sb urg, C. F. von . & • & . \\
\hline r. . . & & & & & - 82 & olfsen, $\mathrm{H}$. & - & : \\
\hline $\mathrm{n}$ & & & & & 54 & . & & $\therefore$ \\
\hline P. . . & & & & & · 16 & $\therefore$ & . & $\therefore$ \\
\hline v. d. & & & . & & - 163 & Vood, E. . & . & · \\
\hline br. & & & - & & - $2 I_{4}$ & R. ... . . & - & . \\
\hline & & & & & - $2 \mathrm{I}_{2}$ & \& Caldwell & & · \\
\hline land & & & & & . 214 & Vright . • & . & . \\
\hline$\therefore$ & & & & & . 17 & J. . & . & · \\
\hline & & & & & I & Fr., da Rovigo . & . & $\cdot \cdot 30$ \\
\hline & & & & & $3 I$ & 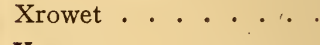 & · & $\cdot \cdot 334$ \\
\hline & & & & & $\begin{array}{l}\cdot I \\
. \\
2\end{array}$ & · · & . & . . \\
\hline d. . & . & & & & $\begin{array}{l}204 \\
159\end{array}$ & ara . . . . . & : & . . 292 \\
\hline . . . & & & & & . 24 & e... & - & . . 28 \\
\hline & & & & & . 3 & oto. . & . & $\therefore$ \\
\hline Co.. & & & & & . $2 \mathrm{I}$ & $\cdot \cdot \therefore$ & . & $\therefore$ \\
\hline & & & & & . I74 & . . $\cdot$ & 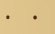 & 282. \\
\hline $\mathrm{Cl}$. & & & & & . $32 \mathrm{I}$ & bi & . & . \\
\hline ag. & & & & & 34 & hi & & . $\quad 285$ \\
\hline & & & & & 3 & . & . & 290 \\
\hline & & & & & 26 & toyo . & • & . . 287 \\
\hline ing . & & & & & - 34 & 1. & • & $\therefore 28 c$ \\
\hline . . . & & & & & . 3 & potio & - & . . 346 \\
\hline & & & & & 2 & nastie & $:$ & $\cdot \cdot$ \\
\hline d . . & & & & . 175 . & 5. I & n-hsing & • & 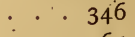 \\
\hline d. . : & & & & . I75. & . 2 & . · · & & \\
\hline$\&$ Bentle & & & & . I75. & . $I$ & hêng & & .266 .267 \\
\hline \& Co. . & & & & $\therefore$ & . $I^{\prime}$ & lel . • • & - & : 3 \\
\hline de. . & & & & & . I & . & & $\cdot$ \\
\hline . & & & & . I99. & . 3 & . . . . & & \\
\hline · . . : & & & & . . & & enigo da Ve & & \\
\hline r, M. J. & & & & & . 3 & - & 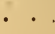 & \\
\hline . . & & & & & & . & & \\
\hline & & & & & & $r$ & & . . \\
\hline & & & & & & . & & \\
\hline & & & & & & W & & \\
\hline & & & & & & W & & \\
\hline & & & & & & J. M. . & & \\
\hline
\end{tabular}




\section{Verzeichnis \\ der auf Marken vorkommenden bildlichen Darstellungen.}

Adler 59, 62, I $4 \mathrm{I}, \mathrm{I} 74$, 199, 200, 205, 2 เ3, $214,228,229,260$, $26 \mathrm{r}$.

Anker 45, 47, 63, roo, I4I, I46, 2I $2,2 \mathrm{I} 3$, $215,224,230,235$, $242,244,25 \mathrm{I}, 254$, $26 \mathrm{r}$.

Arm 7, 63, 212, 218 .

Auge 23I, 26I.

Axt 232.

Baldachin 275 .

Band (siehe auch: Schleife, Bandgeschlinge).

Bandgeschlinge $4,5,7$, 10, $40,54,56,67,88,230$, $273,276,277$ (siehe auch: Schleife).

Baum 37, I39, 230.

Beil I53, I54.

Biene 187 .

Bienenkorb 178 .

Bischofsmütze $2 \mathrm{I}_{4}$.

Blatt (Blätter) 82 , I II, I54, 207, 208, 212, $214,248,276,277$.

Blume (siehe auch: Nelke, Lilie, Lotos) 5, I 53 , I94, 207, 275 .

Bogen 244.

Buch 49.

Burg IOI, I 84 .

Chinese II 5 .

Chinesische Ornamentik I94, I95, 245, 273.
Chinesische Schriftzeichen Glas 230.

I73, I80, 194, r 95, Glocke' I5I, 275.

216, 242, 245, 246, Grabmal 42.

249, 250, 263-274, Greif 25I.

$278-292$.

Hahn I63, 207, 255.

Dolch 6, 23r.

Drachen 195, 196.

Dreieck I8, 58, 69, I75, I9I, 230, 242, 246, 259, $26 \mathrm{I}$.

Dreifuß 243, 277.

Dreizack 5, 22, 23, 26, 83 , 250.

Eichel 231.

Engel 162 .

Erdkugel $25 \mathrm{r}$.

Fahne (Fahnen) 2 I2.

Fackel (Fackeln) 23I, 236.

Fasan 198.

Federn 184 .

Felsen 50, 56.

Fisch (Fische) 63, 65, I4I, $222,225,276$.

Flasche I6I,

Flïgel $8,30,38$.

Fuchs 215, 233.

Füllhorn I3.

Gabel (siehe auch: Dreizack) 127, 206, 215.

Gans 70.

Gebäude (siehe: Haus, Kirche, Leuchtturm, Turm).

Gesetzrad 275.

Gesicht (siehe: Kopf).

Geweih I23, 203, 218.
Halbmond 4, 55, 56, 214, 23I, 238, 244, 245, 247,249 .

Hammer 214, 218, 219.

Hand $19,68$.

Hase 277.

Haus 58, 99, I6 1230.

Hausmarke 234.

Helm 34 .

Helmschmuck I 84 .

Herz 89, I75, I8I, 23 I.

Horn 226.

Hund, Turm und Leièr 252.

Kanne I3.

Keule 94 .

Kind 218.

Kirche 253.

Knospe 194.

Kopf 2, 8, 49, 65, I43.

Kranz 90, 227, 260.

Kreis 4, 22, 67, 69, 87, I75, 232, 246 .

Kreuz 3, 7, 8, 9, I 2, I3, 16, I8, 19, 20, 22, $25,27,35,39,40$, $42, \quad 45,52,54,55$. $56,58,59,6 \mathrm{r}, 63$, $70,74,77,78,80$, $82,85,89,92,93$, I 75, I97, 200, 204, $215,217,222,231$, $246,248,256,258$, 
Krone (Kronen) 36, 40, Pferd 206.

44, 5I, 52, 54, 55, Pinsel 273.

56, 57, 58, 59, 60, Punkt (Punkte) I96, 232.

$62,65,66,69,86$,

99, II 9, I 21, I 4 I, I77,

I79, I80, I8I, I85,

I90, 200, 20I, 203,

205, 2IO, 2I2, $2 I_{4}$,

2I5, 219, 220, 223,

$224,226,227,228$,

$229,237,238,242$,

$243,247,25 \mathrm{I}, 253$,

$255,256,258,259$,

260.

Kugel (Kugeln) 4, 253, 277.

Kuppel 253.

Lanzenspitze 220:

Leier $185,252$.

Leuchtturm 62 .

Licht 215 .

Lilie $70,73,74,78,86$, $96,99,183,225,227$, $232,255,257$.

Löwe I25, I8I, 20I, 205, 213,214 .

Lotos 275 .

Mensch (siehe auch: Chinese) 35.

Merkurstab I95, 23I, 246.

Molch I 49.

Muschel 49, 275, 288.

Nelke 47.

Note 232, 236.

Notenschliussel 230.

Oval 28, 35, 5I, 64, 93.

Papierdrachen 195.

Pentagramm 6o, 62, 65, 68, I 4I, I87, 204, 255.

Pfeifen 236.

Pfeil (Pfeile) 20, I76, I 84, 203, 2I4, 2I8, 232, $236,244,246,249$.
Quadrat (Quadrate) I75, I95.

Rad 4, 87, 92, I21, 129, I33, 200, 217, 246 .

Räuchergefäß 243, 277.

Ranke 86.

Raute 232.

Reh 213.

Reichsapfel $58,62,63,81$, 200.

Ring 232.

Rosette 2, 25, 85, 87, I94, $206,247,273$.

Schiff 177.

Schild (siehe auch: Wappen) $9,27,45,64,90$.

Schirm 275.

Schlange 94 .

Schleife 72 (siehe: Bandgeschlinge).

Schlïssel I22, I4I.

Schwert (Schwerter) I95 bis $197,204,207,208$, $2 \mathrm{I} 2,2 \mathrm{I} 3,2 \mathrm{I} 4,220$, 22I, 24I, 242, 243, $244,246,249,260$,

Sonne 58, 6I, 2I3, 225, $227,23 I$.

Spirale 4, I9, 26.

Stäbe, gekreuzte 242, 243.

Stange 166.

Stein 8, 28, 247. Pentagramm) 2, 3, 10, $35,50,53,55,59$, $62,87, \mathrm{I} 32, \mathrm{I} 54, \mathrm{I} 8 \mathrm{O}$, 196, 200, 207, 208, 225, 230, 23I, 24I, $254,255,256,260$, $26 \mathrm{I}, 262$.

Storch 220.
Stern (Sterne, siehe auch:
Striche 230.

Strick 25, $23 \mathrm{I}$.

Szepter 39, 199 .

Tafel 6, 7, 8, 9, 19, 26, $29,32,33,36,37$, $38,40,4 \mathrm{I}, 46,48$, 49 .

Thyrsos 35 .

Topf I6I, I75, 23I (siehe auch: Vase).

Trompete 63 .

Turm (siehe auch: Leuchtturm) 58, 99; ror, I45, 22I, 224, 252, 262.

Turnierbalken $232, \quad 235$, 240.

Vase 275 (siehe auch: Topf).

Verzierungen 198 (siehe auch: Chinesische Ornamentik).

Vogel (siehe auch: Adler, Gans, Storch, Fasan, Hahn) 4I, II 5, I45, I 8I, I99, 230 .

Waffen 48.

Wappen $45,50,52,55$, $57,58,59,6 \mathrm{r}, 69$, 70,85 , II 7 , I 24, I $4 \mathrm{I}$, I43, I45, I60, 20I, 203, 204, 205, 206, 2I3, 2I4, 2I5, 2I6, $218,219,222,247$, $248,259$.

Weintraube $122,212$.

Wellenlinien 222, 223 .

Windmühle 240 .

Zirkel 23r.

Zweig (Zweige) 25, 26, $44,45,55,86$, I $4 \mathrm{I}$, $230,232$.

Zwiebel I94. 


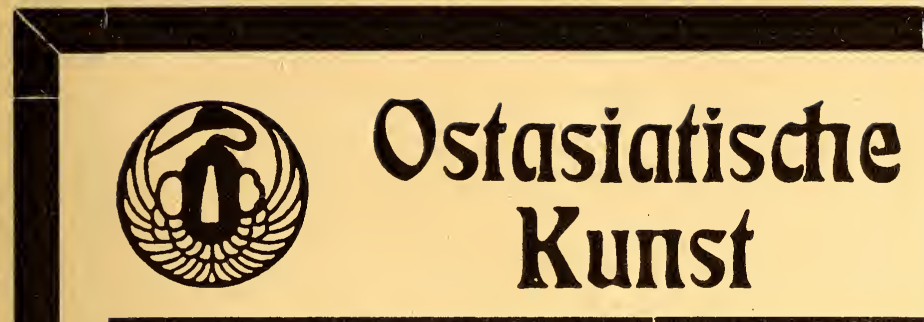

\section{Japan}

\section{China}

Persien

Grobe Sammlung japanischer Fayencen, Bronzen, lacke, Stoffe usw. - Herdorragende chinesische Porzellane, Arbeiz ten in Stein, Kristall usw. usळ.

Persische Fayencen - syrische Bläser

R.Wagner, Berlin Wo Potsdamer Straßze 20a 


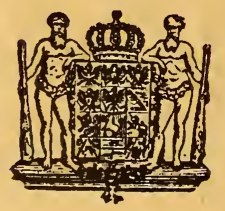

Hoflieferant

Sr. Majestät des Kaisers und Königs

\title{
Alte China-Porzellane,
}

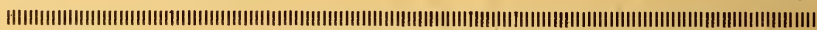

\section{Ausgrabungen}

\section{ANTIQUITÄTEN ALLER ART:}

Bronzen, Stoffe, Holzschnitzereien, Möbel, Elfenbein-Schnitzereien, Stichblätter, Lacke

\author{
Antike Möbel
}

_ Gobelins und alte Stickereien

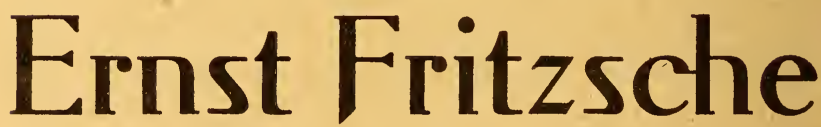

BERLIN W 8, Wilhelmstraße 49 Telephon: Centr. 1189 

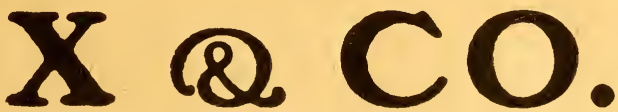

Gegründet 1854

BERLIN W 8

Mohrenitraße 7-8 (Rex-Haus)

Große Auswahl alter CHINA-

PORZELLANE der verfchiedenften Epochen

Keramifche Ausgrabungen der Han-, Tang- und SungDynaftien

Alte japanifche Keramiken

Alle Arten

chinefifcher und japanifcher Kunftarbeiten und Antiquitäten

Auskunft und Anfichtsfendungen bereitwilligit.

Bei Anwefenheit in Berlin Befuch erbeten. 


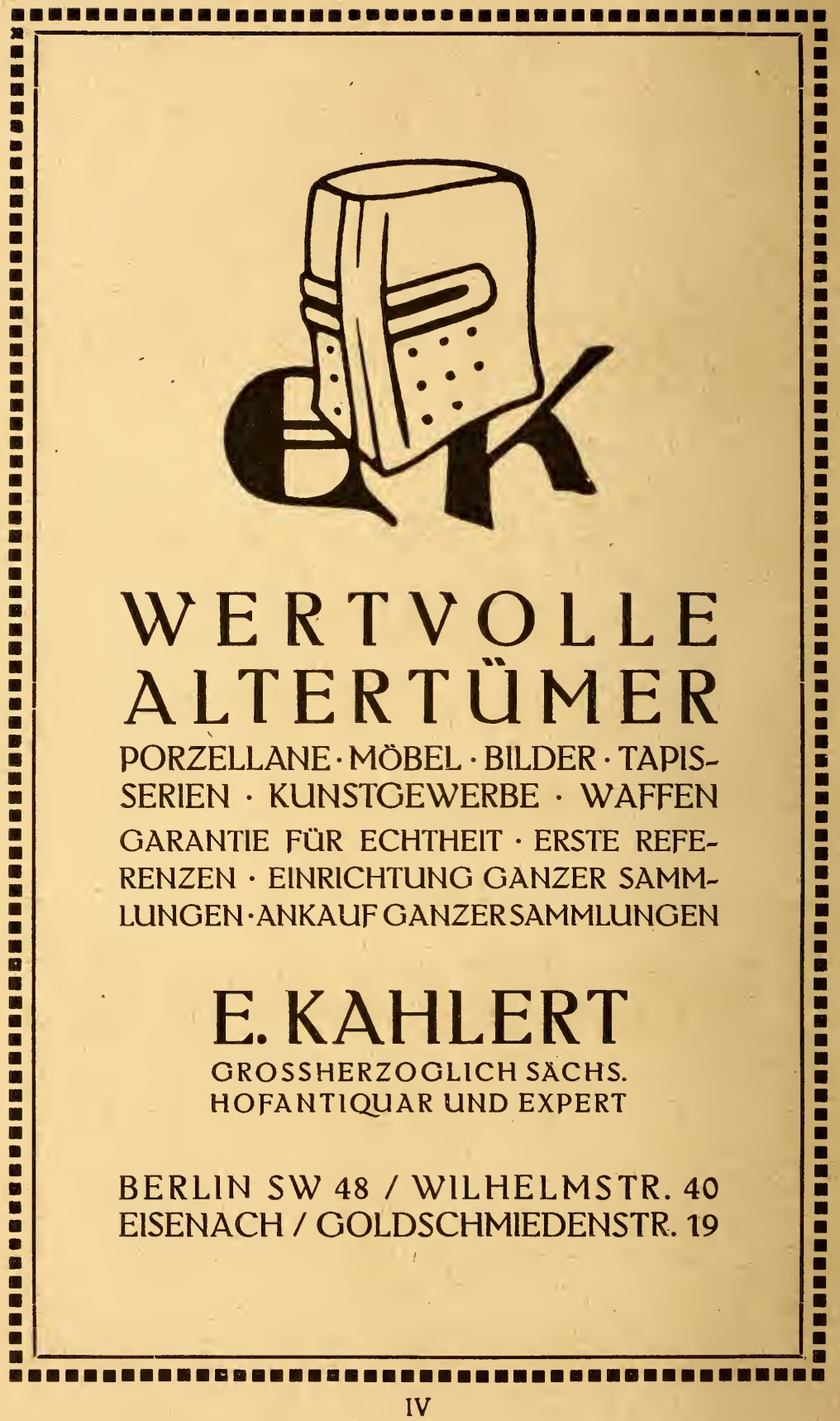




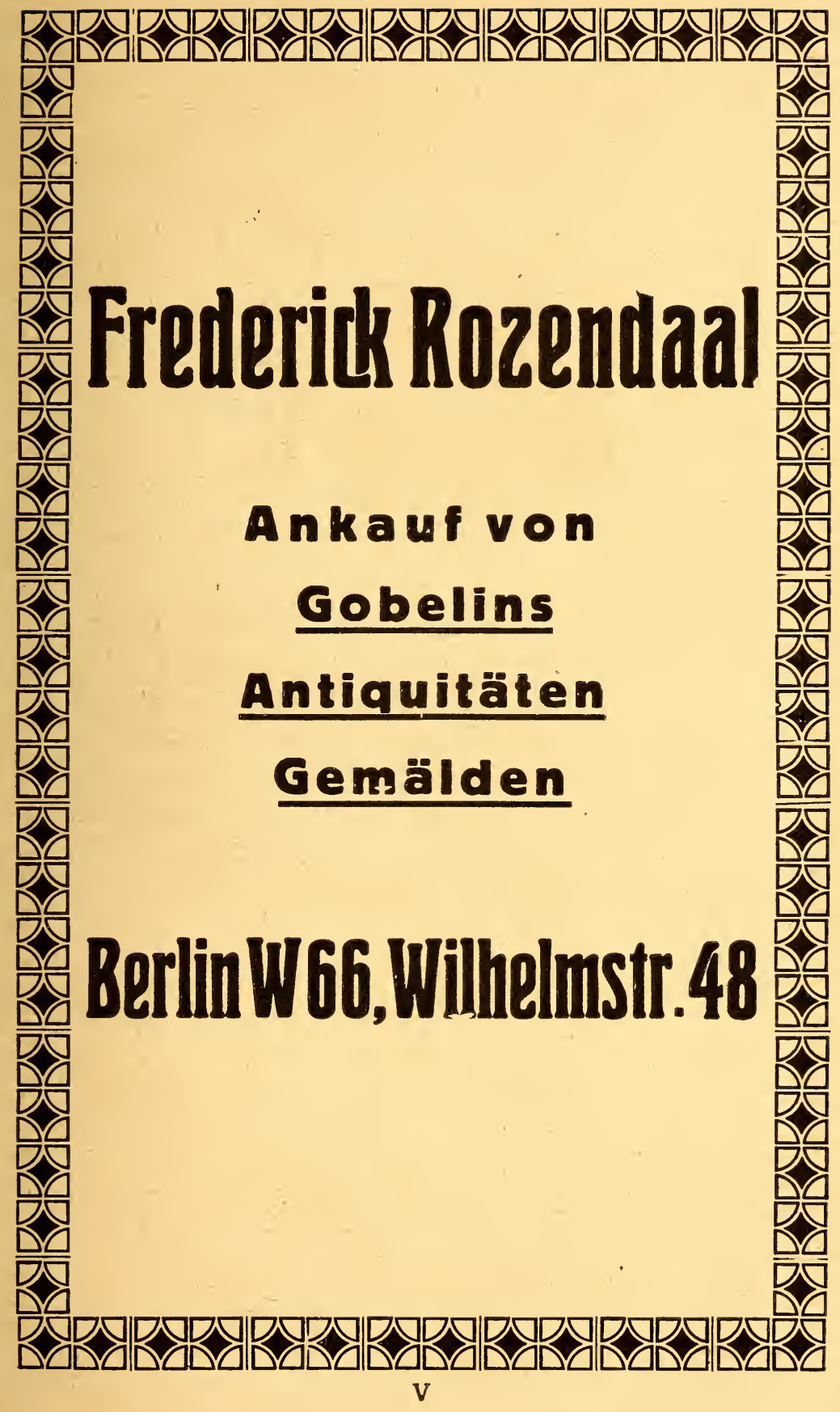




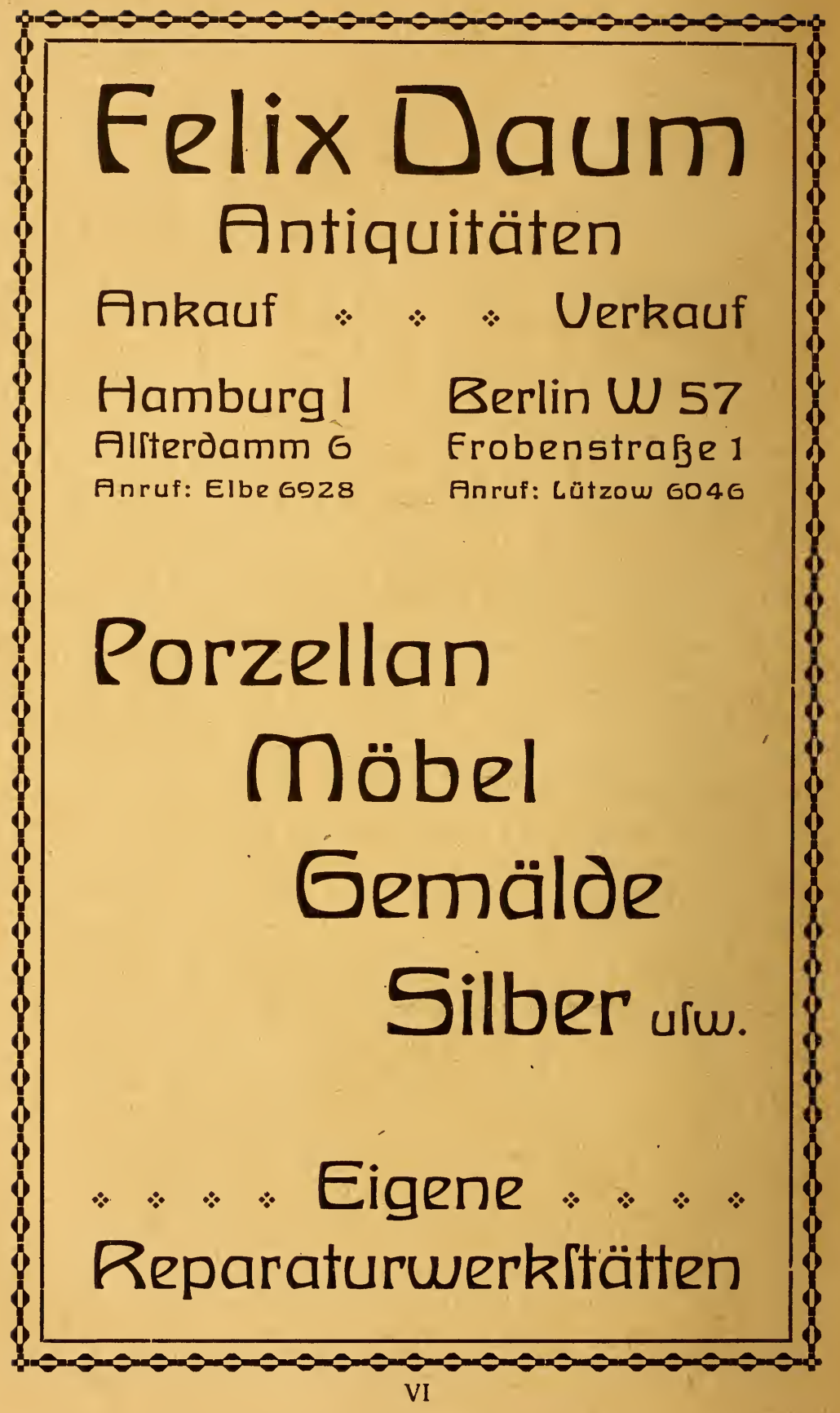




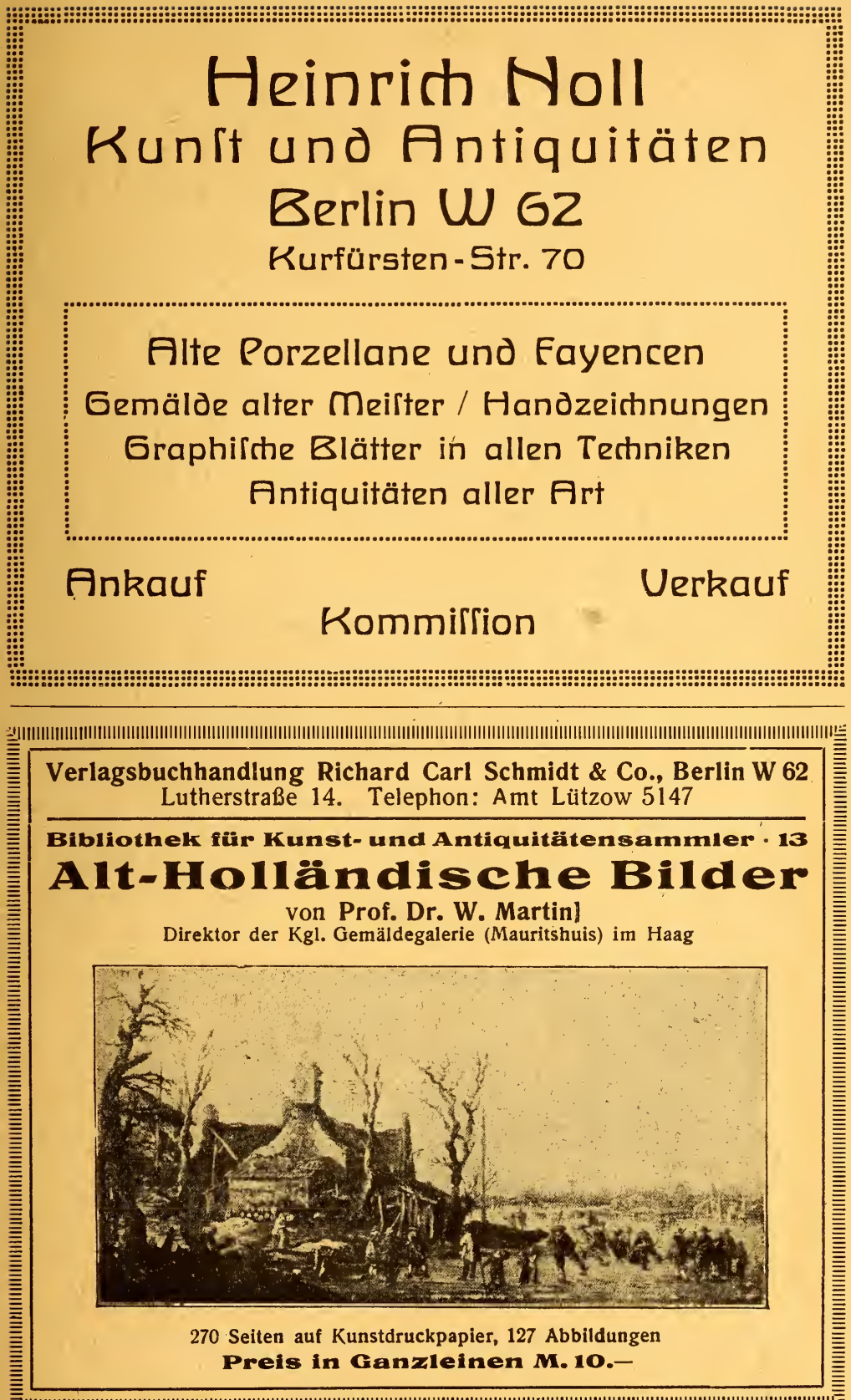

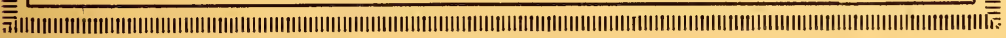
VII 
Theodoor Rozendaal

$$
\text { Berlin W } 50
$$

Kurfürstendamm 242

Gegenüber dem Zoolog. Garten

$$
\text { Antiquitäten }
$$




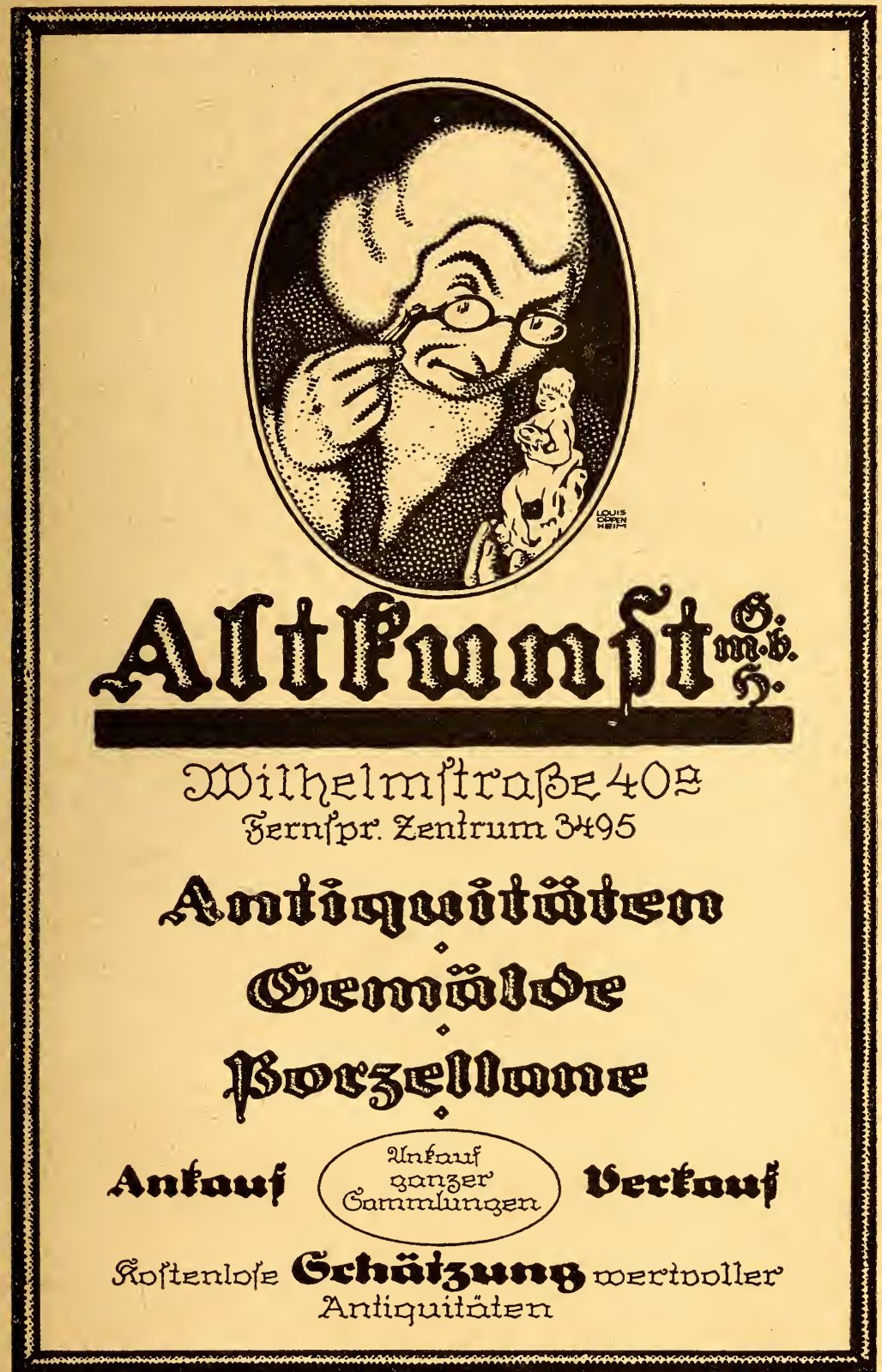




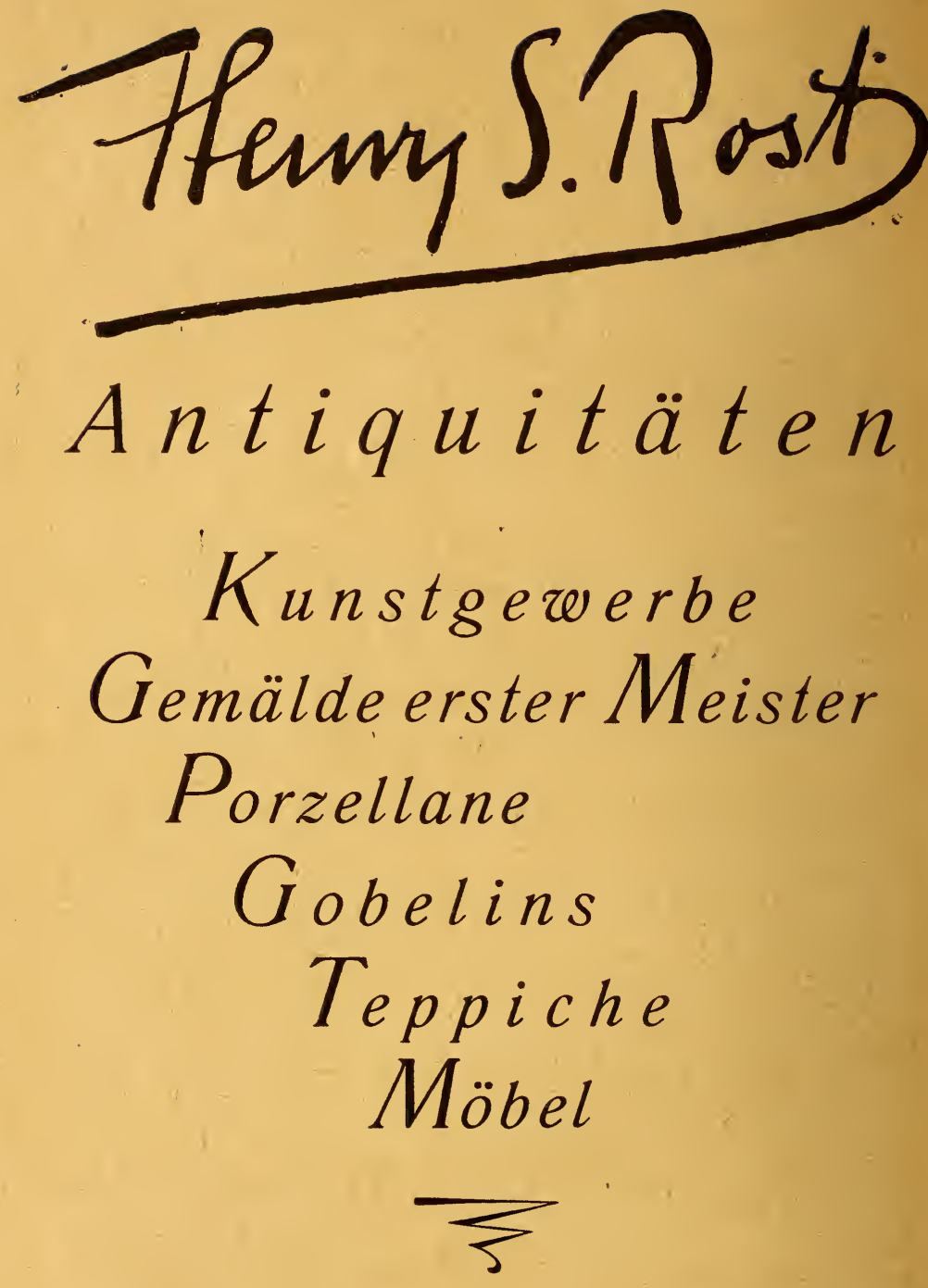

Berlin W, Nettelbeckstraße 20 Nollendorf 3511 


\section{EDMUND MEYER}

Buchhändler und Antiquar

BERLIN W 35, Potsdamer Straße 27 ${ }^{\text {b }}$

LUXUSDRUCKE

Leder - und Luxuseinbände / Moderne Erstausgaben / Privatpressen / lllustrierte Bücher des XV. $-X X$. lahrh. / Bibliophile Seltenheiten jeder Art / Kunstwissenschaftliche Werke usw. in deutscher, engl., franz. Sprache / Graphik Kataloge auf Wunsch Ankauf einzelner Werke und ganzer Bibliotheken

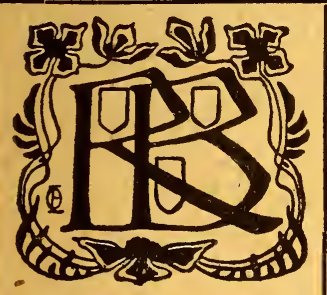

RUDOLF BANGEL'S KUNST-AUKTIONS-HAUS FRANKFURT A.M./NEUE BÖRSE 00000000000000000000000000 GEGRÜNDET 1869

Beeidigte Sachverftändige für die Gerichte des Landgerichtbezirksu. für das OberlandesgerichtFrankfurta.M.

Vorteilhafte Verwertung von Gemälden aller Schulen, Antiquitäten und Kunftgewerbe

Bereits gegen 1000 Kataloge erfchienen - Auflage 1000 bis 2000 


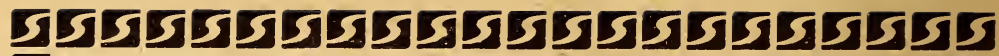

\section{5}

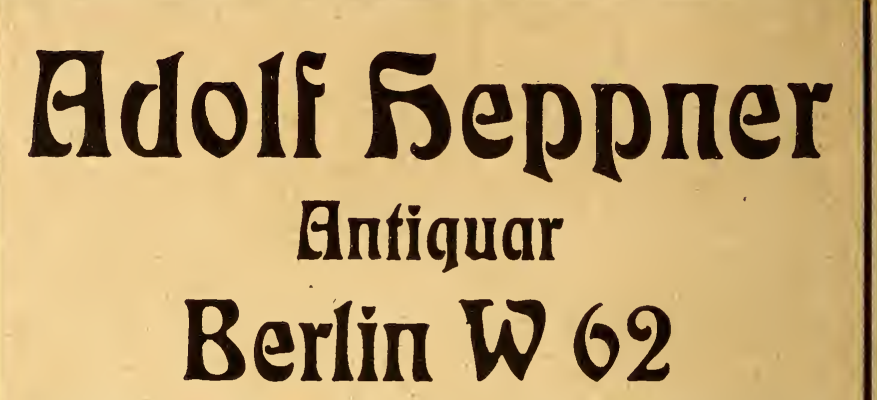

Kurfürítenítraße 98, am Zoo • Fernruf Stpl.6877

(c)

Seltenheiten iür Sammler und lsiebhaber:

Porzellane Fayencen

Feine alte möbel

Kupferifictie . . Silber

(5)

Bnkauí guter Stücke N

Fachmänniiche Beratung

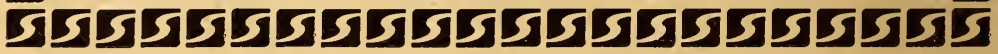
XII 


\section{RUDOLPH LEPKE'S KUNST-AUCTIONS-HAUS}

BERLIN W 35, POTSDAMER STR. 122 A/B

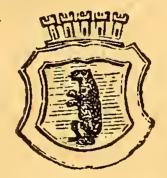

Vom September bis Mai finden Versteigerungen von Gemälden alter und neuer Meister, Kupferstichen, Antiquitäten, Möbeln und Kunstgegenständen aller Art statt

Die Kataloge werden auf Verlangen zugesandt

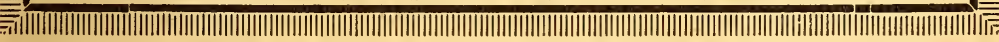

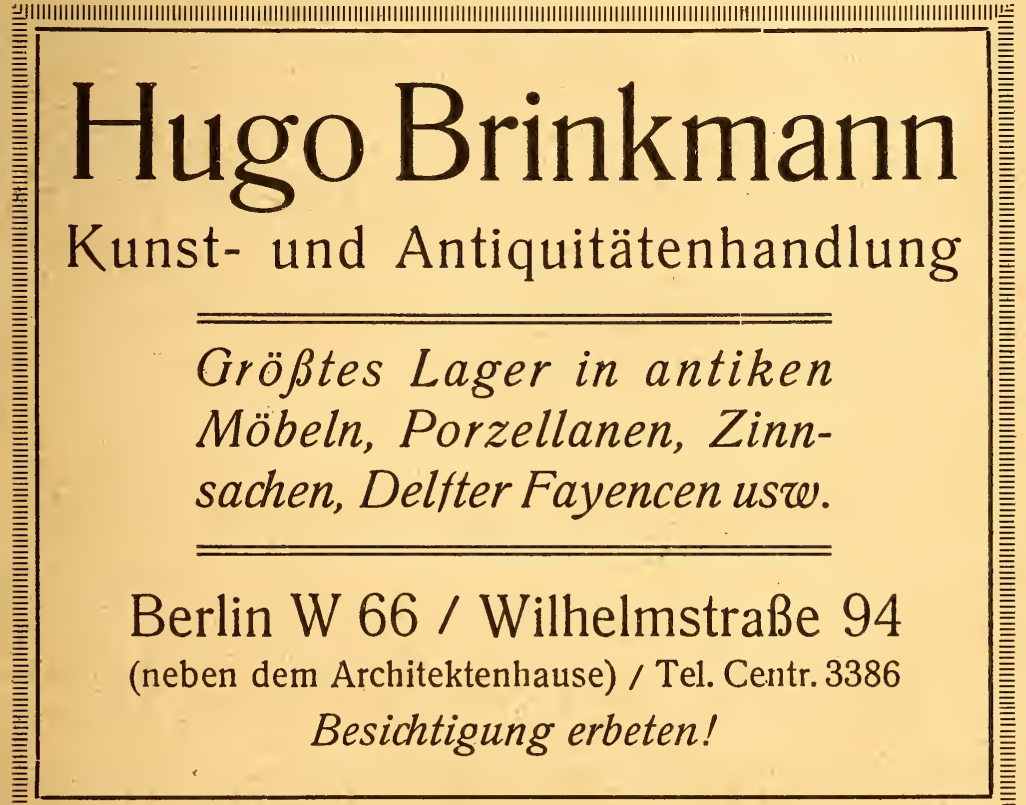

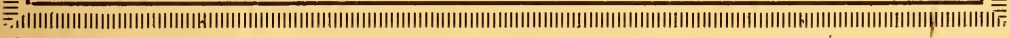




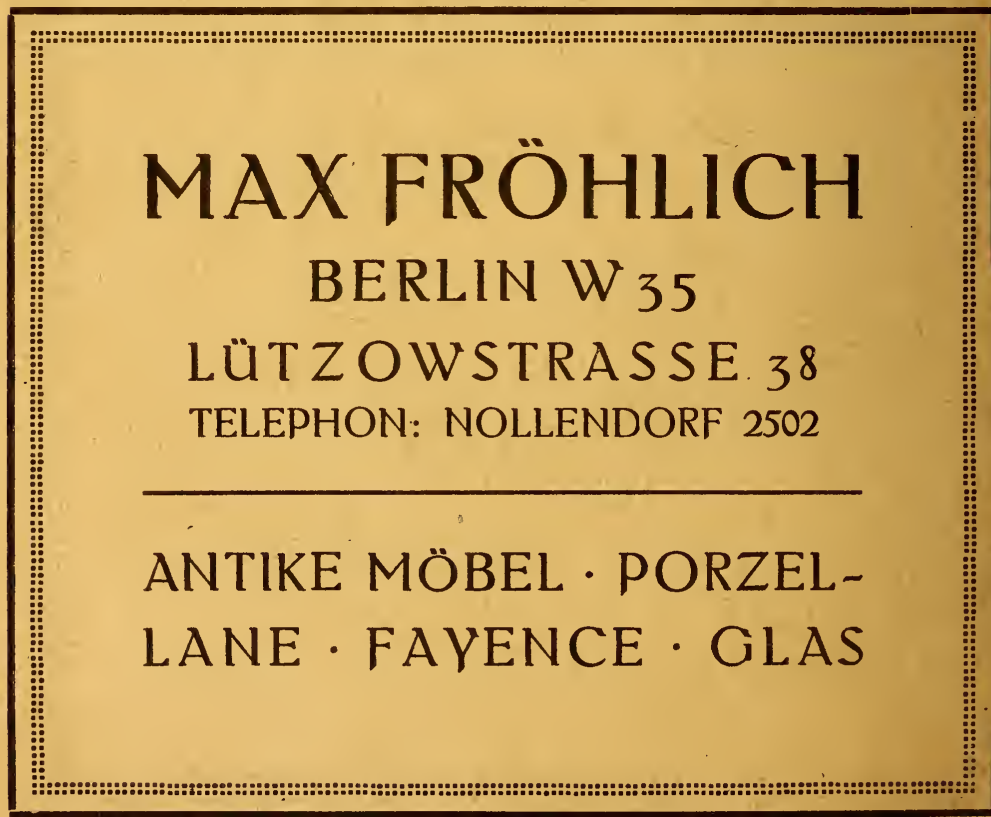




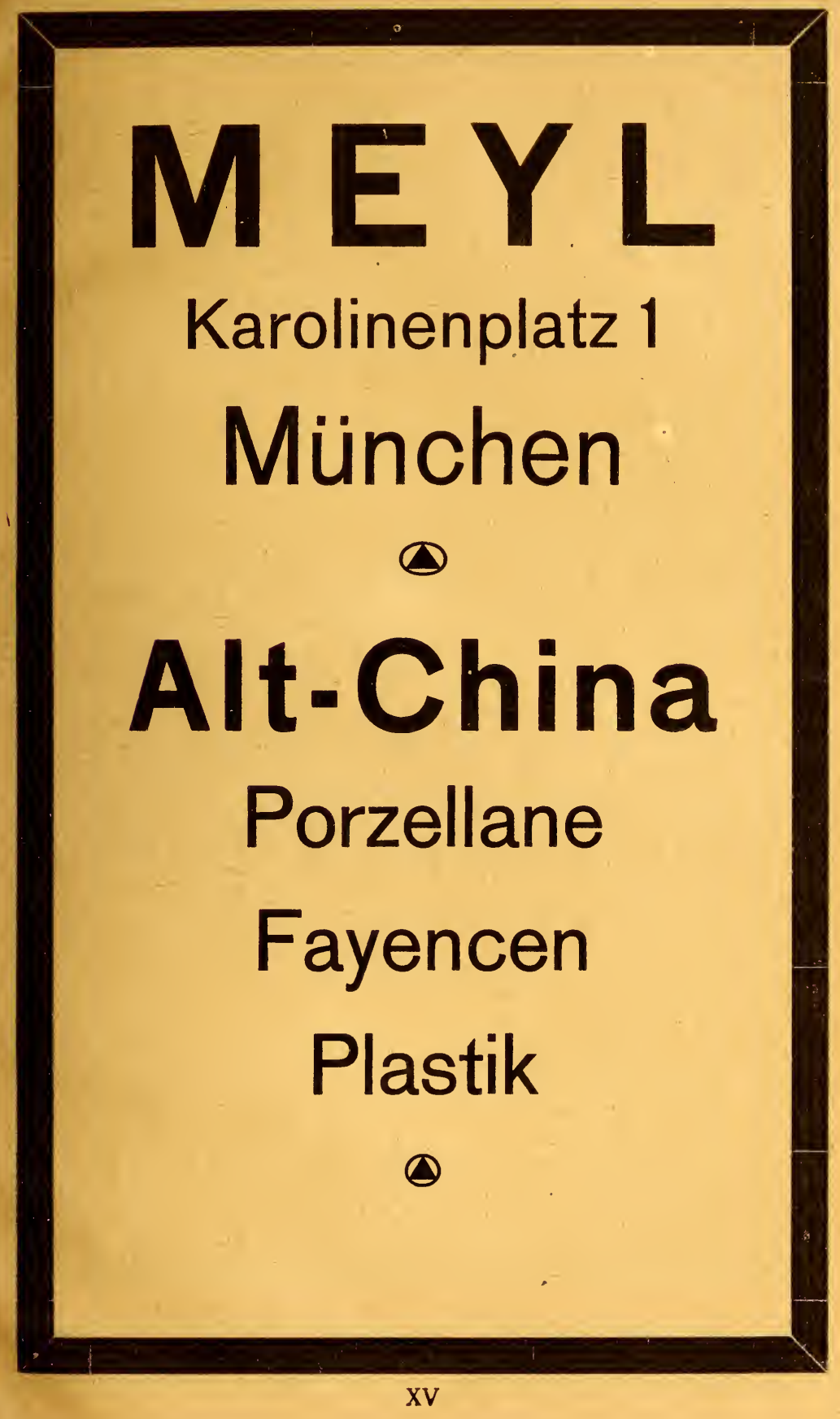




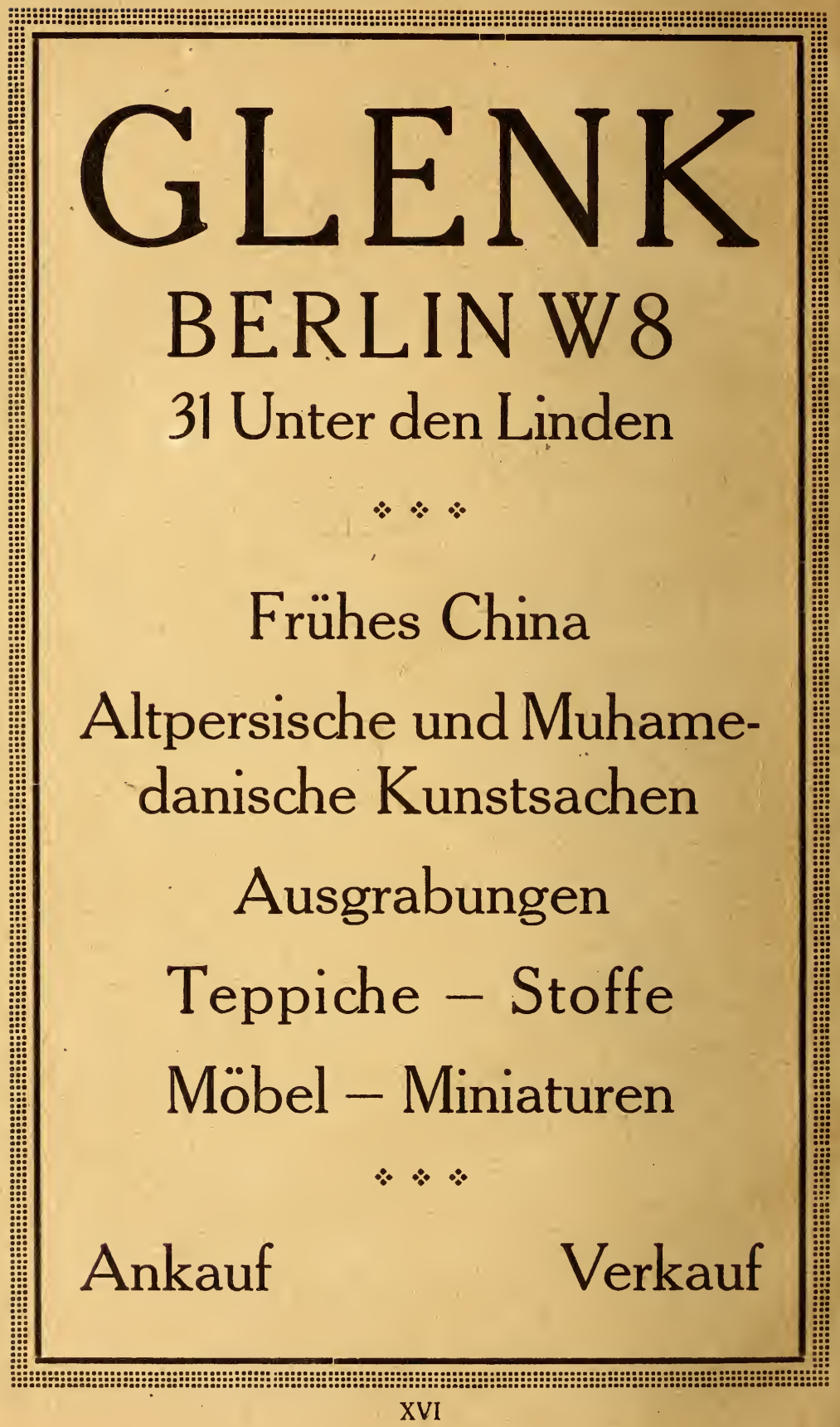




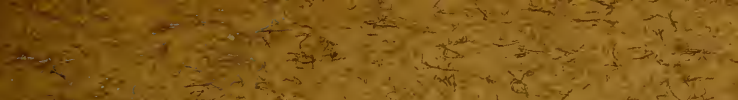

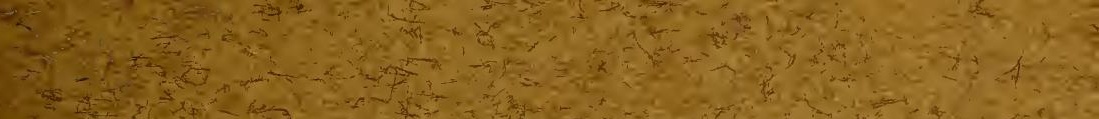

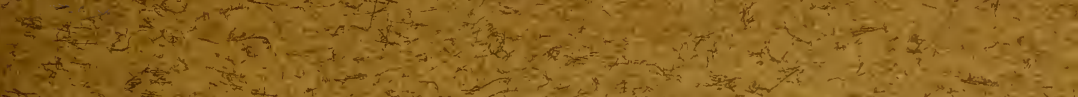

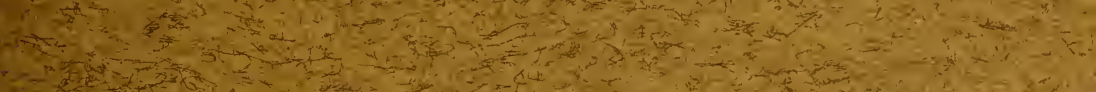

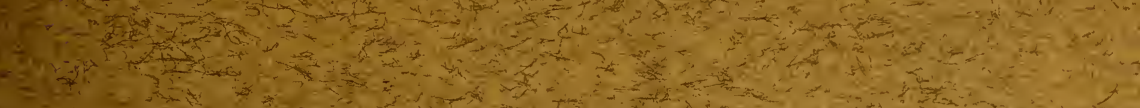

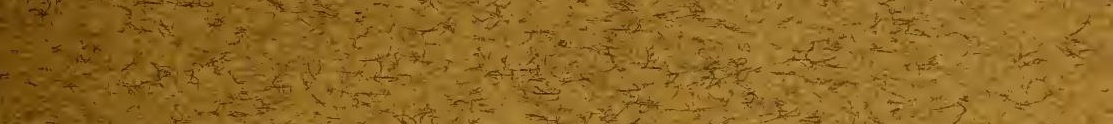

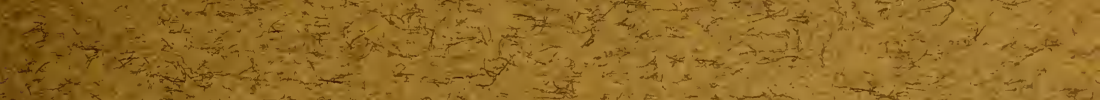
E

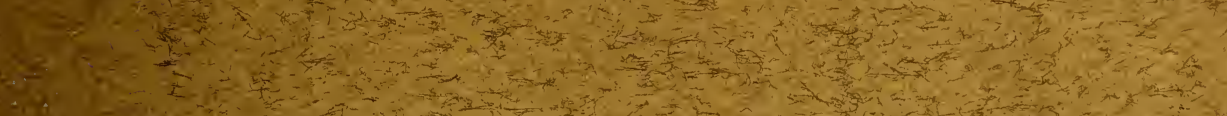

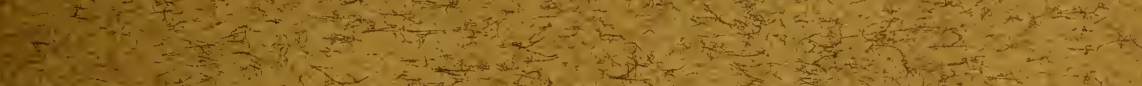

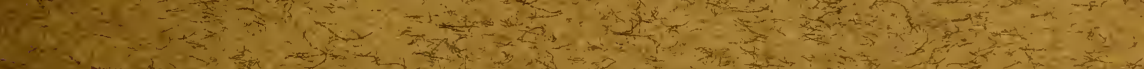

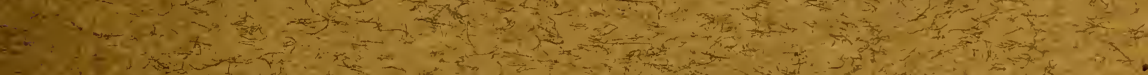

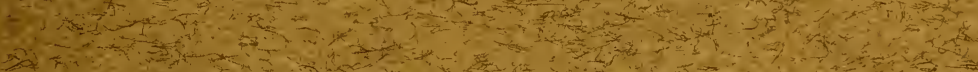

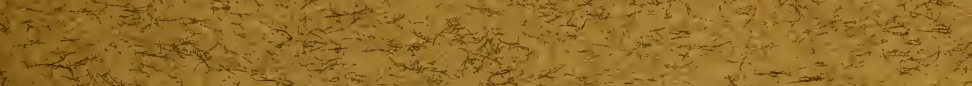

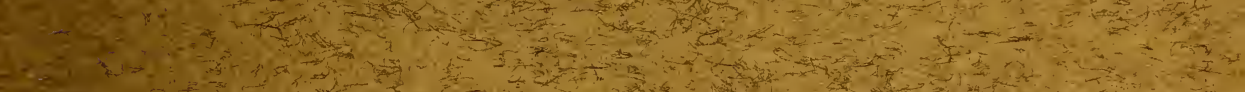

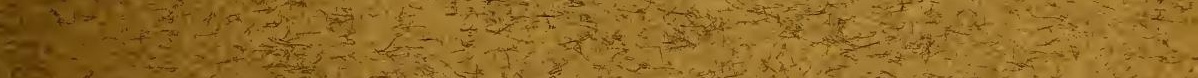

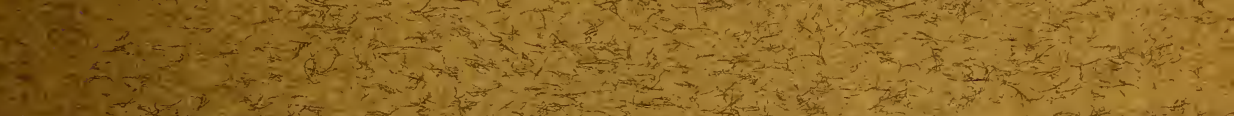

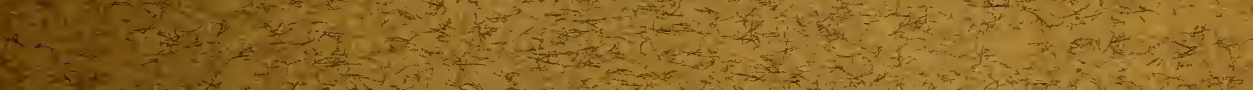

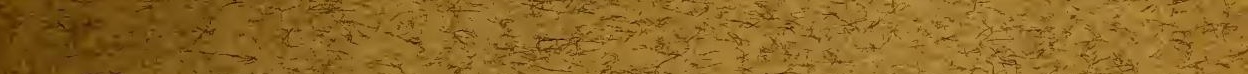

5

(3)

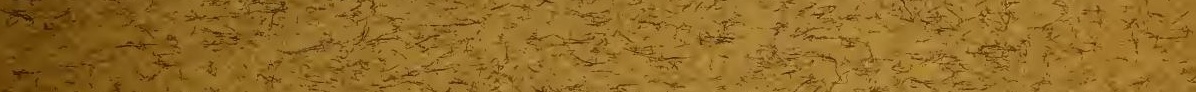

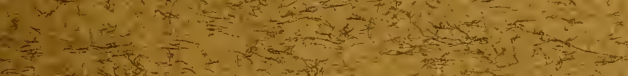

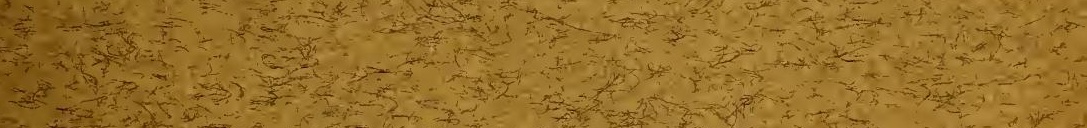

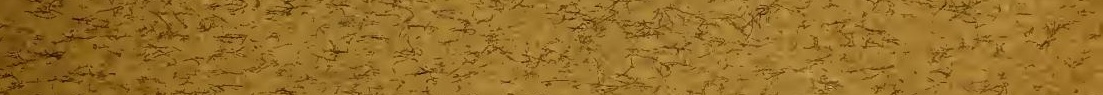

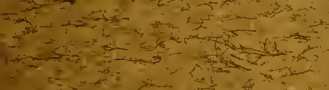

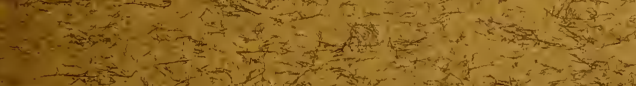

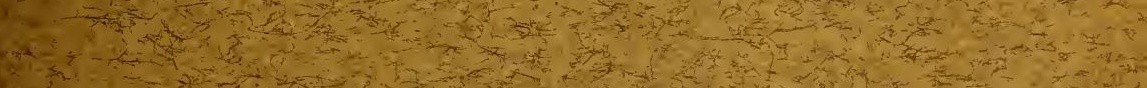

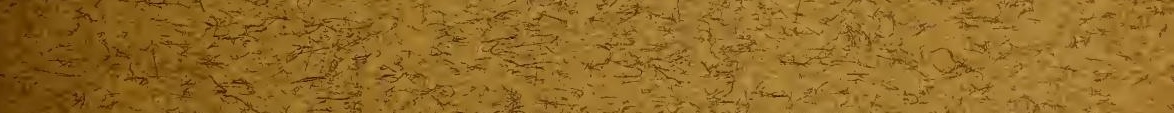

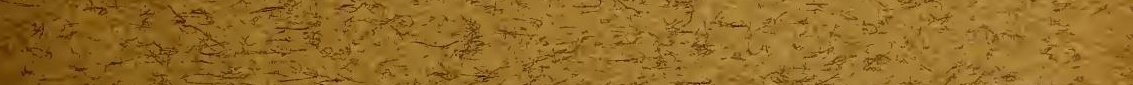

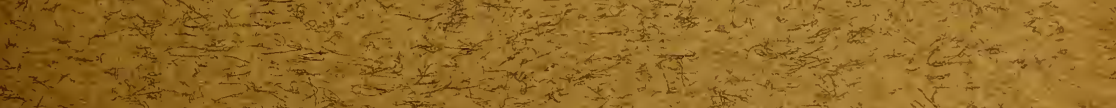

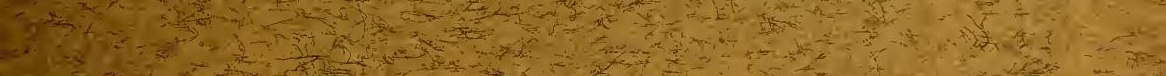

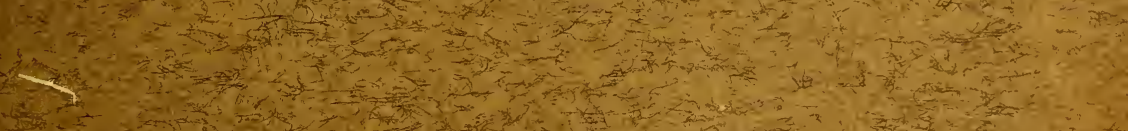

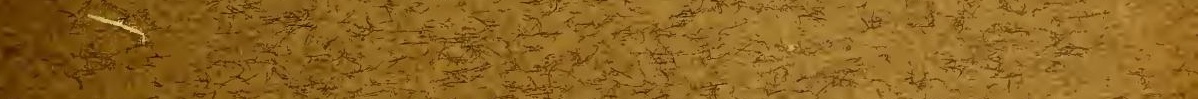

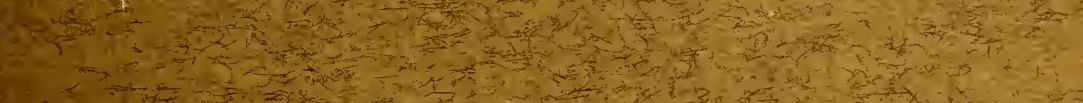

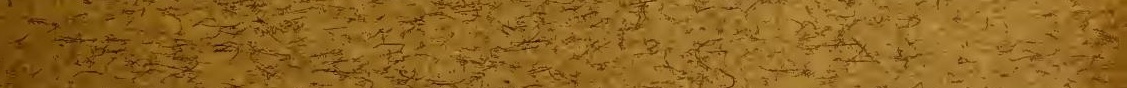
misher 


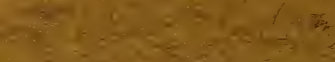

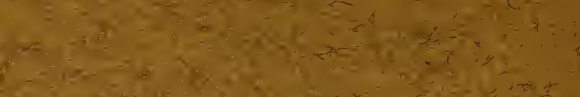

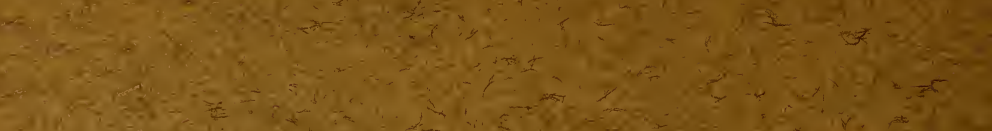

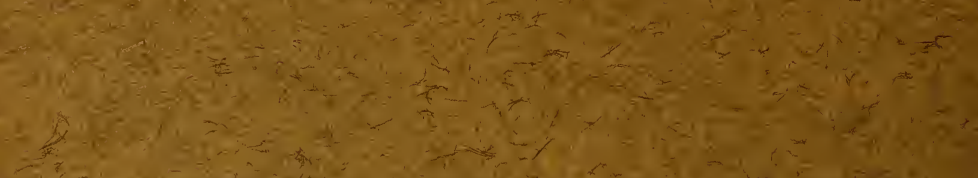

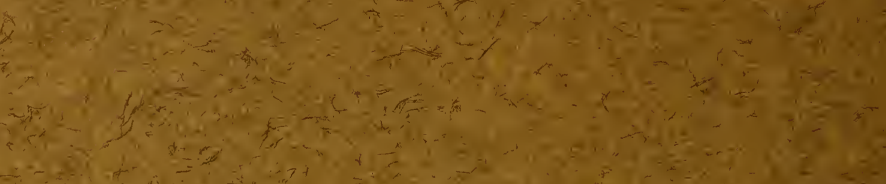

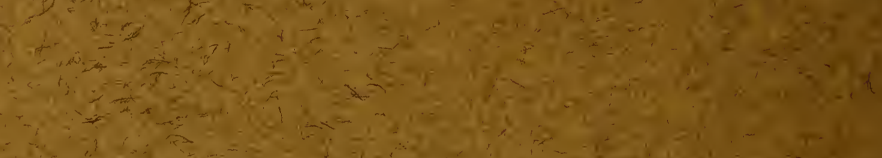
tents

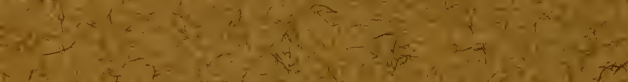

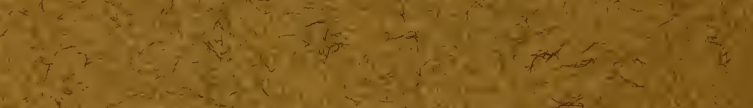

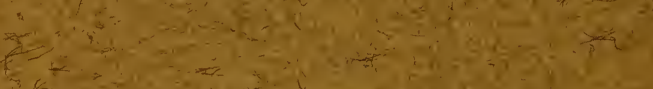
(i)

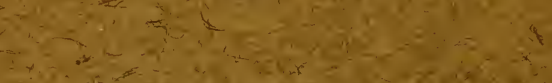

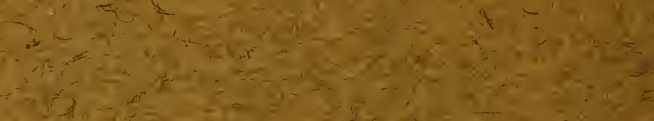

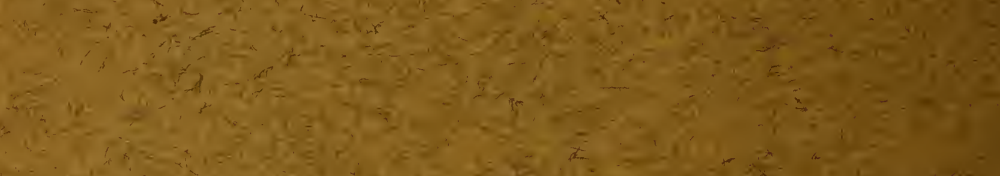

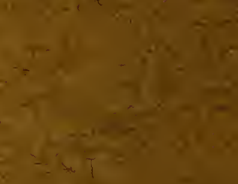
2.0.

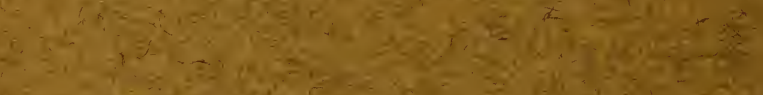

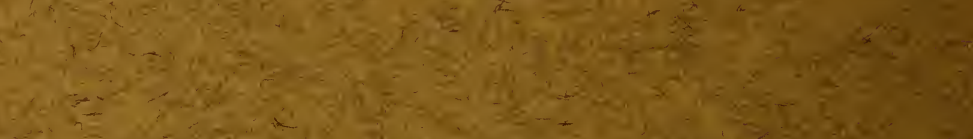

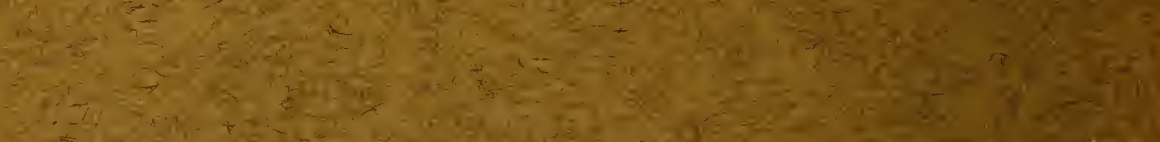

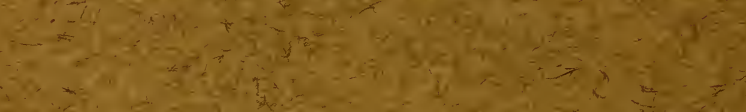

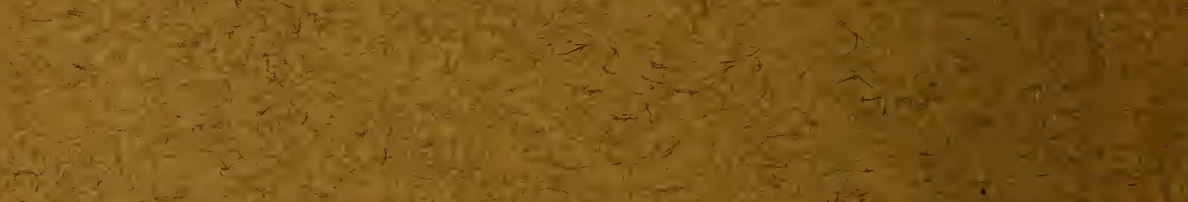

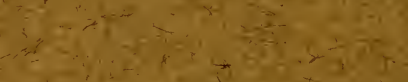

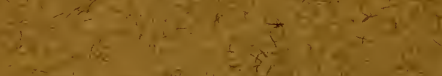

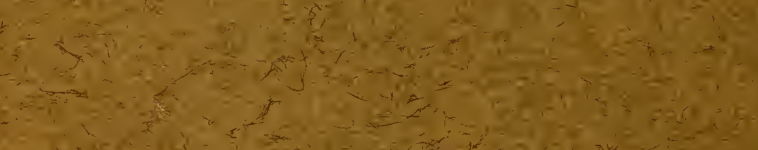

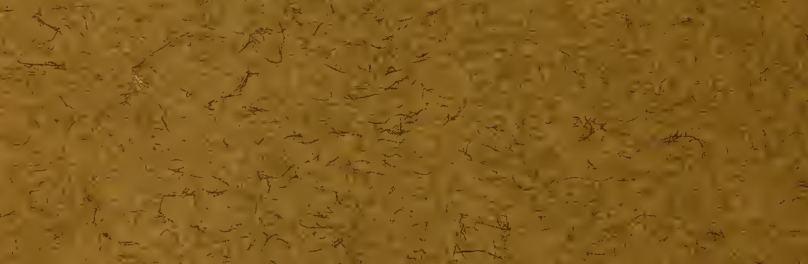

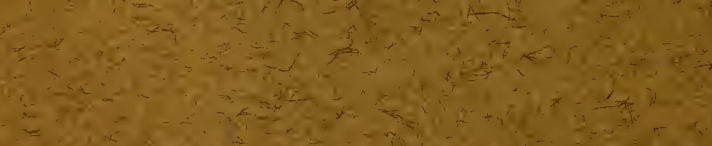
$\therefore \quad+7$

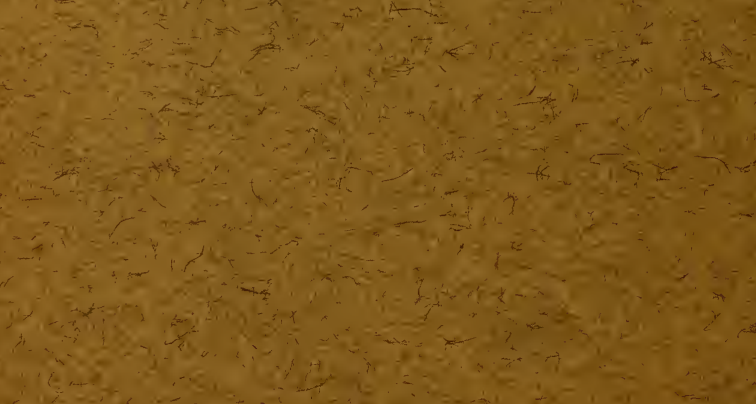





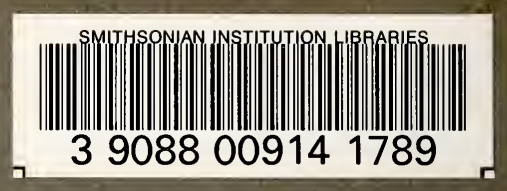

